\title{
Precipitation Assessment and Hydrological Implications of Climate Change in the High-Altitude Indus Basin
}

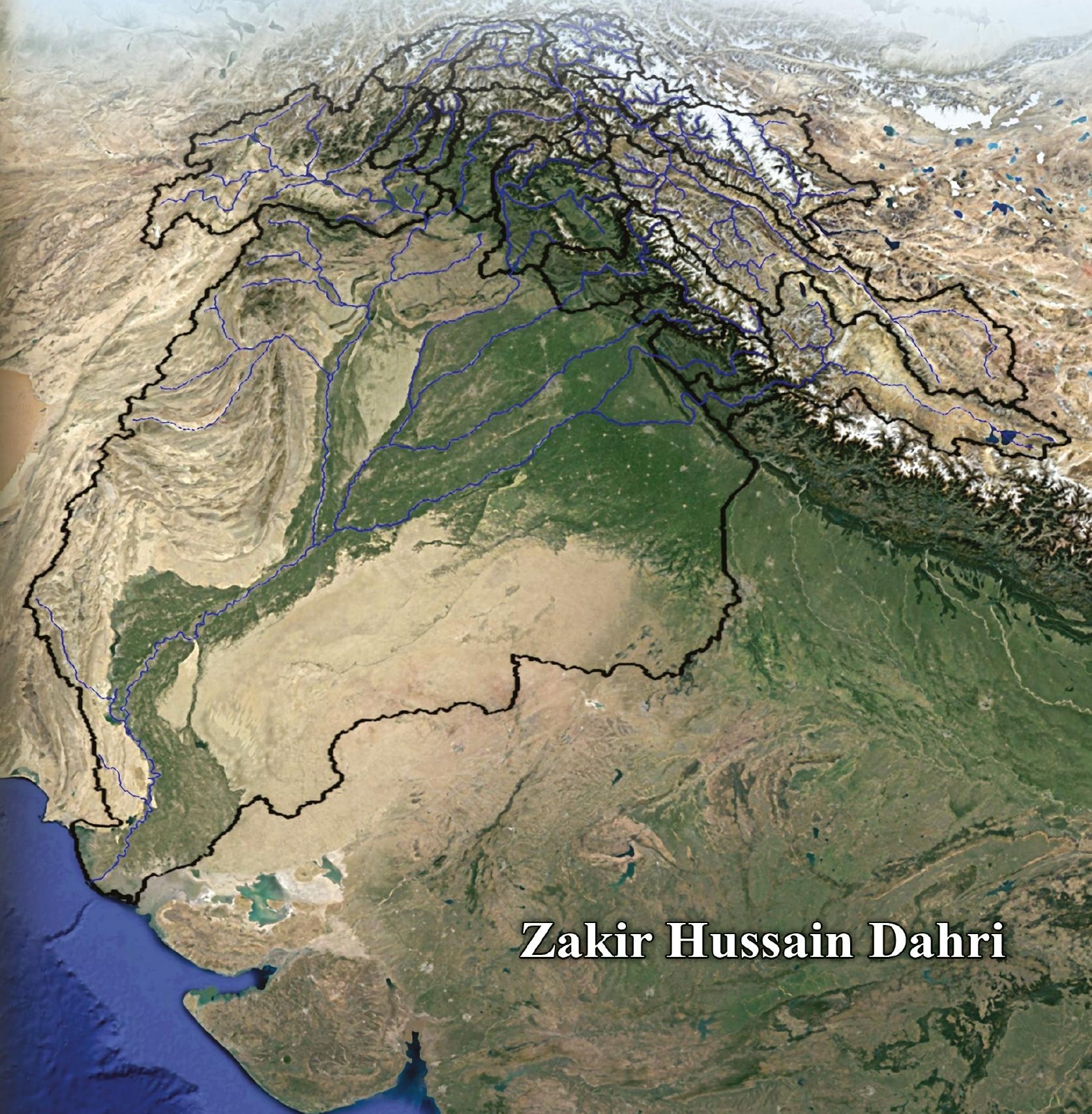




\section{Propositions}

1. Improvements in meteorological forcing datasets are more important than improved analysis methods for accurate analysis of climate change and hydrological implications in the Indus basin.

(this thesis)

2. Contrasting climate change and hydrological signals across the highaltitude Indus basin require significant modifications in the strategies and action plans for optimal river basin management. (this thesis)

3. Propagating myths like yoga practice, cow urine or a eucalyptus neckless to fight COVID-19 is hampering healthcare workers to contain the virus.

4. Adoption of low-delta crops and high-efficiency irrigation systems is triggered by the potential water savings but ignoring the governance, economic and technological aspects.

5. Patients in the Netherlands really need to be patient.

6. A sandwich $\mathrm{PhD}$ student is really a sandwich between university, employing organization and family.

Propositions belonging to the thesis, entitled:

Precipitation Assessment and Hydrological Implications of Climate Change in the High-Altitude Indus Basin

Zakir Hussain Dahri

Wageningen, 13 October 2020 


\section{Precipitation Assessment and Hydrological Implications of Climate Change in the High-Altitude Indus Basin}




\section{Thesis Committee}

\section{Promotors}

Prof. Dr P. Kabat

Special Professor, Global Water and Climate Systems,

Wageningen University \& Research

Chief Scientist and Director Research,

World Meteorological Organization (WMO), Geneva, Switzerland

Prof. Dr F. Ludwig

Professor, Water and Climate Change, Wageningen University \& Research

\section{Co-promotor}

Prof. Dr E.J. Moors

Rector, IHE Delft Institute for Water Education

Professor, Water and Climate, VU University Amsterdam

\section{Other members}

Prof. Dr R. Uijlenhoet, Wageningen University \& Research

Prof. Dr N. van de Giesen, TU Delft

Prof. Dr W.W. Immerzeel, Utrecht University

Dr N. Forsythe, Newcastle University, UK

This research was conducted under the auspices of the Graduate School for Socio-Economic and Natural Sciences of Environment (SENSE). 


\title{
Precipitation Assessment and Hydrological Implications of Climate Change in the High-Altitude Indus Basin
}

\author{
Zakir Hussain Dahri
}

\section{Thesis}

Submitted in fulfilment of the requirements for the degree of doctor

at Wageningen University

by the authority of Rector Magnificus,

Prof. Dr A.P.J. Mol,

in the presence of the

Thesis Committee appointed by the Academic Board

to be defended in public

on Tuesday 13 October 2020

at 4 p.m. in the Aula. 
Zakir Hussain Dahri

Precipitation Assessment and Hydrological Implications of Climate Change in the High-Altitude Indus Basin, 176 pages.

$\mathrm{PhD}$ thesis, Wageningen University, Wageningen, the Netherlands (2020)

With references, with summary in English

ISBN: $\quad 978-94-6395-538-6$

DOI: https://doi.org/10.18174/530921 


\begin{abstract}
In this $\mathrm{PhD}$ dissertation, the direct measurements of precipitation from different sources are integrated with the indirect estimates of precipitation at the major glacier zones to appraise spatial and altitudinal distribution of precipitation in the high-altitude Indus basin. These data are further adjusted for measurement errors and high-quality reference data of spatially distributed precipitation is developed to reconcile precipitation distribution. The reference data of temperature are developed using elevation and latitude dependent regression models. Performance of 27 widely used gridded precipitation products is evaluated at subregional scale. The best performing product is bias-corrected using the reference data of precipitation and temperature. Similarly, precipitation estimates of 75 GCM outputs are evaluated and two best performing GCMs representing warm-wet and cold-dry extremes under three RCPs $(2.6,4.5 \& 8.5)$ are bias-corrected. The historical and future datasets developed therein are analysed to detect climate change and variability at sub-regional scale. A fully-distributed physically-based energy-balance Variable Infiltration Capacity (VIC) hydrological model is forced with these datasets to simulate the hydrologic regime of the study area at sub-basin scale. River inflows are analysed for change and variability in water availability, shifts in seasonality and annual cycle of river water, and changes in future hydrological extremes at Kabul-Nowshera, Indus-Tarbela, Jhelum-Mangla and Chenab-Marala rim stations.
\end{abstract}

The results indicated an increase of $0.6{ }^{\circ} \mathrm{C}$ in the median annual air temperature and $11.9 \%$ decline in median annual precipitation during the last 40 years. The corresponding changes in river inflows remained highly variable but consistently declined. Indus-Tarbela, JhelumMangla, Chenab-Marala and Kabul-Nowshera rivers inflows experienced 4.9\%, 19.6\%, $11.9 \%$ and $4.5 \%$ decline respectively. Future projections however show gradual increase in temperature but highly variable precipitation indicating increasing trends in Karakoram and parts of Kharmong and W-Hindukush regions and declining trends in the remaining areas. Future river inflows are likely to increase between 17.0-73.6\% at Indus-Tarbela river gauge under wet-warm scenarios and between $1.2-9.7 \%$ under cold-dry scenarios, while river inflows at the remaining three rim stations show increases for wet-warm and decreases for cold-dry scenarios. Most of the ensembles show increasing high flows and decreasing low flows at all gauging stations implying intensification of future hydrological extremes of floods and droughts. The contrasting climate change and hydrological signals across the river basin require important modifications in the strategies and action plans for river basin management. 


\section{Contents}

1 Introduction 1

1.1 Background and Problem Statement 1

1.2 Climate and Hydrological Regime of the High-Altitude Indus Basin 3

1.3 Hydrological Modelling 5

1.4 Research Questions and Study Objectives 7

1.5 Thesis Outline and Methods 8

2 An Appraisal of Precipitation Distribution 11

2.1 Introduction 12

2.2 Study Area 14

$\begin{array}{lll}2.3 & \text { Data and Methods } & 15\end{array}$

$\begin{array}{lll}2.3 .1 & \text { Station based point observations } & 15\end{array}$

$\begin{array}{lll}\text { 2.3.2 Gridded datasets } & 17\end{array}$

\begin{tabular}{ll}
2.3 .3 & River flow data \\
\hline
\end{tabular}

$\begin{array}{lll}2.3 .4 & \text { Methods } & 18\end{array}$

2.3.5 Uncertainty analysis 20

2.4 Results 22

2.4.1 Altitudinal variation of precipitation 22

2.4.2 Spatial interpolation of precipitation observations 22

2.4.3 Validation of KED-based estimated precipitation 26

2.4.4 Evaluation of the gridded precipitation products 28

2.5 Discussion 31

2.6 Conclusions 33

$3 \quad$ Adjustment of Measurement Errors to Reconcile Precipitation 35

Distribution

3.1 Introduction 36

2.2 Study Area 38

3.3 Data and Methods 39

3.3.1 Precipitation observations $\quad 39$

3.3.2 Temperature and wind speed observations 40

3.3.3 River flows 40

3.3.4 Precipitation measurement error adjustment methods 41

3.3.5 Adjustment of net snow accumulations 42

3.3.6 River flow adjustments 43

3.3.7 Spatial interpolation $\quad 44$

3.3.8 Cross validation of corrected precipitation 44

3.4 Results 45

3.4.1 Precipitation adjustments 45 
3.4.2 Snow accumulation adjustments $\quad 47$

3.4.3 Spatial distribution of unadjusted and adjusted precipitation $\quad 48$

$\begin{array}{lll}3.4 .4 & \text { River runoff adjustment } & 48\end{array}$

$\begin{array}{lll}3.4 .5 & \text { Validation of precipitation estimates } & 49\end{array}$

3.5 Discussion 51

3.6 Conclusions $\quad 55$

$4 \quad$ Evaluation of Gridded Precipitation Products 57

4.1 Introduction 58

$\begin{array}{lll}4.2 & \text { Data and Methods } & 61\end{array}$

$\begin{array}{lll}\text { 4.2.1 Reference dataset } & 61\end{array}$

$\begin{array}{lll}\text { 4.2.2 Gridded precipitation products } & 61\end{array}$

$\begin{array}{lll}4.2 .3 & \text { River flows } & 61\end{array}$

4.2.4 Potential evapotranspiration $\quad 62$

$\begin{array}{lll}\text { 4.2.5 Evaluation approach } & 62\end{array}$

4.3 Results 65

4.3.1 Spatial distribution of mean annual precipitation \& residual 65

$\begin{array}{lll}\text { 4.3.2 } & \text { Residual errors } & 67\end{array}$

$\begin{array}{lll}4.3 .3 & \text { KGE scores } & 69\end{array}$

$\begin{array}{lll}\text { 4.3.4 Annual cycle of monthly means } & 69\end{array}$

$\begin{array}{lll}\text { 4.3.5 Comparison and cross validation against adjusted streamflows } & 72\end{array}$

$\begin{array}{lll}\text { 4.3.6 Skill scores and rankings } & 74\end{array}$

$\begin{array}{lll}4.4 & \text { Discussion } & 75\end{array}$

$\begin{array}{lll}4.5 & \text { Conclusions } & 78\end{array}$

5 Climate Change and Hydrological Regime under Extreme Climate 79 Scenarios

$\begin{array}{lll}5.1 & \text { Introduction } & 80\end{array}$

5.2 The Indus River Basin $\quad 82$

$\begin{array}{lll}5.3 & \text { Data and Methods } & 82\end{array}$

5.3.1 Historical climate data $\quad 82$

$\begin{array}{lll}\text { 5.3.2 Future climate projections } & 84\end{array}$

5.3.3 Description, setup and performance of VIC hydrological model 87

$\begin{array}{ll}\text { 5.3.4 Methods for analysing climate and hydrological changes } & 89\end{array}$

$\begin{array}{lll}5.4 & \text { Results } & 89\end{array}$

$\begin{array}{lll}\text { 5.4.1 Climate change and variability } & 89\end{array}$

5.4.2 Performance of the VIC hydrological model 93

5.4.2 Changes and variability in overall water availability 95

5.4.3 Shifts in seasonality and annual cycle of water availability 97

5.4.4 Changes in future hydrological extremes 99

$\begin{array}{ll}5.5 \text { Discussion } & 100\end{array}$ 
5.5.1 Climate change 100

5.5.2 Hydrological regime 102

5.5.3 Uncertainties and limitations 103

5.5.4 Implications for water management 105

$\begin{array}{lll}5.6 & \text { Conclusions } & 105\end{array}$

6 Synthesis 107

$\begin{array}{lll}6.1 & \text { Introduction } & 107\end{array}$

6.2 Discussion of Main Results 110

6.2.1 An appraisal of precipitation distribution 110

6.2.2 Adjustment of measurement errors 111

6.2.3 Evaluation of gridded precipitation products 112

6.2.4 Climate change and hydrological regime 113

$\begin{array}{lll}6.3 & \text { Scientific Contribution } & 114\end{array}$

6.4 Uncertainties and Limitations 115

6.4.1 Observational uncertainties 115

6.4.2 GCM structure and parameterization $\quad 115$

$\begin{array}{ll}\text { 6.4.3 Downscaling and bias correction } & 116\end{array}$

6.4.4 Hydrological modeling structure and parameterization 116

$\begin{array}{ll}\text { 6.4.5 Uncertainties in river flow measurements } & 117\end{array}$

$\begin{array}{ll}6.5 & \text { Implications for Water Management } \\ 6\end{array}$

6.6 Outlook and Direction for Further Research 118

$\begin{array}{lr}\text { Supplementary Material } & 119\end{array}$

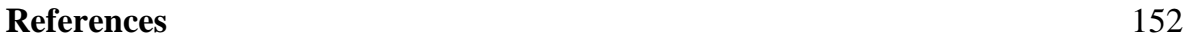

$\begin{array}{ll}\text { Summary } & 168\end{array}$

$\begin{array}{ll}\text { Acknowledgements } & 170\end{array}$

$\begin{array}{lr}\text { Curriculum Vitae } & 172\end{array}$

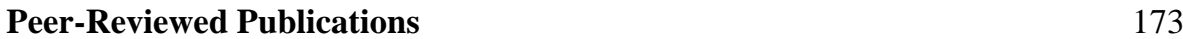

$\begin{array}{ll}\text { Sense Education Certificate } & 174\end{array}$ 


\section{Chapter 1}

\section{Introduction}

\subsection{Background and Problem Statement}

Water is essential for life, sustainable ecosystem services and continuous socio-economic developments. It forms a relationship based on the complexities of both the hydrologic cycle and the interdependencies of life on Earth (Eckstein, 2010). However, freshwater is finite and more often a shared resource. It is also unevenly distributed in space and time. The Hindukush Karakoram Himalayan (HKH) mountain region and adjoining ranges of Pamirs and Tibetan Plateau (TP) hold the world's largest repositories of snow and ice mass outside the Polar Regions (Qiu, 2008; UNESCO-SCOPE-UNEP, 2011). Precipitation and snow/glacier-melt in these areas are the important sources of freshwater supplies for the downstream areas. The IPCC through its third assessment report (Lal et al., 2001) issued the wakeup call stating that the average annual runoff in the Indus river would decline by $27 \%$ by the year 2050 due mainly to glacier retreat. Even greater thrill was moulded when the IPCC in its fourth assessment report (Cruz et al., 2007) reported lack of data to support accurate assessments for the HKH region but declared that "glaciers in Himalayas are receding faster than in any other part of the world and, if the present rate continues, the likelihood of their disappearing by the year 2035 is very high". Later, this unrealistic statement was traced back to an inaccurate citation of the grey literature and the IPCC withdrew its original statement but stayed with the broader conclusion of the report. Nevertheless, the controversy that ensued over the statement highlighted how little was known about the HKH region (Singh et al., 2011) and paved the way for increased and concerted research efforts to study hydrometeorology of this highly underexplored region. However, majority of the subsequent research is mainly focused on improved methods using more or less the same commonly available datasets that use low-altitude, directionally biased and largely unrepresentative observations in their development or validation. This $\mathrm{PhD}$ research study however recognized the vital nature of the issue and put more emphasis on the development of improved precipitation and temperature datasets to facilitate accurate analysis of climate change and water balance studies in the highaltitude Indus basin.

The Indus river system (IRS) had been the lifeline for the Indus valley civilization since time immemorial and currently sustains livelihoods of over 300 million inhabitants. Yet, there is limited understanding of quantitative and spatiotemporal distribution of the key climatic variables (Immerzeel et al., 2015; Mishra, 2015; Ragettli and Pellicciotti, 2012; Hewitt, 2005; Winiger et al., 2005) leading to a large uncertainty in the hydro-climatic predictability in the basin (Lutz et al., 2016a). The greatest uncertainties are associated with very high variability in seasonality, magnitude, coverage, and altitudinal distribution of precipitation in the high-altitude Indus basin. Generally, scarcity and biased distribution of observed data at the higher altitudes (Fowler and Archer, 2006) and measurement errors in precipitation observations (Kochendorfer et al., 2017a; Wolff et al., 2015; Adam and Lettenmaier, 2003) are the primary causes of such uncertainties. Hence, there are significant errors in the current estimation of basin and sub-basin scale precipitation as most of the meteorological stations are located at elevations lower than $2500 \mathrm{~m}$, whereas 
about $74 \%$ of the study domain lies above this elevation range. Accurate assessment of precipitation is essential as relatively small errors in precipitation estimates may translate into considerable changes in surface runoff estimates and associated water allocations. However, precipitation estimates by earlier studies (e.g. Krakauer et al., 2019; Ullah et al., 2019; Ahmed et al., 2019; Khan et al., 2018; Iqbal et al., 2018; Anjum et al., 2018; Ghulami et al., 2017; Hussain et al., 2017; Immerzeel et al., 2015; CWC and NRSC, 2014; Ali et al., 2012; Bocchiala et al., 2011) are highly contrasting but consistent in underestimating precipitation in most parts of the high-altitude Indus basin. The global/regional scale gridded precipitation products also tend to show highly variable estimates for the Indus basin (Baudouin et al., 2020; Palazzi et al., 2013). The gridded precipitation products often fail to capture the strong gradients and large and abrupt changes in precipitation over short distances due to their coarser resolution and pronounced orographic effects in the high mountain areas (Reggiani and Rientjes, 2015; Immerzeel et al., 2015)

Similarly, there are significant paradoxes on the reported behaviour of snow/glacier cover in the HKH region. Many of the global/regional studies (e.g. Pritchard, 2019; Kraaijenbrink et al., 2017; ICIMOD, 2009; Immerzeel et al., 2009; WNEP \& WGMS, 2008; Zemp et al., 2008; Cruz et al., 2007; WWF, 2005; Lal et al., 2001) indicated glacier retreat in HKH region while other (e.g. Farinotti et al., 2020; Tahir et al., 2011; Armstrong, 2010; Fowler and Archer, 2006; Hewitt, 2005) reveal expansion or immobility of HKH snow/glacier cover. The main reasons behind such contrasting claims and results are: lack of data and insufficient in-situ measurements to support accurate assessments, use of nonrepresentative climate data, projecting the results of a small-scale study over the entire basin or one region to another, errors in up-scaling and/or down-scaling of results, and neglecting the impacts of debris cover and land use change. However, it is important not to generalize climate and glacier changes across the HKH region (ICIMOD, 2011). In fact, variable retreat rates (Scherler et al., 2011; Armstrong, 2010; Raina, 2009; Hewitt, 2005; Young and Hewitt, 1993), presence of debris cover (Kraaijenbrink et al., 2017; Scherler et al., 2011; Young and Hewitt, 1993), paucity of glacial mass-balance data (UNEP \& WGMS, 2008) and influence of multiple weather systems interacting with high orography make it difficult to develop a coherent picture of climate change impacts in the HKH region (Scherler et al., 2011). Armstrong (2010) noticed that glaciers at elevations greater than $4000 \mathrm{~m}$ have not responded to the recent climate warming in the same way as the glaciers at lower elevations, simply because the glaciers at higher elevations remain below freezing during most of the year. This is particularly true for the Indus basin glaciers where over $80 \%$ of the ice cover is concentrated between 4000 and $5500 \mathrm{~m}$ elevation (Hewitt, 2011) and $50 \%$ of the area has elevation greater than $4000 \mathrm{~m}$.

Assessing climate change and associated hydrological implications require high-quality and long-term data of important climatic variables. However, the data quality issues are often inadequately addressed that contribute to uncertainty in the results of hydrometeorological studies. Therefore, the basin and sub-basin scale changes in the observed and future climate estimated by the previous studies (e.g. Hasson et al., 2019 \& 2017; Bokhari et al., 2018; Bashir et al., 2017; Lutz et al., 2016a; Ali et al., 2015; Khan et al., 2015) are highly variable due to use of inconsistent and uncertain datasets and a large spread in the outcomes of global climate models (Lutz et al., 2016b; Palazzi et al., 2014). Similarly, the consequential river inflows also show high variability, which may partly be attributed to use of different hydrological models. Indus is predominantly a snow/glacier-fed river basin, therefore the simple degree-day or temperature-index based hydrological models often inadequately 
represent the prevailing energy balance, which is the key driving force for melting of seasonal snow and perennial glaciers in the snow/glacier-fed systems. Several hydrological models are currently under use to assess hydrological implications of climate change. The choice of hydrological model is often based on the model structure to represent the complex natural system, model performance under site-specific conditions, data availability and requirements, and study objectives (Kay et al., 2009). A substantial portion of uncertainty often stems from the use of suboptimal input parameters (Ragettli et al., 2013; Kay et al., 2009). Therefore, this $\mathrm{PhD}$ research study has been undertaken to reduce the uncertainties associated with climatic variables and hydrological modeling for better assessment of the current and future hydrometeorological regime of the high-altitude Indus basin.

\subsection{Climate and Hydrological Regime of the High-Altitude Indus Basin}

The Indus river originates in the high-mountain ranges of Tibetan Plateau (TP) and HKH region with a drainage area of about 112 million hectares in four counties (8\% in China, $39 \%$ in India, 6\% in Afghanistan and 47\% in Pakistan). This research study is undertaken in the high-altitude watersheds of the Indus river encompassed by its six major tributaries (i.e. Indus river at Tarbela dam, Kabul river at Nowshera, Jhelum river at Mangla dam, Chenab river at Marala, Ravi river at Thein dam, Beas river at Pong dam, and Sutlej river at Bhakra dam), stretched over an area of 40.3 million hectares (Figure 1.1).

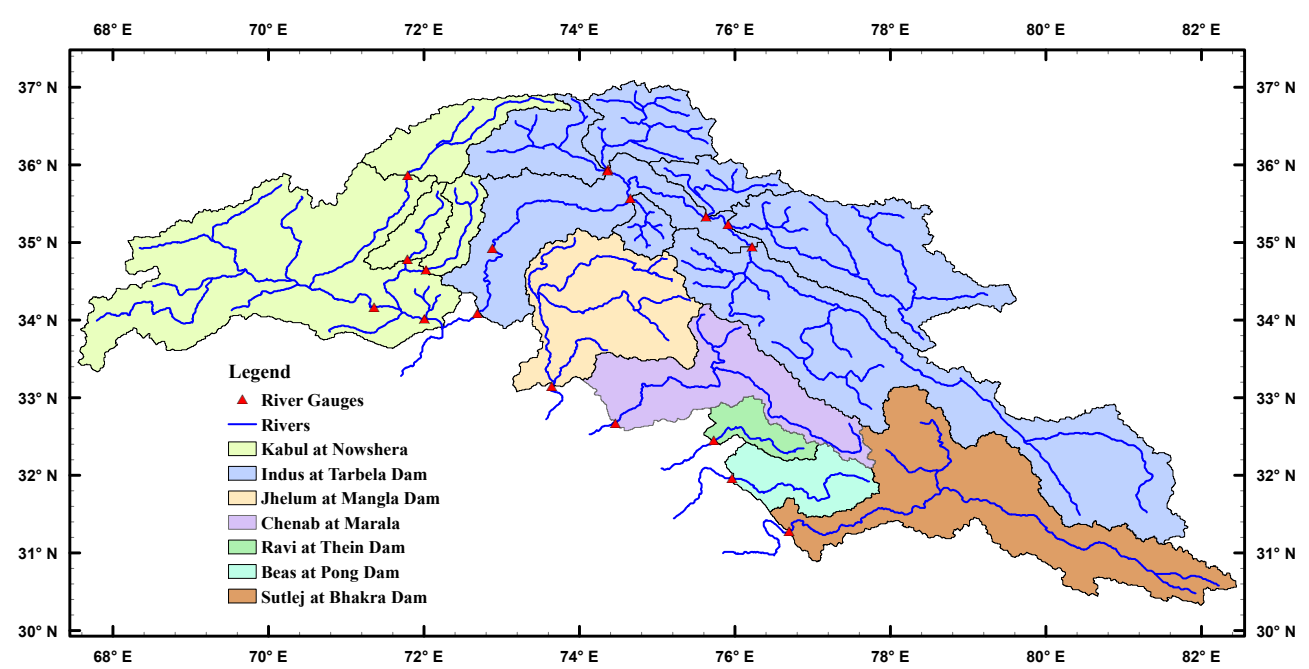

Figure 1.1: Geographic location of the Study area showing river networks and watersheds of Indus river and it six major tributaries.

The extensive Eurasian continent and the Indian and Pacific oceans play an important role in atmospheric circulation and monsoon formation of the world's largest and most powerful monsoon system in South Asia (Saha, 2010). The climate of the high-altitude Indus basin is complex and significantly influenced by the intricate interplay between synoptic-scale atmospheric circulations and valley-scale topography-atmosphere interaction resulting in orographic precipitation and funnelling of air movement (Barros et al., 2004; Hewitt, 2013). The synoptic-scale climate and precipitation are largely modulated by the Indian summer monsoon and the westerly systems (Pang et al., 2014; Yao et al., 2012; Ding and Chan, 2005; Wang and Lin, 2002) (Figure 1.2). The Indian summer monsoon advects moisture through several trajectories originating from the Bay of Bengal, Indian Ocean and Arabian 
Sea due to the differential heating between land and sea (Pang et al., 2014; Bolch et al., 2012; Yao et al., 2012; Bohner, 2006; Hodges, 2006). It causes heavy rainfall in southeastern areas during June-September and moves north-westward along the Himalayan Arc with decreasing strength. The westerly systems transport large masses of moist air from the Caspian, Black \& Mediterranean seas and North Atlantic Ocean throughout the year and are the dominant source of precipitation in the Hindukush, Karakoram and to a lesser extent in the W-Himalayan regions during December-April months (Filippi et al., 2014; Pal et al., 2014; Mayer et al., 2014; Bohner, 2006; Treydte et al., 2006; Syed et al., 2006). The winter westerlies usually bifurcate into the northern, central and southern branches around the Karakoram and western TP regions due to topographic blocking (Pang et al., 2014). Wintertime precipitation in the $\mathrm{HKH}$ region is mainly related to water vapour transport by the southern and to a lesser extent by the central branch of westerly systems (Yihui and Zunya, 2008; Wei and Gasse, 1999). Seldom, relatively weak storms of East-Asian summer monsoon also enter into the Ladakh region from the eastern end (Ding and Chan, 2005; Wang and Lin, 2002). Moreover, significant amount of moisture in the air is added to the atmosphere by evapotranspiration from the vast irrigated plains and forestlands (de Kok et al., 2018; Harding et al., 2013; Wie et al., 2013; Tuinenburg et al., 2012). Heavy precipitation events are often encountered whenever these systems coincide and interact with each other (Zaidi, 2014; SUPARCO and FAO, 2010; WMO, 2010).

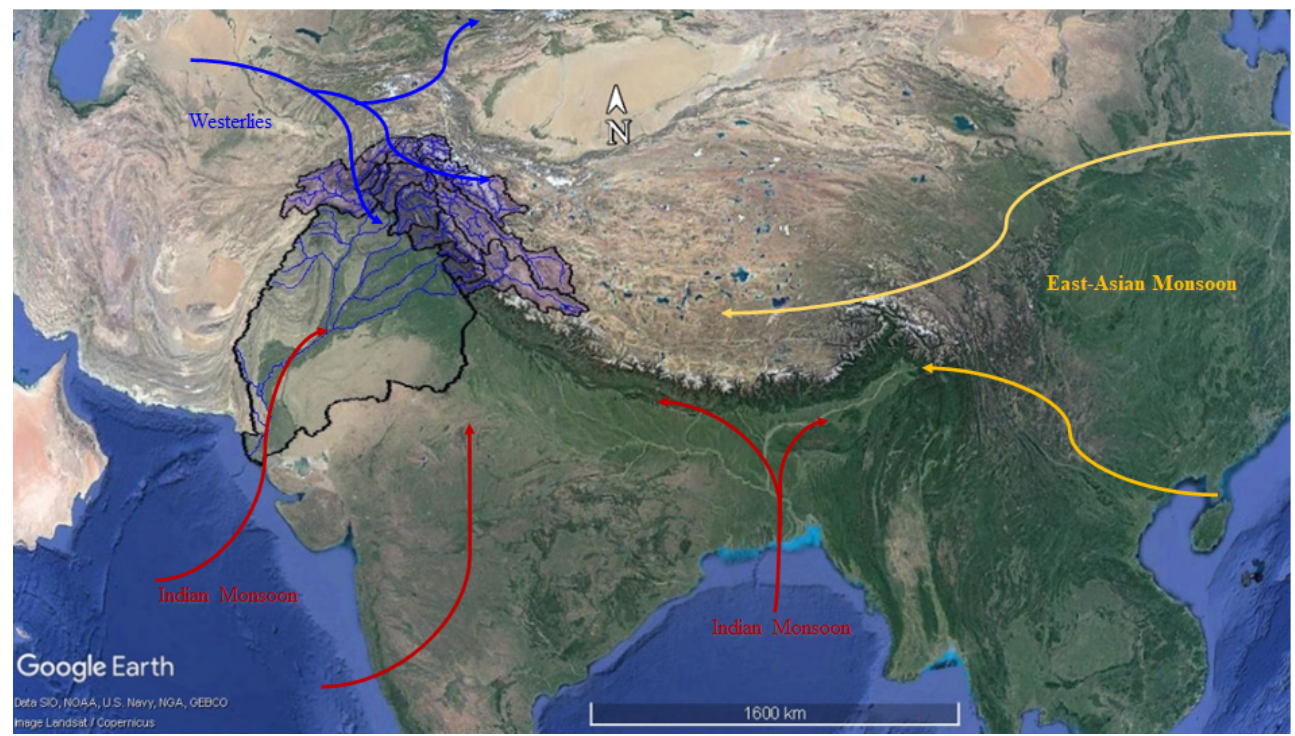

Figure 1.2: Geographic map of the Study area showing trajectories of major circulation systems. Blue arrows indicate the trajectories of winter westerlies, red arrows represent Indian summer monsoon trajectories and yellow line arrows show East-Asian monsoon trajectories. Map source: google earth.

The Indus river system (IRS) is the largest source of freshwater supply (153 BCM year $\left.{ }^{-1}\right)$ in Pakistan and plays a crucial role in water, energy and food security of the region. The hydrological regime of the IRS is largely modulated by monsoon precipitation, winter snowfall and subsequent melting of snow and glacial ice mass accumulated in the highmountains. Generally, a glacial regime at very high altitudes where flow occurs due to melting of perennial snow and glaciers during late summer, a nival regime at mid altitudes where flow is largely dependent on the melting of seasonal snow accumulated during the preceding winter and spring seasons, and a pluvial regime dependent on runoff from 
concurrent rainfall mainly during the monsoon season are the characteristic river flow regimes of the high-altitude Indus basin (Hasson et al., 2019; NESPAK-AHT-DELTARES, 2015; Archer et al., 2010). About $59 \%$ of the total precipitation in the study domain is received during Oct-Apr months, majority of which falls in the form of snow. As a result, large repositories of glacial ice mass have been deposited over the centuries in these highmountain ranges. Around $50 \%$ of the basin area can be covered with snow during winter months. However, most of it melts during the subsequent summer season leaving only about $10 \%$ of the basin area covered with perennial snow or glaciers by the end of August (Tahir, 2011). Conversely, about $84 \%$ of the river flows are received during May-Sep months mainly due to monsoon precipitation and melting of seasonal snow and perennial glaciers. Hence, perennial snow and glaciers are largely acting as repository of the glacial ice mass to store or release rainwater depending upon the winter precipitation and summer temperatures (Pritchard, 2019; Thayyen and Gergan, 2010, Archer, 2003). This shows the importance of seasonal snow cover in the water budget of the basin, which usually receives heavy floods whenever high precipitation in winter season is followed by a warm and wet monsoon season. Interannual variability of river flows is extremely high and generally responsive to annual precipitation and summer temperatures. The observed data show that maximum annual flows can be double of minimum flows. Nevertheless, the IRS is predominantly a snow- and glacier-fed system (Lutz et al., 2016a \& 2014; Yu et al., 2013), which makes it more sensitive and vulnerable to climate change and variability.

\subsection{Hydrological Modeling}

A hydrological model is a simplified representation of the complex natural system. Hydrological models are mainly used for predicting system behaviour and understanding various hydrological processes. The preferable model is the one which is simpler and easier to execute and that gives results closer to reality requiring least input. The simple empirical models are based on observed relationships rather than on simulated physical processes. These models are often lumped, treating a complete watershed as a single homogeneous unit. Physically-based models are often distributed, dividing a watershed into uniform sized grid cells and calculating flows between them. These models are generally complex with large input data requirements. Conceptual models are transition between empirical and physically-based models in terms of data requirements and details to represent the physical processes. Similarly, semi-distributed models are transition between lumped and distributed models, dividing a watershed in different elevation zones, homogeneous sub-basins or hydrological response units. Nevertheless, a number of hydrological models ranging from lumped to fully-distributed, simple temperature-index to complex energy-balance, and from standalone catchment-scale to global-scale land surface schemes are currently under use. Each model has got its own unique characteristics, specific applications and drawbacks like large data requirements, operational complexities and structural limitations. Generally, physically-based distributed models are better suited for the accurate simulation of spatial and temporal patterns in surface runoff. Accuracy of model outputs is usually dependent on the precisions in the physical processes, mathematical representations and details of the input parameters. However, model outputs are more affected by the underlying input parameters rather than the model structure (Islam and Déry, 2017; Kay et al., 2009).

This PhD study used the Variable Infiltration Capacity (VIC) hydrological model, 4.2d version (Liang et al., 1994; 1996). The VIC model is an evolving macroscale, fullydistributed, physically-based hydrologic model that can solve water balance as well as full water-and-energy balance to compute surface runoff, baseflow, evapotranspiration and 
other turbulent fluxes for each individual grid. The VIC model was originally designed to provide boundary conditions at the land surface schemes for global and regional climate models and is equally applicable for catchment- to global-scale hydrological modeling. The model is characterized by heterogeneous topography (elevation and vegetation) and multiple soil layers (Figure 1.3a). The land surface is modelled as uniform grids of any size. Sub-grid variability of precipitation, temperature and land cover is considered by further dividing the surface grids into an arbitrary number of bands/tiles with variable elevation and land cover types. Temperature is lapsed from the grid cell average elevation to sub-grid band elevation, while altitudinal variability of precipitation within each grid cell is specified through fractional areal coverage. Sub-grid variability in infiltration capacity is represented by a fast runoff response to precipitation through a variable infiltration curve and slow runoff response via a nonlinear relationship between baseflow and deep soil moisture. Water can only enter to a grid cell via the atmosphere. The VIC model considers snow in several forms: ground snow pack, snow in the vegetation canopy, and snow on top of lake ice. Ground snowpack accumulation and ablation is solved by energy balance approach while sublimation, drip and release of intercepted snow by the vegetation is covered by an explicit canopy snow interception scheme described by Andreadis et al. (2009). Additionally, blowing snow sublimation is considered by the Bowling et al. (2004) model. Evapotranspiration (ET) from each vegetation type is characterized by the PenmanMonteith formulation. Total evapotranspiration is the sum of evaporation from the canopy layer and transpiration from each vegetation tiles and evaporation from the bare soil.

An offline Lohmann routing model (Lohmann et al., 1998) processes the surface runoff and baseflow fluxes generated for each grid cell and routes the accumulated flows at the specified locations in the model domain using elevation-based flow direction (Figure 1.3b). The Lohman routing model uses the unit hydrograph principle within the grid cells and linearized St. Venant's equations to simulate river flow through the stream channel. Comprehensive technical descriptions and mathematical formulations of the VIC model are provided at https://vic.readthedocs.io/en/master/Overview/ModelOverview/ and by Gao et al. (2010).
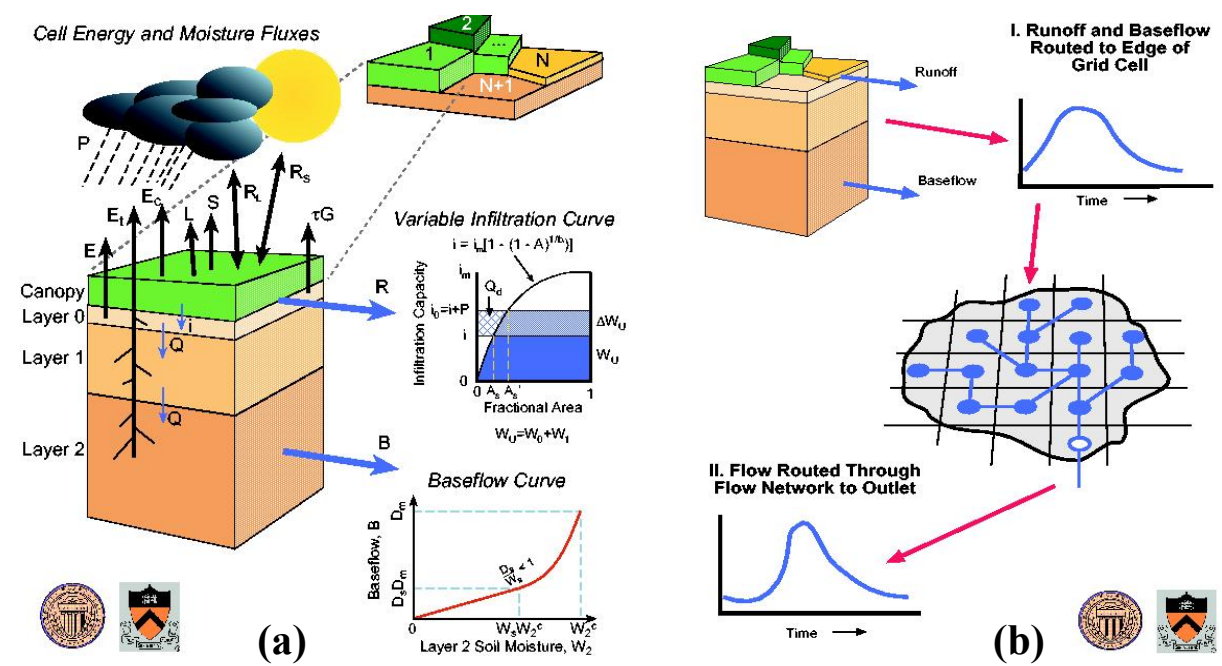

Figure 1.3: Schematic representation of the basic features of VIC hydrological model (a), and routing model (b). Source: Gao et al., 2009. 
The selection of VIC hydrological model in this $\mathrm{PhD}$ research study is mainly inspired to its proven and successful applications in a variety of topographical and hydrometeorological conditions and its ability to model full water-and-energy balance in a fully-distributed manner, which is particularly crucial for simulation of snow and glacier dominated land surfaces. Generally, relatively simple hydrological models calibrated against sparse and short records of mountain climatic variables and runoff have been applied in the study domain (e.g. Hasson et al., 2019; Lutz et al., 2016, Tahir et al., 2011; Immerzeel et al., 2010). Whereas, the application of VIC hydrological model is very rare for hydrological simulations in the study area. Therefore, this study will add to the knowledge and practice of the hydrological modeling in the study domain.

\subsection{Research Questions and Study Objectives}

The high-altitude Indus basin is one of the most complex and largely underexplored regions in the World. The region is also recognized as the 'hotspot' of climate change due to significant transformations in its hydrometeorological regime (Krishnan et al., 2019; Wijngaard et al., 2018; Lutz et al., 2018; Kraaijenbrink et al., 2017; De Souza et al., 2015). However, an authentic assessment of climate change and variability and associated hydrological implications in the basin are seriously constrained by paucity of observed data and their directional biases. Quality of observed data and delicate investigation of the current and future hydrometeorology also add uncertainties and limit our understanding of the basin's hydrometeorological regime. Therefore, the overarching aim of this $\mathrm{PhD}$ research study is to reduce the underlying uncertainties in precipitation and temperature observations to develop high-quality long-term data for historical and future time periods and improved simulation of the water balance and hydrological regime at regional and subbasin scale under extreme climate scenarios. This research study therefore was designed to highlight and address the underlying issues in precipitation observations and existing datasets by investigating \& answering the following interconnected research questions $(\mathrm{Q})$ :

Q1. How precipitation in the basin area is distributed in space and time?

Q2. How strong are the vertical gradients of precipitation at sub-basin scale?

Q3. What uncertainties are present in the existing observations and knowledge in precipitation distribution of the study area?

Q4. To what extent precipitation measurement errors can affect total precipitation?

Q5. How monthly-scale temperature gradients vary with elevation and latitude?

Q6. What is the contribution of net glacier mass balance to river inflows?

Q7. How accurate and representative are the global/regional gridded precipitation products for the study area?

Q8. What uncertainties are present in the precipitation estimates of different gridded precipitation products for the study area?

Q9. How closely the observed precipitation in the study area is simulated by the Global Circulation Models (GCMs)?

Q10. To what extent climate has changed in the study area over the recent past and what are the prospects for climate change under various climate change scenarios by the end of $21^{\text {st }}$ century? 
Q11. What shifts in precipitation and water availability can be expected at monthly, seasonal or annual scales?

Q12. How climate change is likely to affect hydrological regime of the basin?

Q13. What will be the frequency, intensity and distribution of floods and droughts by mid-century and end-century with respect to baseline reference period?

Q14. What uncertainties are present in the current understanding of the basin's hydrological regime and overall assessment of the hydrological process and how these can be minimized?

Given the importance of precipitation and a large uncertainty over its distribution, a twopronged strategy is adopted to investigate and address these research questions. In the $1^{\text {st }}$ step the above-outlined research questions are integrated and framed in the four broadbased research objectives. Secondly, each objective is achieved through a separate scientific chapter using the appropriate datasets and specific procedures. The four broadbased and specific objectives of this research study are:

1. Appraise the current state of precipitation distribution in the high-altitude Indus basin and highlight the underlying issues and uncertainties in precipitation observations and quality of gridded precipitation products (Q1, Q2, Q3, addressed in Chapter 2)

2. Adjust measurement errors in precipitation observations and develop high-quality reference climatologies of precipitation and temperature (Q1, Q3, Q4, Q5, Q6, addressed in Chapter 3)

3. Evaluate quality of regional/global scale precipitation products for the study area with respect to the reference dataset (Q7, Q8 addressed in Chapter 4)

4. Develop long-term datasets of precipitation and temperature for the historical and future time periods and assess past and future climate change and variability and associated hydrological implications (Q9, Q10, Q11, Q12, Q13, Q14 addressed in Chapter 5).

\subsection{Thesis Outline and Methods}

Investigations to answer the above-outlined research questions and achieve the four broadbased and specific objectives of the study are pursued in the subsequent four chapters (Chapter 2 to 5). Each chapter is the product of a peer-reviewed scientific paper published in the journals of international repute.

Chapter 2 appraises improved precipitation distribution of mean monthly, seasonal and annual precipitation in the study domain using observed precipitation data from multiple sources combined with the indirect precipitation estimates at the accumulation zones of major glaciers. This chapter highlighted the data gaps in the in-situ observations and underlined the uncertainties associated with the existing precipitation datasets. Altitudinal dependency of precipitation is analyzed at sub-basin scale. The issues related to direct use of gridded precipitation products are also underscored.

In chapter 3, precipitation distribution is reconciled by incorporating additional observations and a better approach for cross-validation. The WMO recommended standard methods are adopted to adjust systematic errors in precipitation measurements. The net snow accumulations were adjusted for the ablation losses using standard ablation gradients, 
while the river inflows were adjusted for the contribution of net mass balance using mass balance estimates of Kääb et al., 2012 and the glacier areas from the Randolf Glacier Inventory (RGI) version 5.0 (Arendt et al., 2015). Reference climatologies of mean monthly precipitation are derived from the adjusted observations and are cross-validated with the adjusted river inflows at sub-basin scale using Turc-Budyko non-dimensional analysis.

Chapter 4 highlighted and corroborated the underlying issues and uncertainties associated with a wide range of gridded precipitation products in the high-mountain Indus basin. Performance of 27 widely used gridded precipitation products belonging to three different categories (gauge-based, reanalyses and merged) is evaluated with respect to high-quality reference climatologies of mean monthly precipitation developed in the previous chapter using the widely used statistical measures and quantitative analyses techniques. Cross validation is accomplished with the corresponding specific runoffs using Turc-Budyko nondimensional analysis.

In Chapter 5, the best performing gridded precipitation product (ERA5) evaluated in the $4^{\text {th }}$ chapter is statistically downscaled and bias-corrected with respect to the reference dataset developed in the $3^{\text {rd }}$ chapter. The temperature data of the same product are bias corrected against the observational-based monthly-scale temperature dataset derived from the elevation and latitudue dependent lapse rates. Similarly, representing warm-wet and cold-dry climates, the outputs of two best performing GCMs used in CMIP5 for RCP2.6, RCP4.5 \& RCP8.5 are statistically downscaled and bias-corrected. A fully-distributed physically-based energy-balance VIC hydrological model is forced with these novel datasets to simulate the water balance at regional and sub-basin scale. Hydrometeorological analyses are conducted to determine the changes and variability in climate and overall water availability and examine the shifts in the timings and peaks of hydrological extremes during the entire $21^{\text {st }}$ century.

Finally, the main results of the four scientific chapters are synthesized in Chapter 6. This chapter also highlights the scientific contribution of this research study, discusses the underlying uncertainties, signifies the implications for water management, and presents the outlook and direction for further research. 


\title{
Chapter 2
}

\section{An Appraisal of Precipitation Distribution}

\begin{abstract}
Scarcity of in-situ observations coupled with high orographic influences has prevented a comprehensive assessment of precipitation distribution in the high-altitude Indus basin. Available data are generally fragmented and scattered with different organizations and mostly cover the valleys. Here, we combine most of the available station data with the indirect precipitation estimates at the accumulation zones of major glaciers to analyse altitudinal dependency of precipitation in the high-altitude Indus basin. The available observations signified the importance of orography in each sub-hydrological basin but could not infer an accurate distribution of precipitation with altitude. We used Kriging with External Drift (KED) interpolation scheme with elevation as a predictor to appraise spatiotemporal distribution of mean monthly, seasonal and annual precipitation for the period of 1998-2012. The KED-based annual precipitation estimates are verified by the corresponding basin-wide observed specific runoffs, which show good agreement. In contrast to earlier studies, our estimates reveal substantially higher precipitation in most of the sub-basins indicating two distinct rainfall maxima; 1st along southern and lower most slopes of Chenab, Jhelum, Indus main and Swat basins, and 2nd around north-west corner of Shyok basin in the central Karakoram. The study demonstrated that the selected gridded precipitation products covering this region are prone to significant errors. In terms of quantitative estimates, ERA-Interim is relatively better close to the observations followed by WFDEI and TRMM, while APHRODITE gives highly underestimated precipitation estimates in the study area. Basin-wide seasonal and annual correction factors introduced for each gridded dataset can be useful for lumped hydrological modelling studies, while the estimated precipitation distribution can serve as a basis for bias correction of any gridded precipitation products for the study area.
\end{abstract}

This chapter has been published as:

Dahri, Z. H., Ludwig, F., Moors, E., Ahmad, B., Khan, A., \& Kabat, P. (2016). An appraisal of precipitation distribution in the high-altitude catchments of the Indus basin, Science of the Total Environment, 548-549: 289-306, http://dx.doi.org/10.1016/j.scitotenv.2016.01.001. 


\subsection{Introduction}

The Hindukush Karakoram Himalayan (HKH) mountain region and adjoining ranges of Pamirs and Tibetan Plateau (TP) hold the world's largest repositories of snow and ice mass outside the Polar Regions (Qiu, 2008; UNESCO-SCOPE-UNEP, 2011). The Indus River System (IRS), originating from TP and HKH mountain region and crossing through China, India, Afghanistan and Pakistan, sustains livelihoods of over 215 million people. Yet, little is known about environmental change and mountain hydrology in this highly diversified and complex mountain region (Immerzeel et al., 2012; Karki et al., 2011). There is limited understanding of quantitative and spatiotemporal distribution of precipitation, which provides the basic and critical input for hydrological assessment, mass balance and climate change studies. The current knowledge is mainly constrained by limited in-situ hydrometeorological and cryospheric mass balance observations in the high-altitude catchments of Indus basin (Pellicciotti et al., 2012; Wake, 1987). Political environments, poor accessibility and harsh weather conditions pose serious challenges for such observations in this region. As a result, there are significant data, information and knowledge gaps in hydro-climatic aspects.

Precipitation in the high-altitude catchments of Indus basin is predominantly controlled by large-scale orography and remains highly variable in time, space and altitude. Its variability and distribution pattern mainly depend on the interactions and interplay of orographic features with large-scale atmospheric circulation systems, regional climatic processes and local evapotranspiration rates. Large changes in precipitation over short distances and within short periods of time are common and high amplitude events are often localized (Nesbitt and Anders, 2009). The zone of maximum precipitation is usually the function of enhanced moisture condensation and exponential reduction in the quantity of available moisture with increasing barrier height (Alpert, 1986). Hence, rainfall gradients in the complex terrains are often not linearly correlated with altitude (Singh and Kumar, 1997; Loukas and Quick, 1996). Nevertheless, several other studies indicated that precipitation in the HKH region exhibits a considerable vertical gradient (e.g. Pang et al., 2014; Winiger et al., 2005; Hewitt, 2011; Weiers, 1995; Wake, 1989; Dhar and Rakhecha, 1981; BIG, 1979; Decheng, 1978).

Precipitation is an important component of the hydrological cycle that governs the renewable water resources affecting agro-economic development, hydropower generation and environmental integrity. Therefore, accurate assessment of precipitation is essential as small errors in precipitation estimates may translate into major changes in surface runoff estimates and associated water allocations. Accurate assessment of precipitation requires good quality observations with adequate spatiotemporal coverage to assess the sub-basin or local scale variability. However, the existing rain gauge network in this region is not only inadequate but also biased towards valley bottoms (Fowler and Archer, 2006). The solid precipitation (snowfall) at higher altitudes is often difficult to accurately measure and generally susceptible to undercatch by $20-50 \%$ (Rasmussen et al., 2012). Furthermore, the Indus is an international river basin and the available observational data are usually fragmented and scattered with different organizations in four countries and are not freely accessible. Therefore, there is an ever-increasing trend of using the easily available global/regional scale gridded datasets for hydro-climatic assessment and mass balance studies (e.g. Lutz et al., 2014a; Sakai et al., 2014; Immerzeel et al., 2012, 2010, 2009; Tahir et al., 2011; Bookhagen and Burbank, 2006). 
Indeed, the gridded datasets provide better information in terms of spatial coverage and temporal consistency, but with potentially large errors particularly in high-mountains where the resolution of the data is often larger than the spatial variability of precipitation and the adopted interpolation schemes add further uncertainty. Also, satellite observations underestimate precipitation in areas with significant snowfall (Andermann et al., 2011). Moreover, the gridded datasets covering the high-altitude areas of Indus basin use station data of only a few commonly available old observatories predominantly located at the valley floors, which do not reflect the topographical complexity and spatial variability of precipitation in these areas (Reggiani and Rientjes, 2015). Hence, the accuracy of gridded datasets is particularly questionable in this region requiring their correction and validation before use. However, the limitations and internal inconsistencies of the gridded datasets are often overlooked in the hydro-climate studies; where underestimated precipitation is often compensated by underrated evapotranspiration and/or overestimated snow/glacier melt rates (Lutz et al., 2014a; Pellicciotti et al., 2012; Schaefli et al., 2005). Ultimately, the inferences regarding precipitation distribution, snow/glacier cover dynamics and associated melt water contributions are inaccurately adjudicated. Point observations, on the other hand, provide relatively accurate local information, but their wider-scale use in hydroclimate studies is constrained by their restricted accessibility, limited spatiotemporal coverage and uneven distribution in both horizontal and vertical directions. Paucity of precipitation measurements in the high-altitude areas, where the bulk of precipitation falls, provides an incomplete picture of precipitation distribution. Auspiciously, there are few mass balance studies (e.g. Mayer et al., 2014, 2006; Hewitt, 2011; Shroder et al., 2000; Bhutiyani, 1999; Wake, 1989; Mayewski et al., 1984, 1983; Kick, 1980; BIG, 1979; Decheng, 1978; Qazi, 1973) that indirectly estimated net precipitation (as water equivalent) using snow pillows, snow pits, and ice cores from the accumulation zones of few important large glaciers in this region. These sparse but relatively accurate and high-altitude point observations can be integrated with the low-mid altitude observations to derive highaltitude precipitation and to verify and correct the gridded datasets developed through various means.

In addition, the specific runoffs (measured flow/drainage area) from all the high altitude catchments of Indus basin are significantly higher than the corresponding precipitation estimates by earlier studies (Immerzeel et al., 2012, 2015). This indicates that either the estimated precipitation is lower than the actual or these basins are receiving bulk of their runoff from snow/glacier melt in the absence of an adequate precipitation (snowfall) inputto sustain the snow/glacier systems. The latter case certainly recognizes for tangible glacier retreat and loss of glacial mass. However, the scientific research on precipitation inputs and associated snow/glacier mass balance in the study area is uncertain and largely contradicting due mainly to paucity of in-situ precipitation and glacier mass balance data (Kääb et al., 2012; Immerzeel et al., 2009). Moreover, mass balance studies in this region are always difficult as most of the glaciers based at the high-altitude areas (above $4000 \mathrm{~m}$ ) are often nourished by avalanches and redistribution by wind in addition to seasonal snow (Hewitt, 2013, 2011). While Kääb et al. (2012), Wiltshire (2014), Gardner et al. (2013), Jacob et al. (2012), Cogley (2011) and Immerzeel et al. (2009) noticed loss of ice mass and consistent decrease in glacier extent in the HKH region, several other studies (e.g. Bhambri et al., 2013; Minora et al., 2013; Gardelle et al., 2013, 2012; Bolch et al., 2012; Scherler et al., 2011; Tahir et al., 2014, 2011; Schmidt and Nusser, 2012; Mayer et al., 2006; Hewitt, $2005)$ indicated 'Karakoram anomaly' advocating stability or even growth of HindukushKarakoram glaciers. The possible reasons for such an anomaly have been linked to the role 
of debris-covered areas in reducing ice ablation (Scherler et al., 2011) and favourable changes in winter precipitation and summer temperatures (Mathison et al., 2013; Hewitt, 2011, 2005; Fowler and Archer, 2006; Archer and Fowler, 2004).

Given the importance of precipitation and a large uncertainty over its distribution, the major aim of this study is to analyse altitude dependency of precipitation and derive its spatiotemporal distribution by using the observed data available from different sources. Therefore, we collected precipitation data of 118 meteorological stations; more than half of these are located at mid to high-altitudes and have never been used for formation or calibration of precipitation datasets. These station observations are further supported by 16 virtual stations over major glacier accumulation zones, where average net annual precipitation is estimated through mass balance studies. We focus separately on each sub hydrological basin and explain how precipitation amounts, seasonality and patterns are represented. The study provides much improved estimates of precipitation distribution, which are comparable with the corresponding observed runoffs at sub-basin scale.

\subsection{Study Area}

The Indus basin originates from the TP and the HKH region and spreads over parts of China (8\%), India (39\%), Afghanistan (6\%) and Pakistan (47\%). The study area extends over the high-mountain sub-basins of Indus basin (Figure 2.1). The total area of these highaltitude catchments is $259,913 \mathrm{~km} 2$ of which $57.5 \%$ is laid above $4000 \mathrm{~m}$ a.s.1. Although, there is no definite boundary among the three mountain ranges but it is generally assumed that the river Indus bisects the Himalayan range from the Hindukush, Karakoram and TP. The eastern boundary of Shyok basin limits the Karakoram range in the east, while the boundary between Gilgit and Hunza basins separates it from the Hindukush range. The study area is the largest source of fresh water resources (153 BCM year ${ }^{-1}$ ) of Pakistan and plays a crucial role in water, energy and food security of the region.

The extensive Eurasian continent and the Indian and Pacific oceans play an important role in atmospheric circulation and monsoon formation of the world's largest and most powerful monsoon system in South Asia (Saha, 2010). The climate of Indus basin is characteristic of the South Asian atmospheric circulation that is associated with the summer monsoon evolution and extra-tropical cyclonic/anticyclonic circulations around troughs of low/high pressure areas during winter. Thus, precipitation in the study area is predominantly influenced by the two principal weather systems: the Indian summer monsoon (ISM) advecting moisture from the Indian Ocean, Arabian Sea and Bay of Bengal due to the differential heating between land and sea during summer (e.g., Palazzi et al., 2013; Ahmad et al., 2012; Krishnamurti and Kishtawal, 2000; Wu and Zhang, 1998; Li and Yanali, 1996), and the western disturbances (WDs) bringing moisture from the Mediterranean and Caspian sea as an extratropical frontal system during winter and early spring (Filippi et al., 2014; Pal et al., 2014; Mayer et al., 2014; Treydte et al., 2006; Syed et al., 2006; Archer and Fowler, 2004; Archer, 2001; Singh et al., 1995). Seldom, relatively weak storms of East Asian summer monsoon (Ding and Chan, 2005; Wang and Lin, 2002) also enter into the Ladakh region from the eastern end.

The summer monsoon in the Indus basin, extending from July- September, is the northwestern limit of the ISM. There are three monsoon moisture trajectories: $1^{\text {st }}$ from the Indian Ocean across the Arabian Sea, $2^{\text {nd }}$ along the Indian river valley to the western Himalayas and TP, and $3^{\text {rd }}$ from the Bay of Bengal moving northward to the eastern 
Himalayas and TP along the Brahmaputra river valley (Pang et al., 2014; Liu, 1989; Lin and $\mathrm{Wu}, 1990)$. The WDs enter the north-west Indus basin during late November mostly in a diffused state with distorted structure, but regain their frontal structure and strength by interacting with the pre-existing orographically- maintained trough of low pressure. They usually bifurcate into the northern and southern branches around the Karakoram and western TP regions due to topographic blocking (Pang et al., 2014). Wintertime precipitation in the $\mathrm{HKH}$ region is mainly related to water vapor transport by the southern branch of WDs (Yihui and Zunya, 2008; Wei and Gasse, 1999). The interplay between these regional- scale atmospheric circulation systems and the local climatic and topographic features usually determine the amount and distribution pattern of precipitation in the highaltitude catchments of Indus basin.

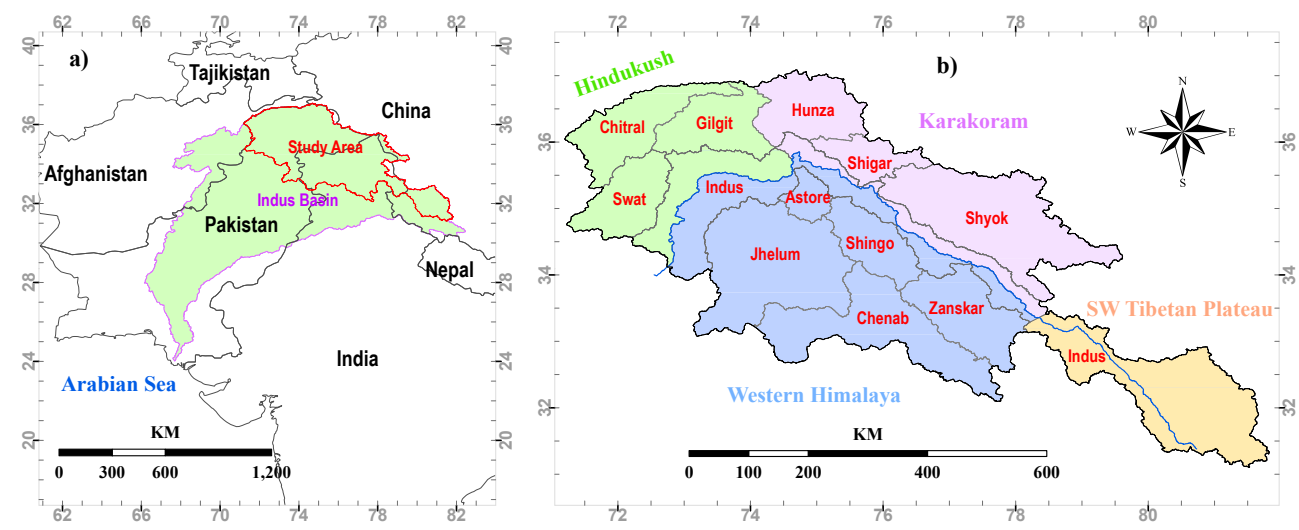

Figure 2.1: a) Location of the study area, and b) location of sub-basins and mountain ranges. The mountain ranges are separated by different colour schemes.

\subsection{Data and Methods}

\subsubsection{Station based point observations}

Meteorological data of the Indus basin is scattered among different organizations [e.g. Pakistan Meteorological Department (PMD), Water and Power Development Authority (WAPDA) of Pakistan, Indian Meteorological Department (IMD), University of Boon under the Culture Areas Karakoram (CAK) programme in the Bagrot valley and Yasin catchment of Gilgit basin during 1990-91, and Ev-K2-CNR (an Italian based organization) under the SHARE project]. However, not all these data are freely accessible. PMD operates a number of meteorological stations in Pakistan but their network of observatories in the high-altitude catchments of Indus basin is sparse and mainly concentrated in the valleys with elevations less than $2500 \mathrm{~m}$ a.s.l. WAPDA installed a network of meteorological observatories in various sub-basins of Indus basin under the Surface Water Hydrology Project and more recently (199499) under the Snow and Ice Hydrology Project mainly at the higher altitudes. We collected climatic data of 21 stations from PMD and 44 stations from WAPDA located in the study area. Monthly summaries of the observed precipitation at 41 observatories located in the Indian Territory available from NOAA-NCDC's website http://www.ncdc.noaa. gov/cdo-web/datasets (NOAA-NCDC) were downloaded in June, 2014. Meteorological data of 2 observatories installed by Ev-K2-CNR in Shigar basin were downloaded from http://data.eol.ucar.edu/codiac/dss/id?76.200 in June, 2014. The meteorological data collected under the CAK project in Gilgit and Hunza basins are not 
publicly available therefore we derived average precipitation of 10 observatories from Winiger et al. (2005), Miehe et al. (2001, 1996) and Eberhardt et al. (2007). Finally, we assumed 16 virtual stations located at the accumulation zones of major glaciers where average annual net precipitation is estimated from mass balance studies (Table 2.1). The observed station data used in this study are detailed in Figure 2.2 and Table S-2.1.

Table 2.1: Annual precipitation as water equivalent (we) at major glacier accumulation zones.

\begin{tabular}{|c|c|c|c|c|c|c|}
\hline $\begin{array}{l}\text { Sr. } \\
\#\end{array}$ & $\begin{array}{l}\text { Virtual } \\
\text { Station }\end{array}$ & $\begin{array}{l}\text { Latitude } \\
\text { (dd) }\end{array}$ & $\begin{array}{l}\text { Longitude } \\
\text { (dd) }\end{array}$ & $\begin{array}{l}\text { Altitude } \\
\text { (m) }\end{array}$ & $\begin{array}{l}\text { we } \\
(\mathbf{m m})\end{array}$ & $\begin{array}{l}\text { Data } \\
\text { Source } \\
\end{array}$ \\
\hline 1 & Sentik & 33.996667 & 75.95000 & 4908 & 620 & Mayewski et al. (1984) \\
\hline 2 & Nun Kun North & 34.121927 & 76.10142 & 5200 & 900 & Mayewski et al. (1983) and Qazi \\
\hline 3 & Batura & 36.666667 & 74.38333 & 4840 & 1034 & Batura Investigation Group (1979) \\
\hline 4 & Baldor & 35.877780 & 76.55079 & 5500 & 1600 & Mayer et al. (2006) and Decheng \\
\hline 5 & Urdok & 35.766876 & 76.70253 & 5400 & 1060 & Mayer et al. (2014) \\
\hline 6 & Whaleback & 36.057170 & 75.59149 & 4900 & 1790 & Hewitt $(2011,2006)$ and Wake \\
\hline 7 & Approach & 36.067780 & 75.63310 & 5100 & 1880 & Hewitt $(2011,2006)$ and Wake \\
\hline 8 & Hispar East & 35.849533 & 75.50639 & 4830 & 1070 & Hewitt $(2011,2006)$ and Wake \\
\hline 9 & Hispar Dome & 36.010910 & 75.51872 & 5450 & 1620 & Hewitt $(2011,2006)$ and Wake \\
\hline 10 & Hispar Pass & 36.028070 & 75.52151 & 5100 & 1420 & Hewitt $(2011,2006)$ \\
\hline 11 & Khurdopin & 36.133770 & 75.61969 & 5520 & 2240 & Hewitt (2011) \\
\hline 12 & Nanga Parbat & 35.167250 & 74.44442 & 4500 & 2000 & Shroder et al. (2000) and Kick \\
\hline 13 & Siachin A & 35.470730 & 77.03757 & 4800 & 484 & Bhutiyani (1999) \\
\hline 14 & Siachin B & 35.523490 & 76.99150 & 4950 & 526 & Bhutiyani (1999) \\
\hline 15 & Siachin C & 35.518660 & 76.91160 & 5050 & 662 & Bhutiyani (1999) \\
\hline 16 & Siachin D & 35.624230 & 76.85924 & 5350 & 855 & Bhutiyani (1999) \\
\hline
\end{tabular}

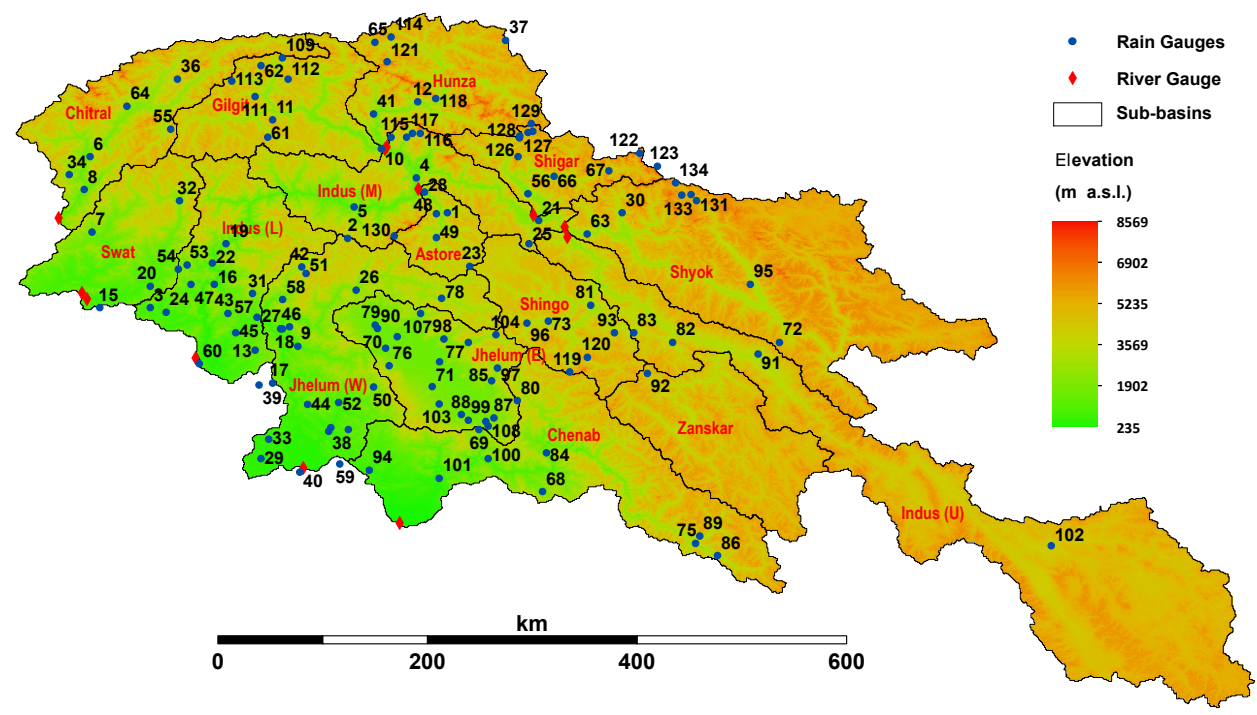

Figure 2.2: Elevation distribution, sub-basins considered for altitudinal variation of precipitation, and location of rain gauges and river gauges (the numbers refer to the respective rain gauges mentioned in Table S-2.1). 


\subsubsection{Gridded datasets}

Substantial progress has been made during the last three decades in constructing the analysed fields of precipitation over global land areas from multiple sources. As such, a wide variety of global and/or regional scale gridded precipitation products derived through various means is currently available for climate change and hydrological assessment studies. The most common and widely used products can broadly be classified into four categories; (i) based on climate models' reanalysis, (ii) merged model (reanalysis) and station observations, (iii) merged satellite estimates and station observations, and (iv) derived solely from station observations. In this study, we selected at least one dataset from each basic category to underline the inherent uncertainties in these datasets and highlight the importance of their bias correction before use in hydro-climate studies in the study area.

ERA-Interim: ERA-Interim (Dee et al., 2011) is a third generation global atmospheric reanalysis product with an improved atmospheric model and assimilation system, produced by the European Centre for Medium- range Weather Forecasts (ECMWF) providing data from 1979 to present. Estimates of precipitation associated with the reanalysis are produced by the forecast model, based on temperature and humidity information derived from assimilated observations. These data are available at sub-daily, daily and monthly intervals and at spatial resolution of $0.75^{\circ}$ latitude-longitude grid, but we used monthly means of daily means re-gridded at $0.125^{\circ}$ available at http://apps.ecmwf.int/ datasets/data/interimfull-moda/, accessed in January, 2015. Berrisford et al. (2011) provides a detailed description of the ERA-Interim product.

WFDEI: The WATCH Forcing Data-ERA Interim (WFDEI) dataset (Weedon et al., 2014) is derived from ERA-Interim reanalysis product (Dee et al., 2011) via sequential interpolation to a $0.5^{\circ}$ resolution, elevation correction and monthly-scale adjustments based on CRU TS3.1/TS3.21 (Harris et al., 2013) and GPCCv5/v6 (Schneider et al., 2013) monthly precipitation observations for 1979-2012 combined with new corrections for varying atmospheric aerosol-loading and separate precipitation gauge corrections for rainfall and snowfall under the Water and Global Change (WATCH) programme of the European Union. The WFDEI is an open access dataset at ftp://rfdata:forceDATA@ftp.iiasa.ac. at/. We accessed the data in December, 2014 and used CRU TS3.1/ TS3.21 adjusted WFDEI product.

TRMM: The Tropical Rainfall Measuring Mission (TRMM), launched in November 1997 as a joint project by NASA and the Japanese Space Agency (JAXA), is instrumented with Precipitation Radar (PR), TRMM Microwave Imager (TMI), and Visible Infrared Scanner (VIRS). The PR provides three-dimensional maps of storm structure giving information on the intensity, distribution and type of rain, storm depth and the height at which the snow melts into rain. The TMI quantifies water vapor and cloud water content as well as the rainfall intensity in the atmosphere, while the VIRS provides the cloud context of the precipitation and connects microwave precipitation information to infrared- based precipitation estimates from geosynchronous satellites. The TRMM Multi-satellite Precipitation Analysis (TMPA) combines all the available precipitation datasets from different satellite sensors and monthly surface rain gauge data to provide a "best" estimate of precipitation at spatial resolution of $0.25^{\circ}$ for the $50^{\circ} \mathrm{N}-\mathrm{S}$ areas (Huffman et al., 2007). We used TRMM 3B43 version 7 monthly precipitation product released by TMPA in May 2012. Huffman et al. (2007) provide detailed information on the algorithms and different processing steps. The dataset available at http://disc.sci.gsfc.nasa.gov/daacbin/DataHoldingsPDISC.pl?LOOKUPID_List=3B43 was accessed in December, 2014. 
APHRODITE: Asian Precipitation-highly Resolved Observational Data Integration Towards Evaluation of Water Resources (APHRODITE) is the state-of-the-art highresolution daily precipitation dataset developed by a consortium between the Research Institute for Humanity and Nature (RIHN) Japan and the Meteorological Research Institute of Japan Meteorological Agency (MRI/JMA) from a dense rain gauge observational network in Asia. We used the latest and improved version of daily dataset for Monsoon Asia (APHRO_MA_V1101) covering 60.0E-150.0E, 15.0S-55.0N at a high spatial resolution of $0.25^{\circ}$ for the period extending from 1951-2007 (Yatagai et al., 2012). The precipitation data from a dense network of rain gauges is $1^{\text {st }}$ interpolated on to a grid of $0.05^{\circ}$ using the modified version of the distance-weighting interpolation method (Shepard, 1968), which considers sphericityand orography by the Spheremap (Willmott et al. 1985) and the Mountain Mapper (Schaake, 2004) methods respectively. This dataset is then regridded to $0.25^{\circ}$ and $0.5^{\circ}$ products using the area-weighted mean. The algorithm is improved in that the weighting function considers the local topography between the raingauge and interpolated point (Yatagai etal., 2012). The veryhigh resolution $\left(0.05^{\circ}\right)$ dataset is restricted to the partner institutes only and is not publicly available. Therefore, we used the latest and improved version (APHRO_MA_V1101) with spatial resolution of $0.25^{\circ}$. The dataset, available at http://www. chikyu.ac.jp/precip/, was accessed in July, 2014.

\subsubsection{River Flows}

Historical daily discharge data at the sub-basin level for twelve stations (Figure 2.2; Indus at Kharmong, Shyok at Yugo, Shigar at Shigar, Hunza at Dainyor, Gilgit at Gigit, Astore at Doyian, Indus at Tarbela, Chitral at Chitral, Swat at Chakdara, Panjkora at Zulam bridge, Jhelum at Mangla, and Chenab at Marala) in the study area are available from WAPDA. The current study used river discharge data for the 1998-2012 period for consistency with the observed and gridded precipitation products.

\subsubsection{Methods}

The pre-processed void free Shuttle Radar Topography Mission (SRTM) digital elevation data of $90 \mathrm{~m}$ resolution freely available from http://hydrosheds.cr.usgs.gov/ are used to delineate the watershed boundaries according to the methodology explained by Khan et al. (2014). However, for consistency with the precipitation datasets, the boundaries are also delineated from $1 \mathrm{~km}(30 \mathrm{~s})$ DEM available from the same site.

We selected all the stations that covered at least three years of data to cover the recent installations and keeping in view the paucity of the observed data. Daily precipitation observations were converted into monthly totals if no more than three days were missing in a month. Similarly, seasonal and annual totals were calculated if no month was missing in a season or year. The study used station observations of average monthly, seasonal and annual precipitation totals from 134 points located within the study area to analyze altitudinal dependency and derive spatiotemporal distribution of precipitation averaged over the 1998-2012 period. In order to appraise the influence of elevation on precipitation, the average annual precipitation of a group of stations located within or closest to each subbasin for the common time period are plotted.

For estimation of precipitation distribution, we selected the best suited spatial interpolation scheme based on literature review and specific geo-hydro-climatological conditions of our study area. While going through the literature, we noticed that with wide and increasing 
applications of the spatial interpolation methods, there is also a growing concern about their accuracy and precision for a given set of conditions (Hartkamp et al., 1999). In general, when quality and amount of sampled data is sufficiently high, most of the spatial interpolation methods are accurate and produce almost similar estimates (Burrough and McDonnell, 1998). Minasny and McBratney (2007) however argued that improvements in prediction rely more on representativeness and quality of input data rather than on more sophisticated methods. A thorough review of spatial interpolation methods by Li and Heap (2014) could not infer any simple answer or consistent findings regarding the choice of best method, but it provided guidelines and suggestions by describing and comparing the features, strengths and weaknesses of a number of interpolators. Li and Heap (2011) analysed the performance of 32 spatial interpolation methods and observed that their performance depends not only on the structure of the method itself, but also on the nature of interpolating surface as well as quality and amount of the input data. They found kriging methods better than nongeostatistical methods and recommended Kriging with External Drift (KED) method. Many other studies (e.g. Tobin et al., 2011; Haberlandt, 2007; Verfaillie et al., 2006; ICES, 2005; Hengl et al., 2003; Rivoirard and Wieland, 2001; Bourennane et al., 2000; Bishop and McBratney, 2001; Goovaerts, 2000) also compared different geostatistical and non-geostatistical methods in a variety of situations and noticed that Kriging with KED usually provided better estimates than all other methods.

The KED interpolation method (Schabenberger and Gotway, 2005) allows the processing of non-stationary random functions taking into account the spatial dependence of a primary variable known only at a small set of points as well as its linear relation to one or more additional covariates (secondary variables/predictors) exhaustively known at all points over the whole domain. It uses semivariograms or covariances, cross-covariance, transformations, trend removal, and allows for error/ uncertainty check. It is most appropriate when there is an overriding trend in the sampled data, which can be modelled by a deterministic polynomial function. Moreover, Masson and Frei (2014) observed simple one-predictor KED model markedly better than the multilinear regression model with nine predictors and noticed only marginal improvement with inclusion of complex physiographic predictors. Therefore, we selected KED interpolation method with elevation as a predictor to predict unknown values from these observations, as our study area is largely an under-sampled and complex high-mountain terrain exposed to three main circulation systems leading to reasonable spatial (directional) and altitudinal biases in precipitation distribution.

The KED model includes a component of spatial autocorrelation and a component for multilinear dependence on pre-defined variables (predictors). It considers the observations (Y) at sample locations (s) as a random variable of the form (e.g. Diggle and Ribeiro, 2007):

$$
\begin{aligned}
& Y(s)=\mu(s)+Z(s) \\
& \mu(s)=\beta_{0}+\sum_{k=1}^{K} \beta_{k} \cdot x_{k}(s)
\end{aligned}
$$

Here, $\boldsymbol{\mu}(\boldsymbol{s})$ describes the deterministic component of the model (external drift or trend) and is given as a linear combination of $\mathrm{K}$ predictor fields $\boldsymbol{x}_{\boldsymbol{k}}(\boldsymbol{s})$ (trend variables) plus an intercept $\boldsymbol{\beta}_{\mathbf{0}}$. The $\boldsymbol{\beta}_{\boldsymbol{k}}$ are denoted as trend coefficients, while $\boldsymbol{Z}(\boldsymbol{s})$ describes the stochastic part of the KED model and represents a random Gaussian field with a zero mean and a $2^{\text {nd }}$ 
order stationary covariance structure. The latter is conveniently modelled by an eligible parametric semi-variogram function describing the dependence of semi-variance as a function of lag (possibly with a directional dependence). To derive the climatology of mean monthly, seasonal and annual cycle of precipitation from the point observations, we applied KED interpolation method with elevation as a predictor separately for monthly, seasonal and annual precipitation totals averaged over the period of 1998-2012. The KED-based estimated precipitation distribution was further converted into grid format (1 km size) for sub-basin scale precipitation computations and comparisons with the gridded datasets.

Daily river discharge data at the available outlets are used to compute the average monthly, seasonal and annual specific runoff (measured flow/drainage area) for each sub-basin. The KED-based estimated annual precipitation totals from each sub-basin are validated by the corresponding average specific runoff and the pattern of glacier cover using ICIMOD glacier inventory (Bajracharya and Shrestha, 2011) and compared with earlier studies.

The selected gridded precipitation products are re-gridded and processed to compute mean monthly, seasonal and annual precipitation totals at sub-basin scale. Afterwards, their accuracy relative to the KED-based estimated precipitation is evaluated for each subhydrological basin. For evaluation of precipitation patterns, the Taylor diagram is used for the re-gridded precipitation values ofallthe products to acom- mon grid of $0.05^{\circ}$; while for quantitative assessment, the seasonal and annual biases relative to the KED-based estimated precipitation at the sub-basin scale are analysed.Basin-wide seasonaland annual correction factors are introduced to account for the inherent errors in each gridded product.These correction factors are determined by dividing the respective grid values of the estimated precipitation by the gridded datasets and averaging them at sub-basin level. These factors simply need to be multiplied with the respective gridded datasets for the area of interest.

\subsubsection{Uncertainty Analysis}

The major uncertainties involved in this study are associated with the quality and amount of the observed data and the interpolation technique used to predict the unknown values from these observations. The organizations operating weather stations in the study area generally indicate to apply WMO standards for collection of meteorological data. Yet, in many cases, the quality of data is affected by instrumental problems, station locality and interruption of timeseries (Miehe etal.,1996). PMD, WAPDA and Ev-K2-CNR use the tipping bucket rain gauges to record liquid precipitation in the low- to mid-altitude areas. In the case of occasional snowfall, the water equivalent calculated manually is usually added to the daily precipitation records. The automatic data collection platforms (DCPs) installed by WAPDA in the high-altitude areas during 1994-95 use snow pillows to measure both solid and liquid precipitation as water equivalent. However, most of the installed snow pillows encountered technical issues of interfacing with the transmission system as well as unexpected "jumps" due to possible ice bridging and rupture effects (SIHP, 1997). Although, the problem was substantially minimized in 1996 by attaching a precision potentiometer to convert the shaft encoders from a digital output to an analogue, the snow pillows are still subjected to underestimate solid precipitation under strong wind conditions (Hasson et al., 2014). The automatic weather stations installed within the framework of the CAK project measure precipitation using data logger, tipping bucket and snow depth gauge (Miehe et al., 1996). Yet, measurement of solid precipitation in strong windy conditions is subject to considerable errors due to constant blowing away of snow from the ultrasonic sensors. GHCN-monthly 
summaries of the observed precipitation for the study area are based on data from IMD, which also follows WMO standards, and are subjected to a suite of quality assurance reviews.

Another source of uncertainty is inconsistency in the precipitation observations due to late installation of instruments, temporary sensor failures or non-collection of data. The time series of the observed data is variable, ranging from more than 30 years for a few stations to at least 3 years for the most recently installed stations (Table S-2.1). We used average precipitation during the period of 1998-2012, because the majority of data is available for this period except the GHCN dataset, which contains precipitation data of some old observatories operational between 1901 and 1970. To check for possible temporal change, we compared these stations' records with the nearest stations with up to date data. We only found an insignificant trend. Similarly, the net precipitation estimated from glacier accumulation studies is also inconsistent in temporal terms.

KED interpolation model produces both prediction as well as error/ uncertainty surfaces, giving an indication or measure of how good the predictions are. It estimates an interpolated surface from randomly varied small set of measured points and recalculates estimated values for these measured points to validate the estimates and determine extent of errors. Since, we used all of the available observations; there is no more ground truth available to validate the performance of this method. However, we used leave-one-out cross validation strategy to assess the performance of the employed interpolation scheme. We applied cross validation on the observed and predicted values from all the stations to assess the errors/uncertainty associated with the interpolation scheme by using error scores of the relative bias (B) and the relative mean root-transformed error (E), which are defined as:

$$
\begin{aligned}
B & =\frac{\sum_{i=1}^{n} P_{i}}{\sum_{i=1}^{n} o_{i}} \\
E & =\frac{\frac{1}{n} \sum_{i=1}^{n}\left(\sqrt{P}_{i}-\sqrt{o}_{i}\right)^{2}}{\frac{1}{n} \sum_{i=1}^{n}\left(\sqrt{\bar{o}}-\sqrt{0}_{i}\right)^{2}}
\end{aligned}
$$

Here $\mathrm{P}_{\mathrm{i}}$ and $\mathrm{O}_{\mathrm{i}}$ are the predicted and observed precipitation values respectively, while $\overline{\boldsymbol{O}}$ is the spatial average of the observations over all (or a subset of $n$ ) stations. The crossvalidation results (Table 2.2) depict relative bias values of slightly higher than for all months, indicating only a small overestimation of the predicted values but at annual scale it is almost zero. Similarly, E values less than 1 suggest typical errors smaller than the spatial variations except for pre-monsoon season. In summary, there are no serious uncertainties or constraints but further improvements in the estimated precipitation distribution can be achieved by using higher quality observed data with more spatiotemporal coverage, particularly at higher-altitudes.

Table 2.2: Relative bias (B) and relative mean root-transformed error (E) calculated over all observation points. PMSN is pre-monsoon (Apr-Jun), MSN is monsoon (Jul-Sep), WIN is winter (Oct-Mar) and ANN is annual.

JAN FEB MAR APR MAY JUN JUL AUG SEP OCT NOV DEC PMSN MSN WIN ANN

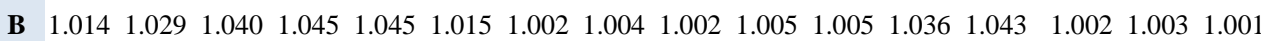

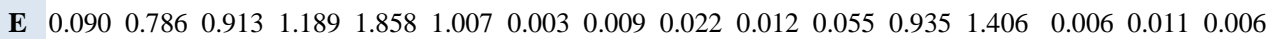




\subsection{Results}

\subsubsection{Altitudinal variation of precipitation}

The analysis of observed precipitation records revealed significant altitude dependency of precipitation in all the sub-basins (Figure 2.3), which supports earlier studies (e.g. Pang et al., 2014; Hewitt, 2011; Winiger et al., 2005; Weiers, 1995; Wake, 1989; Dhar and Rakhecha, 1981; BIG, 1979; Decheng, 1978). However, there is substantial difference in the rate and magnitude of variation from one basin to another due to significant directional bias (spatial autocorrelation) and influence of highly diversified orography (topography and exposure) interacting with multiple weather systems. Therefore, the complex altitudinal variation of precipitation in the high-altitude Indus basin cannot be represented by a single relation. Such an elusive behaviour of precipitation gradient was also found by Immerzeel et al. (2014) in Nepalese Himalayas, where a uniform valley wide precipitation gradient could not be established due to influence of several scale-dependent mechanisms. Although, we attempted a separate analysis for each sub-hydrological basin, yet the spatial variability in each sub hydrological basin is so high that the number of available observations is inadequate to infer an accurate distribution of altitudinal precipitation. Rather complex and nonlinear trend of precipitation increase with altitude is evident in most sub-basins. The south-west TP and eastern Karakoram regions display an elusive trend mainly due to higher variability and a small number of observation points. Astore and Chitral basins depict mixed trend, while Shigar, Hunza and Gilgit basins infer relatively strong positive vertical gradients. The southern basins like Chenab, Jhelum, Swat and Lower most reach of Indus main experience the zone of maximum precipitation at an altitude of around $2500 \mathrm{~m}$. Pang et al. (2014) and Dhar and Rakhecha (1981) also observed that the monsoon precipitation above $2400 \mathrm{~m}$ elevation in the central Himalayas decreases significantly with rising elevation. The height of maximum precipitation in rest of the subbasins is not clear but tends to increase with latitude. Hence, the assumptions of linear increase in precipitation with elevation by the earlier studies (e.g. Immerzeel et al., 2012; Mayer et al., 2006 and Winiger et al., 2005) could not be confirmed by this study as the available observations are highly inadequate to infer an accurate distribution of altitudinal precipitation.

\subsubsection{Spatial interpolation of precipitation observations}

The KED-based interpolation of the point observations revealed some important characteristics of precipitation distribution in the study area. Monthly distribution of precipitation indicates largely bimodal weather system in the study area reflecting the wintertime precipitation associated with the westerly systems and the impact of Indian summer monsoon. The south-western Himalayan catchments (Chenab, Jhelum \& Indus-L) are dominated by the summer monsoon but also receive considerable amounts of precipitation during winter and pre-monsoon seasons. The Hindukush and Karakoram basins receive most of their precipitation during winter (40-60\%) and pre-monsoon (25-45\%) seasons. The winter precipitation usually strengthens in December, peaks in March and starts receding during April and is very important for accumulated summer flows particularly in the Hindukush and Karakoram regions (Figure 2.4). 

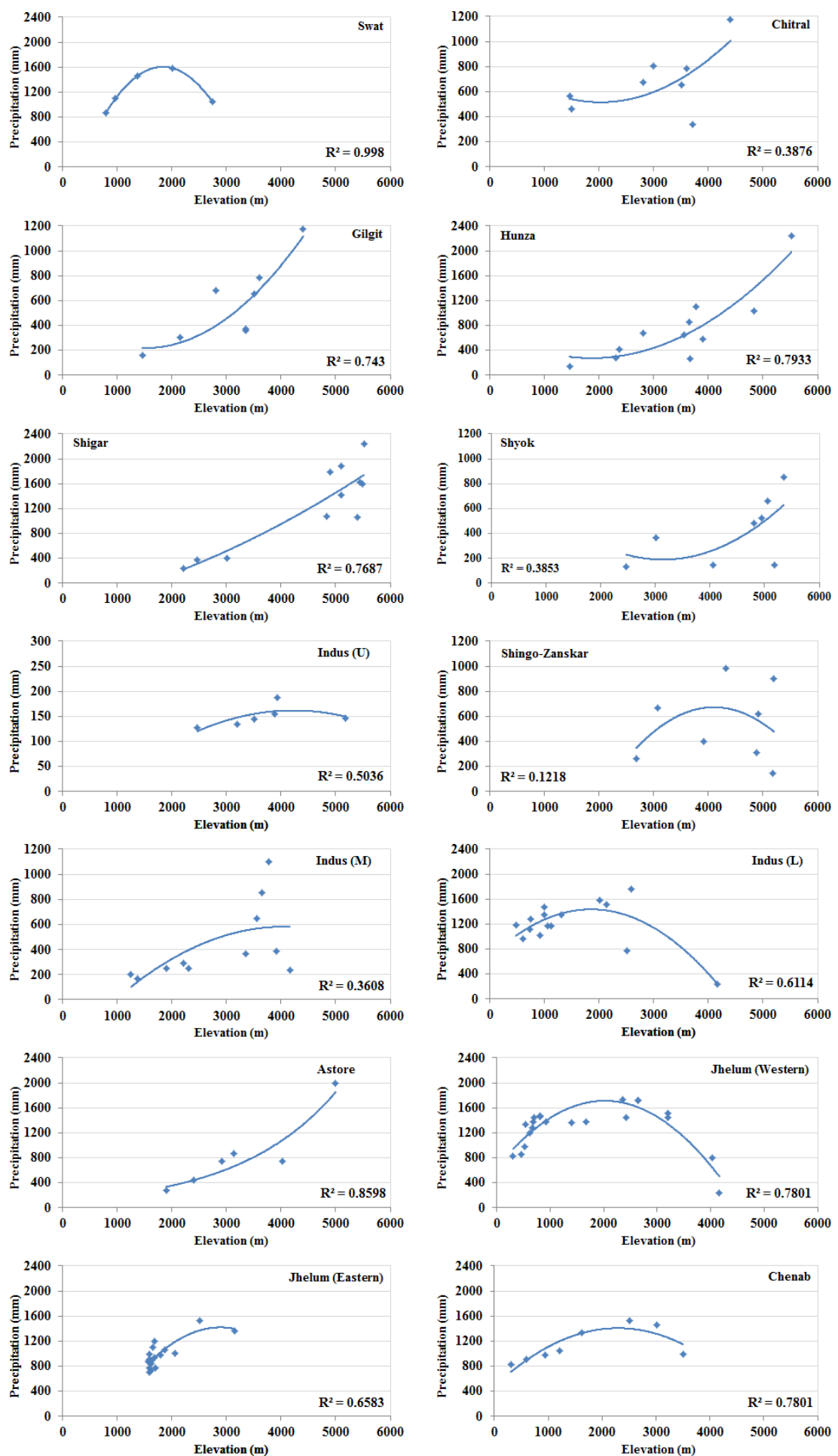

Figure 2.3: Altitudinal variation of annual precipitation in each sub-basin. 

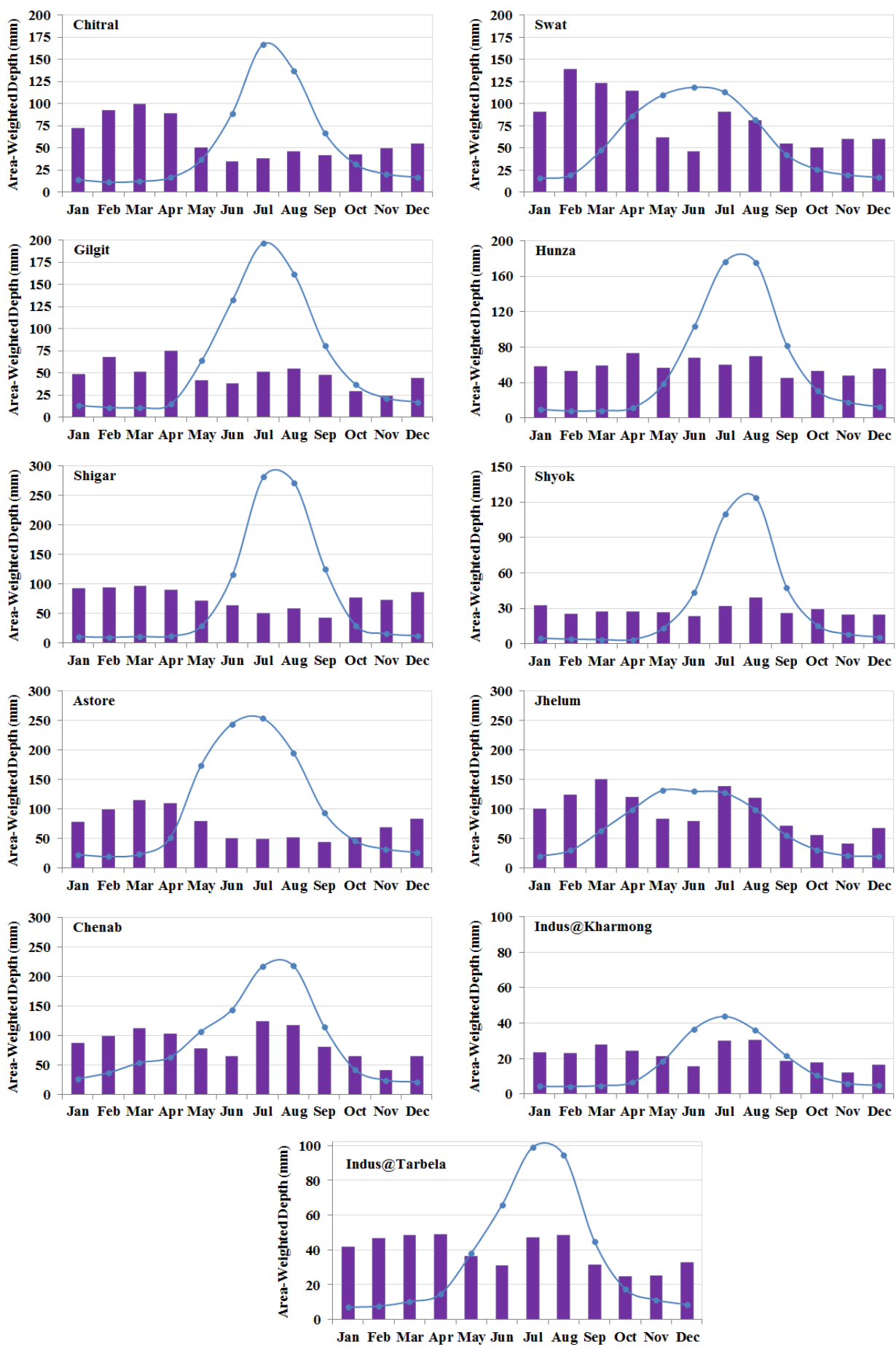

Figure 2.4: Monthly distribution of area-weighted depths of estimated precipitation and specific runoffs at sub-basin level. 
The hydrographs of estimated precipitation and specific runoff (Figure 2.4) indicate dominancy of snow/glacier melt contribution during May-September. Since, snowfields and glaciers often perform an important function of regulating stream flows, the downstream areas usually receive heavy floods whenever higher precipitation in winter season is followed by a relatively warm and wet monsoon season. Due to varying inputs of precipitation and snowmelt components, there is large variability in the amount (depth) of peak flows from different sub-basins but the timing tends to be in late July for most of the basins. Generally, the river flows are very low during winter, start rising in May, peak in July-August and descend sharply until the start of next winter. The high-altitude western and northern basins (Chitral, Gilgit, Hunza Shigar, Shyok, Indus at Kharmong and Astore) are more dominated by snow/glacier melt while the low-altitude southern basins (Swat, Indus-lower, Jhelum and Chenab) receive substantial flows from direct rainfall.
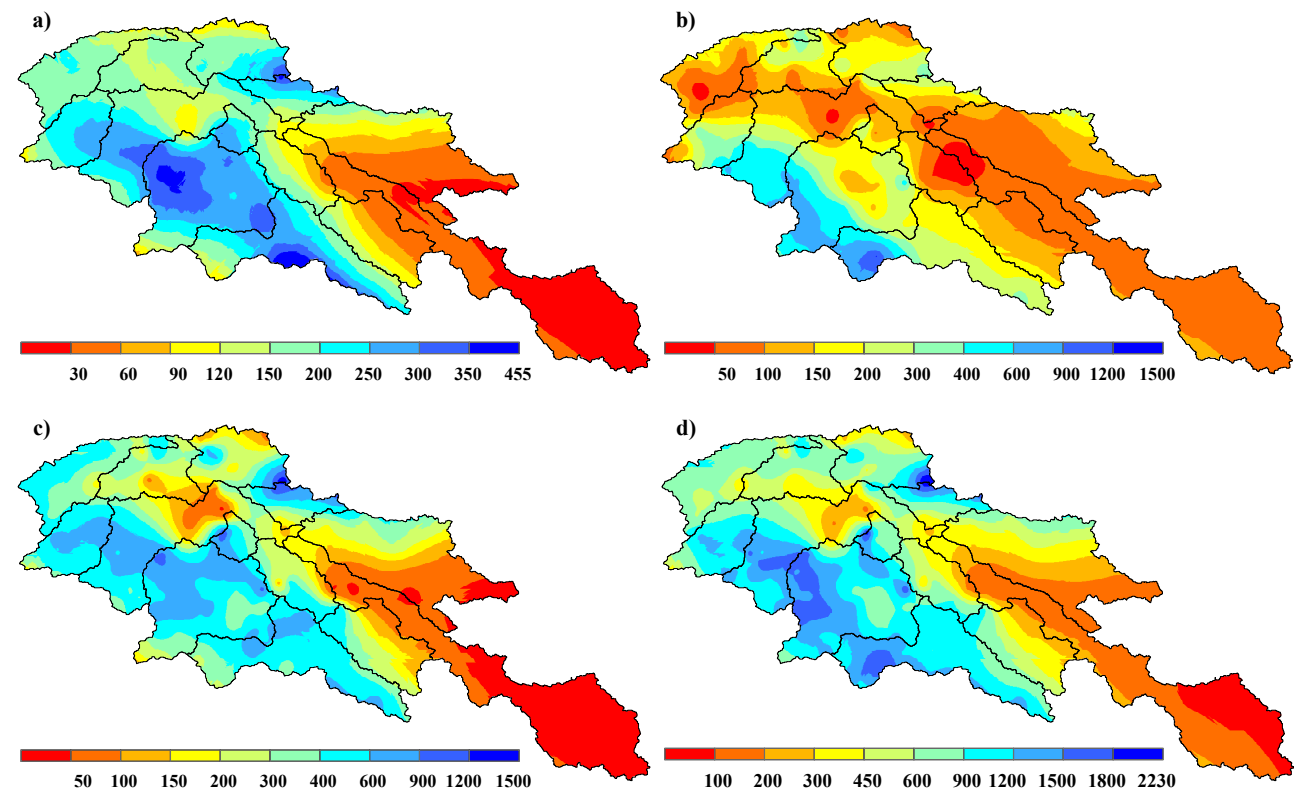

Figure 2.5: Spatial distribution of KED based estimated precipitation for a) pre-monsoon (Apr-Jun), b) monsoon (Jul-Sep), c) winter (Oct-Mar) and d) annual basis. All values are in mm (note different scales for each panel).

The estimated precipitation distribution (Figure 2.4 and 2.5) signifies the key features of mean annual cycle and seasonality of precipitation. Moisture-laden westerly winds are intercepted by high mountains in the west and north, leading to moisture condensation and precipitation at higher altitudes. As such, winter precipitation tends to be stronger in Chitral, Swat, Gilgit, Hunza, Astore and Shigar basins, which receive significant precipitation in the form of snowfall during winter and spring (pre-monsoon) seasons. The Indian summer monsoon mainly dominates at southern parts (i.e. Chenab, Jhelum, Swat and Indus-lower basins). Northwardly oriented Astore, Shingo and Zanskar basins are on the leeward side of western Himalayan range and thus receive lower precipitation as compared to Chenab and Jhelum basins in monsoon season. The Tashain glacier and Nanga Parbat massif located in the south-west of Astore basin hinder further north-west movement of the monsoon. However, stronger storms often divert northwardly and penetrate in to the central Karakoram region. Highly elevated boundary between Chenab and Zanskar basins 
hardly allows monsoon rains to penetrate further northward; as such the Zanskar range and Ladakh region in the TP are relatively drier. The East Asian summer monsoon seldom reaches to the Karakoram from the east. However, whenever it does penetrate significantly, it interacts dramatically with the features of the already present Indian summer monsoon and westerly systems causing heavy downpours and extensive floods (e.g. Jul-Aug 2010 floods in Pakistan). The Indus main up to Chilas (climatic station number 5 in Figure 2.2), which remains under the rain shadow of the surrounding high mountains on both sides, is least affected by both summer monsoon and western disturbances.

\subsubsection{Validation of KED-based estimated precipitation}

The basin-wide KED-based estimated precipitation is validated by the specific runoff (measured flow/drainage area) of respective subbasins (Figure 2.6a). The specific runoff in snow/glacier fed basins is usually affected by precipitation losses and the dynamics of snow/glacier mass balance as the river flows are often regulated by changes in storage of snow/glacier mass. In the absence of comprehensive and reliable mass balance estimates, the estimated precipitation and the corresponding specific runoffs can be used to infer the change in snow/glacial mass balance. Positive changes in storage are expected when the net precipitation (excluding losses) is markedly greater than river runoff. Conversely, higher runoff compared to the net precipitation may point to loss of storage indicating negative mass balance. However, reliable estimates on evapotranspiration, interception, sublimation and percolation losses in the study area are lacking, forcing earlier studies (e.g. Immerzeel et al., 2009; Tahir et al., 2011) to ignore these losses. The assumption that these components in water balance studies may be negligible particularly in the Karakoram region are supported by the fact that the majority of the landscape in this region is rocky with scarce vegetative cover resulting in minor evapotranspiration, interception and percolation. Nevertheless, these losses will result in reduced net precipitation. We used net precipitation from the glacier accumulation zones, which already excludes the losses from snowfields and glaciated areas.

(a)

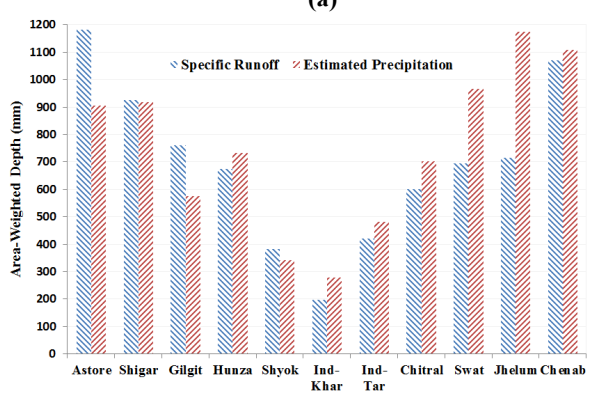

(b)

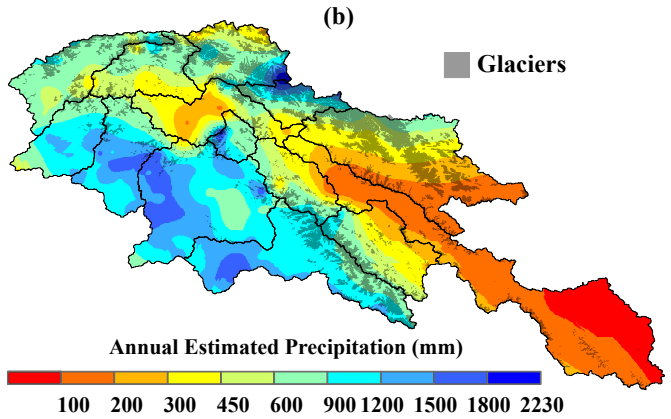

Figure 2.6: Validation of estimated precipitation with specific runoff (a) and glacier cover (b).

Moreover, there may be some compensating errors because the solid precipitation in the high-altitude and windyareas is generally susceptible to undercatch by $20-50 \%$ (Rasmussen et al., 2012). Therefore, we assume that the potential losses (evapotranspiration, sublimation, interception and deep percolation) and possible gains (undercatch of snowfall) cancel each other out and the net difference is insignificant particularly in the Karakoram and north-west Hindukush regions. Another approximation to validate the estimated 
precipitation is superimposition of glacier cover over the estimated precipitation (Figure 2.6b) since an adequate amount of precipitation is essential to sustain and surge the glaciers in this area. The estimated precipitation coherently follows the pattern of glacier cover in high-altitude areas except the eastern Shyok basin. Finally, the KED-based estimated precipitation is compared with the estimates of earlier studies derived either from station observations or gridded datasets. The comparative analysis, summarized in Table 2.3, shows that the precipitation estimates by earlier studies are highly contrasting but consistent in underestimating precipitation in majority of the areas. These earlier studies have used non-representative precipitation data and/or overestimated basin boundaries resulting in highly biased precipitation estimates.

Table 2.3: Comparison of KED-based estimated precipitation with the estimates of earlier studies

\begin{tabular}{|c|c|c|c|}
\hline River Basin & $\begin{array}{c}\text { Precipitation } \\
(\mathbf{m m})\end{array}$ & Dataset Used & Reference Study \\
\hline \multirow{3}{*}{$\begin{array}{l}\text { Indus- } \\
\text { Kharmong }\end{array}$} & 388.0 & Terrestrial Precipitation V2.01 & Mukhopadhyay, 2012 \\
\hline & 277.3 & Station data + KED interpolation & This Study \\
\hline & 161.0 & APHRODITE $* 1.17$ & Lutz et al., $2014 \mathrm{a}$ \\
\hline \multirow{3}{*}{ Shyok } & 341.5 & Station data + KED interpolation & This Study \\
\hline & 251.2 & Terrestrial Precipitation V2.01 & Mukhopadhyay, 2012 \\
\hline & 175.5 & APHRODITE $* 1.17$ & Lutz et al., 2014a \\
\hline \multirow{5}{*}{ Shigar } & 917.2 & Station data + KED interpolation & This Study \\
\hline & 882.0 & India-WRIS & CWC and NRSC, 2014 \\
\hline & 550.0 & Model & Bocchiala et al., 2011 \\
\hline & 264.0 & APHRODITE $* 1.17$ & Lutz et al., 2014a \\
\hline & 201.7 & Terrestrial Precipitation V2.01 & Mukhopadhyay, 2012 \\
\hline \multirow{8}{*}{ Hunza } & 828.0 & Glaciers as proxy \& station data & Immerzeel et al., 2012 \\
\hline & 732.8 & Station data + KED interpolation & This Study \\
\hline & 692.0 & APHRODITE+Glacier as proxy & Lutz et al., 2014b \\
\hline & 582.6 & India-WRIS & CWC and NRSC, 2014 \\
\hline & 229.7 & Terrestrial Precipitation V2.01 & Mukhopadhyay, 2012 \\
\hline & 205.0 & APHRODITE $* 1.17$ & Lutz et al., $2014 \mathrm{a}$ \\
\hline & 176.0 & APHRODITE & Tahir et al., 2011 \\
\hline & 162.5 & Station Observations & Akhtar et al., 2008 \\
\hline \multirow{5}{*}{ Gilgit } & 582.6 & India-WRIS & CWC and NRSC, 2014 \\
\hline & 575.4 & Station data + KED interpolation & This Study \\
\hline & 326.0 & APHRODITE $* 1.17$ & Lutz et al., 2014a \\
\hline & 315.0 & Terrestrial Precipitation V2.01 & Mukhopadhyay, 2012 \\
\hline & 162.5 & Station Observations & Akhtar et al., 2008 \\
\hline \multirow{4}{*}{ Astore } & 904.6 & Station data + KED interpolation & This Study \\
\hline & 882.0 & India-WRIS & CWC and NRSC, 2014 \\
\hline & 496.0 & Station Observations & Akhtar et al., 2008 \\
\hline & 430.5 & APHRODITE $* 1.17$ & Lutz et al., $2014 \mathrm{a}$ \\
\hline \multirow{7}{*}{$\begin{array}{l}\text { Indus- } \\
\text { Tarbela }\end{array}$} & 675.0 & ERA-Interim, NCEP/NCAR & Reggiani \& Rientjes, 2015 \\
\hline & 671.0 & APHRODITE+Glacier as proxy & Lutz et al., $2014 \mathrm{~b}$ \\
\hline & 481.6 & Station data + KED interpolation & This Study \\
\hline & 315.0 & Terrestrial Precipitation V2.01 & Mukhopadhyay, 2012 \\
\hline & 311.0 & TRMM 3B43 & Immerzeel et al., 2009, 2010 \\
\hline & 300.0 & TRMM 2B31 & Bookhagen \& Burbank, 2010 \\
\hline & 218.9 & APHRODITE $* 1.17$ & Lutz et al., 2014a \\
\hline \multirow{2}{*}{ Jhelum } & 1175.2 & Station data + KED interpolation & This Study \\
\hline & 1052.5 & India-WRIS & CWC and NRSC, 2014 \\
\hline \multirow{2}{*}{ Chenab } & 1333.8 & India-WRIS & CWC and NRSC, 2014 \\
\hline & 1107.5 & Station data + KED interpolation & This Study \\
\hline
\end{tabular}




\subsubsection{Evaluation of the gridded products}

The gridded precipitation products often fail to capture the large and abrupt changes in precipitation over short distances due to their coarse resolution and pronounced orographic effects in the high mountain areas. In this study, we evaluated accuracy of important precipitation products derived through four different means for the high-altitude areas of the Indus basin. The spatial distribution of mean seasonal and annual precipitation totals from ERA-Interim, WFDEI, TRMM and APHRODITE products show contrasting timings and amplitudes (Table 2.4) and patterns (Figure 2.7) relative to the KED-based estimated precipitation. In quantitative terms, ERA-Interim largely overestimates precipitation in all the sub-basins except Shigar and Hunza, while the other three datasets consistently underestimate precipitation in all the areas barring Ladakh region of the TP (Indus at Kharmong). However, the inter-comparison of the four gridded products show a reasonable consistency between TRMM and APHRODITE, while WFDEI tend to be slightly different and ERA-Interim displays large overestimates. Within the ambit of overall dry bias, WFDEI gives relatively better quantitative estimates for Hindukush, Karakoram and northwestern Himalayan regions but seems less accurate for the south-western Himalaya, whereas TRMM shows opposite estimates for these areas. Similarly, TRMM gives better estimates during monsoon but WFDEI is better for the other seasons. The APHRODITE product is the least accurate among the four datasets showing strong dry bias for almost all seasons and all areas, particularly for winter and in the high-altitude catchments.

Table 2.4: Basin-wise mean seasonal and annual precipitation totals (mm) from Estimated (EST), ERA-Interim (ERAI), WFDEI (WEI), TRMM (TRM), and APHRODITE (APH) precipitation products during 1998-2012.

\begin{tabular}{|c|c|c|c|c|c|c|c|c|c|c|c|c|c|c|c|c|c|c|c|c|}
\hline \multirow{2}{*}{$\begin{array}{l}\text { River } \\
\text { Basin }\end{array}$} & \multicolumn{5}{|c|}{ Pre-monsoon } & \multicolumn{5}{|c|}{ Monsoon } & \multicolumn{5}{|c|}{ Winter } & \multicolumn{5}{|c|}{ Annual } \\
\hline & EST & ERAI & WEI & TRM & APH & EST & ERAI & WEI & TRM & APH & EST & ERAI & WEI & TRM & APH & EST & ERAI & WEI & TRM & APH \\
\hline Indus-U & 29.2 & 129.5 & 79.8 & 56.6 & 33.0 & 69.4 & 206.2 & 128.2 & 124.7 & 81.9 & 46.4 & 112.1 & 124.6 & 90.8 & 41.9 & 145.0 & 447.8 & 332.6 & 272.1 & 156.8 \\
\hline anskar & 92.6 & 247.7 & 92.7 & 84.2 & 53.3 & 126.8 & 191.7 & 132.8 & 146.8 & 80.7 & 254.8 & 339.5 & 147.5 & 131.3 & 87.5 & 474.2 & 778.9 & 373.0 & 362.3 & 221.5 \\
\hline Shingo & 135.1 & 281.3 & 110.3 & 121.1 & 78.3 & 98.0 & 174.1 & 117.2 & 119.3 & 58.1 & 322.6 & 516.6 & 208.3 & 190.8 & 115.7 & 555.7 & 972.0 & 435.8 & 431.2 & 252.1 \\
\hline Shyok & 77.0 & 148.4 & 59.4 & 59.6 & 40.2 & 100.1 & 116.8 & 27.3 & 79.1 & 41.2 & 164.4 & 157.3 & 143.0 & 69.3 & 56.8 & 341.5 & 422.5 & 229.7 & 208.0 & 138.2 \\
\hline Shigar & 224.7 & 206.8 & 88.5 & 67.6 & 90.8 & 160.4 & 120.9 & 31.7 & 101.8 & 46.8 & 532.2 & 318.5 & 202.1 & 117.2 & 87.9 & 917.2 & 646.2 & 322.3 & 286.6 & 225.5 \\
\hline Hunza & 198.6 & 251.6 & 92.4 & & 70.6 & 188.6 & & & & 46.1 & & & & & 59.0 & & 737.3 & 327.0 & 356.1 & 175.7 \\
\hline Gilgit & 156.2 & 371.7 & 133.7 & 97.8 & 133.2 & 162.2 & 234.1 & 86.3 & 109.5 & 61.6 & 257.0 & 559.8 & 286.8 & 117.1 & 83.4 & 575.4 & 1165.6 & 506.8 & 324.4 & 278.2 \\
\hline Astore & 235.5 & 352.1 & 124.6 & 129.7 & 135.6 & 153.0 & 262.1 & 116.6 & 138.8 & 64.3 & 516.2 & 590.7 & 241.1 & 173.1 & 134.5 & 904.6 & 1204.9 & 482.3 & 441.6 & 334.4 \\
\hline Indus-M & 151.9 & 362.6 & 127.9 & 94.4 & 117.6 & 101.5 & 295.2 & 111.8 & 119.2 & 53.9 & 199.4 & 557.6 & & 109.9 & 89.8 & 452.9 & 1215.5 & 481.6 & 323.5 & 261.3 \\
\hline Indus-L & 237.6 & 343.1 & 22.7 & 187.2 & 214.9 & 355.8 & 662.4 & 330.1 & 347.3 & 338.2 & 542.9 & 620.0 & 368.9 & 306.8 & 364.0 & 1136.3 & 1625.6 & 721.6 & 841.3 & 917.1 \\
\hline Indus-Tar & 115.7 & 228.0 & 102.2 & 86.0 & 77.7 & 133.1 & 231.6 & 109.6 & 134.6 & 86.9 & 232.9 & 317.7 & 191.8 & 127.6 & 94.9 & 481.6 & 777.3 & 403.6 & 348.2 & 259.5 \\
\hline Chitral & 173.2 & 344.4 & 168.2 & 104.2 & 141.9 & 124.8 & 145.2 & 104.9 & 94.6 & 54.3 & 404.4 & 595.1 & 371.9 & 218.6 & 184.2 & 702.3 & 1084.7 & 645.0 & 417.4 & 380.4 \\
\hline Swat & 218.5 & 332.1 & 218.5 & 172.8 & 200.3 & 224.9 & 620.5 & 250.1 & 234.6 & 222.6 & 522.7 & 655.7 & 393.6 & 368.7 & 415.7 & 966.1 & 1608.4 & 862.2 & 776.1 & 838.6 \\
\hline Jhelum & 278.5 & 314.8 & 181.5 & 211.9 & 179.5 & 337.6 & 496.6 & 329.2 & 370.0 & 252.1 & 559.0 & 641.2 & 295.8 & 367.4 & 333.0 & 1175.2 & 1452.6 & 806.5 & 949.3 & 764.6 \\
\hline Chenab & 242.4 & 289.9 & 140.1 & 162.7 & 137.5 & 353.6 & 401.6 & 303.3 & 427.1 & 272.6 & 511.6 & 563.4 & 198.5 & 290.8 & 288.3 & 1107.5 & 1254.9 & 641.9 & 880.6 & 698.4 \\
\hline
\end{tabular}

The pattern statistics of the mean annual precipitation in the study area (Figure 2.8) show normalized RMSE values ranging from 0.6 for APHRODITE to 0.62 for TRMM, 0.72 for WFDEI and 0.8 for ERA-Interim products. The APHRODITE and TRMM products show a relatively higher correlation coefficient of around $80 \%$ against $73 \%$ by ERA-Interim and WFDEI products. It is important to note that these statistics only evaluate the pattern of the gridded datasets. Overall, there is significant spatiotemporal (basin to basin and season to annual) bias in the precipitation totals of gridded datasets (Figure 2.9). ERA-Interim largely displays positive bias (overestimation) while the other three datasets show substantial negative bias (underestimation) in most parts of the study area. The highest negative bias is observed in the central Karakoram region consistently by all the datasets, whereas the 
positive bias is mainly concentrated in the Ladakh region. However, the estimated precipitation is very close to net precipitation, whereas the gridded precipitation products give gross precipitation amounts, which are subjected to some losses from precipitation. Hence, some room for overestimation can be permitted. Nevertheless, the extent of absolute bias suggests the importance of bias correction of the gridded datasets before their use in hydroclimate studies in the study area. To support such a bias correction, we analysed the seasonal and annual biases relative to the estimated precipitation at the sub-basin scale and introduced appropriate correction factors to account for the inherent errors in each gridded dataset. These basin-wide seasonal/annual correction factors (Table 2.5) may be multiplied with the respective gridded datasets and sub-basins. This will ensure reasonably good estimates of actual precipitation that can be used to avoid or minimize suboptimal calibration of model input parameters in the hydrological modelling/water balance studies.

a)
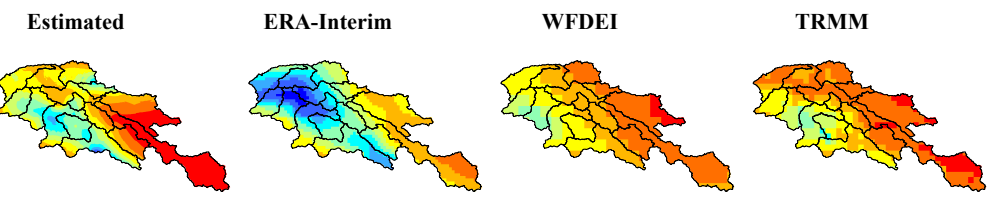

APHRODITE
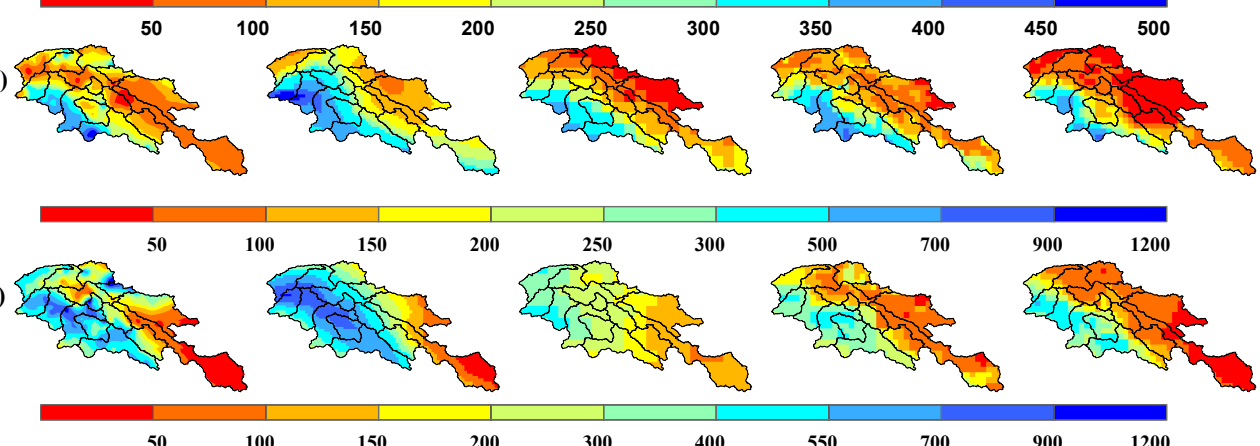

d)
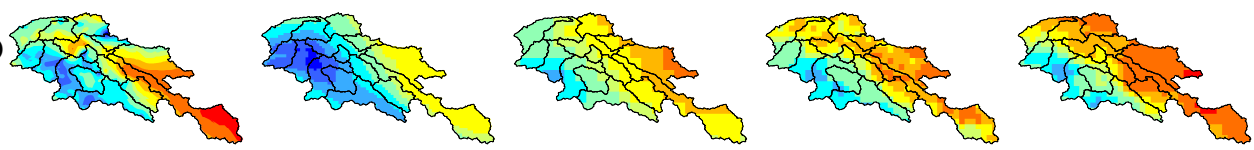

100

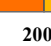

300

450

600

900

1200

1500

1800

2230

Figure 2.7: Spatial distribution of mean precipitation $(\mathrm{mm})$ by the estimated, ERA-Interim, WFDEI, TRMM and APHRODITE datasets for a) pre-monsoon, b) monsoon, c) winter, and d) annual basis. All values are in $\mathrm{mm}$ (note the different colour scales). 


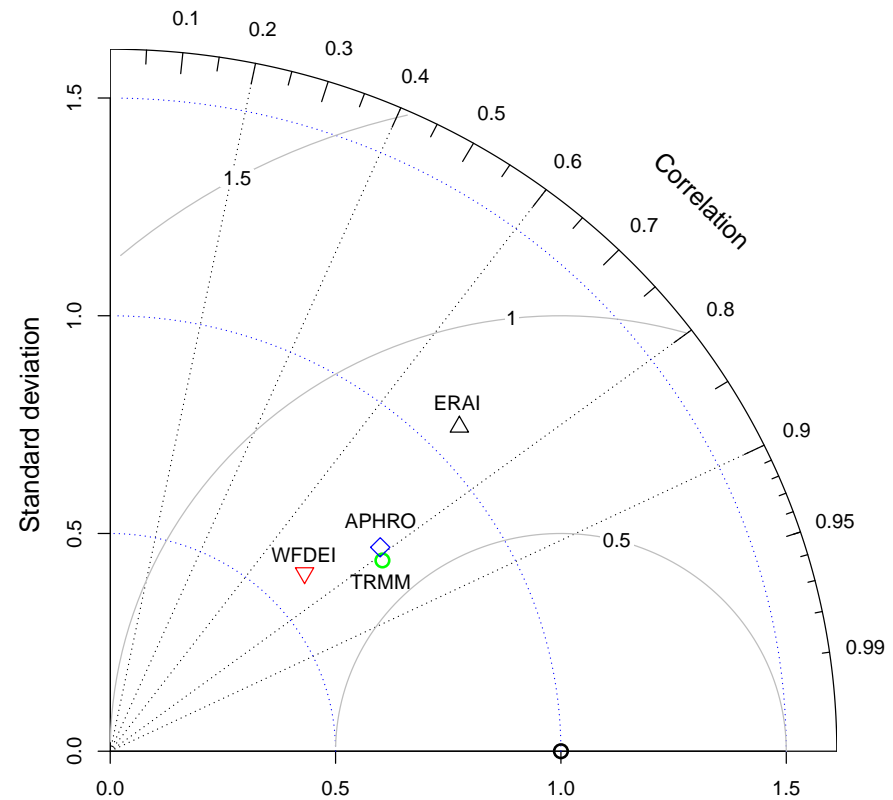

Figure 2.8: Pattern statistics of mean annual precipitation in the study area for the four gridded products. The RMSE and standard deviations are normalized by those of the estimated precipitation.

a)
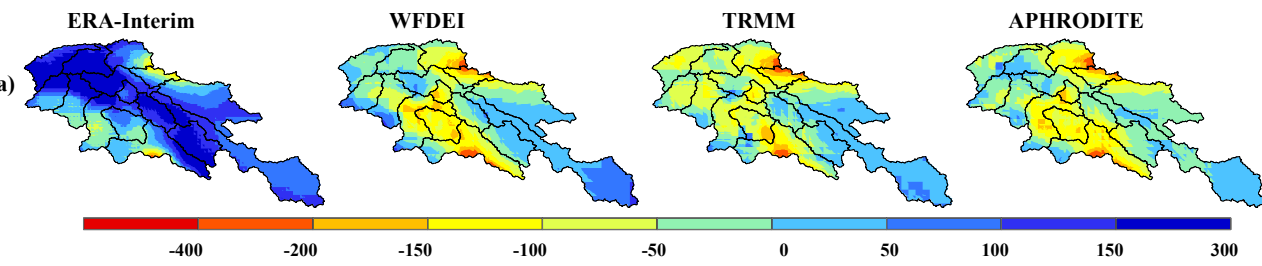

b)
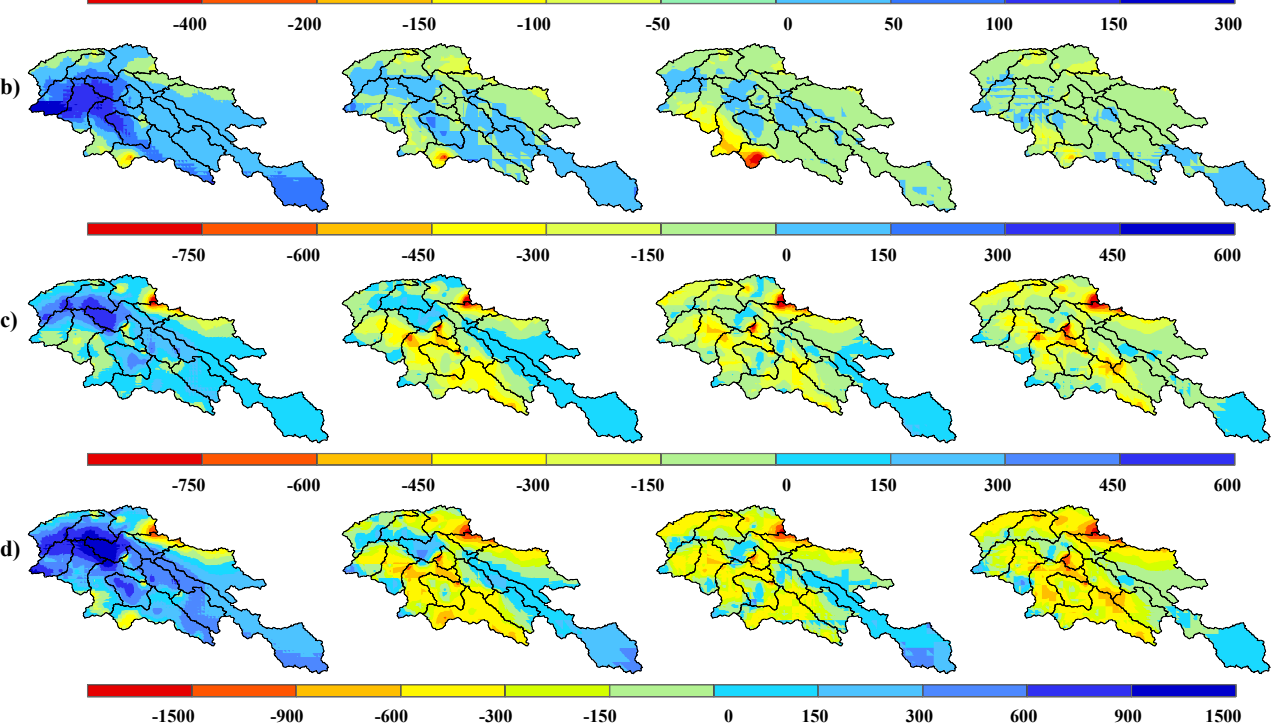

Figure 2.9: Absolute bias (in $\mathrm{mm}$ ) of ERA-Interim, WFDEI, TRMM and APHRODITE precipitation relative to the KED-based estimated precipitation for a) pre-monsoon, b) monsoon, c) winter and d) annual basis (note the different colour scales). 
Table 2.5: Basin-wide, seasonal and annual correction factors for each gridded precipitation product

\begin{tabular}{l|cccc|cccc|cccc|cccc}
\hline River & \multicolumn{3}{|c|}{ Pre-monsoon } & \multicolumn{5}{|c|}{ Monsoon } & \multicolumn{5}{c}{ Winter } & \multicolumn{5}{c}{ Annual } \\
Basin & ERAI & WEI & TRM & APH & ERAI & WEI & TRM & APH & ERAI & WEI & TRM & APH & ERAI & WEI & TRM & APH \\
\hline Indus-U & 0.21 & 0.37 & 0.52 & 0.86 & 0.36 & 0.57 & 0.63 & 0.96 & 0.47 & 0.35 & 0.59 & 1.05 & 0.32 & 0.45 & 0.59 & 0.95 \\
Zanskar & 0.33 & 0.89 & 1.01 & 1.70 & 0.61 & 0.84 & 0.83 & 1.80 & 0.66 & 1.53 & 1.72 & 2.81 & 0.59 & 1.23 & 1.30 & 2.41 \\
Shingo & 0.46 & 1.18 & 1.11 & 1.84 & 0.53 & 0.79 & 0.80 & 1.67 & 0.60 & 1.53 & 1.97 & 3.02 & 0.56 & 1.26 & 1.35 & 2.41 \\
Indus-K & 0.27 & 0.60 & 0.71 & 1.19 & 0.44 & 0.66 & 0.70 & 1.25 & 0.53 & 0.78 & 1.04 & 1.72 & 0.42 & 0.74 & 0.86 & 1.49 \\
Shyok & 0.49 & 1.22 & 1.35 & 1.73 & 0.84 & 8.74 & 1.45 & 2.32 & 1.08 & 1.03 & 2.38 & 2.61 & 0.82 & 1.53 & 1.75 & 2.41 \\
Shigar & 1.12 & 2.55 & 3.29 & 2.57 & 1.38 & 7.67 & 1.56 & 3.35 & 1.81 & 2.61 & 4.52 & 6.30 & 1.53 & 2.93 & 3.17 & 4.23 \\
Hunza & 0.77 & 2.06 & 2.27 & 2.77 & 1.07 & 9.75 & 1.62 & 3.87 & 1.12 & 1.60 & 2.33 & 5.49 & 1.04 & 2.29 & 2.11 & 4.23 \\
Gilgit & 0.42 & 1.16 & 1.58 & 1.22 & 0.74 & 2.11 & 1.46 & 2.72 & 0.48 & 0.88 & 2.23 & 3.23 & 0.52 & 1.17 & 1.79 & 2.22 \\
Astore & 0.65 & 1.85 & 1.82 & 1.75 & 0.57 & 1.26 & 1.17 & 2.37 & 0.84 & 2.08 & 3.22 & 4.11 & 0.74 & 1.86 & 2.07 & 2.82 \\
Indus-M & 0.45 & 1.24 & 1.70 & 1.33 & 0.42 & 1.05 & 0.92 & 1.93 & 0.38 & 0.85 & 1.82 & 2.21 & 0.40 & 0.99 & 1.43 & 1.73 \\
Indus-L & 0.77 & 1.09 & 1.32 & 1.11 & 0.51 & 1.03 & 1.01 & 1.11 & 0.92 & 1.51 & 2.01 & 1.80 & 0.71 & 1.27 & 1.47 & 1.37 \\
Indus-Tar & 0.47 & 1.09 & 1.30 & 1.50 & 0.64 & 3.43 & 1.07 & 1.94 & 0.76 & 1.08 & 1.84 & 2.60 & 0.63 & 1.24 & 1.43 & 2.09 \\
Chitral & 0.50 & 1.03 & 1.73 & 1.27 & 0.96 & 1.42 & 1.56 & 2.47 & 0.72 & 1.09 & 4.44 & 2.98 & 0.69 & 1.10 & 1.88 & 2.14 \\
Swat & 0.70 & 1.03 & 1.28 & 1.11 & 0.39 & 0.88 & 0.93 & 1.02 & 0.86 & 1.38 & 1.43 & 1.36 & 0.62 & 1.18 & 1.27 & 1.22 \\
Jhelum & 0.91 & 1.56 & 1.32 & 1.54 & 0.68 & 0.98 & 0.96 & 1.41 & 0.87 & 1.90 & 1.51 & 1.80 & 0.82 & 1.51 & 1.27 & 1.63 \\
Chenab & 0.84 & 1.70 & 1.47 & 1.83 & 0.80 & 1.05 & 0.76 & 1.35 & 0.87 & 2.58 & 1.75 & 2.11 & 0.89 & 1.77 & 1.28 & 1.84 \\
\hline
\end{tabular}

\subsection{Discussion}

The altitudinal analysis of precipitation distribution demonstrates the typical orographic precipitation trend, which increases up to a certain height of maximum precipitation and thereafter decreases, in most of the sub-basins. However, the basin to basin difference in the rate and magnitude of change is considerable. These results are in good agreement with earlier studies for the Chenab basin (Arora et al., 2006 and Singh et al., 1995). The altitudinal dependency of precipitation expressed by the $2^{\text {nd }}$ order polynomial functions indicates only the generalized trend of precipitation variation with altitude. The exact behaviour of precipitation is too complex to be represented by such functions. Presence of spatial autocorrelation and very high uncertainty beyond the altitudinal extent of the point observations, particularly higher than $4000 \mathrm{~m}$ which is attained by $57 \%$ of the study area, are the major complexities. Generally, precipitation tends to decrease with increasing latitude (from south to north), while longitude has seasonal influence, positive in monsoon and negative in winter season. Similarly, the southeast ward and southwestward orientated locations mostly receive more precipitation in monsoon and winter seasons respectively. However, the areas under the influence of rain shadow are notable exceptions, where precipitation tends to be far less throughout the year.

The core characteristics and spatial pattern of mean seasonal and annual precipitation estimates show strong south-north precipitation gradients containing the general rainfall maxima along the southern and lower most slopes of Chenab, Jhelum, Indus main and Swat basins (Figure 2.5), which was also observed in previous studies (e.g. Palazzi et al., 2013; Bookhagen and Burbank, 2006). However, the unique distribution revealed by this study is the emergence of an unusually wet zone containing the $2^{\text {nd }}$ precipitation maxima along the northern boundary of central Karakoram region, which had never been detected by the earlier datasets or studies. Despite the fact that this zone in the central Karakoram region accommodates some of the largest glaciers (e.g. Baltoro, Approach, Whaleback, Hispar, Biafo and Khurdopin), most of which are believed to be stable or even surging with a net positive glacier mass balance, the earlier datasets consistently and significantly underestimated precipitation in this region. However, to sustain and surge, the glaciers in this area essentially require more precipitation than their ablation/discharges. Our estimates 
of higher precipitation coherently follow the pattern and extent of the glacier cover in the high-altitude areas. Contrary to the inconsistent and contrasting estimates by the earlier studies, this study estimated significantly higher precipitation in all the sub-basins, which are comparable and consistent with the corresponding specific runoffs (measured flows). Similarly, the drier areas under the influence of rain shadow, which are often ignored and usually overestimated by the gridded datasets, are also well recognized.

The basin-wide estimated precipitation and corresponding values of specific runoff shown in Table 2.3 do not support the idea of a positive mass balance in the study area. Higher values of specific runoff for Gilgit, Astore, Shyok and Shigar basins suggest essentially a negative mass balance in these basins. Similarly, Chenab, Hunza and Chitral basins show slightly higher precipitation and may have neutral to slightly negative mass balance. Swat, Jhelum and Chenab basins indicate precipitation greater than river flows. However, evapotranspiration and percolation losses from these basins may be relatively large due to higher temperatures (large area below the $0{ }^{\circ} \mathrm{C}$ isotherm), greater vegetative cover and availability of moisture for evapotranspiration/percolation (more runoff from rainfall and seasonal snow). Thus, these basins may also be considered to have neutral to negative mass balance. The estimates for Zanskar basin and Ladakh region in the TP are relatively uncertain due to very low number of observation points in these areas. The precipitation estimates relative to the corresponding river flow for the Indus at Kharmong basin seem to be on the higher side. Therefore, a neutral to negative mass balance can be expected for this catchment. The Indus at Tarbela combines drainage of the upstream catchments, which are either neutral or experience a negative mass balance. However, the net impact is likely to be a negative mass balance as precipitation is only marginally higher than the specific runoff. Our results are in good agreement to available glacier mass balance studies (e.g. Gardelle et al., 2012; Kääb et al., 2012, 2015).

The selected gridded precipitation products provide only a marginal resemblance of the actual precipitation. ERA-Interim largely overestimates precipitation in all the sub-basins except Shigar and Hunza, while the other three datasets consistently underestimate precipitation in all the areas barring Ladakh region of the TP (Indus-U up to Kharmong). The overestimated precipitation in the TP region by the APHRODITE and TRMM 3B43 products was also observed by Palazzi et al. (2013), Prakash et al. (2013), Andermann et al. (2011) and Yin et al. (2008). ERA-Interim is prone to underestimate precipitation by up to $40 \%$ in the areas with low evaporation rates and overestimate by about $150 \%$ under conditions with high evaporation rates (Bumke, 2015). The overall underestimated precipitation by WFDEI and TRMM datasets, also observed by Li et al. (2013), may be attributed to the fact that their correction/validation is done mainly by the use of stations predominantly located in valley bottoms. This was also reported by Reggiani and Rientjes (2015) who observed uncorrected reanalysis data from ERA-Interim and NCEP/NCAR products as the better option in terms of quantitative estimates of precipitation in the UIB up to Besham Qila. Several studies (e.g. Andermann et al., 2011; Rajeevan and Bhate, 2009; Krishnamurti et al., 2009; Yatagai and Kawamoto, 2008; Yatagai and Xie, 2006) consider APHRODITE as an accurate dataset, but its accuracy greatly depends on the density of station data in the area of interest. In the high-altitude Indus basin, the APHRODITE product uses non-representative low-altitude stations to derive the spatial distribution of high-altitude precipitation. Therefore, it reflects highly underestimated precipitation in all of the sub-basins. Moreover, the four gridded products completely fail to reproduce the zone of $2^{\text {nd }}$ precipitation maxima in the central Karakoram and could not 
properly detect the drier areas under the influence of rain shadow. They tend to smooth the precipitation due to their lower spatial resolution resulting in significant overestimated precipitation in these areas. This study incorporates high-altitude observations, which have never been used in the formation or validation of precipitation datasets.

The KED-based interpolation scheme further amplifies the precipitation at the higher altitudes by taking into account the spatial autocorrelation and elevation effects at local scale. The pattern statistics indicate that despite better quantitative estimates, ERA-Interim and WFDEI products are relatively poor in reproducing the spatial pattern of estimated precipitation mainly due to their lower spatial resolution and use of nonrepresentative data in their formation and/or validation. The relatively better patterns shown by APHRODITE are due to the fact that this dataset is derived from station observations.

In view of significant biases in the gridded precipitation products covering this region, we determined basin-wide seasonal and annual correction factors for each dataset. These correction factors can be used for lumped hydrological modeling studies. Like, Lutz et al. (2014a) appropriately multiplied APHRODITE precipitation by a constant factor of 1.17 to account for the inherent underestimation and avoid undue compensation by suboptimal input parameters. However, this factor is still on the lower end as our analysis suggests an average correction factor of 2.1 for the UIB up to Tarbela dam, which varies significantly for all other sub-basins. Hence, the use of underestimated precipitation by Lutz et al. (2014a) might have resulted in an exaggerated snow/glacier melt contribution and a biased conclusion of the associated snow/glacier cover extent. Nevertheless, our KED-based precipitation estimates and correction factors can efficiently be used for bias correction of these gridded precipitation products and improved hydroclimate assessments for the study area.

Although, the methods employed in this study are straightforward and robust, further improvements in precipitation estimation can be expected once higher quality observed data with more spatiotemporal coverage, particularly above $4000 \mathrm{~m}$ a.s.l., become available. Moreover, the employed methods are equally applicable for other regions of the world, especially with similar geo-hydro-climatological conditions.

\subsection{Conclusions}

Precipitation in the high-altitude areas of the Indus basin governs the renewable water resources and associated developments, but a comprehensive assessment of precipitation distribution in this region is largely lacking. Here, we attempt to explain how precipitation amounts, seasonality and patterns are represented in the study area. The altitudinal analysis of precipitation observations in each subbasin demonstrated the important role of orographic precipitation. Yet, the topographical variability even at the sub-basin and local scale is so high that the available observations are insufficient to infer an accurate distribution of altitudinal precipitation. Instead, rather complex and nonlinear trends of precipitation increase with altitude are evidently depicted.

The study provides much improved estimates of precipitation distribution, which are comparable and consistent with the corresponding observed runoffs from the 12 sub-basins. The geo-statistical analysis of precipitation observations revealed substantially higher precipitation in most of the sub-basins compared to earlier studies. The study area largely experiences a bimodal weather system reflecting wintertime precipitation associated with the westerly systems and the impact of Indian summer monsoon. The analysis 
demonstrated two distinct rainfall maxima; $1^{\text {st }}$ along southern and lower most slopes of Chenab, Jhelum, Indus main and Swat basins, and $2^{\text {nd }}$ around north-west corner of Shyok basin in the central Karakoram. Moreover, the estimates better recognize the drier areas under the influence ofrain shadow, which are often overlooked by the gridded datasets.

Our analysis shows that the selected gridded precipitation products derived from four different sources are prone to significant errors providing only a marginal resemblance of the actual precipitation in the study area. We conclude that the uncorrected gridded precipitation products are highly unsuitable to estimate precipitation distribution and to derive glacio-hydrological models in water balance studies in the high-altitude areas of Indus basin. The suggested basin-wide seasonal and annual correction factors for the four gridded precipitation products can be useful for lumped hydrological modelling studies. The estimated precipitation distribution can effectively serve as a basis for bias correction of any gridded precipitation products for the study area.

\section{Acknowledgements}

This research work is supported by the Dutch Ministry of Foreign Affairs through the Netherlands Fellowship Program (NFP-PhD.11/898) under the budget for development cooperation and partially carried out under the Himalayan Adaptation Water and Resilience (HIAWARE) consortium supported by the Collaborative Adaptation Research Initiative in Africa and Asia (CARIAA) with funding from the UK Government's Department for International Development and the International Development Research Centre, Ottawa, Canada. The views expressed in this work do not necessarily represent those of the supporting organizations. The authors express their deepest gratitude to WAPDA and PMD for sharing the hydro-meteorological data of the study region, especially to all the anonymous observers who have collected the data. Ev-K2-CNR and GHCN are also appreciated for ensuring online availability of their climatic data. The authors also acknowledge the institutions and the teams responsible for the creation and distribution of the ERA-Interim, WFDEI, TRMM and APHRODITE precipitation products. Useful input offered by the anonymous reviewers is also duly acknowledged. 


\title{
Chapter 3
}

\section{Adjustment of Measurement Errors to Reconcile Precipitation Distribution}

\begin{abstract}
Precipitation in the high-altitude Indus basin governs its renewable water resources affecting water, energy and food securities. However, reliable estimates of precipitation climatology and associated hydrological implications are seriously constrained by the quality of observed data. As such, quantitative and spatiotemporal distributions of precipitation estimated by previous studies in the study area are highly contrasting and uncertain. Generally, scarcity and biased distribution of observed data at the higher altitudes and measurement errors in precipitation observations are the primary causes of such uncertainties. In this study, we integrated precipitation data of 307 observatories with the net snow accumulations estimated through mass balance studies at 21 major glacier zones. Precipitation observations are adjusted for measurement errors using the guidelines and standard methods developed under the WMO's international precipitation measurement intercomparisons, while net snow accumulations are adjusted for ablation losses using standard ablation gradients. The results showed more significant increases in precipitation of individual stations located at higher altitudes during winter months, which are consistent with previous studies. Spatial interpolation of unadjusted precipitation observations and net snow accumulations at monthly scale indicated significant improvements in the quantitative and spatio-temporal distribution of precipitation over the unadjusted case and previous studies. Adjustment of river flows revealed only a marginal contribution of net glacier mass balance to river flows. The adjusted precipitation estimates are more consistent with the corresponding adjusted river flows. The study recognized that the higher river flows than the corresponding precipitation estimates by the previous studies are mainly due to underestimated precipitation. The results can be useful for water balance studies and bias correction of gridded precipitation products for the study area.
\end{abstract}

This chapter has been published as:

Dahri, Z. H., Moors, E., Ludwig, F., Ahmad, S., Khan, A., Ali, I, \& Kabat, P. (2018). Adjustment of measurement errors to reconcile precipitation distribution in the high-altitude Indus basin, International Journal of Climatology, 2018;1-19. DOI: 10.1002/joc.5539 


\subsection{Introduction}

High mountain ranges around the world are important sources of freshwater storage and subsequent supplies to downstream areas. Indus basin contains one of the most diversified and complex mountain terrains in the world. Precipitation in its high-altitude areas governs the renewable water resources determining water, energy and food securities in the region. Runoff regime of the basin is predominantly controlled by winter- and summer-monsoon precipitations and summer temperatures ( $\mathrm{Yu}$ et al., 2013). Yet, there is limited understanding and reliable evidence of quantitative and spatiotemporal distribution of the key climatic variables, particularly the precipitation (Immerzeel et al., 2015; Mishra, 2015; Ragettli and Pellicciotti, 2012; Hewitt, 2005; Winiger et al., 2005) leading to a large uncertainty in the hydro-climatic predictability in the basin (Lutz et al., 2016). Overall scarcity and biased spatial and altitudinal distribution of the in-situ observations are the primary reasons for this uncertainty and knowledge gap. Substantial increase in research on glacio-hydro-climatology of the Hindukush Karakoram Himalayan (HKH) region is observed since the International Panel on Climate Change (IPCC) released its 4th assessment report, which claimed that "glaciers in Himalayas are receding faster than in any other part of the world and, if the present rate continues, the likelihood of their disappearing by the year 2035 is very high" (Cruz et al., 2007). Later, IPCC withdrew this statement due to an inaccurate citation of the grey literature. Yet, most of the subsequent research is mainly focused on improved methods using more or less the same commonly available datasets that use low-altitude and largely unrepresentative observations in the development or validation of these datasets.

Adequate monitoring of climatic variables to better represent the entire range of a diverse climate of this complex mountain terrain is essential for reducing uncertainties and inferring informed policy decisions. However, such an observational network in the study region is lacking mainly due to resource constraints and logistical limitations. To overcome the observational data gaps, the hydro-climatologists generally rely on numerous global/regional scale gridded products derived through various means (e.g. climate models reanalysis, merged model and station observations, merged satellite estimates and station observations, and derived solely from station observations). However, the strong gradients and extreme heterogeneity of this complex mountain terrain are inadequately captured by the gridded products due to their coarse resolution and use of non-representative climate data in their development or validation (Dahri et al., 2016; Reggiani and Rientjes, 2015; Immerzeel et al., 2015). As such, the precipitation estimates by a number of earlier studies (e.g. Reggiani and Rientjes, 2015; Lutz et al., 2014a; Lutz et al., 2014b; CWC and NRSC, 2014; Mukhopadhyay, 2012; Immerzeel et al., 2012a, 2010, 2009; Bocchiala et al., 2011; Tahir et al., 2011; Bookhagen \& Burbank, 2010; Akhtar et al., 2008) that used the gridded datasets show highly contrasting but consistently underestimated precipitation in most parts of the high-altitude Indus basin.

Numerous efforts to accurately estimate precipitation in this region only partially succeeded due to lack of observed data but significantly underlined the relevance and severity of the problem. In many hydrological modelling studies, the underestimated precipitation is often compensated for with other parameters like evapotranspiration and/or snow/glacier melt factors (Lutz et al., 2014a; Pellicciotti et al., 2012; Schaefli et al., 2005). This results in inaccurate and suboptimal inferences regarding precipitation distribution, snow/glacier 
cover dynamics and associated melt water contributions. Adam et al. (2006) used a water balance approach to indirectly correct monthly precipitation in mountain regions from an existing global dataset and provided reasonable approximations at basin level. However due to inaccuracies in water balance components and use of biased gridded datasets developed from limited observations, their results show large differences in precipitation amounts and distribution patterns at sub-basin scale in the study area. For example, precipitation in the high-mountain Karakorum region is largely underestimated due to lack of stations in this area, whereas higher precipitation amounts are shown for the southern parts of western Himalayan region that hosts many precipitation gauges. Lutz et al. (2014a) recognized underestimation of APHRODITE precipitation and multiplied it with an arbitrary constant factor of 1.17 to account for the inherent underestimations.

Recently, Immerzeel et al. (2015) and Dahri et al. (2016) used other sources of data/information to cover the observational gaps and provided relatively better estimates of precipitation amounts and distribution in the high-altitude Indus basin. The approach adopted by Immerzeel et al. (2015) used the glacier mass balance estimates of Kääb et al. (2012) to inversely infer the high-altitude precipitation. Using APHRODITE as the basis, they computed vertical precipitation gradients until observed mass balance matched the simulated mass balance for the 550 major glacier systems in the Indus basin. However, precipitation in the basin does not have constant and linear gradients (Dahri et al., 2016), APHRODITE precipitation distribution is highly biased (Dahri et al., 2016; Palazzi et al., 2013), and their mass balance computations are uncertain due to the use of extremely elusive direct evapotranspiration losses and negligence of percolation, interception and sublimation losses from the precipitation. Moreover, precipitation estimates of Immerzeel et al. (2015) might be affected by the overestimated basin boundaries of Shyok and Indus at Tarbela sub-basins. Whereas, Dahri et al. (2016) integrated the available station observations with the indirect precipitation estimates at the accumulation zones of major glacier systems. They employed Kriging with external drift (KED) interpolation scheme with elevation as predictor to derive the spatiotemporal distribution of mean monthly and annual precipitation climatologies. They validated their precipitation estimates by the individual station observations and the observed specific runoff at sub-basin scale. However, if the net mass balance (i.e. slightly negative as estimated by Kääb et al., 2012) and precipitation losses (direct evapotranspiration, percolation, interception and sublimation) in the basin are taken into account, the Dahri et al. (2016) estimates still seem to be on lower side.

The underestimated precipitation relative to the corresponding specific runoff in most subbasins may be attributed to three possible reasons: i) overestimated river flows, ii) significant contribution of snow/glacier melt without an adequate amount of precipitation to feed/sustain the glacier systems, and iii) underestimated precipitation. Given the technological advancements and relative precision of discharge measurement techniques and quality control ensured by the data collecting agencies, river flows are generally considered to be adequately accurate. However, there is considerable speculation but little analysis and evidence regarding the contribution of net glacier mass imbalance to the river flows. Although, Immerzeel et al. (2015) attributed the observed gap between precipitation and streamflow to the underestimated precipitation rather than the observed glacier mass balance, there is an emergent need to quantify the contribution of net glacier mass imbalance to the river flows. The underestimated precipitation by Dahri et al. (2016) is probably due to the use of net precipitation estimates from the glacier accumulation zones 
and the raw/uncorrected precipitation gauge observations which are subject to significant measurements errors (Wolff et al., 2015; Chen et al., 2015; Goodison et al., 1998; Legates and Willmot, 1990; Legates, 1987; Sevruk and Hamon, 1984).

The IPCC in its $5^{\text {th }}$ assessment report stressed the need for adjustment of precipitation measurement errors and declared that observational uncertainties in precipitation may limit the confidence in the assessment of climatic change impacts (Bindoff et al., 2013). The measurement errors in precipitation observations, particularly the wind-induced undercatch of solid precipitation in windy conditions can be substantial (Kochendorfer et al., 2017a\&b; Wolff et al., 2015; Adam and Lettenmaier, 2003). This is particularly important in the high-altitude Indus basin where moderately strong winds are a common phenomenon; temperature mostly remains below the freezing point and the majority of precipitation falls in the form of snow. Legates (1987), Legates and Wilmot (1990) and Adam and Lattenmaier (2003) adjusted the systematic biases of global precipitation products including the Indus basin but these datasets included only a few stations located in relatively dry valleys in the study area. The uncertainties in precipitation estimates may significantly affect the outcomes of hydrological/land surface models and mass balance studies. A systematic error of over $3 \%$ in rainfall measurement could lead to substantial underestimation of water in the hydrologic system (e.g. Sevruk, 1982; Biemans et al., 2009). Therefore, the systematic errors in precipitation observations must be corrected if the measurements are to be used for climate change, hydrological modelling, and water balance studies (Wolff et al., 2015; Voisin et al., 2008; Legates and Willmott, 1990). This study attempts to address the above concerns by adjustment of the systematic measurement errors in precipitation observations, adjustment of net snow accumulation for the ablation losses, and adjustment of river flows for the net mass balance contributions. The ultimate goal of this research is to facilitate creation of an accurate and consistent gridded precipitation product for the highly under-explored region of Indus basin. The results will have considerable implications for water resources planning and management in both upstream (high-altitude) and downstream (low-altitude) areas of the Indus basin.

\subsection{Study Area}

The study area covers the high-altitude catchments of the Indus river, which originates from the Tibetan Plateau (TP) and the Hindukush Karakoram Himalayan (HKH) mountain regions (Figure 3.1). The total area of the study region is about $4.03 \times 105 \mathrm{~km} 2$ of which $50 \%$ is above $4000 \mathrm{~m}$ a.s.1. and another $24 \%$ between $2500-4000 \mathrm{~m}$ a.s.l. Precipitation in the study area is influenced by multiple weather systems. The Indian summer monsoon brings moisture from the Indian Ocean and Bay of Bengal and is the dominant system in the south-eastern areas. The western disturbances originating from the Mediterranean and Caspian Sea dominate the south-western and north-western areas bringing winter monsoon during December-April months. During spring and early summer, irregular collapses of the Tibetan anticyclone sometimes allow monsoonal air masses to penetrate into the Karakoram Range (Wake, 1989). Direct transport of moisture from the Arabian Sea and local evapotranspiration also have considerable influence as about 5-40\% of the precipitation falling in the Himalayas originates from the irrigated areas in northern India and Pakistan (Tuinenburg et al., 2012; Harding et al., 2013: Wie et al., 2013). However, the hydrological cycle in the study region is usually intensified when all or some of these systems interact with each other. 


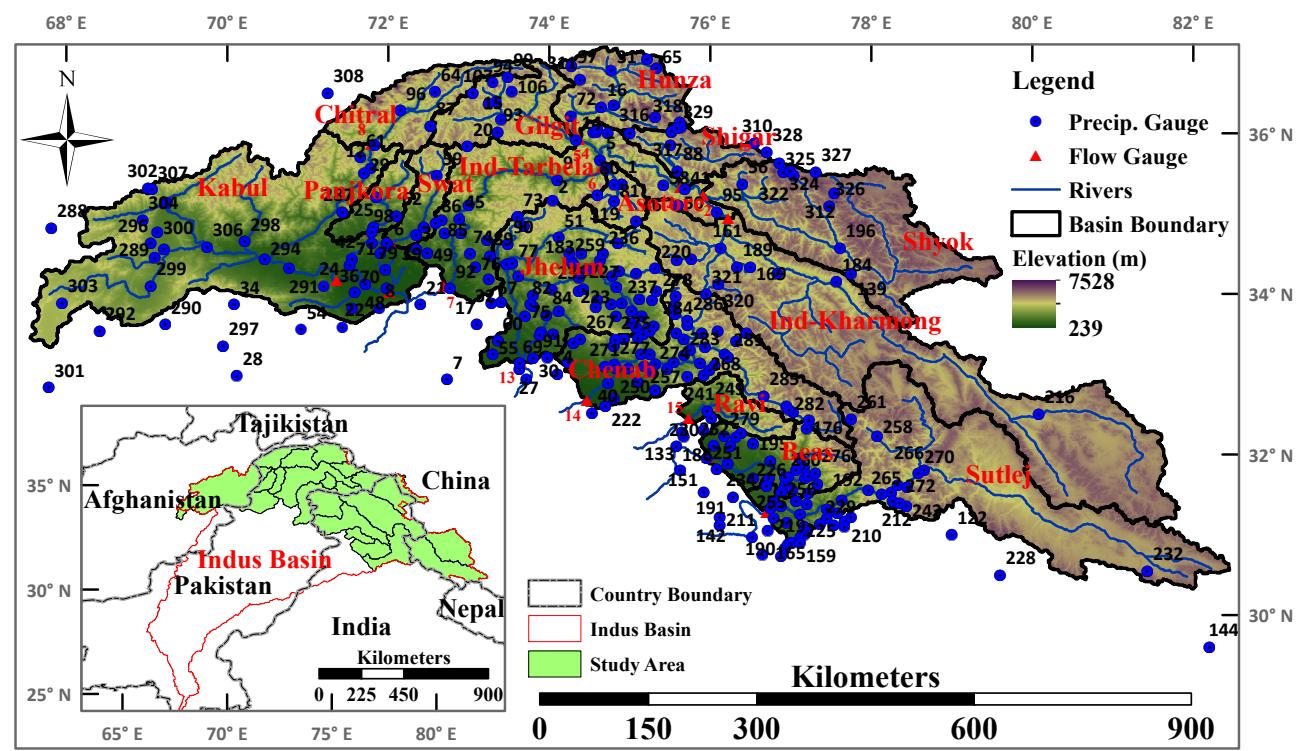

Figure 3.1 Location of study area (bottom) and description of sub-basins, river network and location of precipitation and flow measuring gauges (top). The red triangle and associated numbers refer to flow measuring gauges on various tributaries, which are (1) Indus at Kharmong, (2) Shyok at Yogo, (3) Shigar at Shigar, (4) Hunza at Dainyor, (5) Gilgit at Gilgit, (6) Astore at Doyian, (7) Indus at Tarbela dam, (8) Chitral at Chitral, (9) Panjgora at Zulum Br., (10) upper swat at Chakdara, (11) Kabul at Warsak, (12) Kabul at Nowshera, (13) Jhelum at Mangla dam, (14) Chenab at Marala, (15) Ravi at Thein dam, (16) Beas at Pong dam and (17) Sutlej at Bhakra dam. The blue circles and associated numbers refer to the precipitation gauges, details of which are given at Table S-3.1

\subsection{Data and Methods}

\subsubsection{Precipitation observations}

Indus is a transboundary river basin, as such its meteorological data are scattered in four countries (i.e. Afghanistan, China, India and Pakistan). The meteorological data of Pakistani parts were collected from Pakistan Meteorological Department (PMD) and Pakistan Water and Power Development Authority (WAPDA). Precipitation data of the station located in Afghanistan are available with Afghan-Agriculture UCDAVIS (http://afghanag.ucdavis.edu/natural-resource-management/weather), NOAA Central Library of US (https://docs.lib.noaa.gov/rescue/data_rescue_afghanistan.html) and US Geological Survey (http://edcintl.cr.usgs.gov/downloads/sciweb1/shared/afghan/downloads/documents/), while precipitation data of Indian and a couple of Chinese stations were downloaded from KNMI Climate Explorer (https://climexp.knmi.nl). In addition, we derived monthly precipitation data of many stations from Winiger et al. (2005), Miehe et al., (2001, 1996), Eberhardt (2007), Arora et al (2006), Singh and Kumar (1997), and Singh et al. (1995).

Information regarding the gauge type, use of wind shield if any, orifice area and height of the gauge orifice were taken from Sevruk and Klemm (1989), BIS (1992a\&b) and from PMD and WAPDA through personal communications. Until 1969, the most extensively used rain-gauge in India was non-recording (Symon's gauge or MK2 model) with orifice area of $127 \mathrm{~cm}^{2}$ and instrument height of $0.3 \mathrm{~m}$ (Sevruk and Klemm, 1989). Thereafter, Indian standards adopted by the Bureau of Indian Standards (BIS) for design and manufacturing of meteorological instruments are strictly followed and Indian rain-gauge 
(20-22-P) reinforced with fibreglass polyester is predominantly used (BIS, 1992a\&b). Similarly, the most widely used rain-gauge type by PMD has been non-recording MK2 (1315-C) model with orifice area of $127 \mathrm{~cm}^{2}$ and instrument height of $0.3 \mathrm{~m}$. In 2010, PMD started using its own model, which is Tipping Bucket Rain Gauge (TBRG) type equipped with logger and standalone method of monitoring rainfall, with $0.2 \mathrm{~mm}$ (moderate rain) tipping bucket, orifice area of $400 \mathrm{~cm}^{2}$ and gauge height of $0.6 \mathrm{~m}$. WAPDA uses both automatic weighing and standard meteorological service manual rain gauges. The automatic gauges have an orifice area of $127 \mathrm{~cm}^{2}$, tipping capacity of $0.254 \mathrm{~mm}$ and gauge height of $0.3 \mathrm{~m}$ (WAPDA, 2003). A manual gauge is read in conjunction with each automatic gauge as a check on the total rainfall. In 1994-95, WAPDA installed 20 automatic data collection platforms (DCPs) in the high-altitude areas that use snow pillows to measure both solid and liquid precipitation as water equivalent (SIHP, 1997). The observatories installed and maintained by the University of Bonn under the CAK program used the automatic weather stations including data logger, tipping bucket and snow depth gauge to measure precipitation (Miehe et al., 1996). Afghanistan mainly uses the Tretyakov (20-24-G) type of rain-gauge without windshield having orifice area of $200 \mathrm{~cm}^{2}$ and $0.4 \mathrm{~m}$ height (Sevruk and Klemm, 1989). The metadata of 305 precipitation observatories and 21 glacier observation points used in this study are outlined and described in the supplementary material (Table S-3.1).

\subsubsection{Temperature and wind speed observations}

The adjustments for wind-induced under-catch of precipitation observations require corresponding data of temperature and wind speed. However, out of 307 stations, temperature data was available for only 114 stations (Table S-3.1). We therefore derived monthly lapse rates based on elevation and latitude and estimated the maximum and minimum temperatures for the remaining stations. The observed data of wind speed was available for only 25 stations. Wind speed for the remaining stations is taken from the Japanese 55-year Reanalysis (JRA55) dataset (Kobayashi et al., 2015). JRA55 provides wind speed estimates at the standard anemometer height of $10 \mathrm{~m}$, whereas the station-based observed wind speed is measured at $2 \mathrm{~m}$ height. In order to get an idea of the accuracy of the JRA55 wind speed data, we compared it with the observed wind speed for the 25 stations. For this purpose, we computed wind speed from the U- and V-components at $10 \mathrm{~m}$ height and down-scaled it to match the $2 \mathrm{~m}$ height of stations using the Monin Obukhov theory (Obukhov, 1971; Businger and Yaglom, 1971). Although, we could not detect large differences and/or any definite and strong trends, a tendency of slightly underestimated wind speed in low-altitude areas and vice versa in high-altitude areas is noticed. We also observed marginally increased wind speeds during Nov-Feb months and slightly decreased wind speeds during Mar-Oct months for the JRA55 data. Due to insufficient observed data of wind speed, we have neglected these minor differences and used wind speed data of JRA55 as such. Nevertheless, such minor differences of wind speeds in JRA55 data might result in slight overestimation of precipitation adjustments in the higher-altitude areas during four (Nov-Feb) winter months and slight underestimation of precipitation adjustments in the lower-altitude areas during the remaining months.

\subsubsection{River flows}

Daily data of the observed river flows at sub-basin level for 14 hydrological stations (Figure 3.1) in the study area were collected from WAPDA. We used flow data of Jhelum and Chenab rivers for 1961-1970 period and all the rivers in the western part sub-basins for 
1999-2011 period to coincide with the precipitation data periods. Ravi, Beas and Sutlej basins are located in India and their inflow data are not publicly available. Therefore, we extracted mean monthly river flows from Adeloye et al. (2016) for the Beas river at Pong dam for 2000-2008 period and from ADB (2010) for the Sutlej river at Bhakra dam for 1962-1971 period. The river discharge data for the Ravi at Mukesar (near Thein dam) is collected from the Global River Discharge Database (RivDIS v1.1) for the period of 19681979. It is worth to note that there are considerable diversions in some sub-basins on the upstream side of their rim stations (e.g. at Warsak, Nowshera and Tarbela), which are often overlooked by previous studies. We also collected the data of these upstream diversions and added them to the flows of the respective sub-basins. River flow data of coinciding time periods are used to validate the adjusted precipitation at sub-basin scale.

\subsubsection{Precipitation measurement error adjustment methods}

The amount of actual precipitation reaching the ground is generally higher than what is measured in precipitation gauges due to measurement errors, which usually depend on the form of precipitation, gauge type, topography, vegetation around the gauge site and the exposure of the gauges to prevailing temperatures and winds. Wind-induced under-catch is by far the most dominant source of errors in gauge-measured precipitation observations (Wolff et al., 2015; Adam and Lattenmaier, 2003; Michelson, 2004; Goodison et al., 1998), yet most of the widely used global precipitation datasets are not adjusted for such errors (Adam and Lattenmaier, 2003). While recognizing the significance of measurement errors in precipitation observations, the WMO initiated a comprehensive program of international precipitation measurement intercomparisons during 1960-1993 and established the pit gauge (Sevruk and Hamon, 1984) and the Double-Fence International Reference (DFIR; Goodison et al., 1998) as the standard reference gauges for liquid (rain) and solid (snow) precipitation respectively. Sevruk and Hamon (1984) and Goodison et al. (1998) also underlined the need for gauge calibration and adjustment of errors to increase reliability of the precipitation data. However, the agencies involved in measurement of precipitation in the Indus basin generally indicate to follow the WMO standards for design, construction, installation and operation of precipitation gauges but hardly or inadequately adjust the systematic measurement errors at the source, which signifies the need for correction of measurement errors.

Sevruk (1982) related and statistically analysed various components of the systematic measurement errors to the meteorological and instrumental factors and proposed a general equation for adjustment of gauge-measured precipitation errors. Legates (1987) later modified it to account for both liquid and solid precipitation components separately. The modified equation is expressed as:

$$
\mathbf{P}_{\mathrm{a}}=(\mathbf{1 - R}) K_{r}\left(\mathbf{P}_{\mathrm{m}}+\Delta \mathbf{P}_{\mathrm{wr}}+\Delta \mathbf{P}_{\mathrm{tr}}+\Delta \mathbf{P}_{\mathrm{er}}\right)+\mathbf{R} K_{s}\left(\mathbf{P}_{\mathrm{m}}+\Delta \mathbf{P}_{\mathrm{ws}}+\Delta \mathbf{P}_{\mathrm{ts}}+\Delta \mathbf{P}_{\mathrm{es}}\right)
$$

Where, $\mathbf{P}_{\mathbf{a}}$ is adjusted precipitation $(\mathrm{mm}), \mathbf{R}$ is proportion of solid precipitation, $\boldsymbol{K}$ is correction coefficient that accounts for wind-induced losses, $\mathbf{P}_{\mathbf{m}}$ is measured precipitation $(\mathrm{mm}), \boldsymbol{\Delta} \mathbf{P}_{\mathbf{w}}$ is wetting losses $(\mathrm{mm}), \boldsymbol{\Delta} \mathbf{P}_{\mathbf{e}}$ is evaporation losses $(\mathrm{mm}), \boldsymbol{\Delta} \mathbf{P}_{\mathbf{t}}$ is trace precipitation $(\mathrm{mm})$, and sub-scripts $\mathbf{r}$ and $\mathbf{s}$ denote rain and snow components respectively. Legates (1987) model was developed for a variety of manual rain gauges including Nipher, Tretyakov and MK1/MK2 models with and without windshields. However, significant 
uncertainties remained for wind induced under-catch of solid precipitation particularly by automatic precipitation gauges. Nitu and Wong (2010) observed much larger variation between gauges and windshield configurations for automatic stations than for manual stations.

Wolff et al. (2015) compared precipitation data from the standard automatic Geonor precipitation gauge with data from a reference configuration consisting of an automatic precipitation gauge (Geonor T200-BM) and an Alter wind shield with double-fence construction. They derived an adjustment model to determine catch efficiency as a continuous function of both wind speed and air temperature using Bayesian statistics to more objectively choose the model that best describes the data. Wolff's model allows solid precipitation adjustments at wind speeds greater than $7.0 \mathrm{~ms}^{-1}$. However, it is also gauge/shield specific and different site specificities and gauge/shield configurations might result in different adjustment functions.

Kochendorfer et al. (2017a) analysed precipitation measurements from eight different WMO-SPICE sites for both unshielded and single-Alter-shielded OTT Pluvio ${ }^{2}$ and Geonor T-200B3 types of weighing gauges. They grouped unshielded and single-alter-shielded precipitation gauge configurations separately irrespective of gauge types and created a single transfer function of air temperature and wind speed using the corresponding measurements from the reference gauge. They also derived the coefficient fits for both unshielded and single-alter-shielded precipitation gauges at gauge height as well as $10 \mathrm{~m}$ height. The derived transfer function is expressed as:

$$
C E=e^{-a(U)\left(1-T A N^{-1}\left(b\left(T_{a i r}\right)\right)+c\right)}
$$

Where $\boldsymbol{T}_{\text {air }}$ is mean air temperature $\left({ }^{0} \mathrm{C}\right) ; \boldsymbol{U}$ is wind speed $\left(\mathrm{ms}^{-1}\right) ; \mathrm{a}, \mathrm{b}$, and c are the coefficients fit to the data, and $\mathrm{TAN}^{-1}$ is the inverse of tangent function.

Our method of adjusting systematic errors in precipitation measurements largely follows the approach by Adam and Lattenmaier (2003) using the 'liquid' part of the model by Legates (1987) and uses the model by Kochendorfer et al. (2017a) for adjustment of the solid precipitation component. The detailed methods for computation of the required variables in equations (1) are described in the supplementary material. The coefficient values in equation (2) $(\mathrm{a}=0.0623, \mathrm{~b}=0.776, \mathrm{c}=0.431)$ are taken as determined at $10 \mathrm{~m}$ height by Kochendorfer et al. (2017a). We used the coefficient values of $10 \mathrm{~m}$ height because most of our wind speed data belonged to the JRA55 dataset, which provides wind speed data at $10 \mathrm{~m}$ height. The observed wind speed at 25 stations is converted from observation height to $10 \mathrm{~m}$ height using the Monin Obukhov theory (Obukhov, 1971; Businger and Yaglom, 1971).

\subsubsection{Adjustment of net snow accumulations methods}

The meteorological stations in the study area are unevenly distributed in both horizontal and vertical direction. Scarcity of precipitation measurements at higher-altitude areas, where the bulk of precipitation falls, seriously limits an accurate assessment of precipitation climatology and its hydrological implications. In order to overcome this observational data gap, we assumed 21 virtual stations at the major glaciers where the net snow accumulations were estimated through mass balance studies using snow pillows, snow pits, and ice cores (e.g. Mayer et al., 2014, 2006; Hewitt, 2011; Shroder et al., 2000; Bhutiani, 1999; Wake, 1989; Mayewski et al., 1984, 1983; Kick, 1980; BIG, 1979; Decheng, 1978; Qazi, 1973). 
However, most of these mass balance studies were undertaken in the active ablation zones of the glaciers, where ablation and accumulation processes are happening simultaneously. Generally, glacier ablation is the function of ablation rate, altitude of the Equilibrium Line Altitude (ELA) and the elevation difference between mean ELA and the glacier observation point. Ablation zones are the areas below the Equilibrium Line Altitude (ELA), which is the elevation at which the annual net mass of the glacier remains zero and the area above this elevation is known as the accumulation zone (Cuffey and Paterson, 2010). Hence, the estimated net glacier mass accumulations are subject to ablation losses until the next accumulation period. The ablation gradients can be variable depending on debris cover and surface albedo or energy availability to melt the exposed glaciers. Wagnon et al. (2007) observed ablation gradients of 0.60-0.81 m w.e. (water equivalent) for each $100 \mathrm{~m}$ with a mean value of $0.69 \mathrm{~m}$ w.e. over a period of four year of mass balance studies at the Chhota Shigri Glacier, western Himalaya. Yu et al. (2013), based on glacier studies by Mayer et al. (2006) and Wagnon et al. (2007) in the Karakoram and western Himalaya, assumed an ablation gradient of $1 \mathrm{~m}$ w.e. per $100 \mathrm{~m}$ for the upper Indus basin. Hewitt et al. (1989) however, estimated an ablation gradient of $0.5 \mathrm{~m}$ per $100 \mathrm{~m}$ for the middle portion of the ablation zone on the Biafo glacier in the central part of the Karakoram. No ablation above ELA is assumed. We selected the rather conservative estimates of ablation gradient by Hewitt et al. (1989) and adjusted the net accumulations by taking the Equilibrium Line Altitude (ELA) as the boundary for the ablation process. However, the location of ELA can vary from location to location. In temperate glaciers, usually the Snow Line Elevation (SLE) and ELA are often assumed to be the same. The estimates for mean ELA at subbasin scale are taken from Khan et al. (2015), who estimated ELA values based on SLE.

\subsubsection{River flow adjustments}

WAPDA uses standard flow measuring devices to ensure high quality river flow data. The primary river flow measuring technique uses area velocity measurements to determine the stage-discharge relationships and associated rating tables. The results are verified by areavelocity method, area-slope method, contracted opening measurements, or computation of flow over dams or weirs (WAPDA, 2012). The daily mean discharge values are computed from the mean gauge heights and corresponding calibrated rating tables. In case of extremely high discharges, the rating curves are extrapolated by applying simple linear regression between the gauge height and discharge measurements. The actual measurements are however taken 4-8 times per month. The intermediate daily values are estimated from the rating tables. The accuracy of stream flow measurements depends primarily on stability of the stage-discharge relationship, frequency of discharge measurements if the relationship is unstable, and accuracy in the observation of the stage and measurement of discharges. In general, monthly and annual mean values are more accurate than daily values because of compensation of random errors. WAPDA evaluates the probable accuracy of discharge measurements as excellent (error $<5 \%$ ), good (error < $10 \%$ ), fair (error $<15 \%$ ), and poor (error $>15 \%$ ). In general, a probable accuracy of $0-5 \%$ is aimed for. Although river flow data may still be subject to some degree of uncertainty due to measurement errors, we assumed river flows as adequately accurate considering the relative precision of discharge measurement techniques and quality control ensured by the data collection agencies.

To account for the contribution of net glacier mass imbalance in each sub-hydrological basin, we adjusted the measured river flows. Kääb et al. (2012) used satellite laser altimetry and a global elevation model and observed a slightly negative mass balance of $-0.21 \pm$ 
$0.05 \mathrm{~m} \mathrm{yr}^{-1}$ w.e. for $\mathrm{HKH}$ region during 2003-2008 with maximum rates of $-0.66 \pm$ $0.09 \mathrm{~m} \mathrm{yr}^{-1}$ w.e. in the western Himalayan (Jammu-Kashmir) areas. We derived the specific net mass balance rates at sub-basin scale from the mass balance estimates of Kääb et al. (2012) and took glacier areas from the Randolf Glacier Inventory (RGI) version 5.0 (Arendt et al., 2015) to compute the contribution of the changes in the net glacial mass imbalance to the observed river flows. The adjusted river flows are used for validation of the adjusted precipitation estimates at sub-basin scale.

\subsubsection{Spatial interpolation}

The actual and error-adjusted point measurements of mean monthly precipitation are spatially interpolated following Dahri et al. (2016), who used the Kriging with External Drift (KED) interpolation scheme (Schabenberger and Gotway, 2005) with elevation as a predictor to derive spatiotemporal distribution of precipitation in the high-altitude Indus basin. The KED model includes a component of spatial autocorrelation and a component for multilinear dependence on pre-defined variables (predictors). It considers the observations $(\boldsymbol{Y})$ at sample locations $(\boldsymbol{s})$ as a random variable of the form (e.g. Diggle and Ribeiro, 2007):

$$
\begin{aligned}
& Y(s)=\mu(s)+Z(s) \\
& \mu(s)=\beta_{0}+\sum_{k=1}^{K} \beta_{k} \cdot x_{k}(s)
\end{aligned}
$$

Where, $\boldsymbol{\mu}(\boldsymbol{s})$ describes the deterministic component of the model (external drift or trend) and is given as a linear combination of $\boldsymbol{K}$ predictor fields $\boldsymbol{x}_{\boldsymbol{k}}(\boldsymbol{s})$ (trend variables) plus an intercept $\left(\boldsymbol{\beta}_{0}\right)$. The $\boldsymbol{\beta}_{\boldsymbol{k}}$ are denoted as trend coefficients, while $\boldsymbol{Z}(\boldsymbol{s})$ describes the stochastic part of the KED model and represents a random Gaussian field with a zero mean and a $2^{\text {nd }}$ order stationary covariance structure. The latter is conveniently modelled by an eligible parametric semi-variogram function describing the dependence of semi-variance as a function of lag (possibly with a directional dependence). Dahri et al. (2016) provided a detailed account of the KED interpolation method including model description and functionalities, reasons for its selection, and comparative advantages of its use in the highaltitude Indus basin.

\subsubsection{Cross validation of the adjusted precipitation}

We used exactly the same approach of interpolation and cross validation as adopted by Dahri et al. (2016), where the cross validation applied on the observed and predicted values from all the stations is used to assess the errors/uncertainty associated with the interpolation scheme by using error scores of the relative bias $(B)$ and the relative mean root-transformed error $(E)$, which are defined as:

$$
\begin{aligned}
& B=\frac{\sum_{i=1}^{n} P_{i}}{\sum_{i=1}^{n} O_{i}} \\
& E=\frac{\frac{1}{n} \sum_{i=1}^{n}\left(\sqrt{P}_{i}-\sqrt{\sigma_{i}}\right)^{2}}{\frac{1}{n} \sum_{i=1}^{n}\left(\sqrt{o}-\sqrt{\sigma_{i}}\right)^{2}}
\end{aligned}
$$


Where $\boldsymbol{P}_{i}$ and $\boldsymbol{O}_{i}$ are the predicted and observed precipitation values respectively, while $\boldsymbol{O}$ is the average of all (or a subset of) the station observations and $n$ refers to the number of precipitation values.

Under ideal conditions, the overall performance of the employed regression models and interpolation estimates at basin/sub-basin scale can also be cross validated by applying the continuity equation suggested by Budyko (1974), which is given by:

$$
\frac{\Delta \mathbf{S}}{\Delta \mathbf{t}}=\boldsymbol{P}-\boldsymbol{Q}-\boldsymbol{E T}-\boldsymbol{G}
$$

Where $P, Q, E T$ and $G$ are the basin-average precipitation, runoff, evapotranspiration and net groundwater discharge respectively, while $\Delta S$ is the net change in storage for a given time increment $(\Delta \mathrm{t})$. Equation (7) can be modified by adding interception $(I)$, sublimation $(S)$ and net mass balance $(\triangle M B)$ contributions for the highly glacierized and snowpack dependent river basins as follows:

$$
\frac{\Delta S}{\Delta \mathrm{t}}=P-Q-E T-G-I-S+\Delta M B
$$

Unfortunately, there are no independent datasets of actual evapotranspiration, sublimation, interception and the net groundwater discharge for the study area. The global scale datasets of these variables are generally more uncertain than precipitation itself; therefore, it would be unwise to validate the estimated precipitation with these extremely uncertain datasets. Nevertheless, surface storage and groundwater recharge are mostly very low in highaltitude areas, which are mostly rocky bare mountains with steep slopes and no groundwater. Precipitation may travel long distances through breaches but ultimately joins the river streams as base flow. Although, there might be considerable delay effects, these may be considered negligible for long term average conditions. Similarly, the surface storage due to topographical undulations may also have a delaying effect. Interception by the vegetation cover and sublimation (direct evaporation from the snow glacier fields) are included in the total direct evapotranspiration. Direct evapotranspiration is notoriously complex to measure as it is among others a function of water availability as well as water demand. The available global scale gridded datasets of actual evapotranspiration are highly inconsistent in quantitative as well as spatial distribution terms and generally reflect overestimated values. We therefore rely mainly on the specific runoff and net mass balance data to validate our adjusted precipitation estimates.

\subsection{Results}

\subsubsection{Precipitation adjustments}

To facilitate adjustment of measurement errors in precipitation observations, the corresponding air temperature is determined from elevation and latitude-based lapse rates. The results revealed a strong correlation of temperature with elevation and considerable correlation with latitude (Figures S-3.2-S-3.5). Significantly different gradients for each month and substantial difference among the gradients for maximum and minimum temperatures were observed (Table 3.1). Hence, use of a universally assumed or time independent site-specific observed gradient of mean annual temperature to estimate maximum and minimum temperatures (e.g. Immerzeel et al., 2012a\&b; Lutz et al., 2013) is probably not correct in the high-altitude Indus basin. Comparison of Table 3.1 and Figures S-3.2 and S-3.3 indicate that incorporation of latitude as an additional predictor improves the correlation of the regression models by up to $6.0 \%$ for maximum temperature and up to 
1.5\% for minimum temperature during 1999-2011. Almost similar trends are observed for 1961-1970 period. The contribution of elevation to the correction is positive in the summer and negative in the winter months, while the contribution of latitude is positive throughout the year. The highest improvement is achieved during the monsoon season (Jul-Sep).

Table 3.1: Multiple regressions for maximum and minimum temperatures for the two time periods of 1999-2011 and 1961-1970. $\mathrm{T}_{\mathrm{x}} 1-12$ and $\mathrm{T}_{\mathrm{n}} 1-12$ refer to the calendar months for maximum and minimum temperatures respectively. E denotes elevation $(\mathrm{m})$ and $\mathrm{L}$ represents latitude (decimal degrees) of the meteorological stations. $\mathrm{R}^{2}$ is the combined correlation of temperature with $\mathrm{E}$ and $\mathrm{L}$.

\begin{tabular}{|c|c|c|c|}
\hline Regression Equation for $T_{x}$ & $\mathbf{R}^{2}(\%)$ & Regression Equation for $T_{n}$ & $\mathbf{R}^{2}(\%)$ \\
\hline \multicolumn{4}{|c|}{ 1999-2011 } \\
\hline $\mathrm{T}_{\mathrm{x}} 1=31.5-0.00688 \mathrm{E}-0.318 \mathrm{~L}$ & 96.7 & $\mathrm{~T}_{\mathrm{n}} 1=17.4-0.00534 \mathrm{E}-0.307 \mathrm{~L}$ & 91.1 \\
\hline $\mathrm{T}_{\mathrm{x}} 2=38.1-0.00691 \mathrm{E}-0.455 \mathrm{~L}$ & 97.5 & $\mathrm{~T}_{\mathrm{n}} 2=19.1-0.00559 \mathrm{E}-0.285 \mathrm{~L}$ & 92.3 \\
\hline $\mathrm{T}_{\mathrm{x}} 3=41.3-0.00712 \mathrm{E}-0.383 \mathrm{~L}$ & 96.6 & $\mathrm{~T}_{\mathrm{n}} 3=23.4-0.00567 \mathrm{E}-0.278 \mathrm{~L}$ & 93.8 \\
\hline $\mathrm{T}_{\mathrm{x}} 4=44.5-0.00739 \mathrm{E}-0.303 \mathrm{~L}$ & 97.5 & $\mathrm{~T}_{\mathrm{n}} 4=33.2-0.00567 \mathrm{E}-0.428 \mathrm{~L}$ & 94.1 \\
\hline $\mathrm{T}_{\mathrm{x}} 5=41.0-0.00790 \mathrm{E}-0.025 \mathrm{~L}$ & 96.9 & $\mathrm{~T}_{\mathrm{n}} 5=37.3-0.00599 \mathrm{E}-0.404 \mathrm{~L}$ & 94.5 \\
\hline $\mathrm{T}_{\mathrm{x}} 6=19.1-0.00817 \mathrm{E}+0.719 \mathrm{~L}$ & 96.2 & $\mathrm{~T}_{\mathrm{n}} 6=34.3-0.00591 \mathrm{E}-0.220 \mathrm{~L}$ & 95.6 \\
\hline $\mathrm{T}_{\mathrm{x}} 7=-9.47-0.00713 \mathrm{E}+1.48 \mathrm{~L}$ & 90.5 & $\mathrm{~T}_{\mathrm{n}} 7=22.2-0.00575 \mathrm{E}+0.166 \mathrm{~L}$ & 95.4 \\
\hline $\mathrm{T}_{\mathrm{x}} 8=-5.13-0.00685 \mathrm{E}+1.30 \mathrm{~L}$ & 90.9 & $\mathrm{~T}_{\mathrm{n}} 8=22.6-0.00567 \mathrm{E}+0.136 \mathrm{~L}$ & 95.5 \\
\hline $\mathrm{T}_{\mathrm{X}} 9=8.60-0.00727 \mathrm{E}+0.876 \mathrm{~L}$ & 96.0 & $\mathrm{~T}_{\mathrm{n}} 9=35.2-0.00532 \mathrm{E}-0.341 \mathrm{~L}$ & 95.1 \\
\hline $\mathrm{T}_{\mathrm{X}} 10=20.4-0.00780 \mathrm{E}+0.444 \mathrm{~L}$ & 97.0 & $\mathrm{~T}_{\mathrm{n}} 10=30.7-0.00518 \mathrm{E}-0.380 \mathrm{~L}$ & 91.8 \\
\hline $\mathrm{T}_{\mathrm{x}} 11=39.0-0.00721 \mathrm{E}-0.291 \mathrm{~L}$ & 97.8 & $\mathrm{~T}_{\mathrm{n}} 11=22.7-0.00515 \mathrm{E}-0.300 \mathrm{~L}$ & 90.3 \\
\hline $\mathrm{T}_{\mathrm{x}} 12=38.8-0.00689 \mathrm{E}-0.459 \mathrm{~L}$ & 96.8 & $\mathrm{~T}_{\mathrm{n}} 12=16.7-0.00519 \mathrm{E}-0.246 \mathrm{~L}$ & 90.3 \\
\hline \multicolumn{4}{|c|}{$1961-1970$} \\
\hline $\mathrm{T}_{\mathrm{x}} 1=38.2-0.00673 \mathrm{E}-0.529 \mathrm{~L}$ & 98.0 & $\mathrm{~T}_{\mathrm{n}} 1=15.9-0.00536 \mathrm{E}-0.267 \mathrm{~L}$ & 89.3 \\
\hline $\mathrm{T}_{\mathrm{x}} 2=39.3-0.00691 \mathrm{E}-0.495 \mathrm{~L}$ & 97.9 & $\mathrm{~T}_{\mathrm{n}} 2=15.9-0.00572 \mathrm{E}-0.188 \mathrm{~L}$ & 92.8 \\
\hline $\mathrm{T}_{\mathrm{x}} 3=45.3-0.00686 \mathrm{E}-0.524 \mathrm{~L}$ & 97.3 & $\mathrm{~T}_{\mathrm{n}} 3=21.8-0.00582 \mathrm{E}-0.232 \mathrm{~L}$ & 93.8 \\
\hline $\mathrm{T}_{\mathrm{x}} 4=53.2-0.00713 \mathrm{E}-0.589 \mathrm{~L}$ & 97.7 & $\mathrm{~T}_{\mathrm{n}} 4=30.0-0.00592 \mathrm{E}-0.334 \mathrm{~L}$ & 94.7 \\
\hline $\mathrm{Tx} 5=48.7-0.00766 \mathrm{E}-0.281 \mathrm{~L}$ & 97.8 & $\mathrm{~T}_{\mathrm{n}} 5=35.1-0.00612 \mathrm{E}-0.346 \mathrm{~L}$ & 95.4 \\
\hline $\mathrm{T}_{\mathrm{x}} 6=20.0-0.00828 \mathrm{E}+0.703 \mathrm{~L}$ & 96.6 & $\mathrm{~T}_{\mathrm{n}} 6=31.6-0.00608 \mathrm{E}-0.129 \mathrm{~L}$ & 94.7 \\
\hline $\mathrm{T}_{\mathrm{x}} 7=-9.23-0.00727 \mathrm{E}+1.48 \mathrm{~L}$ & 90.3 & $\mathrm{~T}_{\mathrm{n}} 7=17.1-0.00590 \mathrm{E}+0.328 \mathrm{~L}$ & 95.1 \\
\hline $\mathrm{T}_{\mathrm{x}} 8=-6.80-0.00701 \mathrm{E}+1.37 \mathrm{~L}$ & 88.3 & $\mathrm{~T}_{\mathrm{n}} 8=17.0-0.00588 \mathrm{E}+0.316 \mathrm{~L}$ & 95.2 \\
\hline $\mathrm{T}_{\mathrm{x}} 9=2.74-0.00751 \mathrm{E}+1.06 \mathrm{~L}$ & 95.4 & $\mathrm{~T}_{\mathrm{n}} 9=27.1-0.00560 \mathrm{E}-0.088 \mathrm{~L}$ & 94.4 \\
\hline $\mathrm{T}_{\mathrm{x}} 10=25.2-0.00765 \mathrm{E}+0.288 \mathrm{~L}$ & 98.0 & $\mathrm{~T}_{\mathrm{n}} 10=22.8-0.00546 \mathrm{E}-0.136 \mathrm{~L}$ & 91.7 \\
\hline $\mathrm{T}_{\mathrm{x}} 11=38.0-0.00706 \mathrm{E}-0.281 \mathrm{~L}$ & 98.3 & $\mathrm{~T}_{\mathrm{n}} 11=20.7-0.00530 \mathrm{E}-0.228 \mathrm{~L}$ & 89.4 \\
\hline $\mathrm{T}_{\mathrm{x}} 12=44.0-0.00654 \mathrm{E}-0.632 \mathrm{~L}$ & 96.9 & $\mathrm{~T}_{\mathrm{n}} 12=14.2-0.00524 \mathrm{E}-0.174 \mathrm{~L}$ & 87.8 \\
\hline
\end{tabular}

To illustrate the precipitation biases over the high-altitude Indus basin, the results for each individual station are presented. The applied bias adjustments significantly increased the gauge-measured precipitation. The highest increments are computed for wind-induced under-catch of solid precipitation followed by liquid precipitation under-catch, wetting losses and precipitation losses during trace events (Figure 3.2a-d). The solid precipitation under-catch generally dominates the higher-altitude stations, i.e. elevations greater than $2000 \mathrm{~m}$ and during the Dec-Apr months. The range of liquid precipitation under-catch is much lower and mainly concentrates in the summer monsoon dominated low-altitude areas. The wetting losses and unmeasured trace precipitation depend on the number of precipitation events. In many cases, particularly for the low-altitude stations experiencing 
lower wind speeds, the wetting losses exceeded the wind induced under catch of liquid precipitation due to the fact that it covers all the stations and both forms of precipitation (liquid and solid). The total bias between the gauge-measured and error-adjusted precipitation ranged from 12 to $773 \mathrm{~mm} \mathrm{yr}^{-1}$ for various individual stations and up to $1000 \mathrm{~mm} \mathrm{yr}^{-1}$ for the glacier points (Figure 3.2e). The total absolute biases (corrections) for all the stations at monthly and annual scale are given at Table S-3.2. The largest increases are found for the stations receiving greater precipitation amounts, located at higher-altitudes and encountering higher wind speeds. Based on the above-mentioned corrections, we introduced monthly scale correction factors (CFs) for each station (Table S-3.3). These station-based correction factors vary over space and time, with stronger magnitude in higher-altitude areas (Figure 3.2f) and during winter months (Table S-3.3).

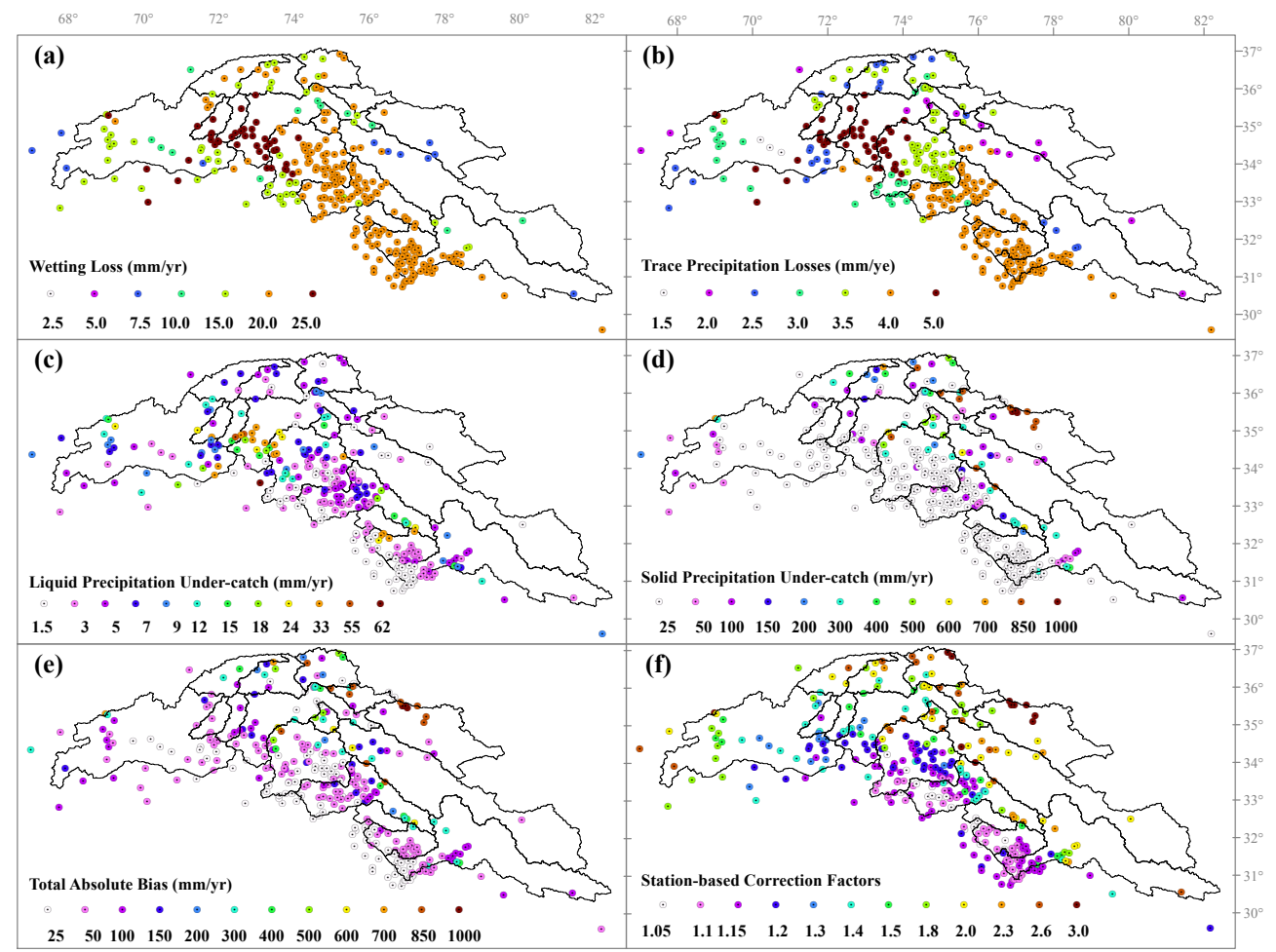

Figure 3.2: Adjusted station observations for (a) wetting loss, (b) trace precipitation loss, (c) liquid precipitation under-catch, (d) solid precipitation under-catch, (e) total absolute bias between gaugemeasured and error-adjusted annual precipitation, (f) station-based CFs for under-catch of gaugemeasured precipitation. The different scales are to be noted.

\subsubsection{Snow accumulation adjustments}

The total ablation losses at a given ablation rate from a glacier zone depend on the ablation gradient and $\triangle E L A$ (the difference between the mean elevation of a glacier zone and ELA). Assuming that the practical ablation above ELA is insignificant, the potential ablation losses from the selected glacier zones vary from 0-1000 $\mathrm{mm} \mathrm{yr}^{-1}$ (Table 3.2). These ablation losses are added to the original estimates of the net accumulations to account for the ablation losses from the actual precipitation. 
Table 3.2: Adjusted net snow water equivalent at the major glacier accumulation zones. Lon is longitude, Lat is latitude, Ele is elevation, ELA is equilibrium line altitude, $\triangle E L A$ is the net elevation contributing to ablation, and $\Delta \mathrm{A}$ is adjustment in the net accumulation.

\begin{tabular}{lcccccrrrr}
\hline $\begin{array}{l}\text { Glacier } \\
\text { Name }\end{array}$ & $\begin{array}{l}\text { Lon } \\
(\mathbf{d d})\end{array}$ & $\begin{array}{c}\text { Lat } \\
(\mathbf{d d})\end{array}$ & $\begin{array}{c}\text { Ele } \\
(\mathbf{m})\end{array}$ & $\begin{array}{c}\text { River } \\
\text { Basin }\end{array}$ & $\begin{array}{c}\text { ELA } \\
(\mathbf{m})\end{array}$ & $\begin{array}{c}\Delta \text { ELA } \\
(\mathbf{m})\end{array}$ & $\begin{array}{c}\Delta \mathbf{A} \\
(\mathbf{m m})\end{array}$ & $\begin{array}{c}\text { Net Acc. } \\
\left(\mathbf{m m} \mathbf{~ y r}^{-1}\right)\end{array}$ & $\begin{array}{r}\text { Adj. Acc. } \\
\left(\mathbf{m m} \mathbf{~ y r} \mathbf{}^{-1}\right)\end{array}$ \\
\hline Approach & 75.6331 & 36.0678 & 5100 & Shigar & 5050 & 0 & 0 & 1880 & 1880 \\
Baltoro & 76.5508 & 35.8778 & 5500 & Shigar & 5050 & 0 & 0 & 1600 & 1600 \\
Batura & 74.3833 & 36.6667 & 4840 & Hunza & 5000 & 160 & 800 & 1034 & 1834 \\
Chogolungma & 75.0000 & 36.0000 & 4850 & Hunza & 5000 & 150 & 750 & 1070 & 1820 \\
Chong Kumdan & 77.5448 & 35.2532 & 5330 & Shyok & 5500 & 170 & 850 & 484 & 1334 \\
Hispar Dome & 75.5187 & 36.0109 & 5450 & Shigar & 5050 & 0 & 0 & 1620 & 1620 \\
Hispar East & 75.5064 & 35.8495 & 4900 & Shigar & 5050 & 150 & 750 & 1070 & 1820 \\
Hispar West & 75.5064 & 35.8495 & 5100 & Shigar & 5050 & 0 & 0 & 1620 & 1620 \\
Hispar Pass & 75.5215 & 36.0281 & 5000 & Shigar & 5050 & 50 & 250 & 1420 & 1670 \\
Khurdopin & 75.6197 & 36.1338 & 5520 & Shigar & 5050 & 0 & 0 & 2240 & 2240 \\
Nanga Parbat & 74.4444 & 35.1672 & 4600 & Astore & 4700 & 100 & 500 & 2000 & 2500 \\
Nun Kun North & 76.1014 & 34.1219 & 5200 & Shingo & 5250 & 50 & 250 & 900 & 1150 \\
Sentik & 75.9500 & 33.9967 & 5100 & Shingo & 5250 & 150 & 750 & 620 & 1370 \\
Siachin A & 77.0376 & 35.4707 & 5300 & Shyok & 5500 & 200 & 1000 & 484 & 1484 \\
Siachin B & 76.9915 & 35.5235 & 5300 & Shyok & 5500 & 200 & 1000 & 526 & 1526 \\
Siachin C & 76.9116 & 35.5187 & 5320 & Shyok & 5500 & 180 & 900 & 662 & 1562 \\
Siachin D & 76.8592 & 35.6242 & 5350 & Shyok & 5500 & 150 & 750 & 855 & 1605 \\
South Terong & 77.4516 & 35.1384 & 5330 & Shyok & 5500 & 170 & 850 & 484 & 1334 \\
Terong & 77.3120 & 35.5177 & 5350 & Shyok & 5500 & 150 & 750 & 855 & 1605 \\
Urdok & 76.7025 & 35.7669 & 5400 & Shigar & 5050 & 0 & 0 & 1060 & 1060 \\
Whaleback & 75.5915 & 36.0572 & 4900 & Shigar & 5050 & 150 & 750 & 1790 & 2540 \\
\hline
\end{tabular}

\subsubsection{Spatial distribution of unadjusted and adjusted precipitation}

Continuous fields of precipitation generated through KED-based interpolation of the adjusted station observations and adjusted snow accumulations at monthly scale show how precipitation patterns and amounts are spatially distributed in the study area (Figure 3.3a-1). Monthly precipitation distributions largely confirm the bimodal weather system reflecting the wintertime precipitation associated with the westerlies and the impact of Indian summer monsoon in the study area. Overall climatology and distribution patterns of the adjusted precipitation (Figure 3.3m) match very well to the unadjusted case (Figure 3.3n) or estimates of Dahri et al. (2016). However, the adjustments revealed significant improvement in terms of quantitative and spatio-temporal distribution of precipitation in the study area (Figure 3.3o). An overall increase of $21.3 \%$ in average annual precipitation is realized at basin (study area) level, while at sub-basin scale it ranged from 6 to 77\% (Table 3.3). Greatest improvements are achieved in the high-altitude areas of Astore, Shyok, Shigar, Hunza, Gilgit, and Chitral sub-basin and during the winter months.

\subsubsection{River runoff adjustments}

The net mass balance estimates of Kääb et al. (2012) for the study area are translated into the amount of runoff generated at sub-basin scale. As a result of slightly negative mass balance estimates for all sub-basins, their contributions to river runoff are also negative and relatively small ranging from $0.4-6.1 \%$. The adjustments in river specific runoff depend on the net mass balance as well as glacier area and varied from $-51.5 \mathrm{~mm}$ in the Chenab subbasin to $-2.5 \mathrm{~mm}$ in the Panjkora sub-basin (Table 3.4). 
(a)

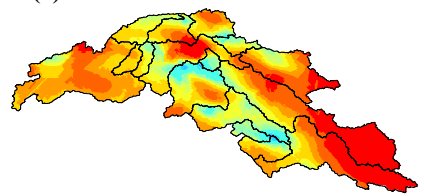

(d)

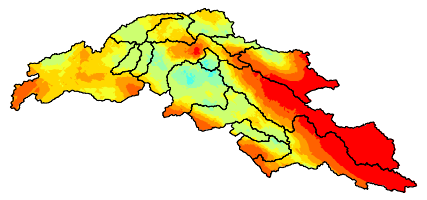

(g)

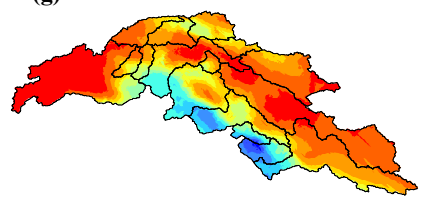

(j)

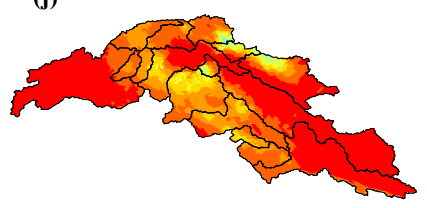

(b)

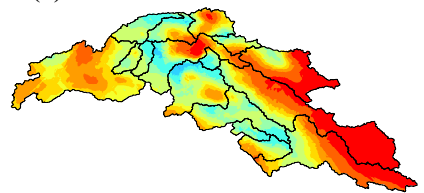

(e)

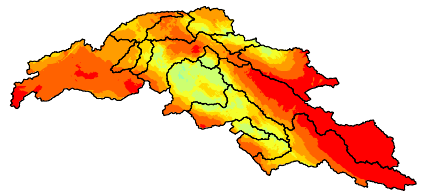

(h)

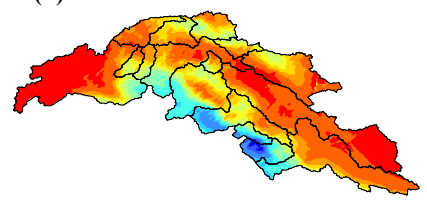

(k) (c)

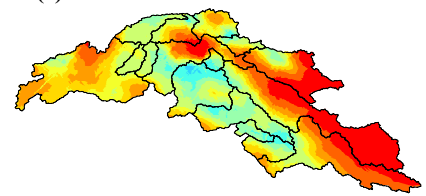

(f)

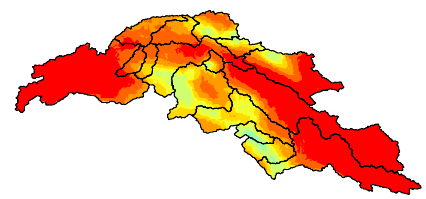

(i)

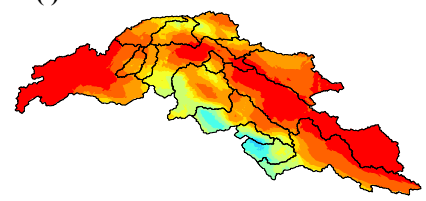

(l)
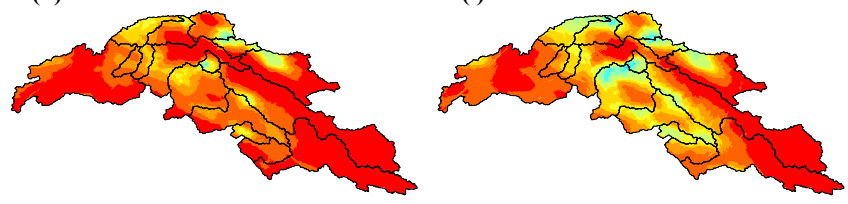

Mean monthly adjusted precipitation (mm)
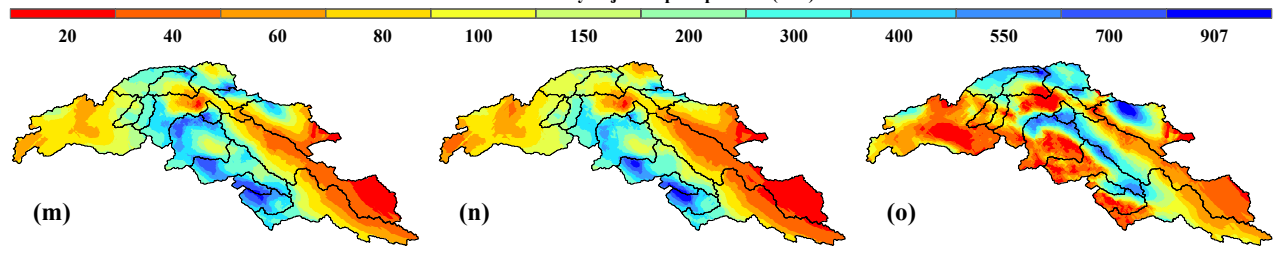

Absolute difference (mm/year) Mean annual adjusted and unadjusted (obseved) precipitation (mm)

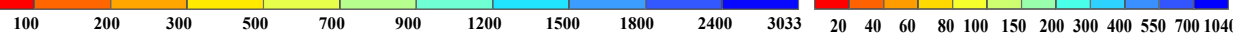

Figure 3.3: Estimated precipitation distribution, (a-1) are mean monthly (Jan-Dec) error-adjusted precipitation, (m) is error adjusted annual precipitation, (n) is unadjusted annual precipitation, and (o) is the absolute difference between adjusted and unadjusted annual precipitation distributions

\subsubsection{Validation of precipitation estimates}

The estimated precipitation distributions can be validated by evaluating the accuracy of the employed interpolation scheme and the output interpolated fields. For accuracy assessment of the interpolation scheme, the KED interpolation model produces both prediction as well as error/uncertainty surfaces, giving an indication or measure of how good the predictions are. The cross validation applied on the observed and predicted values from all the stations resulted in relative bias (B) error scores of less than 1, suggesting a negligible underestimation of the predicted values for all months except August, which shows a slight overestimation (Table 3.5). Similarly, the relative mean root-transformed error (E) scores of less than 1 for the months Jan-May suggest excellent results. While, the remaining months of Jun-Dec experience E values of greater than 1, which depict typical errors slightly greater than the spatial variations. Almost similar trends are observed for the unadjusted case. In general, the cross-validation results depict excellent/good agreement between the observed and predicted values. 
Table 3.3: Precipitation estimates at sub-basin scale. $\mathrm{P}_{\mathrm{obs}}$ is observed precipitation derived through actual measurements and net glacier accumulations, $P_{\text {adj }}$ is adjusted precipitation derived through adjusted precipitation observations and glacier accumulations, and $\Delta \mathrm{P}$ is the difference between them.

\begin{tabular}{llrrrr}
\hline S. \# & River Basin & $\mathbf{P}_{\text {obs }}(\mathbf{m m})$ & $\mathbf{P}_{\text {adj }}(\mathbf{m m})$ & $\boldsymbol{\Delta P} \mathbf{( m m )}$ & Increase $(\boldsymbol{\%})$ \\
\hline 1 & Gilgit at Gilgit & 566.7 & 749.3 & 182.6 & 32.2 \\
2 & Hunza at Dainyor & 634.1 & 845.5 & 21.4 & 33.3 \\
3 & Shigar at Shigar & 847.3 & 986.5 & 139.2 & 16.4 \\
4 & Shyok at Yugo & 254.2 & 426.2 & 172.0 & 67.7 \\
5 & Indus at Kharmong & 177.5 & 272.0 & 94.5 & 53.3 \\
6 & Astore at Doyian & 879.5 & 1214.1 & 334.6 & 38.0 \\
7 & Indus at Tarbela Dam & 393.8 & 521.8 & 128.0 & 32.5 \\
8 & Chitral at Chitral & 642.3 & 830.1 & 187.9 & 29.3 \\
9 & Panjkora at Zulum Br. & 757.0 & 798.4 & 41.4 & 5.5 \\
10 & Swat at Chakdara & 960.5 & 1034.7 & 74.3 & 7.7 \\
11 & Kabul at Warsak & 372.8 & 448.4 & 75.5 & 20.3 \\
12 & Kabul at Nowshera & 448.6 & 515.0 & 66.4 & 14.8 \\
13 & Jhelum at Mangla Dam & 1119.4 & 1223.3 & 103.9 & 9.3 \\
14 & Chenab at Marala & 1101.2 & 1247.7 & 146.5 & 13.3 \\
15 & Ravi at Thein Dam & 1334.8 & 1552.3 & 217.5 & 16.3 \\
16 & Beas at Pong Dam & 1504.1 & 1611.0 & 106.9 & 7.1 \\
17 & Sutlej at Bhakra Dam & 357.1 & 428.4 & 71.3 & 20.0 \\
\hline & Whole Basin & $\mathbf{5 6 5 . 7}$ & $\mathbf{6 6 8 . 7}$ & $\mathbf{1 0 3 . 0}$ & $\mathbf{1 8 . 2}$ \\
\hline
\end{tabular}

Table 3.4: Contribution of net glacier mass balance (GMB) to river flows \& adjusted specific runoff.

\begin{tabular}{|c|c|c|c|c|c|c|}
\hline $\begin{array}{l}\text { S. } \\
\text { No. }\end{array}$ & $\begin{array}{l}\text { River } \\
\text { Basin } \\
\text { Name } \\
\end{array}$ & $\begin{array}{c}\text { Glacier } \\
\text { Area } \\
\left(\mathbf{k m}^{2}\right) \\
\end{array}$ & $\begin{array}{c}\text { Net } \\
\text { GMB } \\
\left(\mathbf{m ~ y r}^{-1}\right) \\
\end{array}$ & 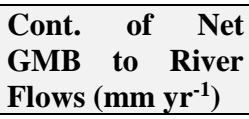 & $\begin{array}{l}\text { Obs. Sp. } \\
\text { Runoff } \\
\left(\mathrm{mm} \mathrm{yr} \mathbf{y r}^{-1}\right) \\
\end{array}$ & $\begin{array}{c}\text { Adj. Sp. } \\
\text { Runoff } \\
\left(\mathrm{mm} \mathrm{yr}^{-1}\right) \\
\end{array}$ \\
\hline 1 & Gilgit at Gilgit & 1212.5 & -0.350 & $-33.3(4.4 \%)$ & 758.0 & 724.7 \\
\hline 2 & Hunza at Dainyor & 4268.7 & -0.113 & $-35.4(5.2 \%)$ & 680.1 & 644.7 \\
\hline 3 & Shigar at Shigar & 2974.1 & -0.090 & $-38.1(4.1 \%)$ & 924.9 & 886.8 \\
\hline 4 & Shyok at Yugo & 7400.4 & -0.060 & $-13.0(3.6 \%)$ & 365.5 & 352.5 \\
\hline 5 & Indus at Kharmong & 2164.7 & -0.326 & $-9.9(4.9 \%)$ & 201.3 & 191.4 \\
\hline 6 & Astore at Doyian & 257.7 & -0.540 & $-35.1(3.1 \%)$ & 1136.7 & 1101.6 \\
\hline 7 & Indus at Tarbela & 19355.3 & -0.150 & $-16.7(4.0 \%)$ & 421.2 & 404.6 \\
\hline 8 & Chitral at Chitral & 1736.3 & -0.320 & $-44.8(6.1 \%)$ & 737.2 & 692.4 \\
\hline 9 & Panjkora at Zulum Br. & 41.0 & -0.350 & $-2.5(0.4 \%)$ & 616.5 & 614.0 \\
\hline 10 & Swat at Chakdara & 202.6 & -0.400 & $-14.1(1.2 \%)$ & 1186.3 & 1172.2 \\
\hline 11 & Kabul at Warsak & 1851.5 & -0.340 & $-8.9(5.7 \%)$ & 154.8 & 145.9 \\
\hline 12 & Kabul at Nowshera & 2095.0 & -0.340 & $-7.9(2.5 \%)$ & 305.6 & 297.7 \\
\hline 13 & Jhelum at Mangla & 262.7 & -0.550 & $-4.3(0.5 \%)$ & 792.8 & 788.5 \\
\hline 14 & Chenab at Marala & 2667.4 & -0.560 & $-51.5(5.0 \%)$ & 1026.4 & 975.0 \\
\hline 15 & Ravi at Thein dam & 166.9 & -0.386 & $-10.5(0.8 \%)$ & 1391.0 & 1380.5 \\
\hline 16 & Beas at Pong dam & 511.0 & -0.213 & $-8.7(0.9 \%)$ & 986.5 & 977.8 \\
\hline 17 & Sutlej at Bhakra dam & 1411.9 & -0.359 & $-9.3(3.5 \%)$ & 264.2 & 254.9 \\
\hline
\end{tabular}

Table 3.5: Relative bias (B) and relative mean root-transformed error (E) for all observation points.

\begin{tabular}{llllllllllllll}
\hline & JAN & FEB & MAR & APR & MAY & JUN & JUL & AUG & SEP & OCT & NOV & DEC & ANN \\
\hline B & 0.85 & 0.89 & 0.89 & 0.88 & 0.87 & 1.03 & 0.94 & 0.97 & 0.91 & 0.78 & 0.79 & 0.83 & 0.94 \\
$\mathbf{E}$ & 0.86 & 0.75 & 0.74 & 0.73 & 0.79 & 0.68 & 0.59 & 0.58 & 0.6 & 0.82 & 0.82 & 0.85 & 0.71 \\
\hline
\end{tabular}


Another means of validation is the comparison of the estimated precipitation with the corresponding observed river flows (specific runoffs). Dahri et al. (2016) demonstrated that the previous estimates of precipitation distribution in the study area are not only highly contrasting but largely underestimating the actual precipitation. Likewise, in the Dahri et al. (2016) study, precipitation estimates derived from the unadjusted precipitation observations provided relatively better estimates than the previous studies. Yet, slightly lower precipitation than the measured specific runoff in 9 out of 17 sub-basins (Figure 3.4) is absolutely counterintuitive implying underestimated precipitation or an unaccounted source of water (e.g. glacier melt contribution). Long term annual mean precipitation must always be greater than the corresponding specific runoff if a positive or neutral mass balance is prevalent in any river basin. In case of a negative mass balance, its contribution to river flows has to be subtracted from the actually observed river flows and the adjusted flows must be lower than the corresponding mean annual precipitation. Cross validation of adjusted precipitation estimates with the corresponding adjusted specific runoffs (Figure 3.4) revealed adjusted specific runoff well below the adjusted precipitation estimates for all the sub-basins except Swat, which reflects underestimated precipitation or a bigger contribution of a negative mass balance to river flows.

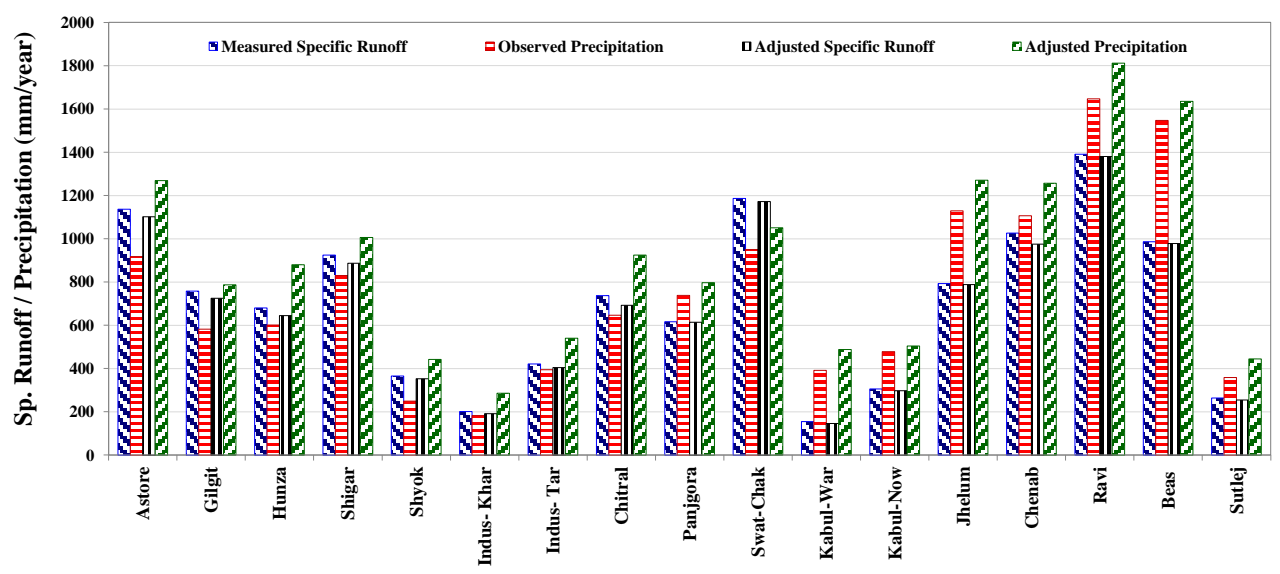

Figure 3.4: Annual measured and adjusted specific run-off and annual observed and adjusted precipitation at sub-basin scale

\subsection{Discussion}

Precipitation is an integral component of the hydrological cycle and usually the most important input to water balance assessments and climate change studies. Hence, its accuracy is essential as errors in precipitation estimates may translate into major changes in the water budget of a particular region. However, in many areas, precipitation measurements are still subject to significant errors and a large uncertainty (Kochendorfer et al., 2017a \& b) often leading to a substantial underestimation of the actual precipitation. The situation is particularly serious in the high-altitude Indus basin where biased distribution and lack of the observed data further worsen the problem. As such the precipitation products derived from or validated by the observed data covering this region are prone to significant errors (Dahri et al., 2016; Reggiani and Rientjes, 2015). Scientists have used different approaches to overcome the observational data gaps. For example, Adam et al. (2006) used a water balance approach to indirectly estimate precipitation. However, large uncertainties in the different water balance components limit wider 
application of this approach. Immerzeel et al. (2015) used mass balance estimates to inversely compute precipitation in the major snow/glacier zones and applied a linear lapse rate of precipitation increase with elevation up to $5000 \mathrm{~m}$ using APHRODITE as the reference dataset. Uncertainties in mass balance and water balance components and assumption of linear precipitation increase with altitude are the major drawbacks of this method. Dahri et al. (2016) integrated station observations with the net snow accumulations estimated through mass balance studies and applied KED interpolation scheme to derive precipitation in the ungauged areas. Measurement errors in precipitation observations and negligence of snow/glacier ablations in the net snow accumulations are the key shortcomings of this approach.

The approach adopted in this study is based on catch adjustments of precipitation observations for systematic measurement errors, adjustment of net snow accumulations for the ablation losses, and adjustment of river flows for the contribution of net glacier mass balance. Mean monthly precipitation climatologies are derived from i) actual precipitation observations and actual net snow accumulations, ii) adjusted precipitation observations \& the adjusted net snow accumulations following Dahri et al. (2016).

The results presented in this study further support the wind-induced under-catch as the largest source of errors in gauge-measured precipitation observations. The catch corrections have increased the gauge-measured precipitation values ranging from $12-773 \mathrm{~mm} \mathrm{yr}^{-1}$ for various stations, while net snow accumulations at the glacier points increased up to $1000 \mathrm{~mm} \mathrm{yr}^{-1}$. A large part of precipitation in the high-altitude Indus basin falls as snow, which is more susceptible to under-catch even at moderate wind speeds. The largest corrections were found for wind-induced under-catch of solid precipitation, which is in line with the results of previous studies (e.g. Kochendorfer et al., 2017a \& b; Wolff et al., 2015; Chen et al., 2015; Yang et al., 2005; Michelson, 2004; Ye et al., 2004; Adam and Lattenmaier, 2003; Goodison et al., 1998; Legates and Willmot, 1990). However, liquid precipitation under-catch, wetting loss and trace precipitation loss are also important, particularly in low-altitude and relatively dry areas.

The large differences between the observed precipitation and the corresponding specific runoff observations (usually greater specific runoff than precipitation) in previous estimates are often attributed to the contribution of snow/glacier melt. Indeed, the high-altitude Indus basin receives considerable snow/glacier melt contributions, which largely come from the melting of temporary/seasonal snow cover and may vary from year to year depending on the quantity and timing of winter snowfall and snowmelt during the subsequent summer. However, quantitative estimates of net glacier mass balance contributions to river flows are largely lacking. Therefore, the accuracy of the estimated net glacier mass balance contributions to the river flows is mainly depending on the uncertainties in glacier area and the ablation rates of mass balance. Our methodology of adjusting river flows for the net mass balance contributions is straight forward and the adjustments are slightly less than what is modelled by Lutz et al. (2016). For example, we estimated net glacier mass balance contribution of $-17.3 \mathrm{~mm} \mathrm{yr}^{-1}$ for the Indus at Besham Qila against $-25.0 \mathrm{~mm} \mathrm{yr}^{-1}$ modelled by Lutz et al. (2016). The difference might be due to the use of different approaches and different glacier inventories having different glacier areas. Lutz et al. (2016) pointed out a $23 \%$ difference in the glacier areas from three different inventories implying considerable differences in the water balance components.

The precipitation distribution derived through actual station observations combined with the actual net glacier accumulations is almost similar to that derived by Dahri et al. (2016) 
except for the addition of a few sub-basins and the use of additional and updated observed data. The catch corrections and snow accumulation adjustments significantly increased the total gauge-measured as well as basin scale precipitation (Figs. 3.2, 3.3-o \& 3.4; Table 3.3). The overall distribution patterns of precipitation remained largely the same as identified by Dahri et al. (2016), but substantial increases in the magnitude of precipitation amounts are realized. One of the advantages of the KED interpolation method is that it estimates an interpolated surface from a randomly varied small set of measured points and recalculates estimated values for these measured points to validate the estimates and determine the extent of errors. When compared with the corrected precipitation derived by Immerzeel et al. (2015), our estimates show significantly smaller root mean square error and a stronger correlation with the error-adjusted station observations (Figure 3.5). The corrected precipitation estimates by Immerzeel et al. (2015) show considerable differences with significantly lower values at the majority of station locations including the points at the major glaciers, where actual measurements of net snow accumulations were taken. At the basin scale their estimates are relatively better but seem to be on the higher side in about half of the sub-basins. This discrepancy between station-based point observations and basin scale precipitation estimates by Immerzeel et al. (2015) may be attributed to the higher and linear lapse rates of precipitation increase applied to compute the precipitation fields. Also, they did not validate their estimates with the observed precipitation of the individual stations. Instead, they used the Turc-Budyko representation to show the physical realism of their estimates and attributed some of the estimates that fall on the right side (inside) of the theoretical Budyko curve to the possible contribution of the negative mass balance to river flows and uncertainties in the potential evapotranspiration $\left(\mathrm{ET}_{\mathrm{p}}\right)$ dataset.

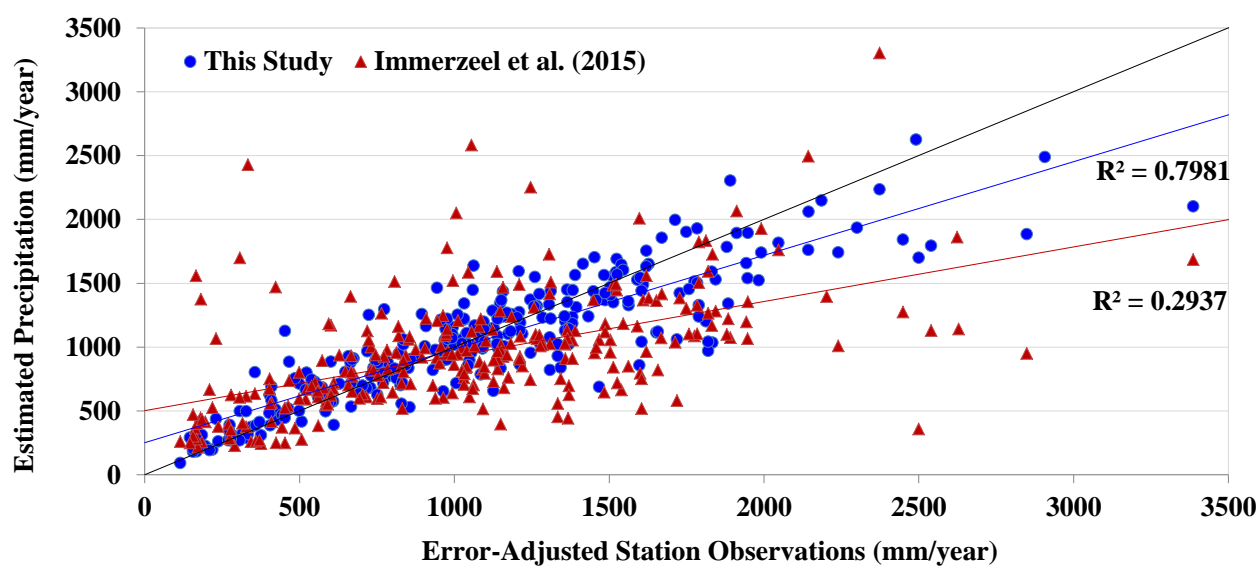

Figure 3.5: Comparison of error adjusted station observations with the corresponding estimated values under this study and by Immerzeel et al. (2015)

In this study, we used accurate runoff observations (specific runoffs), which are further improved by adjusting for the net glacier mass balance contributions, and improved ET $_{P}$ estimates from JRA55 reanalysis dataset (Figure 3.6) to evaluate the physical realism of our estimated precipitation compared to the precipitation estimates from Immerzeel et al. (2015). Over one-third of the points representing estimated precipitation by Immerzeel et al. (2015) in various sub-basins (e.g. Gilgit, Chitral, Panjkora, Kabul at Warsak and Nowshera, and Sutlej) lay inside the theoretical Budyko curve indicating higher values than the theoretically expected. Whereas, the estimates of unadjusted precipitation in our study, 
which are almost similar to the estimates of Dahri et al. (2016), show 10 out of 17 subbasins above the line of moisture limit indicating underestimated precipitation in these subbasins. The adjusted precipitation derived in our study shows relatively better fits in the Turc-Budyko representation except for the Swat sub-basin. The greater specific runoff than precipitation in the Swat basin may be attributed to yet an underestimated precipitation and/or greater negative mass balance than what is presently assumed.

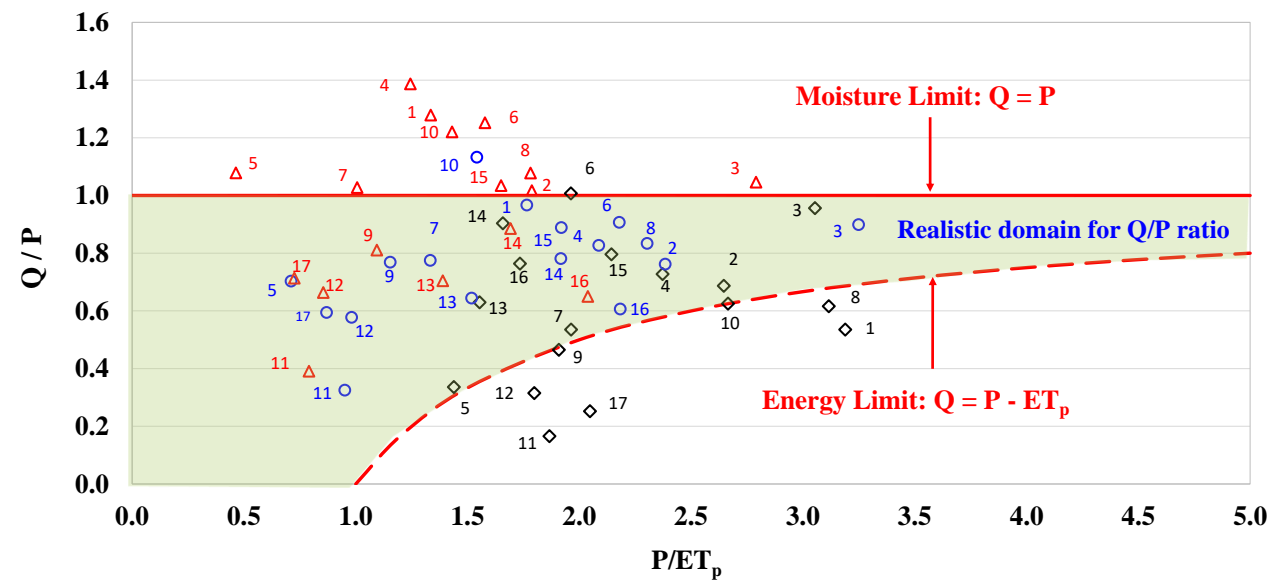

Figure 3.6: Turc-Budyko representation of run-off ratio $(\mathrm{Q} / \mathrm{P})$ and aridity index $\left(\mathrm{P} / \mathrm{ET}_{\mathrm{p}}\right)$. The red triangles display estimates of unadjusted case or Dahri et al. (2016), black diamonds show estimates of Immerzeel et al. (2015) and blue circles indicate adjusted estimates under this study. The numbers refer to the sub-basins as given in Table 4.

The runoff ratio $(\mathrm{Q} / \mathrm{P})$ determines the amount of precipitation converted into overland flow or surface runoff. It is mainly controlled by largely stable natural factors including climate, soil and topography and to some extent by the human alterations to landscapes. Relatively higher runoff ratios are produced for areas with shallow or clay soils, steeper slopes and devoid of vegetation cover. Snow covered areas hold winter precipitation as snow/ice and produce higher runoff ratios during the subsequent snow melting periods. Over $50 \%$ of the study area possesses slopes steeper than $40 \%$ and about $81 \%$ of the surface soil type is leptosol (47.4\%), cambisol (22.5\%), and rock outcrop (11.1\%). Dominant land cover types are closed to open herbaceous vegetation $(34.6 \%)$, bare rocky areas $(25.3 \%)$ and permanent snow and glaciers (13.4\%) (Figure S-3.6). All these topographical properties infer the highaltitude Indus basin as a typical case of an area that accelerates rapid runoff generation. Therefore, relatively high rates of runoff ratios are to be expected. Table S-3.4 and Figure 3.6 show the improved runoff ratios $(\mathrm{Q} / \mathrm{P})$ and aridity indices $\left(\mathrm{P} / \mathrm{ET}_{\mathrm{p}}\right)$ if compared to the datasets of Dahri et al. (2016) and Immerzeel et al. (2015).

Although, the error-adjusted precipitation derived in this study seems to be more consistent, yet there are a few uncertainties that need to be understood and taken care of in future investigations. The major uncertainties associated with the results of our study may arise from four possible sources: i) uncertainties in regression models due to their imprecision and uncertainties in the input data, ii) uncertainties arising from the estimated temperature and wind speed for many observatories, iii) uncertainty in the gauge type of the basin's gauge network, and iv) uncertainties in spatial interpolation of the point observations to derive gridded fields of precipitation. The error estimation of the regression models employed in this study are tested at different locations and the relationships with the best fit 
are also applicable for similar situations in other areas. Nevertheless, regression models are in essence approximations of reality and some degree of uncertainty will always remain in the results. Relatively more accurate adjustments of precipitation under-catch for any precipitation event can be made by using the corresponding data of temperature and wind speed. However, hourly or daily data of these parameters are not available for many observatories in the study area. Also, there are many stations for which such data are not available at all. For locations without these data, temperature may be derived from the lapse rates of the available observations and wind speed from JRA55 dataset. However as shown, the use of these data may add to the uncertainties in the catch corrections. The meteorological data collecting agencies in the Indus basin generally indicate to follow the WMO standards but we found inconsistencies in the use of precipitation measurement instruments and techniques. As the correction coefficients to account for wind-induced under-catch of precipitation depend on the type and orifice area of the precipitation gauge, incorrect gauge configuration information has consequences for the catch corrections. Although we tried our best to obtain the maximum possible information regarding the type and specs of precipitation gauges, we cannot exclude the chances of different precipitation gauges than the actual ones in some cases. However, we also think that the possibility of slight differences in gauge type will only have a small impact on the final results. The uncertainties resulting from spatial interpolation techniques described by Dahri et al. (2016) are equally applicable for this study. Importantly, the cross-validation results infer high accuracy of the corrections and indicate excellent agreement between the adjusted precipitation and adjusted specific runoff at sub-basin scale.

\subsection{Conclusions}

Reliable estimates of precipitation climatologies and amounts in the high-altitude Indus basin are seriously constrained by the quality and number of observed data (e.g. scarcity of in-situ observations, measurement errors, and space-time breaks). This study attempted to address these core issues by improved estimates of the precipitation measurement errors and integrating precipitation data from multiple sources with the net snow accumulations at major glacier zones. The study employed WMO recommended standard methods to adjust systematic errors in precipitation measurements. Simple methods to adjust net snow accumulation for the ablation losses and adjustment of river flows for the net mass balance contributions are introduced. Mean monthly adjusted and unadjusted precipitation observations and net snow accumulations are spatially interpolated using the Kriging with external drift interpolation scheme. Analysis of temperature variations with elevation and latitude revealed significantly different gradients for each month and substantial differences among the gradients at different locations for maximum and minimum temperatures. Hence, the use of a universal annual gradient or a time independent gradient of mean temperature to estimate maximum and minimum temperatures or vice versa is a major source of uncertainty for the high-altitude Indus basin.

The applied error-adjustments significantly increased the gauge-measured precipitation, which is in line with previous studies. The total bias between gauge-measured and error-

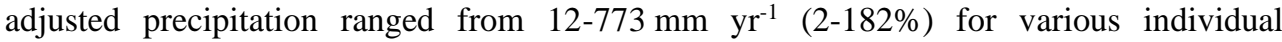
stations. The highest increments are computed for wind-induced under-catch of solid precipitation, particularly in higher-altitude areas and during winter months. The range of liquid precipitation under-catch is much smaller concentrating mainly in the low-altitude areas during summer monsoon. Similarly, notable increases varying from $0-1000 \mathrm{~mm} \mathrm{yr}^{-1}$ $(0-200 \%)$ are estimated for net snow accumulations. Precipitation increase at the basin 
(study area) scale is $21.3 \%$, while at sub-basin scale it ranged from 6-77\% with greater increments at higher-altitude areas and during winter months. Contrary to the general understanding, the contribution of net glacier mass balance to river flows is only marginal ranging from $0.5-6.1 \%$ of the observed flows. The highest contributions are revealed for the Chenab, Chitral, Shigar, Hunza, Astore and Gilgit basins.

The cross-validation results (Figure 3.4) and the Turc-Budyko representation of the runoff ratios and aridity indices at sub-basin scale (Figure 3.5) show that the adjusted precipitation amounts and distribution patterns derived in this study are more accurate than the unadjusted data and previous estimates. The catch corrections provided new insights in the magnitude and distribution patterns of precipitation implying potential hydrological implications for water resources assessment, planning and management. The actual precipitation is considerably greater than what has been previously thought. These increases are mainly realized in the higher-altitude areas of Chitral, Gilgit, Hunza, Shigar, Shyok and Astore basins. The study recognizes that the data-quality driven underestimated precipitation may be the major source of uncertainty in the water balance estimates in the high-altitude Indus basin. The improved climatologies of mean monthly precipitation developed in this study can be used for basin or sub-basin scale water balance studies and bias correction of gridded precipitation products, thereby paving the way for the development of an accurate, consistent and high-resolution gridded precipitation product for this highly under-explored region of the Indus basin.

Although, our estimates of precipitation distribution can easily be regarded as much better than currently available estimates, the uncertainties elaborated at the end of the previous section recognize the need for further improvement. Further improvements can be achieved by calibration of the already installed precipitation gauges with the WMO recommended reference gauges and development of site and gauge specific error adjustment models, use of observed data with better spatio-temporal coverage, use of daily or even sub-daily time steps, use of corresponding observed wind speed and temperature datasets, selection of any better spatial interpolation technique, accuracy assessment and precise determination of other components of the water balance to validate precipitation, and a better integration of precipitation data with mass balance data.

\section{Acknowledgements}

This research work is supported by the Dutch Ministry of Foreign Affairs through the Netherlands Fellowship Program (NFP) and carried out by the Himalayan Adaptation, Water and Resilience (HI-AWARE) consortium. A significant part of this work was undertaken at the International Institute for Applied System Analysis (IIASA), Laxenburg, Austria under the Young Scientist Summer Program (YSSP) 2016 with financial support provided by the Dutch National Member Organization (NMO). The views expressed in this work do not necessarily represent those of the supporting organizations. The authors express their deepest gratitude to WAPDA and PMD for sharing the hydro-meteorological data of the study region. We also acknowledge Arthur F Lutz and Jennifer C Adam for providing their precipitation datasets for comparison and further analysis in this study. The authors declare that there is no conflict of interest. 


\title{
Chapter 4
}

\section{Evaluation of Gridded Precipitation Products}

\begin{abstract}
The high-altitude Indus basin is one of the most complex and inadequately explored mountain terrains in the World, where reliable observations of precipitation are highly lacking. Therefore, spatially distributed precipitation products developed at global/regional scale are often used in several scientific disciplines. However, large uncertainties in precipitation estimates of such precipitation datasets often lead to suboptimal outcomes. In this study, performance of 27 widely used gridded precipitation products belonging to three different categories of gauge-based, reanalysis and merged (combined) is evaluated with respect to high-quality reference climatologies of mean monthly precipitation. Widely used statistical measures and quantitative analyses techniques are used to analyze the spatial patterns and quantitative distribution of mean monthly, seasonal and annual precipitation at sub-regional scale. Mean annual precipitation estimates of the gridded datasets are cross validated with the corresponding adjusted streamflows using Turc-Budyko nondimensional analysis. Results reveal poor to moderately good performance of the gridded datasets. Marked differences in spatiotemporal and quantitative distribution of precipitation are found among the datasets. All datasets are consistent in their patterns showing negative or dry bias in wet areas and positive or wet bias in dry areas, although considerable differences in the magnitudes of the biases are noticed at sub-regional scale. None of the datasets is equally good for all sub-regions due to very high spatiotemporal variability in their performance at sub-regional scale. Gauge-based and merged products performed better in dry regions and during monsoon season, while reanalysis products provided better estimates in wet areas and during winter months. GPCC V8, ERA5 and MSWEP2.2 are found better than their counter-grouped datasets. Overall, ERA5 is found most acceptable for all sub-regions, particularly at higher-altitudes, in wet areas and during winter months.
\end{abstract}

\section{This chapter has been accepted as:}

Dahri, Z. H., Moors, E., Ludwig, F., Ahmad, S., Ahmad, B., Shoaib, M., Ali, I., Iqbal, M.S., Pomee, M.S., Mangrio, A.G., Ahmad, M.M., \& Kabat, P. (2020). Spatio-Temporal Evaluation of Gridded Precipitation Products for the High-Altitude Indus Basin, International Journal of Climatology, 2020; JOC-19-0591. 


\subsection{Introduction}

Precipitation is the most important climatic variable associated with atmospheric circulations and hydrological cycle and unequivocally the principal source of freshwater supplies. It plays a crucial role in socioeconomic developments, environmental integrity and sustaining life on earth. Naturally, precipitation is discontinuous in space and time, has complex characteristics, can occur in several forms, and its causal mechanisms can influence precipitation from cloud to cyclone scales. Errors in precipitation data can have significant implications for climate and water balance studies. It is therefore essential to accurately measure/estimate precipitation at higher spatiotemporal resolutions. This is especially the case in topographically complex high-mountain ranges where precipitation often changes abruptly over short distances (Anders et al., 2006) and majority of it falls as snow. A high-quality, dense and adequately representative network of observations is essential to precisely measure occurrence, quantity and type of precipitation.

The high-altitude Indus basin is one of the most complex and largely underexplored regions in the World. Its climate and precipitation are largely modulated by a couple of synopticscale atmospheric circulation systems: the Indian summer monsoon and the winter westerlies (Pang et al., 2014; Yao et al., 2012; Ding and Chan, 2005; Wang and Lin, 2002). The Indian summer monsoon advects moisture through several trajectories originating from the Bay of Bengal, Indian Ocean and Arabian Sea due to the differential heating between land and sea (Pang et al., 2014; Bolch et al., 2012; Yao et al., 2012; Bohner, 2006; Hodges, 2006). It causes heavy rainfall in south-eastern areas during June-September and moves north-westward along the Himalayan Arc with decreasing strength. The winter westerlies transport large masses of moist air from the Caspian, Black \& Mediterranean seas and North Atlantic Ocean throughout the year and are the dominant source of precipitation in the Hindukush, Karakoram and to a lesser extent in the W-Himalayan regions during December-April months (Filippi et al., 2014; Pal et al., 2014; Mayer et al., 2014; Bohner, 2006; Treydte et al., 2006; Syed et al., 2006). Moreover, significant amount of moisture in the air is added to the atmosphere by evapotranspiration from the vast irrigated plains and forestlands (de Kok et al., 2018; Harding et al., 2013; Wie et al., 2013; Tuinenburg et al., 2012). Heavy precipitation events are encountered whenever these systems coincide and interact with each other (Zaidi, 2014; SUPARCO and FAO, 2010; WMO, 2010).

Precipitation distribution in the high-altitude Indus basin is extremely variable due to varying influence and interplay of the prevailing synoptic-scale atmospheric circulation systems with the local climate and topographic features. Highly sparse and directionally biased network of existing in situ observations insufficiently represents the entire range of a diverse climate in the study area (Dahri et al., 2018, 2016; Immerzeel et al., 2015; Reggiani and Rientjes, 2015; Fowler and Archer, 2006). Hence, our understanding of the prevailing hydro-meteorological processes in this region is seriously uncertain (Lutz et al., 2014; Andermann et al., 2011). Therefore, reliance on gridded datasets has been increased due to inadequate in situ observations and increasing demand for precipitation data in spatially distributed format. As such, a wide range of gridded precipitation products have been developed over the recent decades. The available datasets can broadly be categorized into four groups: gauge-based, reanalysis, satellite-derived and merged products. The most important are the gauge-based datasets derived from the on-ground direct measurements, which provide relatively precise occurrences, amounts and types of precipitation at the measuring points. These point measurements are often used for calibration, validation and bias correction of reanalysis and satellite estimates. However, the gauge-based precipitation 
datasets are also prone to observational uncertainties resulting from measurement errors, insufficient spatiotemporal coverage, uneven distribution and directional biases of the gauges, difficulties in snowfall measurements in windy conditions, and the applied interpolation methods. The magnitude of these uncertainties can be significant in orographically influenced complex mountain terrains (Dahri et al., 2018; Prein et al., 2017; Boers et al., 2016; Lundquist et al., 2010).

Alternatively, several precipitation estimates modelled through Retrospective weather forecast model analysis (Reanalysis) or derived from satellite data provide gaugeindependent estimates. These datasets offer viable substitutes for homogeneous, consistent, near-real-time and fairly reliable estimates of a wide range of climatic variables at global scale (Ghodichore et al., 2018). A typical reanalysis system objectively integrates observations, a global forecast model, and an assimilation scheme to generate synthesized estimates of the past atmospheric states at global scale (Fujiwara et al., 2017). Conversely, precipitation products derived from satellite data have gone through gradual improvements since their inception and currently incorporate data from several instruments and satellites (e.g. Ciabatta et al., 2018; Huffman et al., 2018, 2007; Xie et al., 2017; Ushio et al., 2009; Joseph et al., 2009). Yet these products are poor in precisely capturing the solid precipitation (Rasmussen et al., 2012; Putkonen, 2004). The satellite-based precipitation products vary considerably in terms of their source and processing algorithms as several sensors aboard geostationary earth orbiting (GEO) and low-earth orbiting (LEO) satellites observe precipitation passively or actively. A few studies observed that satellite-based products are better at estimating convective precipitation, whereas frontal system precipitation is better characterized by reanalysis (e.g. Vila et al., 2010; Sapiano and Arkin, 2009; Tian et al., 2009; Ruane and Roads, 2007; Ebert et al., 2007). This indicates that reanalysis and satellite-derived datasets are complementary, particularly for the areas where validations are inadequate or impossible due to lack or absence of in situ observations. (Beck et al., 2017; Pena-Arancibia et al., 2013).

Owing to the underlying issues in the available datasets to precisely estimate extreme heterogeneity of precipitation, several attempts have been made to take full advantage of the complementary nature and comparative advantages of the gauge-based observations, satellite data and reanalysis. Numerous merged (gauge-based, reanalysis and/or satellitederived) precipitation products have been developed over the recent time (e.g. Beck et al., 2019, Xie et al., 2017; Karger et al., 2017; Ashouri et al., 2015; Funk et al., 2015a; Weedon et al., 2014; Huffman et al., 2007; Janowiak and Xie, 1999; Xie and Arkin, 1997). These datasets mostly rely on merging algorithms to limit the shortcomings of the source datasets and produce higher quality end products.

Although, gridded datasets provide better information in terms of spatiotemporal consistency, their inadequacy to precisely estimate occurrence, quantity and type of precipitation is still a major concern. Recent innovations in weather forecasting models, satellite sensors and retrieval methods, and multi-source merging techniques coupled with high-quality observations have significantly improved the quality of resultant precipitation products. Yet, their spatiotemporal accuracy at basin/catchment scales particularly in orographically influenced and topographically complex mountain terrains is highly variable (Beck et al., 2019, 2017; Sun et al., 2018; Henn et al., 2018; Maggioni et al., 2016;). There are also seasonal biases and difficulties in capturing the low intensity and snowfall events. Many existing precipitation products exhibit differences that are often larger than can be explained by observational or methodological uncertainties (Yin et al., 2015; Aghakouchak 
et al., 2012). Several evaluation studies have been undertaken at varying spatial scales using a variety of approaches, performance metrics and statistical indices (e.g. reviews by Sun et al., 2018; Maggioni et al., 2016; and Gebremichael, 2010). However, inconsistency in terms of reference dataset against which the accuracy is to be evaluated is an important issue. Many studies relied on spatially inconsistent point observations to assess the accuracy of gridded datasets; while others re-used many gauge observations already incorporated in development or validation of precipitation datasets, thereby precluding independent validation (Beck et al., 2019). Even though it is well-recognized that the gauge observations are prone to significant measurement errors (Goodison et al., 1998; Legates and Willmot, 1990; Sevruk and Hamon, 1984), these point-based gauge observations are often used without addressing uncertainties. Such reference point observations generally lack the required density to accurately represent the spatial heterogeneity of precipitation. Hydrological modeling is also used to evaluate quality of precipitation data by comparing observed and simulated flows obtained through varying precipitation inputs. However, the uncertainties associated with the modeling structure and other input data are the major drawbacks of this approach.

Performance of gridded precipitation products may often be satisfactory at global/continental scale, but they generally lack the accuracy and precision required at subregional and catchment scale studies, especially over regions of high spatio-temporal heterogeneity (Ghodichore et al., 2018; Gamp and Ludwig, 2017). The Indus river basin traversing through the high mountain ranges of the Tibetan Plateau (TP) and HindukushKarakoram-Himalaya $(\mathrm{HKH})$ regions is experiencing significant transformations in its hydrometeorology (Lutz et al., 2016) and is recognized as climate change hotspot (Krishnan et al., 2019; Lutz et al., 2018; De Souza et al., 2015). There is lack of comprehensive studies that could evaluate performance of gridded datasets in this area. Few studies evaluated the performance of some gridded precipitation products against a limited number of point observations (e.g. Krakauer et al., 2019; Ullah et al., 2019; Ahmed et al., 2019; Khan et al., 2018; Iqbal et al., 2018; Hussain et al., 2017; Ali et al., 2012) or using spatially distributed fields of mean annual precipitation derived from a limited number of point observations (e.g. Anjum et al., 2018; Ghulami et al., 2017). Dahri et al. (2016) integrated precipitation data from different sources to derive better estimates of spatially distributed precipitation and corroborated the underlying issues related to the use of four important gridded precipitation products in this region. Other studies (e.g. Reggiani and Rientjes, 2015; Palazzi et al., 2013) have relied on inter-comparison of a few datasets in the absence of reference dataset.

This study therefore comprehensively and rigorously evaluates the applicability, robustness and limitations of 27 widely used precipitation products for the complex and high-mountain Indus basin in the HKH region. The study is unique in that it assesses the performance and reliability of a wide range of products over a finer spatial scale. Quantitative and spatial variability of precipitation products is investigated at monthly, seasonal and annual scales against high-quality reference climatologies derived by Dahri et al. (2018). In addition, it cross validates the precipitation estimates of all gridded products using adjusted river flows through Turc-Budyko non-dimensional analysis. The study will provide useful inputs and guidelines for development, bias correction and improvement of gridded datasets. It will also serve as the basis for selection and use of appropriate datasets for hydrological and water assessment studies in the study area. 


\subsection{Data and Methods}

\subsubsection{Reference dataset}

The accuracy of a product is usually assessed against a high-quality reference benchmark. Here we used a recently developed high-resolution $(1 \mathrm{~km})$ dataset of mean monthly precipitation climatologies described in Dahri et al. (2018). This dataset was derived by integration of several precipitation data sources with indirect estimates of precipitation from snow accumulations measured at the major glacier zones to cover the observational gaps. The precipitation observations were adjusted for measurement errors, snow accumulations for the ablation losses, and river flows for the contribution net glacier mass balance. Precipitation estimates at sub-basin scale were validated by the corresponding adjusted specific runoff. Accuracy of the selected gridded precipitation products in this study is evaluated for each common grid cell with respect to this novel and high-resolution reference dataset of mean monthly precipitation at basin and sub-regional scale.

\subsubsection{Gridded precipitation products}

The selection of gridded datasets for this evaluation study is primarily based on availability of long-term ( 20 years) records, which must coincide with the period of the reference dataset (1999-2011). The three types of data evaluated in this study include the gaugebased, reanalysis and merged precipitation products. We excluded the satellite-derived products due to their short-term records, which do not coincide with the reference period and their large uncertainty to estimate solid precipitation (Rasmussen et al., 2012; Putkonen, 2004), which is dominant in the study area. An ensemble of 27 gridded precipitation products (nine from each of the three groups) available for the historical periods is selected to evaluate their accuracy in the study area. The major characteristics of the selected datasets are summarized in Table 4.1. For a detailed description of the input datasets and techniques used in development of these datasets, the corresponding references and/or concerned websites are suggested.

The datasets available at finer temporal resolutions (daily/sub-daily) were aggregated to monthly scale. Mean monthly precipitation climatologies for the reference period of 19992011 for each gridded dataset were regridded to 30 arc-seconds to match the temporal and spatial resolution of the reference dataset using the nearest neighbour interpolation technique. Extended winter (Oct-May) and monsoon (Jun-Sep) seasons are regarded keeping in view the onset and continuity of precipitation during the two major circulation systems (winter westerlies and summer monsoon) prevailing in the study area.

\subsubsection{River flows}

Dahri et al. (2018) collected river flow data for all major sub-basins in the study area and accounted for the diversions upstream of each rim station (river gauge). These observed river flows were adjusted for the contributions of net mass balances using mass balance estimates provided by Kääb et al. (2012) and glacier areas estimated by Randolf Glacier Inventory (RGI) version 5.0 (Arendt et al., 2015). These adjusted river flows are used in this study for analysis and cross validation of the precipitation from the selected products through Turc-Budyko non-dimensional analysis. The adjusted river flows for the five subregions are computed from the area-weighted mean adjusted flows of the concerned susbasins. 
Table 4.1: Summary of the basic characteristics of the selected gridded precipitation products used in this study.

\begin{tabular}{|c|c|c|c|c|c|}
\hline $\begin{array}{l}\text { S. } \\
\#\end{array}$ & Product Name and Details & $\begin{array}{l}\text { Data } \\
\text { Sourc }\end{array}$ & $\begin{array}{l}\text { Grid } \\
\text { Size }\end{array}$ & $\begin{array}{l}\text { Temporal } \\
\text { Coverage }\end{array}$ & Reference \\
\hline & Gauge-based Products & & & & \\
\hline 1 & GPCC V8, Global Precipitation Climatology Centre (GPCC) Full Data Monthly Product Version 8 & G & $0.25^{\circ}$ & $1891-2016$ & Schneider et al. (2018) \\
\hline 2 & GPCC V7, Global Precipitation Climatology Centre (GPCC) Full Data Monthly Product Version 7 & G & $0.5^{\circ}$ & $1901-2013$ & Schneider et al. (2014) \\
\hline 3 & UDEL V5.01, University of Dilawere, Terestrial Precipitation: Gridded Monthly Time Series (V 5.01) & G & $0.5^{\circ}$ & $1900-2017$ & Mats. \& Willm. (2018) \\
\hline 4 & APHRODITE V1801R1, Asian Precipitation - Highly-Resolred Observational Data Integration Towards Evaluation V1801R1 & G & $0.25^{\circ}$ & 1998-2015 & Yatagai et al. (2018) \\
\hline 5 & APHRODIIE V1101, Asian Precipitation - Highly-Resolved Observational Data Integration Towards Evaluation V1101 & G & $0.25^{\circ}$ & 1951-2015 & Yatagai et al. (2012) \\
\hline 6 & PREC-Land, NOAA's PRECipitation REC onstruction orer Land (PREC/L) & G & $0.5^{\circ}$ & 1948-2012 & Chen et al. (2002) \\
\hline 7 & CRU TS4.02, Climate Research Unit TS4.02 & G & $0.5^{\circ}$ & $1901-2017$ & Harris et al. (2014) \\
\hline 8 & GPCP V2.3, Global Precipitation Climatology Project (GPCP) Version 2.3 Monthly Analysis & G & $2.5^{\circ}$ & 1979-NRT & Adler et al. (2018) \\
\hline \multirow[t]{2}{*}{9} & CPC Unified, Climate Prediction Center Unified gauge-based analysis V1.0 & G & $0.5^{\circ}$ & 1979-NRT & Chen et al. (2008) \\
\hline & Reanalyses Products & & & & \\
\hline 1 & ERA5, ECMWF's 5th generation Atmospheric ReAnalysis & $\mathbf{R}$ & $\sim 0.281^{\circ}$ & 1979-NRT & Hersbach et al. (2018) \\
\hline 2 & CFSR, National Centers for Environmental Prediction (NCEP) Climate Forecast System Reanalysis & $\mathbf{R}$ & $\sim 0.31^{\circ}$ & 1979-2010 & Saha et al. (2010) \\
\hline 3 & JRA-55, Japanese 55-year ReAnalysis & $\mathbf{R}$ & $0.5625^{\circ}$ & 1958-NRT & Kobayashi et al. (2015) \\
\hline 4 & ERA 20C, ECMWF Atmospheric Reanalysis of the 20th Century & $\mathbf{R}$ & $\sim 1.406^{\circ}$ & $1900-2010$ & Stickler et al. (2014) \\
\hline 5 & MERRA-2, Modern-Era Retrospective analysis for Research and Applications, Version 2 & $\mathbf{R}$ & $0.5^{\circ}$ & 1980-NRT & Gelaro et al. (2017) \\
\hline 6 & ERAI, European Centre for Medium-range Weather Forecasts ReAnalysis Interim & $\mathbf{R}$ & $\sim 0.75^{\circ}$ & 1979-NRT & Dee et al. (2011) \\
\hline 7 & DOE-R2, NCEP-Department of Energy (DOE) Reanalysis 2 & $\mathbf{R}$ & $\sim 1.875^{\circ}$ & 1979-NRT & Kanamitsu et al. (2002) \\
\hline 8 & NCAR-R1, NCEP-National Center for Atmospheric Research (NCAR) Reanalysis 1 & $\mathbf{R}$ & $\sim 1.875^{\circ}$ & 1948-NRT & Kalnay et al. (1996) \\
\hline \multirow[t]{2}{*}{9} & 20CR V2C, NOAA-CIRES Twentieth Century Reanalysis Version 2C & $\mathbf{R}$ & $\sim 1.875^{\circ}$ & $1851-2011$ & Compo et al. (2011) \\
\hline & Merged (combined gauge, reanalyses, satellite) Products & & & & \\
\hline 1. & MSWEP V2.2, Multi-Source Weighted-Ensemble Precipitation Version 2.2 & $\mathrm{G}, \mathrm{S}, \mathrm{R}, \mathrm{A}$ & $0.1^{\circ}$ & 1979-NRT & Beck et al. $(2017,2019)$ \\
\hline 2 & TMPA 3B42 V7, TRMM Multi-satellite Precipitation Analysis (TMPA) 3B42 V7 & $\mathrm{G}, \mathrm{S}$ & $0.25^{\circ}$ & 1998-NRT & Huffman et al. (2007) \\
\hline 3 & $\begin{array}{l}\text { PERSLANN-CDR VIRl, Precipitation Estimation from Remotely Sensed Information using Artificial Neural Networks- } \\
\text { Climate Data Record VIRI }\end{array}$ & G, $\mathrm{S}$ & $0.25^{\circ}$ & 1983-2016 & Ashouri et al. (2015) \\
\hline 4 & CHELSA V1.2, Climatologies at High resolution for the Earth Land Surface Areas, Version 1.2 & $\mathrm{G}, \mathrm{R}, \mathrm{A}$ & $0.0083^{\circ}$ & $1979-2013$ & Karger et al. (2017) \\
\hline 5 & CHIRPS V2.0, Climate Hazards group Infrared Precipitation with Stations Version 2.0 & $\mathrm{G}, \mathrm{S}, \mathrm{R}, \mathrm{A}$ & $0.05^{\circ}$ & 1981-NRT & Funk et al. (2015) \\
\hline 6 & WFDEI-CRU, WATCH Forcing Data ERA-Interim corrected by CRU & G, $R$ & $0.5^{\circ}$ & 1979-2015 & Weedon et al. (2014) \\
\hline 7 & CMAP, CPC Merged Analysis of Precipitation & $\mathbf{G}, \mathrm{S}, \mathbf{R}$ & $2.5^{\circ}$ & 1979-NRT & Xie and Arkin (1997) \\
\hline 8 & CMORPH V1.0, CPC MORPHing technique (CMORPH) Vl.0 & $\mathbf{G}, \mathbf{S}$ & $0.5^{\circ}$ & 1998-NRT & Xie et al. (2017) \\
\hline 9 & CAMSOPI, Climate Anomaly Monitoring System ("CAMS") and OLR Precipitation Index ("OPI") & $\mathrm{G}, \mathrm{S}$ & $2.5^{\circ}$ & 1979-NRT & Janowiak \& Xie (1999) \\
\hline
\end{tabular}

\subsubsection{Potential evapotranspiration}

There is no observational-based independent dataset of potential evapotranspiration (PET) for the study area. Therefore, previous studies have relied on global-scale gridded datasets using PET data from a single product (e.g. Dahri et al., 2018) or ensemble mean of several datasets (e.g. Immerzeel et al., 2015). Importantly, PET does not have crucial use in this study. It is only employed to estimate aridity index (P/PET) in Turc-Budyko nondimensional analysis. Recently, a $5^{\text {th }}$ generation reanalysis (ERA5) dataset including a number of atmospheric variables at global scale has been released. The evaluation of precipitation products undertaken in this study reveals that precipitation estimates of ERA5 are much better than the rest of reanalysis products for the study area. Therefore, as a complementary climate variable, PET from ERA5 reanalysis is selected for this study.

\subsubsection{Evaluation approach}

The study area is stretched over vast mountain and sub-mountain ranges of extremely variable topographic features (relief, aspect, soil, land cover, etc.) in the Indus basin. The confluence of three mountain ranges (Hindukush, Karakoram and Western-Himalaya) adds significant complexities in characterizing the connection between precipitation and topographic features (Palazzi et al., 2013). These three mountain ranges are influenced differently by the underlying atmospheric circulation systems and possess unique hydrometeorological and geomorphological characteristics. Therefore, in order to have a better idea of the quality of spatial distribution depicted by various gridded precipitation products and based on the availability of observed streamflows for ultimate evaluation and cross validation; the study area is divided into five sub-regions (Figure 1), which possess considerable differences in their precipitation patterns and magnitudes, and landscape morphologies. 


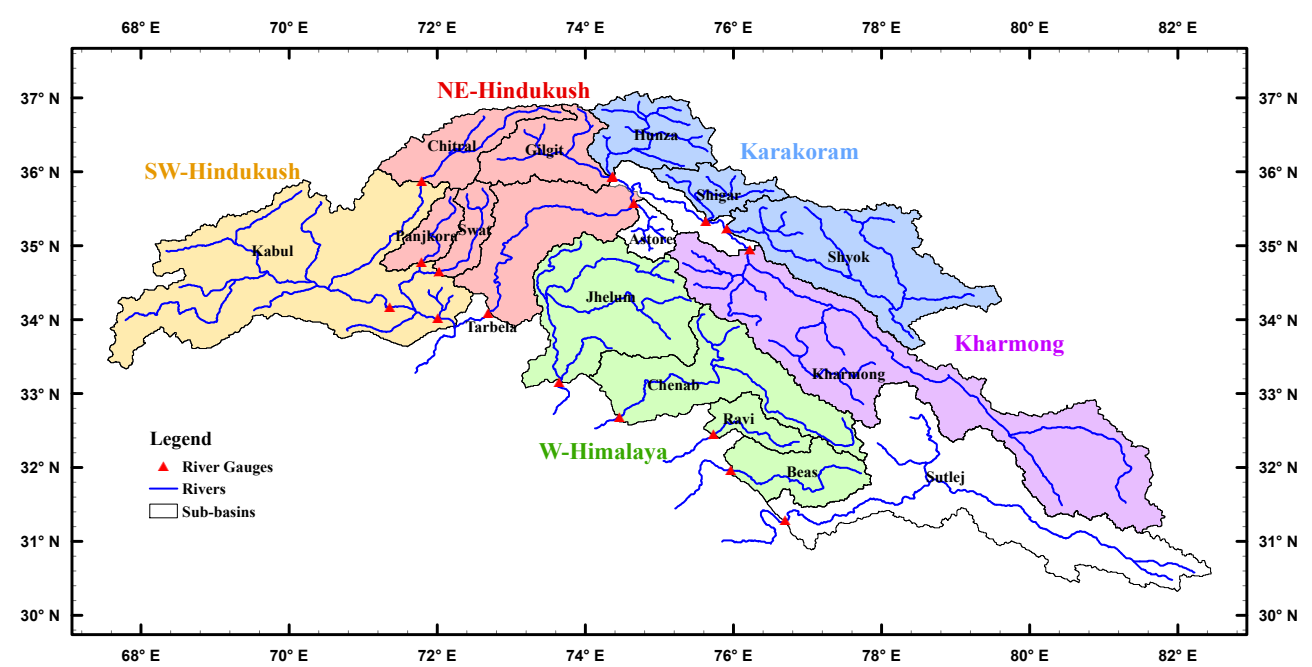

Figure 4.1: Study area and location of five regions analyzed in this study

Widely used statistical measures and quantitative analyses techniques are used to evaluate performance of the selected gridded precipitation products. All analyses are performed on the common grids for the reference period of 1999-2011. Mean annual precipitation for the reference period over the study area is plotted for each gridded dataset to visually examine their spatial distribution. The biases between mean annual precipitation of each gridded dataset and the corresponding grid of the reference dataset are plotted to show the spatial distribution of their residuals over the study area. The biases between mean monthly precipitation of each gridded dataset and reference dataset at each corresponding grid are analyzed through Box-Whisker charts for extended winter (Oct-May) and monsoon (Jun-Sep) season, which are further extended at annual scale for the study area as well as its five sub-regions to examine their median, distribution and spread at $1^{\text {st }}$ and $3^{\text {rd }}$ quarters and extremes. This is followed by computation of mean absolute error (MAE) in mean monthly precipitation of all gridded datasets against the reference dataset. The MAE computes the magnitude of the mean differences between two datasets without considering the direction of the error and is given by Eq. 1. The MAE is generally a preferred metric over widely used root mean square error (RMSE) when the errors are unlikely to follow a normal distribution (Beck et al., 2017; Willmott et al., 2017; Chai and Draxler, 2014).

Modified Kling-Gupta Efficiency (KGE) scores are computed using Eq. 2 (Kling et al., 2012; Gupta et al., 2009) at monthly scale for the study area and monthly precipitation extended at annual scale for study area and five sub-regions to examine how closely the spatio-temporal precipitation estimates of the gridded datasets are statistically matched with those of the reference dataset. Any value of KGE gives the lower limit of its three components, meaning that the worst component is $\geq$ to that value. The KGE has primarily been used for evaluating the quality of climate or hydrological models' outputs against the observed data. However, it can also be used to evaluate the performance of gridded precipitation estimates with respect to the corresponding observed or reference data (e.g. Beck et., 2019). The mathematical expressions of the employed performance evaluation metrics are given by:

$$
\text { MAE }=\frac{1}{n}+\sum_{i=1}^{n}\left|G_{i}-R_{i}\right|
$$




$$
\begin{aligned}
\mathbf{K G E}=1 & -\sqrt{(\boldsymbol{r}-\mathbf{1})^{2}+(\boldsymbol{\beta}-\mathbf{1})^{2}+(\gamma-\mathbf{1})^{2}} \\
\mathbf{r} & =\frac{\sum_{i=1}^{n}\left(G_{i}-\bar{G}\right)\left(R_{i}-\overline{\boldsymbol{R}}\right)}{\sqrt{\sum_{i=1}^{n}\left(G_{i}-\bar{G}\right)^{2} \sum_{i=1}^{n}\left(R_{i}-\bar{R}\right)^{2}}} \\
\boldsymbol{\beta} & =\frac{\mu_{\text {grd }}}{\mu_{\text {ref }}} \\
\gamma & =\frac{\sigma_{\text {grd }} / \mu_{\text {grd }}}{\sigma_{\text {ref }} / \mu_{\text {ref }}}
\end{aligned}
$$

Where: $\boldsymbol{G}_{\boldsymbol{i}}$ and $\boldsymbol{R}_{\boldsymbol{i}}$ represent the gridded and reference datasets at $\boldsymbol{i}_{\boldsymbol{t}}$ grid and $\boldsymbol{n}$ denotes number of grid cells, $\mathbf{r}$ in Eq. 2 is Pearson's correlation coefficient to measure the degree of linear relation between two datasets, $\boldsymbol{\beta}$ is bias computed by the ratio of gridded and reference means $(\boldsymbol{\mu}), \boldsymbol{\gamma}$ is variability ratio given by the ratio of the gridded and reference dataset's coefficients of variation $(\boldsymbol{\sigma} / \boldsymbol{\mu}), \boldsymbol{\sigma}$ is standard deviation, and subscripts grd and $r e f$ indicate gridded and reference datasets respectively. The optimum values of KGE, $r, \beta$, and $\gamma$ are at unity.

For robust quantitative assessment, the study further evaluated the annual cycle of areaweighted mean monthly precipitation of each gridded dataset for the reference period against that of the reference dataset. This comparison evaluates how well the gridded datasets follow the mean monthly and seasonal cycle of precipitation during the reference period in the study area and in each sub-region. The goodness-of-fit of these monthly cycles is ascertained through coefficient of determination $\left(\mathrm{R}^{2}\right)$ and MAE.

Isolated measures of performance evaluation are often associated with their specific uncertainties and limitations resulting in contrasting inferences. Therefore, instead of relying on a single measure, the outcomes of the above-described performance metrics and quantitative analyses techniques are integrated through a simple ranking system to evaluate the performance of the gridded datasets in a better and more consistent manner. For this purpose, the originally estimated values of these performance metrics for each gridded dataset are normalized and rescaled between 0 and 1 . However, in contrast to KGE and $\mathrm{R}^{2}$, lower values of MAE infer better performance. Therefore, the normalized values of MAE are subtracted from one to synchronize them with KGE and $\mathrm{R}^{2}$. The integrated skill scores are obtained by sum of the normalized values of KGE and $\mathrm{R}^{2}$ and subtracted normalized values of MAE for each dataset. The larger values infer higher rankings. This simple ranking system greatly reduces the contradictions and complexities in interpretation of the evaluation results. Furthermore, the wet and dry areas are defined by combining the subregions where annual mean precipitation is more and less than $600 \mathrm{~mm}$ respectively. Hence, the wet area adds the skill scores of W-Himalaya, Karakoram and NE-Hindukush, while dry area combines Kharmong and SW-Hindukush sub-regions. Similarly, the rankings and skill scores are also calculated for Indus basin upstream of Tarbela dam (Figure S-4.1 and Table S-4.3).

Finally, the mean annual precipitation estimates of all datasets are cross-validated by the corresponding adjusted streamflows (specific runoff) using Turc-Budyko non-dimensional analysis (Andreassian and Perrin, 2012; Valery, Andreassian and Perrin 2010; Budyko, 1974; Turc, 1954). Adjusted river flows determined in Dahri et at., 2018, potential evapotranspiration (PET) from ERA5 reanalysis product and precipitation estimates of gridded datasets for the whole study area and five sub-regions are used to compute run-off ratio $(\mathrm{Q} / \mathrm{P})$ and aridity index $(\mathrm{P} / \mathrm{PET})$. The Turc-Budyko non-dimensional analysis 
approach was originally introduced by Turc (1954) and Budyko (1974) to represent the relationships between actual and potential evapotranspiration (AET/PET) and between precipitation and potential evapotranspiration (P/PET). However, actual evapotranspiration is difficult to measure and spatially distributed data are very rare and often highly biased. Therefore, it was later on modified and further elaborated by an equivalent and alternative representation between $\mathrm{Q} / \mathrm{P}$ and $\mathrm{P} / \mathrm{PET}$ and introducing water and energy limits (e.g. Coron et al., 2015; Andreassian and Perrin, 2012; Valery, Andreassian and Perrin, 2010). Since then, the approach has been extensively applied in hydrometeorological and water balance assessments in several regions. The rankings and integrated skill scores of the datasets for each region are recognized only if a particular dataset falls within the theoretically feasible domain of $\mathrm{Q} / \mathrm{P}$ ratio in Turc-Budyko representation. The order of the ranking is updated accordingly after exclusion of the underperforming datasets in this criterion.

\subsection{Results}

\subsubsection{Spatial distribution of mean annual precipitation and residual errors}

Spatial distribution of mean annual precipitation estimates from various gridded datasets presented in Figure 4.2A and area-weighted seasonal and annual precipitation totals provided in Table 4.2 reveal significant variability of mean annual precipitation. Compared to annual mean precipitation of $697 \mathrm{~mm}$ of the reference dataset, the minimum estimates of $374 \mathrm{~mm}(-46 \%)$ are depicted by CPC Unified and maximum estimates of $976 \mathrm{~mm}(+40 \%)$ by ERAI datasets. However, the magnitudes vary considerably at sub-regional and seasonal scale. None of the selected gridded precipitation products could accurately distinguish and capture the zone of $2^{\text {nd }}$ precipitation maxima present in the central Karakoram. All gridded datasets also failed to detect the drier areas under the influence of rain shadows. However, significant consistency in terms of spatial patterns showing negative or dry bias in wet areas and positive or wet bias in dry areas with considerable difference in the magnitude of biases is noticed. Most prominent are the two swaths/paths of negative bias: $1^{\text {st }}$ from upper Chitral basin and passing through Gilgit, Hunza, Shigar \& Shyok basins; and $2^{\text {nd }}$ along the western Himalayan foothills from Beas across Chitral sub-basin. A large spread of residual errors (from -2600 to $3000 \mathrm{~mm}$ ) compared to the reference dataset (Figure 4.2B) is also evident.

The gauge-based and merged products show strong tendency of underestimation; while except ERA20C, reanalysis products tend to overestimate precipitation in most parts of the study area, with considerable difference at sub-regional level. This overestimation by the reanalysis products is more pronounced in the drier areas (e.g. Kharmong and SWHindukush). With the exception of a few reanalysis products (e.g. ERA5, CFSR, JRA-55, MERRA-2 and 20CR), the largest underestimates of gridded datasets are observed in the Karakoram region. Interestingly, DOE R2 provides the best quantitative estimate for the whole study area but its quantitative estimates, correlations and KGE scores at sub-regional scale are below par. The inter-regional variations are offset when aggregated for the whole study area. This underlines the importance of consistency in accuracy of precipitation estimates at varying spatial scales. 

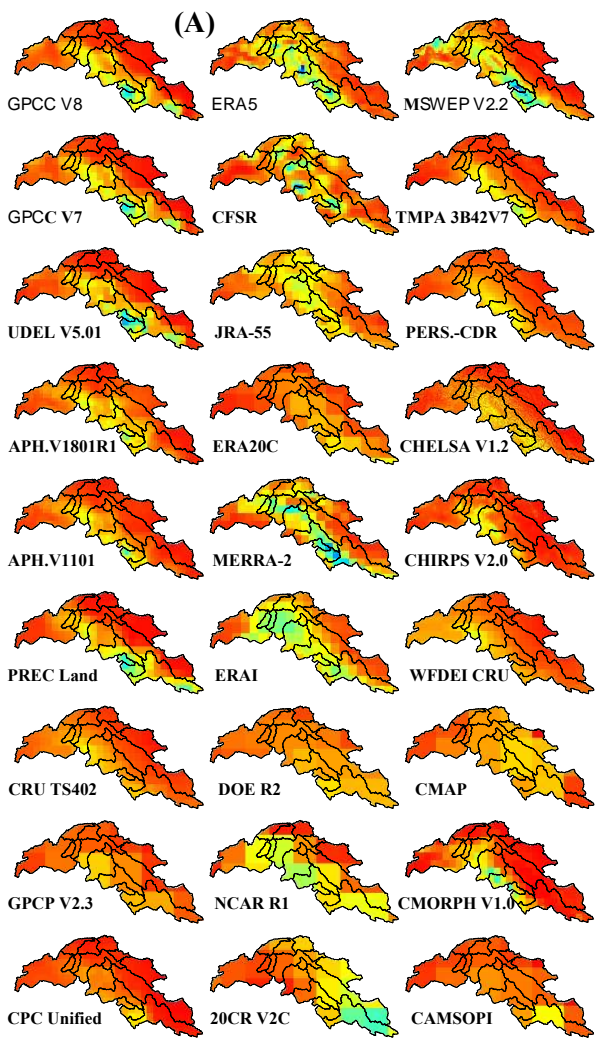

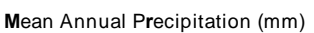
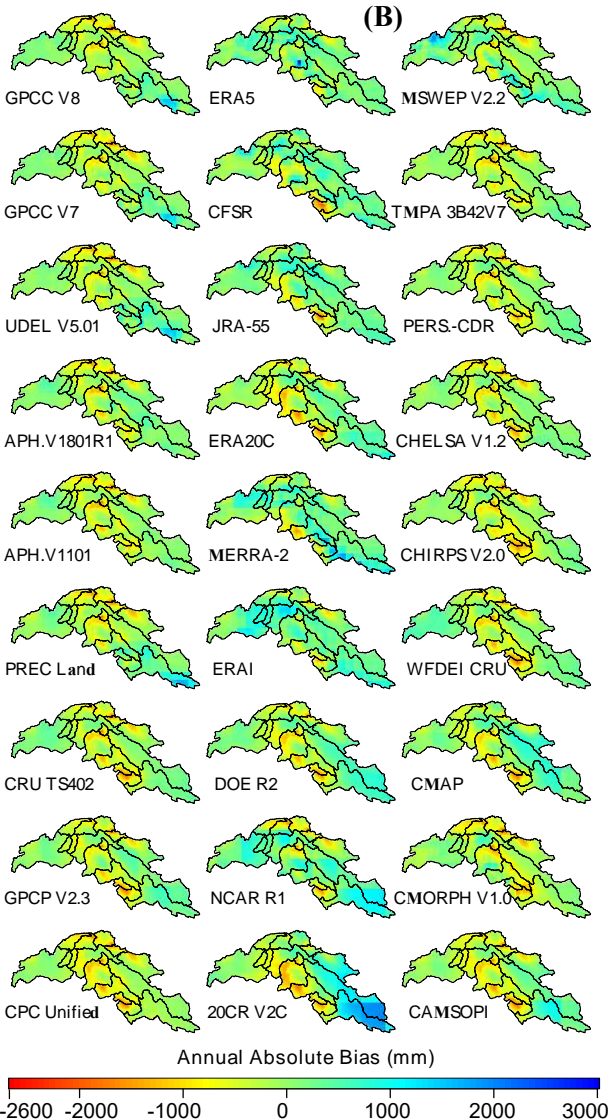

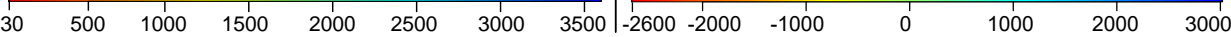

Figure 4.2: Spatial distribution of (A) mean annual precipitation illustrated by various gridded datasets, and (B) absolute bias between mean annual precipitation of gauge-based (left column), reanalysis (middle column), and merged (right column) products compared to the reference dataset.

Table 4.2: Area-weighted seasonal \& annual precipitation of the reference dataset and difference in the precipitation estimates of various gridded datasets.

\begin{tabular}{|c|c|c|c|c|c|c|c|c|c|c|c|c|c|c|c|c|c|c|c|}
\hline \multicolumn{2}{|r|}{ S. Precipitation } & \multicolumn{3}{|c|}{ Study Area } & \multicolumn{3}{|c|}{ W-Himalaya } & \multicolumn{3}{|c|}{ Karakoram } & \multicolumn{3}{|c|}{ Kharmong } & \multicolumn{3}{|c|}{ NE-Hindukush } & \multicolumn{3}{|c|}{ SW-Hindukush } \\
\hline H & Pro & $-\mathbf{M}$ & $\mathbf{J}-\mathbf{S}$ & ANN & O- $\mathbf{M}$ & $\mathbf{J}-\mathbf{S}$ & ANN & $\mathbf{O}-\mathbf{M}$ & $\mathbf{J}-\mathbf{S}$ & ANN & - M & $\mathbf{J}-\mathbf{S}$ & ANN & O- $\mathbf{M}$ & $\mathbf{J}-\mathbf{S}$ & ANN & O- M & $\mathbf{J}-\mathbf{S}$ & ANN \\
\hline 0 & REFERENCE (mm) & 457.7 & 239.6 & 697.3 & 778.7 & 584.2 & 1362.9 & 434.7 & 187.3 & 622.0 & 210.4 & 73.6 & 284.0 & 628.7 & 246.4 & 875.1 & 350.5 & 87.0 & 437.5 \\
\hline 1 & PC & 23.9 & 12.0 & 11.6 & -29.8 & 5.3 & 4.7 & 73.8 & -76.3 & 74.5 & -6. & 42.5 & 5.9 & -40.3 & 13.2 & 32.7 & 11.3 & 2.6 & 23.5 \\
\hline 2 & $G$ & 4. & .9 & 8 & -30.7 & 4 & 5.8 & 73.7 & -76.2 & 4 & -2.4 & .4 & 11.5 & 41.3 & 14.6 & 33.8 & 11.3 & 2.4 & 23.5 \\
\hline 3 & U & -18.6 & & 0 & -22.1 & .8 & .7 & 72.3 & -76.8 & 6 & 2 & 3.6 & 6 & 3.7 & 24.8 & 2.0 & 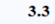 & 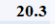 & 6.7 \\
\hline 4 & AP & -2 & & & -36 & & & -4 & & & & & 54.1 & & & & .2 & & 5.0 \\
\hline 5 & A & -37.3 & & & -43.5 & & & -62.3 & - 59 & -61.5 & -28.4 & 1.4 & -5.1 & -41.4 & -19.8 & 35.4 & 7.6 & ..5 & 0.1 \\
\hline 6 & $\mathbf{P}$ & -32.3 & 3.0 & & -41.2 & $x^{2}>$ & 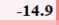 & -82.3 & -7 & -79.4 & 14.5 & 3.2 & 18.6 & -50.2 & -0. & 36.3 & 1.2 & 48.0 & 6.2 \\
\hline 7 & CR & -36.9 & 1. & 3.8 & -59.6 & -22.7 & 8 & -57 & -76 & -63.4 & -15.0 & 7.9 & 22.0 & -34.1 & -4.1 & .7 & 1.1 & 03.8 & 37.6 \\
\hline 8 & G & -40.1 & .6 & 18.5 & -57.1 & -13 & 38.3 & -51 & -25.8 & -43.6 & -6.4 & 300.1 & 74.7 & -43.9 & -1 & .1 & .1 & 64 & 3.0 \\
\hline 9 & CPC & -59.9 & 0.5 & -46.4 & -63.9 & -28.7 & -48.8 & -76.4 & -64.7 & -72.8 & -56.5 & 24.9 & -35.4 & -62.6 & -44.1 & -57.4 & -26.5 & 57.5 & -9.8 \\
\hline 1 & $\mathbf{I}$ & 6.9 & & 19.3 & -0.6 & 10.6 & 4.2 & -7 & 12. & -1.4 & 17.6 & 6.1 & 63.8 & 8.5 & 43 & 8.3 & 9.4 & 43.7 & 2.2 \\
\hline 2 & & 19.1 & & 1.2 & -12.7 & -27.3 & .9 & 39 & -9 & & .6 & 7.4 & 9.4 & 4.0 & -3 & .4 & 5.7 & & 2.3 \\
\hline 3 & JR & 18. & & & -10.6 & -26 & .3 & & & 4 & & .7 & .1 & .5 & -1 & .7 & 5.3 & & 6.4 \\
\hline 4 & $\mathbf{E}$ & -31.3 & 12.5 & -10 & -53.9 & -26.2 & -42.0 & -42 & -14 & -3 & & 3 & 103.9 & -34.6 & -4 & -37.0 & -29.4 & & -27.1 \\
\hline 5 & MEI & 11. & $74.0-3$ & & -1 & 18 & 7 & 20 & 3 & 1 & .2 & 7.1 & 90.1 & 9.7 & 64. & .1 & .6 & 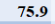 & 3.0 \\
\hline 6 & ERA & 20.9 & 76 & 40. & -3. & 8.6 & 1 & -20. & 3. & -1. & 0.0 & 0.3 & 11.9 & 37.7 & 15.2 & .5 & .9 & 261 & 4.3 \\
\hline 7 & DO & 2. & & & -35.3 & -42.9 & -38.6 & -4 & 48. & -1 & 11.4 & 3.3 & 70.4 & -22.0 & $-4 c$ & -29.0 & .0 & 56.8 & 3.3 \\
\hline 8 & $\mathrm{NC}$ & 34.4 & 30.1 & 2.9 & 7.4 & 21.2 & -4.9 & -58.1 & -33.7 & -50.8 & 163.2 & 31.7 & 206.8 & 35.9 & -26.7 & 18.3 & 60.1 & 22.2 & 92.3 \\
\hline 9 & $20 \mathrm{C}$ & 10.1 & .1 & 4.1 & -55.6 & -38.5 & -48.3 & 78.8 & 48. & 69.6 & 187.5 & $\mathbf{5 4 7 . 3}$ & 384.4 & -18.7 & -76.9 & -35.1 & 5.1 & -89.8 & -13.8 \\
\hline 1 & MSI & -7.9 & 39.4 & 8. & -23.0 & 30.3 & -0.1 & -60 . & -63.7 & -61.7 & -31.2 & 67.7 & -5.6 & -0.6 & 21.8 & 5.7 & 6.8 & 31. & 77.8 \\
\hline 2 & TMP & -44.1 & 9.6 & 5.6 & -47.0 & -4.4 & -28.8 & -68.2 & -44.8 & -61.1 & -32.0 & 09.4 & 4.6 & 51.2 & -7.5 & 38.9 & -21.7 & 8.9 & -7.6 \\
\hline 3 & PEF & -40.3 & & 1.8 & -54.8 & -6.6 & 4.2 & -51.1 & -50.2 & -50.8 & -20.6 & 191.0 & 34.2 & -41.1 & -0.9 & 29.8 & -11.0 & 8 & 5.1 \\
\hline 4 & $\mathrm{CHE}$ & -31.8 & & -25 & -39.8 & -27.1 & & -66.0 & -60 & -64.3 & -0 & & & -40.0 & -28.6 & & .0 & 0 & 0.6 \\
\hline 5 & $\mathrm{CH}$ & -47 & -17 & -37 & -61. & -2 & & -65 & -62 & -6 & -38. & & -17.0 & & & & 3 & & .0 \\
\hline 6 & WFI & -28 & & -1 & -58 & & & -49 & -78 & & -6 & & & & - & & & & 81.6 \\
\hline 7 & $\mathrm{CM}$ & -7. & & & -42 & & & 39 & -3 & & 28 & 295.7 & & & & & & & 4.5 \\
\hline 8 & $\mathrm{CM}$ & & -3 & & -40 & -16 & & -92 & 56 & & & 0 & -47.8 & & .0 & -30.0 & 28.2 & 128.6 & 8.0 \\
\hline 20 & CAMISUPI & -55.5 & 3.9 & 11.4 & 69.0 & 22.8 & 49.2 & 62.7 & 18.6 & 38.2 & 31.4 & 14.0 & 34.0 & 53.2 & 30.6 & 46.9 & 33.8 & 60.2 & 15. \\
\hline
\end{tabular}




\subsubsection{Residual errors}

Residual errors in mean monthly precipitation of the gridded datasets summarized in Figure 4.3 suggest large deviations from the reference mean. However, the errors vary considerably among the datasets at seasonal scale and over the sub-regions. The largest spreads of residual errors are found in W-Himalayan region for all products, partly due to highest precipitation.
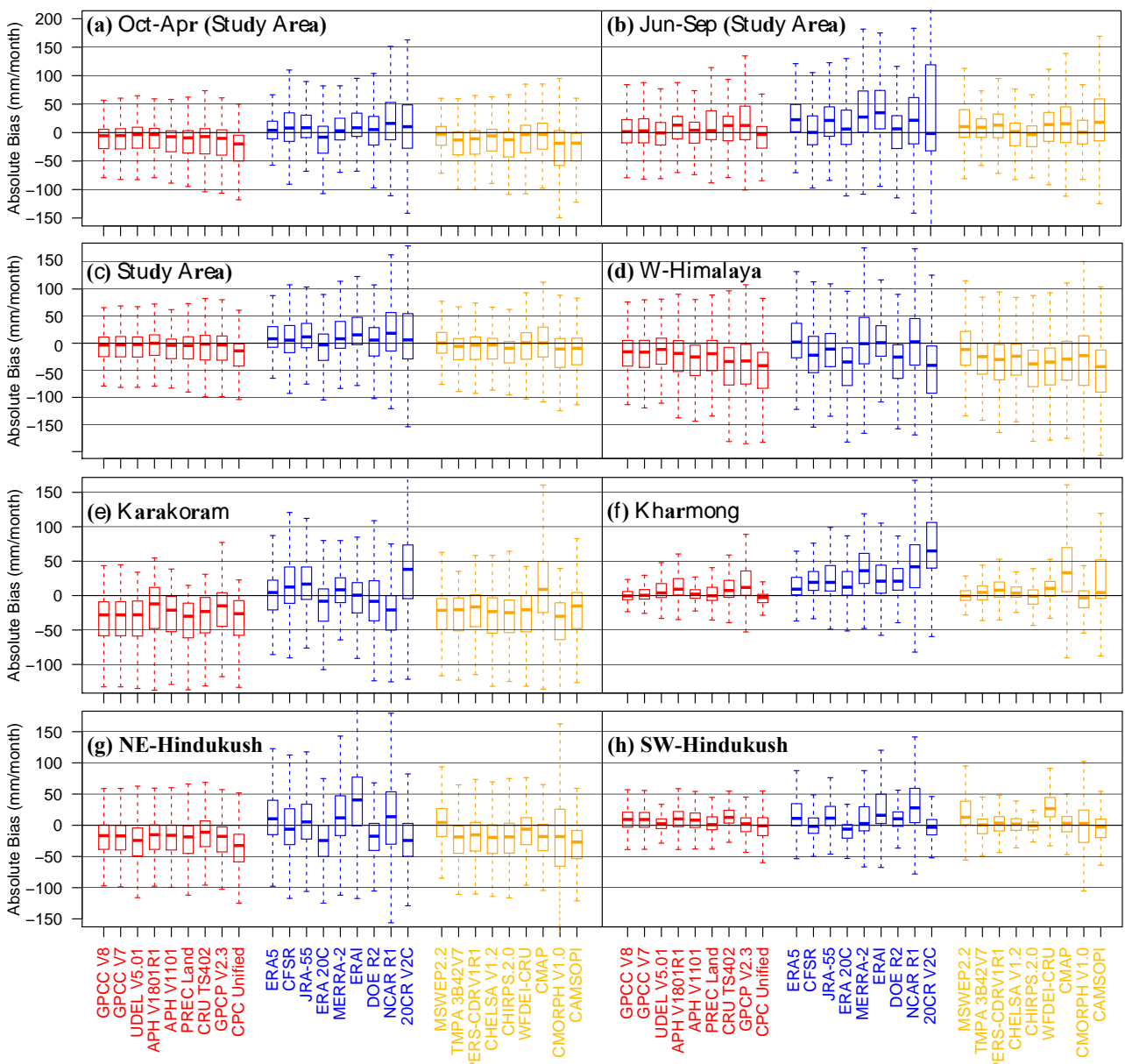

Figure 4.3: Box-Whisker plots of the absolute bias of mean monthly precipitation of each gridded dataset against the reference dataset for Oct-May months and monsoon (Jun-Sep) season for the study area and extended at annual scale for the study area and its five regions. Red colour indicates datasets developed from gauge observations, blue colour represents reanalysis products and orange colour are the merged datasets. The zero line represents the mean precipitation of reference dataset, the thick lines in the middle of boxes show the median differences, the bottom and top edges of the box represent the deviations from the reference mean at $25^{\text {th }}$ and $75^{\text {th }}$ percentiles respectively, while the "whiskers" represent the extreme values for each gridded dataset. Outliers are removed.

The gauge-based and merged products perform relatively better during monsoon season and reanalysis products during the winter months. Generally, reanalysis products show larger variability and wider spread of residuals than gauge-based and merged products, 
which is understandable and attributed to their independence from direct measurements of precipitation, use of varying type and number of assimilated observations, and use of different atmospheric models and assimilation schemes. The gauge-based and merged products significantly underestimate precipitation in relatively wet regions of W-Himalaya, NE-Hindukush and Karakoram. None of the selected products could be singled out as the best product for all regions as their accuracy varies considerably from one region to another. However, ERA5, GPCC V8, and MSWEP2.2 provide better estimates among their counterpart grouped products.

Mean absolute error (MAE) magnitudes of the gridded datasets (Figure 4.4) depict almost similar patterns of the errors as in Figure 4.3. The largest MAEs are observed in WHimalayan region and during monsoon season. Gauge-based products show relatively small absolute errors except high-altitude Karakoram region, where reanalysis products perform much better. However, reanalysis products show larger errors during monsoon season, probably due to the convective nature of monsoon precipitation and high uncertainties in deep convection parameterization schemes applied in the reanalysis models, which is in line with the findings of Beck et al. (2019). ERA5 is found best during winter and premonsoon (Oct-May) months and in higher-altitude Karakoram region, while GPCC V8 provides the least MAE values in W-Himalaya and Kharmong, WFDEI-CRU in NEHindukush, and CHIRPS V2.0 in SW-Hindukush regions.
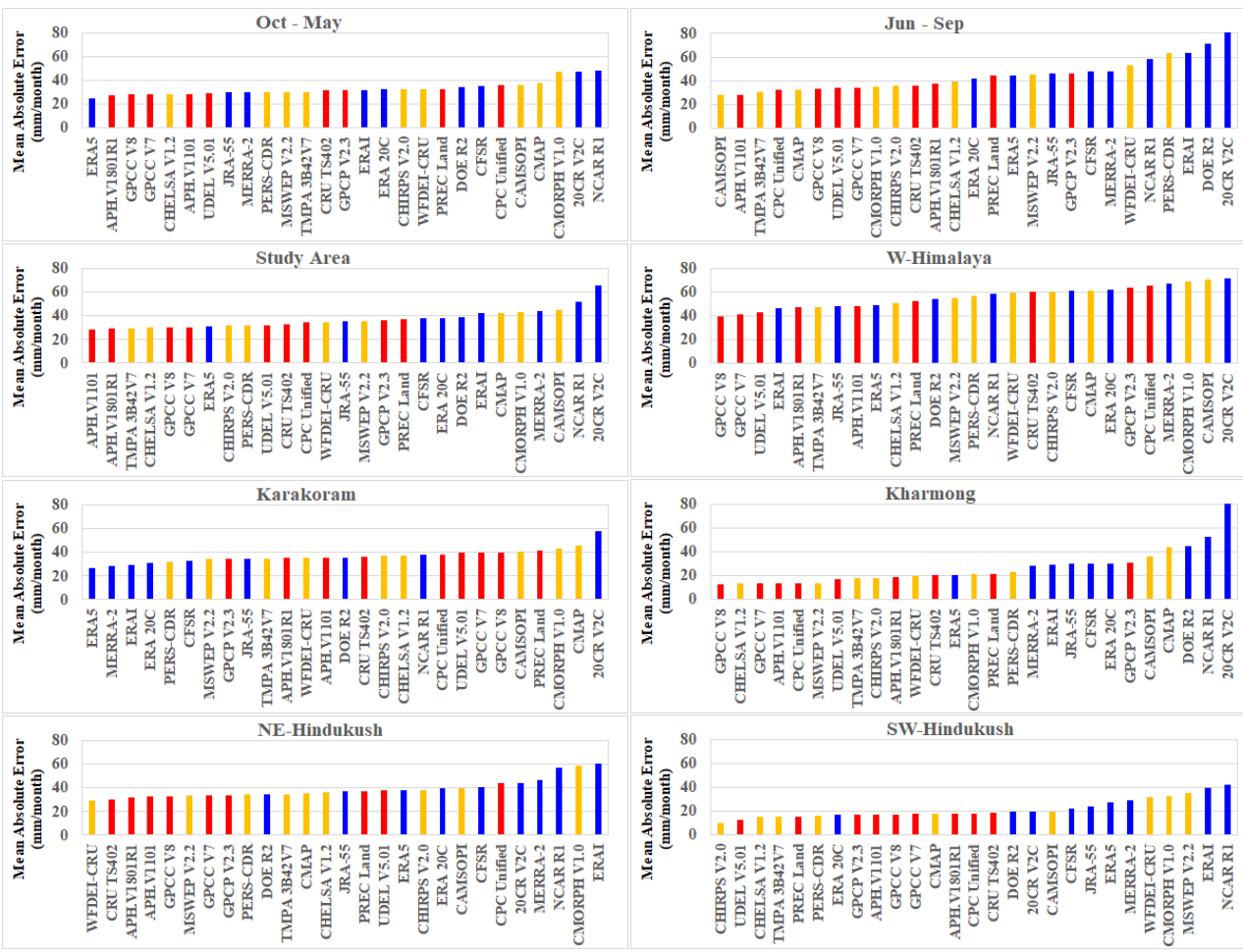

Figure 4.4: Mean absolute errors with the same group colour schemes and spatiotemporal scales as in Figure 4.3, but ranked according to error magnitudes. 


\subsubsection{KGE scores}

Very low to moderately high KGE scores ranging from -0.76 to 0.80 for various months (Figure $4.5 \mathrm{~A}$ ) and from -2.91 to 0.86 for various regions (Figure $4.5 \mathrm{~B}$ ) indicate very poor to moderately good performance of gridded datasets to match the precipitation of reference dataset. Negative KGE values reflect negative correlation and/or large deviation of bias and variability ratio from their optimum values. Except ERA5, CFSR, JRA-55, MERRA-2 and ERAI; all datasets provide lowest KGE scores during winter (Oct-Mar) months. ERA5 outperforms all datasets during Feb-May and MERRA2 during Oct-Jan. The gauge-based and few merged products depict better KGE scores during monsoon season (Jun-Sep).

In case of extended monthly data at annual scale for the whole study area (Figure 5B), GPCC V8 produces the highest KGE score of 0.674 followed by GPCC V7 (0.673), MSWEP2.2 (0.634), UDEL5.01 (0.633), TMPA 3B42V7 (0.630), APHRODITE V1801R1 (0.617), APHRODITE V1101 (0.613), and ERA5 (0.590). However, KGE scores vary considerably at sub-regional level. GPCC V8 outperforms all the datasets in W-Himalaya and Kharmong, MERRA-2 in Karakoram, MSWEP2.2 in NE-Hindukush, and CHIRPS V2.0 in SW-Hindukush regions. Details of monthly scale KGE scores, correlations, biases and variability ratios for the study area are provided in Table S-4.1, while Table S-4.2 presents the same metrics for extended time scale for study area and five sub-regions. The low KGE scores in Karakoram region can be attributed to the more complex topography and greater spatio-temporal heterogeneity of precipitation in this complex mountain region.

\subsubsection{Annual cycle of monthly means}

The observational-based reference climatologies of area-weighted mean monthly precipitation exhibit a strong seasonality with biomodal pattern clearly reflecting the influence of winter westerlies and summer monsoon, but many gridded datasets have difficulties in efficiently reproducing this seasonality and biomodal pattern as shown in Figure 4.6. Few datasets show very weak seasonality, and one merged dataset (CMORPH) even displays a 'negative' correlation. The strength of gridded products to reproduce the annual cycle of area-weighted mean monthly precipitation of the reference dataset is ascertained by coefficient of determination $\left(\mathrm{R}^{2}\right)$ and MAE and presented in Table 4.3. Correlation coefficient and coefficient of determination primarily indicate patterns and linear trend between two data but lack in quantifying the margin of errors, which is determined by MAE. None of the dataset is equally best for all sub-regions due to a large variability in $R^{2}$ and MAE values.

All gauge-based products underestimate precipitation during Oct-May in all sub-regions except relatively dry sub-region of SW-Hindukus. They show mix trends during monsoon season with PREC Land, UDEL V5.01 and two versions of GPCC slightly overestimating precipitation in W-Himalaya, Kharmong and SW-Hindukush. However, in the higeraltitude regions of Karakoram and NE-Hindukush, the gauge-based products significantly underestimate precipitation throughout the year. An important discrpancy in attainment of the lowest and heighest peaks is also evident. Almost all gauge-based datasets show the lowest and heighest peaks during May and July respectively against June and August dipicted by the reference dataset in most parts except NE-Hindukush and SW-Hindukush, where heighest peack is achieved during February and April respectively. The reanalysis products better reflect the wintertime precipitation but exhibit relatively large variability among them and in different regions. They record higher correlations and lower MAEs than the gauge-based and merged products particularly in higher altitdue and wetter regions. 


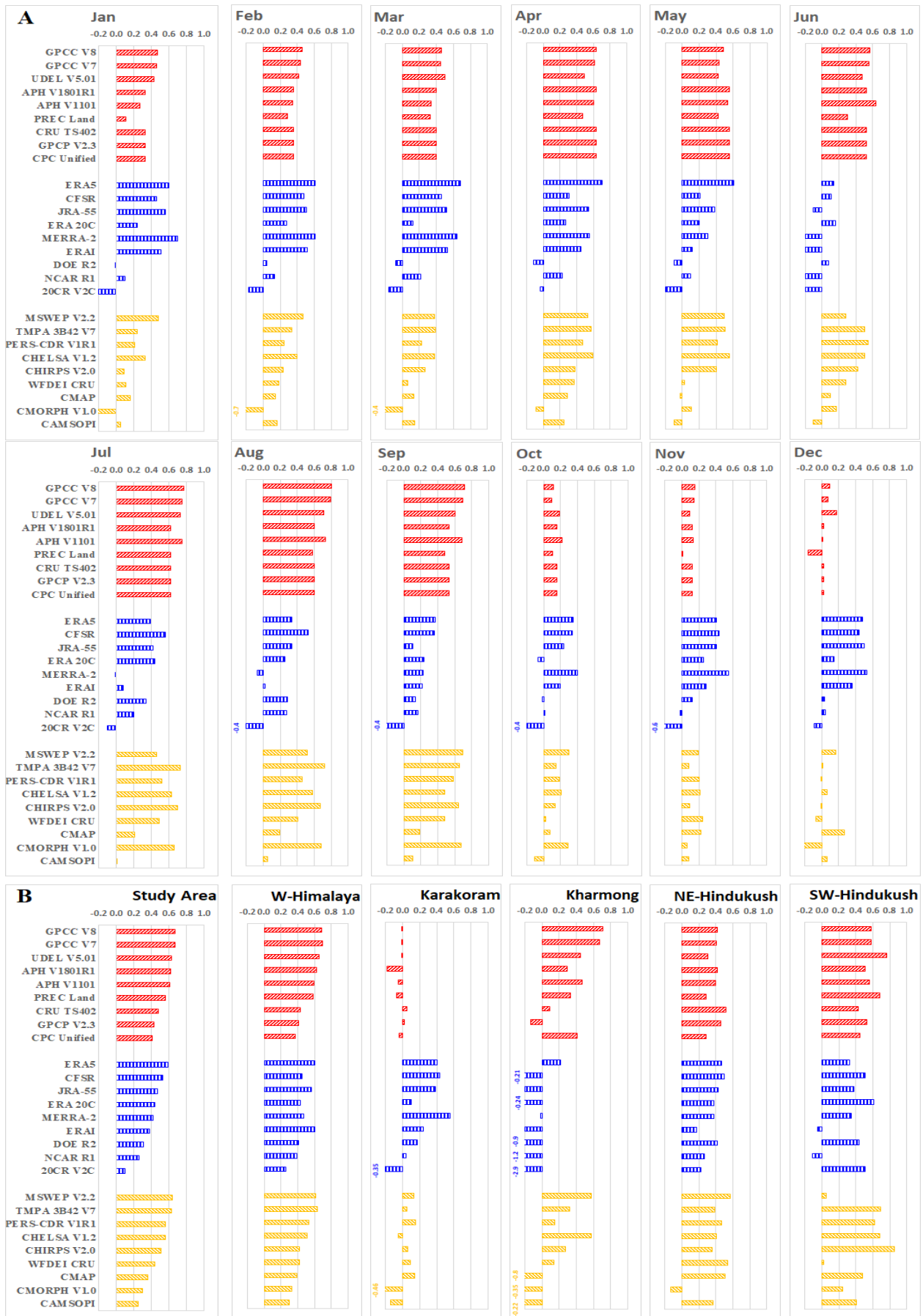

Figure 4.5: KGE scores based on mean monthly precipitation totals at monthly scale for the study area (A), and extended at annual scale for the study area and five regions (B). The red, blue and orange colours represent KGE scores for gauge-based, reanalysis and merged datasets respectively. 


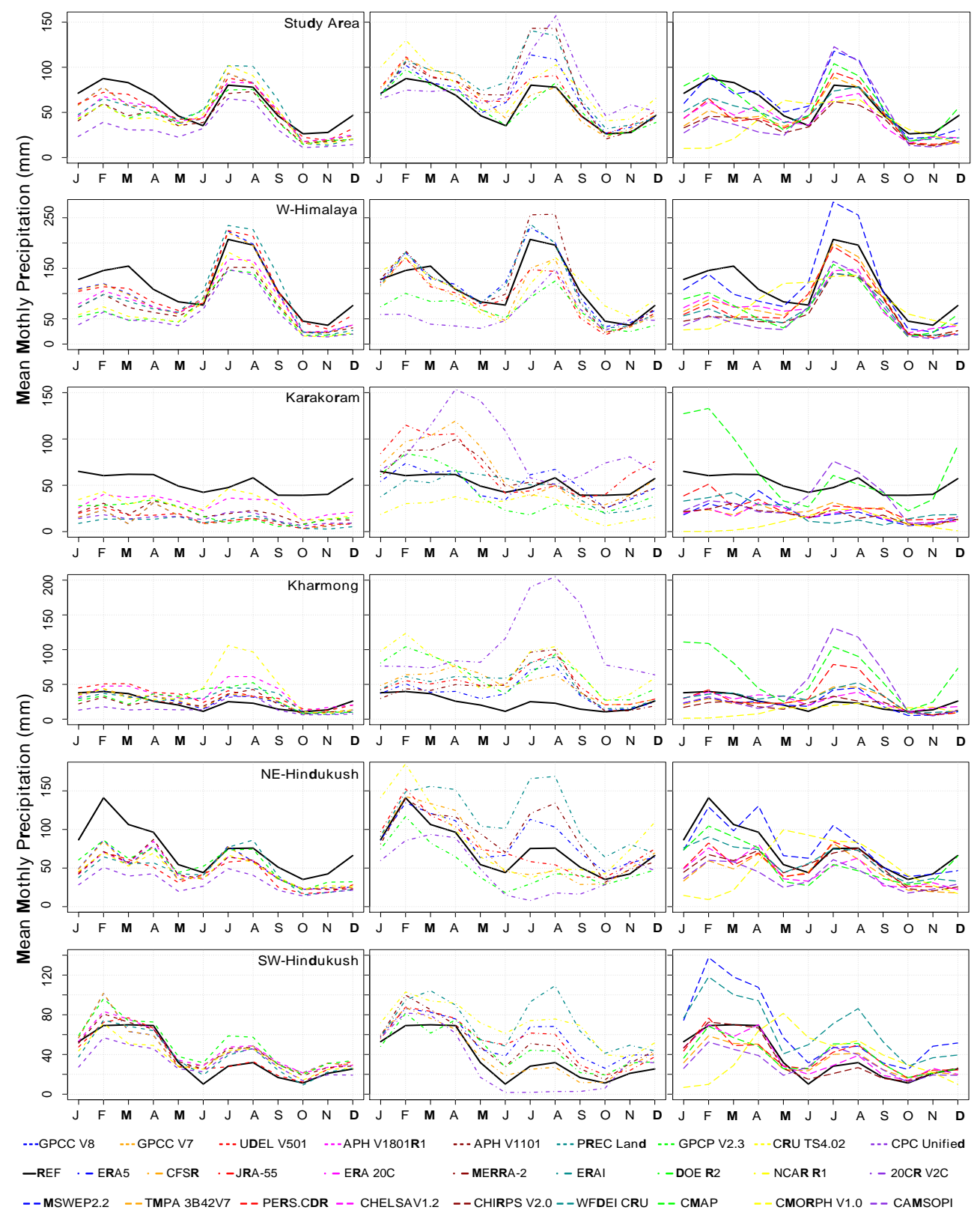

Figure 4.6: Annual cycle of mean monthly precipitation for the study area and five regions. Each row represents a particular region. Left column shows gauge-based datasets, middle column indicates reanalysis products and right column shows merged products.

Nevertheless, they consistently overestimate precipitation in drier regions of Kharmong and SW-Hindukush throughout the year but more significantly during monsoon season. The merged products largely follow the same patterns as gauge-based products, which is understandable due to the reason that these products are derived by taking input from the 
gauge observations. However, the merged products exhbit larger variability as comapred to gauge-based products. They also show larger spread of error margins among themselves. CMAP and MSWEP2.2 provided higher $\mathrm{R}^{2}$ and lower MAE as compared to their counterpart grouped datasets, except in SW-Hindukush where CHIRPS V2.0 proved to be the best in terms of both these performance metrics. The lowest MAE values in Karakoram, W-Himalaya and NE-Hindukush regions are obtained by ERA5, while CHIRPS V2.0 in SW-Hindukush and GPCC V8 in Kharmong outperform all other datasets with the lowest MAE and highest $\mathrm{R}^{2}$ values. Interestingly, for the whole basin DOE R2 provides the best values of $\mathrm{R}^{2} \&$ MAE despite its poor performance at regional scale in terms of KGE scores.

Table 4.3: Coefficient of determination (R2) and MAE (mm) values based on area-weighted monthly means presented in Figure 4.6.

\begin{tabular}{|c|c|c|c|c|c|c|c|c|c|c|c|c|c|}
\hline \multirow{2}{*}{\multicolumn{2}{|c|}{$\begin{array}{l}\text { S. Precipitation } \\
\text { \# Product }\end{array}$}} & \multicolumn{2}{|c|}{ Study Area } & \multicolumn{2}{|c|}{ W-Himalaya } & \multicolumn{2}{|c|}{ Karakoram } & \multicolumn{2}{|c|}{ Kharmong } & \multicolumn{2}{|c|}{ NE-Hindukush } & \multicolumn{2}{|c|}{ SW-Hindukush } \\
\hline & & $\mathbf{R}^{2}$ & MAE & $\mathbf{R}^{2}$ & MAE & $\mathbf{R}^{2}$ & MAE & $\mathbf{R}^{2}$ & MAE & $\mathbf{R}^{2}$ & MAE & $\mathbf{R}^{2}$ & MAE \\
\hline 1 & GPCC V8 & 0.776 & 11.5 & 0.888 & 22.3 & 0.373 & 38.6 & 0.623 & 6.1 & 0.656 & 24.4 & 0.739 & 12.1 \\
\hline 2 & GPCC V7 & 0.769 & 11.6 & 0.886 & 22.6 & 0.369 & 38.6 & 0.611 & 6.7 & 0.650 & 25.0 & 0.738 & 12.1 \\
\hline 3 & UDEL V5.01 & 0.847 & 9.0 & 0.902 & 17.7 & 0.561 & 38.2 & 0.713 & 10.6 & 0.723 & 30.6 & 0.937 & 4.5 \\
\hline 4 & APH.V1801R1 & 0.725 & 12.2 & 0.872 & 30.7 & 0.264 & 22.4 & 0.189 & 14.9 & 0.581 & 26.5 & 0.889 & 9.7 \\
\hline 5 & APH.V1101 & 0.706 & 16.2 & 0.853 & 39.1 & 0.339 & 31.9 & 0.151 & 9.2 & 0.579 & 25.8 & 0.904 & 8.2 \\
\hline 6 & PREC Land & 0.527 & 18.9 & 0.739 & 36.5 & 0.195 & 41.2 & 0.128 & 11.0 & 0.332 & 28.5 & 0.877 & 6.3 \\
\hline 7 & CRU TS402 & 0.553 & 16.9 & 0.665 & 52.0 & 0.573 & 32.8 & 0.018 & 14.3 & 0.665 & 20.1 & 0.829 & 13.7 \\
\hline 8 & GPCP V2.3 & 0.468 & 19.8 & 0.736 & 44.5 & 0.289 & 22.6 & 0.011 & 22.4 & 0.643 & 27.1 & 0.732 & 9.8 \\
\hline 9 & CPC Unified & 0.472 & 27.0 & 0.732 & 55.4 & 0.204 & 37.8 & 0.051 & 11.7 & 0.672 & 41.8 & 0.622 & 12.3 \\
\hline 1 & ERA5 & 0.824 & & 0.888 & 15.9 & 0.577 & 9.2 & & 15.6 & 0.834 & 14.8 & 0.779 & 19.0 \\
\hline 2 & CFSR & 0.839 & 10.0 & 0.854 & 25.7 & 0.546 & 19.5 & 0.308 & 25.9 & 0.760 & 15.5 & 0.909 & 7.3 \\
\hline 3 & JRA-55 & 0.894 & 11.0 & 0.757 & 25.6 & 0.632 & 19.9 & 0.073 & 26.1 & 0.820 & 13.5 & 0.743 & 16.9 \\
\hline 4 & ERA 20C & 0.596 & 14.5 & 0.788 & 47.7 & 0.351 & 18.0 & 0.073 & 24.6 & 0.887 & & 0.865 & 10.5 \\
\hline 5 & MERRA-2 & 0.685 & 21.0 & 0.926 & 20.8 & 0.493 & 14.8 & 0.020 & 24.1 & 0.622 & 21.4 & 0.886 & 13.8 \\
\hline 6 & ERAI & 0.662 & 24.0 & 0.899 & 16.4 & 0.198 & 12.8 & & 26.5 & 0.437 & 43.4 & 0.422 & 34.4 \\
\hline 7 & DOE R2 & 0.896 & 5.4 & 0.814 & 43.8 & 0.631 & 16.8 & 0.652 & 40.3 & 0.847 & 21.2 & 0.857 & 10.7 \\
\hline 8 & NCAR R1 & 0.881 & 19.1 & 0.602 & 26.5 & 0.177 & 26.3 & 0.671 & 49.0 & 0.682 & 24.9 & 0.695 & 33.7 \\
\hline 9 & 20CR V2C & 0.596 & 24.6 & 0.528 & 56.6 & 0.029 & 37.3 & 0.030 & 91.0 & 0.455 & 28.3 & 0.815 & 12.6 \\
\hline 1 & MSWEP V2.2 & 0.721 & 13.1 & 0.819 & 29.8 & 0.423 & 32.0 & 0.203 & 9.6 & 0.736 & 13.2 & 0.930 & 28.4 \\
\hline 2 & TMPA 3B42 V7 & 0.469 & 18.8 & 0.779 & 35.1 & 0.116 & 31.7 & 0.029 & 12.7 & 0.290 & 29.6 & 0.755 & 10.8 \\
\hline 3 & PERS-CDR V1R1 & 0.531 & 18.1 & 0.689 & 42.5 & 0.461 & 26.3 & 0.054 & 16.2 & 0.511 & 23.2 & 0.674 & 10.9 \\
\hline 4 & CHELSA V1.2 & 0.761 & 15.0 & 0.872 & 39.0 & 0.343 & 33.3 & 0.427 & 7.4 & 0.695 & 26.8 & 0.936 & 4.7 \\
\hline 5 & CHIRPS V2.0 & 0.651 & 21.6 & 0.701 & 53.0 & 0.399 & 33.6 & 0.104 & 9.7 & 0.388 & 24.2 & 0.969 & 3.3 \\
\hline 6 & WFDEI-CRU & 0.722 & 12.9 & 0.738 & 51.2 & 0.534 & 30.2 & 0.168 & 11.1 & 0.729 & 15.7 & 0.750 & 29.7 \\
\hline 7 & CMAP & 0.803 & 11.3 & 0.874 & 41.1 & 0.666 & 24.0 & 0.643 & 40.6 & 0.916 & 17.3 & 0.615 & 11.2 \\
\hline 8 & CMORPH V1.0 & 0.009 & 28.3 & 0.121 & 52.2 & 0.194 & 42.5 & 0.586 & 13.8 & 0.193 & 43.3 & 0.028 & 29.0 \\
\hline 9 & CAMSOPI & 0.253 & 29.9 & 0.666 & 55.9 & 0.001 & 26.2 & 0.005 & 31.3 & 0.679 & 34.2 & 0.454 & 14.4 \\
\hline
\end{tabular}

\subsubsection{Comparison and cross validation against adjusted streamflows}

Mean annual precipitation of all gridded datasets is cross validated by the Turk-Budyko non-dimensional analysis (Figure 4.7), which is based on the factual logic that streamflow can never be greater than precipitation provided there is no contribution from the negative mass balance (glaciers) and groundwater. This study used the adjusted streamflows for the contribution of negative mass balance determined by Dahri et al. (2018), so there is no question of galcier mass balance contributions. For longterm analysis, groundwater contribution to river flows is negligible in the study area. Therefore, the logic of lower streamflow than the precipitation is absolutely valid. 

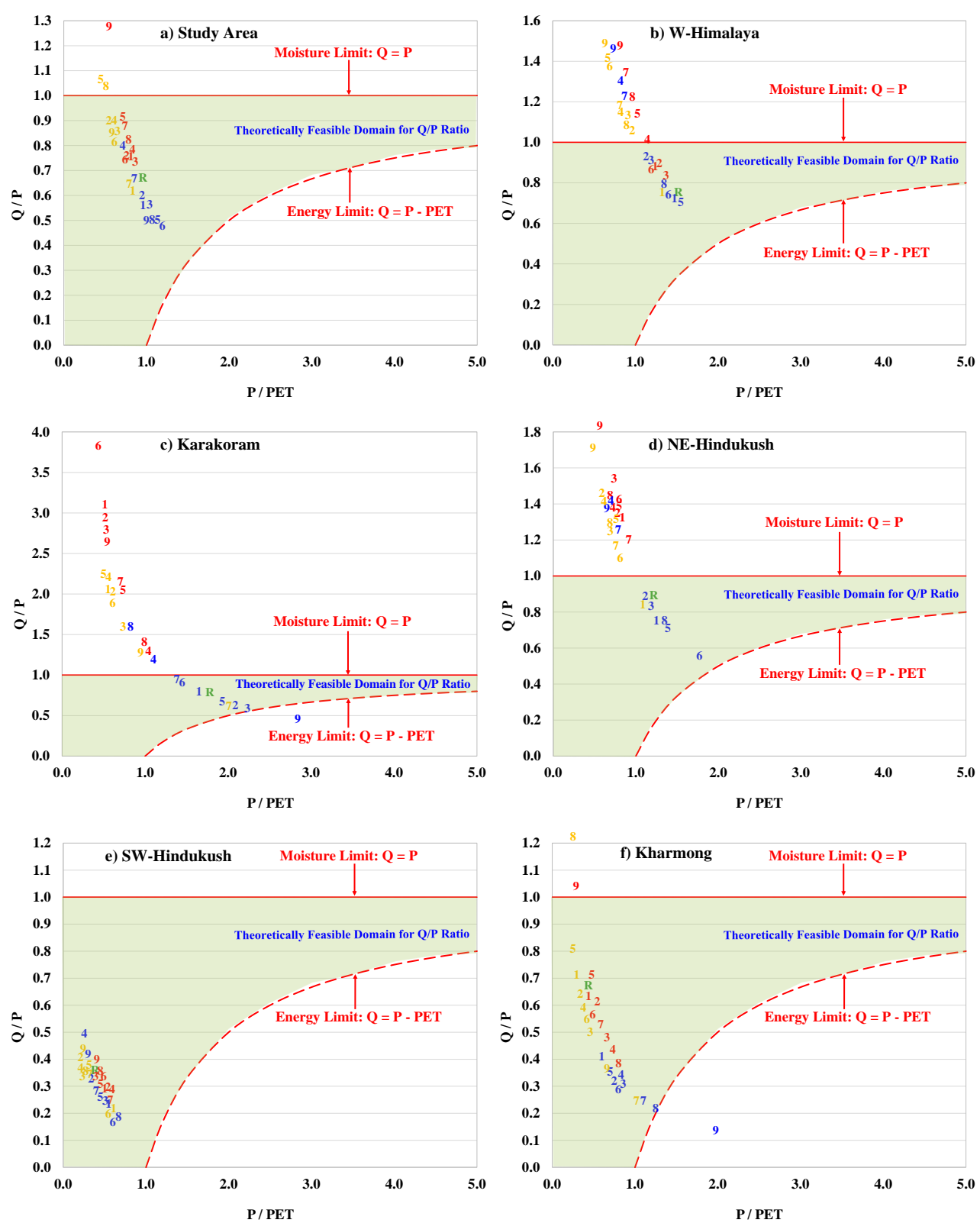

Figure 4.7: Turc-Budyko representation of runoff ratio $(\mathrm{Q} / \mathrm{P})$ and aridity indices $(\mathrm{P} / \mathrm{PET})$ for various regions of study area. Red, blue and orange coloured numbers reflect the respective names of gaugebased, reanalysis and merged datasets summarized in Table 1 . The green colored capital $\mathrm{R}$ represents the reference dataset.

The Turc-Budyko diagrams presented in indicate that precipitation estimates by most of the gridded datasets, particularly gauge-based and merged products in higher altitude regions, are less than the corressponding streamflows, which is quite unrealistic and counterintuitive. It is also evident that most of the datasets are within the acceptable limits 
for the drier regions of Kharmong and SW-Hindukush. However, in more complex highmountain Karakoram and NE-Hindukush regions, all gauge-based and merged products (except CMAP in Karakoram and MSWEP2.2 in NE-Hindukush) fail to fall within the feasible domain of the Budyko curve due to their unrealistically lower precipitation than the corressponding streamflows. Similarly, for the monsoon dominated and relatively wetter region of W-Himalaya, half of the datasts show unaccpetable Q/P ratios. However, such inter-regional variations offset each other when aggregated for the whole study area and except CPC-Unified, CHIRPS V2.0 \& CAMSOPI, all datasets provide acceptable representation. Only ERA5, CFSR, JRA-55, MERRA-2 and ERAI proved realitic for all regions. The other datasets fail due to underestimated precipitation in one or another region.

\subsubsection{Skill scores and rankings}

Interestingly, a considerable inconsistency in the outcome of the applied performance metrics is noticed using the same data at the selected spatiotemporal scales. For example, AHPRODITE V1101 is found to be the best dataset in terms of MAE for the whole study area at annual scale (Figure 4c), whereas GPCC outperformed all datasets in terms of KGE score (Figure $4.5 \mathrm{~m}$ ). Similarly, MAE and $\mathrm{R}^{2}$ obtained for the annual cycle of mean monthly precipitation (Table 3) suggest DOE R2 as the best dataset. However, the integrated skill scores and rankings derived from the normalized values of the selected statistical indices suggest considerably different inferences than presented in the results of the individual metrics. For the whole study area, UDEL V5.01 provided the best skill score of 3.629 followed by GPCC V8 (3.579), GPCC V (3.55) and ERA5 (3.443). However, there is considerable variation in the skill scores at sub-regional scale. GPCC V8 outperforms all datasets in W-Himalaya and Kharmong, ERA5 in Karakoram, MSWEP2.2 in NEHindukush and CHIRPS V2.0 in SW-Hindukush regions. For the combined wet and dry areas, ERA5 and UDEL V5.01 provided the highest skill scores respectively. The 20CR V2C and CMORPH V1.0 proved to be the worst performers in almost all regions. Overall, reanalysis products perform better in wet areas while gauge-based and merged products excelled in dry areas. Similarly, reanalysis products outperformed other datasets in higheraltitudes and gauge-based datasets provided better estimates in plain areas.

The ultimate rankings presented in Table 4.4 reveal that none of the gauge-based products could fall within the Budyko curve for the Karakoram and NE-Hindukush regions mainly due to their lower precipitation estimates than the corresponding streamflows. Out of merged products, only MSWEP2.2 in NE-Hindukush and CMAP in Karakoram show acceptable $\mathrm{Q} / \mathrm{P}$ ratios, while majority of reanalysis retain their rankings with ERA5 performing the best. The drier regions of SW-Himalaya and Kharmong are trivially affected as most of the datasets show acceptable $\mathrm{Q} / \mathrm{P}$ ratios and are laid within the Budyko curve. Similarly, about half of the datasets are excluded in the monsoon dominated WHimalaya due to higher Q/P ratios, with GPCP V8 retaining the top ranking. However, for the whole study area, the shortcomings exhibited at regional scale are offset and only CPC Unified, CHIRPS V2.0 and CMORPH V1.0 are excluded. The Turc-Budyko nondimensional analysis is found an effective performance indicator to single out the datasets that underestimate precipitation due to a definite upper limit of $\mathrm{Q} / \mathrm{P}$ ratio, while aridity index appears to be a softer indicator to highlight the overestimating datasets. 
Table 4.4: Final rankings (R) and skill scores (SS) of gridded datasets derived through integration of performance metrics for study area and its various regions. Red, blue and orange colors represent gauge-based, reanalysis and merged products respectively.

\begin{tabular}{|c|c|c|c|c|c|c|c|c|c|c|c|c|}
\hline $\mathbf{R}$ & Study Area & & W-Himala & & Kara & & NE-Hindı & & SW-Hindukus & & Kharmong & \\
\hline \# & Dataset & SS & Dataset & SS & Dataset & SS & Dataset & SS & Dataset & SS & Dataset & SS \\
\hline 1 & UDEL V5.01 & 3.63 & GPCC V8 & 3.77 & ERA5 & 3.71 & MSWEP V 2.2 & 3.63 & CHIRPS V2.0 & 4.00 & GPCC V8 & 3.87 \\
\hline 2 & GPCC V8 & 3.58 & UDEL V5.01 & 3.74 & MERRA-2 & 3.51 & ERA5 & 3.42 & UDEL V5.01 & 3.76 & GPCC V7 & 3.83 \\
\hline 3 & GPCC V7 & 3.55 & GPCC V7 & 3.72 & JRA-55 & 3.20 & JRA-55 & 3.40 & CHELSA V1.2 & 3.59 & UDEL V5.01 & 3.82 \\
\hline 4 & ERA5 & 3.44 & ERAI & 3.52 & CFSR & 3.19 & CFSR & 3.27 & PREC Land & 3.47 & CHELSA V1.2 & 3.53 \\
\hline 5 & APH.V1801R1 & 3.40 & ERA5 & 3.45 & DOE R2 & 3.05 & MERRA-2 & 2.51 & APH.V1101 & 3.27 & MSWEP V2.2 & 3.18 \\
\hline 6 & CFSR & 3.24 & APH.V1801R1 & 3.15 & ERAI & 2.78 & NCAR R1 & 1.98 & TMPA 3B42 V7 & 3.21 & APH.V1101 & 3.09 \\
\hline 7 & JRA-55 & 3.24 & JRA-55 & 2.98 & CMAP & 2.53 & ERAI & 0.77 & ERA 20C & 3.21 & APH.V1801R1 & 2.96 \\
\hline 8 & APH.V1101 & 3.24 & MSWEP V2.2 & 2.85 & & & & & APH.V1801R1 & 3.11 & WFDEI-CRU & 2.92 \\
\hline 9 & MSWEP V2.2 & 3.23 & PREC Land & 2.61 & & & & & CFSR & 3.07 & CHIRPS V2.0 & 2.91 \\
\hline 10 & CHELSA V1.2 & 3.22 & MERRA-2 & 2.49 & & & & & PERS-CDR V1R1 & 3.04 & PREC Land & 2.91 \\
\hline 11 & DOE R2 & 3.08 & CFSR & 2.43 & & & & & GPCP V 2.3 & 2.99 & ERA5 & 2.85 \\
\hline 12 & WFDEI-CRU & 2.93 & NCAR R1 & 2.04 & & & & & GPCC V8 & 2.97 & TMPA 3 B 42 V7 & 2.79 \\
\hline 13 & TMPA 3B42 V7 & 2.87 & & & & & & & GPCC V7 & 2.96 & CFSR & 2.73 \\
\hline 14 & PERS-CDR V1R1 & 2.78 & & & & & & & DOE R2 & 2.93 & CMAP & 2.70 \\
\hline 15 & CMAP & 2.72 & & & & & & & 20CR V2C & 2.90 & DOE R2 & 2.69 \\
\hline 16 & CRU TS402 & 2.68 & & & & & & & CRU TS402 & 2.81 & PERS-CDR V1R1 & 2.67 \\
\hline 17 & ERA 20C & 2.63 & & & & & & & CMAP & 2.75 & CRU TS402 & 2.65 \\
\hline 18 & PREC Land & 2.61 & & & & & & & CPC Unified & 2.68 & ERAI & 2.46 \\
\hline 19 & GPCP V2.3 & 2.29 & & & & & & & MERRA-2 & 2.44 & NCAR R1 & 2.43 \\
\hline 20 & MERRA-2 & 2.28 & & & & & & & JRA-55 & 2.40 & MERRA-2 & 2.42 \\
\hline 21 & ERAI & 2.09 & & & & & & & CAMSOPI & 2.33 & ERA 20C & 2.40 \\
\hline 22 & NCAR R1 & 2.09 & & & & & & & ERA5 & 2.23 & JRA-55 & 2.40 \\
\hline 23 & CAMSOPI & 1.10 & & & & & & & MSWEP V2.2 & 1.53 & GPCP V2.3 & 2.36 \\
\hline 24 & 20CR V2C & 0.88 & & & & & & & WFDEI-CRU & 1.37 & CAMSOPI & 2.16 \\
\hline 25 & & & & & & & & & CMORPH V1.0 & 0.83 & & \\
\hline 26 & & & & & & & & & NCAR R1 & 0.73 & & \\
\hline 27 & & & & & & & & & ERAI & 0.56 & & \\
\hline
\end{tabular}

\subsection{Discussion}

This study provides a comprehensive evaluation and accuracy assessment of 27 global scale gridded precipitation products for a transboundary high-mountain Indus basin. Widely used performance metrics are applied to evaluate and quantify the accuracy in sub-regional scale precipitation estimates of the gridded datasets with respect to a high-quality reference dataset at monthly, seasonal and annual timescales. The results revealed significant errors and uncertainties in the precipitation estimates of the selected gridded precipitation datasets. Most of the gridded datasets provided reasonably good patterns of seasonal precipitation distribution but displayed large differences in their precipitation magnitudes at monthly, seasonal and annual timescales in the study area. These differences are inconsistent and more pronounced at sub-regional scale (Figure 4.3). Overall, a large uncertainty in quantitative and spatio-temporal distribution of precipitation is evident in all gridded datasets. The results are in line with the findings of Sun et al. (2018), who reviewed and intercompared 30 global scale precipitation datasets. The most important attributions for the large differences and uncertainties in gridded precipitation products are different structural characteristics, diverse input data and observational densities, variable quality control and gauge under-catch corrections, spatiotemporal resolution, and use of different interpolation schemes. The landscape heterogeneities further add to the uncertainties. Generally, precipitation differences among gridded datasets and their biases with respect to the reference data can be explained by the uncertainties in the ways and means by which these datasets are produced. The similarities in precipitation estimates of two or more datasets can often be attributed to the similar input data and methods. However, if the 
dissimilar datasets depict consistencies then it is very likely that such signal is present in the actual situations.

The study area is highly deficient in precipitation observations and even worse is the fact that only one-third of the observed data are actually used by the existing gridded precipitation datasets due to strict data sharing policies of the national meteorological agencies. The situation is further worsened by the coarser spatial resolution of many gridded datasets, which limits the representation of precipitation in the high-mountain areas due to smoothing leading to underestimated peaks. Quality of the reference and gridded datasets can be improved by measuring and sharing precipitation data of the higher-altitude ranges and robust integration of mass balance data at corresponding timescales. Gaugebased datasets generally underestimate precipitation in the study area except relatively dry regions of Kharmong and SW-Hindukush during monsoon season. This is obvious because higher altitude regions of Karakoram and NE-Hindukush are highly deficient in groundbased observations. Therefore, the gauge-based datasets are derived from lower-altitude stations located in the dry valleys. The underestimated precipitation in the W-Himalaya region during winter months can be explained by lack of stations at the higher altitudes and significant under-catch of solid precipitation by the existing gauges (Dahri et al., 2018).

APHRODITE dataset uses the largest number of station observations in the study area. Whereas, the evaluation results reveal that both of its versions significantly underestimate precipitation in most parts of the study area. GPCC and UDEL V5.01 use about half of the station observations compared to APHRODITE but provide better estimates, probably due to their efficient interpolation schemes and 5-10\% correction factors applied to account for measurement errors in GPCC data (Schneider et al., 2014). However, the study conducted by Dahri et al., (2018) revealed that the corrections factors applied in GPCC are still on the lower side for the high-mountain Indus basin where under-catch of individual precipitation gauges varied between $2-182 \%$ with greater under-catches at higher altitude areas and during winter months. GPCP V2.3 also applied bulk correction factors for monthly climatological conditions but its precipitation estimates are even lower than APHRODITE due to very coarse grid size, use of different data and interpolation techniques, and possibly lower correction factors. PREC Land, CRU TS4.02 and CPC-Unified use the station data shared with WMOs Global Telecommunication System (GTS), which is about half of the stations used by GPCC in the study area and employ different interpolation schemes. The gauge-based products exhibited almost similar spatial patterns but with significant differences in their precipitation magnitudes. Generally, lack of observed data at higher elevations, biased distribution of the existing stations, and measurement errors seriously limit the accuracy of gauge-based precipitation products.

Unlike gauge-based precipitation datasets, reanalysis products are significantly different from each other, because each reanalysis uses its own atmospheric model, modeling technique and data assimilation scheme (Ghodichore et al., 2018; Fujiwara et al., 2017). The type and number of assimilated observations also varies from one model to another. Reanalysis products mostly suffer from uncertainties in the assimilated observations, physical aspects of the reanalysis system and the model parameterizations used for weather forecast (Bosilovich et al., 2008). Reanalysis products exhibit larger variability and wider spread of residual errors than the gauge-based and merged products because these datasets are independence of the direct measurements of precipitation and use different atmospheric models and assimilation schemes. Nevertheless, winter months and higher altitude Karakoram and NE-Hindukush regions are better covered by the reanalysis products, which 
is in line with the findings of Beck et al. (2019) and general characteristics of reanalysis providing better estimates for the frontal system precipitation during cooler seasons. The largest errors are observed during monsoon season, which is probably due to the convective nature of monsoon precipitation and high uncertainties in deep convection parameterization schemes applied in the reanalysis models. They also better follow the peaks. Annual cycle of area-weighted mean monthly precipitation is also better reflected in wet areas. Yet, precipitation in dry areas is largely overestimated by reanalysis products.

The development of merged precipitation products aims to exploit the complementary nature and comparative advantages of ground-based observations, reanalysis and/or satellite data leading to higher quality end products. However, the merged products inherit the limitations of their source data and are also affected by the uncertainties in the merging algorithms. Most of the gauge-observations used to calibrate satellite products and develop merged products are extracted from GTS network with poor spatial coverage in the HKH region (Yatagai et al., 2012). This could explain the underestimated precipitation by the resultant products at higher altitudes. Since, the merged products take inputs from the ground observations, they are closer to the gauge-based products. Yet, they exhibit a larger variability and error spreads among themselves due to differences in other data sources. Surprisingly, the performance of merged products in most parts of the study area is worse than many gauge-based and reanalysis products. This can partly be attributed to use of different and/or less observations. However, the major reason might be the problem in merging techniques, which are unable to preserve the comparative advantages of the gauge, reanalysis and satellite data. The quality of a merged product should be better than its parent input datasets. This might be true in many data rich regions, but is not the case in a complex high-mountain Indus basin.

Generally, errors or uncertainties in terms of over- and/or under-estimation of precipitation are ascertained in relative terms against a reference dataset. However, if the reference data differ in their precipitation patterns and magnitudes, the performance of the gridded datasets may change accordingly. The reference dataset used in this study is unique and has never been used before for evaluation purpose. Therefore, the magnitudes and errors in precipitation estimates of the gridded datasets deduced in this study cannot be compared to earlier studies, which used different benchmarks. It is also pertinent to note that the reference dataset used in this study includes monthly means of the error-adjusted precipitation observations for the period of 1999-2011, the best evaluation results can be achieved at similar temporal resolution scale. The data beyond this temporal scale are assumed to follow similar trends in the biases and are liable to some degree of uncertainty. It would be advisable to extend the evaluation using long term daily and monthly timeseries once the high-quality reference dataset at such temporal scales is made available. The evaluation at daily timestep would be useful to select the better datasets for assessing precipitation extremes and subsequent droughts or floods. Moreover, the Turc-Budyko nondimensional analysis largely depends on the Q/P ratio, which is subject to uncertainties in the quality of river flows data. Study conducted by

The global/continental scale datasets seem to compromise over the techniques used to derive/infer precipitation from various sources as the efficiency of the techniques varies with site-specific conditions. While the underlying efforts to develop merged datasets have been focusing on merging/combining data from various sources, another alternative may be to develop national/basin scale datasets using optimum data and techniques first and then merge these products to form a high-quality product at regional/global scale. 


\subsection{Conclusions}

This study highlighted and corroborated the underlying issues and uncertainties associated with a wide range of gridded precipitation products in the high-mountain Indus basin. The results clearly indicate that all gridded datasets evaluated in this study contain significant errors in their precipitation estimates and cannot be used directly without careful bias correction. The following major conclusions are drawn.

1. All gridded datasets tend to underestimate precipitation in wet areas and overestimate precipitation in dry areas, implying considerable implications for hydrological extremes of floods and droughts. GPCC V8, ERA5 and MSWEP2.2 provided better estimates than their counter-groups of gauge-based, reanalysis and merged datasets. ERA5 and UDEL V5.01 provided the highest skill scores for wet and dry areas respectively.

2. None of the dataset is equally best for all sub-regions of the study area. A particular dataset performing very well in one sub-region is found worse in the other subregion. Nevertheless, ERA5 is found most acceptable for all sub-regions. This study therefore would provide useful guidance for selection and use of the best dataset for a particular sub-region or sub-basin for hydrometeorological assessments.

3. Due to large uncertainties in the gauge-based precipitation products in the complex Karakoram and NE-Hindukush regions, a general perception is that precipitation from uncalibrated reanalysis products might be closer to the actual precipitation. This study however revealed that reanalysis datasets provide relatively better estimates for the higher-altitude areas where observations are generally scarce. However, not all reanalysis products can serve the purpose due to large differences in their precipitation patterns and magnitudes. Therefore, a careful selection is deemed essential.

4. Relatively poor performance of the merged datasets in the study region highlights their weaknesses and inability to accurately estimate precipitation and underlines the need to develop more advanced and accurate merging techniques, which can preserve the comparative advantages of input datasets and which are equally accurate at smaller (basin/catchment) scales.

\section{Acknowledgements}

This research work is supported by the Dutch Ministry of Foreign Affairs through the Netherlands Fellowship Program and partially carried out under the Himalayan Adaptation, Water and Resilience (HI-AWARE) consortium supported by the Collaborative Adaptation Research Initiative in Africa and Asia (CARIAA). The views expressed in this work do not necessarily represent those of the supporting organizations. Deepest gratitude is expressed to the institutions and the teams responsible for the development and distribution of the gridded precipitation datasets used in this study. The authors declare that there is no conflict of interest. 


\title{
Chapter 5
}

\section{Climate Change and Hydrological Regime of the High- Altitude Indus Basin under Extreme Climate Scenarios}

\begin{abstract}
Climate change is recognized as one of the greatest challenges of $21^{\text {st }}$ century and its impacts are particularly important for water systems. This study investigated climate and hydrological regimes of the high-altitude Indus basin for the historical period and extreme scenarios of future climate until the end of $21^{\text {st }}$ century. Improved datasets of precipitation and temperature for historical and future periods were developed and forced to a fully-distributed physically-based energy-balance hydrological model to simulate the water balance at regional scale. Relative to historical baseline, results revealed highly contrasting signals of climate and hydrological regime changes. Against an increase of $0.6{ }^{\circ} \mathrm{C}$ during the last 40 years, the median annual air temperature is projected to increase further between $0.8-5.7^{\circ} \mathrm{C}$ by the end of century. Similarly, a decline of $11.9 \%$ in annual precipitation is recorded, but future projections are highly conflicting and spatially variable. The Karakoram region is anticipated to receive more precipitation, while SW-Hindukush and parts of W-Himalayan region may experience decline in precipitation. The MIROC5 model generally shows increases, while MPI-ESM-LR ensembles indicate decreases in precipitation and river inflows. Indus-Tarbela inflows are more likely to increase compared to Kabul, Jhelum and Chenab river inflows. Substantial increase in the magnitudes of peak flows and one-month earlier attainment is projected for all river gauges. High flows are anticipated to increase further under most scenarios, while low flows may further decrease for MPI-ESM-LR in Jhelum, Chenab and Kabul river basins. Hence, hydrological extremes are likely to be intensified. Critical modifications in strategies and action plans for hydropower generation, construction and operation of storage reservoirs, irrigation withdrawals, flood control and drought management will be required to optimally manage water resources in the basin.
\end{abstract}

This chapter has been submitted as:

Dahri, Z. H., Ludwig, F., Moors, E., Ahmad, S., Ahmad, B., Ahmad, S., Riaz, M., \& Kabat, P. (submitted). Climate Change and Hydrological Regime of the High-Altitude Indus Basin under Extreme Climate Scenarios, Science of the Total Environment, STOTEN-D20-16914. 


\subsection{Introduction}

The Indus river system (IRS) had been the lifeline for the Indus valley civilization since time immemorial. Wiping out of this ancient civilization is attributed to drying up of the rivers due to prolonged drought for about 900 years during 2330-1430 BC (Dutt et al., 2018). Even in the modern era of technological innovations, climate change is considered to be one of the major drivers of demographic changes and expansion or weakening of national economies across the globe (WEF, 2016). Climate change is also alleged to impede nations' abilities to achieve sustainable development goals (IPCC, 2007). Evidence of the impacts and risks associated with climate change is strongest for natural systems (IPCC, 2014). Yet, there are considerable uncertainties in the observed and modelled climate particularly for precipitation, which preclude reliable modeling of the changes and variability in the current and future water cycles at global and regional scales (IPCC, 2013). Hindukush-Karakoram-Himalayan (HKH) region and concomitant high-altitude Indus basin is recognized as a 'hotspot' of climate change due to significant transformations in its hydrometeorological regime (Krishnan et al., 2019; Wijngaard et al., 2018; Lutz et al., 2018; Kraaijenbrink et al., 2017; De Souza et al., 2015). However, an authentic assessment of climate change and variability and its underlying impacts on the hydrological regime of the basin are seriously constrained by paucity of observed data and delicate hydrometeorological investigations.

Climate change research in the Indus basin is a recent campaign and still at its infancy. As such, there are significant data, information and knowledge gaps. Thus, our understanding of the basin's hydrometeorological regimes particularly at sub-basin scale is limited. Numerous studies have attempted to analyze recent climate change (e.g. Bashir et al., 2017; Hasson et al., 2017; Fowler and Archer 2006; Archer and Fowler, 2004), project future changes (e.g. Lutz et al., 2018; Hasson et al., 2013;) and model the underlying impacts on hydrological regime and/or water availability (e.g. Hasson et al., 2019 \& 2016; Lutz et al., 2016a \& 2014; Ali et al., 2015; Khan et al., 2015; Immerzeel et al., 2013, 2010 \& 2009; Akhtar et al., 2008) in the Indus basin. Few studies (e.g. Hasson et al., 2017; Khattak et al., 2011; Sheikh et al., 2009; Fowler and Archer, 2006) noted cooling of monsoon season and slight warming during winter and spring months, while Archer and Fowler (2004) detected a considerable increase in precipitation throughout the year. In contrast to a worldwide decline of mountain glaciers, Hewitt (2005) observed widespread evidence of glacier expansion in the central Karakoram mountain range. This anomalous behaviour of the glacier surge in the Karakoram mountain range is supported by Bashir et al. (2017) who detected regional-scale increases in humidity, cloud cover and precipitation along with decreased net radiation, near-surface wind speed, potential evapotranspiration and river flows and termed the high-mountain watersheds of the Indus basin as moisture surplus and energy deficient. Generally, summer runoff in the highermountain catchments of the Indus basin is strongly correlated with the preceding winter snowpack (Archer, 2003; De Scally, 1994) and prevailing summer temperatures (Archer, 2004). Nevertheless, significant uncertainties still exist in precise representation of the basin's hydrometeorological regime due to lack of observed meteorological data and extremely complex orography interacting with synoptic-scale atmospheric circulations (Lutz et al., 2014; Andermann et al., 2011). The greatest uncertainties are associated with the spatiotemporal distribution of precipitation and dynamics of glacial ice mass in the higheraltitude areas, which are the dominant source of streamflows in the IRS.

Accuracy of future hydrometeorological projections on the other hand is constrained by a high variability and large spread of the available general circulation model (GCM) outcomes (Kraaijenbrink et al., 2017; Lutz et al., 2016b), structural limitations of the climate and 
hydrological modeling frameworks, and lack of reliable and representative observations for bias corrections and validations. Despite improvements in climate models to better represent the climate processes, significant uncertainties in precisely reproducing the current and projected climates still remain the subject of ample concern (Knutti and Sedláček, 2012). All GCMs project consistent but slightly varying increases in temperature, whereas their precipitation projections are highly variable ranging from considerably drier to moderately wetter precipitation regimes (Lutz et al., 2016b). Most GCMs have difficulties in simulating the South-Asian monsoon and its variability on a range of timescales (Turner and Annamalai, 2012). Even greater changes and uncertainties are estimated with regard to snow/glacier dynamics under different climate change scenarios (Kraaijenbrink et al., 2017). The "Paris Agreement" signed in 2015 urged the 195 signatory nations to limit the global temperature increase well below $2{ }^{\circ} \mathrm{C}$ relative to pre-industrial (1861-1890) level and stabilize it at $1.5{ }^{\circ} \mathrm{C}$ by 2100 . However, the road to realize such an ambitious goal would require unprecedented efforts to increase the current levels of nationally determined contributions (NDCs) by 3 and 5 times respectively (Kabat et al., 2019). Lutz et al., (2018) argued that regional changes in temperature and other climate change indicators are stronger, more realistic and plausible under Representative Concentration Pathways (RCPs) 4.5 \& 8.5 scenarios than for the 1.5 and $2{ }^{\circ} \mathrm{C}$ global warmings and showed that in a 1.5 and $2{ }^{\circ} \mathrm{C}$ warmer world, the temperature in the $\mathrm{HKH}$ region may rise to 2.1 and $2.7^{\circ} \mathrm{C}$ respectively. Relatively accelerated rise in temperature of high-altitude regions is attributed to elevation-dependent warming explained by Pepin et al., (2015). The anticipated impacts of 1.5 and $2{ }^{\circ} \mathrm{C}$ global warmings on basinscale water balance project 34 and $43 \%$ increase in river runoff from upper Indus basin (upstream of Besham Qila), Jhelum and Kabul (Hasson et al., 2019). Similarly, the projected loss of present-day glacial ice mass in high-mountain Asia by 2100 is $26 \pm 7 \%$ under $1.5{ }^{\circ} \mathrm{C}$ global warming, which may increase to $49 \pm 7,51 \pm 6$ and $64 \pm 5 \%$ under RCP $4.5,6.0$ and 8.5 scenarios respectively (Kraaijenbrink et al., 2017). Hence, significant and somewhat accelerated transformations in the basin's hydrometeorological regime can be anticipated under various climate change scenarios. Under such circumstances, precision of the baseline meteorological forcing and energy input data and application of appropriate hydrological models play a vital role in the assessment of catchment scale water budgets.

It is now well-recognized that actual precipitation in the high-altitude Indus basin is much higher than what had been previously thought and used in the earlier studies (Dahri et al., 2016 \& 2018; Immerzeel et al., 2015). In hydrological modeling frameworks, the underestimated precipitation is often compensated through sub-optimal alteration of other parameters (e.g. evapotranspiration, soil properties, snow/glacier melt factors, etc.) during model calibration and validation (Lutz et al., 2014; Pellicciotti et al., 2012; Schaefli et al., 2005). Therefore, the inferences regarding the dynamics and underlying impacts of these suboptimally altered parameters may be uncertain and inaccurately adjudicated. Moreover, since the Indus is predominantly a snow/glacier-fed basin, the degree-day or temperature-index based hydrological models often inadequately represent the prevailing energy balance, which is the key driving force for melting of glacial ice mass in the snow/glacier-fed systems.

Therefore, in this study a much-improved and high-resolution meteorological forcing datasets of precipitation and temperature have been developed for the historical period and various future climate change scenarios to better analyse recent and future climate changes in the high-altitude Indus basin. This new dataset is used to force a fully-distributed physically-based energy-balance hydrological model to simulate the water balance at subbasin scale. Climate change analyses and hydrological model executions are attained for a baseline historical period and a set of six scenarios of future climate. The study further 
determines the changes and variability in overall water availability and examines the shifts in the timings and peaks of hydrological extremes. The results of this study will curtail the uncertainties to understand the current and future hydrometeorological regime of the highmountain Indus basin and help take informed policy decisions for hydropower development, climate change adaptation and optimal management of the Indus river water resources.

\subsection{The Indus River Basin}

The IRS, primarily nourished by precipitation and snow/glacier melt in the high-mountain ranges of Tibet-China, northwest India, northeast Afghanistan and north Pakistan, is one of the largest irrigation systems in the world with drainage area of about 112 million hectares and irrigated command area of over 26 million hectares. The water flowing through the IRS is a vital source of existing hydropower projects with massive untapped potential across its headwater tributaries and for agricultural production in valleys and downstream arable lands. The river water plays a crucial role in prosperity and growth of national economies and livelihoods of over 300 million inhabitants in Afghanistan, India and Pakistan. However, water, food and energy securities in this region are inextricably linked with sustainable water supplies in the IRS, which are prone to alteration by the future climate change and/or variability (Lutz et al., 2016a; Hasson, 2016; Bocchiola and Diolaiuti, 2013) and stressed by increasing demands for rapidly growing population, socioeconomic development and ecosystem services (Wijngaard et al., 2018).

This study is conducted in the high-mountain watersheds of the Indus river, which is gauged at many locations along its main course and at the confluence of major tributaries (Figure 5.1). Except the Kabul river, the other watersheds receive largely uninterrupted naturalized flows at the selected discharge outlets. The canal diversions from the Kabul river upstream of Nowshera are also included in the Kabul river observed flows at Nowshera. Similarly, the diversions from the Beas river to the Sutlej river through the Beas-Sutlej link canal are also accounted for to determine the actual river flows from these two tributaries.

\subsection{Data and Methods}

\subsubsection{Historical climate data}

The study area is highly deficient in observed data of important climatic variables. This is especially the case for precipitation which is the most important variable for climate change and water balance studies. The quality of spatially distributed precipitation dataset for the high-mountain Indus basin is generally poor due to scarcity and very high directional bias (predominantly low-altitude valley-based station observations) of observed data (Dahri et al., 2018 \& 2016; Reggiani and Rientjes, 2015; Immerzeel et al., 2015). Hence, the strong gradients and extreme heterogeneity of precipitation in this complex and orographically influenced high-mountain terrain are inadequately captured by the existing global and regional scale gridded datasets leading to insufficient representation of the actual precipitation regime. Dahri et al. (2020, under review) evaluated precipitation estimates of 27 widely used gridded precipitation products with respect to the reference climatologies of mean monthly precipitation developed by Dahri et al. (2018) for the 1999-2011 period and cross validated these estimates by the corresponding river flows at sub-basin scale in the high-altitude Indus basin. Precipitation estimates of ERA5 (Hersbach et al., 2018) gridded dataset were found relatively better than the other products and equally acceptable for all regions of the study area. ERA5 also offers spatially distributed and long-term data of a range of climate variables required for hydrological modeling and climate change studies. 
Therefore, precipitation data of this $5^{\text {th }}$ generation reanalysis product is statistically downscaled and bias-corrected with respect to the reference climatologies of mean monthly precipitation developed by Dahri et al. (2018) using the simple delta change method, which uses linear scaling to perfectly match the monthly means of corrected values with those of observed ones (Lenderink et al., 2007). The precipitation estimates beyond the reference period are cross validated by the corresponding observed river inflows at annual scale. For bias correction of temperature dataset, the same method in additive/subtractive form is applied. The reference climatologies of mean monthly maximum and minimum temperature are derived by following the approach of Dahri et al. (2018), who used multiple regressions to derive elevation and latitude dependent monthly lapse rates for the study area. The area however is divided into three parts of southern, northern and western zones (Figure S-5.1) to achieve better accuracy. The resultant lapse rates to derive mean monthly climatologies of maximum and minimum temperature for the reference period are given in Table S-5.1.

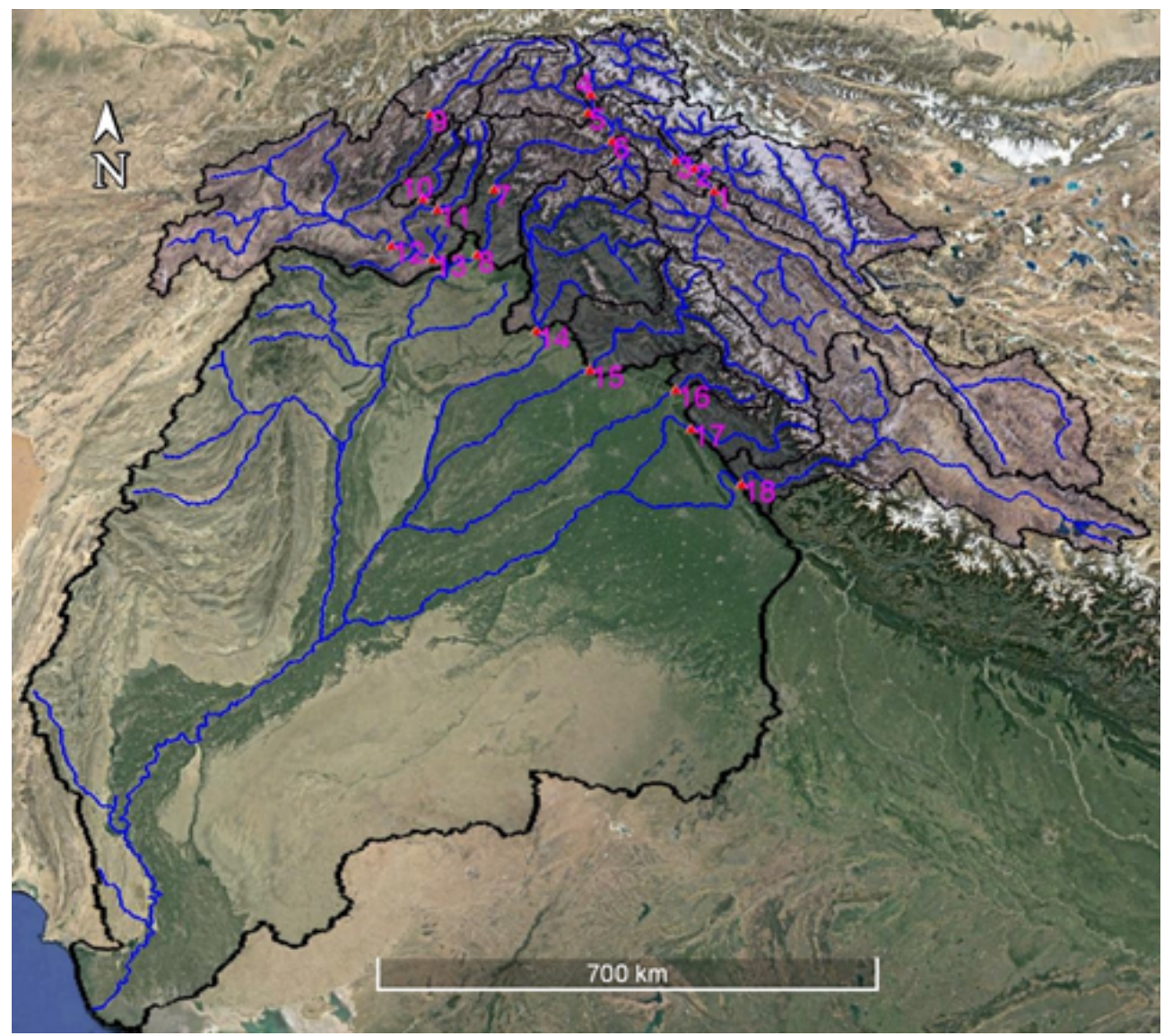

Figure 5.1: Indus basin and its high-mountain watersheds. The black lines show basin boundaries and blue lines represent river network. The pink coloured numbers indicate the discharge measuring river gauges installed at: 1) Indus-Kharmong, 2) Shyk-Yogo, 3) Shigar-Shigar, 4) Hunza-Dainyor, 5) GilgitGilgit, 6) Astore-Doyian, 7) Indus-Besham, 8) Indus-Tarbela, 9) Chitral-Chitral, 10) Panjkora-Zulam Bridge, 11) Swat-Chakdara, 12) Kabul-Warsak, 13) Kabul-Nowshera, 14) Jhelum-Mangla, 15) Chenab-Marala, 16) Ravi-Thein dam, 17) Beas-Pong dam, 18) Sutlej-Bhakra dam. Watersheds numbered 1-6 and 8-11 are tributaries of Indus at Tarbela and Kabul at Nowshera respectively. 


\subsubsection{Future climate projections}

Projected changes in global climate are highly uncertain and depend on future socioeconomic development and technological innovations to mitigate greenhouse gas emissions in the atmosphere. The Coupled Model Intercomparison Project Phase 5 (CMIP5), coordinated by the World Climate Research Programme in support of the $5^{\text {th }}$ assessment report (AR5) of the IPCC, used a set of four RCPs, which provided wider range of future climate change scenarios including a mitigation scenario (RCP2.6), two medium stabilization scenarios (RCP4.5 \& RCP6.0) and an extreme emission scenario (RCP8.5). After the Paris Agreement in 2015, global community committed an ambitious target of climate change mitigation to stabilize global warming at $1.5^{\circ} \mathrm{C}$ by the end of $21^{\text {st }}$ century. Therefore, we selected an optimistic scenario of RCP2.6, which is very close to $1.5-2{ }^{\circ} \mathrm{C}$ global warming; a medium emission scenario of RCP4.5, under which global warming may continue business as usual; and an extreme scenario of RCP8.5, which predicts accelerated and continued global warming throughout $21^{\text {st }}$ century. The intermediate scenario of RCP6.0 is excluded.

Projections of future climate are generally provided by global climate models commonly known as GCMs. However, with a large number of GCM outputs for single or multiple ensembles under various experiments or RCPs available within CMIP5, the spread and variability in their outcomes is also large (Lutz et al., 2016b), particularly at regional and catchment scale (Cai et al., 2009). Therefore, GCM selection is tricky and can be based on multiple criteria using several variables. Generally, the simultaneous use of all GCMs is discouraged and commonly replaced by an ensemble mean. However, this may lump the outcome of worse performing models with that of better performing models resulting in overall poor-quality outcomes. Ensemble mean also significantly increases the frequency of precipitation events at the cost of its intensity, which may result in unusual and unrealistic hydrological responses particularly related to extremes. Mostly, the best representative climate model or a small ensemble of relatively better performing GCMs for the region of interest is selected (Lutz et al., 2016a). Temperature projections by GCMs are often regarded as much more reliable as compared to their precipitation outputs (Houghton et al., 1990), which was also observed during initial screening in this study. Therefore, we only used historical estimates of GCM precipitation for their evaluation and ultimate selection. The monthly scale GCM precipitation was downloaded from the Royal Netherlands Meteorological Institute (KNMI) Climate Explorer (https://climexp.knmi.nl) in May 2019.

The initial selection of GCMs is based on the availability of precipitation as well as temperature (maximum \& minimum) data at daily time scale for the three ensembles or experiments of climate change scenarios (RCP 2.6, RCP4.5 \& RCP8.5). Only 75 GCM ensemble runs (Table 5.1) fulfilled these criteria of initial selection.

The $2^{\text {nd }}$ step evaluated the past performance of these 75 GCM runs to precisely reproduce the quantitative and spatiotemporal distribution of mean monthly precipitation over the study domain. The evaluation approach adopted by Dahri et al. (2020, under review) for accuracy assessment of gridded precipitation products is largely adopted to cross-validate GCM precipitation against the bias corrected ERA5 precipitation data for the period of 1981-2005. Monthly precipitation climatologies of the selected GCMs are regridded to match the spatial resolution of bias-corrected ERA5 dataset, but their original estimates are preserved. Annual cycle of the area-weighted monthly precipitation of each GCM is analysed against that of the reference dataset using coefficient of determination $\left(\mathrm{R}^{2}\right)$. Modified Kling-Gupta Efficiency (KGE, Kling et al., 2012) is computed to assess its spatial patterns, correlation and spread of biases and deviations between the reference and GCM precipitation. The $\mathrm{R}^{2}$ and KGE values 
are normalized and rescaled between 0 and 1, which are then added to yield the final skill score (rank) of each GCM (Table 5.1).

Table 5.1: Performance of GCM precipitation against the reference precipitation data (bias corrected ERA5) shown in blue colour ( $\mathrm{mm}$ ). The monthly and yearly values in graduated coloured cells highlight the biases $(\%)$ of GCM precipitation. $\mathrm{R}^{2}$ is coefficient of determination and KGE is Kling-Gupta Efficiency between precipitation of reference dataset and each GCM run; $\mathrm{N}$ denotes normalized values and skill is the final score for each GCM run.

\begin{tabular}{|c|c|c|c|c|c|c|c|c|c|c|c|c|c|c|c|c|c|c|c|}
\hline k & GCM Dat & $\mathbf{J}$ & $\mathbf{F}$ & M & $\mathbf{A}$ & M & $\mathbf{J}$ & $\mathbf{J}$ & $\mathbf{A}$ & $\mathbf{S}$ & $\mathbf{O}$ & $\mathbf{N}$ & D & $\mathbf{Y}$ & $\mathbf{R}^{2}$ & $\mathbf{N R}^{2}$ & KGE & $\mathrm{KG}$ & kill \\
\hline REF & ERA5 (BC) & 71.7 & 81.2 & 108.3 & 77.8 & 66.9 & 49.7 & 79.2 & 68.7 & 34.2 & 38.3 & 29.8 & 54.8 & 760.6 & -- & -. & - & - & \\
\hline 1 & MIROC5_r1i1p1 & 10.6 & 24.4 & 32.4 & 54.0 & 3.3 & -30.4 & -15.9 & 24.9 & 31.6 & -22.7 & 21.1 & 10.2 & 14.5 & 0.82 & 1.00 & 0.35 & 0.90 & 1.90 \\
\hline 2 & MIROC & 4.5 & 34.2 & 23.4 & 50.5 & 5.1 & -9.1 & -12.1 & -8.7 & 8.2 & -10.7 & 19.5 & 34.1 & 13.4 & .82 & 1.00 & 0.34 & 0.89 & 189 \\
\hline 3 & MIROC5_r2i1p1 & 31.8 & 23.3 & 3.0 & 5.6 & -7.8 & -41.9 & -11.6 & 10.5 & 26.3 & -19.3 & 7.7 & 8.4 & 12.9 & 0.79 & 0.95 & 0.37 & 0.93 & 1.89 \\
\hline 4 & MPI-E & 29.6 & 48.9 & 16.3 & 75.3 & 5.4 & -4.4 & 6.2 & 33.8 & 52.3 & 34.7 & 61.1 & 20.4 & 29.9 & 0.75 & 0.91 & 0.07 & 50 & 1.42 \\
\hline 5 & R_r2i1p1 & 15.1 & 22.9 & 17.1 & 51.9 & -7.9 & 9.5 & 7.1 & 23.9 & 102.0 & 3.7 & 59.4 & 28.3 & 23.6 & 0.76 & 0.93 & 0.05 & 0.48 & 1.40 \\
\hline 6 & MPI-ES & 7.5 & 9.5 & 10.4 & 40.1 & -15.4 & -1.6 & 26.1 & 19.2 & 74.9 & 32.4 & 4.5 & 35.9 & & 0.77 & 0.93 & 0.04 & 0.46 & 1.39 \\
\hline 7 & HadG & -7.4 & -13.2 & 14.5 & 86.0 & 88.9 & 74.2 & 50.1 & 34.2 & 22.2 & 33.7 & 73.2 & -11.9 & 34.4 & 0.51 & 0.62 & 0.20 & 0.69 & 1.32 \\
\hline 8 & CMC & 19.9 & 23.6 & 33.6 & 68.0 & 16.9 & -1.6 & -17.9 & -6.3 & 54.7 & 88.0 & 189.6 & 66.6 & 34.2 & 0.43 & 0.53 & & 0.79 & 1.31 \\
\hline 9 & EC-EA & -37.1 & -22.2 & -5.0 & 3.2 & -12.6 & 109.1 & 62.0 & 54.9 & 124.9 & 14.9 & -0.3 & -34.7 & & .25 & 0.30 & 0.42 & .00 & 1.29 \\
\hline 10 & EC-I & 3.4 & -24.6 & -11.2 & 2.2 & -8.8 & 85.3 & 68.2 & 53.6 & 107.3 & 9.1 & 55.7 & 35.2 & 13.6 & 0.22 & 0.26 & 0.41 & .98 & 1.24 \\
\hline 11 & CES & 4.4 & -26.1 & -11.7 & 8.7 & 21.2 & 34.0 & 67.6 & 102.0 & 94.7 & 36.3 & 62.1 & -5.7 & 21.6 & .24 & 0.29 & & & 1.23 \\
\hline 12 & HadG & -45.5 & -21.9 & 21.2 & 71.3 & 93.1 & 58.6 & 61.6 & 47.3 & 41.5 & 38.1 & 70.5 & -0.2 & & 44 & 0.53 & 20 & 69 & 1.22 \\
\hline 13 & EC-I & -44.9 & -25.4 & -14.9 & 14.1 & -0.4 & 111.3 & 63.9 & 65.5 & 120.2 & 27.2 & 9.7 & -37.0 & & .18 & 0.22 & 0.42 & .00 & 1.22 \\
\hline 14 & CES & 6.9 & -34.0 & -4.2 & 33.8 & 16.0 & 55.7 & 75.0 & 82.5 & 87.4 & 38.6 & 78.5 & 10.4 & & .25 & 0.30 & & & 1.21 \\
\hline 15 & & -44.9 & 25.4 & -14.9 & 14.1 & -0.4 & 111.3 & 63.9 & 65.5 & 120.2 & 27.2 & 9.7 & -37.0 & 16.7 & 18 & 0.22 & & 97 & 1.18 \\
\hline 16 & CES & -58.7 & -37.6 & -13.4 & 15.9 & 6.1 & 55.1 & 70.5 & 89.4 & 79.2 & 19.3 & 57.4 & -17.5 & 153 & .20 & 0.24 & 0.38 & 0.94 & 1.18 \\
\hline 17 & HadC & -32.8 & -24.8 & 9.7 & 77.2 & 04.9 & 83.9 & 45.7 & 41.9 & 26.3 & 101 & 65.8 & 16.8 & & .39 & 0.47 & & & 1.14 \\
\hline 18 & HadC & -30.3 & -23.5 & 6.5 & 73.4 & 96.1 & 71.8 & 58.5 & 37.6 & 47.1 & 42.3 & 72.8 & -12.0 & 31 & .38 & 0.46 & & & 1.13 \\
\hline 19 & GISS & -7.8 & -7.4 & 7.3 & 24.8 & 54.7 & 23.1 & -33.1 & -31.4 & 9.9 & $\mathbf{5 0 . 7}$ & 127.5 & 31.0 & 12 & .38 & 0.47 & 0.18 & 66 & 1.13 \\
\hline 20 & HadC & -28.5 & -15.5 & 15.0 & 70.7 & 106.4 & 97.8 & 78.7 & 39.7 & 54.7 & 56.9 & 62.4 & 35 & 40 & 39 & 0.48 & 0 & & .11 \\
\hline 21 & 10 & 31.5 & 2.2 & 12 & 42.3 & -17.9 & -15.3 & 19.4 & 23.9 & 82.7 & 18.3 & 25.2 & 71 & 18.2 & .55 & 0.66 & & & 1.10 \\
\hline 22 & HadG & -36.3 & -16.4 & -3 & 78.3 & 87.0 & 79.5 & 73.6 & 52.5 & 50.3 & 31.9 & 84.9 & -26.3 & & 32 & 0.39 & & 71 & 1.10 \\
\hline 23 & C & 18.7 & 24.5 & 50. & 89.1 & 47.5 & 38.8 & 9.2 & 22.1 & 135.4 & 97.9 & 163.8 & 40 & & 57 & 0.69 & & & 1.09 \\
\hline 24 & & 21. & 11. & 31 & 47.0 & 67.7 & 48.9 & 41.2 & -38.7 & 41.8 & 49.9 & 57.7 & 87.4 & & 31 & 0.38 & & & 1.07 \\
\hline 25 & & -24.0 & -14 & 5.2 & 62.2 & 95.1 & 98.8 & 47.9 & 39.0 & 34.2 & 78.1 & 0.1 & -1.6 & & 33 & 0.39 & & 67 & 1.06 \\
\hline 26 & & 37.9 & 45.4 & 41. & 75.1 & 24.4 & -25.8 & -35.4 & 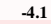 & & 5 & 3.0 & & & 52 & .63 & & & 1.05 \\
\hline 27 & & 4.2 & & 7 & 55.0 & 59 & 56.7 & -19.3 & & & 5 & 1.4 & & & 36 & 43 & & & 1.03 \\
\hline 28 & G & 1 & 11 & 13 & 46. & 82 & 41.6 & -39.8 & -26.5 & 20.5 & & & & & .27 & 0.33 & & 60 & 0.93 \\
\hline 29 & & & -2 & & 54.0 & 62 & 25 & -43.4 & -44.3 & -1 & 4 & .8 & & & 19 & .23 & & 69 & 0.92 \\
\hline 30 & & 1. & -17. & 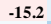 & & 9 & 33.2 & 108.2 & 16.2 & 45 & & 5 & & & 10 & & & & .86 \\
\hline 31 & & -5.0 & 2.2 & 12 & 76 & & 39.6 & -40.7 & -35.8 & 22.8 & .0 & 25.2 & 44.9 & & 15 & 8 & & & .82 \\
\hline 32 & & -37.4 & -19.0 & 5.9 & 42.2 & 17 & 48.9 & 90.9 & 127.5 & 205.3 & 86.9 & 163.1 & 25.5 & 4 & 10 & & & & .78 \\
\hline 33 & & 21. & 7 & & & & -8 & 8 & .7 & & & & & & E. & & & & .78 \\
\hline 34 & & 1. & 2.1 & & & 2.2 & -22.5 & 17.2 & 95.1 & & .0 & 2.0 & & & 20 & 0.24 & & 52 & 0.76 \\
\hline 35 & 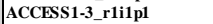 & -44.8 & -28.4 & 10.5 & 83.7 & 142.0 & 184.3 & 91.0 & 76.9 & 62.6 & -5.5 & 96.3 & 5 & 49.7 & 20 & 0.25 & & & 0.75 \\
\hline 36 & G & 9 & $=$ & -46.6 & -186 & & -19.7 & -21.3 & 12.2 & 9 & 106.0 & & -1 & -3 & 12 & 4 & & & .74 \\
\hline 37 & & 6.5 & -25.9 & 4.3 & 16 & 1. & 27.4 & 110.1 & 139.7 & n & 89.6 & 02.3 & 0.9 & 44 & .07 & .08 & 0 & 66 & 0.74 \\
\hline 38 & C & -7.4 & -193 & -5.1 & 44.1 & -1 & 48.3 & 87.2 & 34.5 & 227.2 & 79.1 & 39.9 & 30.7 & 47.2 & .08 & 0.09 & & & 0.74 \\
\hline 39 & $\mathrm{~N}$ & 1 & 1 & 1 & 23 & 3 & -32.2 & 52.1 & 92.3 & , & & 0 & & & 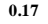 & 0 & & & .73 \\
\hline 40 & CCs & 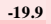 & -23 & -4.4 & 25. & 5 & 60.0 & 102.4 & 148.0 & 04.4 & 99.2 & 2.7 & 30 & 48 & .05 & .06 & 0. & 64 & 0.70 \\
\hline 41 & & -42.7 & -27.6 & 5.4 & 28.7 & 26 & 55.7 & 96.2 & 8.0 & 230.0 & 89.3 & 31.9 & 30.7 & 48.6 & .05 & 0.06 & & 64 & 0.70 \\
\hline 42 & & 10.2 & 64 & 211 & -94 & 2 & 165 & & -74.7 & -14.9 & & & & & & & & & .70 \\
\hline 43 & IPS & 23. & -2.5 & 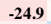 & -4.5 & 15 & 11.6 & .1 & 5.4 & -7.9 & -13 & 65. & 87.2 & 13 & 12 & 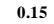 & & .55 & 0.70 \\
\hline 44 & & -3.1 & -26.7 & & 50.4 & & 34 & 90.5 & 145.1 & 242.0 & 69.7 & 168.1 & 28.1 & 51 & 03 & 3 & 0 & 0 & 0.63 \\
\hline 45 & & 2.4 & -30.5 & 86 & 333 & 181 & & & & & & & & . & & & & & 63 \\
\hline 46 & II & 19 & -1 & . & 17. & 6 . & .0 & -89.3 & -7 & & -5 & 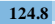 & & -3 & 05 & 6 & & .53 & .60 \\
\hline 47 & & 1.7 & -9.4 & 6.6 & 4.2 & 18.4 & 11.7 & -40.2 & -27.1 & 10.5 & 94.5 & 25.2 & 28 & 3.9 & 20 & 5 & -0 & 33 & .58 \\
\hline 48 & II & 1.0 & -16.4 & -6.5 & 27.2 & 17.0 & -40.6 & -85.6 & -76.6 & 295 & & & & 29 & 05 & 06 & & & .58 \\
\hline 49 & BNU & 60 & -17 & & & & & & -35.4 & & -1 & & & & .14 & 7 & -0 & & 54 \\
\hline 50 & & 8.1 & -26.8 & 0 & 4.2 & -2 & .5 & & -2.0 & 61.7 & 100.5 & 3.4 & & & 01 & 1 & & & .52 \\
\hline 51 & & 5.1 & -15.6 & 108 & & & & -63.4 & -64.2 & 4.4 & 38.6 & 51.0 & -10 & -5.4 & 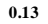 & 15 & 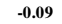 & 29 & .44 \\
\hline 52 & II & 0 & 19.3 & 17 & 77 & 54 & 8 & -75.6 & & 36.8 & 115.1 & & & & & & & & 42 \\
\hline 53 & & & & & & & -20. & -64 & & 96.5 & & & & & 111 & 13 & 1 & & J \\
\hline 54 & & 4.5 & 55.9 & & & & .8 & 3.2 & 3.0 & & & 23.8 & 4.4 & & 12 & 15 & 13 & 3 & .38 \\
\hline 55 & & $=$ & -66.7 & -55 & -35.7 & 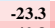 & 475 & & & -48.2 & -5 & -14.8 & & -57.4 & & & & & 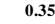 \\
\hline 56 & & 9. & -70 & & 29 & 10 & & & & 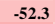 & & 6 & & & & 0 & & & 3 \\
\hline 57 & & 0.9 & -67.7 & & & 30 & & & & 3 & 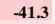 & & & & 20 & 24 & 0.23 & .09 & 1.33 \\
\hline 58 & & 9.5 & -60.8 & & & -23 & -4 & & -7 & A & -23.8 & -21.1 & & & & & & & .32 \\
\hline 59 & & 7 & -57 & & & 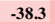 & & & & -2 & -36.8 & 14 & & & & 0 & & & 13. \\
\hline 60 & & 0.1 & -68.3 & -44.5 & -31 & -5 & -57.1 & -6 & -72.2 & 13.7 & & 4.0 & & -4 & 12 & 0.14 & 0.18 & & .30 \\
\hline 61 & & 80 & 52 & 3.9 & 17 & & 2.0 & & -30.3 & 124.0 & & 235.2 & 116 & 3 & & & & & .2 \\
\hline 62 & & & & & & & & & & & & & & & 14 & 7 & & & 0 \\
\hline 63 & & -20.1 & -43.8 & & 30 & 61 & 49 & -32 & & 35.7 & 142.6 & 2 & & & 00 & 0.00 & -0.09 & & .29 \\
\hline 64 & & -9.9 & -22.9 & -36 & -23.9 & -23 & -54.7 & -77.5 & -76.9 & -59.4 & 21.9 & 146.3 & 35.6 & -2 & 44 & .04 & -0 . & & 0.28 \\
\hline 6 & & & & & & & & & & & & & & & & & & & 0 \\
\hline 66 & & & & & & & & & & -41.5 & & & & & 14 & 0.16 & 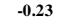 & 99 & 0.26 \\
\hline 67 & & 9.1 & -69.1 & -58.4 & 17.5 & -29.6 & & & & -64.6 & -31.3 & 11.4 & -40.7 & 8 & 11 & 0.13 & 0.21 & & .24 \\
\hline so & & 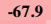 & 60 & & & & & & & 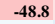 & & 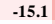 & & & & & & & .24 \\
\hline 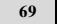 & & & & & & & & & & 13 & & & & & & & & & 1.10 \\
\hline 70 & C & -72.9 & -60.5 & -57 & -2 & -53 & & & -4 & 6.1 & -9.7 & & 8 & -44.2 & 06 & 0.07 & 2 & 10 & 0.17 \\
\hline 71 & MIRO & -10.0 & 2 & 10 & 73 & 82 & 201.4 & 119. & 88.4 & 200.6 & 148.8 & 128.2 & & & 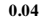 & 05 & & & 0.17 \\
\hline 72 & & -68 & 58. & & & 51 & & 6 & -56 & & & & & 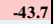 & & & & & 0.14 \\
\hline 73 & & -10.9 & -4.7 & -37 & -12 & -24 & 49.5 & 39 & 5 & 221.3 & 60.1 & 126.5 & 55 & 24.2 & 00 & 0.00 & -0.21 & 11 & 0.11 \\
\hline 74 & & -67.4 & -62.8 & -52 & -45 & & -43 & & -58 & 30 & -0.3 & & -47 & -47 & 0.03 & 0.03 & -0.25 & 06 & 0.09 \\
\hline 75 & MIROC-ESM_r1i1p & -6.3 & -4.6 & 10.4 & & & 228.0 & 109.2 & & 232.5 & 169.7 & 166.8 & 49. & 77.3 & 0.00 & 0.00 & -0.29 & 0.00 & 0.0 \\
\hline
\end{tabular}


In the $3^{\text {rd }}$ step, top 24 GCMs ranked in the previous step are evaluated based on the changes in climatic means between 1971-2000 and 2071-2100 for RCP4.5. Following Lutz et al. (2016b), four corners of warm-dry, warm-wet, cold-dry and cold-wet spectrum are determined from the range of projected changes in mean annual air temperature $(\Delta T)$ and annual precipitation $(\Delta \operatorname{Pr})$ using the $10^{\text {th }}$ and $90^{\text {th }}$ percentile values (Figure 5.2). However, unlike Lutz et al. (2016a \& b), who selected different GCMs for RCP4.5 and RCP8.5, we evaluated the projected changes only for a more realistic scenario of RCP4.5 to select the best GCM for each corner. The selected GCMs were sustained for other RCPs to ensure consistency in their projections. If a GCM run projects more extreme conditions but does not perform well to reproduce the past climate of the study area is eliminated. The analysis in this study is limited to two GCMs representing extreme precipitation and temperature for the warm-wet and cold-dry corners represented by MIROC5_r1i1p1 and MPI-ESM-LR_r1i1p1 respectively under RCP2.6, RCP4.5 and RCP8.5. Coincidently, both these GCMs are also the best performers based on the past performance summarized in Table 5.1. Lutz et al. (2016a) also selected these two GCMs for RCP 8.5. We also ascertained the end of century global warmings projected by both these GCMs under three RCPs over the pre-industrial period. The projected increases in global mean air temperature are $1.51,2.13 \& 3.61^{\circ} \mathrm{C}$ by MIROC5_r1i1p1 and 1.69, $2.49 \& 4.26^{\circ} \mathrm{C}$ by MPI-ESM-LR_r1i1p1 under RCP2.6, RCP4.5 \& RCP8.5 respectively. However, MIROC5 is found about $1^{\circ} \mathrm{C}$ warmer than MPI-ESM-LR for the study area.

\begin{tabular}{clrr}
\hline S.\# & \multicolumn{1}{c}{ GCM } & \multicolumn{1}{c}{$\boldsymbol{\Delta P ( \% )}$} & $\boldsymbol{\Delta} \mathbf{T}\left({ }^{\circ} \mathbf{C}\right)$ \\
\hline 1 & MPI-ESM-LR_r2i1p1 & -14.636 & 3.017 \\
2 & MPI-ESM-LR_r1i1p1 & $-\mathbf{1 0 . 1 4 3}$ & $\mathbf{2 . 9 2 5}$ \\
3 & CESM1-CAM5_r3i1p1 & -4.175 & 3.450 \\
4 & MPI-ESM-LR_r3i1p1 & -3.981 & 3.050 \\
5 & CESM1-CAM5_r1i1p1 & -3.656 & 3.408 \\
6 & CESM1-CAM5_r2i1p1 & -1.295 & 3.392 \\
7 & CMCC-CM_r1i1p1 & -0.534 & 3.558 \\
8 & HadGEM2-ES_r1i1p1 & 0.633 & 3.733 \\
9 & EC-EARTH_r2i1p1 & 1.699 & 2.658 \\
10 & CNRM-CM5_r1i1p1 & 1.739 & 2.483 \\
11 & HadGEM2-ES_r3i1p1 & 1.879 & 3.350 \\
12 & GISS-E2-R_r1i1p1 & 2.399 & 2.600 \\
13 & HadGEM2-ES_r4i1p1 & 2.620 & 3.433 \\
14 & HadGEM2-ES_r2i1p1 & 3.328 & 3.600 \\
15 & MPI-ESM-MR_r1i1p1 & 3.951 & 3.017 \\
16 & HadGEM2-AO_r1ilp1 & 4.591 & 3.875 \\
17 & EC-EARTH_r8i1p1 & 4.771 & 2.583 \\
18 & HadGEM2-CC_r1i1p1 & 5.277 & 3.267 \\
19 & EC-EARTH_r9i1p1 & 7.299 & 2.508 \\
20 & EC-EARTH_r12i1p1 & 7.919 & 2.367 \\
21 & GISS-E2-H_p3_r1i1p1 & 8.171 & 3.333 \\
22 & MIROC5_r1i1p1 & $\mathbf{1 4 . 1 7 1}$ & $\mathbf{3 . 9 1 7}$ \\
23 & MIROC5_r2i1p1 & 16.348 & 3.992 \\
24 & MIROC5_r3i1p1 & 18.839 & 3.717 \\
\hline
\end{tabular}

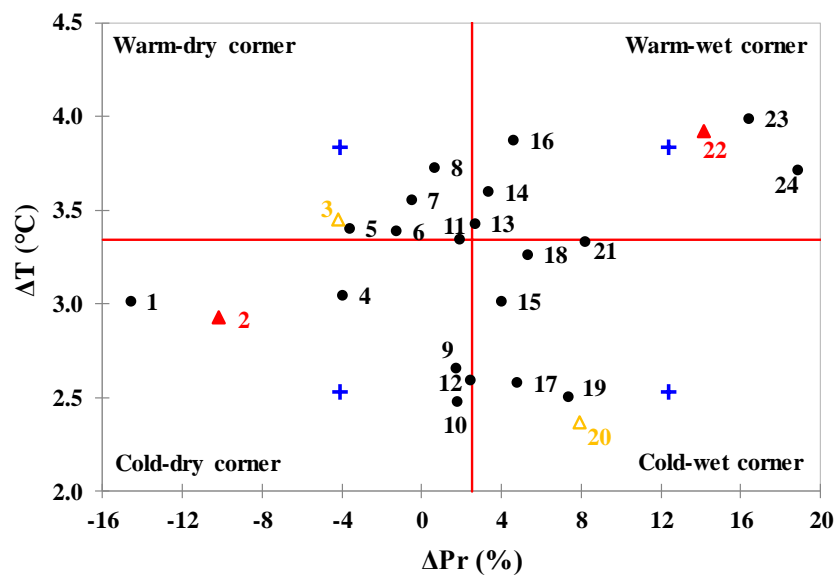

Figure 5.2: Projected changes in mean annual air temperature $(\Delta \mathrm{T})$ and annual precipitation $(\Delta \operatorname{Pr})$ between $1971-2000$ and $2071-2100$ by top 24 ranked GCMs in the study area, numbers reflect the respective GCMs in the adjoined table. Blue plus signs represent the $10^{\text {th }}$ and $90^{\text {th }}$ percentile values of $\Delta \mathrm{T}$ and $\Delta \mathrm{Pr}$ by the $24 \mathrm{GCMs}$. The diamonds show the GCMs representing four extreme corners. Two red coloured filled diamonds are the GCMs selected for this study.

Finally, the precipitation and temperature data of the two selected GCMs under three RCPs at daily timescale are statistically downscaled and bias-corrected against the bias-corrected ERA5 dataset for the period of 1981-2005 using the same approach as illustrated in the preceding section. The data of other parameters (e.g. wind speed, incoming longwave and shortwave radiations, specific humidity and pressure are directly used for hydrological modeling. 


\subsubsection{Description, setup and calibration of VIC hydrological model}

The Variable Infiltration Capacity (VIC) model (Liang et al., 1994; 1996) is an evolving macroscale, fully-distributed, physically-based hydrologic model that can solve water balance as well as full water-and-energy balance to compute surface runoff, baseflow, evapotranspiration and other turbulent fluxes for each individual grid. The land surface is modelled as uniform grids of any size. Sub-grid variability of precipitation, temperature and land cover is considered by further dividing the surface grids into an arbitrary number of bands/tiles with variable elevation and land cover types. Temperature is lapsed from the grid cell average elevation to sub-grid band elevation, while altitudinal variability of precipitation within each grid cell is specified through fractional areal coverage. Sub-grid variability in infiltration capacity is represented by a fast runoff response to precipitation through a variable infiltration curve and slow runoff response via a nonlinear relationship between baseflow and deep soil moisture. Water can only enter to a grid cell via the atmosphere. An offline Lohmann routing model (Lohmann et al., 1998) processes the runoff and baseflow fluxes generated for each grid cell and routes the accumulated flows at the specified locations in the model domain using elevation-based flow direction. Comprehensive technical descriptions and mathematical formulations of the VIC model are provided at https://vic.readthedocs.io/en/master/Overview/ModelOverview/ and by Gao et al. (2010).

The VIC model (VIC4.2d) used in this study requires input of a wide range of climatic, soil, vegetation and snow parameters in spatially distributed form for each grid cell. Most of these model input parameters are physically-based either directly measured or calculated from the observed data and the standard lookup tables. However, sensitivity of the model outcome is largely limited to only a few important parameters, which are usually adjusted during model calibration and validation. Apart from the meteorological forcing data described in preceding sections, soil physical and hydraulic properties were derived from the harmonized world soil database (HWSD, FAO/IIASA/ISRIC/ISSCAS/JRC, 2012) and HiHydroSoil-V1.2 database obtained from FutureWater (https://www.futurewater.eu/2015/07/soil-hydraulic-properties). The soil column was represented by three soil layers extending downward from the land surface to capture baseflow and the vertical distribution of soil moisture. Vegetation characteristics are extracted from the $500 \mathrm{~m}$ MODIS Terra+Aqua Land Cover Product (MCD12Q1.006/), while leaf area index (LAI) data are extracted from MODIS Terra+Aqua LAI product (MCD15A2H.006) available at 8-day timestep and $500 \mathrm{~m}$ grid size for the same year (https://e4ftl01.cr.usgs.gov/MOTA/). Monthly scale standard values of vegetation height, albedo, canopy resistance, stomatal resistance and the relative fraction of roots are specified based on vegetation types following Mao and Cherkauer (2009).

After derivation and preparation of required model input data, the VIC hydrological model was setup to full water-and-energy balance mode and executed at daily timestep over the study domain at a grid cell resolution of $0.08333333^{\circ}(\approx 10 \mathrm{~km}$ at the equator $)$ with 25 elevation bands. Hence, the model is practically run at a high resolution of less than $2 \mathrm{~km}$ grid size. The VIC model is forced with the statistically downscaled and bias-corrected ERA5 precipitation and temperature data, while wind speed, incoming shortwave and longwave radiations, specific humidity and atmospheric pressure were directly used as other input climatic variables for the period of 1979-2018 to accomplish model calibration and validation. For the Indus at Tarbela dam and the Kabul at Nowshera, the model is calibrated in a cascaded and sequential manner against the daily observed river flows at 8 and 4 upstream locations respectively, while Jhelum and Chenab watersheds are calibrated against daily river inflows observed at Mangla dam and Marala headworks respectively (Figure 5.1). 
Ravi, Beas and Sutlej river basins are excluded from hydrological analysis due to nonavailability of their river flow data. The river flow data were acquired from Pakistan's Water and Power Development Authority. Depending upon the availability of observed river flow timeseries at various locations, the model was calibrated for an intermediate period and validated for both the preceding and succeeding time slices of the observed river flows for each flow gauge.

Calibration of the VIC model was accomplished through an iterative process by sequentially changing each of the selected parameters to obtain best possible match between the observed and simulated values of daily river inflows. Six important soil parameters were adjusted during model calibration and validation process. These parameters included: i) variable infiltration parameter, which separates the rainfall into direct runoff and infiltration; ii-iii) thicknesses of two bottom soil layers, which control the availability of water for transpiration and baseflow; iv) maximum velocity of baseflow from the bottom soil layer; v) fraction of maximum baseflow velocity where the rapidly increasing nonlinear baseflow starts; and vi) fraction of the maximum soil moisture where non-linear baseflow occurs. The snow component was calibrated for maximum air temperature at which snowfall can occur, minimum air temperature at which rainfall can occur, and surface roughness of the snowpack, which is very important in snow/glacier-fed river basins.

The quality of model calibration and validation is evaluated by employing the standard and most widely used statistical indices using the observed and simulated river flows at daily and monthly time scales. These performance indicators included modified Kling-Gupta Efficiency (KGE; Kling et al. 2012; Gupta et al. 2009) and percent bias (PBIAS), which are expressed as under:

$$
\begin{gathered}
\text { KGE }=1-\sqrt{(\boldsymbol{r}-\mathbf{1})^{2}+(\boldsymbol{\beta}-\mathbf{1})^{2}+(\gamma-\mathbf{1})^{2}} \\
\mathbf{r}=\frac{\sum_{i=1}^{n}\left(\boldsymbol{Q}_{s}-\overline{Q_{s}}\right)\left(\boldsymbol{Q}_{o}-\overline{Q_{o}}\right)}{\sqrt{\sum_{i=1}^{n}\left(Q_{s}-\overline{Q_{s}}\right)^{2} \sum_{i=1}^{n}\left(Q_{o}-\overline{Q_{o}}\right)^{2}}} \\
\boldsymbol{\beta}=\frac{\mu_{s}}{\mu_{o}} \\
\gamma=\frac{\sigma_{s} / \mu_{s}}{\sigma_{o} / \mu_{o}} \\
\text { PBIAS }=\frac{\overline{Q_{s}}-\overline{Q_{o}}}{\bar{Q}_{o}} \mathbf{1 0 0}
\end{gathered}
$$

Here: $\boldsymbol{Q}$ is river discharge while subscripts $\mathbf{o}$ and $\mathbf{s}$ represent observed and simulated discharges respectively. $\mathbf{r}$ in eq. 1 is Pearson's correlation coefficient to measure the degree of linear relation between observed and simulated values, $\boldsymbol{\beta}$ is bias computed by the ratio of the simulated to the observed means $(\boldsymbol{\mu}), \boldsymbol{\gamma}$ is variability ratio given by the ratio of the simulated and observed dataset's coefficients of variation $(\boldsymbol{\sigma} / \boldsymbol{\mu}), \boldsymbol{\sigma}$ is standard deviation, and $\boldsymbol{n}$ is number of observations. The optimum values of KGE, r, $\beta$, and $\gamma$ are at unity, while PBIAS values are desired to be closer to zero. 


\subsubsection{Methods for analysing climate and hydrological changes}

Apart from the methods employed for the development of high-resolution historical and future climatic datasets described in the sections 5.3.1 and 5.3.2, several techniques have been applied to analyse climate and hydrological changes and variability in the study domain. Monthly and annual medians of temperature, precipitation and river inflows are calculated for both the historical and different scenarios of future climate. The data availability of 120 years is analysed for three equal time-slices representing historical baseline (1981-2020) midcentury (2021-2060) and end-century (2061-2100) periods. At the time of analysis, the baseline data (bias-corrected ERA5) was available until 2018, therefore the data for 2019 and 2020 are derived by computing mean from two GCMs under RCP2.6 and RCP4.5, while RCP8.5 is excluded due to unrealistically extreme projections for these initial years.

The climate change and variability analysis for precipitation is conducted for five regions considered by Dahri et al. (2020, under review), while for temperature the whole study area is considered as a single unit because of its much lower spatial variability. Monthly and annual scale temperature and precipitation statistics are analysed for their variability, trends and overall shift in magnitudes during the future time periods relative to historical baseline data. The change and variability in the overall water availability, shifts in seasonality and annual cycle of hydrographs and changes in future hydrological extremes are examined for the four major sub-river basins (i.e. Kabul at Nowshera, Indus at Tarbela, Jhelum at Mangla and Chenab at Marala). The analysis particularly focuses on the trends in historical and future climate and river flows, changes in median flows, shifts in the timings and magnitudes of the future hydrographs, changes in the flow duration curves for each considered future climate change scenario, and changes in high flow (Q5 \& Q10) and low flow (Q90 \& Q95) conditions.

\subsection{Results}

\subsubsection{Climate change and variability}

In the presence of significant interannual variability, median annual air temperature during the last 40 years has increased almost linearly with an overall increase of $0.6{ }^{\circ} \mathrm{C}$ (Figure 5.3 and Table 5.2), and is likely to increase further throughout the $1^{\text {st }}$ half of the projection period (2021-2060). However, after 2060, it shows slight reduction under RCP2.6 and tends to stabilize under RCP4.5. The extreme scenario of RCP8.5 projects an alarming and gradual increase in temperature throughout the $21^{\text {st }}$ century under both the extreme conditions represented by two GCM outputs.
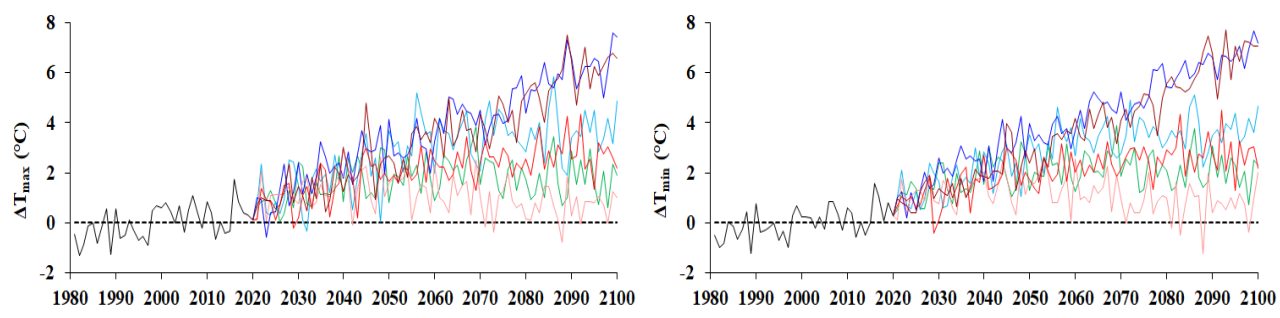

-Baseline (ERA5-BC) -MIROC5_r1i1p1_RCP2.6 - MIROC5_r1i1p1_RCP4.5 - MIROC5_r1i1p1_RCP8.5
-MPI-ESM-LR_r1i1p1_RCP2.6 -MPI-ESM-LR_r1i1p1_RCP4.5 - MPI-ESM-LR_r1i1p1_RCP8.5

Figure 5.3: Median annual maximum \& minimum temperature anomalies averaged over study area. 
Table 5.2: Change in projected maximum and minimum temperatures $\left({ }^{\circ} \mathrm{C}\right)$ in the study area during 2021-2060 and 2061-2100 relative to the baseline period of 1981-2020. The graduated colour scheme highlights the changes as per grid cell values.

\begin{tabular}{|c|c|c|c|c|c|c|c|c|c|c|c|c|c|c|c|c|c|c|c|c|c|c|c|c|c|c|}
\hline $\mathbf{J}$ & $\mathbf{F}$ & M & A & M & J & $\mathbf{J}$ & $\mathbf{A}$ & $\mathbf{S}$ & $\mathbf{0}$ & $\mathbf{N}$ & D & $\mathbf{Y}$ & GCM / RCP & $\mathbf{J}$ & $\mathbf{F}$ & M & A & M & $\mathbf{J}$ & $\mathbf{J}$ & A & $\mathbf{S}$ & $\mathbf{0}$ & $\mathbf{N}$ & D & $\mathbf{Y}$ \\
\hline \multicolumn{12}{|c|}{$2021-2060$} & \multicolumn{3}{|c|}{$\Delta \mathrm{T}_{\max }\left({ }^{\circ} \mathrm{C}\right)$} & \multicolumn{12}{|c|}{$2061-2100$} \\
\hline 1. & 1.3 & 1.5 & 1.8 & 1.6 & 1.91 & 1.6 & 1.1 & 1.3 & 1.8 & 0.8 & 1.6 & 1.5 & MIROC5_RCP2.6 & 2.3 & 1.5 & 1.2 & 2.0 & 2.0 & & 2.21 & 1.72 & 2.2 & 1.9 & 1.8 & 2.1 & 1.9 \\
\hline 1.3 & 1.9 & 1.1 & 1.3 & 0.7 & & 1.5 & 1.2 & 1.2 & 1.1 & 1.1 & 1.4 & 1.3 & MPI-ESM-LR_RCP2.6 & 1.1 & 1.6 & 0.9 & 0.5 & 0.1 & & 0.91 & 1.01 & 1.0 & 0.5 & 0.9 & 1.0 & 0.8 \\
\hline 1.9 & 1.5 & 2.2 & 2.4 & 2.7 & 2 & 2.2 & 1.7 & 1.7 & 2.1 & 1.7 & 1.8 & 2.0 & MIROC5_RC & 3.8 & 3.4 & 3.4 & 3.6 & 4.1 & 4.73 & 3.92 & 2.93 & 3.1 & 3.1 & 3.1 & 4.2 & 3.6 \\
\hline 2.3 & 1.7 & 1.4 & 1.5 & 1.0 & 1.72 & 2.0 & 1.6 & 1.5 & 1.2 & 1.4 & 1.7 & 1.6 & MPI-ESM-LR_ & 3.0 & 2.6 & 3.0 & 2.7 & 2.2 & & 2.52 & 2.32 & 2.4 & 2.1 & 2.3 & 2.8 & 2.6 \\
\hline 2. & 1.9 & 1.8 & 2.3 & 2.6 & 2.72 & 2.4 & 1.9 & 2.1 & 2.3 & 2.0 & 2.4 & 2.2 & 8.5 & 5.8 & 5.1 & 4.9 & 5.1 & 6.7 & & 5.23 & 3.94 & 4.4 & 5.0 & 4.1 & 5.5 & 5.2 \\
\hline & 2.2 & 1.7 & 1.9 & 1.2 & 1.92 & 2.22 & 2.2 & 2.0 & 1.5 & 1.9 & 2.1 & 1.9 & MPI-ESM-LR_RCP8.5 & 5.4 & 4.7 & 4.7 & 4.3 & 4.3 & & 5.44 & 4.95 & 5.0 & 4.5 & 5.1 & 5.4 & 4.9 \\
\hline \multicolumn{12}{|c|}{ 2021-2060 } & \multicolumn{3}{|c|}{$\Delta \mathbf{T}_{\min }\left({ }^{\circ} \mathbf{C}\right)$} & \multicolumn{12}{|c|}{ 2061-2100 } \\
\hline & 1.4 & 1.6 & 1.9 & 1.8 & 2.32 & 2.4 & 1.6 & 1.7 & 2.1 & 1.2 & 1.6 & 1.8 & MIROC5_RCP2.6 & 2.9 & 1.6 & 1.3 & 2.2 & 2.0 & & 2.81 & 1.92 & 2.2 & 2.3 & 2.0 & 2.2 & 2.2 \\
\hline 1.2 & 1.4 & 1.1 & 1.6 & 1.0 & 1.71 & 1.2 & 1.1 & 1.3 & 1.3 & 1.5 & 1.4 & 1.3 & MPI-ESM-LR_RCP2.6 & 1.1 & 1.0 & 1.3 & 1.3 & 0.7 & & 0.40 & 0.71 & 1.1 & 0.5 & 0.8 & 1.1 & 0.9 \\
\hline 2.0 & 1.5 & 2.0 & 2.1 & 2.7 & 3.02 & 2.7 & 1.9 & 1.8 & 2.1 & 1.8 & 2.1 & 2.2 & MIROC5_RCP4.5 & 3.9 & 3.5 & 3.5 & 3.4 & 4.0 & 4.7 & 4.33 & 3.02 & 2.9 & 3.0 & 3.2 & 4.0 & 3.6 \\
\hline 2.0 & 1.7 & 1.3 & 1.6 & 1.4 & 2.11 & 1.6 & 1.4 & 1.6 & 1.5 & 1.2 & 1.6 & 1.6 & MPI-ESM-LR_RCP4.5 & 2.4 & 2.1 & 3.2 & 3.1 & 2.6 & 3.02 & 2.42 & 2.12 & 2.6 & 2.4 & 2.3 & 3.1 & 2.6 \\
\hline 2.6 & 2.0 & 2.1 & 2.6 & 3.0 & 3.23 & 3.12 & 2.4 & 2.3 & 2.7 & 2.4 & 2.6 & 2.6 & MIROC5_RCP8.5 & 6.1 & 5.3 & 5.1 & 5.1 & 7.1 & 7.8 & 6.64 & 4.94 & 4.9 & 5.3 & 4.7 & 5.4 & 5.7 \\
\hline 1.9 & 1.8 & 2.0 & 2.4 & 1.6 & 1.81 & 1.82 & 2.0 & 2.1 & 1.9 & 1.8 & 2.8 & 2.0 & MPI-ESM-LR_RCP8.5 & 5.6 & 4.8 & 5.0 & 4.8 & 5.3 & 5.95 & 5.14 & 4.85 & 5.3 & 5.4 & 5.9 & 6.0 & 5.3 \\
\hline
\end{tabular}

The deviations of maximum and minimum temperatures in the study area projected by various GCM runs for mid-century (2021-2060) and end-century (2061-2100) relative to the baseline historical period (1981-2020) presented in Figure 5.4 and Table 5.2 indicate MIROC5 output as considerably warmer than MPI-ESM-LR output almost throughout the year and during the entire $21^{\text {st }}$ century for all RCPs. The comparative increase in the warming of MIROC5 is the largest for RCP2.6 (about 2.3 times higher at annual scale) followed by RCP4.5 until the end-century. Increase in mean annual air temperature in the study domain may range between $1.3-2.6^{\circ} \mathrm{C}$ during mid-century and between $0.8-5.7^{\circ} \mathrm{C}$ by the endcentury. The highest increases are projected by MIROC5_RCP8.5 and vice versa by MPIESM-LR_RCP2.6. Monthly-scale variability of temperature increase is also highly variable. MIROC5 projects higher increases during Apr-Jul and Dec-Jan months throughout the projection period, while MPI-ESM-LR projects relatively higher increases during Dec-Feb months until mid-century, which are lowered down thereafter. Moreover, slightly higher increases are projected in minimum temperature as compared to maximum temperature resulting in trivial narrowing-down of diurnal temperature range in future, which might be due to increased cloud cover and reduced pan evaporation (Hasson et al., 2019).

Compared to temperature, precipitation in the study area has remained highly variable and extremely uncertain during the last 40 years. Median annual precipitation experienced considerable decline during the famous and prolonged drought of recent time (1999-2003). It tried to recover thereafter but still lagged behind by $11.9 \%$ of the median annual precipitation for the $1^{\text {st }}$ half of the baseline period. Definite and strong increasing trend in the projections of median annual precipitation is only evident for MIROC5-RCP8.5, while MPIESM-LR shows declining trends for RCP4.5 \& RCP8.5 (Figure 5.4). The remainder of GCM runs show mixed trends. Compared to the baseline historical period, the interannual variability of the projected precipitation by all GCM runs is considerably higher particularly during monsoon season. Marked variability in quantitative and spatio-temporal distribution of precipitation is clearly depicted by all GCM runs at regional as well as basin scale for both the future time-slices (Figure $5.5 \&$ Table 5.3). MIROC5 generally projects positive changes in precipitation at annual scale and during most of the months under all RCPs, while MPIESM-LR mostly indicates reduced precipitation in all regions except Karakoram. Median annual precipitation in the study domain may change between -12.9 and $21.8 \%$ during 20212060 and between -19.6 and 43.4\% during 2061-2100 relative to baseline level. Substantial 
increases in monthly precipitation at basin scale are expected during Jul-Oct and Dec-Jan months and vice versa during the remaining months, whereas regional scale variability in precipitation distribution will remain significantly high.
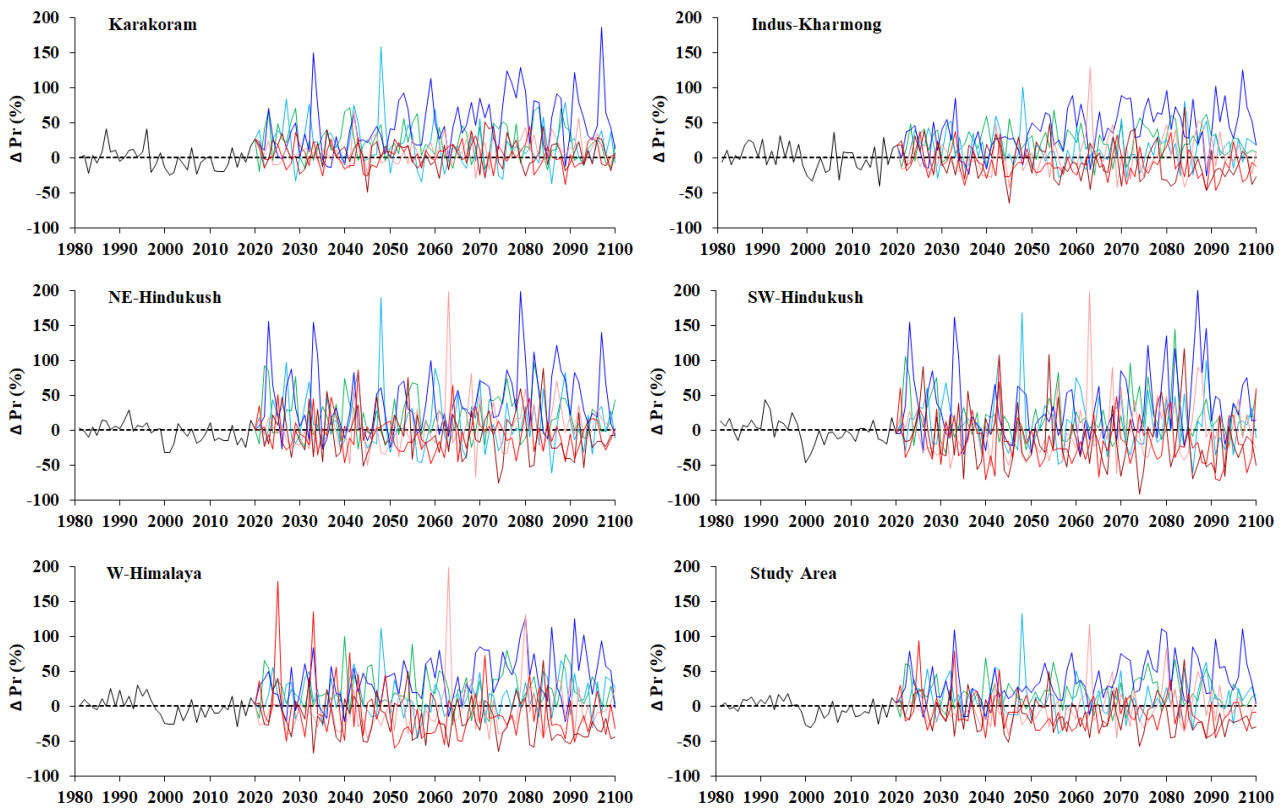

- Baseline (ERA5-BC) —MIROC5_r1i1p1_RCP2.6

-MIROC5_r1i1p1_RCP4.5

-MIROC5_r1i1p1_RCP8.5 -MPI-ESM-LR_r1i1p1_RCP2.6 —MPI-ESM-LR_r1i1p1_RCP4.5 —MPI-ESM-LR_r1i1p1_RCP8.5

Figure 5.4: Median annual precipitation anomalies against the median annual precipitation during the baseline reference period averaged over five regions and study area.

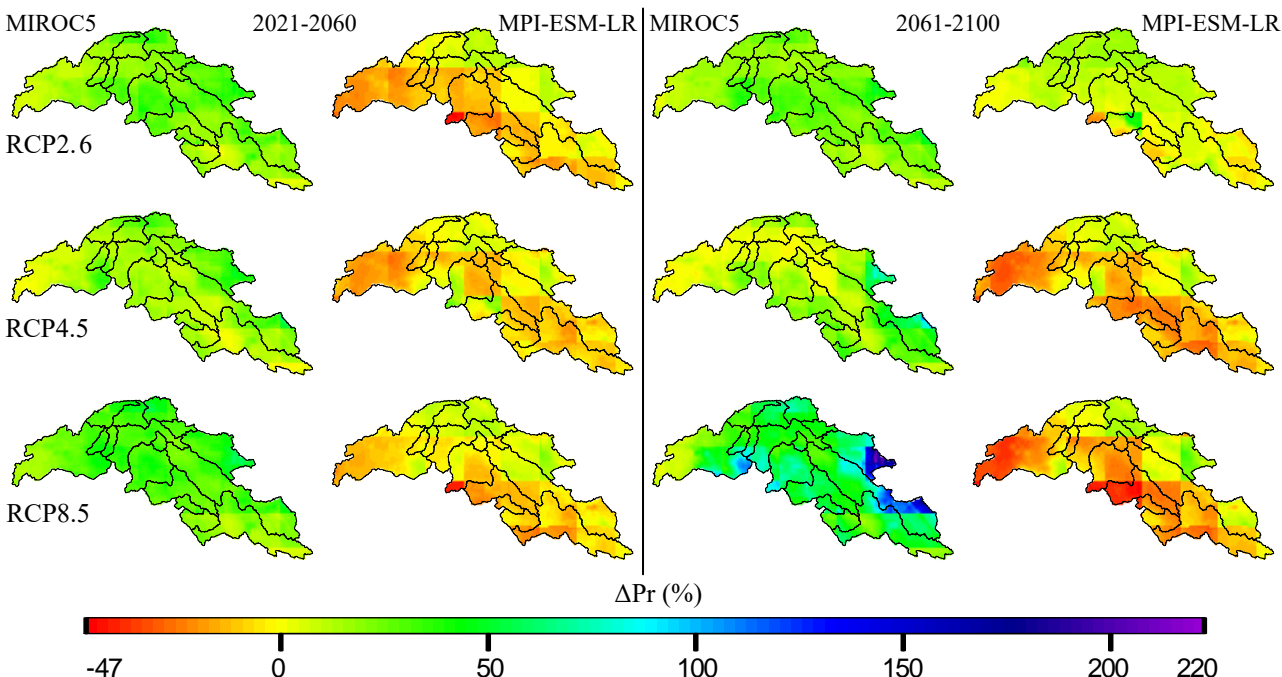

Figure 5.5: Projected changes (\%) in mean annual precipitation relative to baseline (1981-2020) data by two GCMs for three RCPs for mid-century (2021-60) and end-century (2061-2100) time-slices. 
Table 5.3: Projected changes (\%) in median monthly and annual precipitation by 2 GCMs under 3 RCPs in the study area and its five regions for 2021-2060 and 2061-2100 time-slices relative to baseline period of 1981-2020. The blue and red colour stretches highlight the positive and negative changes respectively.

\begin{tabular}{|c|c|c|c|c|c|c|c|c|c|c|c|c|c|c|c|c|c|c|c|c|c|c|c|c|c|c|}
\hline $\mathbf{J}$ & $\mathbf{F}$ & M & $\mathbf{A}$ & M & $\mathbf{J}$ & $\mathbf{J}$ & $\mathbf{A}$ & S & O & $\mathbf{N}$ & D & $\mathbf{Y}$ & CM / RCP & $\mathbf{J}$ & $\mathbf{F}$ & M & $\mathbf{A}$ & M & J & $\mathbf{J}$ & $\mathbf{A}$ & $\mathbf{S}$ & $\mathbf{O}$ & $\mathbf{N}$ & D & $\mathbf{Y}$ \\
\hline \multicolumn{13}{|c|}{$2021-2060$} & Karakoram & \multicolumn{13}{|c|}{$2061-2100$} \\
\hline 10.2 & -19.9 & 16.6 & -7.3 & 9 & 29.4 & 57.2 & 4.3 & 15.4 & 9 & 41.0 & 9 & 9 & IROC5_RCP2.6 & 1 & -16.6 & 3 & 0 & 9.0 & 3.7 & 68.0 & -32.9 & 6.9 & 34.3 & -24.6 & 8 & 5.2 \\
\hline 30.7 & -13.2 & -0.5 & 1.7 & & -8.7 & - & 3.6 & 0.2 & 6.5 & 29 & 8.7 & 1.3 & MPI-ESI & & 1.1 & 4.7 & 25.3 & 5.4 & 7.0 & -6.8 & 2.7 & 15.0 & 51.4 & 5.8 & 3.8 & 7.6 \\
\hline 6.5 & -8.7 & .3 & -18.4 & 4 & 341 & 82.1 & 10.5 & & 9 & .8 & & 19 & & 9.9 & -1.0 & 2 & -12.6 & 13.6 & -0.6 & 36.9 & 8.9 & -31.8 & 6.9 & 4.9 & .1 & 12.4 \\
\hline 0.5 & 9.1 & -10.2 & 50 & .7 & 3 & -0.7 & -20.2 & 00 & 4 & 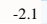 & 7 & .3 & & 0.1 & -5.6 & 15 & 6.5 & 40.5 & 6.9 & 11.7 & 4.5 & 11.3 & 27.7 & .0 & 8 & 56 \\
\hline 1.0 & -12.7 & 20.3 & 1.6 & 5.5 & 9.9 & 71.1 & 25.7 & .5 & 7.6 & 0.1 & .1 & 29.8 & & 9.8 & 10.8 & .7 & 3.6 & 28.3 & 89.8 & 17.4 & 45.5 & 32.5 & 130.4 & 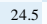 & 4.7 & 6.7 \\
\hline 25 & -3.4 & 9 & 86 & .5 & -14.8 & -1.5 & -2.3 & -5.0 & 34.2 & -64 & 0 & 3.5 & 8.5 & 1.4 & 11.2 & 4.3 & 2.0 & 61.7 & -6.7 & -19.5 & -9.8 & -13.0 & 31.5 & 1 & 1.1 & 70 \\
\hline \multicolumn{13}{|c|}{ 2021-2060 } & Indus-Kharmong & \multicolumn{13}{|c|}{$2061-2100$} \\
\hline 9.7 & -3.4 & 28.2 & -11.4 & 14.2 & -3.2 & 58.8 & 37.2 & 39.8 & 104.4 & 36.0 & 11.7 & 21.9 & MIROC5_RCP2.6 & 29.8 & -2.6 & 22.4 & -2.8 & $\begin{array}{l}-5.7 \\
\end{array}$ & 37.7 & 103.8 & 25.1 & 8.8 & 75.6 & $\begin{array}{l}-59.7 \\
\end{array}$ & 8.9 & 21.0 \\
\hline 2.3 & -36.3 & 3.7 & -31.8 & -3.6 & -13.5 & -4.8 & -0.3 & 8.8 & 33.3 & -4.7 & 5.2 & -5.4 & MPI-ES & 15.7 & -27.5 & 2.4 & 16.9 & 24.9 & -2.6 & -18.6 & 1.2 & -3.2 & 92.9 & 10.5 & 5.5 & 3.8 \\
\hline 10.6 & -9.6 & 0.5 & -28.5 & 11.9 & 25.3 & 103.1 & 39.5 & 12.0 & 100.4 & -27.9 & 14.9 & 12.4 & MIR & -8.2 & 7.2 & 15.4 & -22.2 & -3.4 & -3.7 & 97.6 & 48.4 & 25.5 & 154.2 & -39.5 & 2.8 & 10.3 \\
\hline-1.1 & -20.0 & -2.4 & -21.4 & -25.5 & -7.9 & -10.2 & 4.6 & 6.0 & 3.7 & -20.5 & 3.7 & -10.2 & MPI- & -19.5 & -41.9 & -10.9 & -35.0 & -31.6 & 6.9 & 10.2 & -6.3 & -6.5 & 96.4 & 4.3 & 4.3 & 9.3 \\
\hline 29.0 & 3.8 & 5.0 & -14.3 & 21.9 & 32.5 & 87.6 & 55.6 & 10.6 & 117.4 & -17.8 & 3 & 27.0 & & 3.1 & 10.3 & 9.2 & -18.6 & 26.3 & 76.5 & 150.0 & 105.1 & 66.2 & 176.0 & 1.3 & 8.9 & 51.7 \\
\hline & & & 0 & 60 & 7.4 & -13.8 & 11.0 & 0.0 & 833 & -3.9 & & 0.4 & RCP 8.5 & -16.6 & -28.9 & -26.4 & -48.4 & -16.8 & -16.4 & -9.3 & 40 & 4.2 & 118.5 & & 8.4 & \\
\hline \multicolumn{13}{|c|}{$2021-2060$} & $\mathrm{NE}$ - & \multicolumn{13}{|c|}{ 2061-2100 } \\
\hline 0.5 & -3.6 & 19.6 & -0.6 & 13.8 & -10.9 & 7.0 & \begin{tabular}{|l|}
-31.3 \\
\end{tabular} & 3 & 1 & 33.5 & 38.6 & 17.0 & & 2 & 2.1 & .0 & -4.7 & 11.9 & 28.5 & -16.2 & -67.1 & 1.9 & 38.5 & -4 & 9 & 17.3 \\
\hline 1.6 & -30.2 & -22.4 & -20.9 & -39.1 & & -39.1 & & & & 6.3 & -28.3 & -7.7 & & 3 & -28.9 & 8 & -0.2 & 9 & -13.3 & -48.6 & & .4 & 4.0 & 6 & .8 & 2.5 \\
\hline 11.9 & 2.0 & 7.4 & -26.8 & 4.8 & -13.3 & -10.1 & -32.2 & -6.7 & 35.0 & -18.2 & 40.3 & 79 & .5 & -13.8 & 12.5 & 19.2 & -27.6 & -22.8 & -22.5 & -53.2 & -32.4 & -0.4 & 37.1 & -34.7 & .2 & 3.2 \\
\hline-13.4 & -15.1 & -35.1 & -30.0 & -37.0 & -11.0 & 12.3 & -44.6 & 3.5 & -54.0 & -13.5 & -16.3 & -12.1 & MPI-E & -20.5 & -20.6 & -28.8 & -32.8 & -40.6 & -23.5 & -9.4 & -15.4 & 5.4 & -42.8 & -20.3 & 9.3 & -3.2 \\
\hline 14.7 & -3.4 & 25.2 & 0.9 & 7.3 & 1.4 & -19.1 & -38 & -3.6 & 94.0 & -20.5 & 12.9 & 23.4 & & 24.5 & 7.5 & 6.3 & -16.0 & 15.9 & 15.0 & 12.4 & 69.3 & 73.4 & 173.0 & 9.5 & 8.0 & 36.4 \\
\hline 17. & -20.6 & -18.0 & -18.3 & -37.3 & -30.8 & -35.0 & -40.4 & -18.7 & -45.5 & -22.7 & 52.6 & -2.0 & MPI-E & -12.8 & -35.3 & -37.1 & -58.5 & -52.0 & -26.5 & -15.1 & -12.0 & 15.6 & -53.2 & - & 9 & 4.7 \\
\hline \multicolumn{13}{|c|}{$2021-2060$} & St & \multicolumn{13}{|c|}{$2061-2100$} \\
\hline-11.6 & -5.0 & . & 2.3 & -2.6 & -21.7 & -73.6 & -55.5 & 23.7 & 89.6 & 1.9 & 21.1 & t & 2.6 & & 6.0 & 17.7 & 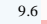 & -3.5 & -10.6 & -43.3 & -70.0 & 81.7 & 53.0 & & .4 & 11.2 \\
\hline-27.6 & -52.3 & -14.9 & -25.0 & -58.8 & -75.0 & -88.0 & -28.7 & 25.3 & -48.5 & -37.4 & -48.0 & -22.8 & MPI & -14.1 & -54.8 & -14.0 & 10.1 & -47.8 & -59.4 & -84.0 & -43.2 & -7.4 & 5.0 & & -43.2 & 1.4 \\
\hline-5.4 & 10.9 & 14.5 & .1 & -10.1 & -37.8 & -49.2 & -64.2 & 43.6 & 53.5 & -42.4 & 18.2 & 9.0 & & -21.1 & 7.6 & 25.6 & -15.9 & -34.2 & -54.4 & -44.3 & -18.5 & 61.1 & 62.4 & - & -2 & 5.5 \\
\hline-33.7 & -45.9 & -38.9 & -46.0 & -65.3 & -33.9 & 6.5 & -32.8 & 8.9 & -62.9 & -53.6 & -46.9 & -21.1 & RCP4.5 & -18.7 & -50.5 & -39.2 & -62.0 & -80.4 & -67.2 & -39.3 & -25.2 & 56.0 & -39.1 & -50.8 & -51.8 & -20.1 \\
\hline 15.0 & -3.4 & 35.3 & 3.2 & 19.8 & -35.6 & -57.5 & -72.0 & 82.5 & 132.7 & -24.9 & 1.6 & 18.3 & $\mathrm{RCP} 8.5$ & 5.9 & 7.8 & 28.9 & -14.5 & -17.1 & -47.8 & -20.5 & 25.2 & 115.6 & 161.9 & -26.2 & -32.2 & 24.0 \\
\hline & -22.5 & -21.0 & -26.4 & -74.5 & -77.2 & -49.9 & -29.0 & 37.0 & -55.0 & -58.9 & 9 & -13.7 & RCP 8.5 & -48.5 & -64.7 & -55.2 & -79.1 & -82.0 & -80.6 & -37.2 & 25.7 & 90.8 & -36.6 & & & \\
\hline \multicolumn{13}{|c|}{$2021-2060$} & & \multicolumn{13}{|c|}{$2061-2100$} \\
\hline 16.3 & -21.7 & 9.7 & 5.5 & -6.8 & -17.9 & 53.8 & -8.0 & 56.6 & 116.2 & 2.5 & 24.6 & 16.5 & & & -15.9 & 1 & 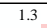 & 0.3 & 19.2 & 61.4 & -7 & 9.0 & 120.4 & -8 & 5.4 & 1.9 \\
\hline & -60.0 & -26.6 & -57.3 & -66.0 & -44.5 & -11.9 & -1 & -6.6 & -31.4 & -17.8 & -17.6 & -15.3 & & 12.7 & -43.9 & -37.0 & 9 & -33.2 & -55.9 & -28.3 & -11.1 & -19.0 & -1.0 & 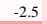 & -18.3 & 60 \\
\hline$-2 c$ & -14.1 & -16.5 & -32.2 & -3.9 & 21.9 & 38.2 & 12 & 23.4 & 69.0 & -48.7 & 37.1 & 66 & & -31.0 & -4.9 & 3.3 & -25.6 & -3.7 & 8.3 & 55.6 & 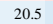 & 30.0 & 86.7 & -6 & 1 & 71 \\
\hline-17.6 & -38.2 & -57.2 & -52.1 & -84.8 & -24.3 & -14.4 & -15.0 & -8.8 & & -55.1 & -42.2 & -12 & & -5.6 & -54.9 & -48.5 & -68.7 & -73.2 & -29.4 & 0.6 & -16.0 & -9.7 & -25.2 & -30.3 & 3.2 & -17.0 \\
\hline 9.4 & -1.4 & 11.7 & -5.7 & 33.5 & -9.4 & 47.5 & 12.9 & -2.2 & 148.7 & -52.8 & 9.9 & 26.3 & $\mathrm{RCP} 8.5$ & -19.2 & 1.5 & -0.8 & -23.7 & 27.4 & 55.5 & 67.4 & 46.4 & 103.0 & 164.4 & -26.2 & -40.4 & 48.1 \\
\hline-19.1 & -38.1 & -21.4 & -40.7 & -68.7 & -59.0 & -23.0 & 6.9 & -3.5 & -30.1 & -45.9 & 18.9 & -7.0 & MPI-ESM-LR_RCP8.5 & -42.5 & -58.9 & -62.9 & -74.6 & -68.7 & -63.8 & -26.2 & -9.9 & 13.6 & -22.0 & -52.3 & -45.9 & -29.6 \\
\hline \multicolumn{13}{|c|}{ 2021-2060 } & Whole Study Area & \multicolumn{13}{|c|}{ 2061-2100 } \\
\hline-10.7 & -15.4 & 13.8 & -0.1 & 2.2 & 2.2 & 53.0 & 6.6 & 29.1 & 102.8 & 24.3 & 25.0 & 16.1 & MIROC5_RCP2.6 & 33.5 & -4.5 & 8.0 & 4.3 & 13.8 & 27.4 & 47.1 & -28.1 & 3.0 & 58.5 & -49.6 & 15.0 & 17.7 \\
\hline-3.4 & -47.9 & -19.2 & -25.9 & -29.3 & -23.4 & -13.7 & 5.2 & -5.0 & -0.4 & -7.7 & -15.8 & -10.1 & MPI & 13.3 & -45.5 & -18.6 & 6.9 & 18.0 & -26.9 & -28.5 & -14.1 & -15.1 & 41.8 & 4.2 & 1.5 & 1.1 \\
\hline 3.0 & -6.5 & -2.3 & -27.7 & -2.7 & 17.1 & 44.5 & 14 & 3. & 59.7 & -19.8 & 24 & 9.5 & & -14.4 & -5.2 & 6.1 & -21.0 & -7.3 & 14.1 & 5 & 4.1 & 6.7 & 58.5 & 4 & 6.0 & 8.7 \\
\hline 18.3 & -35.6 & -37.8 & -30.2 & -39.9 & -13.8 & 5.9 & -18.2 & 9 & -11.2 & -29.8 & -21.2 & -12.9 & CP4. & -8.0 & -40.5 & -32.2 & -42.8 & -40.1 & -31.8 & -15.9 & -10.5 & -12.3 & 5.9 & -12.3 & -2.3 & -13.2 \\
\hline 25. & -6.3 & 17.3 & 1.4 & 33 & 8.8 & & 2. & & 118.1 & -32 & 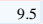 & & & 9.1 & 7.0 & 9.3 & -9.9 & 16.0 & 51.9 & 90.8 & 45.1 & 104.6 & 157.4 & -17.8 & -24.4 & 43.4 \\
\hline-5.0 & -21.8 & -15.1 & -10.1 & -19.4 & -42.0 & -14.4 & 3.9 & 4.6 & 2.6 & -29.8 & 27.0 & -7.9 & MPI-ESM-LR_RCP8. 5 & -20.3 & -45.2 & -50.4 & -53.3 & -31.6 & -39.1 & -12.3 & -2.3 & 19.7 & 4.5 & -30.0 & -31.9 & -19.6 \\
\hline
\end{tabular}

The Karakoram region witnessed about $9.3 \%$ decrease in winter precipitation and $3.6 \%$ increase in monsoon precipitation during the $2^{\text {nd }}$ half of the baseline period (2001-2020) relative to its $1^{\text {st }}$ half (1981-2000). However, median annual precipitation in this region is projected to increase under all GCM runs throughout the remainder of $21^{\text {st }}$ century, except a trivial decrease during mid-century by MPI-ESM-LR-RCP2.6. Maximum increases of $29.8 \%$ and 56.7\% are projected by MIROC5-RCP8.5 during 2021-2060 and 2061-2100 times-slice respectively. At monthly scale, increase in precipitation is projected for all months except Feb, Apr and Sep. Seasonal precipitation however largely follows the pattern of increase observed by Bashir et al. (2017) with about 13.3-34.2\% increases in winter (Oct-Jan) and 7.0-38.0\% increase in summer (May-Aug) precipitation, while spring (Feb-Apr) season may experience slightly declined precipitation. Onset of monsoon is also shifted towards May, which might be due to the interplay between westerlies and East-Asian monsoon systems. Similarly, positive changes in precipitation are projected in Indus-Kharmong region during Jul-Oct and Dec-Jan months by majority of GCM runs. MIROC5 projects an overall increase of $12.4-27.0 \%$ in median annual precipitation of the Kharmong region, while MPI-ESM-LR predicts a decline of up to $10.2 \%$ by mid-century. 
Highest variability in median monthly and annual precipitation is projected for the SWHindukush region in the Kabul river basin. It may experience the largest reductions in precipitation during Jun-Aug and Nov-Dec months under all considered scenarios. Median monthly precipitation in the NW-Hindukush region also follows the similar pattern but at a sluggish rate with slight increases in winter precipitation in the Chitral sub-basin. However, at annual scale overall positive change by MIROC5 and negative change by MPI-ESM-LR in both the regions of Hindukush are quite evident. MIROC5 projects an increase of 11.2, 5.5 \& $24.0 \%$, while MPI-ESM-LR forecasts a decrease of 4.4, 20.1 and $22.0 \%$ in median annual precipitation of the SW-Hindukush region under RCP2.6, 4.5, 8.5 respectively during the end-century time slice. Similarly, precipitation change in the NW-Hindukush region may vary from $17.3,3.2 \& 36.4 \%$ and $2.5,-3.2 \&-14.7 \%$ under the same scenarios.

Median annual precipitation in the W-Himalayan region declined by $12.1 \%$ during the $2^{\text {nd }}$ half of the baseline period relative to its $1^{\text {st }}$ half. Future precipitation projected by various GCM runs in this region however may be extremely variable. MPI-ESM-LR projects significant reductions (-6.0, -17.0 \& -29.6\%), while MIROC5 suggests substantial increases $(24.9,17.1 \& 48.1 \%)$ in median annual precipitation under RCP2.6, 4.5 \& 8.5 respectively by the end of century. The largest increases in median monthly precipitation are predicted during Jul-Oct months (monsoon season), whereas Jan-Jun (winter and spring) months will experience scarce and scanty precipitation under all future scenarios.

\subsubsection{Performance of the VIC hydrological model}

The calibrated and validated model setup for the study domain was executed for the entire timeseries of the baseline historical data at daily timestep under full water-and-energy balance mode. The resultant hydrographs of the observed and simulated river flows at daily timescale are plotted for each of the 14 sub-hydrological basins (Figure 5.6), which show excellent matching of the simulated river flows with the observed river flows throughout the year and simulation period at all river gauges. The VIC model efficiently reproduces the lowest baseflows during winter season and extremely high flows during monsoon season. The results of the VIC model performance ascertained through the selected statistical indices for calibration and validation periods at daily and monthly timescale are presented in Tables $5.4 \& 5.5$ respectively.

The performance indicators (statistical indices) for the calibration and validation periods show that the VIC model performed quite well in all sub-basins. The performance indicators are significantly better at monthly scale than at daily timestep, which is understandable due to smoothing of monthly values. The model performs much better in snow/glacier dominant river basins as compared to the Indian summer monsoon dominated western Himalayan catchments. Model performance is also adversely affected in relatively dry areas of Kharmong and south-west Kabul basin. However, it is important to note that relatively poor performance of the VIC model in a few river basins can mainly be attributed to a combination of uncertainties in input climatic variables, observed river inflows and calibrated parameters in these areas rather than the model structure. Based on these validation results, we conclude that the calibrated model setup sufficiently simulates the hydrology of the study domain and can be executed to model hydrological changes under different scenarios. 

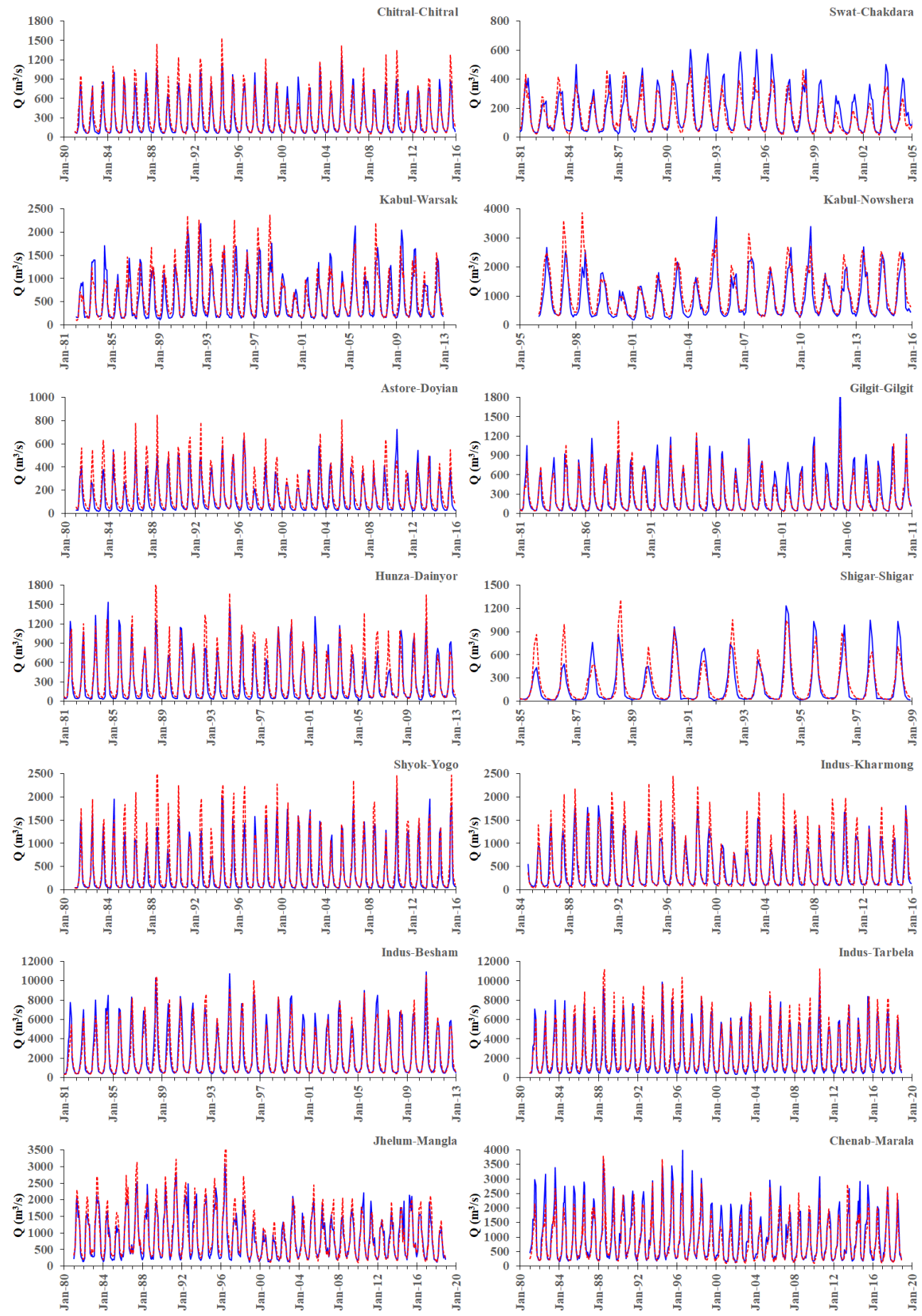

Figure 5.6: Performance of VIC hydrological model against the daily-scale observed river flows at 14 locations. 
Table 5.4: Model performance at daily timescale for calibration and validation periods.

\begin{tabular}{|c|c|c|c|c|c|c|c|c|c|c|c|c|c|c|c|}
\hline \multirow{2}{*}{ Sub-basin } & \multicolumn{5}{|c|}{ Validation (Back ward) } & \multicolumn{5}{|c|}{ Calibration } & \multicolumn{5}{|c|}{ Validation (Forward) } \\
\hline & $\mathbf{r}$ & $\beta$ & $\gamma$ & KGE & PBIAS & $\mathbf{r}$ & $\boldsymbol{\beta}$ & $\gamma$ & KGE & PBIAS & $\mathbf{r}$ & $\boldsymbol{\beta}$ & $\gamma$ & KGE & PBIAS \\
\hline Chitral & 0.91 & 1.22 & 0.92 & 0.75 & 21.92 & 0.91 & 1.09 & 0.96 & 0.87 & 8.91 & 0.89 & 1.10 & 0.99 & 0.85 & 9.79 \\
\hline Swat & 0.75 & 1.05 & 0.86 & 0.71 & 4.76 & 0.72 & 1.13 & 0.87 & 0.67 & -15.23 & 0.64 & 0.81 & 0.97 & 0.59 & -19.54 \\
\hline Kabul-W & 0.76 & 1.10 & 0.87 & 0.71 & 10.27 & 0.76 & 1.17 & 0.94 & 0.70 & 16.93 & 0.72 & 1.06 & 0.82 & 0.66 & 5.51 \\
\hline Kabul-N & 0.81 & 1.29 & 0.97 & 0.65 & 29.03 & 0.83 & 1.17 & 0.82 & 0.70 & 17.14 & 0.79 & 1.17 & 0.80 & 0.66 & 16.59 \\
\hline Astore & 0.87 & 1.39 & 0.89 & 0.57 & 39.30 & 0.86 & 1.18 & 0.93 & 0.76 & 17.72 & 0.79 & 1.10 & 0.90 & 0.75 & 9.86 \\
\hline Gilgit & 0.83 & 0.98 & 0.97 & 0.83 & -2.04 & 0.88 & 0.95 & 0.92 & 0.85 & -5.42 & 0.85 & 0.78 & 0.94 & 0.73 & -22.04 \\
\hline Hunza & 0.84 & 1.11 & 0.88 & 0.77 & 11.44 & 0.83 & 1.21 & 0.89 & 0.70 & 21.39 & 0.81 & 1.14 & 0.99 & 0.77 & 13.62 \\
\hline Shigar & 0.81 & 1.37 & 0.99 & 0.58 & 37.23 & 0.84 & 1.08 & 0.89 & 0.79 & 8.35 & 0.84 & 0.95 & 0.82 & 0.75 & -5.23 \\
\hline Shyok & 0.81 & 1.30 & 1.01 & 0.65 & 30.23 & 0.82 & 1.23 & 0.94 & 0.71 & 22.93 & 0.80 & 1.19 & 0.95 & 0.72 & 18.86 \\
\hline Indus-K & 0.83 & 0.70 & 0.89 & 0.64 & -29.65 & 0.84 & 1.01 & 1.23 & 0.72 & 0.69 & 0.87 & 1.08 & 1.06 & 0.83 & 8.19 \\
\hline Indus-B & 0.85 & 0.93 & 0.93 & 0.82 & -7.08 & 0.89 & 1.03 & 0.93 & 0.86 & 2.58 & 0.87 & 0.93 & 0.95 & 0.85 & -7.15 \\
\hline Indus-T & 0.88 & 1.06 & 0.92 & 0.84 & 5.61 & 0.86 & 1.11 & 0.89 & 0.79 & 10.97 & 0.92 & 1.15 & 0.86 & 0.78 & 14.60 \\
\hline Jhelum & 0.80 & 1.01 & 1.08 & 0.79 & 1.19 & 0.77 & 1.00 & 1.01 & 0.77 & -0.30 & 0.79 & 0.99 & 0.97 & 0.79 & -1.19 \\
\hline Chenab & 0.74 & 0.91 & 1.00 & 0.72 & -9.49 & 0.77 & 0.96 & 0.96 & 0.77 & -4.25 & 0.72 & 0.88 & 0.89 & 0.68 & -11.87 \\
\hline
\end{tabular}

Table 5.5: Model performance at monthly timescale for calibration \& validation periods.

\begin{tabular}{|c|c|c|c|c|c|c|c|c|c|c|c|c|c|c|c|}
\hline \multirow{2}{*}{ Sub-basin } & \multicolumn{5}{|c|}{ n (Back ward) } & \multicolumn{5}{|c|}{ Calibration } & \multicolumn{5}{|c|}{ Validation (Forward) } \\
\hline & $\mathbf{r}$ & $\beta$ & $\gamma$ & KGE & PBIAS & $\mathbf{r}$ & $\beta$ & $\gamma$ & KGI & & $\mathbf{r}$ & $\beta$ & $\gamma$ & KGE & PBIAS \\
\hline hitral & 0.95 & 1.23 & 0.93 & 6 & 1 & 0 . & 1.09 & 0 & & 000 & 0.93 & & 02 & 38 & 9.78 \\
\hline wat & 0.86 & 1.05 & 0.89 & 0.82 & 4.89 & & 0.85 & 0.89 & 79 & 15.14 & & & 02 & 78 & $-19.4 c$ \\
\hline 00 & 34 & 1.10 & 8 & 0.77 & 10.38 & $T$ & 6 & 15 & 76 & 16.41 & 0 & 1.07 & 36 & .79 & 6.60 \\
\hline & & & & 0.69 & 000 & & & & 0.76 & 170 & & & & 0.10 & 167 \\
\hline st & 0 & 1.38 & 0 & 0.60 & 38.01 & 0.91 & 1 & 0 & 79 & 17.68 & 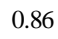 & 1.11 & 0.90 & 0.19 & 10.69 \\
\hline Silgit & 0.90 & 098 & 0.97 & 0.89 & -2.12 & 0.95 & 0.94 & 0.91 & 0.88 & -5.50 & 0.92 & 0. & 0.94 & 0.76 & -22.05 \\
\hline Hunza & 0.93 & 1.11 & 0 & 0.82 & 11.45 & 0. & 1.21 & 0 & 33 & 21.49 & 0.88 & 1. & 0.96 & 0.82 & 13.6 \\
\hline 111 & 0.86 & & 1 & 0 & 51.11 & & 1 & & & 0.2 & & & & 0 & -5.0 \\
\hline hy & 0.91 & 1.3 & 0.99 & 0.67 & 31.35 & 0.93 & 1. & & 0.75 & 23.00 & 0 & & & 0.80 & 17.0 \\
\hline Indus-K & 0.87 & 0.97 & 1.16 & 0.79 & -2.54 & 0.89 & 1.01 & 1.24 & 0.73 & 0.50 & 0.91 & 1.08 & 1.09 & 0.85 & 8.11 \\
\hline ndu & 0.91 & 0.9 & 0.97 & 0.89 & -5.16 & 0.95 & 1.00 & 0.95 & 0.93 & 0.1 & 0.95 & 0.96 & 0.95 & 0.92 & -4.1 \\
\hline ndus-T & 0.92 & 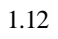 & 0.91 & 0.83 & 12.04 & 0.95 & 1.10 & 0.93 & 0.86 & 10.25 & 0.95 & 1.1 & 0.91 & 0.84 & $\begin{array}{l}41.77 \\
+\end{array}$ \\
\hline Thelum & 0.94 & 1.09 & 0.98 & 0.89 & 8.95 & 0.93 & 1.10 & 1.02 & 0.88 & 10.40 & 0.91 & 0.99 & 1.05 & 0.89 & -0.94 \\
\hline Chenab & 0.92 & 0.90 & 0.94 & 0.86 & -10.26 & 0.94 & 0.86 & 1.02 & 0.85 & -13.77 & 0.92 & 0.91 & 0.99 & 0.88 & -8.70 \\
\hline
\end{tabular}

\subsubsection{Changes and variability in water availability}

In order to emphasize and maintain the focus of hydrological assessment at regional scale, we limit our analysis to four downstream river gauging stations (Jhelum-Mangla, ChenabMarala, Kabul-Nowshera \& Indus-Tarbela). Historical median annual river inflows at these four locations in the study domain remained highly variable and experienced significant decline during the $2^{\text {nd }}$ half of baseline period as compared to its $1^{\text {st }}$ half (Figure 5.7). This interannual variability and decline in median annual river inflows during the $2^{\text {nd }}$ half of baseline period is largely responsive to the similar trends in annual precipitation. The maximum inflows in the W-Himalayan basins (Jhelum and Chenab) were about three-times higher than the minimum inflows. A reduction of $19.6 \%$ and $11.9 \%$ is observed for Jhelum and Chenab rivers respectively during the $2^{\text {nd }}$ half of the baseline period as compared to its $1^{\text {st }}$ half. The Kabul river in the Hindukush region also exhibited similar interannual variability of median annual river inflows and difference between the maximum and minimum inflows. 
However, the $2^{\text {nd }}$ half median river inflows fell short by only $4.5 \%$ as compared to the $1^{\text {st }}$ half. The Indus-Tarbela rim station receiving bulk of its inflows from the Karakoram region experienced relatively stable river inflows as maximum annual inflows were 1.7-times higher than the minimum inflows. Tarbela inflows also declined by $4.9 \%$ during the $2^{\text {nd }}$ half of the last 40 years, but a modest increasing trend is observed during this period.
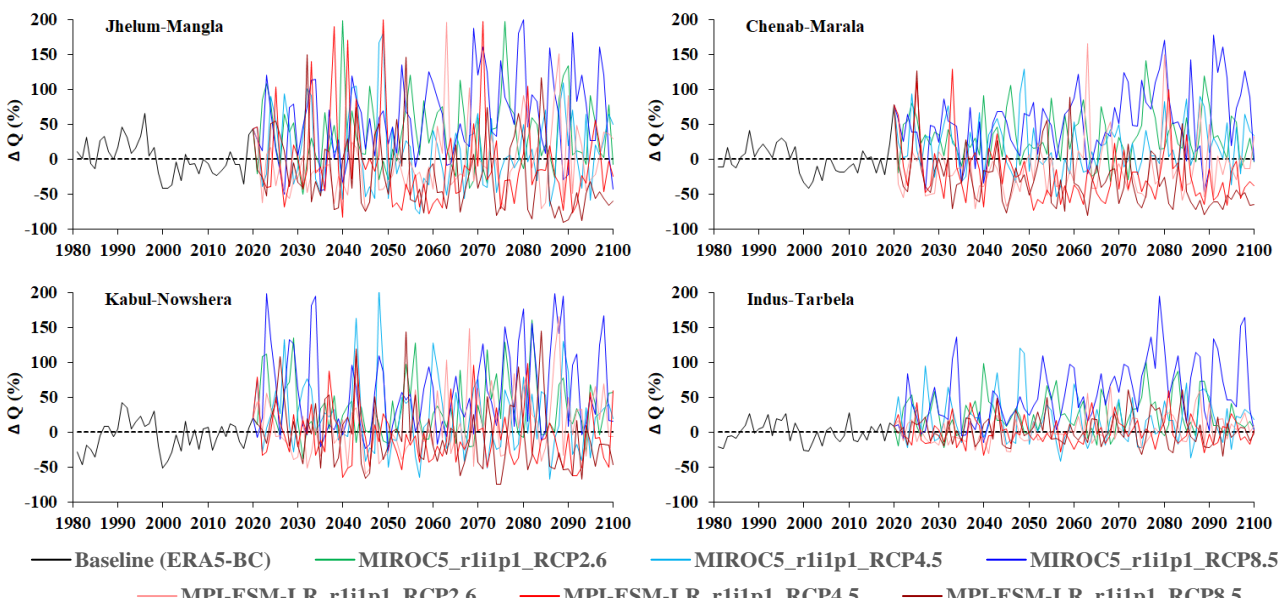

Figure 5.7: Projected median annual river discharge anomalies relative to baseline at four outlets

Stronger interannual variability of the projected river inflows during $21^{\text {st }}$ century is anticipated under all future scenarios as compared to the baseline historical period at all the four locations. MIROC5 projects pronounced increases in median annual river flows under all RCPs, while MPI-ESM-LR suggests substantial reductions under RCP4.5\&8.5. Like median annual precipitation, a definite and strong increasing trend of median annual river inflows is only evident for MIROC5 under RCP8.5 at all locations, while MPI-ESM-LR shows declining trends for RCP4.5 \& RCP8.5 at Jhelum, Chenab and Kabul river gauges. The remaining GCM runs show slightly increasing trends. Tarbela inflows show the strongest increasing trends under all scenarios except MPI-ESM-LR_RCP4.5\&8.5, which suggest almost no trend.

Median annual river inflows of the Jhelum river at Mangla rim station are anticipated to increase by $29.2,14.0 \& 55.7 \%$ during mid-century and by $26.2,12.4 \& 71.7 \%$ during endcentury time-slices under MIROC5 ensembles (RCP2.6, $4.5 \& 8.5$ ) respectively (Table 5.6), while MPI-ESM-LR projects reductions of $-22.1,-16.5,-15.1$ and $-1.2,-17.7,-47.4 \%$ under the same scenarios. Almost similar trends but slightly different magnitudes in the changes in median annual river inflows of Chenab river at Marala rim station can be expected. Under extreme dry (wet) conditions, the largest reductions (gains) of $-51.3 \%$ (73.6\%) in median annual river inflows can be expected at Chenab-Marala (Indus-Tarbela) rim station during end-century time-slice. Indus-Tarbela inflows are anticipated to increase at varying levels under all scenarios, while Kabul-Nowshera inflows may increase under MIROC5 ensembles and decrease under MPI-ESM-LR ensembles except under RCP2.6 by the end of century.

Monthly scale river inflows are more variable and uncertain particularly in the monsoon dominated W-Himalayan river basins. The Jhelum-Mangla river inflows show large reductions under all ensembles of MPI-ESM-LR throughout the year during the entire $21^{\text {st }}$ century except slight increases in Apr-Jun inflows for RCP2.6 during end-century time-slice. 
Similarly, Chenab-Marala inflows also depict large reductions throughout the year but considerable increases are prominent during Apr-June months for both the time-slices. Under extreme wet-warm scenario, the MIROC5 ensembles project moderate to high increases in most of the monthly river inflows except Feb-Mar months under RCP4.5 and Jul-Aug months under RCP4.5\&8.5 during the entire $21^{\text {st }}$ century.

Table 5.6. Projected changes (\%) in median monthly and annual river flows at 4 locations by 2 GCMs under 3 RCPs during 2021-2060 \& 2061-2100 time-slices relative to baseline period (1981-2020). The red \& blue colour stretches highlight the magnitudes of negative $\&$ positive changes respectively.

\begin{tabular}{|c|c|c|c|c|c|c|c|c|c|c|c|c|c|c|c|c|c|c|c|c|c|c|c|c|c|c|}
\hline J & $\mathbf{F}$ & IM & A & 10 & $\mathbf{J}$ & J & A & $\mathbf{S}$ & & & & & & & & & & & & & & & & & & \\
\hline \multicolumn{13}{|c|}{ 2021-2060 } & Jhe & \multicolumn{13}{|c|}{$2061-2100$} \\
\hline 45.5 & & & & & -2.2 & 5.1 & 10.2 & & 3 & & & & & 3.7 & .5 & 9 & 17.6 & & 9.5 & 17.5 & 3.3 & & & & & \\
\hline & 8.5 & & & .4 & -35.5 & & & -26.1 & & & & -22.1 & & & & & 5.5 & & ( & & & & & & & 1.2 \\
\hline 1.2 & -4.6 & 7.6 & -8.0 & 5.3 & 4.4 & -22.0 & -14.7 & 80 & & & & & & & & .3 & 4.3 & .3 & & & & & & 0.3 & & 2 \\
\hline 9.5 & & -39.5 & & 6.8 & -28.9 & & & & & & & & & & & & & & - & & & & 1.3 & 0.0 & & \\
\hline 5.9 & & & & & & & & & & & & & & 9 & & & & 2.4 & -16.9 & -1 & & & 0.6 & & & \\
\hline & & & & & & & & & & & & & & & & & & & & & & & & & & \\
\hline \multicolumn{13}{|c|}{ 2021-2060 } & & \multicolumn{13}{|c|}{ 2061-2100 } \\
\hline & & & & & 5.8 & 6 & 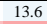 & & & & & & & & & & .5 & 7.9 & 41.7 & 3.1 & & & & & & \\
\hline-35.8 & & & & & & -43.1 & & & -42.0 & & & & & & & & 0.0 & 5.5 & 7.0 & -26.6 & & 2 & & .3 & & -13 \\
\hline 12.5 & & & & & & & & & & & & & & & & & & .6 & 5 & & 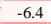 & & & & & 4 \\
\hline 6.0 & -3 & -44 & & & 5.5 & . & -61.8 & -56.1 & -44 & -3 & & $3-2$ & & & & & & 7.6 & -38.7 & -56.4 & 6.1 & - & 1 & . & & 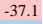 \\
\hline 36.3 & & & & & 0.3 & & & 3 & & & & & & & & & 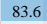 & 2.5 & 66.5 & -7.8 & 1.1 & 34.8 & 5.5 & 1 & & 2. \\
\hline & & & & & 3 & -57.3 & -64 & & & & & & 1 & & & & & & 6.6 & -74.9 & -77.4 & & & & & \\
\hline \multicolumn{13}{|c|}{2021} & & \multicolumn{13}{|c|}{206} \\
\hline 35.6 & & & & & 32.2 & & & & & & & & & & & & & & 38.8 & & & & & & & 8 \\
\hline-4 & & -2 & & & & & & & & & & & & & & & & & ${ }^{\circ}$ & & & & & & & 9 \\
\hline-5.0 & 2 & 47.4 & & & 22.7 & 2 & -12.8 & & & & & & & & 1 & 3.7 & & 9.6 & 4.9 & & & & & & & 20. \\
\hline 2.0 & -28 & -44.7 & -33.2 & -2 & 7.6 & & -1.3 & & 5 & -11 & & & & .1 & 3.2 & -46.1 & 7.0 & 2.8 & -8.5 & .5 & 1.2 & & 1.4 & 3.9 & 4.6 & -11 \\
\hline 31.2 & 4 & 77.0 & & & 40.2 & 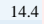 & & & & & 35. & & & & & & .1 & & 5.4 & 1.0 & 2.6 & 2.3 & 178.7 & 8.6 & 2.1 & 50 \\
\hline & & 29 & & & -1.8 & & 11 & & & & 61 & & & & & & & & -29.2 & & 5 & & & & & \\
\hline \multicolumn{13}{|c|}{ 2021-2060 } & & \multicolumn{13}{|c|}{ 2061-2100 } \\
\hline 20.5 & & & & & & & & & & & & & & & & & & & 50.7 & 80 & & & & & & 17. \\
\hline-5.6 & -17.0 & -18.8 & 3. & & 2.1 & 4.3 & -20.8 & -6.4 & 1.5 & -5.5 & -0 & .3 & & 1.0 & & 3 & .7 & 3 & 36.6 & 8.1 & -2.9 & 5.1 & & & & \\
\hline-2.1 & & 5.1 & & & 3.6 & 4.7 & -0.9 & 6.5 & -1.6 & -7.8 & -6.6 & & & .0 & & & & & 54.0 & 24.8 & & & & & .7 & 19. \\
\hline-8 . & & -19 & & & & & -14 & 6.5 & 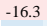 & -16 & & & & & & & & 47.1 & 30.8 & & & & & 9.9 & & \\
\hline & & & & & & & & & & & & & & & & & & & a & & & & & & & \\
\hline $2 \pi$ & & & & & 9 & & & & & & & & & & & & & & 40.3 & & & & 50 & -3.7 & 6 & \\
\hline
\end{tabular}

Indus-Tarbela on the other side show considerable gains in future river inflows for most of the moths under all scenarios, particularly for Apr-Jul months during both the time-slices and except Jul-Aug flows under RCP4.5 during end-century time-slice. Median monthly river inflows of the Kabul river at Nowshera gauging station may experience significant reductions under MPI-ESM-LR ensembles except Jun-Jul months during mid-century time-slice and Jul month under RCP4.5 during end-century time-slice, while RCP2.6 may result in increased river inflows throughout the year except Feb-Mar months during the end-century time-slice. MIROC5 ensembles on the other hand predict modest increases in median monthly river inflows throughout the year except Aug-Sep during mid-century time-slice. However, for end-century time-slice, slightly reduced inflows can be expected during Aug-Sep under RCP2.6 and Jun-Oct under RCP4.5.

\subsubsection{Shifts in seasonality and annual cycle of water availability}

As a result of future climate change, large changes and shifts in the seasonality and annual cycle of hydrological peaks are expected for all the four rim stations under different scenarios (Figure 5.8). The peaks are mainly affected by intensity and duration of precipitation, snow/glacial ice reserves and the amount of energy available for melting of seasonal snow and perennial glaciers. The hydrographs of median monthly river discharges projected by two GCMs under three RCPs indicate that the study area has distinct climate and associated hydrological regime at regional or sub-basin scale. The highest peaks are generally achieved during summer months when monsoon precipitation is coincided with snowmelt runoff and the lowest flows are attained during winter season when most of the precipitation falls in solid form and baseflows are mainly augmented by groundwater. 

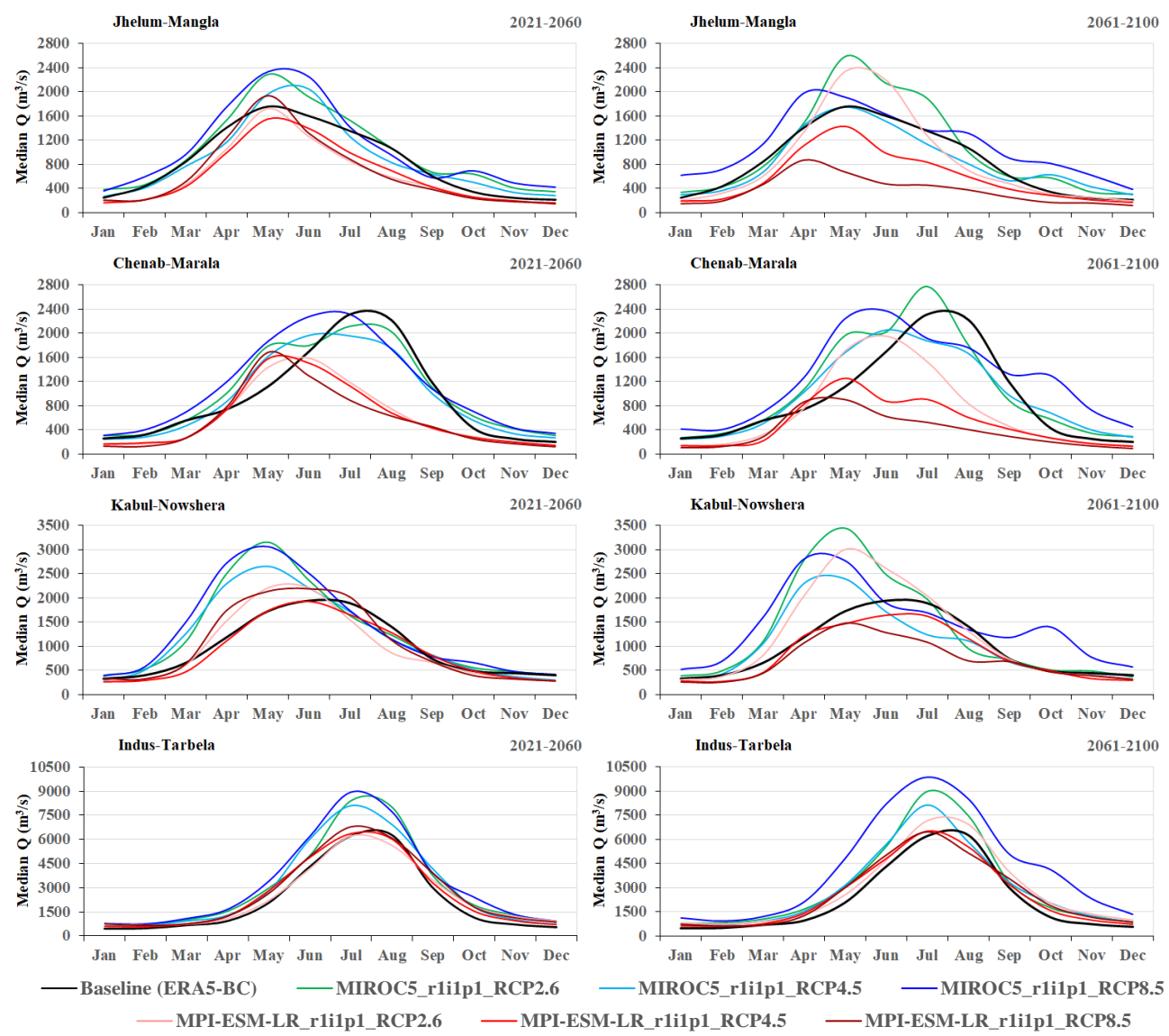

Figure 5.8: Shifts in seasonality and annual cycle of hydrological peaks.

For the Jhelum-Mangla river gauge during mid-century time-slice, the highest peak flow tends to shift from Jun to May under all ensemble scenarios except MIROC5-RCP4.5, which maintains the peak in Jun as per historical period. There are large differences in the magnitudes of peaks, with ample underestimates by the MPI-ESM-LR ensembles and vice versa by the MIROC5 ensembles. The wintertime lowest discharges representing baseflow however, are affected differently, with increasing levels by MIROC5 ensembles and further lowering by MPI-ESM-LR ensembles. The situation becomes highly uncertain during the end-century time-slice, when MPI-ESM-LR-4.5 \& 8.5 depict extreme reduction of river flows throughout the year and flattening of the peaks. MIROC5-RCP8.5 shows shifting of peak from Jun to Apr and substantial dipping of Jun-Jul flows, while MIROC5-RCP2.6 and MPI-ESM-LR-RCP2.6 project about 38.8 and $25.2 \%$ increases in peak flows in May thereby alerting for increased floods during this month. Almost similar trends in attainment of peak flows can be noticed for Chenab-Marala rim station. Significant increases in Apr-Jun flows are projected by MIROC5 ensembles with shifting of peaks from Jul to Jun, whereas MPIESM-LR ensembles show massive reductions in monsoon (Jul-Sep) flows for both the timeslices. Peak flows of Kabul-Nowshera river gauge are anticipated to slightly shift from late May to early May under MIROC5 ensembles with substantial and certain increases in spring and pre-monsoon (Feb-Jun) river inflows during both the time-slices. Indus-Tarbela inflows generally attain peak during mid Aug, which is very likely to be shifted to mid Jul under all 
ensemble scenarios. Significant increases in monthly peaks during Apr-Jul are also evident under all scenarios. The baseflow during winter is slightly increased under MIROC5 ensembles and vice versa under MPI-ESM-LR ensembles. An increase of 28.3, $16.0 \& 41.3 \%$ over the baseline peak flow level can be anticipated under RCP2.6, $4.5 \& 8.5$ of MIROC5 by the end-century time-slice.

\subsubsection{Changes in future hydrological extremes}

The changes in future hydrological extremes are assessed by analysing changes in the flow duration curves (FDCs) and extreme discharge levels of high flows (Q5 \& Q10) and low flows (Q90 \& Q95) at the four river discharge gauges. The FDCs presented in Figure 5.9 represent the percentage of time for baseline historical and two future time-slices of GCM outputs during which the river flows are equal to or greater than the corresponding rates. Flow duration curves have many hydrological and water management applications and are particularly helpful for design of hydropower projects. The high flow and low flow durations provide essential data and information for optimal selection and design of minimum and maximum hydropower units. Persistence and intensity of high and low flow periods (floods and droughts) can also be determined through the flow duration curves.
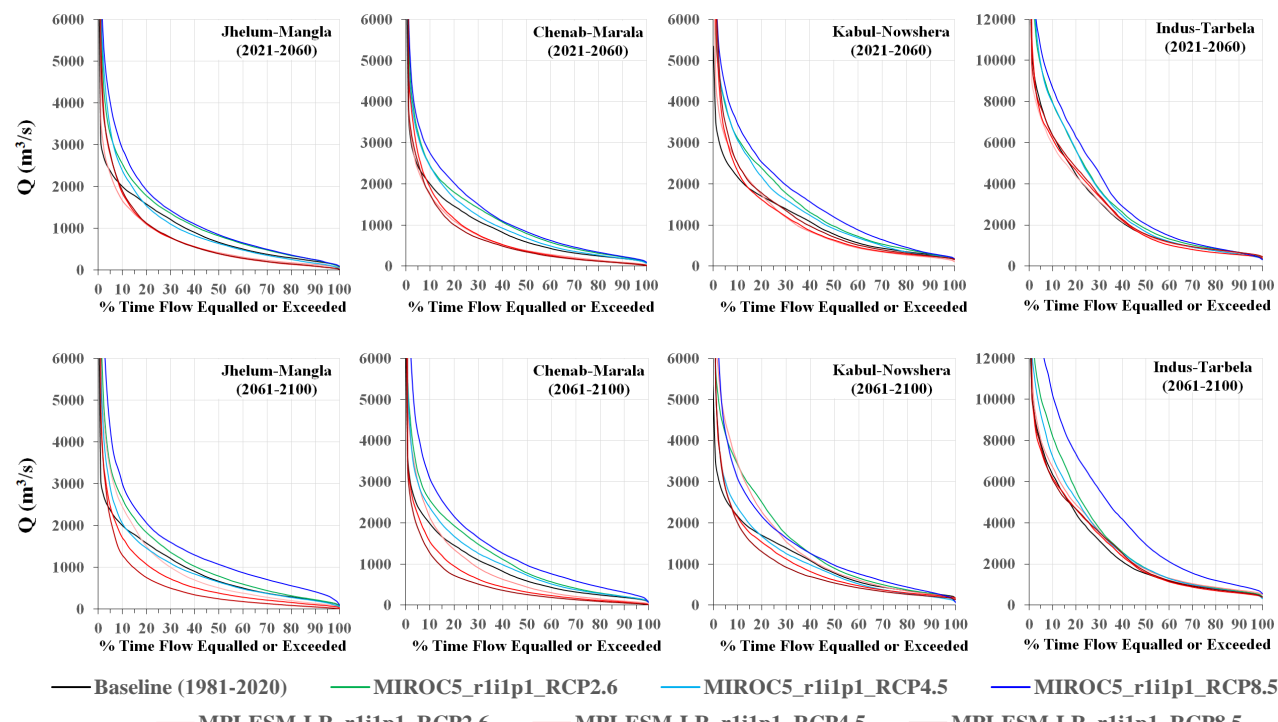

- MPI-ESM-LR_r1i1p1_RCP2.6

-MPI-ESM-LR_r1i1p1_RCP4.5

—MPI-ESM-LR_r1i1p1_RCP8.5

Figure 5.9: Flow duration curves for the four sub-basins during historical and future time-slice.

Figure 5.9 indicates considerable variability and shift in the FDC of each considered watershed under different scenarios of future climate. The FDCs are further analysed and quantified in terms of relative changes in the extreme discharge levels of high flows and low flows with respect to the baseline scenario at four river gauges (Table 5.7). Positive signs indicate increases, while negative signs infer reductions in the projected high or low flows. Most of the ensembles show increasing high flows and decreasing low flows implying intensification of future hydrological extremes. High flows are projected to increase at all gauging stations under all scenarios. The exceptions are MPI-ESM-LR-RCP4.5 for IndusTarbela and MPI-ESM-LR-RCP8.5 for Jhelum-Mangla, Chenab-Marla and Indus-Tarbela gauging stations. MIROC5-RCP8.5 infers the most significant and consistent increase in high 
flows, while MIROC5-RCP2.6 also shows similar pattern and consistency of increase in high flows but at much lower magnitudes. The low flows on the other hand are very likely to decrease further under MPI-ESM-LR ensembles except RCP2.6 \& RCP8.5 in the IndusTarbela watershed. Whereas, MIROC5 ensembles depict increasing lows flows except RCP4.5 in Jhelum-Marala, Kabul-Nowshera and Indus-Tarbela watersheds. Hence, more intensive floods are more likely and certain than droughts.

Table 5.7: Projected changes (\%) in high flow (Q5 and Q10) and low flow (Q90 and Q95) durations under various climate change scenarios for 2021-2060 and 2061-2100 relative to 1981-2020.

\begin{tabular}{|c|c|c|c|c|c|c|c|c|}
\hline \multirow{2}{*}{ GCM / RCP } & \multicolumn{2}{|c|}{ 2021-2060 } & \multicolumn{2}{|c|}{ 2061-2100 } & \multicolumn{2}{|c|}{ 2021-2060 } & \multicolumn{2}{|c|}{ 2061-2100 } \\
\hline & Q5 & Q10 & Q5 & Q10 & Q90 & Q95 & Q90 & Q95 \\
\hline \multicolumn{9}{|c|}{ Jhelum-Mangla } \\
\hline MIROC5_RCP2.6 & 36.8 & 27.2 & 44.3 & 32.2 & 17.7 & 5.7 & 6.6 & 3.9 \\
\hline MPI-ESM-LR_RCP2.6 & -3.3 & -17.1 & 40.1 & 22.6 & -39.1 & -39.3 & -32.1 & -32.5 \\
\hline MIROC5_RCP4.5 & 42.2 & 19.1 & 21.8 & 2.6 & -20.1 & -24.8 & -5.1 & -10.4 \\
\hline MPI-ESM-LR_RCP4.5 & 15.9 & -8.2 & 3.3 & -14.9 & -54.6 & -60.7 & -51.3 & -53.3 \\
\hline MIROC5_RCP8.5 & 68.3 & 45.0 & 80.6 & 50.0 & 17.8 & 5.0 & 98.3 & 92.4 \\
\hline MPI-ESM-LR_RCP8.5 & 17.6 & -4.9 & -8.9 & -35.2 & -52.0 & -58.9 & -71.6 & -76.0 \\
\hline \multicolumn{9}{|c|}{ Chenab-Marala } \\
\hline MIROC5_RCP2.6 & 34.3 & 22.7 & 35.5 & 28.6 & 24.4 & 21.5 & 11.6 & 9.9 \\
\hline MPI-ESM-LR_RCP2.6 & -3.5 & -13.8 & 26.2 & 7.6 & -49.3 & -52.5 & -48.7 & -51.9 \\
\hline MIROC5_RCP4.5 & 30.3 & 21.7 & 25.7 & 20.0 & 7.6 & 6.2 & 16.2 & 13.9 \\
\hline MPI-ESM-LR_RCP4.5 & 12.8 & -3.9 & -7.4 & -21.6 & -55.6 & -62.1 & -56.3 & -63.5 \\
\hline MIROC5_RCP8.5 & 44.0 & 37.8 & 75.7 & 55.7 & 28.7 & 25.3 & 71.0 & 61.6 \\
\hline MPI-ESM-LR_RCP8.5 & 3.9 & -12.7 & -21.1 & -35.5 & -58.0 & -63.0 & -67.7 & -71.1 \\
\hline \multicolumn{9}{|c|}{ Kabul-Nowshera } \\
\hline MIROC5_RCP2.6 & 50.3 & 43.4 & 61.5 & 58.1 & 4.2 & -5.5 & 0.2 & -5.3 \\
\hline MPI-ESM-LR_RCP2.6 & 19.9 & 15.3 & 72.6 & 59.7 & -20.8 & -23.7 & -8.3 & -9.7 \\
\hline MIROC5_RCP4.5 & 53.2 & 41.3 & 17.0 & 9.9 & -6.2 & -5.8 & -24.7 & -31.9 \\
\hline MPI-ESM-LR_RCP4.5 & 23.2 & 6.7 & 10.6 & -1.3 & -15.1 & -12.9 & -14.4 & -14.4 \\
\hline MIROC5_RCP8.5 & 67.5 & 59.4 & 63.5 & 42.6 & 8.3 & 2.3 & 10.8 & -3.4 \\
\hline MPI-ESM-LR_RCP8.5 & 33.6 & 15.3 & 10.6 & -7.5 & -12.1 & -14.9 & -24.1 & -23.9 \\
\hline \multicolumn{9}{|c|}{ Indus-Tarbela } \\
\hline MIROC5_RCP2.6 & 24.0 & 25.7 & 28.7 & 29.6 & 9.5 & 9.2 & 11.5 & 12.1 \\
\hline MPI-ESM-LR_RCP2.6 & -7.9 & -6.7 & 2.6 & 6.1 & 4.3 & 7.2 & 18.0 & 21.7 \\
\hline MIROC5_RCP4.5 & 25.1 & 25.1 & 18.1 & 13.9 & -5.5 & -7.4 & 3.2 & -0.9 \\
\hline MPI-ESM-LR_RCP4.5 & -6.6 & -2.5 & -3.8 & -2.2 & -12.3 & -9.8 & -4.0 & -3.6 \\
\hline MIROC5_RCP8.5 & 34.8 & 36.8 & 64.3 & 61.2 & 8.9 & 0.5 & 53.4 & 50.8 \\
\hline MPI-ESM-LR_RCP8.5 & -1.5 & 0.4 & -1.4 & -4.1 & 6.8 & 7.5 & 0.3 & 1.0 \\
\hline
\end{tabular}

\subsection{Discussion}

Hydrometeorological assessments by the previous studies for the high-altitude Indus basin mostly relied on highly uncertain climate data and use of lumped and/or temperature-index based hydrological models. This study, however, has developed high-quality precipitation and temperature datasets for the historical (Section 5.3.1) and future (Section 5.3.2) periods. A fully-distributed, physically-based, energy-balance hydrological model is forced with these improved datasets to simulate the changes and variability in the sub-basin scale hydrometeorological regimes of the study area for the baseline historical period and plausible conditions of extreme climate (cold-dry \& warm-wet) covering a wider range of future climate scenarios represented by RCPs 2.6, $4.5 \& 8.5$.

\subsubsection{Climate change}

Accurate assessments of climate change in the high-altitude Indus basin have remained daunting task due to highly scarce in situ observations and strong influence of the innately 
complex climate systems interacting with very high orographic features. For instance, the coupled influence of very high orography, winter westerlies, Indian summer monsoon, EastAsian summer monsoon, and significant moisture released by the vast irrigated plains and forest lands in the Himalayan foothills may be inadequately captured by the global climate models. As such, there is large variability in quantitative and spatio-temporal distribution of the projected precipitation by various GCM outputs (Lutz et al., 2016b; Cai et al., 2009). This is confirmed by evaluating the precipitation estimates of 75 GCM runs in this study. None of the GCM run was able to precisely capture the influence of predominant weather systems resulting in large variability and biases in their monthly, seasonal and annual precipitation estimates (Table 5.1). Precipitation distribution estimates of only a few runs were close and comparable to the reference data. Hence, the results of previous studies that have used different GCM outputs and compared with uncertain reference data may be completely different. The existing gridded precipitation products also suffer from observational and other inadequacies and errors (Dahri et al. 2020; Baudouin et al. 2020) leading to large uncertainties and poor understanding of the prevailing hydrometeorological regimes in the study domain (Lutz et al., 2016a).

Lutz et al. (2018) and Kraaijenbrink et al. (2017) showed that the climate in this region is changing at a rate greater than the global average. Whereas, a multi-model study conducted by Su et al. (2016) revealed increasing annual and summer temperatures, increasing monsoon precipitation, and decreasing winter and spring precipitation. Our analysis depicts significant interannual variability and increase (decrease) of median annual air temperature (precipitation) by $0.6^{\circ} \mathrm{C}(11.9 \%)$ in the study area during the last 20 years (2001-2020) relative to the preceding 20 years (1981-2000). Future climate may be more variable in space and time under each scenario. MIROC5 output is considerably warmer than MPI-ESM-LR output almost throughout the year and about $1.2,1.0 \& 0.35^{\circ} \mathrm{C}$ warmer by the end of century under RCPs2.6, 4.5 \& 8.5 respectively. Precipitation changes in most parts of the basin at annual scale and during most of the months are generally positive for MIROC5 and negative for MPI-ESM-LR except Karakoram and western part of Kharmong regions, where both GCMs show positive changes. Monsoon precipitation may get stronger in the IndusKharmong region, which might be due to stronger influence of Tibetan Anticyclone and EastAsian monsoon in the future (Zhu et al., 2013; Wang et al., 2008). Farinotti et al. (2020) concluded that in the past two decades, the Karakoram region has shown balanced to slightly positive glacier budgets, an increase in glacier ice flow speeds, stable to partially advancing glacier termini and widespread glacier surge activity. The authors however were unclear and uncertain about the future of this Karakoram Anomaly, and termed its long-term sustainability unlikely in light of the anticipated warming of future climate. Our study can explain the future of peculiarly anomalous behavior of glacier activity in the Karakoram region. It shows strong signals of considerable precipitation gains even under extreme dry and warm conditions of future climate. Of particular importance is substantial increases in future precipitation during winter and summer seasons, and probably the accompanying cloud cover and reduced sublimation. The projected increases in temperature may only result in slightly upward shift of the snowline. The melting of this transition zone and gains in the accumulation zones will actually determine the net loss of glacial ice mass.

Precipitation projections for the Kabul river basin (SW-Hindukush and NE-Hindukush) are extremely variable at both monthly and annual scales and show largest reductions during JunAug and Nov-Dec months. Similar to the findings of Hasson et al. (2019), an overall increase in median annual precipitation is projected by MIROC5 ensembles, while MPI-ESM-LR ensembles show declining trend which supports the findings of Lutz et al. (2016a). This is in 
contrast to the findings of Bokhari et al. (2018), who examined the future climatic changes in the Kabul River basin using multi-model ensemble mean derived from NASA Earth Exchange Global Daily Downscaled Projections (NEX-GDDP) taking Global Meteorological Forcing Dataset (GMFD) as a reference and noticed an overall decrease in mean annual precipitation over the entire Kabul river basin under RCP4.5 \& RCP8.5. The influence of Indian summer monsoon seems to be weakened in future and may seldom reach to extreme SW-Hindukush region. W-Himalayan precipitation may also experience similar variability and uncertainty as MPI-ESM-LR projects significant reductions, while MIROC5 suggests substantial increases. Hasson et al. (2019) also project slight increase particularly along the northern high mountain ranges. Lutz et al. (2016a) however predicted slight (considerable) reductions (gains) in median annual precipitation for RCP4.5 (RCP8.5), which is amplified by the use of different models for the two RCPs. The contrasting precipitation estimates of the previous studies relative to this study can be attributed to use of different datasets for projection as well as baseline reference. Moreover, our projections primarily represent extreme scenarios conditions rather than median conditions.

\subsubsection{Hydrological regime}

Numerous studies have attempted to assess and model the hydrological regime of highaltitude Indus basin (e.g. Hasson et al., 2019 \& 2016; Lutz et al., 2016a; Archer, 2003). Yet, there are significant uncertainties and our understanding of the basin's hydrological regime remains poor. The greatest uncertainties are associated with the use of non-representative (mostly underestimated) precipitation in the higher altitude areas, which receive bulk of precipitation and have major contributions in streamflows. The basin is predominantly a snow and glacier fed system. Therefore, the temperature-index or degree-day based hydrological models may not accurately simulate the prevailing energy balance, which is the main driving force for streamflow generation from the snow and glacier systems. Whereas, assessment of the future hydrological regime is primarily constrained by very high uncertainties in the GCM outputs for the study domain.

This study used improved climate datasets and employed a fully-distributed physically-based energy-balance hydrological model to investigate hydrological regime of the high-altitude Indus basin at four strategic river gauging stations under extreme climate change scenarios. The existing hydrological regime of the high-altitude Indus basin is largely modulated by timing, intensity, duration, and form of precipitation; snow and glacial ice reserves; and amount of energy available for melting of seasonal and perennial snow and glacial ice (Archer, 2003). Hence, the seasonal pattern of river inflows is significantly modified by the melting of snow and glaciers. However, climate change is expected to weaken the modulating effect of snow and glacier fields with potentially strong effects on hydropower generation, floods and droughts, irrigation water supplies and associated food production (Biemans et al., 2019). Future projections by two GCMs under three RCPs indicate that the study area has distinct climate and associated hydrological regime at regional or sub-basin scale. Unprecedented and highly contrasting hydrological signals across the river basin at the four gauging station are anticipated under these scenarios.

The Jhelum-Mangla river inflows show shifting of peak from Jun to May and large reductions under all ensembles of MPI-ESM-LR throughout the year except slight increases in Apr-Jun inflows for RCP2.6, while MIROC5 ensembles project substantial increases in most of the monthly river inflows except Feb-Mar under RCP4.5 and Jul-Aug under RCP4.5\&8.5. Large reductions in low-flows are also prominent in MPI-ESM-LR ensembles and MIROC5RCP4.5. Such changes in Jhelum-Mangla river inflows are in sheer contrast with the findings 
of Hasson et al. (2019), who anticipated no shifting of peak flows and around $10 \%$ decrease (increase) in Mar-Jun (Jul-Sep) inflows with overall decrease of $3 \%$ under $1.5{ }^{\circ} \mathrm{C}$ and no change under $2.0^{\circ} \mathrm{C}$ global warmings. Strong decreases in Jun-Jul inflows are projected by Lutz et al. (2016a). Similarly, Chenab-Marala inflows also depict large reductions (increases) under MPI-ESM-LR (MIROC5) ensembles throughout the year but considerable increases are anticipated during Apr-June months under all scenarios. Shifting of peak from Jul to Jun can be expected and drastic reductions in low-flows are prominent under MPI-ESM-LR ensembles. Lutz et al., (2016a), however projected considerable reductions under RCP4.5 and vice versa under RCP8.5 with no shift in peak. Indus-Tarbela inflows are projected to significantly increase during Apr-Jul months with overall annual increase of 17.0-73.6\% under MIROC5 ensembles and 1.2-9.7\% under MPI-ESM-LR ensembles. Lutz et al. (2016a) on the other hand show slight increases for the upstream Karakoram river inflows but no change at the downstream Tarbela gauging station against 38\% and 52\% increase anticipated by Hasson et al. (2019). They also expected no shift in peak, which is likely to be shifted from Aug to Jul in this study. The lower altitude Kabul river basin is expected to experience an intermediate pattern of seasonal shifts, with slight reductions in river inflows under MPIESM-LR ensembles and modest increases (20.3-50.3\%) depicted by MIROC5 ensembles. Aug-Sep river flows may get slight decline and there is also a slight shift in Peak from end of May to start of May. Substantial increases in spring and pre-monsoon (Feb-Jun) river inflows however are certain. These findings are contrasted by Hasson et al., (2019) with projected increase of about $60 \%$ in annual flows and unchanged peaks. Lutz et al., (2016a) on the other hand expected strong decreases in Jun-Jul flows and slight decreases spring months, with overall slight decrease in annual flows and no change in attainment of peak. Most of the ensembles show increasing high flows and decreasing low flows at all gauging stations implying intensification of future hydrological extremes.

\subsubsection{Uncertainties and limitations}

Observational uncertainties: This study developed and used much improved precipitation and temperature datasets and employed state-of-the-art interpolation technique to minimize observational uncertainties that are commonly present in the previous datasets. Yet, the observational uncertainties in the hydrometeorological assessment undertaken in this study may still hold a sizable share in the total uncertainties due to imprecision in the observed data and techniques used to adjust measurement errors and spatial interpolation of the point observations. These observational uncertainties can be addressed by incorporating additional observed data with better spatio-temporal coverage particularly in higher-altitude areas, calibration of the precipitation gauges with the WMO recommended references and development of site and gauge-specific error adjustment models, use of daily or even subdaily time steps, use of corresponding observed wind speed and temperature data, selection of better spatial interpolation technique, precise computation of other components of the water balance to validate precipitation, and a better integration of the observed precipitation data with mass balance and remote sensing data.

GCM structure and parameterization: GCM related uncertainties arise mainly from formulation and discretization of the governing mathematical models, from parameterization of unresolved processes, and from imperfect understanding of the climate system (Palmer et al., 2005). Since each GCM is developed based on its own specific assumptions and unique mathematical models to represent the physical processes of climate systems, their outcomes are innately different (Hawkins and Sutton, 2011). Hence, with availability of a large number of GCM outputs, the spread and variability in their outcomes is also large (Lutz et al., 2016b), 
particularly at regional and catchment scale (Cai et al., 2009). GCM related uncertainty is often a dominating source (Kay et al., 2009) constating about 50-85\% of the total uncertainty (Prein et al., 2011), and is generally more pronounced at longer timescales (Hawkins and Sutton, 2011). Parameterization related uncertainty is much smaller than GCM related uncertainty (Her et al., (2019). Performance evaluation of 75 GCM runs undertaken in this study revealed a large variability in precipitation estimates of the GCM projections. Hence, future water availability resulting from GCM precipitation in the high-altitude Indus basin will be even more uncertain.

Statistical downscaling and bias-correction: Statistical downscaling establishes empirical relationships between coarser-scale historical outputs of gridded datasets or GCMs and local climatic observations. These relationships are then applied to the GCM projections to correct their systematic biases. A wide variety of bias-correction methods are currently under use, but all methods are based on the fundamental assumption that the empirical relationship derived from the present climate conditions is also valid for the future scenarios (Dobler et al., 2012; Wilby et al., 2004; Zorita and von Storch, 1999). This assumption of stationarity is the fundamental limitation of this approach but often neglected in hydrological studies. This study used the delta change approach for bias correction of gridded dataset and precipitation and temperature outputs of two GCMs under three RCPs. The delta change approach uses linear scaling to perfectly match the monthly means of estimated climate variables with those of the observed values (Lenderink et al., 2007). This approach is widely used to assess climate change and future water availability, while more advanced methods are preferred for analysis of climate and hydrological extremes (e.g. Lutz et al., 2016a). However, Dobler et al. (2012) and Key et al. (2009) noticed comparatively small uncertainty related to the choice of the bias-correction method.

Hydrological modeling structure and parameterization: Ragettli et al., (2013) showed that the effects of uncertainty sources are variable in space and time and hydrological model input parametric uncertainty in heavily glacierized subregions exceeds the effect of climate model uncertainty and natural internal climate variability, but often decreases with time. The accuracy of the model outcome is largely dependent on the quality of input variables and modeling structure to represent the natural processes. The VIC hydrological model used in this study is validated and extensively used in a variety of conditions and there is no serious drawback in its modeling structure. Its characteristics of physically-based, fully-distributed, energy-balance, and representation of a wide range of physical processes are particularly important. However, the model also suffers from a couple of limitations to precisely model the glacierized catchments. Firstly, it does not take into account the already stored volume of snow and glacial ice mass, and then it simulates snow and glaciers using the same mathematical model. While, glaciers possess considerably different hydraulic properties.

Uncertainties in river flow measurements: River flows usually have indirect use in hydrometeorological studies. Primary use of river flow data is for calibration and validation of the hydrological models at the strategic river gauges. In contrast to most climatic variables, river flow data are generally assumed to be adequately accurate considering the relative precision of discharge measurement techniques and quality control ensured by the data collection agencies. Yet, river flow data may still be subject to some degree of uncertainty due to measurement errors. NESPAK-AHT-DELTARES (2015) assessed river/canal discharge measurement protocols adopted in the IRS and observed overall uncertainties in the rage of 3-8\% at five canal headworks. The river flow uncertainties may slightly vary at each gauge but will remain constant with time. 


\subsubsection{Implications for water management}

The complex hydrology and transboundary nature of the high-altitude Indus basin coupled with significant impacts of climate change highlight serious threats to the basin's integrity and sustainability of the future hydrological regime. The analysis undertaken in this study focuses two best performing GCMs projecting extreme conditions of warm-wet and cold-dry climates under three RCPs. As such, there are significant differences in the magnitudes and trends of future river inflows mainly due to differences in the GCM outputs. The projected changes in monthly, interannual and overall water availability at the four rim stations under different climate change scenarios would have serious implications for future water resources planning, development and management. Strategies and action plans for hydropower generation, construction and operation of storage reservoirs, irrigation withdrawals, flood control and drought management will require significant modifications.

In the presence of significant monthly and interannual variability, overall water availability is projected to improve in future, particularly at Indus-Tarbela rim station which constitutes about $47.2 \%$ of the combined river inflows at the four locations. This will result in increased agricultural productivity and improved livelihoods of the downstream rural communities. In case of cold-dry scenarios, W-Himalayan region is most likely to be affected by extreme water shortages. Further reductions in the low-flow magnitudes for expanded durations will amplify the negative effects on an already water stressed environment adversely affecting agricultural production and ecology of riverine ecosystems and exerting increased pressure on the already stressed groundwater resources. The projected increase in low-flows during winter months will improve water availability during Rabi season and lessen the dependency on storage reservoirs to release stored water. Peak flows at all gauging stations are very likely to increase in magnitudes and shift to earlier month due to early melting of seasonal snowpack in a drier and warmer spring and pre-monsoon season. This will expose the riverine areas to encounter more intensive and one-month earlier floods, which will pose higher risk of reduced productivity and failure of standing crops during Apr-June months. However, higher river inflows during these months will positively impact over all water availability during early Kharif (summer) season. This will particularly benefit the extreme lower riparian Sindh province, which usually faces shortages of irrigation water during this period due to one-month earlier start of Kharif crop season than upstream provinces.

\subsection{Conclusions}

This study demonstrated and confirmed large uncertainties associated with CMIP5 GCM outputs for the complex high-mountain Indus basin. Novel and high-quality datasets of precipitation and temperature are developed and forced to a widely used hydrological model for simulation of future hydrological regimes under extreme scenarios of climate change. Accordingly, the study provides unique estimates of historical and future climate change and associated hydrological implications.

The region has already witnessed modest increase in median annual air temperature and a considerable drop in precipitation with corresponding decline in river inflows during the last 40 years. Future projections are highly variable and uncertain. Almost a linier increase in air temperature with varying magnitudes is projected throughout the $21^{\text {st }}$ century. Precipitation and river inflow projections are relatively more uncertain but overall increasing trends are evident for warm-wet scenarios and vice versa for cold-dry scenarios. The Karakoram and parts of the Indus-Kharmong regions are anticipated to receive significant gains in future precipitation. Monsoon precipitation in the Indus-Kharmong may increase due to a stronger 
influence of the Tibetan Anticyclone and East-Asian monsoon in the future. The influence of the Indian summer monsoon is expected to strengthen slightly in the W-Himalayan region but might be weakened as it travels westward and may seldom reach to the extreme SWHindukush region. Under extreme cold-dry conditions, the W-Himalayan region may be worst-affected by extreme water shortages and low flows are very likely to reduce further. This will amplify the negative effects on an already water stressed environment adversely affecting agricultural production and ecology of riverine ecosystems and exerting increased pressure on the already stressed groundwater resources. The Indus-Tarbela inflows are projected to significantly increase during Apr-Jul months with overall annual increase of 17.0-73.6\% under warm-wet scenarios and 1.2-9.7\% under cold-dry scenarios. The lower altitude Kabul river basin is expected to experience an intermediate pattern of seasonal shifts, with slight reductions in river inflows under cold-dry scenarios and modest increases under warm-wet scenarios. Substantial increases in spring and pre-monsoon (Feb-Jun) river inflows however are certain. In some cases, low-flows during winter months will improve water availability during the Rabi season and lessen the dependency on storage reservoirs to release stored water. The projected increases in early Kharif season (Apr-Jun) river inflows will particularly benefit the extreme lower riparian Balochistan and Sindh provinces, which usually face shortages of irrigation water during this period due to one-month earlier start of the Kharif crop season. The projected increases in the magnitudes of peak inflows and their one-month earlier attainments for all river gauging stations cautions for increased risks of floods.

Summarizing, unprecedented and highly contrasting climate change and hydrological signals across the river basin are anticipated for the extreme climate change scenarios considered in this study. The findings suggest significant modifications in the strategies and action for reservoir operations, optimization of hydropower generation and irrigation abstractions, flood control and drought management.

\section{Acknowledgements}

This research work is supported by the Dutch Ministry of Foreign Affairs through the Netherlands Fellowship Program and partially carried out under the Himalayan Adaptation, Water and Resilience (HI-AWARE) consortium supported by the Collaborative Adaptation Research Initiative in Africa and Asia (CARIAA). The views expressed in this work do not necessarily represent those of the supporting organizations. Deepest gratitude is expressed to the institutions and the teams responsible for the development and distribution of climate data and river inflow data and the hydrological model used in this study. The authors declare that there is no conflict of interest. 


\section{Chapter 6}

\section{Synthesis}

\subsection{Introduction}

Climate change is recognized as an emerging threat and the greatest challenge faced by the global community during $21^{\text {st }}$ Century. Precipitation is the most important climatic variable that governs renewable water resources affecting water-energy-food securities and related socioeconomic developments across the globe. The high-altitude Indus basin is one of the most complex mountain regions in the world, where a comprehensive and reliable assessment of precipitation distribution and associated hydrological implications are largely lacking due to scarcity and directional biases of the observed data. While, the majority of the recent hydrometeorological research has been focused on developing and/or using the improved data analysis techniques and hydrological modeling frameworks, less attention was given to precise estimation of the important climatic variables. Consequently, there is a large uncertainty and variability in the quantitative and spatiotemporal distributions of precipitation estimated by previous studies for this region. This $\mathrm{PhD}$ research recognized that improvements in meteorological forcing datasets hold the key for accurate analysis of climate change and associated hydrological implications in the high-altitude Indus basin. Therefore, high-quality meteorological forcing datasets of precipitation and temperature have been developed for the historical period (1979-2018) and various projections of extreme climate change in future to better analyse the recent and projected climate change and variability. A fully-distributed physically-based energy-balance hydrological model is forced with these datasets to simulate the water balance and examine the changes in hydrological regimes at sub-basin scale.

Chapter 1 sets the scene by presenting an overview of the underlying issues related to data gaps in ground-based precipitation and temperature observations and hydrometeorological assessments for the study domain. The hydrological modeling framework used in this dissertation is also described. This is followed by elaborations on the prevailing climate and hydrological regime of the study area and raising a number of interconnected research questions that need to be addressed. The research questions are integrated and framed in the four broad-based research objectives, which are achieved in the subsequent chapters (Chapter 2-5). Chapter 2 presented an appraisal of precipitation distribution in the high-altitude upper Indus basin by integrating a large number of precipitation observations, majority of which have never been used for formation or calibration of precipitation datasets. The uncertainties associated with the existing precipitation datasets were underlined and altitudinal dependency of precipitation was analysed. Chapter 3 further improved the quality of precipitation distribution by extending the area, incorporating additional data and adoption of an improved approach for cross-validation. Precipitation observations were adjusted for measurement errors, net snow accumulations for ablation losses and river inflows for the contribution of the net mass balance. The precipitation distribution was cross-validated by corresponding adjusted river inflows at sub-basin scale using Turc-Budyko's nondimensional analysis. Chapter $\mathbf{4}$ evaluated the performance of a wide range of commonly used precipitation products and corroborated the underlying uncertainties in their precipitation estimates for the high-altitude Indus basin. In Chapter 5 high-quality 
precipitation and temperature datasets are developed for the historical period and two extreme projections of future climate through bias correction of the best performing gridded dataset and GCM outcomes for warm-wet and cold-dry scenarios under three RCPs. Climate change and variability is analysed at the regional scale. The VIC hydrological model is forced with these novel datasets to simulate and analyse the hydrological regimes at sub-basin scale. The summary of main results for each research objective is presented in Table 6.1. The research questions are addressed at sufficient details, the results of which are discussed in more detail in the following section.

Table 6.1: Summary of main results and conclusions for each research objective.

\begin{tabular}{|c|c|}
\hline Research Objective & Main Results and Conclusions \\
\hline $\begin{array}{l}\text { 1. Appraise the current } \\
\text { state of precipitation } \\
\text { distribution in the } \\
\text { high-altitude Indus } \\
\text { basin and highlight } \\
\text { the underlying issues } \\
\text { in precipitation } \\
\text { observations and } \\
\text { quality gridded } \\
\text { precipitation } \\
\text { products (Q1, Q2, \& } \\
\text { Q3 addressed in } \\
\text { Chapter 2) }\end{array}$ & $\begin{array}{l}\text { - The estimated quantitative and spatiotemporal distributions of } \\
\text { precipitation are much better than the previous data and are comparable } \\
\text { and consistent with the corresponding specific runoffs at sub-basin } \\
\text { level. The study area experiences a bimodal weather system with two } \\
\text { distinct rainfall maxima; } 1^{\text {st }} \text { along southern and lower most slopes of } \\
\text { Chenab, Jhelum, Indus main and Swat basins, and } 2^{\text {nd }} \text { around north- } \\
\text { west corner of Shyok basin in the central Karakoram are clearly } \\
\text { evident. } \\
\text { - Considerable dependency of precipitation on elevation is observed. } \\
\text { However, even at sub-basin level the gradients are insufficient to depict } \\
\text { accurate regression models or power functions. } \\
\text { - The selected four gridded precipitation products derived from different } \\
\text { sources are prone to significant errors providing only a marginal } \\
\text { resemblance of the actual precipitation in the study area. Except } \\
\text { reanalysis product, the other products largely underestimate } \\
\text { precipitation in most of the areas. }\end{array}$ \\
\hline
\end{tabular}

2. Adjust measurement

- Analysis of temperature variations with elevation and latitude revealed errors in precipitation observations and significantly different gradients for each month and substantial develop high-quality reference differences among the gradients at different locations for maximum and minimum temperatures. Hence, the use of a universal annual climatologies of precipitation and temperature $(\mathrm{Q} 1, \mathrm{Q} 3$, Q4, Q5, \& Q6 addressed in Chapter 3) gradient or a time independent gradient of mean temperature to estimate maximum and minimum temperatures or vice versa is a major source of uncertainty in temperature datasets of the high-altitude Indus basin.

- The applied error-adjustments significantly increased the gaugemeasured precipitation ranging from $2-182 \%$ for various individual stations. The highest increments were computed for wind-induced under-catch of solid precipitation, particularly in higher-altitude areas and during winter months. Similarly, increases of $0-200 \%$ are estimated for the net snow accumulations.

- Contrary to the general understanding and speculations, the contribution of net glacier mass balance to river inflows is only marginal ranging from $0.5-6.1 \%$ of the observed river inflows.

- The catch corrections of precipitation gauges provided new insights in the magnitude and distribution patterns of precipitation. The adjusted precipitation is considerably greater than what has been previously thought. These increases are mainly realized in the higher-altitude areas of Chitral, Gilgit, Hunza, Shigar, Shyok and Astore basins. The study recognized that the higher river flows than the corresponding 
precipitation estimates by the previous studies are mainly due to underestimated precipitation.

- Although, cross-validation results indicate high-quality of estimated precipitation, yet there are observational uncertainties and eradication or reduction of these would result in improved estimates. The study recognizes that the data-quality driven underestimated precipitation may be the major source of uncertainty in the water balance estimates in the highaltitude Indus basin.

3. Evaluate quality of regional/global scale precipitation products for the study area with respect to the reference dataset (Q7 \& Q8 addressed in Chapter-4)

- This chapter highlighted and corroborated the underlying issues and uncertainties associated with a wide range of gridded precipitation products in the high-mountain Indus basin. The results clearly indicate that all gridded datasets evaluated in this study contain significant errors in their precipitation estimates and cannot be used directly without careful bias correction.

- Majority of the datasets tend to underestimate precipitation in wet areas and overestimate precipitation in dry areas, implying considerable implications for hydrological extremes of floods and droughts. None of the dataset is equally best for all sub-regions of the study area. A particular dataset performing very well in one sub-region is found worse in the other sub-region. Nevertheless, ERA5 is found most acceptable for all sub-regions.

- It is revealed that reanalysis products provide relatively better estimates for the higher-altitude areas where observations are generally scarce. However, not all reanalyses products can serve the purpose due to large differences in their precipitation patterns and magnitudes. Therefore, a careful selection is deemed essential.

- Relatively poor performance of the merged datasets in the study region highlights their weaknesses and inability to accurately estimate precipitation and underlines the need to develop more advanced and accurate merging techniques, which can preserve the comparative advantages of input datasets and which are equally accurate at subcatchment scales.

4. Develop long-term datasets of precipitation \& temperature for the historical and future time periods and assess past and future climate change \& variability and associated hydrological implications (Q9, Q10, Q11, Q12, Q13 \& Q14 addressed in Chapter 5)
- The region has already witnessed a modest increase in median annual temperature $\left(0.6{ }^{\circ} \mathrm{C}\right)$ and a considerable drop $(11.9 \%)$ in precipitation during the last 40 years. The changes in corresponding river inflows remained highly variable but consistently declined. Indus-Tarbela, Jhelum-Mangla, Chenab-Marala and Kabul-Nowshera rivers inflows experienced $4.9 \%, 19.6 \%, 11.9 \%$ and $4.5 \%$ decline respectively.

- This study demonstrated and confirmed large uncertainties associated with CMIP5 GCM outputs for the complex high-mountain Indus basin. As such, the future projections of climate change and hydrological regime are extremely uncertain.

- Almost a linier increase in air temperature with varying magnitudes is projected throughout the $21^{\text {st }}$ century. Precipitation and river inflow projections are relatively more uncertain but overall increasing trends are evident for warm-wet scenarios and vice versa for cold-dry scenarios. The Karakoram and parts of the Indus-Kharmong regions are may receive significant gains, while other areas may experience reduced precipitation during $21^{\text {st }}$ century.

- Indus-Tarbela inflows are likely to increase between $17.0-73.6 \%$ under wet-warm scenarios and between $1.2-9.7 \%$ under cold-dry scenarios, 
while river inflows at the remaining three rim stations show increases for wet-warm and decreases for cold-dry scenarios

- Unprecedented and highly contrasting climate change and hydrological signals across the river basin are projected for warm-wet and cold-dry scenarios under RCP2.6, 4.5 \& 8.5. Hence, important modifications in the strategies and action plans for reservoir operations, optimized hydropower generation and irrigation diversions, flood control, and drought management would be required.

\subsection{Discussion of the Main Results}

\subsubsection{An appraisal of precipitation distribution (Objective 1, Chapter 2)}

Previous studies (e.g. Pang et al., 2014; Hewitt, 2011; Winiger et al., 2005; Glazirin, 1997; Weiers, 1995; Hormann, 1994; Wake, 1989; Dhar and Rakhecha, 1981; BIG, 1979; Decheng, 1978) noticed strong relationship of precipitation with elevation using only limited or selective ground-based observations and suggested regression models or power functions to estimate precipitation from elevation at any point in the upper Indus basin. This study, however, incorporated a large number of ground-based observations and detected considerable altitude dependency of precipitation but demonstrated substantial differences in the rate and magnitude of altitudinal variation of precipitation from one sub-basin to another. Hence, the complex altitudinal variation of precipitation in the high-altitude Indus basin cannot be represented by a single relation. Even at sub-basin scale, the spatial and altitudinal variability of precipitation is so complicated that the computed precipitation gradients are insufficient to depict accurate regression models or power functions. The elevation zone of maximum precipitation is also highly variable. The Western-Himalayan region experiences the zone of maximum precipitation at an altitude of around $2500 \mathrm{~m}$, which supports the findings of Pang et al. (2014) and Dhar and Rakhecha (1981), who observed significant decrease in monsoon precipitation above $2400 \mathrm{~m}$ elevation in the central Himalayas. The height of maximum precipitation in the rest of the sub-basins is unclear but tends to increase with latitude and in winter westerly dominated areas. Hence, the assumptions of linear increase in precipitation with elevation by the earlier studies (e.g. Immerzeel et al., 2012; Mayer et al., 2006 and Winiger et al., 2005) could not be confirmed by this study.

The estimated distribution of mean monthly precipitation indicates a bimodal weather system (Figure 2.4), particularly in the Western-Himalayan catchments reflecting the wintertime precipitation associated with the westerly systems and the impact of Indian summer monsoon. Generally, precipitation tends to decrease with increasing latitude (from south to north), while longitude has seasonal influence, positive in monsoon and negative in winter season. Similarly, the southeast-ward and southwest-ward orientated locations mostly receive more precipitation in monsoon and winter seasons respectively. The study provided much better estimates of precipitation distribution, which are comparable and consistent with the specific runoff at sub-basin scale (Figure 2.6). Highly contrasting but consistently underestimated precipitation estimates of previous studies (Table 2.3) are revealed due to use of precipitation data of the climatic stations predominantly located in low-altitude dry valleys and/or overestimated basin boundaries. 
Evaluation of four important gridded precipitation products (ERA-Interim, WFDEI, TRMM \& APHRODITE) derived from different sources (reanalysis, merged reanalysis and gauge, merged remote sensing and gauge, and gauge) revealed significant differences in their mean monthly precipitation estimates for each sub-hydrological basin. All the four datasets fail to detect maximum precipitation zones along the western Himalayan foothills and the northeast junction of Shigar and Shyok basins in the central Karakoram (Figure 2.9). They also inadequately captured the dry areas under the influence rain shadow with large overestimates. In quantitative terms, ERA-Interim largely overestimates precipitation in all the sub-basins except Shigar and Hunza, while the other three datasets consistently underestimate precipitation in all the areas barring Ladakh region of the TP (Indus at Kharmong). Basinwide seasonal and annual correction factors are derived for each dataset and for each subbasin (Table 2.5) to facilitate hydrological assessments.

\subsubsection{Adjustment of measurement errors to reconcile precipitation distribution (Objective 2, Chapter 3)}

About $73 \%$ of the climatic stations used in this study did not possess temperature data. Therefore, to facilitate adjustment of measurement errors in precipitation observations of such stations, the corresponding air temperature was derived from elevation and latitude of other stations using multiple regression at monthly and sub-regional scale. Strong correlation of temperature with elevation and considerable correlation with latitude is observed. Significantly different gradients for each month and substantial difference among the gradients for maximum and minimum temperatures prohibit the use of a universally assumed or time independent site-specific observed gradient of mean annual temperature to estimate maximum and minimum temperatures (e.g. Immerzeel et al., 2012a \& b; Lutz et al., 2013). The wind-induced under-catch was by far the largest source of errors in gauge-measured precipitation, particularly during winter months in the high-altitude areas where majority of precipitation falls in the form of snow. The catch corrections have increased the gaugemeasured precipitation values ranging from $2-182 \%$ for various individual stations, while net snow accumulations at the glacier points increased up to $200 \%$. The adjustments revealed significant improvement in the quantitative and spatio-temporal distribution of precipitation in the study area (Figure $3.3 \mathrm{~m}-0$ ). An overall increase of $21.3 \%$ in average annual precipitation is realized at basin (study area) level, while at sub-basin scale it ranged from 6 to $77 \%$ (Table 3.3 ).

The contributions of the net glacial mass imbalance to the observed river flows at sub-basin scale were computed from the specific net mass balance rates derived from the mass balance estimates of Kääb et al. (2012) and glacier areas from the Randolf Glacier Inventory (RGI) version 5.0 (Arendt et al., 2015). Contrary to the general understanding and speculations, the contribution of net glacier mass balance to the observed river inflows is only marginal ranging from $0.4-6.1 \%$ of the observed flows (Table 3.4 ). The study recognized that the higher river inflows than the corresponding precipitation estimates by the previous studies are mainly due to use of underestimated precipitation.

The catch corrections and adjustment of net snow accumulations provided new insights to the quantitative and spatiotemporal distribution of precipitation in the study area. The adjusted precipitation is considerably greater than what has been previously thought. These increases are mainly realized in the higher-altitude areas of the Karakoram (Hunza, Shigar, Shyok), Southwest-Hindukush (Chitral \& Gilgit) and along the high-mountain ridge of Northwestern-Himalayan region (Figure 3.3o). Although, the cross-validation results 
confirm the superior quality of estimated precipitation distributions, yet there are few uncertainties that need to be understood and taken care of in the future investigations. The major uncertainties associated with the results of this study may arise from four possible sources: i) uncertainties in regression models due to their imprecision and uncertainties in the input data, ii) uncertainties arising from the estimated temperature and wind speed for many observatories, iii) uncertainty in the gauge type of the basin's gauge network, and iv) uncertainties in spatial interpolation of the point observations to derive gridded fields of precipitation. Further improvements can be achieved by calibration of the already installed precipitation gauges with the WMO recommended reference gauges and development of site and gauge specific error adjustment models, use of observed data with better spatio-temporal coverage, use of daily or even sub-daily time steps, use of corresponding observed wind speed and temperature datasets, selection of any better spatial interpolation technique, precise determination of other components of the water balance to validate precipitation, and a better integration of precipitation data with mass balance data and other non-conventional data sources.

\subsubsection{Evaluation of gridded precipitation products (Objective 3, Chapter 4)}

In chapter 4 , the accuracy of 27 widely used gridded precipitation products belonging to three groups (gauge-based, reanalysis and merged) was assessed against the high-quality reference dataset developed for the study area (chapter 3) at monthly and annual timescales. Widely used statistical measures and performance metrics are applied to evaluate and quantify the accuracy of these datasets at sub-regional scale.

Generally, a large uncertainty in quantitative and spatio-temporal distribution of precipitation is evident in all gridded datasets, which is in line with the findings of Sun et al. (2018), who reviewed and intercompared 30 precipitation datasets at global and regional scale. The most important attributions for the large differences and uncertainties in gridded precipitation products are related to their different structural characteristics, diverse input data and observational densities, variable quality control and gauge under-catch corrections, spatiotemporal resolution, and use of different interpolation schemes. The landscape heterogeneities further add to the uncertainties.

Spatial distribution of mean annual precipitation estimates from various gridded datasets (Figure 4.2) and area-weighted seasonal and annual precipitation totals (Table 4.2) reveal significant variability and differences in precipitation estimates of the gridded datasets in the study area. Compared to annual mean precipitation of $697 \mathrm{~mm}$ of the reference dataset, the minimum estimates of $374 \mathrm{~mm}(-46 \%)$ are depicted by CPC Unified and maximum estimates of $976 \mathrm{~mm}(+40 \%)$ by ERAI datasets. All gridded products are consistent in their patterns showing underestimated precipitation in wet areas and overestimated precipitation in dry areas, implying considerable implications for hydrological extremes of floods and droughts. The gauge-based and merged products show strong tendency of underestimation; while except ERA20C, reanalysis products tend to overestimate precipitation in most parts of the study area, with considerable difference at sub-regional level. The gauge-based and merged products perform relatively better during monsoon season and reanalysis products during the winter months and at the higher-altitude areas where observations are generally scarce. However, the reanalysis products also show larger variability and wider spread of residuals than gauge-based and merged products (Figure 4.3), which is understandable and attributed to their independence from direct measurements of precipitation, use of varying type and number of assimilated observations, and use of different atmospheric models and assimilation schemes. The gauge-based and merged products significantly underestimate 
precipitation in relatively wet regions of W-Himalaya, NE-Hindukush and Karakoram. The observational-based reference dataset exhibits a strong seasonality with biomodal pattern, but many gridded datasets have difficulties in efficiently reproducing this seasonality and pattern (Figure 4.6). The merged products aim to exploit the complementary nature and comparative advantages of the input source datasets, however relatively poor performance of these products for the study region underlines the dire need for developing more advanced and accurate merging techniques, which can preserve the comparative advantages of input datasets and are equally accurate at smaller (basin/catchment) scales. None of the selected products could be singled out as the best product for all regions as their accuracy varies considerably from one region to another. However, ERA5 is found most acceptable for all sub-regions, particularly at higher-altitudes, in wet areas and during winter months (Figure 4.7). While the underlying efforts to develop global/regional scale datasets have been focusing on combining data from various sources and merging algorithms, another alternative may be to develop national/basin/catchment scale datasets using optimum data and techniques first and then merge these products to form a high-quality product at regional/global scale.

\subsubsection{Climate change and hydrological regime (Objective 4, Chapter 5)}

Accurate assessments of climate change in the high-altitude Indus basin have remained daunting task due to highly scarce in situ observations and strong influence of the innately complex climate systems interacting with very high orographic features. As such, there is large variability in quantitative and spatio-temporal distribution of the historical precipitation described in Chapters $2 \& 4$, and future projections by various GCM outputs (Lutz et al., 2016b; Cai et al., 2009), and associated hydrological implications detailed in Chapter 5. This is confirmed by precipitation estimates of 75 GCM runs evaluated in this chapter (Table 5.1). None of the GCM run was able to precisely capture the influence of predominant weather systems resulting in large variability and biases in their monthly and annual precipitation estimates.

In the presence of significant interannual variability, almost linear increase in temperature is observed during the last 40 years, with an overall increase of $0.6{ }^{\circ} \mathrm{C}$. The future projections are more variable and uncertain but an overall increasing trend is prominent until 2060. Thereafter, strong increasing trend continues for RCP8.5, tends to stabilize under RCP4.5 and show slight reduction under RCP2.6. Compared to temperature, median annual precipitation showed extreme uncertainty during the last 40 years ranging from -30.5 to $17.9 \%$, with an overall decrease of 11.9\% during 2001-2020 over 1981-2000. Definite and strong increasing trends in the projections of median annual precipitation are only evident for MIROC5-RCP8.5, while MPI-ESM-LR shows declining trends for RCP4.5 \& RCP8.5. The remainder of GCM runs show mixed trends. Similarly, median annual river inflows at the four river gauges (Kabul-Nowshera, Indus-Tarbela, Jhelum-Mangla, \& Chenab-Marala) also remained highly variable and experienced significant decline during the same period.

Future climate is likely to be more variable in space and time under each scenario. Precipitation changes in most parts of the basin at annual scale and during most of the months are generally positive for MIROC5 and negative for MPI-ESM-LR except Karakoram and western part of Kharmong regions, where both GCMs show positive changes. Farinotti et al. (2020) noticed balanced to slightly positive glacier budgets, increasing glacier ice flow speeds, stable to partially advancing glacier termini and widespread glacier surge activity. The authors however were uncertain about the future of this Karakoram Anomaly, and termed its long-term sustainability unlikely in light of the anticipated warming of future 
climate. This study can explain the future of peculiarly anomalous behavior of glacier activity in the Karakoram region. It shows strong signals of considerable precipitation gains even under extreme cold-dry condition of future climate. Of particular importance is substantial increases in future precipitation during winter and summer seasons in the Karakoram region.

The existing hydrological regime of the study area is largely modulated by timing, intensity, duration, and form of precipitation; snow and glacial ice reserves; and amount of energy available for melting of seasonal and perennial snow and glacial ice (Archer, 2003). Climate change is expected to weaken the modulating effect of snow and glacier fields with potentially strong hydrological implications (Biemans et al., 2019). Peak flows at the four rim stations are likely to be increased and shifted one month earlier, with substantial increases in pre-monsoon river inflows and decreases in post monsoon river inflows mainly due to early melting of seasonal snow. Indus-Tarbela inflows are likely to increase between 17.0$73.6 \%$ under wet-warm scenarios and between 1.2-9.7\% under cold-dry scenarios. Lutz et al. (2016a) on the other hand show slight increases for the upstream Karakoram river inflows but no change at the downstream Tarbela gauging station against 38\% to 52\% increase anticipated by Hasson et al. (2019). They also expected no shift in peak, which is likely to be shifted from Aug to Jul in this study. In contrast, river inflows at the remaining three rim stations show increases for wet-warm and decreases for cold-dry scenarios. Most of the ensembles show increasing high flows and decreasing low flows at all gauging stations implying intensification of future hydrological extremes. The conflicting signals of changes in the hydrological regimes may primarily be attributed to large differences in the baseline reference climate data, significant uncertainties in GCM outputs, use of different scenarios of future climate change and different hydrological modeling frameworks.

\subsection{Scientific Contribution}

Climate change is likely to significantly alter the hydrological regimes of many river basins. The most important scientific contribution of this research is an improved and more reliable assessment of climate change and hydrological implications in the study area. An improved methodological approach was adopted to adjust precipitation observations for measurement errors, while novel techniques were introduced to adjust net snow accumulations for ablation losses and river inflows for the contribution of net mass balance. This study is the first initiative to undertake such adjustments in the Indus basin. Similarly, an improved method for cross-validation of the precipitation estimated with respect to the adjusted specific runoff was adopted. An innovative approach for derivation of temperature climatologies using elevation and latitude dependent lapse rates at monthly scale was introduced. Development of high-quality reference datasets of precipitation and temperature is the other significant scientific contribution of this study. Performance of 27 widely used gridded precipitation products was evaluated and the best performing product was bias-corrected against the reference datasets to develop long-term datasets of precipitation and temperature for the historical period. Similarly, precipitation estimates of 75 GCM ensembles were evaluated. The best performing GCMs under RCP2.6, 4.5 and 8.5 for warm-wet and cold-dry scenarios are also bias-corrected. Hence, long-term novel datasets of precipitation and temperature for historical period and various scenarios of future climate is another significant milestone of this study. The VIC hydrological model at high resolution in the Indus basin is also applied for the first time. The climate change associated hydrological implications deduced in this study will add to our knowledge and understanding of the basin's hydrometeorological regimes. Finally, the study also provides useful guidelines for developing optimal strategies and action plans for efficient river basin management. 


\subsection{Uncertainties and Limitations}

\subsubsection{Observational uncertainties}

The major observational uncertainties can be associated with the density, direction and quality of the observed climate data and imprecision in the interpolation of point observations to derive spatially distributed fields. The dynamics of snow and glacial ice mass at the higheraltitudes may also add to the observational uncertainties (Kraaijenbrink et al., 2017). Climate observations form the essential inputs to the climate and hydrological models to facilitate the intended future projections and impact assessments. This study developed and used much improved precipitation and temperature datasets and employed state-of-the-art interpolation technique to minimize observational uncertainties that are commonly present in the previous datasets. Yet, the observational uncertainties in the hydrometeorological assessment undertaken in this study may still hold a sizable share in the total uncertainties due to imprecision in the observed data and techniques used to adjust measurement errors and spatial interpolation of the point observations. These observational uncertainties can be addressed by incorporating additional observed data with better spatio-temporal coverage particularly in higher-altitude areas, calibration of the precipitation gauges with the WMO recommended references and development of site and gauge-specific error adjustment models, use of daily or even sub-daily time steps, use of corresponding observed wind speed and temperature data, selection of better spatial interpolation technique, precise computation of other components of the water balance to validate precipitation, and a better integration of the observed precipitation data with mass balance and remote sensing data.

\subsubsection{GCM structure and parameterization}

Climate change impact studies generally depend on the projections of future climate commonly provided by the GCMs. Presently, a multitude of GCM outputs under CMIP5 are available for climate research. GCM related uncertainties arise mainly from formulation and discretization of the governing mathematical models, from parameterization of unresolved processes, and from imperfect understanding of the climate system (Palmer et al., 2005). Since each GCM is developed based on its own specific assumptions and unique mathematical models to represent the physical processes of climate systems, their outcomes are innately different (Hawkins and Sutton, 2011). Hence, with availability of a large number of GCM outputs, the spread and variability in their outcomes is also large (Lutz et al., 2016a;), particularly at regional and catchment scale (Cai et al., 2009). GCM related uncertainty is often a dominating source (Kay et al., 2009) constating about 50-85\% of the total uncertainty (Prein et al., 2011), and is generally more pronounced at longer timescales (Hawkins and Sutton, 2011). Parameterization is used to deal with the processes that occur on scales smaller than the GCM grid resolution and the parameterization related uncertainty is much smaller than GCM related uncertainty (Her et al., (2019). Performance evaluation of 75 GCM runs undertaken in this study revealed a large variability in precipitation estimates of the GCM projections. Hence, future water availability resulting from GCM precipitation in the highaltitude Indus basin will be even more uncertain.

\subsubsection{Downscaling and bias-correction}

Due to coarse spatial resolution and greater uncertainty at finer temporal resolutions, GCMs are generally inappropriate for finer scale impact modeling (Kay et al., 2009). Therefore, GCM projections are often downscaled for regional impact assessments using dynamic or statistical downscaling. Dynamic downscaling nests a finer-scale regional climate 
model (RCM) within a GCM. However, most RCMs do not accurately simulate extreme precipitation - a systematic bias that can worsen as the resolution is increased (Trzaska, and Schnarr, 2014). Therefore, in many cases the direct outcome of the best performing GCM is usually preferred over RCM outcomes or RCM outputs are further downscaled using statistical approach. Statistical downscaling on the other hand establishes empirical relationships between coarser-scale historical outputs of GCMs and local climatic observations. These relationships are then applied to the GCM outputs for future to correct their systematic biases. A wide variety of bias-correction methods are currently under use. Some methods address only a mean difference error, while others cover both average and variance. Few others focus on correction of mean and standard deviation together with wetday frequencies and intensity errors. However, all bias-correction methods are based on the fundamental assumption that the empirical relationship derived from the present climate conditions is also valid for the future scenarios (Dobler et al., 2012; Wilby et al., 2004; Zorita and von Storch, 1999). This study used the delta change approach for bias correction of gridded dataset and precipitation and temperature outputs of two GCMs under three RCPs. The delta change approach uses linear scaling to perfectly match the monthly means of estimated climate variables with those of the observed values (Lenderink et al., 2007). This approach is widely used to assess climate change and future water availability, while more advanced methods are preferred for analysis of climate and hydrological extremes (e.g. Lutz et al., 2016a). However, Dobler et al. (2012) and Key et al. (2009) noticed comparatively small uncertainty related to the choice of the bias-correction method.

\subsubsection{Hydrological modeling structure and parameterization}

Several hydrological models ranging from lumped to fully-distributed, simple temperatureindex to complex energy-balance, and from standalone catchment-scale to global-scale land surface schemes can be used to assess hydrological implications of climate change. However, no model is absolutely perfect in its representation of reality, and the choice of model is often based on the objectives, model performance under specific conditions, data availability and requirements, etc. (Kay et al., 2009). Moreover, a substantial portion of uncertainty may also stem from the use of suboptimal hydrological model parameters (Ragettli et al., 2013; Kay et al., 2009). Ragettli et al., (2013) showed that the effects of uncertainty sources are variable in space and time and hydrological model input parametric uncertainty in heavily glacierized subregions exceeds the effect of climate model uncertainty and natural internal climate variability, but often decreases with time. The accuracy of the model outcome is largely dependent on the quality of input variables and modeling structure to represent the natural processes. The VIC hydrological model used in this study is validated and extensively used in a variety of conditions and there is no serious drawback in its modeling structure. Its characteristics of physically-based, fully-distributed, energy-balance, and representation of a wide range of physical processes are particularly important. However, the model also suffers from a couple of limitations to precisely model the glacierized catchments. Firstly, it does not take into account the already stored volume of snow and glacial ice mass, and then it simulates snow and glaciers using the same mathematical model, whereas glaciers possess considerably different hydraulic properties.

\subsubsection{Uncertainties in river flow measurements}

River flows usually have indirect use in hydrometeorological studies. Primary use of river flow data is for calibration and validation of the hydrological models at the strategic river gauges. In contrast to most climatic variables, river flow data are generally assumed to be 
adequately accurate considering the relative precision of discharge measurement techniques and quality control ensured by the data collection agencies. Yet, river flow data may still be subject to some degree of uncertainty due to measurement errors. NESPAK-AHTDELTARES (2015) reviewed river/canal discharge measurement protocols adopted in the IRS and observed overall uncertainties in the rage of 3-8\% at five canal headworks. The river flow uncertainties may slightly vary at each gauge but will remain constant with time.

\subsection{Implications for Water Management}

Pakistan is highly vulnerable to climate change due to its hot and arid geographical location, dependence on climate sensitive sectors like agriculture, knowledge and technological gaps, and low adaptation capacity (PC, 2010). Its reliance on a single river system, that is also transboundary and one of the hotspots of future climate change, puts it at a higher risk of water insecurity. The water resources of the Indus river system are seriously threatened by a variety of stressors including increasing scarcity and variability due to climate change, deteriorating quality, expanding population, rapid urbanization, growing industrialization, and above all, the recurring inability to develop an adequate platform of modern infrastructure and institutions to ensure efficient water governance (FoDP, 2012). These stressors will further escalate the gap between water supply and demand during the $21^{\text {st }}$ century. The country was already categorized as a water deficit country more than a decade ago (PC, 2008) therefore any change and variability in its water budget can have significant implications for hydropower generation, agricultural productivity, economic growth, peoples' livelihood and interprovincial harmony and sovereignty.

This $\mathrm{PhD}$ research study investigated hydrological implications of climate change at the four river gauging stations i.e. Kabul river at Nowshera, Indus river at Tarbela, Jhelum river at Mangla, and Chenab river at Marala. The hydrological modeling focused on the outputs of the two best performing GCMs projecting extreme conditions of warm-wet and cold-dry climates under three RCPs. As such, there are significant differences in the magnitudes and trends of future river inflows mainly due to differences in the GCM outputs. In the presence of significant monthly and interannual variability, overall water availability is projected to improve in future, particularly at the Indus-Tarbela rim station, which constitutes about $47.2 \%$ of the combined river inflows at the four locations. This will result in increased agricultural productivity and improved livelihoods of the downstream rural communities. In case of cold-dry scenarios, the W-Himalayan region (Jhelum and Chenab rivers) is most likely to be adversely affected by extreme water shortages. Further reductions in the lowflow magnitudes for expanded durations will amplify the negative effects on an already water stressed environment adversely affecting agricultural production and ecology of riverine ecosystems and exerting increased pressure on the already stressed groundwater resources. The projected increase in low-flows during winter months will improve water availability during the Rabi season and lessen the dependency on storage reservoirs to release the stored water. Peak flows at all gauging stations are very likely to increase in magnitudes and shift to earlier months due to early melting of seasonal snowpack in a drier and warmer spring and pre-monsoon season. This will expose the riverine areas to encounter more intensive and onemonth earlier floods, which will pose higher risk of reduced productivity and failure of standing crops during Apr-June months. However, higher river inflows during these months will positively impact overall water availability during the early Kharif (summer) season. This will particularly benefit the extreme lower riparian Sindh and Balochistan provinces, which usually face shortages of irrigation water during this period due to a one-month earlier start of the Kharif crop season than upstream provinces. Hence, the projected changes in 
monthly, interannual and overall water availability at the four river gauging stations under different climate change scenarios depict serious implications for future water resources planning, development and management. Strategies and action plans for hydropower generation, construction and operation of storage reservoirs, irrigation withdrawals, flood control and drought management will require significant modifications.

\subsection{Outlook and Direction for Further Research}

Although, precipitation and temperature datasets developed in this study are demonstrated as much better than the previously available estimates, further improvement can be achieved by addressing the remaining uncertainties. For this, there is an urgent need to undertake a comprehensive and site-specific investigation to unravel, evaluate, quantify and eradicate the uncertainties outlined in section 6.4.

The greatest accuracy is required in the baseline datasets of important climatic variables, which are used to bias-correct the future projections and force the hydrological models to simulate water balance. One of the potential alternatives to significantly improve quality of precipitation distribution in the study area is to incorporate more observations, particularly at the higher-altitudes with sufficient coverage, standardize and improve quality of the observed data, and ensure efficient integration of the ground-based observations with nonconventional data sources (e.g. the indirect precipitation estimates from mass balance observations, inversely inferred precipitation from the glacier mass balances [Immerzeel et al., 2015] duly verified by the ground-based mass balances, radar remote sensing, and smart monitoring by drones). Radar remote sensing and use of drones may be the potential solutions for environmental monitoring and mass balance investigations in this extremely inaccessible region.

Improvements are also needed in hydrological modeling frameworks keeping in view the peculiarly specific condition of this region. The ideal hydrological model for simulating the water balance of this snow/glacier-fed river basin would be a fully-distributed, physicallybased, energy-balance model that also takes input of already stored snow/glacier volume and simulates precipitation, snow and glaciers separately as per their specific hydraulic properties. One way to increase our understanding on the impact of the hydrological modeling structures is to study the hydrologic response of the study area using different hydrological modeling frameworks with the same meteorological forcing, soil and vegetation parameters. 


\section{Supplementary Material - Chapter 2}

Table S-2.1: Details of meteorological stations used in this study. The stations mentioned at S. No. 1-21 are operated by PMD, 22-65 by WAPDA, 66-67 by Ev-K2-CNR, 68-108 by IMD (taken from GHCN), 109-118 by University of Boon's CAK project (synthesized from Winiger et al., 2005; Miehe et al., 1996, 2001; and Eberhardt, 2007), and 119-134 are virtual stations detailed at Table 1.

\begin{tabular}{|c|c|c|c|c|c|c|c|c|c|c|c|}
\hline $\begin{array}{l}\text { Sr. } \\
\text { No }\end{array}$ & $\begin{array}{l}\text { Station } \\
\text { Name }\end{array}$ & $\begin{array}{r}\text { Lat } \\
\text { (dd) }\end{array}$ & $\begin{array}{l}\text { Long } \\
\text { (dd) }\end{array}$ & $\begin{array}{c}\text { Elevation } \\
(\mathbf{m})\end{array}$ & $\begin{array}{l}\text { Data } \\
\text { Period }\end{array}$ & $\begin{array}{l}\text { Sr. } \\
\text { No. }\end{array}$ & $\begin{array}{l}\text { Station } \\
\text { Name }\end{array}$ & $\begin{array}{l}\text { Lat } \\
\text { (dd) }\end{array}$ & $\begin{array}{l}\text { Long } \\
\text { (dd) }\end{array}$ & $\begin{array}{c}\text { Elevation } \\
\text { (m) }\end{array}$ & $\begin{array}{l}\text { Data } \\
\text { Period }\end{array}$ \\
\hline 1 & Astore & 35.3667 & 74.9000 & 2394 & 1954-2012 & 68 & Bhadarwah & 32.9667 & 75.7167 & 1690 & 1911-1968 \\
\hline 2 & Babusar & 35.1458 & 74.0444 & 4160 & 2005-2012 & 69 & Banihal & 33.5000 & 75.1700 & 1630 & $1961-1970$ \\
\hline 3 & Balakot & 34.5500 & 72.3500 & 995 & $1957-2012$ & 70 & Baramula & 34.2000 & 74.3700 & 1572 & $1902-1970$ \\
\hline 4 & Bunji & 35.6667 & 74.6333 & 1372 & 1953-2012 & 71 & Charisharif & 33.8700 & 74.7700 & 1616 & $1960-1970$ \\
\hline 5 & Chilas & 35.4167 & 74.1000 & 1251 & $1953-2013$ & 72 & Digar & 34.2500 & 77.7500 & 5182 & 1956-1964 \\
\hline 6 & Chitral & 35.8500 & 71.8333 & 1498 & 1964-2012 & 73 & Dras & 34.4333 & 75.7667 & 3066 & 1901-1968 \\
\hline 7 & Dir & 35.2000 & 71.8500 & 1425 & $1967-2010$ & 74 & Durroo & 33.5700 & 75.2300 & 1790 & 1924-1964 \\
\hline 8 & Drosh & 35.5667 & 71.7833 & 1464 & $1951-2012$ & 75 & Gondla & 32.5200 & 77.0300 & 3144 & 1951-1970 \\
\hline 9 & GD Poto & 34.2167 & 73.6167 & 814 & 1955-2012 & 76 & Gulmarg & 34.0500 & 74.4000 & 2655 & 1951-1971 \\
\hline 10 & Gilgit & 35.9167 & 74.3333 & 1460 & $1951-2012$ & 77 & Gund & 34.2500 & 75.0800 & 2052 & $1956-1970$ \\
\hline 11 & Gupis & 36.1667 & 73.4000 & 2156 & $1955-2012$ & 78 & Gurez & 34.6300 & 74.8500 & 2417 & 1933-1958 \\
\hline 12 & Hunza & 36.3220 & 74.6460 & 2374 & 2007-2012 & 79 & Handwara & 34.4000 & 74.2800 & 1585 & $1958-1970$ \\
\hline 13 & Kakul & 34.1833 & 73.2500 & 1308 & $1952-2012$ & 80 & Inshan & 33.7500 & 75.5000 & 2440 & 1971-1980 \\
\hline 14 & Kotli & 33.5167 & 73.9000 & 614 & $1953-2012$ & 81 & Kargil & 34.5700 & 76.1300 & 2679 & 1908-1966 \\
\hline 15 & Malakand & 34.5500 & 71.9167 & 800 & 2003-2008 & 82 & Khaltse & 34.2500 & 76.8333 & 3205 & $1956-1970$ \\
\hline 16 & Malamjaba & 34.7500 & 72.9000 & 2591 & 2003-2008 & 83 & Khangral & 34.3333 & 76.5000 & 3887 & 1956-1971 \\
\hline 17 & Murree & 33.9000 & 73.4000 & 2168 & 1980-2012 & 84 & Kishtwar & 33.3000 & 75.7500 & 1215 & 1901-1970 \\
\hline 18 & Muzaffarabad & 34.3667 & 73.4833 & 702 & $1955-2012$ & 85 & Kokernagh & 33.9200 & 75.2800 & 1676 & $1960-1970$ \\
\hline 19 & Pattan & 35.1000 & 73.0000 & 752 & 2004-2012 & 86 & Koksar & 32.4160 & 77.2190 & 3507 & $1951-1970$ \\
\hline 20 & Saidusharif & 34.7333 & 72.3500 & 961 & $1974-2010$ & 87 & Kukernag & 33.6000 & 75.3000 & 1865 & 1961-1970 \\
\hline 21 & Skardu & 35.3000 & 75.6833 & 2210 & $1952-2012$ & 88 & Kulgam & 33.6300 & 75.0200 & 1615 & $1902-1970$ \\
\hline 22 & Besham & 34.9333 & 72.8833 & 480 & $1971-2003$ & 89 & Kyelong & 32.5833 & 77.0667 & 3500 & 1903-1970 \\
\hline 23 & Burzil & 34.9056 & 75.0917 & 4030 & 1999-2012 & 90 & Langet & 34.3700 & 74.3000 & 1588 & 1916-1970 \\
\hline 24 & Dagar & 34.5100 & 72.4864 & 732 & 1984-2001 & 91 & Leh & 34.1500 & 77.5667 & 3514 & 1876-1969 \\
\hline 25 & Deosai & 35.1000 & 75.6000 & 3910 & 1995-2011 & 92 & Matsal & 33.9833 & 76.6167 & 4325 & 1971-1981 \\
\hline 26 & Dhudnial & 34.7000 & 74.1170 & 534 & 1984-1997 & 93 & Mulbek & 34.3333 & 76.3333 & 3926 & 1956-1969 \\
\hline 27 & Domel & 34.3678 & 73.4689 & 686 & 1984-2001 & 94 & Nowshera & 33.1500 & 74.2300 & 599 & 1913-1969 \\
\hline 28 & Doyian & 35.5450 & 74.7042 & 2454 & 1979-2003 & 95 & Panamik & 34.7500 & 77.5000 & 4056 & $1956-1970$ \\
\hline 29 & Gujar Khan & 33.2500 & 73.3000 & 457 & 1984-2001 & 96 & Pendras & 34.4167 & 75.5833 & 4880 & 1956-1971 \\
\hline 30 & Hushy & 35.3667 & 76.4000 & 3010 & 1994-2010 & 97 & Phalgam & 34.0300 & 75.3300 & 1707 & $1960-1972$ \\
\hline 31 & Jabbar & 34.6717 & 73.2278 & 2134 & 1984-2001 & 98 & Prang & 34.2800 & 74.8700 & 1588 & 1960-1973 \\
\hline 32 & Kalam & 35.4700 & 72.6010 & 2744 & 1984-2010 & 99 & Qazi Gund & 33.5800 & 75.0800 & 1690 & $1962-1974$ \\
\hline 33 & Kallar & 33.4167 & 73.3667 & 518 & 1984-2001 & 100 & Ramban & 33.2500 & 75.2500 & 945 & 1901-1969 \\
\hline 34 & Kelash & 35.6955 & 71.6547 & 2810 & $2000-2013$ & 101 & Riasi & 33.0800 & 74.8300 & 585 & $1901-1970$ \\
\hline 35 & Khandar & 33.5000 & 74.0500 & 1067 & 1984-2001 & 102 & Shiquanhe & 32.5000 & 80.0830 & 4280 & $1962-2012$ \\
\hline 36 & Khot & 36.5167 & 72.5833 & 3505 & 1994-2012 & 103 & Shopian & 33.7200 & 74.8300 & 1615 & $1960-1970$ \\
\hline 37 & Khunjrab & 36.8500 & 75.4000 & 4730 & 1995-2012 & 104 & Sonemarg & 34.3167 & 75.3167 & 2515 & 1902-1969 \\
\hline 38 & Kotli & 33.4847 & 73.8811 & 610 & $1984-2001$ & 105 & Sopore & 34.3000 & 74.4700 & 1574 & $1930-1970$ \\
\hline 39 & Lora & 33.8833 & 73.2833 & 1482 & 1989-1992 & 106 & Srinagar & 34.0833 & 74.8333 & 1587 & 1993-2013 \\
\hline 40 & Mangla & 33.1333 & 73.6333 & 305 & 1984-2001 & 107 & Uttamchipura & 34.5000 & 74.6700 & 3145 & $1901-1956$ \\
\hline 41 & Naltar & 36.2167 & 74.2667 & 2810 & 1995-2012 & 108 & Verinagh & 33.5300 & 75.2500 & 1646 & $1965-1970$ \\
\hline 42 & Naran & 34.9000 & 73.6500 & 2363 & 1984-2001 & 109 & Alambar & 36.7000 & 73.4833 & 4400 & 1991-1999 \\
\hline 43 & Oghi & 34.5000 & 73.0167 & 1128 & $1984-2001$ & 110 & Bagrot & 36.0167 & 74.5500 & 2310 & 1993-2009 \\
\hline 44 & Palandri & 33.7167 & 73.7000 & 1402 & $1984-2001$ & 111 & Bulibalsirbar & 36.3667 & 73.2500 & 4050 & 1991-1999 \\
\hline 45 & Phulra & 34.3333 & 73.0833 & 915 & 1984-2001 & 112 & Garmashbar & 36.5167 & 73.5333 & 3600 & 1991-1999 \\
\hline 46 & Pir Chenasi & 34.3850 & 73.5450 & 2650 & 2004-2013 & 113 & Khaimetbar & 36.5000 & 73.0500 & 3600 & 1991-1999 \\
\hline 47 & Puran & 34.7500 & 72.7000 & 1067 & $1984-2001$ & 114 & Khunjrab & 36.8800 & 74.4167 & 4700 & 1993-2012 \\
\hline 48 & Rama & 35.3583 & 74.8056 & 3140 & 1999-2012 & 115 & Dadormal & 36.0167 & 74.4167 & 3780 & 1991-1999 \\
\hline 49 & Ratu & 35.1528 & 74.8056 & 2920 & 1999-2013 & 116 & Dame & 36.0500 & 74.6667 & 3560 & 1991-1999 \\
\hline 50 & Rawlakot & 33.8667 & 74.2667 & 1677 & $1984-2001$ & 117 & Diran & 36.0500 & 74.6000 & 3650 & 1991-1999 \\
\hline 51 & Saifulmulk & 34.8438 & 73.6875 & 3200 & $2000-2013$ & 118 & Baldihel & 36.3500 & 74.8000 & 3900 & 1994-1996 \\
\hline 52 & Sehrkakota & 33.7333 & 73.9667 & 915 & $1984-2001$ & 119 & Sentik & 33.9967 & 75.9500 & 4908 & 1963-1980 \\
\hline 53 & Shahpur & 34.9167 & 72.6667 & 2012 & $1984-2001$ & 120 & Nun Kun $\mathrm{N}$ & 34.1219 & 76.1014 & 5200 & 1973-1980 \\
\hline 54 & Shangla & 34.8808 & 72.5908 & 2160 & $2000-2007$ & 121 & Batura & 36.6667 & 74.3833 & 4840 & 1973-1974 \\
\hline 55 & Shendure & 36.0861 & 72.5250 & 3719 & $1994-2012$ & 122 & Baltoro & 35.8778 & 76.5508 & 5500 & 1973-1980 \\
\hline 56 & Shigar & 35.5300 & 75.5917 & 2470 & 1996-2012 & 123 & Urdok & 35.7669 & 76.7025 & 5400 & 2004-2006 \\
\hline 57 & Shinkiari & 34.4667 & 73.2667 & 991 & 1984-2001 & 124 & Whaleback & 36.0572 & 75.5915 & 4900 & 1985-1986 \\
\hline 58 & Shogran & 34.6200 & 73.4856 & 3205 & $2000-2013$ & 125 & Approach & 36.0678 & 75.6331 & 5100 & $1985-1987$ \\
\hline 59 & Tandar & 33.2039 & 73.9764 & 671 & $1984-2001$ & 126 & Hispar East & 35.8495 & 75.5064 & 4830 & 1985-1988 \\
\hline 60 & Tarbela & 34.0667 & 72.7700 & 610 & 1984-2001 & 127 & Hispar Pass & 36.0281 & 75.5215 & 5100 & 1984-1986 \\
\hline 61 & Ushkore & 36.0175 & 73.3583 & 3353 & 1999-2012 & 128 & Hispar Dome & 36.0109 & 75.5187 & 5450 & 1982-1986 \\
\hline 62 & Yasin & 36.6333 & 73.3000 & 3353 & 1999-2013 & 129 & Khurdopin & 36.1338 & 75.6197 & 5520 & 1984-1986 \\
\hline 63 & Yugo & 35.1833 & 76.1000 & 2469 & $1984-2001$ & 130 & Nanga Parbat & 35.1672 & 74.4444 & 4500 & 1984-1997 \\
\hline 64 & Zani & 36.2833 & 72.1500 & 3000 & 1994-2012 & 131 & Siachin A & 35.4707 & 77.0376 & 4800 & 1986-1991 \\
\hline 65 & Ziarat & 36.8333 & 74.2778 & 3669 & $1995-2012$ & 132 & Siachin B & 35.5235 & 76.9915 & 4950 & 1986-1992 \\
\hline 66 & Askole & 35.6806 & 75.8153 & 3015 & $2005-2008$ & 133 & Siachin C & 35.5187 & 76.9116 & 5050 & 1986-1993 \\
\hline 67 & Urdukas & 35.7281 & 76.2861 & 3926 & 2004-2008 & 134 & Siachin D & 35.6242 & 76.8592 & 5350 & 1986-1994 \\
\hline
\end{tabular}




\section{Supplementary Material - Chapter 3}

This supplementary material contains additional information to further illustrate the data and methods. The observed data of daily and monthly precipitation, temperature and wind speed was collected from different sources. Keeping in view scarcity of the observed data in the study area, we selected all the stations that covered at least three years of data to cover the recent installations. Daily precipitation observations were converted into monthly totals if no more than three consecutive days or five intermittent days were missing in a month. Similarly, seasonal and annual totals were calculated if no month was missing in a season or year.

Information regarding precipitation gauge type, use of wind shield if any, orifice area and height of the gauge orifice were taken from Sevruk and Klemm (1989), BIS (1992a\&b) and from PMD and WAPDA through personal communications. The precipitation data available for 326 observatories (including 21 virtual stations located at the accumulation zones of major glaciers where average annual net precipitation is estimated from mass balance studies) are inconsistent in terms of time period. The stations located in Pakistani parts of the basin (western parts) possess relatively long-term and updated data. The data of stations located in upper Kabul basin in Afghanistan are also updated. The precipitation data of the stations located in Indian side are publicly available only from 1901-1971. Therefore, the study area is divided into two parts (i.e. eastern and western part) depending on maximum availability of precipitation data (Figure S-3.1). The eastern part covering Shyok, Indus up to Kharmong, Jhelum, Chenab, Ravi, Beas and Sutlej basins use the data period of 1961-1970, while the western part spread over the remaining sub-basins uses the time period of 1999-2011. The metadata of 305 precipitation observatories and 21 glacier points used in this study are outlined and described in the supplementary material (Table S-3.1).

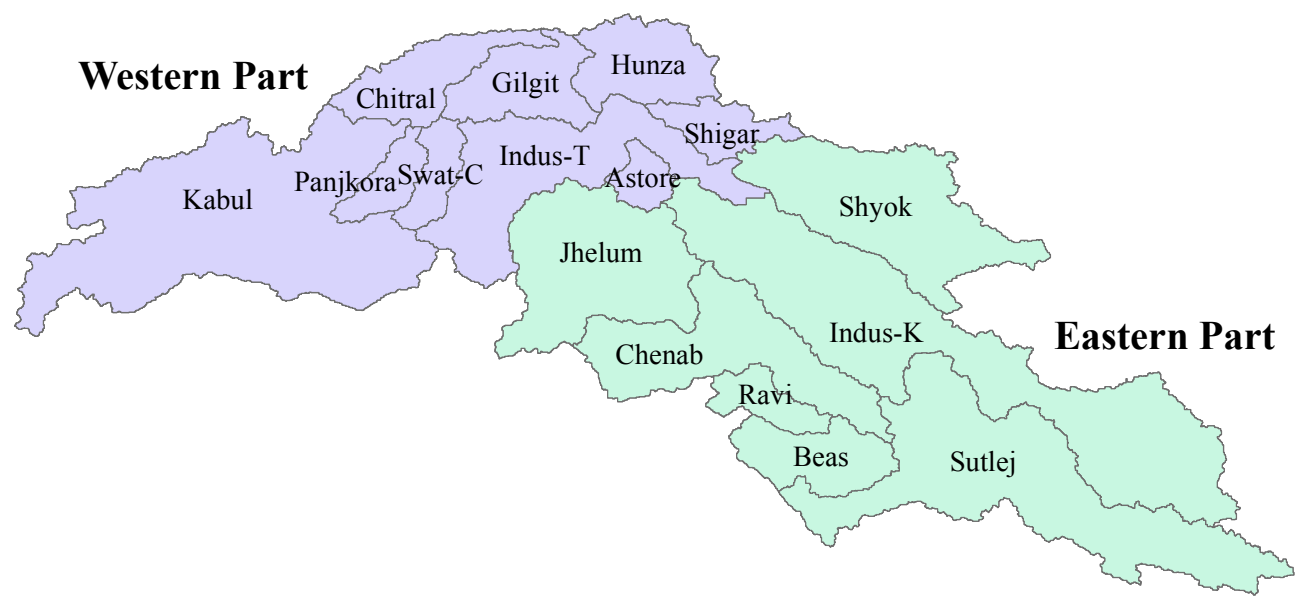

Figure S-3.1: Partition of study area in two zones as per availability of precipitation data

Out of 328 stations, temperature data was available for only 115 stations (Table S-3.1). We therefore derived monthly scale maximum and minimum temperature lapse rates based on elevation and latitude and estimated these parameters for the remaining stations. Air 
temperature varies with elevation due to change in air pressure and the rate of its change is known as the adiabatic lapse rate, which on average is about $6.5{ }^{0} \mathrm{C} \mathrm{km}^{-1}$ globally. Temperature at a particular point also changes with its latitude, its rate of change however varies considerably between tropics, temperate and Polar Regions due to curvature of earth's surface which affects the amount of sunlight received by a particular zone. Yet, latitude is often overlooked in lapse rate-based temperature estimates. Our study area lays in between 30.3 and 37.1 degree latitude, which is in the North Temperate Zone, where the temperature difference between the tropic of cancer (23.5 degree) and 40-degree $\mathrm{N}$ can be about $10{ }^{\circ} \mathrm{C}\left(\sim 0.606{ }^{\circ} \mathrm{C} /\right.$ latitude degree $)$. Keli et al. (2011) however noted latitudinal effect of $-0.36^{\circ} \mathrm{C} /$ latitude degree in the Qinghai-Tibet Plateau. As the observed maximum and minimum temperatures showed significant correlation with both elevation and latitude (Fig. S2-S5), multiple regressions at monthly scale were used to derive the combined lapse rates for the stations that do not have temperature data. Separate lapse rates for mean monthly maximum and minimum temperatures are derived.

Wind-induced under-catch of liquid precipitation is adjusted by following Adam and Lattenmaier (2003), who mainly used Legates (1987) model in which the wind-induced under-catch of liquid precipitation (rain) has been expressed in terms of the correction factor $\left(\boldsymbol{K}_{\boldsymbol{r}}\right)$ defined as the ratio of ground truth precipitation to gauge-measured precipitation. If the ground truth or reference gauge precipitation measurements are not available, the value of $\boldsymbol{K}_{\boldsymbol{r}}$ can be approximated by:

$$
\begin{gathered}
K_{r}=1+0.008 \mu^{2} V_{h p}^{2} \\
K_{r}=1+0.011 \mu^{2} V_{h p}^{2}
\end{gathered}
$$

for gauge orifice area of $127 \mathrm{~cm}^{2}$

for gauge orifice area of $200 \mathrm{~cm}^{2}$

Where $\mathbf{V}_{\mathbf{h p}}$ is wind speed at gauge orifice, and $\boldsymbol{\mu}$ is a transfer coefficient which is given by:

$$
\mu=\frac{p}{100} \cdot \frac{273}{T+273} \cdot \frac{p}{p+0.378 e_{\mathrm{a}}}
$$

Where $\boldsymbol{T}$ is mean air temperature $\left({ }^{0} \mathrm{C}\right), \boldsymbol{p}$ is sea-level pressure $(\mathrm{kPa})$ and $\boldsymbol{e}_{\mathbf{a}}$ is vapour pressure $(\mathrm{kPa})$ expressed by:

$$
e_{a}=0.2 \exp \left[19.0629+0.138952 \ln \left(P_{\mathrm{m}}\right)-\frac{4798.05}{T+273}\right]
$$

It is well recognized that for a given wind speed; gauge under-catch for snow is much higher than that for rain because snow has larger surface area per unit mass. The proportion of solid precipitation $(R)$ is determined by the model also suggested by Legates (1987), which is given by:

$$
R=\frac{1}{1+1.61(1.35)^{T}}
$$

The mathematical models to adjust the wind-induced under-catch of precipitation are relatively more sensitive to wind speed. However, the wind observations needed for adjustment of gauge under-catches are rarely coincident in time and space with precipitation observations. The mean monthly values of wind speed may not match with the wind speed during the precipitation events. Sevruk (1982) referred a mathematical model 
developed by Bogdanova (1969) that relates mean monthly wind speed to wind speed during precipitation event as:

$$
V_{\mathrm{p}}=\boldsymbol{L}_{\boldsymbol{r}} \cdot \boldsymbol{V}
$$

Where $\boldsymbol{V}_{\mathbf{p}}$ is wind speed during precipitation event, $\boldsymbol{V}$ is mean monthly wind speed $(\mathrm{m} / \mathrm{sec})$ and $\boldsymbol{L}_{\boldsymbol{r}}$ is an empirical coefficient which depends on the form of precipitation and number of precipitation days and is expressed as:

$$
\begin{aligned}
& L_{r}=1.12+0.295(0.826)^{M} \\
& L_{r}=1.37-0.0599(1.065)^{M}
\end{aligned}
$$

for solid precipitation

Where $\boldsymbol{M}$ is number of precipitation days per month, which is determined by counting the number of days during each month in which precipitation exceeded the threshold level of $0.1 \mathrm{~mm}$.

The model for adjustment of liquid precipitation requires wind speed at gauge height, which is approximated from the wind speed at the reference height of anemometer $(10 \mathrm{~m})$ using the extensively used (e.g. Mekonnen et al., 2015; Ackere et al., 2015; Stepek and Wijnant 2011; Yang et al., 1998) Monin Obukhov theory (Obukhov, 1971; Businger and Yaglom, 1971), which is given by:

$$
V_{i} \approx V_{\text {ref }} \cdot \frac{\ln \left(\frac{z_{i}}{z_{0}}\right)}{\ln \left(\frac{z_{\text {ref }}}{z_{0}}\right)}
$$

Where $\mathbf{V}_{\mathbf{i}}$ is wind speed $\left(\mathrm{m} \mathrm{s}^{-1}\right.$ ) to be calculated at $\boldsymbol{Z}_{\mathbf{i}}$ height $(\mathrm{m}), \boldsymbol{V}_{\text {ref }}$ is known velocity (m $\left.\mathrm{s}^{-1}\right)$ at the reference height $\boldsymbol{Z}_{\text {ref }}(\mathrm{m})$, and $\boldsymbol{Z}_{\mathbf{0}}$ is roughness length $(\mathrm{m})$ depending upon type of landscape. WMO (2014) recommended $\boldsymbol{Z}_{\mathbf{0}}$ value of $0.01 \mathrm{~m}$ for winter and $0.03 \mathrm{~m}$ for summer months, which were used by Adam and Lattenmaier (2003). We used $\boldsymbol{Z}_{\mathbf{0}}$ values of $0.01 \mathrm{~m}$ for Dec-Mar, $0.02 \mathrm{~m}$ for Apr-May \& Oct-Nov, and 0.03 for Jun-Sep months, which are appropriate for winter snow surfaces and open agricultural or short grassed rangeland areas without fences and hedgerows.

We also considered trace events of precipitation. A precipitation event of less than $0.1 \mathrm{~mm}$ is usually unmeasurable by most gauges and is generally recorded as trace precipitation. Goodison et al. (1998) indicated trace precipitation ranging between 0.0 and $0.2 \mathrm{~mm}$ per event. WMO 2014 recommended value $0.1 \mathrm{~mm}$ for rainfall and $0.2 \mathrm{~mm}$ for snowfall. Ye et al. (2004) recommended a value of $0.1 \mathrm{~mm}$ for each precipitation day, regardless of the number of trace events per day.

Wetting loss depends on geometry and material of collector gauge, form and incidence of precipitation, and frequency of precipitation measurements (Legates (1987). Yang (1988) and Yang et al. (1991) reported a wetting loss of $0.23 \mathrm{~mm}$ for rainfall, $0.30 \mathrm{~mm}$ for snow and $0.29 \mathrm{~mm}$ for mixed precipitation based on precipitation measurements at the Tianshan site. Ren and Li (2007) reported a mean wetting loss of about $0.19 \mathrm{~mm}$ for the total precipitation over eastern China. Legates (1987) compiled and reported mean wetting loss ranging from $0.02-0.30 \mathrm{~mm}$ per precipitation event for various gauge types, with $0.2 \mathrm{~mm}$ for MK2 model and $0.3 \mathrm{~mm}$ for Tretyakov model of rain gauge, which are used for this 
study. However, for automated precipitation gauges, the values are cut by half to account for relatively lower wetting losses in these gauges.

The number of trace and precipitation events is calculated from the available daily records of the stations; while for the stations having only monthly data, we approximated these data from the daily data of the nearby stations. As majority of the precipitation gauges in our study area are manual giving only the daily precipitation amounts. Hence, if a trace or precipitation event had happened multiple times in a day, it is recorded only once. Therefore, there are chances that the actual number of trance and precipitation events may be more than what we have assumed.

The evaporation loss from the precipitation gauge during an event until the measurement of precipitation generally depends on the type of gauge and prevailing climate. Aaltonen et al. (1993) and Zhang et al. (2004) observed only a nominal evaporation rate of $0.10-0.20 \mathrm{~mm}$ $\mathrm{d}^{-1}$ during winter in Finland and Mongolia. The temperature in most parts of the highaltitude Indus basin remains below freezing point throughout the year and there is insufficient information regarding basin level evaporation rates from the national gauges. Therefore, the evaporation loss from the gauges during the precipitation events is considered insignificant and is neglected.

WAPDA is the custodian of all river flow data in Pakistan. The up-stream diversions of river flows (e.g. Warsak left and right bank canals taking off from upstream of Kabul at Warsak; Kabul river canal, upper Swat canal, lower Swat canal and Doaba canal taking off from upstream of Kabul at Nowshera; Pehur high level canal commissioned in 2005, Ichhar canal, upper Siran and lower Siran canals taking off from upstream of Indus at Tarbela; and the Beas-Sutlej Link canal taking off from upstream of Beas at Pong dam), which are often overlooked by the previous studies, are added to the flows of the respective sub-basins. The Beas-Sutlej Link (BSL) project was commissioned in 1977 and the mean annual inflow from the BSL in the period April 1978 to March 2009 was 4.345x109 m3 (ADB, 2010). This flow is diverted into Sutlej river before the river gauge at Bhakra dam, which need to be subtracted if the data used belongs to the period after 1977. River flow data do not have direct use in this study; rather we use it only for validation of our precipitation estimates. Therefore, we have used the flow data only for the corresponding precipitation data periods in each sub-hydrological basin.

Table S-3.1: List of climatic stations used in this study. The stations from S. No. 1-41 are maintained by PMD, from 42-97 by WAPDA, 98-107 by CAK, 108-285 by IMD, 286-307 by Afghanistan, and 308-328 are virtual stations at the major glaciers. OA is orifice area and GH is gauge height. P, T and $\mathrm{W}$ denote precipitation, temperature and wind speed respectively. TBRG is for tipping bucket raingauge, WG is weighing type rain gauge, SP is snow pillow, SD is snow depth gauge, Sn is Symon's gauge, and VS is for virtual stations. All the precipitation gauges are without windshields.

\begin{tabular}{llllllllllc}
\hline $\begin{array}{l}\text { S. } \\
\#\end{array}$ & $\begin{array}{l}\text { Station } \\
\text { Name }\end{array}$ & $\begin{array}{l}\text { Lon } \\
(\mathbf{d d})\end{array}$ & $\begin{array}{l}\text { Lat } \\
(\mathbf{d d})\end{array}$ & $\begin{array}{l}\text { Elev } \\
(\mathbf{m})\end{array}$ & $\begin{array}{l}\text { Data } \\
\text { Period }\end{array}$ & $\begin{array}{l}\text { Para- } \\
\text { meters }\end{array}$ & $\begin{array}{l}\text { Data } \\
\text { Int. }\end{array}$ & $\begin{array}{l}\text { Gauge } \\
\text { Type }\end{array}$ & $\begin{array}{l}\text { OA } \\
\left(\mathbf{c m}^{2}\right)\end{array}$ & $\begin{array}{l}\text { GH } \\
(\mathbf{c m})\end{array}$ \\
\hline 1 & Astore & 74.90000 & 35.36667 & 2394 & $1999-2011$ & P T W & Daily & TBRG & 400 & 60 \\
2 & Babusar & 74.04444 & 35.16000 & 4160 & $2005-2013$ & P T & Daily & MK2 & 127 & 30 \\
3 & Balakot & 72.35000 & 34.55000 & 995 & $1999-2011$ & P T W & Daily & TBRG & 400 & 60 \\
4 & Bhimber & 74.10000 & 33.00000 & 365 & $1941-1947$ & P & Monthly & MK2 & 127 & 30 \\
5 & Bunji & 74.63333 & 35.66667 & 1372 & $1999-2011$ & P T W & Daily & MK2 & 127 & 30 \\
6 & Chakdara & 72.00000 & 34.60000 & 670 & $1970-1975$ & P & Monthly & MK2 & 127 & 30
\end{tabular}




\begin{tabular}{|c|c|c|c|c|c|c|c|c|c|}
\hline 7 & Chakwal & 72.73000 & 32.93300 & 521 & $1999-2011$ & P T W & Daily & MK2 & 127 \\
\hline 8 & Cherat & 71.89050 & 33.82250 & 1372 & $1999-2011$ & P T W & Daily & MK2 & 127 \\
\hline 9 & Chilas & 74.10000 & 35.41667 & 1251 & 1999-2011 & P T W & Daily & MK2 & 127 \\
\hline 10 & Chitral & 71.83333 & 35.85000 & 1498 & 1999-2011 & P T W & Daily & MK2 & 127 \\
\hline 11 & Dir & 71.85000 & 35.20000 & 1425 & 1999-2011 & P T W & Daily & MK2 & 127 \\
\hline 12 & Drosh & 71.78333 & 35.56667 & 1464 & 1999-2011 & P T W & Daily & MK2 & 127 \\
\hline 13 & GD Poto & 73.61667 & 34.21667 & 814 & $1961-1970$ & P T W & Daily & TBRG & 400 \\
\hline 14 & Gilgit & 74.33333 & 35.91667 & 1460 & 1999-2011 & P T W & Daily & TBRG & 400 \\
\hline 15 & Gupis & 73.40000 & 36.16667 & 2156 & 1999-2011 & P T W & Daily & MK2 & 127 \\
\hline 16 & Hunza & 74.64600 & 36.32200 & 2374 & $2007-2015$ & $\mathrm{PT}$ & Daily & MK2 & 127 \\
\hline 17 & Islamabad AP & 73.10000 & 33.61700 & 508 & 1999-2011 & P T W & Daily & TBRG & 400 \\
\hline 18 & Jhelum & 73.71700 & 32.93300 & 234 & $1961-1970$ & P T W & Daily & MK2 & 127 \\
\hline 19 & Kakul & 73.25000 & 34.18333 & 1308 & $1999-2011$ & P T W & Daily & MK2 & 127 \\
\hline 20 & KalamP & 72.98330 & 35.83330 & 2103 & $2004-2015$ & P T W & Daily & MK2 & 127 \\
\hline 21 & Kamra & 72.40000 & 33.86700 & 325 & $2008-2012$ & $\mathrm{P}$ & Monthly & MK2 & 127 \\
\hline 22 & Kohat & 71.43330 & 33.58330 & 513 & $1954-2015$ & P T W & Daily & MK2 & 127 \\
\hline 23 & Kotli-P & 73.90000 & 33.51667 & 614 & $1961-1970$ & P T W & Daily & MK2 & 127 \\
\hline 24 & Landikotal & 71.20000 & 34.10000 & 1068 & $1951-1970$ & $\mathrm{P}$ & Monthly & MK2 & 127 \\
\hline 25 & Lower Dir & 71.81670 & 34.83330 & 786 & $2008-2015$ & P T W & Daily & MK2 & 127 \\
\hline 26 & Malamjaba & 72.90000 & 34.75000 & 2591 & $2003-2015$ & P T W & Daily & MK2 & 127 \\
\hline 27 & Mangla-P & 73.63000 & 33.06000 & 283 & $1999-2011$ & P T W & Daily & MK2 & 127 \\
\hline 28 & Miranshah & 70.12000 & 32.98000 & 912 & $1999-2011$ & $\mathrm{P}$ & Monthly & MK2 & 127 \\
\hline 29 & Mirkhani & 71.70000 & 35.50000 & 1250 & $2008-2015$ & $\mathrm{PT}$ & Daily & MK2 & 127 \\
\hline 30 & Mirpur & 73.80000 & 33.20000 & 361 & $1940-1947$ & P T W & Daily & MK2 & 127 \\
\hline 31 & Misgar & 74.76720 & 36.78837 & 3080 & $1951-1978$ & $\mathrm{P}$ & Monthly & MK2 & 127 \\
\hline 32 & Murree & 73.40000 & 33.90000 & 2168 & $1961-1970$ & P T W & Daily & MK2 & 127 \\
\hline 33 & Muzaffarabad & 73.48333 & 34.36667 & 702 & 1999-2011 & P T W & Daily & TBRG & 400 \\
\hline 34 & Parachinar & 70.08333 & 33.86667 & 1725 & 1999-2011 & P T W & Daily & MK2 & 127 \\
\hline 35 & Pattan & 73.00000 & 35.10000 & 752 & 2004-2015 & P T W & Daily & MK2 & 127 \\
\hline 36 & Peshawar AP & 71.58300 & 34.01700 & 360 & 1999-2011 & P T W & Daily & TBRG & 400 \\
\hline 37 & Rawlakot-P & 73.80000 & 33.85000 & 1680 & $2003-2015$ & P T W & Daily & MK2 & 127 \\
\hline 38 & Risalpur & 71.98300 & 34.06700 & 317 & 1999-2011 & P T W & Daily & MK2 & 127 \\
\hline 39 & Saidusharif & 72.35000 & 34.73333 & 961 & 1999-2011 & P T W & Daily & MK2 & 127 \\
\hline 40 & Sialkot & 74.53333 & 32.51667 & 255 & $1961-1970$ & P T W & Daily & MK2 & 127 \\
\hline 4 & Skardu & 75.68333 & 35.30000 & 2210 & 1999-2011 & P T W & Daily & MK2 & 127 \\
\hline 42 & Abazai & 71.55000 & 34.43333 & 320 & $1985-1997$ & $\mathrm{P}$ & Daily & MK2+WG & 127 \\
\hline 43 & Amandara & 71.98200 & 34.62542 & 666 & $1985-1996$ & $\mathrm{P}$ & Daily & $\mathrm{MK} 2+\mathrm{WG}$ & 127 \\
\hline 44 & Bagh & 73.80000 & 33.98333 & 1159 & 1963-1975 & $\mathrm{P}$ & Monthly & MK2+WG & 127 \\
\hline 45 & Besham & 72.88333 & 34.93333 & 480 & $1995-2006$ & $\mathrm{PT}$ & Daily & MK2+WG & 127 \\
\hline 46 & Burzil & 75.07910 & 34.89940 & 4030 & 1999-2011 & $\mathrm{PT}$ & Daily & SP & 400 \\
\hline 47 & Charbagh & 72.44300 & 34.83420 & 1024 & 1991-1997 & $\mathrm{P}$ & Monthly & MK2+WG & 127 \\
\hline 48 & Charsadda & 71.71667 & 34.11667 & 276 & 1999-2011 & $\mathrm{P}$ & Daily & MK2+WG & 127 \\
\hline 49 & Dagar & 72.48639 & 34.51000 & 732 & 1999-2011 & $\mathrm{PT}$ & Daily & MK2+WG & 127 \\
\hline 50 & Deosai & 75.60000 & 35.10000 & 3910 & 1999-2011 & $\mathrm{PT}$ & Daily & SP & 400 \\
\hline 51 & Dhudnial & 74.11700 & 34.70000 & 534 & 1983-1990 & $\mathrm{PT}$ & Daily & MK2+WG & 127 \\
\hline 52 & Domel & 73.46889 & 34.36778 & 686 & 1984-1990 & $\mathrm{PT}$ & Daily & $\mathrm{MK} 2+\mathrm{WG}$ & 127 \\
\hline 53 & Doyian & 74.70417 & 35.54500 & 2454 & $1999-2011$ & $\mathrm{PT}$ & Daily & MK2+WG & 127 \\
\hline 54 & Fort Lokhart & 70.91864 & 33.55590 & 1996 & 1963-2009 & $\mathrm{PT}$ & Daily & MK2+WG & 127 \\
\hline
\end{tabular}




\begin{tabular}{|c|c|c|c|c|c|c|c|c|c|}
\hline 55 & Gujar Khan & 73.30000 & 33.25000 & 457 & $1984-1990$ & $\mathrm{PT}$ & Daily & MK2+WG & 127 \\
\hline 56 & Hushy & 76.40000 & 35.36667 & 3010 & $1999-2011$ & $\mathrm{PT}$ & Daily & SP & 400 \\
\hline 57 & Jabbar & 73.22778 & 34.67167 & 2134 & 1991-2001 & $\mathrm{PT}$ & Daily & $\mathrm{MK} 2+\mathrm{WG}$ & 127 \\
\hline 58 & Kachura & 75.42000 & 35.35000 & 2341 & 1991-2006 & $\mathrm{PT}$ & Daily & $\mathrm{MK} 2+\mathrm{WG}$ & 127 \\
\hline 59 & KalamW & 72.60100 & 35.47000 & 2744 & $1999-2011$ & $\mathrm{PT}$ & Daily & MK2+WG & 127 \\
\hline 60 & Kallar & 73.36667 & 33.41667 & 518 & 1984-1990 & $\mathrm{PT}$ & Daily & MK2+WG & 127 \\
\hline 61 & Kelash & 71.65472 & 35.69550 & 2810 & $1999-2011$ & $\mathrm{PT}$ & Daily & SP & 400 \\
\hline 62 & Khandar & 74.05000 & 33.50000 & 1067 & $1984-1990$ & $\mathrm{PT}$ & Daily & MK2+WG & 127 \\
\hline 63 & Khot & 72.58333 & 36.51667 & 3505 & $1999-2011$ & $\mathrm{PT}$ & Daily & SP & 400 \\
\hline 64 & Khunjrab-W & 75.33200 & 36.81200 & 4700 & $1999-2011$ & $\mathrm{PT}$ & Daily & SP & 400 \\
\hline 65 & Kotli-W & 73.88111 & 33.48472 & 610 & 1984-1990 & $\mathrm{PT}$ & Daily & MK2+WG & 127 \\
\hline 66 & Lora & 73.28333 & 33.88333 & 1482 & 1989-1992 & $\mathrm{PT}$ & Daily & MK2+WG & 127 \\
\hline 67 & Malakand & 71.90400 & 34.50230 & 603 & 1988-1997 & $\mathrm{P}$ & Daily & MK2+WG & 127 \\
\hline 68 & Mangla-W & 73.63333 & 33.13333 & 305 & 1971-1978 & $\mathrm{PT}$ & Daily & $\mathrm{MK} 2+\mathrm{WG}$ & 127 \\
\hline 69 & Mardan & 71.96667 & 34.30000 & 283 & $1997-2006$ & $\mathrm{PT}$ & Daily & MK2+WG & 127 \\
\hline 70 & Munda dam & 71.52450 & 34.35100 & 380 & $2000-2006$ & $\mathrm{PT}$ & Daily & MK2+WG & 127 \\
\hline 71 & Naltar & 74.26667 & 36.21667 & 2810 & $1999-2011$ & $\mathrm{PT}$ & Daily & SP & 400 \\
\hline 72 & Naran & 73.60700 & 34.96000 & 2363 & $1971-1978$ & $\mathrm{PT}$ & Daily & MK2+WG & 127 \\
\hline 73 & Oghi & 73.01667 & 34.50000 & 1128 & $1995-2006$ & $\mathrm{PT}$ & Daily & $\mathrm{MK} 2+\mathrm{WG}$ & 127 \\
\hline 74 & Palandri & 73.70000 & 33.71667 & 1402 & 1971-1978 & $\mathrm{PT}$ & Daily & $\mathrm{MK} 2+\mathrm{WG}$ & 127 \\
\hline 75 & Phulra & 73.08333 & 34.33333 & 915 & $1995-2006$ & $\mathrm{PT}$ & Daily & $\mathrm{MK} 2+\mathrm{WG}$ & 127 \\
\hline 76 & Pir Chenasi & 73.54500 & 34.38500 & 2650 & $1999-2011$ & $\mathrm{PT}$ & Daily & SP & 400 \\
\hline 77 & Puran & 72.70000 & 34.75000 & 1067 & $1995-2006$ & $\mathrm{PT}$ & Daily & MK2+WG & 127 \\
\hline 78 & Qalangi & 71.80000 & 34.63000 & 688 & $1999-2011$ & $\mathrm{P}$ & Monthly & MK2+WG & 127 \\
\hline 79 & Rama & 74.80556 & 35.35833 & 3140 & $1999-2011$ & $\mathrm{PT}$ & Daily & SP & 400 \\
\hline 80 & Ratu & 74.80556 & 35.15278 & 2920 & $1999-2011$ & $\mathrm{PT}$ & Daily & SP & 400 \\
\hline 81 & Rawlakot-W & 73.76667 & 33.86667 & 1676 & 1971-1978 & $\mathrm{PT}$ & Daily & $\mathrm{MK} 2+\mathrm{WG}$ & 127 \\
\hline 82 & Saifulmulk & 73.68750 & 34.84375 & 3200 & $2000-2011$ & $\mathrm{PT}$ & Daily & SP & 400 \\
\hline 83 & Sehrkakota & 73.96667 & 33.73333 & 915 & $1984-1990$ & $\mathrm{PT}$ & Daily & MK2+WG & 127 \\
\hline 84 & Shahpur & 72.66667 & 34.91667 & 2012 & $1995-2006$ & $\mathrm{PT}$ & Daily & MK2+WG & 127 \\
\hline 85 & Shangla & 72.59083 & 34.88083 & 2160 & $1999-2011$ & $\mathrm{PT}$ & Daily & SP & 400 \\
\hline 86 & Shendure & 72.52500 & 36.08611 & 3719 & 1999-2011 & $\mathrm{PT}$ & Daily & SP & 400 \\
\hline 87 & Shigar & 75.59167 & 35.53000 & 2470 & $1999-2011$ & $\mathrm{PT}$ & Daily & SP & 400 \\
\hline 88 & Shinkiari & 73.26667 & 34.46667 & 991 & $1995-2006$ & $\mathrm{PT}$ & Daily & MK2+WG & 127 \\
\hline 89 & Shogran & 73.48556 & 34.62000 & 3205 & $2000-2008$ & $\mathrm{PT}$ & Daily & SP & 400 \\
\hline 90 & Tandar & 73.97639 & 33.20389 & 671 & $1984-1990$ & $\mathrm{PT}$ & Daily & MK2+WG & 127 \\
\hline 91 & Tarbela & 72.77000 & 34.06667 & 610 & $1999-2011$ & $\mathrm{PT}$ & Daily & MK2+WG & 127 \\
\hline 92 & Ushkore & 73.35833 & 36.01750 & 3353 & 1999-2011 & $\mathrm{PT}$ & Daily & SP & 400 \\
\hline 93 & Yasin & 73.30000 & 36.63333 & 3353 & 1999-2011 & $\mathrm{PT}$ & Daily & SP & 400 \\
\hline 94 & Yugo & 76.08000 & 35.02000 & 2469 & $1999-2011$ & $\mathrm{PT}$ & Daily & MK2+WG & 127 \\
\hline 95 & Zani & 72.15000 & 36.28333 & 3000 & 1999-2011 & $\mathrm{PT}$ & Daily & SP & 400 \\
\hline 96 & Ziarat & 74.27778 & 36.83333 & 3669 & 1999-2011 & $\mathrm{PT}$ & Daily & SP & 400 \\
\hline 97 & Zulam Br. & 71.79388 & 34.76500 & 680 & 2000-2006 & $\mathrm{P}$ & Daily & MK2+WG & 127 \\
\hline 98 & Alambar & 73.48333 & 36.70000 & 4400 & 1991-1999 & $\mathrm{P}$ & Monthly & TBRG+SD & 127 \\
\hline 99 & Bagrot & 74.55000 & 36.01667 & 2310 & 1999-2009 & $\mathrm{P}$ & Daily & TBRG+SD & 127 \\
\hline 100 & Baldihel & 74.80000 & 36.35000 & 3900 & 1991-1999 & $\mathrm{P}$ & Monthly & TBRG+SD & 127 \\
\hline 101 & Bulibalsirbar & 73.25000 & 36.36667 & 4050 & 1991-1999 & $\mathrm{P}$ & Monthly & TBRG+SD & 127 \\
\hline 102 & Dadormal & 74.58333 & 36.01667 & 3560 & 1991-1999 & $\mathrm{P}$ & Monthly & TBRG+SD & 127 \\
\hline
\end{tabular}




\begin{tabular}{|c|c|c|c|c|c|c|c|c|c|}
\hline 103 & Dame & 74.72608 & 36.00293 & 3780 & 1991-1999 & $\mathrm{P}$ & Monthly & TBRG+SD & 127 \\
\hline 104 & Diran & 74.60000 & 36.05000 & 3650 & 1991-1999 & $\mathrm{P}$ & Monthly & $\mathrm{TBRG}+\mathrm{SD}$ & 127 \\
\hline 105 & Garmashbar & 73.53333 & 36.51667 & 3600 & 1991-1999 & $\mathrm{P}$ & Monthly & $\mathrm{TBRG}+\mathrm{SD}$ & 127 \\
\hline 106 & Khaimetbar & 73.05000 & 36.50000 & 3600 & 1991-1999 & $\mathrm{P}$ & Monthly & $\mathrm{TBRG}+\mathrm{SD}$ & 127 \\
\hline 107 & Khunjrab-C & 75.21543 & 36.92630 & 4730 & $1999-2011$ & $\mathrm{PT}$ & Daily & $\mathrm{TBRG}+\mathrm{SD}$ & 127 \\
\hline 108 & Akhnoor & 74.73333 & 32.88333 & 331 & $1960-1970$ & $\mathrm{P}$ & Monthly & $\mathrm{MK} 2 / \mathrm{Sn}$ & 127 \\
\hline 109 & Anantnag & 75.15000 & 33.72000 & 1588 & $1958-1970$ & $\mathrm{P}$ & Monthly & $\mathrm{MK} 2 / \mathrm{Sn}$ & 127 \\
\hline 110 & Arizal & 74.60000 & 33.92000 & 1615 & $1961-1970$ & $\mathrm{P}$ & Monthly & $\mathrm{MK} 2 / \mathrm{Sn}$ & 127 \\
\hline 111 & Arki & 76.95000 & 31.15000 & 1130 & 1959-1969 & $\mathrm{P}$ & Monthly & $\mathrm{MK} 2 / \mathrm{Sn}$ & 127 \\
\hline 112 & Babapura & 75.02000 & 33.78000 & 1589 & $1961-1970$ & $\mathrm{P}$ & Monthly & $\mathrm{MK} 2 / \mathrm{Sn}$ & 127 \\
\hline 113 & Badarwah & 75.71667 & 32.96667 & 1690 & 1944-1968 & $\mathrm{P}$ & Monthly & $\mathrm{MK} 2 / \mathrm{Sn}$ & 127 \\
\hline 114 & Badgam & 74.58000 & 33.83000 & 1587 & $1961-1970$ & $\mathrm{P}$ & Monthly & $\mathrm{MK} 2 / \mathrm{Sn}$ & 127 \\
\hline 115 & Bandipura & 74.63000 & 34.42000 & 1638 & $1961-1970$ & $\mathrm{P}$ & Monthly & $\mathrm{MK} 2 / \mathrm{Sn}$ & 127 \\
\hline 116 & Banihal & 75.17000 & 33.50000 & 1630 & $1961-1970$ & $\mathrm{P}$ & Monthly & MK2/Sn & 127 \\
\hline 117 & Banjar Saraj & 77.33333 & 31.63333 & 1520 & $1959-1969$ & $\mathrm{P}$ & Monthly & $\mathrm{MK} 2 / \mathrm{Sn}$ & 127 \\
\hline 118 & Baramula & 74.37000 & 34.20000 & 1572 & $1961-1970$ & $\mathrm{P}$ & Monthly & $\mathrm{MK} 2 / \mathrm{Sn}$ & 127 \\
\hline 119 & Bashila & 77.67000 & 31.17000 & 2250 & $1958-1969$ & $\mathrm{P}$ & Monthly & $\mathrm{MK} 2 / \mathrm{Sn}$ & 127 \\
\hline 120 & Batote & 75.32000 & 33.12000 & 1751 & $1961-1970$ & $\mathrm{P}$ & Monthly & $\mathrm{MK} 2 / \mathrm{Sn}$ & 127 \\
\hline 121 & Bhagtan & 79.00000 & 31.00000 & 1036 & $1958-1965$ & $\mathrm{P}$ & Monthly & $\mathrm{MK} 2 / \mathrm{Sn}$ & 127 \\
\hline 122 & Bhangrotu & 76.93000 & 31.62000 & 762 & $1959-1969$ & $\mathrm{P}$ & Monthly & $\mathrm{MK} 2 / \mathrm{Sn}$ & 127 \\
\hline 123 & Bhuntar & 77.16667 & 31.83333 & 1067 & $1975-1984$ & $\mathrm{P}$ & Monthly & $\mathrm{MK} 2 / \mathrm{Sn}$ & 127 \\
\hline 124 & Bilaspur & 76.75000 & 31.33333 & 587 & $1959-1969$ & $\mathrm{P}$ & Monthly & $\mathrm{MK} 2 / \mathrm{Sn}$ & 127 \\
\hline 125 & Chachiot & 77.02000 & 31.55000 & 1504 & $1958-1964$ & $\mathrm{P}$ & Monthly & MK2/Sn & 127 \\
\hline 126 & Chandigarh & 76.88300 & 30.73300 & 347 & $1961-1970$ & $\mathrm{PT}$ & Monthly & $\mathrm{MK} 2 / \mathrm{Sn}$ & 127 \\
\hline 127 & Charisharif & 74.77000 & 33.87000 & 1616 & $1961-1970$ & $\mathrm{P}$ & Monthly & $\mathrm{MK} 2 / \mathrm{Sn}$ & 127 \\
\hline 128 & Chenani & 75.28000 & 33.03000 & 1122 & $1960-1969$ & $\mathrm{P}$ & Monthly & $\mathrm{MK} 2 / \mathrm{Sn}$ & 127 \\
\hline 129 & Chini Kalpa & 78.25000 & 31.53333 & 2781 & 1958-1969 & $\mathrm{P}$ & Monthly & $\mathrm{MK} 2 / \mathrm{Sn}$ & 127 \\
\hline 130 & Chowari & 76.01667 & 32.45000 & 716 & $1960-1969$ & $\mathrm{P}$ & Monthly & MK2/Sn & 127 \\
\hline 131 & Dalhousie & 75.96667 & 32.53333 & 1959 & $1958-1965$ & $\mathrm{P}$ & Monthly & $\mathrm{MK} 2 / \mathrm{Sn}$ & 127 \\
\hline 132 & Dasuya & 75.63000 & 31.80000 & 228 & $1961-1970$ & $\mathrm{P}$ & Monthly & $\mathrm{MK} 2 / \mathrm{Sn}$ & 127 \\
\hline 133 & Dehra Gopipur & 76.21667 & 31.88333 & 436 & $1958-1969$ & $\mathrm{P}$ & Monthly & $\mathrm{MK} 2 / \mathrm{Sn}$ & 127 \\
\hline 134 & Dharampur & 77.01667 & 30.90000 & 1986 & $1961-1970$ & $\mathrm{P}$ & Monthly & $\mathrm{MK} 2 / \mathrm{Sn}$ & 127 \\
\hline 135 & Dharamshala & 76.38300 & 32.26700 & 1211 & $1961-1970$ & $\mathrm{P}$ & Monthly & $\mathrm{MK} 2 / \mathrm{Sn}$ & 127 \\
\hline 136 & Dharamshala-L & 76.31667 & 32.21667 & 1745 & $1901-1969$ & $\mathrm{P}$ & Monthly & $\mathrm{MK} 2 / \mathrm{Sn}$ & 127 \\
\hline 137 & Dharamshala-U & 76.08000 & 31.82000 & 899 & $1951-1965$ & $\mathrm{P}$ & Monthly & $\mathrm{MK} 2 / \mathrm{Sn}$ & 127 \\
\hline 138 & Digar & 77.75000 & 34.25000 & 5182 & 1956-1964 & $\mathrm{P}$ & Monthly & $\mathrm{MK} 2 / \mathrm{Sn}$ & 127 \\
\hline 139 & Dras & 75.76667 & 34.43333 & 3066 & $1956-1970$ & $\mathrm{P}$ & Monthly & $\mathrm{MK} 2 / \mathrm{Sn}$ & 127 \\
\hline 140 & Durroo & 75.23000 & 33.57000 & 1790 & 1956-1964 & $\mathrm{P}$ & Monthly & $\mathrm{MK} 2 / \mathrm{Sn}$ & 127 \\
\hline 141 & Garhshankar & 76.12000 & 31.22000 & 273 & $1961-1970$ & $\mathrm{P}$ & Monthly & $\mathrm{MK} 2 / \mathrm{Sn}$ & 127 \\
\hline 142 & Gondhla & 77.03000 & 32.52000 & 3144 & $1961-1970$ & $\mathrm{P}$ & Monthly & $\mathrm{MK} 2 / \mathrm{Sn}$ & 127 \\
\hline 143 & GS Nagar & 82.20000 & 29.60000 & 2133 & $1971-1990$ & $\mathrm{P}$ & Monthly & MK2/Sn & 127 \\
\hline 144 & Gulabgarh & 74.93000 & 33.43000 & 2137 & $1941-1947$ & $\mathrm{P}$ & Monthly & $\mathrm{MK} 2 / \mathrm{Sn}$ & 127 \\
\hline 145 & Gulmarg & 74.36600 & 34.03200 & 2705 & $1961-1970$ & $\mathrm{P}$ & Monthly & $\mathrm{MK} 2 / \mathrm{Sn}$ & 127 \\
\hline 146 & Gund & 75.08000 & 34.25000 & 2052 & $1961-1970$ & $\mathrm{P}$ & Monthly & $\mathrm{MK} 2 / \mathrm{Sn}$ & 127 \\
\hline 147 & Gurez & 74.85000 & 34.63000 & 2417 & $1953-1958$ & $\mathrm{P}$ & Monthly & $\mathrm{MK} 2 / \mathrm{Sn}$ & 127 \\
\hline 148 & Hamirpur & 76.53333 & 31.70000 & 786 & 1958-1969 & $\mathrm{P}$ & Monthly & $\mathrm{MK} 2 / \mathrm{Sn}$ & 127 \\
\hline 149 & Handwara & 74.28000 & 34.40000 & 1585 & $1961-1970$ & $\mathrm{P}$ & Monthly & $\mathrm{MK} 2 / \mathrm{Sn}$ & 127 \\
\hline 150 & Hoshiyarpur & 75.92000 & 31.53000 & 294 & $1961-1970$ & $\mathrm{P}$ & Monthly & $\mathrm{MK} 2 / \mathrm{Sn}$ & 127 \\
\hline
\end{tabular}




\begin{tabular}{|c|c|c|c|c|c|c|c|c|c|c|}
\hline 151 & Jammu & 74.80000 & 32.70000 & 277 & 1961-1970 & $\mathrm{P}$ & Monthly & MK2/Sn & 127 & 30 \\
\hline 152 & Janjehli & 77.21667 & 31.51667 & 2286 & 1958-1969 & $\mathrm{P}$ & Monthly & MK2/Sn & 127 & 30 \\
\hline 153 & Jhungi & 77.06667 & 31.41667 & 1785 & 1958-1969 & $\mathrm{P}$ & Monthly & MK2/Sn & 127 & 30 \\
\hline 154 & Jogindarnagar & 76.75000 & 31.91667 & 1221 & 1959-1969 & $\mathrm{P}$ & Monthly & MK2/Sn & 127 & 30 \\
\hline 155 & Jubal & 77.66667 & 31.10000 & 2000 & 1959-1969 & $\mathrm{P}$ & Monthly & $\mathrm{MK} 2 / \mathrm{Sn}$ & 127 & 30 \\
\hline 156 & Junga & 77.20000 & 31.03000 & 1227 & 1959-1969 & $\mathrm{P}$ & Monthly & MK2/Sn & 127 & 30 \\
\hline 157 & Kalka & 76.93000 & 30.83000 & 686 & 1958-1969 & $\mathrm{P}$ & Monthly & MK2/Sn & 127 & 30 \\
\hline 158 & Kandaghat & 77.12000 & 30.97000 & 1339 & 1958-1969 & $\mathrm{P}$ & Monthly & MK2/Sn & 127 & 30 \\
\hline 159 & Kangra & 76.25000 & 32.10000 & 701 & $1952-1969$ & $\mathrm{P}$ & Monthly & MK2/Sn & 127 & 30 \\
\hline 160 & Kargil & 76.13000 & 34.57000 & 2679 & 1935-1944 & $\mathrm{P}$ & Monthly & MK2/Sn & 127 & 30 \\
\hline 161 & Karsog & 77.20000 & 31.38000 & 1420 & 1958-1969 & $\mathrm{P}$ & Monthly & MK2/Sn & 127 & 30 \\
\hline 162 & Kasauli1 & 76.96667 & 30.88333 & 1783 & $1945-1950$ & $\mathrm{P}$ & Monthly & $\mathrm{MK} 2 / \mathrm{Sn}$ & 127 & 30 \\
\hline 163 & Kasauli2 & 77.00000 & 30.90000 & 1559 & 1958-1969 & $\mathrm{P}$ & Monthly & $\mathrm{MK} 2 / \mathrm{Sn}$ & 127 & 30 \\
\hline 164 & Kasumpti & 77.17000 & 31.00000 & 1700 & 1958-1969 & $\mathrm{P}$ & Monthly & $\mathrm{MK} 2 / \mathrm{Sn}$ & 127 & 30 \\
\hline 165 & Kataula & 77.06667 & 31.80000 & 1762 & 1959-1969 & $\mathrm{P}$ & Monthly & $\mathrm{MK} 2 / \mathrm{Sn}$ & 127 & 30 \\
\hline 166 & Khadrala & 77.58000 & 31.27000 & 2957 & 1958-1969 & $\mathrm{P}$ & Monthly & $\mathrm{MK} 2 / \mathrm{Sn}$ & 127 & 30 \\
\hline 167 & Khalatse & 76.83333 & 34.25000 & 3205 & 1961-1970 & $\mathrm{P}$ & Monthly & MK2/Sn & 127 & 30 \\
\hline 168 & Khangral & 76.50000 & 34.33333 & 3887 & 1961-1970 & $\mathrm{P}$ & Monthly & MK2/Sn & 127 & 30 \\
\hline 169 & Kharar & 76.65000 & 30.75000 & 280 & $1958-1970$ & $\mathrm{P}$ & Monthly & MK2/Sn & 127 & 30 \\
\hline 170 & Kilba & 78.13000 & 31.50000 & 2592 & 1958-1969 & $\mathrm{P}$ & Monthly & $\mathrm{MK} 2 / \mathrm{Sn}$ & 127 & 30 \\
\hline 171 & Kishtwar & 75.75000 & 33.30000 & 1215 & 1961-1970 & $\mathrm{P}$ & Monthly & MK2/Sn & 127 & 30 \\
\hline 172 & Kokernagh & 75.28000 & 33.92000 & 1676 & $1961-1970$ & $\mathrm{P}$ & Monthly & MK2/Sn & 127 & 30 \\
\hline 173 & Koksar & 77.23000 & 32.42000 & 3204 & 1956-1970 & $\mathrm{P}$ & Monthly & $\mathrm{MK} 2 / \mathrm{Sn}$ & 127 & 30 \\
\hline 174 & Kotarh & 77.48000 & 31.30000 & 2000 & $1953-1965$ & $\mathrm{P}$ & Monthly & $\mathrm{MK} 2 / \mathrm{Sn}$ & 127 & 30 \\
\hline 175 & Kothi & 77.20000 & 32.32000 & 2438 & 1961-1970 & $\mathrm{P}$ & Monthly & $\mathrm{MK} 2 / \mathrm{Sn}$ & 127 & 30 \\
\hline 176 & Kotkhai & 77.53333 & 31.11667 & 1560 & 1958-1969 & $\mathrm{P}$ & Monthly & $\mathrm{MK} 2 / \mathrm{Sn}$ & 127 & 30 \\
\hline 177 & Kukernag & 75.30000 & 33.60000 & 1865 & 1961-1970 & $\mathrm{P}$ & Monthly & MK2/Sn & 127 & 30 \\
\hline 178 & Kulgam & 75.02000 & 33.63000 & 1615 & 1961-1970 & $\mathrm{P}$ & Monthly & $\mathrm{MK} 2 / \mathrm{Sn}$ & 127 & 30 \\
\hline 179 & Kulu & 77.11667 & 31.95000 & 1370 & $1959-1969$ & $\mathrm{P}$ & Monthly & MK2/Sn & 127 & 30 \\
\hline 180 & Kumarsain & 77.45000 & 31.31667 & 1700 & 1959-1969 & $\mathrm{P}$ & Monthly & MK2/Sn & 127 & 30 \\
\hline 181 & Kyelong & 76.94500 & 32.60500 & 3166 & 1961-1970 & $\mathrm{P}$ & Monthly & MK2/Sn & 127 & 30 \\
\hline 182 & Langet & 74.30000 & 34.37000 & 1588 & $1961-1970$ & $\mathrm{P}$ & Monthly & $\mathrm{MK} 2 / \mathrm{Sn}$ & 127 & 30 \\
\hline 183 & Leh & 77.56667 & 34.15000 & 3514 & 1955-1969 & $\mathrm{PT}$ & Monthly & $\mathrm{MK} 2 / \mathrm{Sn}$ & 127 & 30 \\
\hline 184 & Malashahibag & 74.78000 & 34.22000 & 1583 & 1961-1970 & $\mathrm{P}$ & Monthly & MK2/Sn & 127 & 30 \\
\hline 185 & Malikpur & 75.67000 & 32.22000 & 302 & 1961-1970 & $\mathrm{P}$ & Monthly & $\mathrm{MK} 2 / \mathrm{Sn}$ & 127 & 30 \\
\hline 186 & Mandi1 & 76.96667 & 31.71667 & 761 & 1961-1970 & $\mathrm{P}$ & Monthly & MK2/Sn & 127 & 30 \\
\hline 187 & Mandi2 & 76.93000 & 31.72000 & 762 & 1958-1969 & $\mathrm{P}$ & Monthly & MK2/Sn & 127 & 30 \\
\hline 188 & Mulbek & 76.33333 & 34.33333 & 3926 & 1956-1969 & $\mathrm{P}$ & Monthly & MK2/Sn & 127 & 30 \\
\hline 189 & Nalagarh & 76.71667 & 31.05000 & 616 & 1958-1969 & $\mathrm{P}$ & Monthly & MK2/Sn & 127 & 30 \\
\hline 190 & Nawanshahr & 76.12000 & 31.12000 & 255 & 1961-1970 & $\mathrm{P}$ & Monthly & $\mathrm{MK} 2 / \mathrm{Sn}$ & 127 & 30 \\
\hline 191 & Nichar & 77.96667 & 31.55000 & 1970 & 1959-1969 & $\mathrm{P}$ & Monthly & $\mathrm{MK} 2 / \mathrm{Sn}$ & 127 & 30 \\
\hline 192 & Nowshera & 74.23000 & 33.15000 & 599 & 1951-1969 & $\mathrm{P}$ & Monthly & $\mathrm{MK} 2 / \mathrm{Sn}$ & 127 & 30 \\
\hline 193 & Nurpur & 75.91667 & 32.30000 & 616 & 1952-1969 & $\mathrm{P}$ & Monthly & MK2/Sn & 127 & 30 \\
\hline 194 & Palampur & 76.53333 & 32.13333 & 1217 & 1958-1969 & $\mathrm{P}$ & Monthly & $\mathrm{MK} 2 / \mathrm{Sn}$ & 127 & 30 \\
\hline 195 & Panamik & 77.61200 & 34.57200 & 3114 & 1956-1970 & $\mathrm{P}$ & Monthly & MK2/Sn & 127 & 30 \\
\hline 196 & Panjain & 77.18000 & 31.70000 & 1828 & 1959-1969 & $\mathrm{P}$ & Monthly & MK2/Sn & 127 & 30 \\
\hline 197 & Pathankot & 75.65000 & 32.28000 & 312 & 1958-1970 & $\mathrm{P}$ & Monthly & MK2/Sn & 127 & 30 \\
\hline 198 & Pendras & 75.58333 & 34.41667 & 4880 & 1956-1970 & $\mathrm{P}$ & Monthly & MK2/Sn & 127 & 30 \\
\hline
\end{tabular}




\begin{tabular}{|c|c|c|c|c|c|c|c|c|c|c|}
\hline 199 & Phalgam & 75.33000 & 34.03000 & 1707 & $1961-1970$ & $\mathrm{P}$ & Monthly & $\mathrm{MK} 2 / \mathrm{Sn}$ & 127 & 30 \\
\hline 200 & Poonch & 74.12000 & 33.78000 & 1067 & $1945-1962$ & $\mathrm{P}$ & Monthly & $\mathrm{MK} 2 / \mathrm{Sn}$ & 127 & 30 \\
\hline 201 & Prang & 74.87000 & 34.28000 & 1588 & $1961-1970$ & $\mathrm{P}$ & Monthly & $\mathrm{MK} 2 / \mathrm{Sn}$ & 127 & 30 \\
\hline 202 & Purbani & 78.33000 & 31.62000 & 2591 & 1958-1969 & $\mathrm{P}$ & Monthly & $\mathrm{MK} 2 / \mathrm{Sn}$ & 127 & 30 \\
\hline 203 & Qazi Gund & 75.08000 & 33.58000 & 1690 & $1961-1970$ & $\mathrm{P}$ & Monthly & $\mathrm{MK} 2 / \mathrm{Sn}$ & 127 & 30 \\
\hline 204 & Rajdhani & 74.30000 & 33.38000 & 924 & $1941-1947$ & $\mathrm{P}$ & Monthly & MK2/Sn & 127 & 30 \\
\hline 205 & Ramban & 75.25000 & 33.25000 & 945 & $1956-1969$ & $\mathrm{P}$ & Monthly & $\mathrm{MK} 2 / \mathrm{Sn}$ & 127 & 30 \\
\hline 206 & Ramnagar & 75.31667 & 32.80000 & 792 & $1956-1969$ & $\mathrm{P}$ & Monthly & $\mathrm{MK} 2 / \mathrm{Sn}$ & 127 & 30 \\
\hline 207 & Rampur & 77.63333 & 31.43333 & 1067 & 1959-1969 & $\mathrm{P}$ & Monthly & $\mathrm{MK} 2 / \mathrm{Sn}$ & 127 & 30 \\
\hline 208 & Riasi & 74.83000 & 33.08000 & 585 & 1958-1969 & $\mathrm{P}$ & Monthly & $\mathrm{MK} 2 / \mathrm{Sn}$ & 127 & 30 \\
\hline 209 & Rohru & 77.75000 & 31.22000 & 1773 & $1959-1969$ & $\mathrm{P}$ & Monthly & MK2/Sn & 127 & 30 \\
\hline 210 & Rupanagar & 76.52000 & 30.97000 & 274 & 1958-1969 & $\mathrm{P}$ & Monthly & MK2/Sn & 127 & 30 \\
\hline 211 & Sangla & 78.26667 & 31.41667 & 1981 & $1958-1969$ & $\mathrm{P}$ & Monthly & $\mathrm{MK} 2 / \mathrm{Sn}$ & 127 & 30 \\
\hline 212 & Sarkahat & 76.73333 & 31.70000 & 914 & 1959-1969 & $\mathrm{P}$ & Monthly & $\mathrm{MK} 2 / \mathrm{Sn}$ & 127 & 30 \\
\hline 213 & Shillaru & 77.45000 & 31.20000 & 2450 & 1958-1969 & $\mathrm{P}$ & Monthly & $\mathrm{MK} 2 / \mathrm{Sn}$ & 127 & 30 \\
\hline 214 & Shimla & 77.16667 & 31.10000 & 2202 & $1961-1970$ & $\mathrm{PT}$ & Monthly & $\mathrm{MK} 2 / \mathrm{Sn}$ & 127 & 30 \\
\hline 215 & Shiquanhe & 80.08300 & 32.50000 & 4280 & $1961-1970$ & $\mathrm{PT}$ & Monthly & $\mathrm{MK} 2 / \mathrm{Sn}$ & 127 & 30 \\
\hline 216 & Shopian & 74.83000 & 33.72000 & 1615 & $1961-1970$ & $\mathrm{P}$ & Monthly & $\mathrm{MK} 2 / \mathrm{Sn}$ & 127 & 30 \\
\hline 217 & Sogam & 74.40000 & 34.50000 & 1760 & $1961-1970$ & $\mathrm{P}$ & Monthly & $\mathrm{MK} 2 / \mathrm{Sn}$ & 127 & 30 \\
\hline 218 & Solan & 77.11667 & 30.90000 & 1459 & 1959-1969 & $\mathrm{P}$ & Monthly & $\mathrm{MK} 2 / \mathrm{Sn}$ & 127 & 30 \\
\hline 219 & Sonemarg & 75.31667 & 34.31667 & 2515 & 1951-1969 & $\mathrm{P}$ & Monthly & $\mathrm{MK} 2 / \mathrm{Sn}$ & 127 & 30 \\
\hline 220 & Sopore & 74.47000 & 34.30000 & 1574 & $1961-1970$ & $\mathrm{P}$ & Monthly & $\mathrm{MK} 2 / \mathrm{Sn}$ & 127 & 30 \\
\hline 221 & SR Sing & 74.70000 & 32.60000 & 273 & 1958-1969 & $\mathrm{P}$ & Monthly & $\mathrm{MK} 2 / \mathrm{Sn}$ & 127 & 30 \\
\hline 222 & Srinagar & 74.83333 & 34.08333 & 1587 & $1961-1970$ & $\mathrm{PT}$ & Monthly & $\mathrm{MK} 2 / \mathrm{Sn}$ & 127 & 30 \\
\hline 223 & Sundarnagar & 76.88333 & 31.53333 & 1193 & $1959-1969$ & $\mathrm{P}$ & Monthly & $\mathrm{MK} 2 / \mathrm{Sn}$ & 127 & 30 \\
\hline 224 & Suni Seoni & 77.11667 & 31.25000 & 668 & 1958-1969 & $\mathrm{P}$ & Monthly & $\mathrm{MK} 2 / \mathrm{Sn}$ & 127 & 30 \\
\hline 225 & Tanda & 76.70000 & 31.60000 & 769 & $1958-1965$ & $\mathrm{P}$ & Monthly & $\mathrm{MK} 2 / \mathrm{Sn}$ & 127 & 30 \\
\hline 226 & Tangmarg & 74.42469 & 34.06090 & 2171 & $1961-1970$ & $\mathrm{P}$ & Monthly & $\mathrm{MK} 2 / \mathrm{Sn}$ & 127 & 30 \\
\hline 227 & Tapoban & 79.60000 & 30.50000 & 2671 & $1951-1958$ & $\mathrm{P}$ & Monthly & $\mathrm{MK} 2 / \mathrm{Sn}$ & 127 & 30 \\
\hline 228 & Theog & 77.36667 & 31.13333 & 1893 & 1958-1969 & $\mathrm{P}$ & Monthly & $\mathrm{MK} 2 / \mathrm{Sn}$ & 127 & 30 \\
\hline 229 & Tibri & 75.58000 & 32.10000 & 270 & $1961-1970$ & $\mathrm{P}$ & Monthly & $\mathrm{MK} 2 / \mathrm{Sn}$ & 127 & 30 \\
\hline 230 & Tral & 75.12000 & 33.93000 & 1615 & $1961-1970$ & $\mathrm{P}$ & Monthly & $\mathrm{MK} 2 / \mathrm{Sn}$ & 127 & 30 \\
\hline 231 & T-K-I-H-Kung & 81.43300 & 30.55000 & 4736 & 1988-1998 & $\mathrm{PT}$ & Monthly & $\mathrm{MK} 2 / \mathrm{Sn}$ & 127 & 30 \\
\hline 232 & Udhampur & 75.10000 & 32.90000 & 236 & 1958-1969 & $\mathrm{P}$ & Monthly & $\mathrm{MK} 2 / \mathrm{Sn}$ & 127 & 30 \\
\hline 233 & Una & 76.28333 & 31.46667 & 346 & $1958-1969$ & $\mathrm{P}$ & Monthly & $\mathrm{MK} 2 / \mathrm{Sn}$ & 127 & 30 \\
\hline 234 & Uri & 74.03000 & 34.05000 & 1628 & $1941-1947$ & $\mathrm{P}$ & Monthly & $\mathrm{MK} 2 / \mathrm{Sn}$ & 127 & 30 \\
\hline 235 & Uttamchipura & 74.67000 & 34.50000 & 3145 & $1951-1956$ & $\mathrm{P}$ & Monthly & $\mathrm{MK} 2 / \mathrm{Sn}$ & 127 & 30 \\
\hline 236 & Vantipura & 74.90000 & 33.88000 & 1600 & $1961-1970$ & $\mathrm{P}$ & Monthly & MK2/Sn & 127 & 30 \\
\hline 237 & Verinagh & 75.25000 & 33.53000 & 1646 & $1965-1970$ & $\mathrm{P}$ & Monthly & $\mathrm{MK} 2 / \mathrm{Sn}$ & 127 & 30 \\
\hline 238 & Arthal & 76.18095 & 33.24808 & 2225 & $1974-1990$ & $\mathrm{P}$ & Monthly & MK2/Sn & 127 & 30 \\
\hline 239 & Bhakra & 76.78750 & 31.41472 & 518 & $1983-1993$ & $\mathrm{P}$ & Monthly & MK2/Sn & 127 & 30 \\
\hline 240 & Bunencha & 75.91829 & 32.98423 & 2600 & 1974-1990 & $\mathrm{P}$ & Monthly & $\mathrm{MK} 2 / \mathrm{Sn}$ & 127 & 30 \\
\hline 241 & Chingaon & 75.57881 & 33.50729 & 1840 & $1974-1990$ & $\mathrm{P}$ & Monthly & $\mathrm{MK} 2 / \mathrm{Sn}$ & 127 & 30 \\
\hline 242 & Chitkul & 78.43600 & 31.35250 & 3841 & 1983-1993 & $\mathrm{P}$ & Monthly & $\mathrm{MK} 2 / \mathrm{Sn}$ & 127 & 30 \\
\hline 243 & Damini & 74.80286 & 33.34395 & 885 & $1974-1990$ & $\mathrm{P}$ & Monthly & $\mathrm{MK} 2 / \mathrm{Sn}$ & 127 & 30 \\
\hline 244 & Darabshala & 75.87560 & 33.13058 & 1095 & $1974-1990$ & $\mathrm{P}$ & Monthly & MK2/Sn & 127 & 30 \\
\hline 245 & Devigol & 76.05492 & 33.10777 & 2450 & $1974-1990$ & $\mathrm{P}$ & Monthly & MK2/Sn & 127 & 30 \\
\hline 246 & Dhamkund & 75.14167 & 33.24670 & 640 & $1974-1990$ & $\mathrm{P}$ & Monthly & $\mathrm{MK} 2 / \mathrm{Sn}$ & 127 & 30 \\
\hline
\end{tabular}




\begin{tabular}{|c|c|c|c|c|c|c|c|c|c|}
\hline 247 & Doda & 75.54745 & 33.14540 & 1140 & $1974-1990$ & $\mathrm{P}$ & Monthly & $\mathrm{MK} 2 / \mathrm{Sn}$ & 127 \\
\hline 248 & Dusadudha & 75.98979 & 33.04808 & 2440 & $1974-1990$ & $\mathrm{P}$ & Monthly & $\mathrm{MK} 2 / \mathrm{Sn}$ & 127 \\
\hline 249 & Gainta & 74.98332 & 33.06661 & 1000 & $1974-1990$ & $\mathrm{P}$ & Monthly & $\mathrm{MK} 2 / \mathrm{Sn}$ & 127 \\
\hline 250 & Ghamroor & 75.95649 & 31.95345 & 436 & $1983-1993$ & $\mathrm{P}$ & Monthly & $\mathrm{MK} 2 / \mathrm{Sn}$ & 127 \\
\hline 251 & Harsur & 76.04476 & 32.10751 & 667 & $1983-1993$ & $\mathrm{P}$ & Monthly & $\mathrm{MK} 2 / \mathrm{Sn}$ & 127 \\
\hline 252 & Hawal & 76.09438 & 33.53781 & 2745 & $1974-1990$ & $\mathrm{P}$ & Monthly & $\mathrm{MK} 2 / \mathrm{Sn}$ & 127 \\
\hline 253 & Inshan & 75.56600 & 33.75000 & 2440 & $1974-1990$ & $\mathrm{P}$ & Monthly & $\mathrm{MK} 2 / \mathrm{Sn}$ & 127 \\
\hline 254 & Kahu & 76.78750 & 31.20361 & 649 & $1983-1993$ & $\mathrm{P}$ & Monthly & $\mathrm{MK} 2 / \mathrm{Sn}$ & 127 \\
\hline 255 & Kasol & 76.87833 & 31.35694 & 662 & $1983-1993$ & $\mathrm{P}$ & Monthly & $\mathrm{MK} 2 / \mathrm{Sn}$ & 127 \\
\hline 256 & Kati & 75.18426 & 33.09472 & 1570 & $1974-1990$ & $\mathrm{P}$ & Monthly & $\mathrm{MK} 2 / \mathrm{Sn}$ & 127 \\
\hline 257 & Kaza & 78.07222 & 32.22500 & 3639 & $1983-1993$ & $\mathrm{P}$ & Monthly & $\mathrm{MK} 2 / \mathrm{Sn}$ & 127 \\
\hline 258 & Kupwara & 74.25000 & 34.51000 & 1609 & $1958-1969$ & $\mathrm{P}$ & Monthly & $\mathrm{MK} 2 / \mathrm{Sn}$ & 127 \\
\hline 259 & Larji & 77.21894 & 31.72514 & 995 & $1983-1993$ & $\mathrm{P}$ & Monthly & $\mathrm{MK} 2 / \mathrm{Sn}$ & 127 \\
\hline 260 & Lossar & 77.75000 & 32.43800 & 4079 & $1983-1993$ & $\mathrm{P}$ & Monthly & $\mathrm{MK} 2 / \mathrm{Sn}$ & 127 \\
\hline 261 & Matsal & 76.44896 & 33.51256 & 4325 & $1974-1990$ & $\mathrm{P}$ & Monthly & $\mathrm{MK} 2 / \mathrm{Sn}$ & 127 \\
\hline 262 & Mau & 76.32062 & 33.40928 & 2900 & $1974-1990$ & $\mathrm{P}$ & Monthly & MK2/Sn & 127 \\
\hline 263 & Mohu & 75.10488 & 33.44952 & 2440 & $1974-1990$ & $\mathrm{P}$ & Monthly & $\mathrm{MK} 2 / \mathrm{Sn}$ & 127 \\
\hline 264 & Moorang & 78.44800 & 31.59060 & 2744 & $1983-1993$ & $\mathrm{P}$ & Monthly & MK2/Sn & 127 \\
\hline 265 & Namgia & 78.65630 & 31.80306 & 3083 & $1983-1993$ & $\mathrm{P}$ & Monthly & MK2/Sn & 127 \\
\hline 266 & Nandan & 74.38528 & 33.43564 & 1910 & $1974-1990$ & $\mathrm{P}$ & Monthly & $\mathrm{MK} 2 / \mathrm{Sn}$ & 127 \\
\hline 267 & Ohli & 75.93484 & 33.33845 & 1585 & $1974-1990$ & $\mathrm{P}$ & Monthly & $\mathrm{MK} 2 / \mathrm{Sn}$ & 127 \\
\hline 268 & Palmar & 75.67044 & 33.43411 & 1585 & $1974-1990$ & $\mathrm{P}$ & Monthly & $\mathrm{MK} 2 / \mathrm{Sn}$ & 127 \\
\hline 269 & Pooh & 78.58890 & 31.76310 & 2896 & $1983-1993$ & $\mathrm{P}$ & Monthly & $\mathrm{MK} 2 / \mathrm{Sn}$ & 127 \\
\hline 270 & Pouni & 74.69560 & 33.09154 & 600 & $1974-1990$ & $\mathrm{P}$ & Monthly & $\mathrm{MK} 2 / \mathrm{Sn}$ & 127 \\
\hline 271 & Rakchham & 78.35556 & 31.39167 & 3282 & $1983-1993$ & $\mathrm{P}$ & Monthly & $\mathrm{MK} 2 / \mathrm{Sn}$ & 127 \\
\hline 272 & Rekenwas & 75.57231 & 33.96589 & 3660 & $1974-1990$ & $\mathrm{P}$ & Monthly & $\mathrm{MK} 2 / \mathrm{Sn}$ & 127 \\
\hline 273 & Rot & 75.46397 & 33.05582 & 1375 & $1974-1990$ & $\mathrm{P}$ & Monthly & $\mathrm{MK} 2 / \mathrm{Sn}$ & 127 \\
\hline 274 & Sain & 74.82142 & 33.42813 & 2240 & $1974-1990$ & $\mathrm{P}$ & Monthly & $\mathrm{MK} 2 / \mathrm{Sn}$ & 127 \\
\hline 275 & Sainj & 77.30556 & 31.77011 & 1348 & $1983-1993$ & $\mathrm{P}$ & Monthly & $\mathrm{MK} 2 / \mathrm{Sn}$ & 127 \\
\hline 276 & Salal & 74.80628 & 33.11661 & 610 & $1974-1990$ & $\mathrm{P}$ & Monthly & $\mathrm{MK} 2 / \mathrm{Sn}$ & 127 \\
\hline 277 & Sarkund & 75.55288 & 33.84823 & 2350 & $1974-1990$ & $\mathrm{P}$ & Monthly & $\mathrm{MK} 2 / \mathrm{Sn}$ & 127 \\
\hline 278 & Shahpur-I & 76.17020 & 32.22650 & 755 & $1983-1993$ & $\mathrm{P}$ & Monthly & $\mathrm{MK} 2 / \mathrm{Sn}$ & 127 \\
\hline 279 & Sirshi & 75.89336 & 33.50897 & 1675 & $1974-1990$ & $\mathrm{P}$ & Monthly & $\mathrm{MK} 2 / \mathrm{Sn}$ & 127 \\
\hline 280 & Sohal & 76.22161 & 33.21381 & 2000 & $1974-1990$ & $\mathrm{P}$ & Monthly & $\mathrm{MK} 2 / \mathrm{Sn}$ & 127 \\
\hline 281 & Tandi & 76.97637 & 32.55639 & 3100 & $1974-1990$ & $\mathrm{P}$ & Monthly & $\mathrm{MK} 2 / \mathrm{Sn}$ & 127 \\
\hline 282 & Thana & 75.67658 & 33.23242 & 2440 & $1974-1990$ & $\mathrm{P}$ & Monthly & $\mathrm{MK} 2 / \mathrm{Sn}$ & 127 \\
\hline 283 & Tillar & 75.71831 & 33.61311 & 2130 & $1974-1990$ & $\mathrm{P}$ & Monthly & $\mathrm{MK} 2 / \mathrm{Sn}$ & 127 \\
\hline 284 & Udaipur & 76.66494 & 32.72437 & 2600 & $1974-1990$ & $\mathrm{P}$ & Monthly & $\mathrm{MK} 2 / \mathrm{Sn}$ & 127 \\
\hline 285 & Yurod & 75.71760 & 33.66478 & 2165 & $1974-1990$ & $\mathrm{P}$ & Monthly & $\mathrm{MK} 2 / \mathrm{Sn}$ & 127 \\
\hline 286 & Asmar & 71.43333 & 35.01667 & 880 & $1975-2012$ & $\mathrm{PT}$ & Monthly & Tretyakov & 200 \\
\hline 287 & Bamiyan & 67.81667 & 34.81667 & 2550 & $1975-2012$ & $\mathrm{PT}$ & Monthly & Tretyakov & 200 \\
\hline 288 & Darullaman & 69.10000 & 34.45000 & 1825 & 1974-1984 & $\mathrm{PT}$ & Monthly & Tretyakov & 200 \\
\hline 289 & Gerdiz & 69.23333 & 33.61667 & 2350 & $1975-2012$ & $\mathrm{PT}$ & Monthly & Tretyakov & 200 \\
\hline 290 & Ghaziabad & 70.76667 & 34.31667 & 510 & $1975-2012$ & $\mathrm{PT}$ & Monthly & Tretyakov & 200 \\
\hline 291 & Ghazni & 68.41667 & 33.53333 & 2183 & $1975-2012$ & $\mathrm{PT}$ & Monthly & Tretyakov & 200 \\
\hline 292 & Jabul Saraj & 69.25000 & 35.13333 & 1630 & $1975-2012$ & P T W & Monthly & Tretyakov & 200 \\
\hline 293 & Jalalabad & 70.46667 & 34.43333 & 580 & $1975-1984$ & $\mathrm{PT}$ & Monthly & Tretyakov & 200 \\
\hline 294 & Kabul AP & 69.21667 & 34.55000 & 1791 & $1975-2012$ & P T W & Monthly & Tretyakov & 200 \\
\hline
\end{tabular}




\begin{tabular}{|c|c|c|c|c|c|c|c|c|c|c|}
\hline 295 & Karizmir & 69.05000 & 34.63333 & 1905 & 1975-2012 & $\mathrm{PT}$ & Monthly & Tretyakov & 200 & 40 \\
\hline 296 & Khost & 69.95000 & 33.35000 & 1146 & 1975-2012 & $\mathrm{PT}$ & Monthly & Tretyakov & 200 & 40 \\
\hline 297 & Laghman & 70.21667 & 34.65000 & 770 & 1975-1984 & $\mathrm{PT}$ & Monthly & Tretyakov & 200 & 40 \\
\hline 298 & Logar & 69.05000 & 34.10000 & 1935 & 1975-1984 & $\mathrm{PT}$ & Monthly & Tretyakov & 200 & 40 \\
\hline 299 & Mirbachakot & 69.13333 & 34.76667 & 1660 & 1975-1980 & $\mathrm{PT}$ & Monthly & Tretyakov & 200 & 40 \\
\hline 300 & Mokur & 67.78333 & 32.83333 & 2000 & $1975-1984$ & $\mathrm{PT}$ & Monthly & Tretyakov & 200 & 40 \\
\hline 301 & North Salang & 69.01667 & 35.31667 & 3366 & 1975-1984 & $\mathrm{PT}$ & Monthly & Tretyakov & 200 & 40 \\
\hline 302 & Okak & 67.95000 & 33.88333 & 3130 & 1975-1979 & $\mathrm{PT}$ & Monthly & Tretyakov & 200 & 40 \\
\hline 303 & Paghman & 68.95000 & 34.91667 & 2114 & 1975-2012 & $\mathrm{PT}$ & Monthly & Tretyakov & 200 & 40 \\
\hline 304 & Pan Jao & 67.03333 & 34.36667 & 2710 & 1975-1979 & $\mathrm{PT}$ & Monthly & Tretyakov & 200 & 40 \\
\hline 305 & Sarobi & 69.75000 & 34.58333 & 1020 & 1975-2012 & $\mathrm{PT}$ & Monthly & Tretyakov & 200 & 40 \\
\hline 306 & South Salang & 69.06667 & 35.30000 & 3172 & 1975-1984 & P T W & Monthly & Tretyakov & 200 & 40 \\
\hline 307 & Zebak & 71.25000 & 36.50000 & 2600 & 1978-1984 & $\mathrm{P} \mathrm{T}$ & Monthly & Tretyakov & 200 & 40 \\
\hline 308 & Approach & 75.63310 & 36.06778 & 5100 & 1985-1987 & $\mathrm{P}$ & Monthly & V S & - & - \\
\hline 309 & Baltoro & 76.55079 & 35.87778 & 5500 & 1973-1980 & $\mathrm{P}$ & Monthly & V S & - & - \\
\hline 310 & Batura & 74.38333 & 36.66667 & 4840 & 1973-1974 & $\mathrm{P}$ & Monthly & V S & - & - \\
\hline 311 & Chong Kumdan & 77.54475 & 35.25317 & 5330 & 1986-1991 & $\mathrm{P}$ & Monthly & V S & - & - \\
\hline 312 & Chogolungma & 75.00000 & 36.00000 & 4900 & 1985-1988 & $\mathrm{P}$ & Monthly & V S & - & - \\
\hline 313 & Hispar Dome & 75.51872 & 36.01091 & 5450 & 1982-1986 & $\mathrm{P}$ & Monthly & V S & - & - \\
\hline 314 & Hispar East & 75.50639 & 35.84953 & 4830 & 1985-1988 & $\mathrm{P}$ & Monthly & V S & - & - \\
\hline 315 & Hispar West & 75.31635 & 36.20336 & 5450 & 1985-1988 & $\mathrm{P}$ & Monthly & V S & - & - \\
\hline 316 & Hispar Pass & 75.52151 & 36.02807 & 5100 & 1984-1986 & $\mathrm{P}$ & Monthly & V S & - & - \\
\hline 317 & Khurdopin & 75.61969 & 36.13377 & 5520 & 1984-1986 & $\mathrm{P}$ & Monthly & V S & - & - \\
\hline 318 & Nanga Parbat & 74.60000 & 35.22500 & 5440 & 1984-1997 & $\mathrm{P}$ & Monthly & V S & - & - \\
\hline 319 & Nun Kun North & 76.10142 & 34.12193 & 5200 & 1973-1980 & $\mathrm{P}$ & Monthly & V S & - & - \\
\hline 320 & Sentik & 75.95000 & 33.99670 & 4908 & 1963-1980 & $\mathrm{P}$ & Monthly & V S & - & - \\
\hline 321 & Siachin A & 77.03757 & 35.47073 & 4800 & 1986-1991 & $\mathrm{P}$ & Monthly & V S & - & - \\
\hline 322 & Siachin B & 76.99150 & 35.52349 & 4950 & 1986-1991 & $\mathrm{P}$ & Monthly & V S & - & - \\
\hline 323 & Siachin C & 76.91160 & 35.51866 & 5050 & 1986-1991 & $\mathrm{P}$ & Monthly & V S & - & - \\
\hline 324 & Siachin D & 76.85924 & 35.62423 & 5350 & 1986-1991 & $\mathrm{P}$ & Monthly & V S & - & - \\
\hline 325 & South Terong & 77.48080 & 35.09276 & 5330 & 1986-1991 & $\mathrm{P}$ & Monthly & V S & - & - \\
\hline 326 & Terong & 77.31197 & 35.51773 & 5350 & 1986-1991 & $\mathrm{P}$ & Monthly & V S & - & - \\
\hline 327 & Urdok & 76.70253 & 35.76688 & 5400 & 2004-2006 & $P$ & Monthly & V S & - & - \\
\hline 328 & Whaleback & 75.59149 & 36.05717 & 4900 & 1985-1986 & $\mathrm{P}$ & Monthly & V S & - & - \\
\hline
\end{tabular}




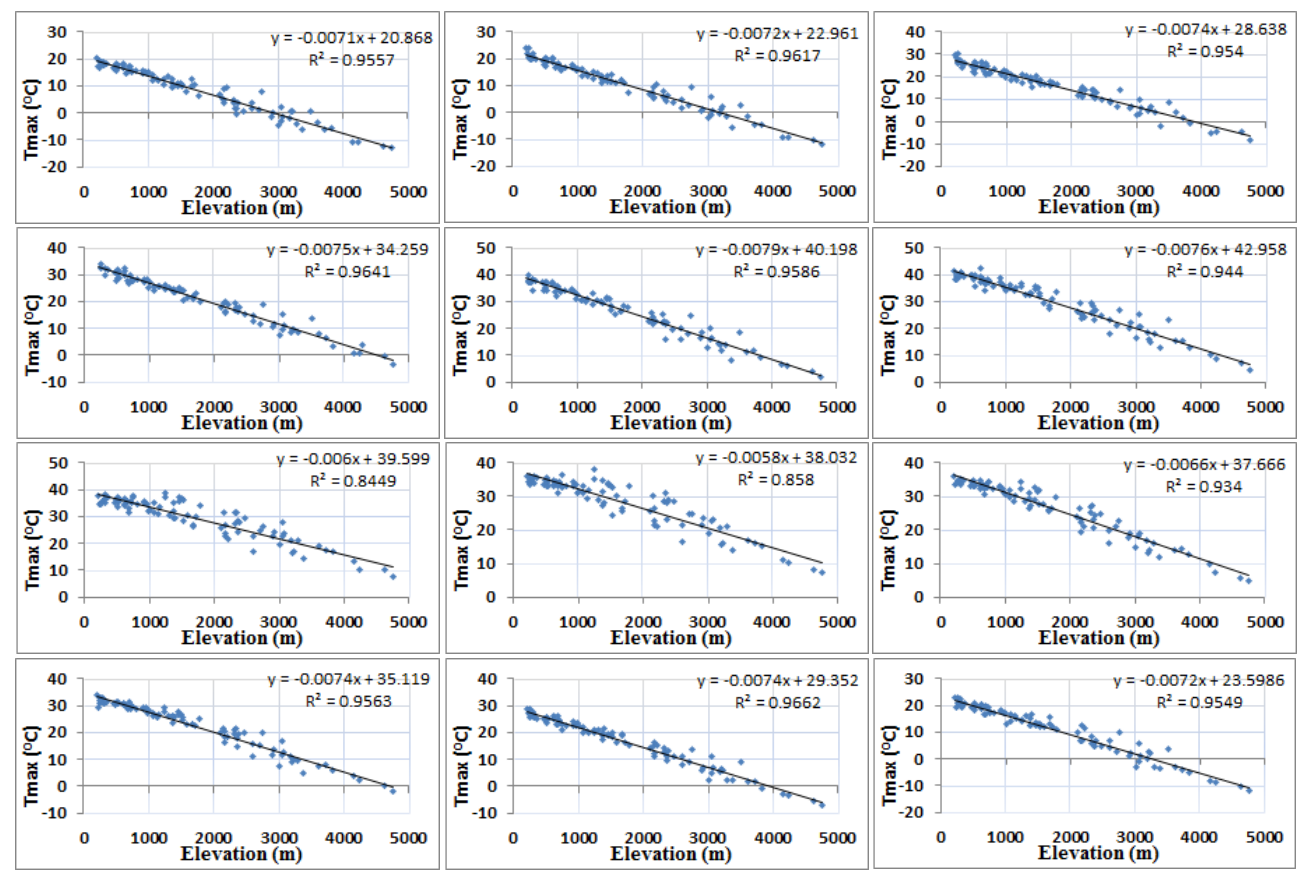

Figure S-3.2: Elevation based lapse rates of mean monthly maximum temperature for 1999-2011 time period. The letters a -1 indicates months from January-December.

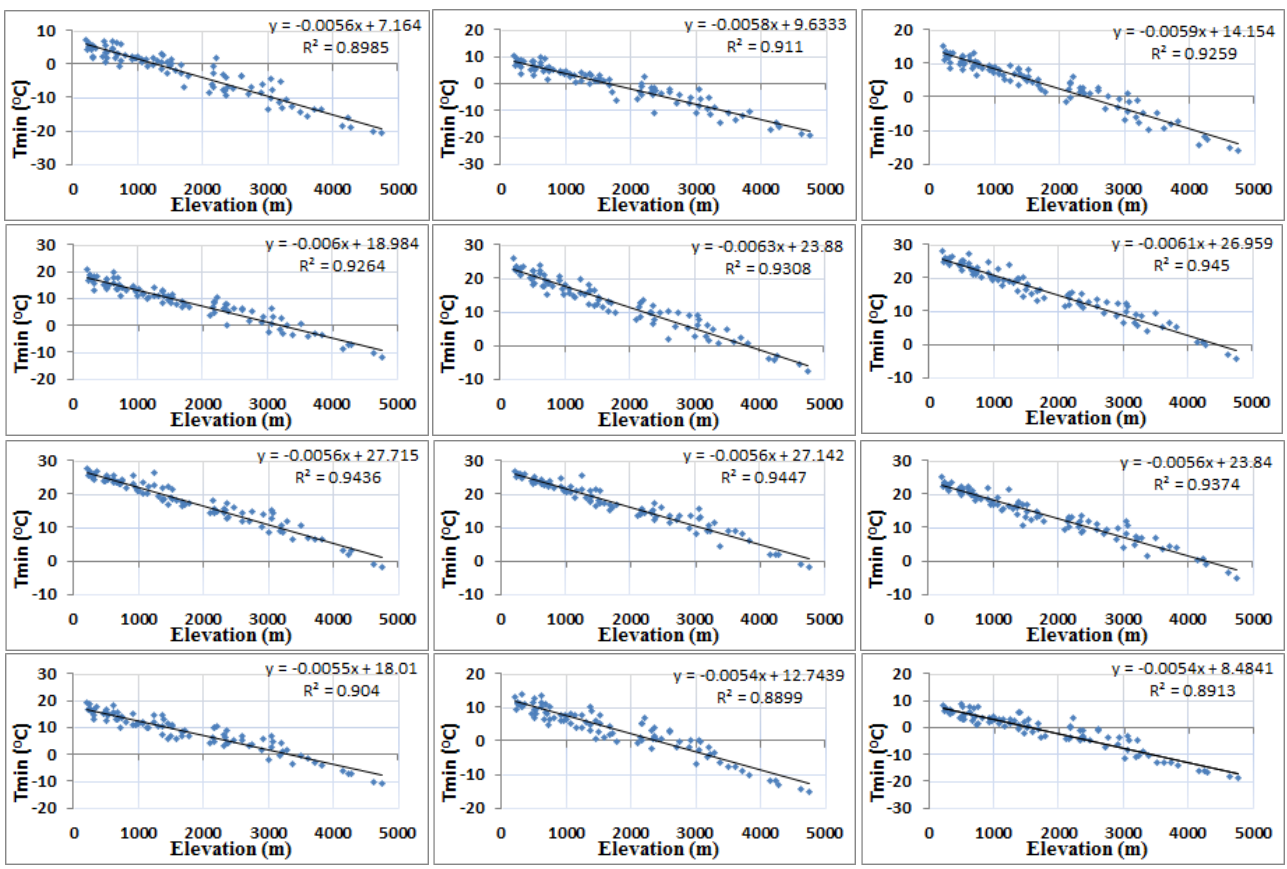

Figure S-3.3: Elevation based lapse rates of mean monthly minimum temperature for 1999-2011 time period. The letters a -1 indicate months from January-December. 


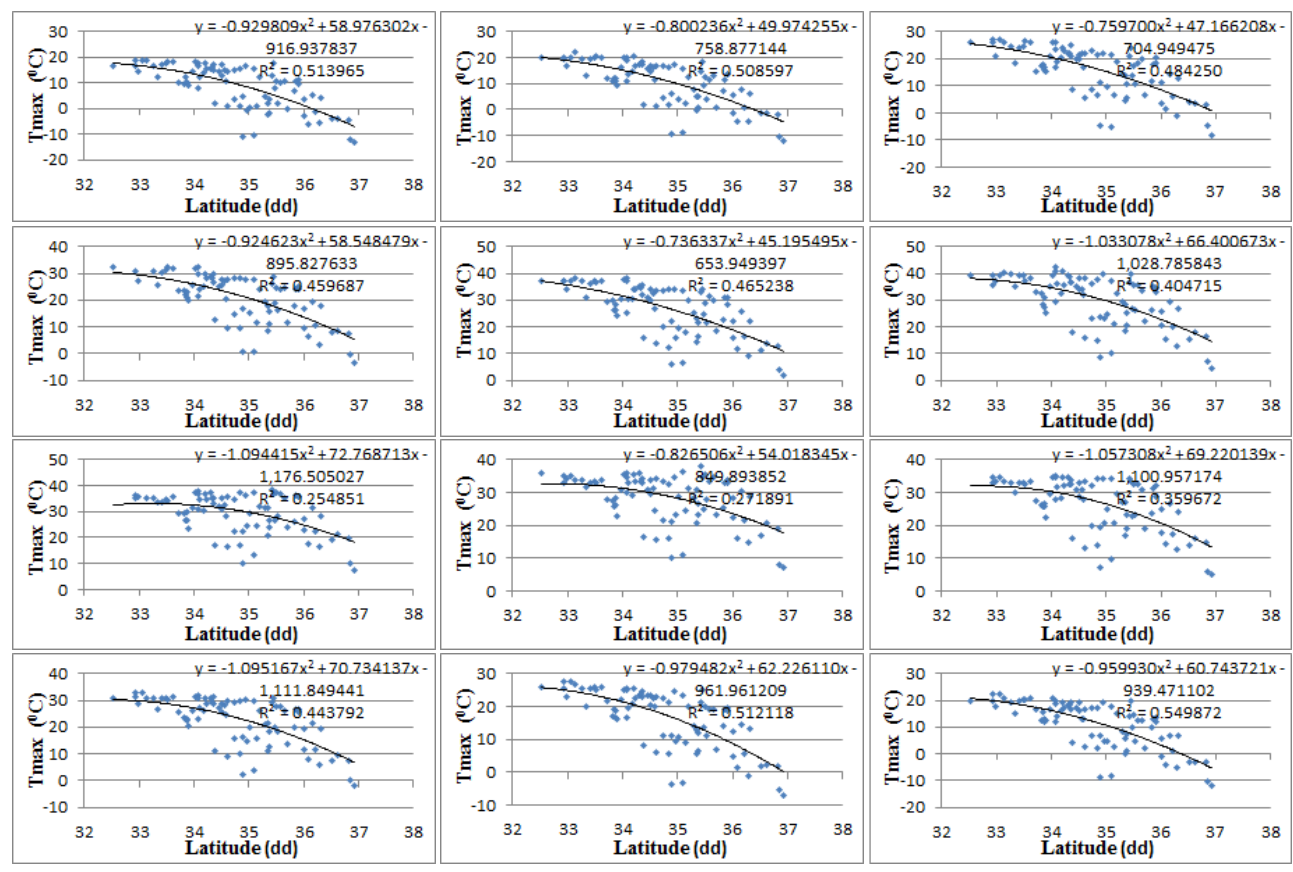

Figure S-3.4: Latitude based lapse rates of mean monthly maximum temperature for 1999-2011 time period. The letters a -1 indicate months from January-December.
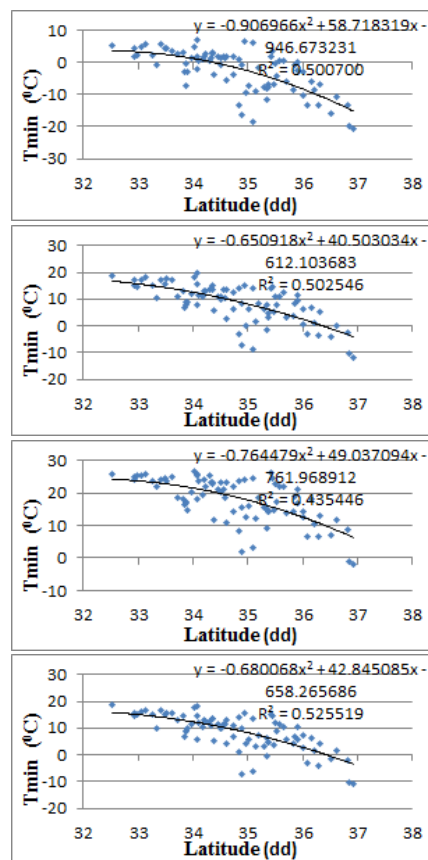
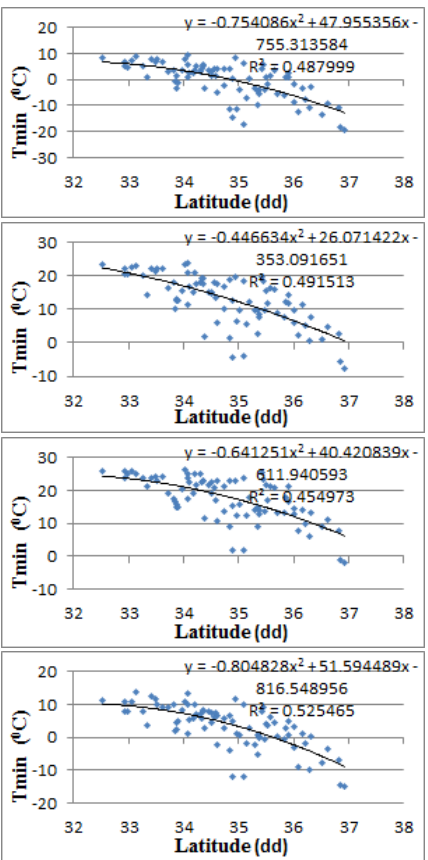
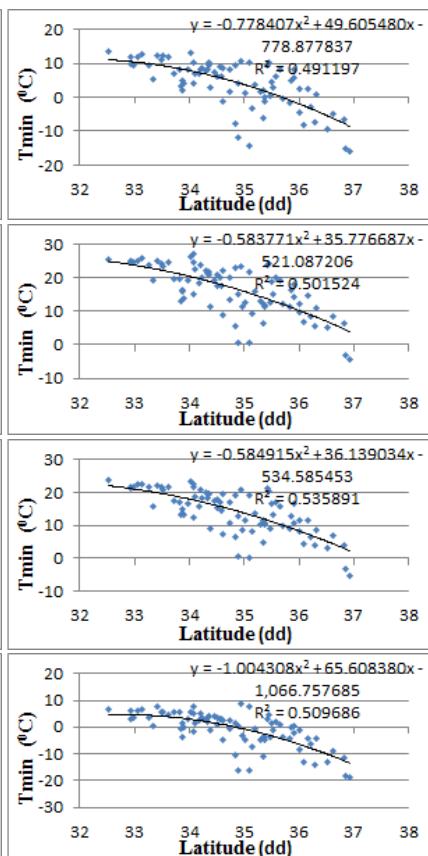

Figure S-3.5: Latitude based lapse rates of mean monthly minimum temperature for 1999-2011 time period. The letters a -1 indicate months from January-December. 


\section{Supplementary Results}

Table S-3.2: Absolute bias (corrected - observed) of the precipitation observations ( $\mathrm{mm})$.

\begin{tabular}{|c|c|c|c|c|c|c|c|c|c|c|c|c|c|c|}
\hline S. \# & Station Name & Jan & Feb & Mar & Apr & May & Jun & Jul & Aug & Sep & Oct & Nov & Dec & ANN \\
\hline 1 & Astore & 20.7 & 14.8 & 4.6 & 4.4 & 3.6 & 2.6 & 2.6 & 2.4 & 2.1 & 1.2 & 1.2 & 3.4 & 63.6 \\
\hline 2 & Babusar & 45.8 & 80.9 & 28.0 & 17.0 & 3.9 & 3.2 & 4.5 & 4.1 & 2.6 & 1.6 & 8.9 & 40.8 & 241.2 \\
\hline 3 & Balakot & 2.0 & 4.1 & 4.6 & 3.5 & 3.5 & 4.9 & 7.0 & 5.0 & 2.6 & 1.8 & 1.0 & 1.3 & 41.5 \\
\hline 4 & Bhimber & 1.2 & 1.5 & 1.5 & 1.0 & 1.3 & 1.6 & 3.3 & 2.9 & 1.4 & 0.5 & 0.3 & 0.4 & 16.9 \\
\hline 5 & Bunji & 0.9 & 0.8 & 1.0 & 1.7 & 1.6 & 1.3 & 1.2 & 1.7 & 1.1 & 0.3 & 0.4 & 0.3 & 12.3 \\
\hline 6 & Chakdara & 2.5 & 4.4 & 4.1 & 2.9 & 2.4 & 2.9 & 4.2 & 3.5 & 2.2 & 1.8 & 1.1 & 2.1 & 34.0 \\
\hline 7 & Chakwal & 1.4 & 1.9 & 1.5 & 1.1 & 1.1 & 2.0 & 3.5 & 3.0 & 1.6 & 1.3 & 1.1 & 0.5 & 19.9 \\
\hline 8 & Cherat & 5.7 & 8.1 & 4.5 & 3.9 & 2.3 & 2.1 & 4.7 & 3.7 & 2.0 & 1.0 & 1.3 & 2.0 & 41.2 \\
\hline 9 & Chilas & 0.9 & 0.8 & 1.2 & 1.7 & 1.5 & 1.3 & 1.1 & 1.5 & 1.1 & 0.3 & 0.4 & 0.3 & 12.2 \\
\hline 10 & Chitral & 4.5 & 5.1 & 3.6 & 3.4 & 2.2 & 1.2 & 1.3 & 1.2 & 1.9 & 1.6 & 1.5 & 1.9 & 29.5 \\
\hline 11 & Dir & 2.8 & 4.3 & 3.7 & 2.6 & 2.6 & 2.3 & 3.4 & 3.2 & 2.6 & 1.4 & 1.2 & 1.5 & 31.6 \\
\hline 12 & Drosh & 3.4 & 4.4 & 4.2 & 3.2 & 2.5 & 1.7 & 2.3 & 1.9 & 2.0 & 1.6 & 1.3 & 1.6 & 30.2 \\
\hline 13 & GD Poto & 1.5 & 2.0 & 2.1 & 1.8 & 1.8 & 2.1 & 3.2 & 2.6 & 1.4 & 1.0 & 0.6 & 0.8 & 20.9 \\
\hline 14 & Gilgit & 1.0 & 1.4 & 1.5 & 2.2 & 2.2 & 1.9 & 2.0 & 2.4 & 2.2 & 0.6 & 0.4 & 0.5 & 18.1 \\
\hline 15 & Gupis & 2.4 & 2.8 & 2.4 & 2.2 & 1.6 & 0.8 & 0.4 & 0.6 & 0.8 & 0.9 & 1.0 & 1.2 & 17.2 \\
\hline 16 & Hunza & 6.6 & 2.8 & 2.5 & 3.6 & 2.4 & 1.9 & 2.6 & 2.5 & 2.9 & 0.6 & 0.6 & 2.1 & 31.3 \\
\hline 17 & Islamabad AP & 4.2 & 10.6 & 8.9 & 4.6 & 4.1 & 8.0 & 15.0 & 10.7 & 4.7 & 1.5 & 0.8 & 1.8 & 74.9 \\
\hline 18 & Jhelum & 1.2 & 1.7 & 1.7 & 1.2 & 1.4 & 1.9 & 3.9 & 3.2 & 1.5 & 0.5 & 0.3 & 0.4 & 18.9 \\
\hline 19 & Kakul & 2.0 & 2.7 & 3.0 & 2.3 & 2.0 & 2.9 & 4.5 & 4.2 & 2.3 & 1.1 & 0.9 & 1.0 & 28.8 \\
\hline 20 & KalamP & 39.3 & 59.6 & 5.2 & 3.5 & 3.8 & 2.9 & 4.2 & 4.0 & 2.3 & 1.3 & 1.5 & 7.0 & 134.7 \\
\hline 21 & Kamra & 0.4 & 1.1 & 1.6 & 1.9 & 2.2 & 2.6 & 4.2 & 4.5 & 2.6 & 1.1 & 0.4 & 0.3 & 22.9 \\
\hline 22 & Kohat & 1.6 & 2.0 & 2.3 & & 1.3 & 0.8 & 6 & 5 & 9 & 0.6 & 0.8 & 9 & 15.8 \\
\hline 23 & Kotli-P & 2.0 & 2.3 & 2.8 & 2.6 & 2.1 & 3.3 & 4.6 & 4.3 & 2.5 & 1.0 & 0.9 & 1.0 & 29.3 \\
\hline 24 & landikotal & 2.8 & 4.7 & 5.2 & 4.1 & 2.8 & 2.8 & 3.7 & 3.2 & 2.1 & 1.8 & 1.5 & 3.8 & 38.5 \\
\hline 25 & Lower Dir & 4.0 & 9.3 & 7.7 & 6.0 & 3.1 & 2.3 & 3.0 & 3.1 & 2.5 & 1.6 & 2.1 & 3.2 & 48.0 \\
\hline 26 & Malamjaba & 55.0 & 36.6 & 11.2 & 6.1 & 3.2 & 3.2 & 4.0 & 3.9 & 2.2 & 1.9 & 2.4 & 11.0 & 140.7 \\
\hline 27 & Mangla-P & 1.3 & 1.5 & 1.4 & 0.9 & 1.0 & 1.8 & .5 & 3.0 & 5 & 1.3 & 1.1 & 5 & 18.8 \\
\hline 28 & Miranshah & 2.1 & 2.9 & 3.2 & 2.3 & 2.8 & 2.3 & 3.1 & 3.5 & 2.3 & 1.1 & 1.1 & 1.6 & 28.3 \\
\hline 29 & Mirkhani & 2.2 & 3.7 & 3.3 & 3.5 & 3.4 & 1.7 & 1.2 & 1.5 & 1.5 & 1.3 & 1.2 & 1.5 & 25.8 \\
\hline 30 & Mirpur & 1.7 & 1.8 & 1.5 & 0.9 & 1.0 & 1.8 & 3.4 & 2.9 & 1.6 & 1.3 & 1.1 & 0.5 & 19.5 \\
\hline 31 & Murree & 14.1 & 13.4 & 18.0 & 3.5 & 1.4 & 1.1 & 1.3 & 1.6 & 1.1 & 0.5 & 2.4 & 7.6 & 76.7 \\
\hline 32 & Muzaffarabad & 3.7 & 5.4 & 3.6 & 3.4 & 2.5 & 3.6 & 5.2 & 4.5 & 2.7 & 1.1 & 1.0 & 1.5 & 38.2 \\
\hline 33 & Parachinar & 1.3 & 2.1 & 2.4 & 2.0 & 1.9 & 2.1 & 3.0 & 2.8 & 1.5 & 1.1 & 0.6 & 0.7 & 21.4 \\
\hline 34 & Pattan & 6.7 & 6.2 & 4.1 & 2.9 & 3.4 & 3.0 & 3.9 & 4.5 & 3.0 & 1.6 & 1.4 & 2.2 & 42.9 \\
\hline 35 & Peshawar AP & 5.8 & 7.8 & 10.0 & 6.9 & 4.4 & 3.5 & 4.5 & 3.8 & 2.2 & 2.1 & 2.2 & 6.1 & 59.3 \\
\hline 36 & Rawlakot-P & 1.1 & 2.6 & 2.9 & 3.3 & 2.2 & 2.1 & 4.2 & 4.1 & 1.7 & 0.5 & 0.5 & 0.6 & 25.8 \\
\hline 37 & Risalpur & 7.6 & 12.7 & 4.5 & 3.0 & 2.3 & 3.3 & 4.5 & 4.2 & 2.5 & 1.0 & 1.2 & 4.0 & 50.7 \\
\hline 38 & Saidusharif & 1.8 & 3.2 & 3.3 & 2.4 & 1.8 & 2.5 & 12.4 & 9.7 & 2.3 & 0.7 & 0.9 & 1.0 & 41.9 \\
\hline 39 & Sialkot & 4.6 & 6.3 & 6.0 & 4.2 & 2.6 & 3.1 & 4.1 & 3.5 & 2.2 & 1.8 & 1.4 & 2.9 & 42.7 \\
\hline 40 & Skardu & 1.3 & 2.0 & 1.8 & 1.1 & 1.4 & 2.6 & 4.4 & 3.2 & 1.8 & 0.6 & 0.3 & 0.9 & 21.5 \\
\hline 41 & Abazai & 20.4 & 5.9 & 2.1 & 2.6 & 1.4 & 1.1 & 1.3 & 1.6 & 1.2 & 0.4 & 0.5 & 3.3 & 41.7 \\
\hline 42 & Amandara & 1.8 & 2.2 & 3.6 & 1.7 & 1.2 & 0.8 & 2.6 & 2.0 & 1.2 & 0.9 & 1.3 & 2.7 & 21.9 \\
\hline 43 & Bagh & 2.4 & 4.1 & 4.7 & 2.7 & 2.5 & 2.1 & 3.1 & 3.1 & 2.4 & 1.2 & 1.2 & 1.8 & 31.4 \\
\hline 44 & Besham & 4.2 & 4.5 & 3.7 & 2.7 & 2.3 & 3.3 & 4.5 & 4.2 & 2.4 & 1.0 & 1.1 & 2.8 & 36.5 \\
\hline 45 & Burzil & 5.2 & 5.9 & 9.9 & 6.1 & 3.8 & 3.6 & 4.2 & 4.1 & 2.3 & 1.3 & 2.1 & 3.6 & 52.0 \\
\hline 46 & Charbagh & 83.8 & 117.0 & 93.0 & 79.4 & 16.6 & 2.4 & 2.5 & 2.6 & 2.9 & 10.8 & 37.7 & 57.6 & 506.1 \\
\hline 47 & Charsadda & 6.9 & 6.3 & 11.5 & 7.5 & 4.0 & 3.2 & 3.8 & 3.4 & 2.2 & 2.1 & 2.1 & 7.7 & 60.8 \\
\hline 48 & Dagar & 1.9 & 3.5 & 2.6 & 1.6 & 1.3 & 0.8 & 1.8 & 1.5 & 0.9 & 0.5 & 0.9 & 1.1 & 18.5 \\
\hline 49 & Deosai & 3.6 & 5.6 & 5.4 & 3.5 & 2.4 & 3.1 & 4.1 & 3.7 & 2.2 & 1.8 & 1.3 & 2.4 & 39.1 \\
\hline 50 & Dhudnial & 42.4 & 50.5 & 39.4 & 34.9 & 8.7 & 2.1 & 2.1 & 2.0 & 1.9 & 3.4 & 16.6 & 35.6 & 239.7 \\
\hline 51 & Domel & 5.8 & 5.2 & 4.0 & 2.7 & 2.8 & 3.1 & 4.4 & 3.8 & 2.2 & 1.7 & 1.3 & 5.8 & 42.9 \\
\hline
\end{tabular}




\begin{tabular}{|c|c|c|c|c|c|c|c|c|c|c|c|c|c|c|}
\hline 52 & Doyian & 5.0 & 6.3 & 7.2 & 4.3 & 3.8 & 4.0 & 5.0 & 4.8 & 2.5 & 1.8 & 2.1 & 11.3 & 58.0 \\
\hline 53 & Fort Lockhart & 2.0 & 1.8 & 2.0 & 2.4 & 2.0 & 1.2 & 1.1 & 1.5 & 1.1 & 0.3 & 0.7 & 1.2 & 17.4 \\
\hline 54 & Gujar Khan & 6.6 & 8.3 & 7.4 & 3.3 & 3.0 & 3.0 & 3.6 & 3.9 & 2.9 & 1.4 & 1.6 & 2.9 & 48.1 \\
\hline 55 & Hushy & 1.2 & 1.5 & 1.4 & 0.9 & 1.0 & 1.8 & 3.5 & 2.9 & 1.6 & 1.3 & 1.1 & 0.5 & \\
\hline 56 & Jabbar & 20.1 & 19.3 & 5.4 & 3.1 & 2.3 & 2.1 & 2.1 & 2.0 & 1.5 & 0.9 & 1.4 & 17.2 & 77. \\
\hline 57 & Kachura & 27.5 & 16.4 & 14.6 & 6.6 & 3.4 & 3.2 & 4.4 & 4.6 & 2.4 & 1.2 & 2.0 & 4.6 & 90. \\
\hline 58 & KalamW & 18.1 & 7.3 & 2.5 & 2.2 & 1.7 & 1.2 & 1.0 & 1.5 & 1.1 & 0.3 & 0.7 & 3.0 & 40.7 \\
\hline 59 & Kallar & 30.8 & 24.5 & 6.7 & 5.0 & 3.6 & 3.0 & 4.2 & 4.0 & 2.3 & 1.2 & 1.6 & 5.1 & 92.1 \\
\hline 60 & Kelash & 1.2 & 1.5 & 1.4 & 0.9 & 1.0 & 1.9 & 3.5 & 2.9 & 1.6 & 1.3 & 1.1 & 0.6 & 18. \\
\hline 61 & Khairabad & 24.4 & 40.3 & 6.7 & 4.8 & 3.9 & 1.9 & 2.1 & 2.2 & 1.5 & 1.5 & 2.4 & 20.0 & 111.6 \\
\hline 62 & Khandar & 1.3 & 1.5 & 1.4 & 1.0 & 1.1 & 1.8 & 3.4 & 2.9 & 1.5 & 1.3 & 1.1 & 0.8 & 19. \\
\hline 63 & Khot & 56.7 & 114.7 & 86.6 & 8.9 & 2.6 & 1.6 & 1.7 & 1.8 & 1.8 & 3.0 & 33.0 & 57.5 & 369. \\
\hline 64 & Khunjrab-W & 40.7 & 49.6 & 51.7 & 54.7 & 19.7 & 4.1 & 2.8 & 2.9 & 5.2 & 20.0 & 31.9 & 66.3 & 349.5 \\
\hline 65 & Kotli-W & 1.4 & 2.0 & 1.7 & 1.0 & 1.1 & 1.8 & 3.5 & 2.9 & 1.6 & 1.3 & 1.1 & 1.1 & 20. \\
\hline 66 & Lora & 3.9 & 4.4 & 4.3 & 3.6 & 2.2 & 3.3 & 4.6 & 4.3 & 2.5 & 0.9 & 0.9 & 2.3 & 37.2 \\
\hline 67 & Malakand & 2.5 & 4.3 & 4.9 & 2.8 & 2.5 & 2.1 & 3.3 & 3.3 & 2.4 & 1.2 & 1.3 & 2.1 & 32.9 \\
\hline 68 & Mangla-W & 1.3 & 1.5 & 1.4 & 0.9 & 1.0 & 1.8 & 3.5 & 3.0 & 1.5 & 1.3 & 1.1 & 0.5 & 18 \\
\hline 69 & Mardan & 2.3 & 2.9 & 3.0 & 1.7 & 1.3 & 0.9 & 2.3 & 2.1 & 1.0 & 0.6 & 1.0 & 1.6 & 20.6 \\
\hline 70 & Munda dam & 2.4 & 2.9 & 3.0 & 1.7 & 1.2 & 0.9 & 2.5 & 2.5 & 1.3 & 0.6 & 1.0 & 1.2 & 21. \\
\hline 71 & Naltar & 22.0 & 40.9 & 6.9 & 4.1 & 2.0 & 2.0 & 2.2 & 2.1 & 1.8 & 1.1 & 2.3 & 20.7 & 108.1 \\
\hline 72 & Naran & 192.9 & 172.6 & 144.2 & 11.5 & 6.0 & 4.1 & 4.5 & 4.0 & 2.3 & 1.2 & 3.7 & 34.3 & 581.2 \\
\hline 73 & Oghi & 5.7 & 5.4 & 7.7 & 4.9 & 2.6 & 3.1 & 4.4 & 4.5 & 2.3 & 1.2 & 1.5 & 3.5 & 46.7 \\
\hline 74 & Palandri & 7.0 & 5.5 & 2.5 & 1.4 & 1.2 & 1.9 & 3.4 & 3.0 & 1.6 & 1.3 & 1.3 & 2.0 & 32.1 \\
\hline 75 & Phulra & 4.2 & 4.6 & 6.0 & 4.5 & 2.5 & 3.0 & 4.3 & 4.6 & 2.3 & 1.1 & 1.4 & 2.7 & 41.3 \\
\hline 76 & Pir Chenasi & 168.5 & 111.1 & 7.8 & 6.0 & 4.9 & 4.7 & 5.0 & 4.6 & 2.5 & 1.8 & 3.5 & 19.9 & 340.2 \\
\hline 77 & Puran & 4.9 & 5.9 & 7.3 & 4.0 & 2.7 & 3.2 & 4.2 & 3.6 & 2.2 & 1.9 & 1.5 & 2.8 & 44 \\
\hline 78 & Qalangi & 2.5 & 4.3 & 4.4 & 2.6 & 2.5 & 2.0 & 3.2 & 3.0 & 2.4 & 1.2 & 1.3 & 1.9 & 31 \\
\hline 79 & Rama & 88.2 & 161.3 & 80.9 & 11.5 & 2.9 & 2.0 & 2.2 & 2.1 & 1.6 & 1.3 & 19.1 & 105.6 & 478.8 \\
\hline 80 & Ratu & 55.2 & 79.9 & 20.3 & 7.1 & 3.2 & 2.0 & 2.1 & 2.0 & 1.6 & 1.1 & 7.4 & 96.5 & 278.5 \\
\hline 81 & Rawlakot-W & 8.3 & 6.3 & 4.2 & 2.7 & 2.8 & 3.2 & 4.5 & 3.8 & 2.2 & 1.7 & 1.2 & 2.8 & 43.6 \\
\hline 82 & Saifulmulk & 241.4 & 212.2 & 103.8 & 12.5 & 5.0 & 4.3 & 4.5 & 4.1 & 2.3 & 1.8 & 18.2 & 162.5 & 772.5 \\
\hline 83 & Sehrkakota & 4.1 & 5.4 & 4.8 & 2.6 & 2.7 & 3.1 & 4.5 & 3.8 & 2.2 & 1.7 & 1.2 & 5.2 & 41 \\
\hline 84 & Shahpur & 42.5 & 25.0 & 19.9 & 11.7 & 5.5 & 3.9 & 3.9 & 3.5 & 2.2 & 2.6 & 4.1 & 14.1 & 139.1 \\
\hline 85 & Shangla & 14.3 & 13.7 & 8.5 & 7.0 & 4.4 & 3.6 & 3.9 & 3.6 & 2.2 & 2.1 & 3.4 & 10.5 & 77.2 \\
\hline 86 & Shendure & 12.5 & 22.2 & 13.4 & 4.5 & 2.0 & 1.2 & 0.7 & 0.7 & 0.8 & 1.3 & 7.4 & 13.4 & 80. \\
\hline 87 & Shigar & 27.4 & 8.7 & 3.6 & 3.2 & 2.7 & 2.0 & 2.1 & 2.0 & 1.6 & 0.9 & 1.5 & 6.4 & 62 \\
\hline 88 & Shinkiari & 4.5 & 4.8 & 6.7 & 4.5 & 2.7 & 3.2 & 4.5 & 4.7 & 2.4 & 1.2 & 1.6 & 3.0 & 43 \\
\hline 89 & Shogran & 144.0 & 131.7 & 16.7 & 5.8 & 4.2 & 3.7 & 4.4 & 4.6 & 2.5 & 1.7 & 6.1 & 135.4 & 460.9 \\
\hline 90 & Tandar & 1.3 & 1.7 & 1.6 & 1.0 & 1.2 & 1.8 & 3.7 & 3.0 & 1.6 & 1.3 & 1.1 & 1.1 & 20.3 \\
\hline 91 & Tarbela & 0.5 & 1.2 & 1.8 & 2.0 & 2.2 & 2.6 & 4.3 & 4.5 & 2.6 & 1.1 & 0.4 & 0.4 & 23 . \\
\hline 92 & Ushkore & 10.1 & 24.4 & 10.1 & 3.2 & 2.1 & 1.1 & 0.6 & 0.6 & 0.8 & 1.1 & 2.1 & 10.9 & 67. \\
\hline 93 & Yasin & 20.3 & 38.6 & 18.3 & 3.8 & 1.7 & 1.2 & 0.8 & 0.8 & 1.0 & 1.1 & 6.9 & 23.3 & 117. \\
\hline 94 & Yugo & 6.4 & 4.8 & 1.6 & 1.9 & 1.6 & 1.2 & 1.0 & 1.4 & 1.1 & 0.3 & 0.5 & 2.3 & 24 . \\
\hline 95 & Zani & 33.6 & 61.8 & 48.4 & 23.1 & 4.4 & 2.3 & 2.2 & 2.3 & 1.9 & 4.6 & 27.0 & 34.8 & 246.4 \\
\hline 96 & Ziarat & 29.8 & 47.4 & 33.7 & 5.8 & 1.8 & 0.9 & 0.6 & 0.8 & 1.3 & 2.0 & 16.5 & 48.8 & 189 \\
\hline 97 & Zulam Br. & 2.8 & 4.4 & 4.7 & 2.8 & 2.6 & 2.1 & 3.2 & 3.1 & 2.4 & 1.2 & 1.2 & 1.7 & 32 \\
\hline 98 & Alambar & 92.5 & 211.1 & 113.5 & 53.0 & 7.3 & 2.5 & 1.2 & 1.3 & 4.8 & 15.8 & 39.8 & 91.1 & 633. \\
\hline 99 & Bagrot & 11.5 & 3.2 & 2.2 & 3.0 & 2.4 & 1.9 & 2.2 & 2.5 & 2.4 & 0.7 & 0.6 & 1.6 & 34 \\
\hline 100 & Baldihel & 24.2 & 31.3 & 26.6 & 37.8 & 4.4 & 2.2 & 2.4 & 2.4 & 2.4 & 3.0 & 17.9 & 25.2 & 179.8 \\
\hline 101 & Bulibalsirbar & 24.1 & 66.0 & 34.4 & 19.6 & 4.4 & 2.1 & 1.5 & 1.2 & 2.2 & 5.3 & 12.7 & 32.6 & 206. \\
\hline 102 & Dadormal & 82.4 & 35.7 & 32.3 & 11.7 & 3.3 & 2.2 & 2.4 & 2.2 & 2.0 & 1.7 & 9.7 & 22.1 & 207. \\
\hline 103 & Dame & 139.8 & 59.5 & 57.4 & 34.4 & 5.4 & 2.4 & 2.7 & 2.4 & 2.7 & 3.4 & 17.7 & 37.1 & 365. \\
\hline 104 & Diran & 108.2 & 46.3 & 43.4 & 18.1 & 4.1 & 2.3 & 2.6 & 2.3 & 2.3 & 2.2 & 13.3 & 28.8 & 273 \\
\hline 105 & Garmashbar & 69.0 & 155.7 & 76.3 & 8.3 & 2.6 & 1.7 & 1.3 & 1.4 & 2.1 & 2.3 & 26.1 & 67.3 & 414.2 \\
\hline 106 & Khaimetbar & 23.5 & 63.7 & 31.2 & 5.9 & 3.1 & 1.8 & 1.5 & 1.2 & 1.7 & 2.1 & 11.2 & 31.4 & 178. \\
\hline 107 & Khunjrab-C & 44.4 & 54.2 & 57.0 & 61.4 & 41.3 & 17.7 & 4.8 & 4.9 & 22.6 & 22.4 & 35.2 & 72.9 & 438.8 \\
\hline 108 & Akhnoor & 1.6 & 2.3 & 2.4 & 2.0 & 1.9 & 2.3 & 3.6 & 3.4 & 2.0 & 1.1 & 0.8 & 1.0 & \\
\hline
\end{tabular}




\begin{tabular}{|c|c|c|c|c|c|c|c|c|c|c|c|c|c|c|}
\hline 109 & Anantnag & 2.8 & 2.2 & 3.2 & 2.8 & 2.3 & 1.4 & 1.9 & 1.7 & 0.9 & 0.7 & 0.7 & 1.9 & 22.5 \\
\hline 110 & Arizal & 2.3 & 2.1 & 2.6 & 2.3 & 2.2 & 1.4 & 1.9 & 1.7 & 0.9 & 0.7 & 0.7 & 1.7 & 20.5 \\
\hline 111 & Arki & 1.6 & 2.1 & 2.3 & 1.9 & 2.0 & 2.4 & 3.6 & 3.4 & 2.1 & 1.1 & 0.8 & 0.9 & 24.2 \\
\hline 112 & Babapura & 2.3 & 2.3 & 2.6 & 2.4 & 2.1 & 1.4 & 1.9 & 1.7 & 0.9 & 0.7 & 0.7 & 1.6 & 20.6 \\
\hline 113 & Badarwah & 4.1 & 3.0 & 2.2 & 1.4 & 1.3 & 1.6 & 3.2 & 2.8 & 1.4 & 0.5 & 0.4 & 1.7 & 23.7 \\
\hline 114 & Badgam & 2.3 & 2.4 & 2.7 & 2.4 & 2.1 & 1.4 & 2.0 & 1.7 & 0.9 & 0.7 & 0.7 & 1.6 & 20.7 \\
\hline 115 & Bandipura & 5.2 & 3.8 & 3.1 & 2.6 & 2.2 & 1.4 & 1.9 & 1.7 & 0.9 & 0.8 & 1.0 & 2.6 & 27.1 \\
\hline 116 & Banihal & 5.4 & 4.1 & 3.0 & 2.7 & 2.2 & 1.4 & 2.0 & 1.7 & 0.9 & 0.7 & 0.8 & 3.3 & 28.1 \\
\hline 117 & Banjar Saraj & 2.4 & 2.5 & 2.5 & 2.1 & 2.0 & 2.5 & 3.7 & 3.3 & 2.0 & 1.1 & 0.8 & 1.2 & 26.2 \\
\hline 118 & Baramula & 2.5 & 3.0 & 2.7 & 2.5 & 2.2 & 1.4 & 1.9 & 1.7 & 0.8 & 0.7 & 0.7 & 1.7 & 21.9 \\
\hline 119 & Bashila & 10.8 & 5.6 & 2.9 & 2.1 & 2.1 & 2.4 & 3.5 & 3.2 & 2.0 & 1.1 & 1.0 & 5.9 & 42.5 \\
\hline 120 & Batote & 1.6 & 1.9 & 1.6 & 1.3 & 1.4 & 1.7 & 3.2 & 2.8 & 1.4 & 0.5 & 0.4 & 0.6 & 18.3 \\
\hline 121 & Bhagtan & 3.4 & 2.8 & 3.3 & 4.7 & 3.1 & 3.0 & 3.6 & 3.5 & 2.2 & 1.3 & 1.5 & 2.8 & 35.1 \\
\hline 122 & Bhangrotu & 1.8 & 2.3 & 2.6 & 2.0 & 2.0 & 2.9 & 4.2 & 3.6 & 2.1 & 1.1 & 0.8 & 1.2 & 26.5 \\
\hline 123 & Bhuntar & 2.3 & 2.3 & 2.7 & 2.1 & 2.0 & 2.4 & 3.5 & 3.3 & 2.0 & 1.1 & 0.8 & 1.2 & 25.7 \\
\hline 124 & Bilaspur & 1.7 & 2.2 & 2.3 & 1.9 & 1.9 & 2.3 & 3.4 & 3.2 & 2.0 & 1.1 & 0.8 & 1.1 & 24.0 \\
\hline 125 & Chachiot & 2.6 & 2.5 & 2.5 & 2.0 & 2.0 & 2.7 & 4.3 & 3.5 & 2.1 & 1.1 & 0.9 & 1.3 & 27.5 \\
\hline 126 & Chandigarh & 1.6 & 2.2 & 2.3 & 1.9 & 1.9 & 2.3 & 3.5 & 3.2 & 2.0 & 1.1 & 0.8 & 1.0 & 23.8 \\
\hline 127 & Charisharif & 2.7 & 2.4 & 2.7 & 2.4 & 2.2 & 1.4 & 2.0 & 1.7 & 0.9 & 0.7 & 0.7 & 1.8 & 21.7 \\
\hline 128 & Chenani & 1.8 & 2.4 & 2.5 & 2.1 & 2.0 & 2.3 & 3.7 & 3.3 & 2.0 & 1.1 & 0.8 & 1.2 & 25.2 \\
\hline 129 & Chini Kalpa & 22.7 & 43.6 & 6.1 & 2.4 & 2.5 & 2.6 & 3.8 & 3.4 & 2.2 & 1.1 & 1.0 & 7.5 & 98.8 \\
\hline 130 & Chowari & 1.7 & 2.3 & 2.6 & 2.0 & 2.0 & 2.3 & 3.7 & 3.5 & 2.0 & 1.1 & 0.8 & 1.1 & 25.2 \\
\hline 131 & Dalhousie & 5.3 & 4.4 & 2.9 & 2.2 & 2.1 & 2.3 & 3.4 & 3.2 & 2.0 & 1.1 & 0.9 & 2.9 & 32.8 \\
\hline 132 & Dasuya & 1.7 & 2.1 & 2.3 & 1.9 & 1.9 & 2.4 & 3.7 & 3.3 & 1.9 & 1.1 & 0.8 & 1.1 & 24.2 \\
\hline 133 & Dehra Gopipur & 1.6 & 2.1 & 2.3 & 1.9 & 1.9 & 2.3 & 3.5 & 3.2 & 2.0 & 1.1 & 0.8 & 1.2 & 24.0 \\
\hline 134 & Dharampur & 2.1 & 2.5 & 2.3 & 1.9 & 2.0 & 2.4 & 3.7 & 3.4 & 2.1 & 1.1 & 0.8 & 1.0 & 25.3 \\
\hline 135 & Dharamshala & 8.6 & 6.7 & 7.3 & 3.0 & 2.1 & 2.7 & 3.6 & 3.5 & 2.4 & 1.5 & 1.4 & 6.7 & 49.3 \\
\hline 136 & Dharamshala-L & 12.8 & 9.6 & 7.7 & 3.7 & 2.2 & 2.7 & 3.7 & 3.6 & 2.5 & 1.4 & 1.7 & 10.8 & 62.4 \\
\hline 137 & Dharamshala-U & 2.1 & 2.2 & 2.3 & 1.9 & 1.9 & 2.3 & 3.8 & 3.4 & 2.0 & 1.1 & 0.9 & 1.4 & 25.5 \\
\hline 138 & Digar & 11.6 & 3.9 & 4.0 & 5.0 & 3.8 & 4.6 & 5.2 & 5.0 & 8.3 & 3.4 & 4.0 & 6.3 & 65.0 \\
\hline 139 & Dras & 27.9 & 20.4 & 16.9 & 3.6 & 2.8 & 2.3 & 3.4 & 3.2 & 2.0 & 1.4 & 2.2 & 14.2 & 100.4 \\
\hline 140 & Durroo & 5.2 & 2.6 & 2.9 & 2.6 & 2.1 & 1.4 & 2.0 & 1.7 & 0.9 & 0.7 & 0.9 & 2.4 & 25.4 \\
\hline 141 & Garhshankar & 1.7 & 2.2 & 2.4 & 1.9 & 1.9 & 2.3 & 3.5 & 3.2 & 2.0 & 1.1 & 0.8 & 1.1 & 24.0 \\
\hline 142 & Gondhla & 145.5 & 81.4 & 34.3 & 9.7 & 2.5 & 2.6 & 3.4 & 3.2 & 2.2 & 0.9 & 4.3 & 42.6 & 332.6 \\
\hline 143 & GS Nagar & 3.4 & 2.7 & 2.4 & 2.0 & 2.4 & 3.3 & 4.2 & 5.7 & 3.5 & 1.3 & 1.1 & 1.2 & 33.4 \\
\hline 144 & Gulabgarh & 20.3 & 5.5 & 2.6 & 2.0 & 2.0 & 2.3 & 3.7 & 3.4 & 2.0 & 1.1 & 0.9 & 4.1 & 49.8 \\
\hline 145 & Gulmarg & 37.9 & 38.8 & 5.9 & 2.6 & 2.5 & 1.5 & 2.0 & 1.7 & 0.9 & 0.8 & 1.5 & 16.7 & 112.7 \\
\hline 146 & Gund & 6.5 & 3.6 & 2.9 & 2.6 & 2.2 & 1.4 & 2.0 & 1.8 & 0.9 & 0.7 & 0.8 & 2.9 & 28.4 \\
\hline 147 & Gurez & 103.4 & 38.4 & 5.8 & 2.8 & 2.6 & 1.4 & 1.9 & 1.7 & 0.9 & 0.8 & 1.4 & 16.1 & 177.3 \\
\hline 148 & Hamirpur & 1.5 & 2.1 & 2.3 & 1.9 & 1.9 & 2.4 & 3.8 & 3.6 & 2.1 & 1.1 & 0.8 & 0.9 & 24.5 \\
\hline 149 & Handwara & 5.1 & 4.1 & 3.2 & 2.6 & 2.2 & 1.4 & 1.9 & 1.7 & 0.9 & 0.8 & 1.1 & 3.5 & 28.4 \\
\hline 150 & Hoshiyarpur & 1.6 & 2.1 & 2.3 & 1.9 & 1.9 & 2.3 & 3.4 & 3.2 & 2.0 & 1.1 & 0.8 & 1.0 & 23.7 \\
\hline 151 & Jammu & 1.6 & 2.2 & 2.4 & 2.0 & 2.0 & 2.3 & 3.6 & 3.3 & 2.0 & 1.1 & 0.8 & 1.0 & 24.2 \\
\hline 152 & Janjehli & 5.2 & 4.1 & 2.9 & 2.1 & 2.0 & 2.8 & 4.0 & 3.5 & 2.1 & 1.1 & 0.9 & 2.6 & 33.4 \\
\hline 153 & Jhungi & 1.8 & 2.4 & 2.3 & 2.0 & 2.0 & 2.5 & 3.6 & 3.4 & 2.1 & 1.1 & 0.8 & 1.0 & 24.9 \\
\hline 154 & Jogindarnagar & 1.6 & 2.2 & 2.3 & 1.9 & 2.0 & 2.5 & 4.1 & 4.0 & 2.2 & 1.1 & 0.8 & 0.9 & 25.6 \\
\hline 155 & Jubal & 4.5 & 3.5 & 2.6 & 2.0 & 2.1 & 2.5 & 3.4 & 3.2 & 2.0 & 1.1 & 0.9 & 1.9 & 29.7 \\
\hline 156 & Junga & 1.6 & 2.1 & 2.3 & 1.9 & 2.0 & 2.4 & 3.6 & 3.3 & 2.1 & 1.1 & 0.8 & 0.9 & 24.3 \\
\hline 157 & Kalka & 1.6 & 2.1 & 2.3 & 1.9 & 2.0 & 2.3 & 3.5 & 3.3 & 2.0 & 1.1 & 0.8 & 0.9 & 23.8 \\
\hline 158 & Kandaghat & 1.6 & 2.1 & 2.3 & 1.9 & 2.0 & 2.4 & 3.6 & 3.3 & 2.1 & 1.1 & 0.8 & 0.9 & 24.2 \\
\hline 159 & Kangra & 7.4 & 4.9 & 7.2 & 2.4 & 2.0 & 2.5 & 3.5 & 3.4 & 2.3 & 1.5 & 1.0 & 5.7 & 43.8 \\
\hline 160 & Kargil & 44.2 & 36.7 & 7.2 & 3.0 & 1.8 & 1.2 & 1.0 & 1.4 & 1.1 & 0.3 & 0.5 & 14.6 & 113.1 \\
\hline 161 & Karsog & 1.6 & 2.2 & 2.3 & 1.9 & 2.0 & 2.4 & 3.5 & 3.3 & 2.0 & 1.1 & 0.8 & 0.9 & 24.1 \\
\hline 162 & Kasauli1 & 2.0 & 2.4 & 2.3 & 1.9 & 1.9 & 2.3 & 3.8 & 3.3 & 2.0 & 1.1 & 0.8 & 1.0 & 24.8 \\
\hline 163 & Kasauli2 & 1.6 & 2.2 & 2.3 & 1.9 & 2.0 & 2.4 & 3.8 & 3.5 & 2.1 & 1.1 & 0.8 & 1.0 & 24.6 \\
\hline 164 & Kasumpti & 1.7 & 2.3 & 2.3 & 1.9 & 2.0 & 2.4 & 3.6 & 3.4 & 2.1 & 1.1 & 0.8 & 1.0 & 24.6 \\
\hline 165 & Kataula & 2.2 & 2.6 & 2.4 & 2.0 & 2.0 & 2.6 & 4.2 & 3.6 & 2.0 & 1.1 & 0.8 & 1.4 & 27.0 \\
\hline
\end{tabular}




\begin{tabular}{|c|c|c|c|c|c|c|c|c|c|c|c|c|c|c|}
\hline 166 & Khadrala & 62.0 & 66.7 & 5.3 & 2.2 & 2.1 & 2.5 & 3.5 & 3.3 & 2.0 & 1.2 & 1.4 & 84.1 & 236.0 \\
\hline 167 & Khalatse & 9.2 & 9.3 & 2.8 & 1.8 & 1.4 & 1.3 & 1.3 & 1.6 & 1.1 & 0.3 & 1.5 & 7.6 & 39.2 \\
\hline 168 & Khangral & 8.3 & 11.7 & 14.2 & 4.7 & 1.5 & 1.2 & 1.1 & 1.4 & 1.3 & 0.6 & 1.6 & 4.8 & 52.3 \\
\hline 169 & Kharar & 1.7 & 2.1 & 2.3 & 1.9 & 1.9 & 2.3 & 3.6 & 3.2 & 2.0 & 1.1 & 0.8 & 1.0 & 24.0 \\
\hline 170 & Kilba & 23.0 & 20.5 & 4.7 & 2.3 & 2.4 & 2.6 & 3.9 & 3.5 & 2.2 & 1.1 & 1.1 & 3.2 & 70.5 \\
\hline 171 & Kishtwar & 2.2 & 3.0 & 3.3 & 3.2 & 2.6 & 2.4 & 3.3 & 3.1 & 2.0 & 1.2 & 1.0 & 1.6 & 28.8 \\
\hline 172 & Kokernagh & 3.6 & 3.4 & 3.1 & 3.0 & 2.6 & 2.3 & 3.4 & 3.1 & 2.0 & 1.2 & 1.0 & 1.8 & 30.5 \\
\hline 173 & Koksar & 253.8 & 125.6 & 43.1 & 8.6 & 2.6 & 2.8 & 3.6 & 3.2 & 2.2 & 2.4 & 6.0 & 100.0 & 553.7 \\
\hline 174 & Kotarh & 4.6 & 3.4 & 2.8 & 2.0 & 2.0 & 2.5 & 3.5 & 3.2 & 2.0 & 1.1 & 0.9 & 2.1 & 30.1 \\
\hline 175 & Kothi & 121.1 & 37.5 & 11.3 & 6.3 & 2.6 & 3.3 & 3.7 & 3.5 & 2.4 & 1.1 & 1.3 & 12.8 & 206.9 \\
\hline 176 & Kotkhai & 2.7 & 2.6 & 2.6 & 2.0 & 2.0 & 2.4 & 3.4 & 3.2 & 2.0 & 1.1 & 0.9 & 1.7 & 26.7 \\
\hline 177 & Kukernag & 5.0 & 4.0 & 2.9 & 2.8 & 2.2 & 1.4 & 2.0 & 1.7 & 0.9 & 0.7 & 0.8 & 2.7 & 27.0 \\
\hline 178 & Kulgam & 2.2 & 2.3 & 2.7 & 2.5 & 2.1 & 1.4 & 2.0 & 1.7 & 0.9 & 0.7 & 0.7 & 1.5 & 20.6 \\
\hline 179 & Kulu & 2.5 & 2.6 & 2.6 & 2.1 & 2.0 & 2.4 & 3.5 & 3.2 & 2.0 & 1.1 & 0.8 & 1.3 & 26.2 \\
\hline 180 & Kumarsain & 3.1 & 2.7 & 2.7 & 2.0 & 2.0 & 2.4 & 3.4 & 3.2 & 2.0 & 1.1 & 0.9 & 1.4 & 27.0 \\
\hline 181 & Kyelong & 93.6 & 119.3 & 33.7 & 5.0 & 2.7 & 2.4 & 3.4 & 3.2 & 2.3 & 1.1 & 8.0 & 46.9 & 321.5 \\
\hline 182 & Langet & 4.8 & 4.2 & 3.2 & 2.6 & 2.2 & 1.4 & 1.9 & 1.7 & 0.9 & 0.7 & 1.1 & 3.2 & 27.9 \\
\hline 183 & Leh & 11.5 & 8.0 & 2.2 & 1.5 & 1.3 & 1.2 & 1.1 & 1.5 & 1.1 & 0.3 & 0.9 & 5.2 & 35.8 \\
\hline 184 & Malashahibag & 2.9 & 2.4 & 2.7 & 2.4 & 2.1 & 1.4 & 2.0 & 1.7 & 0.9 & 0.7 & 0.8 & 1.9 & 21.8 \\
\hline 185 & Malikpur & 1.7 & 2.2 & 2.5 & 2.0 & 1.9 & 2.3 & 3.6 & 3.4 & 2.0 & 1.1 & 0.8 & 1.1 & 24.7 \\
\hline 186 & Mandil & 1.9 & 2.2 & 2.5 & 2.0 & 2.0 & 2.7 & 4.1 & 3.6 & 2.1 & 1.1 & 0.8 & 1.1 & 26.1 \\
\hline 187 & Mandi2 & 1.8 & 2.2 & 2.4 & 2.0 & 2.0 & 2.6 & 4.1 & 3.7 & 2.1 & 1.1 & 0.8 & 1.2 & 25.9 \\
\hline 188 & Mulbek & 33.0 & 21.4 & 23.6 & 9.6 & 2.1 & 2.3 & 3.4 & 3.1 & 2.0 & 1.7 & 6.6 & 19.7 & 128.7 \\
\hline 189 & Nalagarh & 1.7 & 2.1 & 2.3 & 1.9 & 1.9 & 2.3 & 3.4 & 3.2 & 2.0 & 1.1 & 0.8 & 1.1 & 24.0 \\
\hline 190 & Nawanshahr & 1.7 & 2.2 & 2.4 & 1.9 & 1.9 & 2.3 & 3.5 & 3.2 & 2.0 & 1.1 & 0.8 & 1.1 & 24.1 \\
\hline 191 & Nichar & 5.4 & 6.6 & 3.1 & 2.3 & 2.6 & 2.8 & 4.5 & 4.0 & 2.3 & 1.1 & 0.9 & 1.4 & 36.9 \\
\hline 192 & Nowshera & 1.8 & 2.3 & 2.4 & 2.0 & 2.0 & 2.3 & 3.5 & 3.2 & 2.0 & 1.1 & 0.8 & 1.0 & 24.4 \\
\hline 193 & Nurpur & 1.7 & 2.2 & 2.5 & 1.9 & 1.9 & 2.3 & 3.6 & 3.5 & 2.0 & 1.1 & 0.8 & 1.1 & 24.6 \\
\hline 194 & Palampur & 7.5 & 5.4 & 7.4 & 2.9 & 2.0 & 2.5 & 3.6 & 3.4 & 2.4 & 1.8 & 1.9 & 7.6 & 48.3 \\
\hline 195 & Panamik & 4.5 & 5.7 & 1.9 & 1.6 & 1.4 & 1.4 & 1.2 & 1.5 & 1.1 & 0.2 & 0.4 & 9.7 & 30.6 \\
\hline 196 & Panjain & 2.6 & 2.9 & 2.5 & 2.1 & 2.0 & 2.5 & 3.9 & 3.5 & 2.1 & 1.1 & 0.9 & 1.7 & 27.6 \\
\hline 197 & Pathankot & 1.6 & 2.1 & 2.3 & 1.9 & 1.9 & 2.3 & 3.6 & 3.4 & 2.0 & 1.1 & 0.8 & 1.0 & 24.1 \\
\hline 198 & Pendras & 98.4 & 88.4 & 109.6 & 89.9 & 46.9 & 4.4 & 3.6 & 3.7 & 4.8 & 9.1 & 16.8 & 58.2 & 533.8 \\
\hline 199 & Phalgam & 3.2 & 2.7 & 3.1 & 3.1 & 2.5 & 1.4 & 2.0 & 1.7 & 0.9 & 0.8 & 0.8 & 2.0 & 24.1 \\
\hline 200 & Poonch & 2.8 & 2.2 & 2.8 & 2.3 & 2.2 & 1.5 & 2.1 & 1.8 & 0.9 & 0.7 & 0.7 & 1.7 & 21.8 \\
\hline 201 & Prang & 7.2 & 3.9 & 3.2 & 2.5 & 2.2 & 1.4 & 1.9 & 1.7 & 0.9 & 0.8 & 0.9 & 3.6 & 30.3 \\
\hline 202 & Purbani & 33.9 & 15.3 & 3.9 & 2.3 & 2.4 & 2.5 & 3.6 & 3.3 & 2.1 & 1.1 & 1.0 & 3.1 & 74.3 \\
\hline 203 & Qazi Gund & 4.5 & 3.6 & 2.8 & 2.4 & 2.1 & 1.4 & 2.0 & 1.7 & 0.9 & 0.7 & 0.7 & 2.5 & 25.3 \\
\hline 204 & Rajdhani & 2.3 & 2.3 & 2.5 & 2.1 & 2.1 & 2.3 & 3.5 & 3.3 & 2.0 & 1.1 & 0.8 & 1.2 & 25.5 \\
\hline 205 & Ramban & 2.6 & 2.7 & 2.6 & 2.2 & 2.1 & 2.3 & 3.5 & 3.2 & 2.0 & 1.1 & 0.9 & 2.1 & 27.1 \\
\hline 206 & Ramnagar & 1.7 & 2.3 & 2.5 & 2.0 & 2.0 & 2.4 & 4.1 & 3.7 & 2.1 & 1.1 & 0.8 & 1.1 & 25.6 \\
\hline 207 & Rampur & 2.1 & 2.4 & 2.6 & 2.0 & 2.0 & 2.4 & 3.4 & 3.2 & 2.0 & 1.1 & 0.9 & 1.3 & 25.3 \\
\hline 208 & Riasi & 1.7 & 2.4 & 2.4 & 2.0 & 2.0 & 2.3 & 3.7 & 3.5 & 2.0 & 1.1 & 0.8 & 1.0 & 24.9 \\
\hline 209 & Rohru & 3.9 & 3.3 & 2.7 & 2.0 & 2.0 & 2.5 & 3.4 & 3.2 & 2.0 & 1.1 & 0.9 & 1.7 & 28.7 \\
\hline 210 & Rupanagar & 1.8 & 2.2 & 2.3 & 1.9 & 1.9 & 2.3 & 3.4 & 3.2 & 2.0 & 1.1 & 0.8 & 1.0 & 23.9 \\
\hline 211 & Sangla & 17.3 & 5.3 & 5.0 & 3.3 & 2.6 & 2.4 & 3.4 & 3.2 & 2.1 & 1.3 & 1.5 & 4.1 & 51.4 \\
\hline 212 & Sarkahat & 1.5 & 2.1 & 2.3 & 1.9 & 1.9 & 2.5 & 4.0 & 3.8 & 2.2 & 1.1 & 0.8 & 0.9 & 25.2 \\
\hline 213 & Shillaru & 11.2 & 4.9 & 3.1 & 2.0 & 2.1 & 2.5 & 3.5 & 3.2 & 2.0 & 1.1 & 1.0 & 2.0 & 38.7 \\
\hline 214 & Shimla & 2.3 & 2.6 & 2.4 & 1.9 & 2.0 & 2.5 & 3.7 & 3.4 & 2.1 & 1.1 & 0.8 & 1.0 & 25.8 \\
\hline 215 & Shiquanhe1 & 4.6 & 4.5 & 4.0 & 2.3 & 1.5 & 1.3 & 1.3 & 1.6 & 1.1 & 1.1 & 2.5 & 3.8 & 29.6 \\
\hline 216 & Shopian & 2.7 & 2.4 & 2.7 & 2.5 & 2.2 & 1.4 & 2.0 & 1.7 & 0.9 & 0.7 & 0.7 & 1.9 & 21.9 \\
\hline 217 & Sogam & 7.7 & 5.0 & 3.2 & 2.6 & 2.2 & 1.4 & 1.9 & 1.7 & 0.9 & 0.8 & 1.0 & 4.9 & 33.3 \\
\hline 218 & Solan & 1.6 & 2.2 & 2.3 & 1.9 & 2.0 & 2.4 & 3.6 & 3.4 & 2.1 & 1.1 & 0.8 & 1.0 & 24.4 \\
\hline 219 & Sonemarg & 136.6 & 69.5 & 12.7 & 6.2 & 6.0 & 2.0 & 2.0 & 2.0 & 1.3 & 2.2 & 2.2 & 25.4 & 268.0 \\
\hline 220 & Sopore & 2.7 & 3.9 & 3.0 & 2.5 & 2.1 & 1.4 & 1.9 & 1.7 & 0.9 & 0.7 & 1.0 & 2.0 & 23.8 \\
\hline 221 & SR Sing & 1.6 & 2.2 & 2.4 & 1.9 & 2.0 & 2.3 & 3.6 & 3.2 & 2.0 & 1.1 & 0.8 & 1.0 & 24.1 \\
\hline 222 & Srinagar & 3.1 & 2.4 & 2.7 & 2.4 & 2.1 & 1.4 & 1.9 & 1.7 & 0.9 & 0.7 & 0.7 & 2.6 & 22.7 \\
\hline
\end{tabular}




\begin{tabular}{|c|c|c|c|c|c|c|c|c|c|c|c|c|c|c|}
\hline 223 & Sundarnagar & 2.1 & 2.3 & 2.5 & 2.0 & 2.0 & 2.7 & 4.0 & 3.6 & 2.1 & 1.1 & 0.8 & 1.1 & 26.2 \\
\hline 224 & Suni Seoni & 1.5 & 2.1 & 2.3 & 1.9 & 2.0 & 2.4 & 3.6 & 3.3 & 2.0 & 1.1 & 0.8 & 0.9 & 24.0 \\
\hline 225 & Tanda & 1.5 & 2.1 & 2.3 & 1.9 & 1.9 & 2.3 & 3.6 & 3.3 & 2.1 & 1.1 & 0.8 & 0.9 & 23.9 \\
\hline 226 & Tangmarg & 6.1 & 3.8 & 2.9 & 2.4 & 2.3 & 1.4 & 1.9 & 1.7 & 0.9 & 0.7 & 0.8 & 2.7 & 27.7 \\
\hline 227 & Tapoban & 19.9 & 6.6 & 4.4 & 2.5 & 2.1 & 2.7 & 4.0 & 3.6 & 2.2 & 1.3 & 0.9 & 2.2 & 52.5 \\
\hline 228 & Theog & 3.8 & 3.3 & 2.6 & 2.0 & 2.1 & 2.5 & 3.5 & 3.2 & 2.0 & 1.1 & 0.9 & 1.9 & 28.9 \\
\hline 229 & Tibri & 1.6 & 2.1 & 2.3 & 1.9 & 1.9 & 2.3 & 3.4 & 3.2 & 2.0 & 1.1 & 0.8 & 1.0 & 23.8 \\
\hline 230 & Tral & 3.0 & 2.4 & 3.0 & 2.8 & 2.3 & 1.4 & 1.9 & 1.7 & 0.9 & 0.7 & 0.7 & 1.8 & 22.5 \\
\hline 231 & TKIH Kung & 6.7 & 13.7 & 14.2 & 6.0 & 7.3 & 2.6 & 14.7 & 6.9 & 12.1 & 6.6 & 2.8 & 3.4 & 97.1 \\
\hline 232 & Udhampur & 1.7 & 2.3 & 2.4 & 2.0 & 1.9 & 2.3 & 3.9 & 3.7 & 2.1 & 1.1 & 0.8 & 1.0 & 25.2 \\
\hline 233 & Una & 1.5 & 2.1 & 2.3 & 1.9 & 1.9 & 2.3 & 3.7 & 3.5 & 2.1 & 1.1 & 0.8 & 0.9 & 24.2 \\
\hline 234 & Uri & 4.9 & 3.1 & 2.8 & 2.3 & 2.4 & 1.4 & 2.0 & 1.7 & 0.9 & 0.7 & 0.8 & 2.3 & 25.2 \\
\hline 235 & Uttamchipura & 93.8 & 57.8 & 42.3 & 4.4 & 2.5 & 1.5 & 1.9 & 1.7 & 0.9 & 1.1 & 3.3 & 80.6 & 292.0 \\
\hline 236 & Vantipura & 2.5 & 2.3 & 2.6 & 2.4 & 2.1 & 1.4 & 1.9 & 1.7 & 0.9 & 0.7 & 0.7 & 1.6 & 20.8 \\
\hline 237 & Verinagh & 2.8 & 3.2 & 2.9 & 2.6 & 2.3 & 1.4 & 2.0 & 1.7 & 0.9 & 0.7 & 0.7 & 2.3 & 23.5 \\
\hline 238 & Arthal & 16.6 & 7.5 & 3.8 & 2.8 & 2.4 & 2.4 & 3.3 & 3.1 & 1.9 & 1.2 & 1.0 & 4.8 & 50.7 \\
\hline 239 & Bhakra & 1.5 & 2.1 & 2.3 & 2.0 & 2.0 & 2.3 & 3.7 & 3.4 & 2.1 & 1.1 & 0.8 & 0.9 & 24.3 \\
\hline 240 & Bunencha & 44.9 & 27.1 & 5.8 & 2.4 & 2.1 & 2.3 & 3.5 & 3.2 & 2.0 & 1.1 & 1.2 & 8.2 & 103.7 \\
\hline 241 & Chingaon & 9.4 & 5.5 & 3.0 & 2.4 & 2.2 & 2.3 & 3.5 & 3.2 & 2.0 & 1.1 & 0.9 & 2.6 & 38.1 \\
\hline 242 & Chitkul & 92.2 & 64.2 & 82.7 & 9.7 & 3.3 & 2.5 & 3.5 & 3.4 & 2.7 & 2.4 & 15.0 & 63.6 & 345.2 \\
\hline 243 & Damini & 2.7 & 2.9 & 2.8 & 2.2 & 2.1 & 2.3 & 3.6 & 3.3 & 2.0 & 1.1 & 0.9 & 1.4 & 27.3 \\
\hline 244 & Darabshala & 2.6 & 2.6 & 3.0 & 2.2 & 2.0 & 2.3 & 3.4 & 3.1 & 2.0 & 1.1 & 0.9 & 1.4 & 26.6 \\
\hline 245 & Devigol & 27.3 & 12.1 & 4.1 & 2.3 & 2.0 & 2.3 & 3.5 & 3.2 & 2.0 & 1.1 & 1.0 & 3.5 & 64.3 \\
\hline 246 & Dhamkund & 2.7 & 2.9 & 2.9 & 2.2 & 2.1 & 2.3 & 3.5 & 3.2 & 2.0 & 1.1 & 0.9 & 1.6 & 27.3 \\
\hline 247 & Doda & 2.8 & 2.6 & 2.7 & 2.1 & 2.0 & 2.3 & 3.4 & 3.1 & 1.9 & 1.1 & 0.9 & 1.4 & 26.5 \\
\hline 248 & Dusadudha & 23.6 & 10.4 & 3.9 & 2.3 & 2.0 & 2.3 & 3.5 & 3.2 & 2.0 & 1.1 & 1.0 & 3.9 & 59.1 \\
\hline 249 & Gainta & 1.9 & 2.5 & 2.8 & 2.1 & 2.1 & 2.3 & 3.6 & 3.3 & 2.0 & 1.1 & 0.8 & 1.0 & 25.5 \\
\hline 250 & Ghamroor & 1.7 & 2.1 & 2.3 & 1.9 & 1.9 & 2.3 & 3.5 & 3.2 & 2.0 & 1.1 & 0.9 & 1.1 & 24.2 \\
\hline 251 & Harsur & 1.6 & 2.2 & 2.5 & 2.0 & 2.0 & 2.3 & 3.6 & 3.3 & 2.0 & 1.1 & 0.8 & 1.0 & 24.4 \\
\hline 252 & Hawal & 32.9 & 28.4 & 5.7 & 3.1 & 2.5 & 2.4 & 3.4 & 3.1 & 2.0 & 1.2 & 1.5 & 18.8 & 105.1 \\
\hline 253 & Inshan & 25.2 & 10.9 & 3.8 & 2.6 & 2.4 & 2.3 & 3.4 & 3.1 & 2.0 & 1.1 & 1.1 & 5.3 & 63.2 \\
\hline 254 & Kahu & 1.5 & 2.1 & 2.3 & 2.0 & 2.0 & 2.3 & 3.6 & 3.4 & 2.1 & 1.1 & 0.8 & 0.9 & 24.2 \\
\hline 255 & Kasol & 1.5 & 2.1 & 2.3 & 2.0 & 2.0 & 2.3 & 3.6 & 3.4 & 2.1 & 1.1 & 0.8 & 0.9 & 24.3 \\
\hline 256 & Kati & 2.9 & 3.0 & 2.6 & 2.3 & 2.2 & 2.4 & 4.0 & 3.6 & 2.1 & 1.1 & 0.8 & 1.6 & 28.7 \\
\hline 257 & Kaza & 51.1 & 100.1 & 67.7 & 5.1 & 2.3 & 1.4 & 2.0 & 2.1 & 2.4 & 1.3 & 9.0 & 25.3 & 269.7 \\
\hline 258 & Kupwara & 7.2 & 2.1 & 3.5 & 2.5 & 2.3 & 1.5 & 1.9 & 1.7 & 0.9 & 0.7 & 1.2 & 4.7 & 30.2 \\
\hline 259 & Larji & 2.0 & 2.3 & 2.6 & 2.1 & 2.0 & 2.4 & 3.7 & 3.3 & 2.0 & 1.1 & 0.8 & 1.3 & 25.6 \\
\hline 260 & Lossar & 40.2 & 65.4 & 65.2 & 18.9 & 3.2 & 2.2 & 2.0 & 2.1 & 2.7 & 3.0 & 6.4 & 15.0 & 226.2 \\
\hline 261 & Matsal & 153.2 & 203.1 & 204.0 & 69.9 & 8.7 & 3.5 & 3.9 & 3.6 & 2.8 & 15.1 & 25.1 & 44.4 & 737.4 \\
\hline 262 & Mau & 100.2 & 132.3 & 18.0 & 4.9 & 2.6 & 2.5 & 3.6 & 3.3 & 2.1 & 1.4 & 3.2 & 59.4 & 333.4 \\
\hline 263 & Mohu & 48.2 & 21.3 & 4.6 & 2.3 & 2.2 & 2.3 & 3.5 & 3.2 & 2.0 & 1.2 & 1.3 & 13.9 & 105.8 \\
\hline 264 & Moorang & 17.4 & 16.4 & 3.7 & 2.1 & 2.2 & 2.4 & 3.6 & 3.3 & 2.1 & 1.1 & 0.9 & 4.2 & 59.5 \\
\hline 265 & Namgia & 25.3 & 38.1 & 5.7 & 1.9 & 1.3 & 1.2 & 1.7 & 1.9 & 1.4 & 0.5 & 0.9 & 10.7 & 90.7 \\
\hline 266 & Nandan & 8.0 & 5.4 & 2.9 & 2.4 & 2.4 & 2.3 & 3.8 & 3.4 & 2.0 & 1.1 & 0.9 & 2.6 & 37.3 \\
\hline 267 & Ohli & 5.8 & 4.2 & 3.7 & 2.8 & 2.4 & 2.4 & 3.4 & 3.1 & 2.0 & 1.2 & 1.0 & 1.9 & 33.6 \\
\hline 268 & Palmar & 4.5 & 3.7 & 3.3 & 2.8 & 2.4 & 2.4 & 3.4 & 3.1 & 2.0 & 1.2 & 1.0 & 2.5 & 32.1 \\
\hline 269 & Pooh & 27.5 & 38.2 & 4.5 & 1.8 & 1.3 & 1.3 & 1.8 & 1.9 & 1.4 & 0.5 & 0.8 & 9.8 & 90.8 \\
\hline 270 & Pouni & 1.7 & 2.4 & 2.6 & 2.1 & 2.1 & 2.3 & 3.9 & 3.5 & 2.0 & 1.1 & 0.8 & 1.0 & 25.5 \\
\hline 271 & Rakchham & 87.7 & 59.7 & 24.7 & 4.5 & 2.5 & 2.4 & 3.4 & 3.2 & 2.1 & 1.1 & 4.2 & 56.7 & 252.1 \\
\hline 272 & Rekenwas & 28.9 & 28.2 & 27.9 & 5.5 & 2.6 & 2.3 & 3.3 & 3.1 & 2.0 & 1.7 & 5.8 & 22.7 & 134.0 \\
\hline 273 & Rot & 2.3 & 2.6 & 2.5 & 2.1 & 2.1 & 2.4 & 3.5 & 3.2 & 2.0 & 1.1 & 0.8 & 1.2 & 25.9 \\
\hline 274 & Sain & 31.9 & 13.6 & 3.8 & 2.5 & 2.4 & 2.3 & 3.8 & 3.4 & 2.0 & 1.2 & 1.1 & 8.2 & 76.4 \\
\hline 275 & Sainj & 2.6 & 2.5 & 2.7 & 2.1 & 2.0 & 2.5 & 3.7 & 3.3 & 2.0 & 1.1 & 0.8 & 1.4 & 26.6 \\
\hline 276 & Salal & 1.7 & 2.5 & 2.8 & 2.1 & 2.1 & 2.3 & 3.6 & 3.3 & 2.0 & 1.1 & 0.8 & 1.0 & 25.3 \\
\hline 277 & Sarkund & 15.4 & 6.9 & 3.2 & 2.6 & 2.3 & 2.3 & 3.4 & 3.1 & 1.9 & 1.1 & 1.0 & 4.1 & 47.4 \\
\hline 278 & Shahpur-I & 1.6 & 2.2 & 2.5 & 2.0 & 2.0 & 2.3 & 3.6 & 3.3 & 2.0 & 1.1 & 0.8 & 1.0 & 24.5 \\
\hline 279 & Sirshi & 5.7 & 4.1 & 3.4 & 2.9 & 2.5 & 2.4 & 3.4 & 3.1 & 2.0 & 1.2 & 1.0 & 2.0 & 33.6 \\
\hline
\end{tabular}




\begin{tabular}{|c|c|c|c|c|c|c|c|c|c|c|c|c|c|c|}
\hline 280 & Sohal & 15.7 & 11.7 & 7.0 & 3.2 & 2.3 & 2.4 & 3.5 & 3.2 & 2.0 & 1.5 & 1.7 & 4.5 & 58.6 \\
\hline 281 & Tandi & 121.8 & 77.5 & 26.6 & 4.8 & 2.2 & 2.9 & 3.4 & 3.2 & 2.0 & 1.1 & 3.0 & 41.2 & 289.6 \\
\hline 282 & Thana & 61.6 & 24.1 & 6.8 & 2.6 & 2.3 & 2.4 & 3.3 & 3.1 & 1.9 & 1.1 & 1.2 & 5.4 & 115.9 \\
\hline 283 & Tillar & 12.7 & 6.4 & 3.6 & 3.0 & 2.5 & 2.4 & 3.4 & 3.1 & 2.0 & 1.2 & 1.1 & 4.1 & 45.4 \\
\hline 284 & Udaipur & 87.6 & 44.7 & 10.8 & 3.8 & 2.2 & 2.4 & 3.4 & 3.1 & 2.0 & 1.3 & 1.9 & 14.4 & 177.6 \\
\hline 285 & Yurod & 9.7 & 4.8 & 3.1 & 2.5 & 2.2 & 2.3 & 3.3 & 3.1 & 1.9 & 1.1 & 1.0 & 2.6 & 37.8 \\
\hline 286 & Asmar & 6.1 & 4.7 & 5.7 & 6.2 & 4.0 & 2.3 & 2.9 & 2.8 & 2.5 & 1.5 & 1.7 & 2.6 & 43.0 \\
\hline 287 & Bamiyan & 6.6 & 18.6 & 7.5 & 2.8 & 2.4 & 1.3 & 0.5 & 0.2 & 0.3 & 0.4 & 2.5 & 5.7 & 48.7 \\
\hline 288 & Darullaman & 11.7 & 22.7 & 4.2 & 2.9 & 3.7 & 0.6 & 1.7 & 0.8 & 0.5 & 0.7 & 1.1 & 3.4 & 53.9 \\
\hline 289 & Gerdiz & 11.5 & 30.2 & 7.0 & 2.9 & 1.8 & 0.7 & 1.4 & 0.7 & 0.0 & 0.5 & 1.2 & 6.5 & 64.4 \\
\hline 290 & Ghaziabad & 1.3 & 1.8 & 2.3 & 2.3 & 1.1 & 0.2 & 0.3 & 0.7 & 0.5 & 0.2 & 0.5 & 0.9 & 12.1 \\
\hline 291 & Ghazni & 11.3 & 23.0 & 4.7 & 2.5 & 1.4 & 0.1 & 1.1 & 0.1 & 0.0 & 0.7 & 1.4 & 5.8 & 52.3 \\
\hline 292 & Jabul Saraj & 10.2 & 10.0 & 7.9 & 8.9 & 5.0 & 1.0 & 1.6 & 0.8 & 1.1 & 1.9 & 2.0 & 3.2 & 53.5 \\
\hline 293 & Jalalabad & 1.1 & 1.5 & 2.1 & 2.3 & 1.4 & 0.3 & 0.3 & 0.5 & 0.3 & 0.3 & 0.6 & 0.8 & 11.5 \\
\hline 294 & Kabul AP & 9.6 & 12.8 & 5.2 & 4.2 & 3.1 & 0.4 & 1.1 & 0.3 & 0.3 & 0.6 & 1.5 & 2.7 & 41.7 \\
\hline 295 & Karizmir & 17.9 & 24.0 & 4.5 & 3.4 & 4.2 & 1.0 & 2.2 & 0.5 & 0.7 & 0.7 & 1.1 & 3.0 & 63.1 \\
\hline 296 & Khost & 2.2 & 2.9 & 3.1 & 3.3 & 2.9 & 1.4 & 2.8 & 2.1 & 1.5 & 0.7 & 0.6 & 1.7 & 25.1 \\
\hline 297 & Laghman & 1.2 & 1.6 & 2.2 & 2.4 & 1.6 & 0.3 & 0.3 & 0.5 & 0.3 & 0.3 & 0.6 & 0.8 & 12.0 \\
\hline 298 & Logar & 8.6 & 16.1 & 4.4 & 2.8 & 2.6 & 0.4 & 1.0 & 0.4 & 0.3 & 0.5 & 1.1 & 4.6 & 42.7 \\
\hline 299 & Mirbachak & 12.0 & 15.1 & 3.7 & 2.9 & 3.6 & 0.5 & 2.7 & 1.6 & 0. & 0.7 & .0 & 2.6 & 46.8 \\
\hline 300 & Mokur & 15.3 & 25.7 & 4.6 & 2.5 & 1.3 & 0.0 & 0.5 & 0.4 & 0.0 & 0.8 & 1.3 & 4.6 & 56.9 \\
\hline 301 & North Salang & 181.0 & 148.2 & 153.1 & 76.1 & 5.9 & 1.5 & 1.1 & 0.9 & 0.6 & 3.5 & 40.0 & 79.7 & 691.4 \\
\hline 302 & Okak & 26.4 & 23.5 & 27.2 & 5.7 & 1.6 & 0.4 & 1.3 & 0.4 & 0.0 & 0.5 & 9.2 & 10.1 & 106.4 \\
\hline 303 & Paghman & 23.8 & 38.7 & 4.6 & 3.4 & 4.6 & 1.0 & 3.4 & 1.0 & 1.5 & 0.9 & 1.3 & 4.7 & 88.7 \\
\hline 304 & Pan Jao & 20.4 & 98.1 & 46.2 & 6.3 & 1.9 & 0.7 & 0.4 & 0.2 & 0.4 & 0.8 & 10.1 & 21.0 & 206.5 \\
\hline 305 & Sarobi & 2.6 & 2.8 & 3.3 & 3.3 & 3.1 & 0.4 & 0.6 & 0.4 & 0.7 & 0.6 & 1.0 & 1.9 & 20.7 \\
\hline 306 & South Salang & 71.6 & 85.0 & 56.8 & 9.3 & 8.7 & 2.0 & 2.1 & 1.6 & 0.8 & 2.8 & 16.3 & 38.5 & 295.4 \\
\hline 307 & Zebak & 4.4 & 10.7 & 2.9 & 2.8 & 2.2 & 0.9 & 0.9 & 0.3 & 0.1 & 0.6 & 1.8 & 4.6 & 32.0 \\
\hline 308 & Approach & 0.0 & 0.0 & 0.0 & 0.0 & 0.0 & 0.0 & 0.0 & 0.0 & 0.0 & 0.0 & 0.0 & 0.0 & 0.0 \\
\hline 309 & Baltoro & 0.0 & 0.0 & 0.0 & 0.0 & 0.0 & 0.0 & 0.0 & 0.0 & 0.0 & 0.0 & 0.0 & 0.0 & 0.0 \\
\hline 310 & Batura & 68.0 & 84.0 & 112.0 & 76.0 & 64.0 & 60.0 & 72.0 & 80.0 & 56.0 & 36.0 & 32.0 & 60.0 & 800.0 \\
\hline 311 & Chong Kumdan & 86.7 & 86.7 & 91.8 & 85.0 & 63.8 & 63.8 & 38.3 & 57.4 & 31.9 & 81.6 & 76.5 & 86.7 & 850.0 \\
\hline 312 & Chogolungma & 76.5 & 76.5 & 81.0 & 75.0 & 56.3 & 56.3 & 33.8 & 50.6 & 28.1 & 72.0 & 67.5 & 76.5 & 750.0 \\
\hline 313 & Hispar Dome & 0.0 & 0.0 & 0.0 & 0.0 & 0.0 & 0.0 & 0.0 & 0.0 & 0.0 & 0.0 & 0.0 & 0.0 & 0.0 \\
\hline 314 & Hispar East & 76.5 & 76.5 & 81.0 & 75.0 & 56.3 & 56.3 & 33.8 & 50.6 & 28.1 & 72.0 & 67.5 & 76.5 & 750.0 \\
\hline 315 & Hispar West & 0.0 & 0.0 & 0.0 & 0.0 & 0.0 & 0.0 & 0.0 & 0.0 & 0.0 & 0.0 & 0.0 & 0.0 & 0.0 \\
\hline 316 & Hispar Pass & 25.5 & 25.5 & 27.0 & 25.0 & 18.8 & 18.8 & 11.3 & 16.9 & 9.4 & 24.0 & 22.5 & 25.5 & 250.0 \\
\hline 317 & Khurdopin & 0.0 & 0.0 & 0.0 & 0.0 & 0.0 & 0.0 & 0.0 & 0.0 & 0.0 & 0.0 & 0.0 & 0.0 & 0.0 \\
\hline 318 & Nanga Parbat & 51.0 & 51.0 & 54.0 & 50.0 & 37.5 & 37.5 & 22.5 & 33.8 & 18.8 & 48.0 & 45.0 & 51.0 & 500.0 \\
\hline 319 & Nun Kun North & 25.5 & 25.5 & 27.0 & 25.0 & 18.8 & 18.8 & 11.3 & 16.9 & 9.4 & 24.0 & 22.5 & 25.5 & 250.0 \\
\hline 320 & Sentik & 76.5 & 76.5 & 81.0 & 75.0 & 56.3 & 56.3 & 33.8 & 50.6 & 28.1 & 72.0 & 67.5 & 76.5 & 750.0 \\
\hline 321 & Siachin A & 102.0 & 102.0 & 108.0 & 100.0 & 75.0 & 75.0 & 45.0 & 67.5 & 37.5 & 96.0 & 90.0 & 102.0 & 1000. \\
\hline 322 & Siachin B & 102.0 & 102.0 & 108.0 & 100.0 & 75.0 & 75.0 & 45.0 & 67.5 & 37.5 & 96.0 & 90.0 & 102.0 & 1000. \\
\hline 323 & Siachin C & 91.8 & 91.8 & 97.2 & 90.0 & 67.5 & 67.5 & 40.5 & 60.8 & 33.8 & 86.4 & 81.0 & 91.8 & 900.0 \\
\hline 324 & Siachin D & 76.5 & 76.5 & 81.0 & 75.0 & 56.3 & 56.3 & 33.8 & 50.6 & 28.1 & 72.0 & 67.5 & 76.5 & 750.0 \\
\hline 325 & South Terong & 86.7 & 86.7 & 91.8 & 85.0 & 63.8 & 63.8 & 38.3 & 57.4 & 31.9 & 81.6 & 76.5 & 86.7 & 850.0 \\
\hline 326 & Terong & 76.5 & 76.5 & 81.0 & 75.0 & 56.3 & 56.3 & 33.8 & 50.6 & 28.1 & 72.0 & 67.5 & 76.5 & 750.0 \\
\hline 327 & Urdok & 0.0 & 0.0 & 0.0 & 0.0 & 0.0 & 0.0 & 0.0 & 0.0 & 0.0 & 0.0 & 0.0 & 0.0 & 0.0 \\
\hline 328 & Whaleback & 76.5 & 76.5 & 81.0 & 75.0 & 56.3 & 56.3 & 33.8 & 50.6 & 28.1 & 72.0 & 67.5 & 76.5 & 750.0 \\
\hline
\end{tabular}


Table S-3.3: Station-based correction factors for adjusting measurement errors in precipitation observations.

\begin{tabular}{|c|c|c|c|c|c|c|c|c|c|c|c|c|c|c|}
\hline S. \# & tation Name & Jan & Feb & Mar & Apr & May & Jun & Jul & Aug & Sep & Oct & Nov & Dec & ANN \\
\hline 1 & & 399 & 1.261 & .094 & 1.048 & 1.075 & 1.093 & 1.097 & 110 & 91 & 3 & 71 & 06 & 139 \\
\hline 2 & 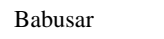 & .970 & 2.096 & .785 & .612 & 124 & 1.143 & .119 & .063 & 1.080 & .424 & .666 & .931 & 1.556 \\
\hline 3 & 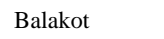 & 021 & .026 & .039 & .040 & & & & 1.020 & 1.020 & & 1.025 & & 1.028 \\
\hline 4 & Bhimbe & 1.018 & 1.045 & 1.031 & 1.026 & 1.087 & 1.039 & .016 & 1.012 & 1.011 & 1.045 & .085 & 1.019 & 1.020 \\
\hline 5 & Bunji & 1.093 & 1.044 & 1.100 & 1.062 & 1.065 & 1.097 & & 1.060 & 1.075 & & & 69 & .071 \\
\hline 6 & $\mathrm{Ch}$ & .048 & 1.045 & 1.052 & 1.046 & 1.044 & 1.112 & 1.030 & 1.030 & 1.036 & 1.186 & .083 & 1.051 & 1.045 \\
\hline 7 & Chakwal & 1.041 & 1.032 & 1.037 & 1.036 & 1.038 & 1.023 & 1.027 & 1.020 & 1.022 & 1.082 & 1.117 & 1.039 & 1.030 \\
\hline 8 & Cherat & 1.097 & 1.096 & 1.072 & 1.080 & 1.108 & 1.051 & 1.045 & 1.040 & 1.056 & 1.082 & 1.098 & 1.103 & 1.069 \\
\hline 9 & Chilas & 1.076 & 1.037 & 1.062 & 1.047 & 1.061 & 1.108 & 1.088 & 1.095 & 1.131 & 1.126 & 1.052 & 1.030 & 1.066 \\
\hline 10 & Chitral & 1.086 & 1.060 & 1.041 & & & & & 1.300 & & & & 1.066 & 1.063 \\
\hline 11 & Dir & 1.026 & 1.022 & 1.022 & 1.019 & 1.043 & 1.037 & 1.022 & 1.025 & 1.036 & & 1.018 & 1.024 & 1.025 \\
\hline 12 & Dr & 1.063 & 1.052 & 1.045 & 1.033 & 1.061 & 1.087 & 1.107 & 1.103 & 1.100 & 1.048 & 1.034 & 1.059 & 1.055 \\
\hline 13 & GD Po & 1.014 & 1.011 & 1.011 & 1.009 & 1.014 & 1.025 & 1.011 & 1.009 & 1.010 & & 1.012 & 1.009 & 1.011 \\
\hline 14 & Gilgit & 1.178 & 1.111 & 1.176 & 1.073 & 1.085 & 1.124 & 1.138 & 1.139 & 1.208 & 1.144 & 1.132 & 1.065 & 1.118 \\
\hline 15 & $\mathrm{Gu}$ & 1.161 & 1.093 & 1.137 & 1.027 & 1.061 & 1.034 & 1.019 & 1.020 & 1.041 & & 1.696 & 1.135 & 1.059 \\
\hline 16 & & 1.851 & 1.377 & 1.105 & 1.053 & 1.060 & 1.040 & 1.026 & 1.055 & 1.045 & & 1.153 & 1.293 & 1.074 \\
\hline 17 & & 1.070 & 1.124 & & & & & & & & & & 62 & .064 \\
\hline 18 & Jhelum & 1.038 & 1.031 & 1.044 & 1.028 & 1.042 & 1.042 & 1.020 & 1.018 & 1.018 & 1.038 & 1.025 & 1.015 & 1.025 \\
\hline 19 & Kakul & 1.027 & 1.023 & 1.032 & 1.027 & 1.038 & 1.027 & 1.018 & 1.021 & 1.026 & 1.030 & 1.026 & 1.024 & 1.024 \\
\hline 20 & & 1.274 & 1.266 & 1.037 & 1.026 & 1.038 & 1.113 & 1.078 & 1.094 & 1.053 & & 1.026 & 1.090 & 1.121 \\
\hline 21 & & 1.016 & 1.017 & 1.070 & 1.100 & 1.262 & 1.036 & 1.023 & 1.022 & 1.043 & & 1.100 & 1.038 & 1.033 \\
\hline 22 & & & & & & & & & & & & & & 024 \\
\hline 23 & $\mathrm{~K}$ & 1.027 & 1.023 & 1.033 & 1.027 & 1.033 & 1.051 & 1.018 & 1.017 & 1.022 & 1.022 & 1.032 & 1.018 & 1.024 \\
\hline 24 & land & 1.107 & 1.088 & 1.040 & 1.034 & 1.045 & 1.643 & 1.136 & 1.264 & 1.178 & 1.184 & 1.057 & 1.063 & 1.070 \\
\hline 25 & & & 1.055 & 1.081 & & & & & 1.026 & & & & & 1.060 \\
\hline 26 & & 1.438 & 1.173 & 1.073 & 1.039 & 1.040 & 1.029 & 1.018 & 1.019 & 1.021 & 1.022 & 1.040 & 1.139 & 1.089 \\
\hline 27 & & & & & & & & & & & & & & 018 \\
\hline 28 & $\mathrm{M}$ & 1.060 & 1.067 & 1.042 & 1.046 & 1.118 & 1.162 & 1.043 & 1.069 & 1.399 & 1. & 1.095 & 1.054 & 1.067 \\
\hline 29 & & 1.093 & 1.042 & 1.047 & 1.062 & 1.100 & 1.161 & 1.159 & 1.066 & 1.091 & 1.095 & 1.054 & 1.115 & 1.068 \\
\hline 30 & & & 1.036 & 1.033 & & & & & 1.014 & & & & 1.032 & 1.024 \\
\hline 31 & & 0,5 & 2.068 & 1.716 & 1.165 & 1.078 & 1.327 & 1.130 & 1.226 & 1.188 & & 1.690 & 1.991 & 1.499 \\
\hline 32 & & & & & & & & & & & & & & .023 \\
\hline 33 & & & 1.015 & 1.018 & & 1.024 & & 1.010 & 1.010 & 1.012 & & & & 1.014 \\
\hline 34 & n & 1.094 & 1.052 & 1.038 & 1.032 & 1.049 & 1.042 & 1.033 & 1.029 & 1.031 & 1.040 & 1.038 & 1.067 & 1.042 \\
\hline 35 & & & 1.033 & & & & & & & & & & & 1.051 \\
\hline 36 & & 1.019 & 1.032 & 1.048 & 1.050 & 1.099 & 1.068 & & 1.051 & 1.044 & & & 1.034 & 1.046 \\
\hline 37 & & & & & & & & & & & & & & 029 \\
\hline 38 & & & 1.037 & 1.063 & & & & & 1.057 & & & & 1.043 & 1.058 \\
\hline 39 & S & 1.051 & 1.047 & 1.059 & 1.041 & 1.059 & 1.042 & 1.022 & 1.030 & 1.029 & 1.051 & 1.042 & 1.071 & 1.041 \\
\hline 40 & & & 1.048 & 1.042 & 1.036 & 1.059 & 1.057 & 1.018 & 1.017 & 1.015 & & 1.036 & 1.030 & 1.025 \\
\hline 41 & & & & 1.071 & & 1.049 & & & 1.112 & 1.108 & & & 1.181 & 1.168 \\
\hline 42 & & & & & & & & & & & & & & .037 \\
\hline 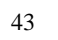 & & & 1.051 & 1.051 & & & & 1.044 & 1.028 & 1.077 & & 1.078 & 1.065 & 1.053 \\
\hline 44 & $\mathrm{Be}$ & 1.067 & 1.045 & 1.034 & 1.036 & 1.027 & 1.030 & 1.015 & 1.020 & 1.034 & 1.031 & 1.041 & 1.054 & 1.030 \\
\hline 45 & & & 1.039 & 1.063 & 1.054 & 1.054 & 1.042 & 1.037 & 1.029 & 1.041 & 1.033 & 1.045 & 1.092 & 1.047 \\
\hline 46 & Charba & 2.240 & 2.357 & 2.019 & 1.882 & 1.210 & 1.050 & 1.036 & 1.034 & 1.051 & 1.275 & 1.881 & 2.247 & 1.640 \\
\hline 47 & & & & & & & & & & & & & & 1.061 \\
\hline 48 & & 1.026 & 1.027 & 1.043 & 1.038 & 1.034 & 1.015 & 1.017 & 1.013 & 1.021 & 1.056 & 1.150 & 1.056 & 1.027 \\
\hline 49 & Deosai & 1.047 & 1.048 & 1.065 & 1.051 & 1.091 & 1.046 & 1.022 & 1.022 & 1.027 & 1.047 & 1.059 & 1.075 & 1.040 \\
\hline 50 & & & 2.230 & 2.004 & 1.977 & 1.259 & 1.097 & 1.076 & 1.050 & 1.075 & & 1.806 & 2.098 & 1.646 \\
\hline 51 & Domel & 1.121 & 1.051 & 1.015 & 1.015 & 1.020 & 1.045 & 1.054 & 1.055 & 1.065 & 1.020 & 1.029 & 1.069 & 1.036 \\
\hline 52 & Doyian & 1.098 & 1.051 & 1.043 & 1.047 & 1.054 & 1.035 & 1.017 & 1.023 & 1.024 & 1.028 & 1.055 & 1.083 & 1.040 \\
\hline
\end{tabular}




\begin{tabular}{|c|c|c|c|c|c|c|c|c|c|c|c|c|c|c|}
\hline 53 & Fort Lockhart & 1.169 & 1.081 & 1.065 & 1.065 & 1.051 & 1.064 & 1.047 & 1.060 & 1.051 & 1.052 & 1.075 & 1.112 & 1.067 \\
\hline 54 & Gujar Khan & 1.099 & 1.097 & 1.056 & 1.031 & 1.037 & 1.037 & 1.021 & 1.021 & 1.030 & 1.034 & 1.069 & 1.086 & 1.043 \\
\hline 5 & Hushy & 1.075 & 1.037 & 1.019 & 1.047 & 1.028 & 1.044 & 1.014 & 1.012 & 1.024 & 1.062 & 1.175 & 1.010 & 1.022 \\
\hline 56 & Jabbar & 1.816 & 1.762 & 1.140 & 1.101 & 1.042 & 1.051 & 1.054 & 1.047 & 1.054 & 1.036 & 1.116 & 1.770 & 1.201 \\
\hline 57 & Kachura & 1.306 & 1.112 & 1.051 & 1.038 & 1.032 & 1.030 & 1.020 & 1.029 & 1.024 & 1.022 & 1.042 & 1.179 & 1.060 \\
\hline 50 & KalamW & 1.994 & 1.469 & 1.069 & 1.080 & 1.076 & 1.140 & 1.098 & 1.100 & 1.083 & 1.075 & 1.088 & 1.301 & 1.214 \\
\hline 59 & Kallar & 1.330 & 1.152 & 1.039 & 1.034 & 1.059 & 1.109 & 1.126 & 1.107 & 1.063 & 1.029 & 1.022 & 1.071 & 1.096 \\
\hline 60 & Kelash & 1.061 & 1.023 & 1.014 & 1.030 & 1.040 & 1.025 & 1.014 & 1.015 & 1.018 & 1.045 & 1.122 & 1.008 & 1.020 \\
\hline 61 & Khairabad & 1.341 & 1.396 & 1.064 & 1.050 & 1.079 & 1.103 & 1.060 & 1.036 & 1.053 & 1.038 & 1.045 & 1.339 & 1.156 \\
\hline 62 & Khandar & 1.040 & 1.019 & 1.011 & 1.019 & 1.016 & 1.021 & 1.013 & 1.017 & 1.026 & 1.029 & 1.034 & 1.007 & 1.017 \\
\hline 63 & Khot & 1.894 & 2.051 & 1.830 & 1.114 & 1.053 & 1.055 & 1.059 & 1.060 & 1.060 & 1.090 & 1.696 & 1.914 & 1.554 \\
\hline 47 & Khunjrab-W & 5.781 & 5.908 & 5.503 & 4.194 & 2.174 & 1.170 & 1.106 & 1.088 & 1.331 & 3.062 & 4.869 & 5.446 & 2.821 \\
\hline 65 & Kotli-W & 1.053 & 1.026 & 1.015 & 1.020 & 1.029 & 1.024 & 1.010 & 1.011 & 1.017 & 1.036 & 1.043 & 1.010 & 1.016 \\
\hline 0 & Lora & 1.040 & 1.034 & 1.023 & 1.026 & 1.052 & 1.067 & 1.021 & 1.020 & 1.014 & 1.042 & 1.093 & 1.028 & 1.027 \\
\hline 67 & Malakand & 1.043 & 1.045 & 1.048 & 1.041 & 1.115 & 1.082 & 1.031 & 1.018 & 1.056 & 1.057 & 1.048 & 1.051 & 1.041 \\
\hline 68 & Mangla-W & 1.022 & 1.027 & 1.023 & 1.041 & 1.031 & 1.024 & 1.013 & 1.012 & 1.020 & 1.086 & 1.253 & 1.025 & 1.020 \\
\hline 69 & Mardan & 1.037 & 1.053 & 1.077 & 1.067 & 1.076 & 1.042 & 1.016 & 1.011 & 1.022 & 1.036 & 1.059 & 1.059 & 1.032 \\
\hline 70 & Munda dam & 1.043 & 1.053 & 1.060 & 1.051 & 1.096 & 1.047 & 1.025 & 1.016 & 1.024 & 1.032 & 1.085 & 1.120 & 1.037 \\
\hline 71 & Naltar & 1.400 & 1.553 & 1.123 & 1.040 & 1.032 & 1.038 & 1.033 & 1.028 & 1.035 & 1.032 & 1.083 & 1.387 & 1.152 \\
\hline 72 & Naran & 2.242 & 1.979 & 1.938 & 1.087 & 1.037 & 1.066 & 1.070 & 1.084 & 1.061 & 1.023 & 1.058 & 1.336 & 1.481 \\
\hline 73 & Oghi & 1.058 & 1.047 & 1.054 & 1.047 & 1.053 & 1.037 & 1.023 & 1.032 & 1.036 & 1.026 & 1.044 & 1.078 & 1.042 \\
\hline 74 & Palandri & 1.059 & 1.045 & 1.027 & 1.016 & 1.025 & 1.015 & 1.011 & 1.009 & 1.015 & 1.045 & 1.050 & 1.057 & 1.022 \\
\hline 75 & Phulra & 1.059 & 1.054 & 1.065 & 1.051 & 1.060 & 1.046 & 1.026 & 1.026 & 1.034 & 1.030 & 1.049 & 1.090 & 1.043 \\
\hline 76 & Pir Chenasi & 1.975 & 1.583 & 1.066 & 1.034 & 1.040 & 1.023 & 1.018 & 1.027 & 1.021 & 1.039 & 1.066 & 1.295 & 1.199 \\
\hline 77 & Puran & 1.045 & 1.046 & 1.052 & 1.043 & 1.046 & 1.036 & 1.020 & 1.022 & 1.027 & 1.033 & 1.037 & 1.064 & 1.036 \\
\hline 78 & Qalangi & 1.041 & 1.045 & 1.057 & 1.050 & 1.114 & 1.624 & 1.043 & 1.039 & 1.139 & 1.161 & 1.061 & 1.058 & 1.058 \\
\hline 79 & Rama & 2.187 & 2.257 & 1.662 & 1.091 & 1.049 & 1.051 & 1.049 & 1.043 & 1.044 & 1.042 & 1.348 & 2.146 & 1.559 \\
\hline 80 & Ratu & 2.152 & 2.129 & 1.197 & 1.055 & 1.037 & 1.057 & 1.068 & 1.075 & 1.058 & 1.030 & 1.082 & 2.019 & 1.359 \\
\hline 81 & Rawlakot-W & 1.074 & 1.056 & 1.032 & 1.026 & 1.033 & 1.019 & 1.020 & 1.018 & 1.024 & 1.042 & 1.038 & 1.072 & 1.032 \\
\hline 8 & Saifulmulk & 2.268 & 1.935 & 1.564 & 1.095 & 1.065 & 1.059 & 1.053 & 1.058 & 1.042 & 1.029 & 1.241 & 2.163 & 1.564 \\
\hline 83 & Sehrkakota & 1.068 & 1.045 & 1.025 & 1.035 & 1.037 & 1.042 & 1.012 & 1.020 & 1.026 & 1.029 & 1.035 & 1.042 & 1.028 \\
\hline 84 & Shahpur & 1.293 & 1.110 & 1.079 & 1.060 & 1.057 & 1.032 & 1.019 & 1.021 & 1.026 & 1.025 & 1.058 & 1.203 & 1.080 \\
\hline 85 & Shangla & 1.230 & 1.130 & 1.103 & 1.071 & 1.067 & 1.039 & 1.022 & 1.018 & 1.031 & 1.046 & 1.062 & 1.189 & 1.070 \\
\hline 86 & Shendure & 1.444 & 1.813 & 1.542 & 1.100 & 1.079 & 1.052 & 1.040 & 1.045 & 1.058 & 1.106 & 1.398 & 1.537 & 1.290 \\
\hline 87 & Shigar & 1.757 & 1.280 & 1.100 & 1.112 & 1.066 & 1.073 & 1.077 & 1.067 & 1.046 & 1.053 & 1.068 & 1.222 & 1.172 \\
\hline 88 & Shinkiari & 1.058 & 1.050 & 1.059 & 1.050 & 1.051 & 1.029 & 1.016 & 1.022 & 1.022 & 1.026 & 1.043 & 1.085 & 1.035 \\
\hline 89 & Shogran & 2.261 & 1.767 & 1.136 & 1.057 & 1.038 & 1.031 & 1.028 & 1.039 & 1.023 & 1.019 & 1.089 & 1.828 & 1.318 \\
\hline 90 & Tandar & 1.068 & 1.024 & 1.015 & 1.030 & 1.025 & 1.028 & 1.009 & 1.009 & 1.017 & 1.044 & 1.052 & 1.010 & 1.015 \\
\hline 91 & Tarbela & 1.008 & 1.015 & 1.029 & 1.040 & 1.075 & 1.037 & 1.018 & 1.022 & 1.027 & 1.064 & 1.020 & 1.017 & 1.025 \\
\hline 92 & Ushkore & 1.403 & 1.652 & 1.338 & 1.048 & 1.073 & 1.046 & 1.020 & 1.018 & 1.031 & 1.049 & 1.201 & 1.526 & 1.189 \\
\hline 93 & Yasin & 1.878 & 2.131 & 1.599 & 1.076 & 1.059 & 1.036 & 1.024 & 1.022 & 1.027 & 1.079 & 1.538 & 1.870 & 1.329 \\
\hline 94 & Yugo & 1.902 & 1.515 & 1.099 & 1.081 & 1.062 & 1.130 & 1.073 & 1.138 & 1.108 & 1.049 & 1.194 & 1.476 & 1.173 \\
\hline 95 & Zani & 1.595 & 1.856 & 1.478 & 1.172 & 1.054 & 1.046 & 1.061 & 1.060 & 1.038 & 1.073 & 1.420 & 1.630 & 1.307 \\
\hline 96 & Ziarat & 2.707 & 3.282 & 2.512 & 1.187 & 1.080 & 1.049 & 1.022 & 1.030 & 1.054 & 1.130 & 2.311 & 2.515 & 1.714 \\
\hline 97 & Zulam Br. & 1.034 & 1.045 & 1.052 & 1.041 & 1.071 & 1.079 & 1.038 & 1.027 & 1.056 & 1.114 & 1.070 & 1.080 & 1.046 \\
\hline 98 & Alambar & 1.980 & 2.359 & 2.042 & 1.573 & 1.135 & 1.039 & 1.009 & 1.010 & 1.034 & 1.321 & 1.866 & 1.960 & 1.543 \\
\hline 99 & Bagrot & 1.372 & 1.272 & 1.132 & 1.071 & 1.059 & 1.052 & 1.063 & 1.076 & 1.119 & 1.054 & 1.118 & 1.180 & 1.116 \\
\hline 100 & Baldihel & 1.963 & 2.250 & 2.004 & 1.396 & 1.061 & 1.031 & 1.042 & 1.028 & 1.049 & 1.124 & 1.799 & 1.997 & 1.314 \\
\hline 101 & Bulibalsirbar & 1.373 & 1.620 & 1.460 & 1.311 & 1.121 & 1.048 & 1.017 & 1.013 & 1.023 & 1.159 & 1.403 & 1.503 & 1.258 \\
\hline 102 & Dadormal & 1.862 & 2.207 & 1.868 & 1.126 & 1.043 & 1.027 & 1.038 & 1.039 & 1.044 & 1.070 & 1.729 & 1.988 & 1.326 \\
\hline 103 & Dame & 1.855 & 2.170 & 1.897 & 1.218 & 1.041 & 1.017 & 1.025 & 1.025 & 1.035 & 1.080 & 1.771 & 1.964 & 1.334 \\
\hline 104 & Diran & 1.857 & 2.184 & 1.880 & 1.149 & 1.040 & 1.021 & 1.030 & 1.031 & 1.038 & 1.069 & 1.749 & 1.972 & 1.325 \\
\hline 105 & Garmashbar & 1.969 & 2.326 & 1.929 & 1.118 & 1.062 & 1.035 & 1.013 & 1.013 & 1.019 & 1.062 & 1.753 & 1.939 & 1.470 \\
\hline 106 & Khaimetbar & 1.370 & 1.610 & 1.425 & 1.095 & 1.086 & 1.043 & 1.017 & 1.013 & 1.017 & 1.064 & 1.363 & 1.494 & 1.227 \\
\hline 107 & Khunjrab-C & 5.719 & 5.861 & 5.490 & 4.261 & 2.658 & 1.441 & 1.111 & 1.098 & 1.809 & 3.099 & 4.875 & 5.436 & 2.625 \\
\hline 108 & Akhnoor & 1.028 & 1.027 & 1.037 & 1.054 & 1.140 & 1.046 & 1.010 & 1.007 & 1.010 & 1.054 & 1.193 & 1.021 & 1.017 \\
\hline 109 & Anantnag & 1.082 & 1.079 & 1.027 & 1.034 & 1.038 & 1.037 & 1.058 & 1.069 & 1.020 & 1.029 & 1.075 & 1.063 & 1.043 \\
\hline
\end{tabular}




\begin{tabular}{|c|c|c|c|c|c|c|c|c|c|c|c|c|c|c|}
\hline 0 & riza & .088 & .060 & .036 & .029 & .025 & 1.044 & 1.052 & 1.066 & 1.029 & 1.015 & 1.041 & 1.057 & 1.040 \\
\hline 11 & arki & 1.034 & 1.036 & 1.034 & 1.068 & 1.042 & 1.018 & 1.010 & 1.013 & 1.016 & 1.090 & 1.075 & 1.036 & 1.021 \\
\hline 2 & abapura & 081 & .040 & .034 & .025 & .027 & 1.044 & .052 & 1.052 & 1.023 & 1.023 & 1.063 & 1.071 & 1.038 \\
\hline 3 & wah & 041 & .027 & .018 & 1.019 & .032 & 1.035 & 1.030 & .026 & 1.025 & 1.026 & 1.015 & 32 & . \\
\hline 14 & $\mathrm{~m}$ & 085 & .036 & 1.030 & 1.025 & 1.031 & 1.048 & 1.042 & 1.043 & 1.023 & 1.018 & 1.036 & 1.069 & 1.036 \\
\hline 5 & Ira & 104 & 1.051 & .035 & 1.020 & 1.038 & 1.051 & 1.057 & 1.050 & 1.023 & 1.017 & 1.039 & 1.103 & 1.043 \\
\hline 16 & an & .037 & .019 & .014 & 1.017 & .023 & 1.027 & 1.024 & 1.029 & 1.012 & 1.016 & 1.015 & 27 & .021 \\
\hline 17 & an & .040 & 1.030 & 1.025 & 1.028 & 1.024 & 1.025 & 1.016 & 1.020 & 1.018 & 1.065 & 1.048 & 1.056 & 1.024 \\
\hline 18 & aramula & 1.062 & 1.023 & 1.020 & 1.013 & 1.028 & 1.043 & 1.046 & 1.080 & & & & 50 & .027 \\
\hline 19 & as & 119 & 1.045 & 1.034 & 1.033 & 1.033 & 1.032 & 1.011 & 1.013 & 1.011 & 1.041 & 1.035 & 1.055 & 1.030 \\
\hline 20 & atote & .043 & 1.030 & 1.015 & 1.009 & 1.015 & 1.039 & 1.072 & 1.090 & 1.037 & 1.012 & 1.015 & 1.018 & 1.026 \\
\hline 21 & hag & 055 & 1.045 & 1.050 & 1.020 & 1.018 & 1.017 & 1.026 & 1.017 & 1.019 & 1.022 & 1.027 & 46 & .025 \\
\hline 22 & ha & 1.038 & 1.028 & 1.022 & 1.056 & 1.051 & 1.011 & 1.008 & 1.009 & 1.009 & 1.049 & 1.049 & 1.041 & 1.015 \\
\hline 23 & 3huntar & 1.021 & 1.023 & 1.018 & 1.027 & 1.029 & 1.046 & 1.027 & 1.027 & 1.039 & 1.051 & 1.029 & 1.035 & 1.027 \\
\hline 24 & 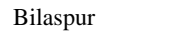 & .047 & 1.038 & 1.035 & 1.110 & 1.069 & 1.030 & 1.011 & 1.012 & 1.013 & 1.052 & 1.057 & 1.042 & 1.022 \\
\hline 25 & ha & 033 & 1.029 & 1.032 & 1.040 & 1.033 & 1.016 & 1.007 & 1.011 & 1.010 & 1.027 & 1.024 & 1.039 & 1.016 \\
\hline 26 & Chan & .043 & 1.040 & 1.055 & 1.159 & 1.125 & 1.029 & 1.013 & 1.012 & 1.011 & 1.061 & 1.103 & 1.038 & 1.023 \\
\hline 27 & ha & 1.063 & 1.038 & 1.022 & 1.021 & 1.024 & 1.031 & 1.036 & 1.040 & 1.021 & 1.017 & 1.028 & 1.050 & 1.030 \\
\hline 28 & Chen & 1.023 & 1.012 & 1.013 & 1.021 & 1.047 & 1.053 & 1.014 & 1.024 & 1.018 & 1.029 & 1.083 & 1.011 & 1.019 \\
\hline 29 & Chir & 489 & 1.348 & 1.046 & 1.027 & 1.038 & 1.121 & 1.071 & 1.074 & 1.034 & 1.075 & 1.073 & 1.199 & 1.138 \\
\hline 30 & he & 019 & 1.021 & 1.021 & 1.032 & 1.030 & 1.016 & 1.006 & 1.006 & 1.008 & 1.053 & 1.031 & 1.018 & 1.012 \\
\hline 31 & Dalhousie & 1.029 & 1.024 & 1.015 & 1.010 & 1.012 & 1.013 & 1.024 & 1.015 & 1.017 & 1.006 & 1.005 & 1.015 & 1.015 \\
\hline 32 & asuya & 1.058 & 1.080 & 1.054 & 1.154 & 1.111 & 1.025 & 1.015 & 1.014 & 1.015 & 1.081 & 1.109 & 1.064 & 1.028 \\
\hline 33 & eh & 1.052 & 1.063 & 1.044 & 1.103 & 1.100 & 1.031 & 1.010 & 1.009 & 1.012 & 1.091 & 1.105 & 1.032 & 1.020 \\
\hline 34 & Shat & 1.027 & 1.033 & 1.039 & 1.110 & 1.045 & 1.017 & 1.009 & 1.012 & 1.011 & 1.047 & 1.088 & 1.031 & 1.019 \\
\hline 35 & Oha & 1.097 & 1.066 & 1.075 & 1.057 & 1.032 & 1.012 & 1.004 & 1.004 & 1.007 & 1.038 & 1.071 & 1.112 & 1.017 \\
\hline 36 & har & 143 & 1.085 & 1.072 & 1.043 & 1.000 & 1.000 & 1.000 & 1.000 & 1.000 & 1.000 & 1.100 & 1.100 & 1.000 \\
\hline 37 & Oharan & 1.014 & 1.025 & 1.021 & 1.035 & 1.040 & 1.021 & 1.004 & 1.004 & 1.009 & 1.023 & 1.041 & 1.019 & 1.009 \\
\hline 38 & Digar & 2.035 & 1.984 & 1.825 & 1.610 & 1.287 & 1.777 & 1.167 & 1.164 & 1.318 & 1.374 & 1.802 & 2.181 & 1.422 \\
\hline 39 & Dras & 2.338 & 2.395 & 1.292 & 1.113 & 1.052 & 1.104 & 1.103 & 1.123 & 1.114 & 1.111 & 1.286 & 2.285 & 1.325 \\
\hline 40 & our & 1.053 & 1.045 & 1.016 & 1.017 & 1.030 & 1.018 & 1.019 & 1.044 & 1.011 & 1.019 & 1.012 & 1.044 & 1.025 \\
\hline 41 & Tarh & 1.059 & 1.088 & 1.057 & 1.292 & 1.159 & 1.032 & 1.014 & 1.016 & 1.017 & 1.147 & 1.143 & 1.059 & 1.031 \\
\hline 142 & Gondhla & 2.290 & 1.724 & 1.223 & 1.058 & 1.023 & 1.095 & 1.076 & 1.062 & 1.028 & 1.057 & 1.128 & 2.190 & 1.353 \\
\hline 43 & GS $\mathrm{N}$ & 1.130 & 1.103 & 1.072 & 1.045 & 1.062 & 1.067 & 1.062 & 1.028 & 1.016 & 1.010 & 1.028 & 1.202 & 1.038 \\
\hline 44 & Sula & 1.096 & 1.048 & 1.027 & 1.030 & 1.047 & 1.045 & 1.009 & 1.008 & 1.016 & 1.048 & 1.036 & 1.039 & 1.030 \\
\hline 45 & Sulm & 1.292 & 1.194 & 1.025 & 1.017 & 1.017 & 1.014 & 1.017 & 1.017 & 1.014 & 1.009 & 1.020 & 1.238 & 1.076 \\
\hline o & iund & 1.120 & 1.049 & 1.025 & 1.014 & 1.019 & 1.027 & 1.028 & 1.018 & 1.014 & 1.015 & 1.023 & 1.060 & 1.029 \\
\hline 47 & Surez & 1.630 & 1.196 & 1.033 & 1.016 & 1.018 & 1.032 & 1.021 & 1.019 & 1.008 & 1.011 & 1.045 & 1.251 & 1.130 \\
\hline 48 & Hami & 1.028 & 1.042 & 1.045 & 1.084 & 1.064 & 1.033 & 1.009 & 1.010 & 1.011 & 1.047 & 1.067 & 1.023 & 1.018 \\
\hline & Hand & 1.084 & 1.033 & 1.019 & 1.012 & 1.025 & 1.044 & 1.039 & 1.058 & 1.029 & 1.010 & 1.023 & 1.077 & 1.029 \\
\hline 50 & Hosh & 1.062 & 1.080 & 1.068 & 1.314 & 1.124 & 1.031 & 1.013 & 1.015 & 1.018 & 1.061 & 1.146 & 1.060 & 1.029 \\
\hline & ammu & 052 & 1.036 & 1.041 & 1.063 & 1.104 & 1.048 & 1.011 & 1.010 & 1.015 & 1.090 & 1.081 & 1.020 & 1.022 \\
\hline 52 & Janjehli & .135 & 1.052 & 1.024 & 1.022 & 1.022 & 1.013 & 1.009 & 1.013 & 1.011 & 1.043 & 1.044 & 1.060 & 1.020 \\
\hline 53 & Jhungi & .036 & 1.029 & 1.023 & 1.031 & 1.024 & 1.015 & 1.009 & 1.013 & 1.015 & 1.037 & 1.046 & 1.029 & 1.017 \\
\hline & ogi & .026 & 1.021 & 1.024 & 1.045 & 1.031 & 1.014 & 1.006 & 1.006 & 1.007 & 1.027 & 1.036 & 1.022 & 1.011 \\
\hline 155 & Jubal & 1.072 & 1.039 & 1.034 & 1.043 & 1.034 & 1.028 & 1.015 & 1.017 & 1.020 & 1.052 & 1.047 & 1.062 & 1.029 \\
\hline 50 & unga & .024 & $1.02 \%$ & .035 & 1.066 & 1.041 & 1.018 & 1.010 & 1.013 & 1.012 & 1.036 & 1.059 & 1.039 & 1.019 \\
\hline 57 & Kalka & .024 & 1.058 & 1.103 & 1.441 & 1.056 & 1.041 & 1.013 & 1.009 & 1.011 & 1.048 & 1.209 & 1.070 & 1.023 \\
\hline 158 & Kandagh & 1.028 & 1.030 & 1.034 & 1.068 & 1.057 & 1.032 & 1.010 & 1.014 & 1.016 & 1.046 & 1.135 & 1.037 & 1.022 \\
\hline 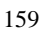 & Kangra & 085 & 1.080 & 1.075 & 1.098 & 1.113 & 1.024 & 1.006 & 1.005 & 1.009 & 1.036 & 1.201 & 1.117 & 1.021 \\
\hline 160 & Kargil & 2.132 & 1.893 & 1.118 & 1.073 & 1.069 & 1.221 & 1.132 & 1.166 & 1.243 & 1.047 & 1.163 & 1.778 & 1.431 \\
\hline 61 & Karsog & 026 & 1.030 & 1.032 & 1.067 & 1.048 & 1.026 & 1.015 & 1.016 & 1.018 & 1.065 & 1.077 & 1.034 & 1.024 \\
\hline 62 & Kasauli 1 & 1.042 & 1.046 & 1.086 & 1.155 & 1.166 & 1.028 & 1.006 & 1.009 & 1.010 & 1.072 & 1.077 & 1.048 & 1.017 \\
\hline 63 & Kasauli2 & 1.038 & 1.039 & 1.038 & 1.179 & 1.057 & 1.029 & 1.007 & 1.008 & 1.010 & 1.025 & 1.132 & 1.033 & 1.016 \\
\hline 64 & Kasumpti & .034 & 1.036 & 1.034 & 1.059 & 1.039 & 1.020 & 1.010 & 1.011 & 1.011 & 1.039 & 1.059 & 1.041 & 1.019 \\
\hline 165 & Kataula & 1.076 & 1.037 & 1.042 & 1.095 & 1.047 & 1.020 & 1.008 & 1.009 & 1.016 & 1.054 & 1.051 & 1.047 & 1.018 \\
\hline 166 & Khadrala & .385 & 1.226 & 1.050 & 1.035 & 1.027 & 1.026 & 1.010 & 1.011 & 1.010 & 1.042 & 1.060 & 1.350 & 1.120 \\
\hline
\end{tabular}




\begin{tabular}{|c|c|c|c|c|c|c|c|c|c|c|c|c|c|c|}
\hline 7 & tse & 977 & 2.053 & .441 & 1.179 & 1.096 & 1.124 & 1.052 & .077 & 1.136 & 107 & 1.280 & 72 & \\
\hline 68 & hangral & .056 & 1.901 & 1.826 & 1.430 & 1.149 & 1.460 & 1.058 & 1.258 & 1.060 & 1.153 & 2.005 & 2.117 & 1.446 \\
\hline 69 & Kharar & 044 & 1.086 & 1.093 & 1.625 & 1.123 & 1.044 & 1.013 & 1.013 & 1.012 & 1.082 & 1.182 & 1.077 & 1.028 \\
\hline 0 & ilba & 3 & .197 & 1.039 & 030 & 1.039 & 1.121 & 1.055 & 1.057 & 1.032 & 56 & 45 & 23 & 99 \\
\hline 71 & ishtwar & 059 & 1.024 & 1.022 & 1.021 & 1.019 & 1.034 & 1.037 & 1.050 & 1.021 & 1.021 & 1.031 & 1.031 & 1.027 \\
\hline 72 & Kokernagh & 075 & 1.044 & 1.024 & 1.022 & 1.022 & 1.031 & 1.035 & 1.048 & 1.026 & 1.027 & 1.031 & 1.053 & 1.033 \\
\hline 73 & Koksar & 291 & 1.728 & 1.269 & 1.064 & 1.022 & 1.071 & 1.025 & 1.032 & 1.020 & 1.025 & 1.148 & 2.200 & 1.397 \\
\hline 174 & Kotarh & 074 & 1.042 & 1.027 & 1.041 & 1.043 & 1.025 & 1.011 & 1.014 & 1.011 & 1.028 & 1.063 & 1.058 & 1.024 \\
\hline 75 & Kothi & 766 & 1.173 & 1.066 & 1.044 & 1.022 & 1.037 & 1.015 & .010 & 1.015 & 1.020 & 1.048 & 1.224 & 116 \\
\hline 76 & Cotkhai & 049 & 1.035 & 1.033 & 1.046 & 1.037 & 1.033 & 1.015 & 1.020 & 1.013 & 1.049 & 1.046 & 1.043 & 1.027 \\
\hline 177 & Kukernag & .066 & 1.030 & 1.018 & 1.014 & 1.023 & 1.037 & 1.029 & 1.023 & 1.022 & 1.008 & 1.020 & 1.044 & .025 \\
\hline 78 & Kulg & 085 & 1.042 & 1.024 & 1.013 & 1.019 & 1.034 & 1.035 & 1.033 & 1.010 & 1.016 & 1.028 & 1.080 & 1.025 \\
\hline 179 & Kulu & 028 & 1.018 & 1.021 & 1.022 & 1.041 & 1.061 & 1.028 & 1.029 & 1.027 & 1.119 & 1.043 & 1.037 & 1.029 \\
\hline 180 & Kumarsain & 052 & 1.039 & 1.031 & 1.049 & 1.037 & 1.029 & 1.015 & 1.020 & 1.022 & 1.095 & 1.060 & 1.070 & 029 \\
\hline 181 & Kyel & 397 & 2.479 & 1.275 & 1.061 & 1.031 & 1.102 & 1.063 & 1.085 & 1.038 & 1.065 & 1.195 & 2.228 & 1.453 \\
\hline 182 & Langet & 087 & 1.032 & 1.019 & 1.013 & 1.027 & 1.046 & 1.045 & 1.073 & 1.029 & 1.011 & 1.023 & 1.082 & 1.030 \\
\hline 183 & Leh & 983 & 1.810 & 1.187 & 1.191 & 1.141 & 1.471 & 1.078 & 1.079 & 1.064 & 1.031 & 1.266 & 2.083 & 1.297 \\
\hline 34 & Mal & 058 & 1.036 & 1.030 & 1.021 & 1.030 & 1.036 & 1.038 & 1.034 & 1.026 & 1.019 & 1.022 & 1.044 & 1.032 \\
\hline 185 & Malikpur & 1.015 & 1.031 & 1.028 & 1.044 & 1.089 & 1.024 & 1.009 & 1.007 & 1.008 & 1.059 & 1.048 & 1.019 & 1.015 \\
\hline 186 & Mandi1 & 030 & 1.034 & 1.036 & 1.048 & 1.035 & 1.016 & 1.008 & 1.009 & 1.014 & 1.046 & 1.045 & 1.048 & 1.017 \\
\hline 87 & Mar & 8 & 1.033 & 1.038 & 1.055 & 1.042 & 1.020 & 1.008 & 1.009 & 1.013 & 1.047 & 1.061 & 1.045 & 1.017 \\
\hline 188 & Mulbek & 978 & 1.915 & 1.836 & 1.419 & 1.388 & 1.332 & 1.192 & 1.396 & 1.311 & 1.200 & 1.862 & 2.078 & 1.687 \\
\hline 39 & Nala & 040 & 1.060 & 1.058 & 1.350 & 1.087 & 1.050 & 1.009 & 1.009 & 1.010 & 1.063 & 1.094 & 1.049 & 020 \\
\hline 190 & Nawans & .060 & 1.086 & 1.053 & 1.401 & 1.157 & 1.031 & 1.014 & 1.013 & 1.017 & 1.122 & 1.130 & 1.052 & 1.029 \\
\hline 191 & Nichar & 084 & 1.047 & 1.033 & 1.034 & 1.033 & 1.064 & 1.031 & 1.026 & 1.026 & 1.042 & 1.053 & 1.062 & 1.039 \\
\hline 2 & Noy & 1 & 1.03 & 1.040 & 1.090 & 1. & 1.0 & 1.014 & 1.013 & 1.020 & 29 & 3 & 1.053 & .026 \\
\hline 193 & Nurpur & 015 & 1.049 & 1.029 & 1.062 & 1.132 & 1.038 & 1.008 & 1.006 & 1.010 & 1.022 & 1.119 & 1.020 & 1.015 \\
\hline 194 & Palam & 101 & 1.075 & 1.074 & 1.063 & 1.050 & 1.020 & 1.005 & 1.005 & 1.007 & 1.024 & 1.051 & 1.110 & 1.020 \\
\hline 95 & Panamik & 010 & 1.868 & 1.276 & 1.163 & 1.118 & 1.138 & 1.059 & 1.070 & 1.122 & 1.069 & 1.306 & 1.860 & 1.264 \\
\hline 196 & Panjain & 064 & 1.033 & 1.027 & 1.032 & 1.023 & 1.024 & 1.011 & 1.013 & 1.013 & 1.042 & 1.021 & 1.041 & 1.020 \\
\hline 197 & Patl & 9 & 1.051 & 1.084 & 1.134 & 1.191 & 1.062 & 1.008 & 1.006 & 1.009 & 1.030 & 0 & 1.043 & 1.017 \\
\hline 88 & Penc & 138 & .947 & 1.905 & 1.965 & 1.884 & 1.307 & 1.225 & 1.168 & 1.288 & 1.479 & 1.872 & 2.088 & 1.878 \\
\hline 199 & Phalgam & 1.093 & 1.052 & 1.031 & 1.025 & 1.028 & 1.022 & 1.025 & 1.022 & 1.020 & 1.015 & 1.027 & 1.070 & 1.031 \\
\hline 0 & Poonch & 020 & 1.017 & 1.017 & 1.027 & 1.035 & 1.015 & 1.006 & 1.008 & 1.008 & 1.052 & 1.022 & 1.026 & 015 \\
\hline 201 & Prang & 083 & 1.046 & 1.033 & 1.023 & 1.033 & 1.046 & 1.038 & 1.036 & 1.019 & 1.018 & 1.042 & 1.076 & 1.041 \\
\hline 202 & Purbani & 404 & 1.211 & 1.049 & 1.034 & 1.041 & 1.154 & 1.123 & 1.133 & 1.050 & 1.134 & 1.067 & 1.129 & 143 \\
\hline 33 & Qazi & 39 & 023 & 1.014 & 1.017 & 1.019 & 1.021 & 1.025 & 1.034 & 1.010 & 1.013 & 1.017 & 1.027 & 1.021 \\
\hline 204 & Rajdhani & 1.017 & 1.033 & 1.028 & 1.034 & 1.038 & 1.032 & 1.012 & 1.009 & 1.012 & 1.041 & 1.038 & 1.022 & 1.018 \\
\hline 55 & Ramban & 022 & 1.019 & 1.022 & 1.025 & 1.031 & 1.058 & 1.022 & 1.026 & 1.018 & 1.037 & 1.048 & 1.018 & 1.024 \\
\hline 206 & Ramn & .017 & 1.021 & 1.017 & 1.032 & 1.052 & 1.020 & 1.008 & 1.008 & 1.011 & 1.013 & 1.066 & 1.018 & 1.013 \\
\hline 207 & Rampur & 043 & 1.034 & 1.034 & 1.038 & 1.046 & 1.043 & 1.018 & 1.018 & 1.019 & 1.045 & 1.069 & 1.057 & 1.029 \\
\hline 8 & Riasi & 19 & 1.019 & 1.033 & 1.048 & 1.053 & 1.031 & 1.007 & 1.006 & 1.008 & 1.034 & 1.047 & 1.014 & .013 \\
\hline 209 & Rohru & 050 & 1.028 & 1.027 & 1.052 & 1.041 & 1.025 & 1.017 & 1.016 & 1.013 & 1.041 & 1.054 & 1.053 & 1.026 \\
\hline 210 & Rupanas & 034 & 1.055 & 1.074 & 1.181 & 1.207 & 1.053 & 1.011 & 1.013 & 1.013 & 1.087 & 1.106 & 1.067 & 1.026 \\
\hline 211 & Sangla & 年 & 1.075 & 1.051 & 1.046 & 1.040 & 1.101 & 1.048 & 1.049 & 1.033 & 1.084 & 1.075 & 1.147 & 1.073 \\
\hline 212 & Sarkahat & 030 & 1.027 & 1.032 & 1.073 & 1.042 & 1.013 & 1.007 & 1.007 & 1.009 & 1.035 & 1.064 & 1.034 & 13 \\
\hline 13 & Shillaru & 253 & 1.093 & 1.040 & 1.045 & 1.030 & 1.020 & 1.009 & 1.013 & 1.012 & 1.047 & 1.050 & 1.137 & 1.030 \\
\hline 214 & Shimla & 042 & 1.049 & 1.038 & 1.059 & 1.037 & 1.015 & 1.009 & 1.012 & 1.010 & 1.034 & 1.076 & 1.056 & 1.018 \\
\hline 215 & Shiquanh & 264 & 2.678 & 2.241 & 1.723 & 1.520 & 1.203 & 1.063 & 1.062 & 1.183 & 1.310 & 2.121 & 2.022 & 1.347 \\
\hline 216 & Shopian & 063 & 1.037 & 1.022 & 1.017 & 1.020 & 1.029 & 1.032 & 1.026 & 1.014 & 1.022 & 1.026 & 1.048 & 1.026 \\
\hline 217 & Sogam & 102 & 1.038 & 1.020 & 1.013 & 1.023 & 1.038 & 1.028 & 1.023 & 1.022 & 1.009 & 1.026 & 1.079 & 1.031 \\
\hline 218 & Solan & 027 & 1.031 & 1.027 & 1.081 & 1.045 & 1.019 & 1.010 & 1.012 & 1.013 & 1.041 & 1.052 & 1.029 & 1.019 \\
\hline 219 & Sonem & 881 & 1.416 & 1.067 & 1.037 & 1.038 & 1.020 & 1.023 & 1.015 & 1.011 & 1.015 & 1.071 & 1.363 & 1.176 \\
\hline 220 & Sopore & 144 & 1.033 & 1.023 & 1.016 & 1.031 & 1.062 & 1.065 & 1.063 & 1.021 & 1.012 & 1.032 & 1.137 & 1.033 \\
\hline 221 & SR Sing & 033 & 1.034 & 1.055 & 1.102 & 1.085 & 1.039 & 1.011 & 1.014 & 1.014 & 1.098 & 1.809 & 1.022 & 1.023 \\
\hline 222 & Srinagar & 075 & 1.035 & 1.027 & 1.022 & 1.031 & 1.036 & 1.045 & 1.038 & 1.026 & 1.018 & 1.029 & 1.048 & .034 \\
\hline 223 & Sundarnagar & 1.031 & 1.029 & 1.033 & 1.067 & 1.036 & 1.017 & 1.009 & 1.011 & 1.012 & 1.063 & 1.046 & 1.046 & 1.018 \\
\hline
\end{tabular}




\begin{tabular}{|c|c|c|c|c|c|c|c|c|c|c|c|c|c|c|}
\hline 24 & Suni Seoni & 1.029 & 1.035 & 1.035 & 1.055 & 1.049 & 1.024 & 1.011 & 1.019 & 1.018 & 1.057 & 1.070 & 1.032 & \\
\hline 225 & Tanda & 1.070 & 1.135 & 1.090 & 1.237 & 1.112 & 1.100 & 1.014 & 1.018 & 1.014 & 1.068 & 1.061 & 1.061 & 1.032 \\
\hline 26 & Tangmarg & 1.163 & 1.062 & 1.028 & 1.016 & 1.024 & 1.034 & 1.044 & 1.024 & 1.022 & 1.016 & 1.033 & 1.076 & 1.037 \\
\hline & Tapoban & 1.512 & 1.198 & 1.069 & 1.058 & 1.063 & 1.047 & 1.018 & 1.021 & 1.026 & 1.013 & 1.128 & 1.296 & 1.061 \\
\hline & Theog & 1.063 & 1.036 & 1.032 & 1.040 & 1.033 & 1.022 & 1.012 & 1.018 & 1.015 & 1.087 & 1.061 & 1.054 & 1.026 \\
\hline 9 & Tibri & 1.036 & 1.072 & 1.044 & 1.083 & 1.130 & 1.058 & 1.017 & 1.013 & 1.032 & 1.105 & 1.085 & 1.040 & 1.031 \\
\hline & Tral & 1.082 & 1.063 & 1.036 & 1.034 & 1.039 & 1.049 & 1.049 & 1.038 & 1.022 & 1.048 & 1.057 & 1.078 & .045 \\
\hline 1 & T-K-I-H-Kung & 1.794 & 1.937 & 1.622 & 1.522 & 1.769 & 1.707 & 1.288 & 1.322 & 1.661 & 1.645 & 1.610 & 1.591 & .533 \\
\hline & Udhampur & 1.017 & 1.021 & 1.022 & 1.058 & 1.178 & 1.048 & 1.010 & 1.008 & 1.008 & 1.048 & 1.031 & 1.021 & 1016 \\
\hline 3 & Una & 1.038 & 1.060 & 1.052 & 1.112 & 1.115 & 1.052 & 1.010 & 1.010 & 1.015 & 1.052 & 1.094 & 1.047 & .022 \\
\hline 34 & Uri & 1.035 & 1.023 & 1.018 & 1.024 & 1.020 & 1.022 & 1.021 & 1.017 & 1.010 & 1.019 & 1.018 & 1.033 & \\
\hline & Uttamchipura & 1.824 & 1.542 & 1.136 & 1.025 & 1.018 & 1.028 & 1.017 & 1.017 & 1.017 & 1.015 & 1.139 & 1.852 & 215 \\
\hline 6 & Vantipura & 1.072 & 1.043 & 1.031 & 1.026 & 1.031 & 1.036 & 1.047 & 1.041 & 1.022 & 1.020 & 1.033 & 1.068 & 1.036 \\
\hline 7 & Verinagh & 1.065 & 1.025 & 1.017 & 1.017 & 1.014 & 1.026 & 1.022 & 1.025 & 1.011 & 1.011 & 1.029 & 1.038 & 1.021 \\
\hline 8 & Arthal & 1.183 & 1.065 & 1.029 & 1.028 & 1.027 & 1.030 & 1.054 & 1.065 & 1.072 & 1.026 & 1.035 & 1.064 & .057 \\
\hline 39 & Bhakra & 1.037 & 1.044 & 1.039 & 1.035 & 1.040 & 1.052 & 1.008 & 1.010 & 1.011 & 1.031 & 1.026 & 1.021 & 017 \\
\hline 0 & Bunencha & 1.324 & 1.172 & 1.029 & 1.023 & 1.022 & 1.028 & 1.020 & 1.023 & 1.025 & 1.031 & 1.037 & 1.180 & .081 \\
\hline 1 & Chingaon & 1.049 & 1.025 & 1.011 & 1.016 & 1.016 & 1.019 & 1.022 & 1.026 & 1.028 & 1.020 & 1.019 & 1.037 & \\
\hline 2 & Chitkul & 2.317 & 1.705 & 1.777 & 1.132 & 1.053 & 1.106 & 1.051 & 1.051 & 1.042 & 1.173 & 1.855 & 2.514 & 49 \\
\hline 3 & Damini & 1.015 & 1.014 & 1.011 & 1.017 & 1.019 & 1.023 & 1.012 & 1.014 & 1.015 & 1.018 & 1.016 & 1.018 & 1.015 \\
\hline 4 & Darabshala & 1.029 & 1.025 & 1.023 & 1.036 & 1.036 & 1.046 & 1.033 & 1.040 & 1.043 & 1.037 & 1.034 & 1.037 & 033 \\
\hline 15 & Devigol & 1.285 & 1.110 & 1.030 & 1.029 & 1.029 & 1.036 & 1.023 & 1.027 & 1.029 & 1.053 & 1.053 & 1.133 & 1.067 \\
\hline 6 & Dhamkund & 1.017 & 1.016 & 1.013 & 1.026 & 1.027 & 1.033 & 1.021 & 1.025 & 1.027 & 1.021 & 1.019 & 1.024 & \\
\hline 47 & Doda & 1.026 & 1.021 & 1.017 & 1.031 & 1.033 & 1.041 & 1.042 & 1.050 & 1.054 & 1.029 & 1.027 & 1.029 & 30 \\
\hline 18 & Dusadudha & 1.281 & 1.108 & 1.032 & 1.030 & 1.030 & 1.038 & 1.022 & 1.026 & 1.028 & 1.043 & 1.044 & 1.121 & 063 \\
\hline 9 & Gainta & 1.012 & 1.014 & 1.013 & 1.024 & 1.026 & 1.033 & 1.011 & 1.012 & 1.013 & 1.019 & 1.016 & 1.014 & 15 \\
\hline 50 & Ghamroor & 1.038 & 1.042 & 1.037 & 1.042 & 1.047 & 1.061 & 1.008 & 1.009 & 1.010 & 1.042 & 1.038 & 1.035 & 1.018 \\
\hline 1 & Harsur & 1.032 & 1.039 & 1.035 & 1.039 & 1.043 & 1.055 & 1.008 & 1.010 & 1.010 & 1.046 & 1.039 & 1.033 & \\
\hline 2 & Hawal & 1.416 & 1.283 & 1.049 & 1.027 & 1.024 & 1.025 & 1.025 & 1.030 & 1.033 & 1.022 & 1.043 & 1.291 & \\
\hline 3 & Inshan & 1.386 & 1.146 & 1.041 & 1.031 & 1.031 & 1.034 & 1.039 & 1.047 & 1.051 & 1.038 & 1.044 & 1.142 & .085 \\
\hline 4 & Kahu & 1.034 & 1.041 & 1.036 & 1.027 & 1.030 & 1.039 & 1.010 & 1.012 & 1.013 & 1.031 & 1.026 & 1.021 & \\
\hline 5 & Kasol & 1.034 & 1.041 & 1.036 & 1.025 & 1.029 & 1.037 & 1.009 & 1.011 & 1.012 & 1.031 & 1.026 & 1.021 & 18 \\
\hline 56 & Kati & 1.018 & 1.015 & 1.011 & 1.010 & 1.011 & 1.014 & 1.008 & 1.009 & 1.010 & 1.010 & 1.011 & 1.013 & 11 \\
\hline 57 & Kaza & 1.910 & 2.370 & 1.792 & 1.086 & 1.046 & 1.077 & 1.036 & 1.039 & 1.047 & 1.114 & 1.644 & 1.749 & \\
\hline 58 & Kupwa & 1.072 & 1.138 & 1.015 & 1.015 & 1.019 & 1.021 & 1.019 & 1.024 & 1.017 & 1.013 & 1.020 & 1.067 & 1.027 \\
\hline 59 & Larji & 1.026 & 1.026 & 1.023 & 1.030 & 1.032 & 1.042 & 1.017 & 1.020 & 1.021 & 1.033 & 1.029 & 1.031 & \\
\hline 50 & Lossar & 1.464 & 1.581 & 1.495 & 1.207 & 1.042 & 1.076 & 1.023 & 1.026 & 1.034 & 1.171 & 1.293 & 1.289 & 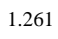 \\
\hline 261 & Matsal & 2.140 & 2.322 & 2.063 & 1.713 & 1.099 & 1.044 & 1.068 & 1.080 & 1.110 & 1.430 & 1.816 & 2.008 & 1.751 \\
\hline 52 & Mau & 2.037 & 2.076 & 1.128 & 1.045 & 1.027 & 1.029 & 1.036 & 1.043 & 1.048 & 1.028 & 1.095 & 1.752 & 2 \\
\hline 63 & Mohu & 1.266 & 1.092 & 1.017 & 1.019 & 1.020 & 1.023 & 1.022 & 1.026 & 1.028 & 1.013 & 1.022 & 1.094 & 1.064 \\
\hline 264 & Moorang & 1.496 & 1.360 & 1.070 & 1.057 & 1.069 & 1.211 & 1.103 & 1.100 & 1.065 & 1.159 & 1.108 & 1.202 & \\
\hline 65 & Namgia & 1.804 & 1.931 & 1.119 & 1.056 & 1.046 & 1.119 & 1.054 & 1.062 & 1.048 & 1.084 & 1.118 & 1.567 & \\
\hline 266 & Nandan & 1.046 & 1.027 & 1.012 & 1.014 & 1.016 & 1.017 & 1.006 & 1.007 & 1.007 & 1.014 & 1.012 & 1.026 & 1.013 \\
\hline 267 & Ohli & 1.040 & 1.025 & 1.018 & 1.027 & 1.026 & 1.029 & 1.034 & 1.040 & 1.044 & 1.028 & 1.027 & 1.037 & 29 \\
\hline 268 & Palmar & 1.045 & 1.029 & 1.022 & 1.028 & 1.027 & 1.029 & 1.030 & 1.036 & 1.039 & 1.023 & 1.029 & 1.030 & .030 \\
\hline 269 & Pooh & 1.760 & 1.812 & 1.081 & 1.048 & 1.041 & 1.106 & 1.049 & 1.055 & 1.043 & 1.071 & 1.086 & 1.452 & \\
\hline 0 & Pouni & 1.016 & 1.019 & 1.017 & 1.024 & 1.026 & 1.032 & 1.005 & 1.006 & 1.006 & 1.024 & 1.020 & 1.017 & \\
\hline 271 & Rakchham & 2.265 & 1.663 & 1.234 & 1.062 & 1.041 & 1.104 & 1.049 & 1.048 & 1.034 & 1.078 & 1.243 & 2.363 & 4 \\
\hline 272 & Rekenwas & 1.528 & 1.405 & 1.350 & 1.101 & 1.053 & 1.053 & 1.084 & 1.101 & 1.112 & 1.057 & 1.302 & 1.507 & 52 \\
\hline 273 & Rot & 1.016 & 1.016 & 1.012 & 1.017 & 1.018 & 1.023 & 1.025 & 1.029 & 1.031 & 1.022 & 1.019 & 1.018 & \\
\hline 274 & Sain & 1.138 & 1.046 & 1.011 & 1.008 & 1.009 & 1.009 & 1.007 & 1.008 & 1.008 & 1.009 & 1.012 & 1.043 & \\
\hline 75 & Sainj & 1.026 & 1.021 & 1.018 & 1.023 & 1.025 & 1.033 & 1.017 & 1.020 & 1.021 & 1.033 & 1.029 & 1.032 & \\
\hline 276 & Salal & 1.012 & 1.015 & 1.013 & 1.022 & 1.024 & 1.030 & 1.010 & 1.012 & 1.012 & 1.020 & 1.017 & 1.015 & \\
\hline 277 & Sarkund & 1.351 & 1.123 & 1.050 & 1.032 & 1.033 & 1.036 & 1.056 & 1.067 & 1.073 & 1.035 & 1.048 & 1.115 & \\
\hline 278 & Shahpur-I & 1.029 & 1.035 & 1.032 & 1.031 & 1.035 & 1.045 & 1.008 & 1.010 & 1.011 & 1.039 & 1.034 & 1.029 & \\
\hline 279 & Sirshi & 1.048 & 1.030 & 1.020 & 1.025 & 1.023 & 1.025 & 1.027 & 1.032 & 1.035 & 1.029 & 1.028 & 1.040 & \\
\hline 280 & Sohal & 1.192 & 1.125 & 1.060 & 1.052 & 1.042 & 1.048 & 1.064 & 1.076 & 1.085 & 1.043 & 1.055 & 1.102 & \\
\hline
\end{tabular}




\begin{tabular}{|c|c|c|c|c|c|c|c|c|c|c|c|c|c|c|}
\hline 281 & Tandi & 2.285 & 1.715 & 1.197 & 1.076 & 1.040 & 1.056 & 1.053 & 1.062 & 1.070 & 1.038 & 1.120 & 2.165 & 1.390 \\
\hline 282 & Thana & 1.312 & 1.107 & 1.024 & 1.031 & 1.031 & 1.035 & 1.069 & 1.082 & 1.090 & 1.030 & 1.035 & 1.113 & 1.100 \\
\hline 283 & Tillar & 1.143 & .057 & 1.028 & 1.023 & 1.022 & 1.023 & 1.029 & 1.034 & 1.038 & 1.019 & 1.026 & 1.057 & 1.041 \\
\hline 84 & Udaipur & 2.011 & 1.452 & 1.087 & 1.049 & 1.033 & 1.039 & 1.052 & 1.062 & 1.069 & 1.044 & 1.075 & 1.391 & .236 \\
\hline 285 & Yurod & 1.175 & .076 & 1.040 & 1.037 & 1.038 & 1.044 & 1.043 & 1.052 & .057 & 1.042 & 1.042 & 1.077 & 1.060 \\
\hline 86 & Asmar & 1.075 & 1.067 & 1.063 & 1.057 & 1.064 & 1.142 & 1.098 & 1.106 & 1.102 & 1.040 & 1.071 & 1.166 & 1.074 \\
\hline 87 & Bamiyan & 1.959 & 2.112 & 1.297 & 1.080 & 1.062 & 1.149 & 1.466 & 1.172 & 1.063 & 1.093 & 1.139 & 1.852 & 1.295 \\
\hline 88 & Darullaman & 1.292 & 1.426 & 1.050 & 1.069 & 1.159 & 1.427 & 1.366 & 1.339 & 1.272 & 1.132 & 1.078 & 1.175 & .186 \\
\hline 89 & Gerdiz & 1.284 & 1.426 & 1.106 & 1.039 & 1.100 & 1.167 & 1.093 & 1.099 & 1.026 & 1.144 & 1.104 & 1.202 & .187 \\
\hline 290 & Ghaziabad & 1.097 & 1.071 & 1.062 & 1.042 & 1.087 & 1.495 & 1.164 & 1.076 & 1.053 & 1.247 & 1.159 & 1.189 & 1.070 \\
\hline 291 & Ghazni & 1.246 & 1.410 & 1.086 & 1.041 & 1.080 & 1.063 & 1.071 & 1.056 & 1.024 & 1.562 & 1.089 & 1.187 & 1.172 \\
\hline 292 & Jabul Saraj & 1.156 & 1.113 & 1.080 & 1.063 & 1.143 & 1.569 & 1.534 & 1.833 & 1.352 & 1.165 & 1.089 & 1.088 & 1.106 \\
\hline 293 & Jalalabad & 1.058 & 1.060 & 1.041 & 1.052 & 1.130 & 1.146 & 1.039 & 1.022 & 1.016 & 1.089 & 1.039 & 1.151 & 1.051 \\
\hline 294 & Kabul AP & 1.289 & 1.219 & 1.081 & 1.050 & 1.127 & 1.345 & 1.208 & 1.282 & 1.154 & 1.246 & 1.079 & 1.115 & 1.131 \\
\hline 295 & Karizmir & 1.248 & 1.349 & 1.049 & 1.028 & 1.137 & 1.302 & 1.320 & 1.516 & 1.200 & 1.142 & 1.056 & 1.147 & 1.142 \\
\hline 296 & Khost & 1.086 & 1.061 & 1.063 & 1.049 & 1.070 & 1.080 & 1.035 & 1.041 & 1.044 & 1.107 & 1.092 & 1.080 & 1.056 \\
\hline 297 & Laghman & 1.047 & 1.049 & 1.038 & 1.051 & 1.100 & 1.239 & 1.033 & 1.021 & 1.025 & 1.044 & 1.031 & 1.088 & 1.047 \\
\hline 298 & Logar & 1.248 & 1.461 & 1.100 & 1.055 & 1.098 & 1.244 & 1.204 & 1.352 & 1.292 & 1.144 & 1.121 & 1.228 & 1.185 \\
\hline 299 & Mirbachakot & 1.210 & 1.243 & 1.046 & 1.067 & 1.165 & 1.540 & 1.299 & 1.271 & 1.387 & 1.134 & 1.070 & 1.125 & 1.144 \\
\hline 300 & Mokur & 1.271 & 1.385 & 1.053 & 1.074 & 1.118 & 1.017 & 1.028 & 1.020 & 1.007 & 1.079 & 1.145 & 1.177 & 1.170 \\
\hline 301 & North Salang & 2.467 & 2.443 & 1.893 & 1.504 & 1.063 & 1.160 & 1.176 & 1.141 & 1.333 & 1.133 & 1.547 & 1.980 & 1.818 \\
\hline 302 & Okak & 1.587 & 1.886 & 1.833 & 1.194 & 1.096 & 1.081 & 1.116 & 1.102 & 1.035 & 1.875 & 1.631 & 1.750 & 1.531 \\
\hline 303 & Paghman & 1.383 & 1.565 & 1.056 & 1.041 & 1.167 & 1.407 & 1.406 & 1.494 & 1.232 & 1.174 & 1.048 & 1.126 & 1.216 \\
\hline 304 & Pan Jao & 2.018 & 2.258 & 2.100 & 1.070 & 1.074 & 1.150 & 1.436 & 1.151 & 1.039 & 1.037 & 1.530 & 2.000 & 1.616 \\
\hline 305 & Sarobi & 1.059 & 1.051 & 1.071 & 1.059 & 1.213 & 1.432 & 1.649 & 1.174 & 1.081 & 1.170 & 1.161 & 1.095 & 1.080 \\
\hline 306 & South Salang & 1.377 & 1.593 & 1.259 & 1.079 & 1.143 & 1.466 & 1.527 & 1.484 & 1.633 & 1.155 & 1.206 & 1.504 & 1.322 \\
\hline 307 & Zebak & 2.754 & 2.395 & 1.508 & 1.059 & 1.058 & 1.090 & 1.181 & 1.151 & 1.053 & 1.100 & 1.320 & 2.213 & 1.239 \\
\hline 308 & Approach & 1.000 & 1.000 & 1.000 & 1.000 & 1.000 & 1.000 & 1.000 & 1.000 & 1.000 & 1.000 & 1.000 & 1.000 & 1.000 \\
\hline 309 & Baltoro & 1.000 & 1.000 & 1.000 & 1.000 & 1.000 & 1.000 & 1.000 & 1.000 & 1.000 & 1.000 & 1.000 & 1.000 & 1.000 \\
\hline 310 & Batura & 1.774 & 1.774 & 1.774 & 1.774 & 1.774 & 1.774 & 1.774 & 1.774 & 1.774 & 1.774 & 1.774 & 1.774 & 1.774 \\
\hline 311 & Chong Kumdar & 2.756 & 2.756 & 2.756 & 2.756 & 2.756 & 2.756 & 2.756 & 2.756 & 2.756 & 2.756 & 2.756 & 2.756 & 2.756 \\
\hline 312 & Chogolungma & 1.701 & 1.701 & 1.701 & 1.701 & 1.701 & 1.701 & 1.701 & 1.701 & 1.701 & 1.701 & 1.701 & 1.701 & 1.701 \\
\hline 313 & Hispar Dome & 1.000 & 1.000 & 1.000 & 1.000 & 1.000 & 1.000 & 1.000 & 1.000 & 1.000 & 1.000 & 1.000 & 1.000 & 1.000 \\
\hline 314 & Hispar East & 1.701 & 1.701 & 1.701 & 1.701 & 1.701 & 1.701 & 1.701 & 1.701 & 1.701 & 1.701 & 1.701 & 1.701 & 1.701 \\
\hline 315 & Hispar West & 1.000 & 1.000 & 1.000 & 1.000 & 1.000 & 1.000 & 1.000 & 1.000 & 1.000 & 1.000 & 1.000 & 1.000 & 1.000 \\
\hline 316 & Hispar Pass & 1.176 & 1.176 & 1.176 & 1.176 & 1.176 & 1.176 & 1.176 & 1.176 & 1.176 & 1.176 & 1.176 & 1.176 & 1.176 \\
\hline 317 & Khurdopin & 1.000 & 1.000 & 1.000 & 1.000 & 1.000 & 1.000 & 1.000 & 1.000 & 1.000 & 1.000 & 1.000 & 1.000 & 1.000 \\
\hline 318 & Nanga Parbat & 1.250 & 1.250 & 1.250 & 1.250 & 1.250 & 1.250 & 1.250 & 1.250 & 1.250 & 1.250 & 1.250 & 1.250 & 1.250 \\
\hline 319 & Nun Kun Nortl: & 1.278 & 1.278 & 1.278 & 1.278 & 1.278 & 1.278 & 1.278 & 1.278 & 1.278 & 1.278 & 1.278 & 1.278 & 1.278 \\
\hline 20 & Sentik & 2.210 & 2.210 & 2.210 & 2.210 & 2.210 & 2.210 & 2.210 & 2.210 & 2.210 & 2.210 & 2.210 & 2.210 & 2.210 \\
\hline 321 & Siachin A & 3.066 & 3.066 & 3.066 & 3.066 & 3.066 & 3.066 & 3.066 & 3.066 & 3.066 & 3.066 & 3.066 & 3.066 & 3.066 \\
\hline 322 & Siachin B & 2.901 & 2.901 & 2.901 & 2.901 & 2.901 & 2.901 & 2.901 & 2.901 & 2.901 & 2.901 & 2.901 & 2.901 & 2.901 \\
\hline 323 & Siachin C & 2.360 & 2.360 & 2.360 & 2.360 & 2.360 & 2.360 & 2.360 & 2.360 & 2.360 & 2.360 & 2.360 & 2.360 & 2.360 \\
\hline 324 & Siachin D & 1.877 & 1.877 & 1.877 & 1.877 & 1.877 & 1.877 & 1.877 & 1.877 & 1.877 & 1.877 & 1.877 & 1.877 & 1.877 \\
\hline 325 & South Terong & 2.756 & 2.756 & 2.756 & 2.756 & 2.756 & 2.756 & 2.756 & 2.756 & 2.756 & 2.756 & 2.756 & 2.756 & 2.756 \\
\hline 326 & Terong & 1.877 & 1.877 & 1.877 & 1.877 & 1.877 & 1.877 & 1.877 & 1.877 & 1.877 & 1.877 & 1.877 & 1.877 & 1.877 \\
\hline 327 & Urdok & 1.000 & 1.000 & 1.000 & 1.000 & 1.000 & 1.000 & 1.000 & 1.000 & 1.000 & 1.000 & 1.000 & 1.000 & 1.000 \\
\hline 328 & Whaleback & 1.419 & 1.419 & 1.419 & 1.419 & 1.419 & 1.419 & 1.419 & 1.419 & 1.419 & 1.419 & 1.419 & 1.419 & 1.419 \\
\hline
\end{tabular}


Table S-3.4: Runoff ratio $(Q / P)$ and aridity index $\left(P / E T_{p}\right)$ for different sub-basins. Qadj is the adjusted specific runoff, $\mathrm{P}_{\text {uadj }}$ is uncorrected precipitation under this study or observational-based estimated precipitation derived by Dahri et al. (2016), $P_{\text {adj }}$ denotes adjusted precipitation under this study and $\mathrm{P}_{\text {corl }}$ are the corrected precipitation estimates by Immerzeel et al. (2015).

\begin{tabular}{llllllll}
\hline S. & River & $\mathbf{P}_{\text {uadj }} /$ & $\mathbf{P}_{\mathbf{a d j}} /$ & $\mathbf{P}_{\text {corI }} /$ & $\mathbf{Q}_{\text {adj }} /$ & $\mathbf{Q}_{\text {adj }} /$ & $\mathbf{Q}_{\text {adj }} /$ \\
No & Basin & $\mathbf{E T}_{\mathbf{p}}$ & $\mathbf{E T}_{\mathbf{p}}$ & $\mathbf{E T}_{\mathbf{p}}$ & $\mathbf{P}_{\text {uadj }}$ & $\mathbf{P}_{\text {adj }}$ & $\mathbf{P}_{\text {corI }}$ \\
\hline 1 & Gilgit at Gilgit & 1.371 & 1.853 & 3.189 & 1.245 & 0.921 & 0.535 \\
2 & Hunza at Dainyor & 1.695 & 2.481 & 2.646 & 1.072 & 0.733 & 0.687 \\
3 & Shigar at Shigar & 2.487 & 3.015 & 2.779 & 1.069 & 0.882 & 0.956 \\
4 & Shyok at Yugo & 1.222 & 2.166 & 2.372 & 1.412 & 0.797 & 0.728 \\
5 & Indus at Kharmong & 0.476 & 0.746 & 1.384 & 1.049 & 0.670 & 0.361 \\
6 & Astore at Doyian & 1.646 & 2.277 & 1.962 & 1.200 & 0.868 & 1.007 \\
7 & Indus at Tarbela Dam & 1.008 & 1.381 & 1.930 & 1.026 & 0.748 & 0.535 \\
8 & Chitral at Chitral & 1.793 & 2.565 & 3.116 & 1.071 & 0.749 & 0.616 \\
9 & Panjkora at Zulum Br. & 1.067 & 1.152 & 1.908 & 0.832 & 0.770 & 0.465 \\
10 & Swat at Chakdara & 1.416 & 1.565 & 2.665 & 1.234 & 1.116 & 0.655 \\
11 & Kabul at Warsak & 0.831 & 1.036 & 1.866 & 0.372 & 0.299 & 0.166 \\
12 & Kabul at Nowshera & 0.910 & 0.960 & 1.798 & 0.623 & 0.591 & 0.315 \\
13 & Jhelum at Mangla Dam & 1.401 & 1.577 & 1.553 & 0.698 & 0.620 & 0.630 \\
14 & Chenab at Marala & 1.700 & 1.931 & 1.658 & 0.881 & 0.776 & 0.904 \\
15 & Ravi at Thein Dam & 2.036 & 2.240 & 2.143 & 0.838 & 0.762 & 0.796 \\
16 & Beas at Pong Dam & 2.096 & 2.216 & 1.734 & 0.632 & 0.598 & 0.764 \\
17 & Sutlej at Bhakra Dam & 0.727 & 0.902 & 2.048 & 0.711 & 0.573 & 0.253 \\
\hline
\end{tabular}

Table S-3.5: Land cover classes shown in Figure S-3.6c.

\begin{tabular}{ll}
\hline Land Cover Class No. & Land Cover Type \\
\hline 0 & Water Bodies \\
1 & Evergreen Needleleaf Forest \\
2 & Evergreen Broadleaf Forest \\
3 & Deciduous Needleleaf Forest \\
4 & Deciduous Broadleaf Forest \\
5 & Mixed Forest \\
6 & Closed Shrublands \\
7 & Open Shrublands \\
8 & Woody Savannas \\
9 & Savannas \\
10 & Grasslands \\
11 & Permanent Wetlands \\
12 & Croplands \\
13 & Urban and Built-Up \\
14 & Cropland/Natural Vegetation Mosaic \\
15 & Snow and Ice \\
16 & Barren or Sparsely Vegetated \\
\hline
\end{tabular}



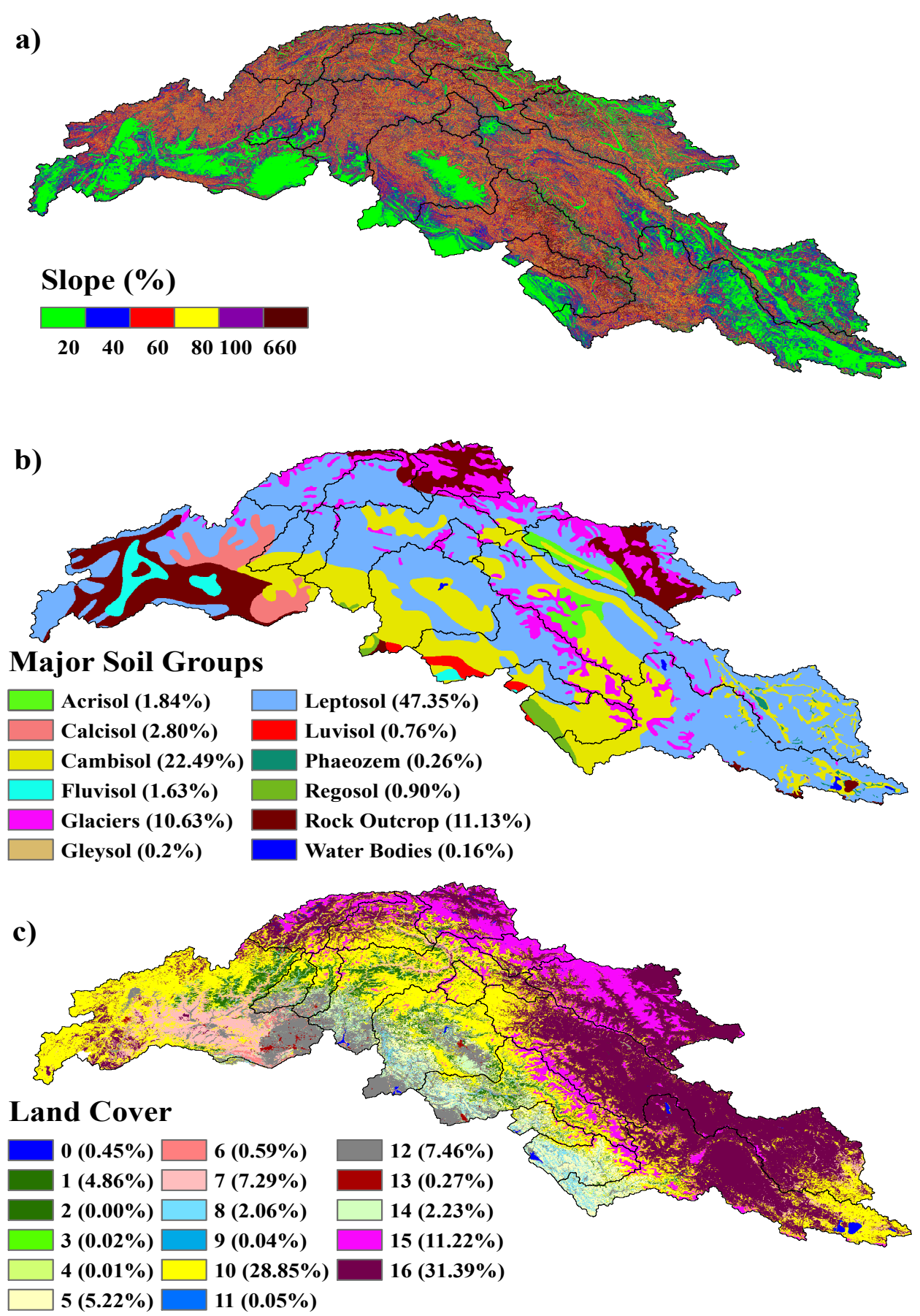

Figure S-3.6: Slope (a), major soil groups (b) and land cover (c) in the study area. The class numbers in bottom figure (c) refer to the major land cover types given in Table S-3.5. Soil types are derived from Fischer et al. (2008) dataset, while land cover types are derived from MODIS Land Cover type product (MCD12Q1; Friedl et al., 2010). 


\section{Supplementary Material - Chapter 4}

Table S-4.1: KGE, correlation, bias \& variability ratios at monthly scale in the study area

\begin{tabular}{|c|c|c|c|c|c|c|c|c|c|c|c|c|c|c|c|c|c|c|c|c|c|c|c|c|c|}
\hline \multirow{2}{*}{$\begin{array}{l}\text { S. } \\
\#\end{array}$} & \multirow{2}{*}{$\begin{array}{l}\text { Precipitation } \\
\text { Product }\end{array}$} & \multicolumn{12}{|c|}{ Kling-Gupta Efficiciency (KGE) } & \multicolumn{12}{|c|}{ Correlation (r) } \\
\hline & & Jan & Feb & Mar & Apr & May & Jun & Jul & Aug & Sep & Oct & Nov & Dec & Jan & Feb & Mar & Apr & r May & Jun & ul & Aug & Sep & Oct & Nov & \\
\hline 1 & CPCCY8 & 0.47 & 6 & 6 & 3 & 8 & 7 & 77 & 30 & 1 & 11 & 15 & 09 & 52 & 0.51 & 0.56 & .71 & 52 & .62 & 83 & 30.81 & 72 & 19 & 25 & ก? \\
\hline & & & 3 & 5 & 1 & 4 & 55 & 75 & 80 & 70 & 10 & 14 & 8 & 51 & 0.50 & 0.55 & 70 & 0.47 & 61 & .81 & 0.80 & 0.71 & 19 & 25 & \\
\hline & & & 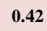 & 50 & .50 & 13 & 48 & 72 & .71 & .61 & 18 & 0.09 & 0.17 & .47 & 0.51 & 0.53 & 0.54 & 0.43 & 0.54 & 0.77 & $7 \quad 0.76$ & 0.65 & 20 & 24 & \\
\hline & & 0.32 & ( & 39 & 63 & & 53 & 62 & 0.60 & 53 & & & 0.02 & \begin{tabular}{|l|}
0.48 \\
\end{tabular} & 0.46 & 0.51 & 0.73 & $\begin{array}{ll}3 & 0.70\end{array}$ & 66 & 0.81 & 0.80 & 0.70 & 33 & 24 & \\
\hline & & 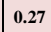 & 0.34 & 34 & 01 & 54 & 64 & 0.75 & 0.73 & 0.68 & 21 & 0.13 & 0.01 & \begin{tabular}{|l|}
0.44 \\
\end{tabular} & 0.46 & 0.51 & 0.72 & $\begin{array}{l}2 \\
0.73\end{array}$ & 0.67 & 0.87 & 7 0.84 & 0.82 & .39 & 27 & \\
\hline , & & 0.1 & 0.00 & 0.33 & 0.47 & .43 & 30 & 0.62 & 0.58 & 0.48 & 0.11 & 0.01 & -0.16 & \begin{tabular}{|l|}
0.18 \\
\end{tabular} & 0.34 & 0.42 & 0.56 & 60.44 & 0.47 & 0.74 & 0.72 & 0.63 & 0.23 & 18 & \\
\hline 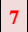 & 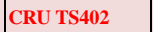 & 0.32 & 0.36 & 0.39 & 0.63 & 55 & 0.53 & 0.62 & 0.60 & 0.53 & 15 & 12 & 0.02 & \begin{tabular}{|l|}
0.48 \\
\end{tabular} & 0.46 & 0.51 & 0.73 & 30.70 & 0.66 & 0.81 & 0.80 & 0.70 & 0.33 & 0.24 & 2. \\
\hline 8 & 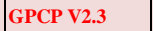 & 0.32 & 0.36 & 0.39 & 0.63 & 55 & 53 & 0.62 & 0.60 & 0.53 & 15 & 0.12 & 0.02 & \begin{tabular}{|l|}
0.48 \\
\end{tabular} & 0.46 & 0.51 & 0.73 & $\begin{array}{ll}3 & 0.70\end{array}$ & 0.66 & 0.81 & 0.80 & 0.70 & 0.33 & 0.24 & .21 \\
\hline 9 & CPC & 0.32 & 0.36 & 0.39 & 0.63 & 0.55 & 0.53 & 0.62 & 0.60 & 0.53 & 0.15 & 0.12 & 0.02 & \begin{tabular}{|l}
0.48 \\
\end{tabular} & 0.46 & 0.51 & 0.73 & $\begin{array}{ll}3 \quad 0.70 \\
\end{array}$ & 0.66 & 0.81 & 0.80 & 0.70 & 0.33 & 0.24 & 0.21 \\
\hline 1 & 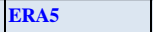 & 0 & 0.61 & 0.68 & 0.71 & 0.61 & 0.14 & 0.39 & 0.34 & 0.37 & 0.35 & 0.41 & 0.48 & .70 & 0.71 & 0.72 & 0.76 & 60.63 & 54 & 0.73 & 30.69 & 0.56 & 37 & 50 & 0.59 \\
\hline 2 & C & 5 & 0. & 0.46 & 0 & 0 & 0 & 0 & 0 & 6 & 4 & 14 & 0.44 & 8 & 0.61 & 52 & 0.42 & $\begin{array}{ll}2 & 0.38\end{array}$ & 14 & 0.62 & 0.56 & 0.39 & 36 & 50 & .58 \\
\hline 3 & J & 56 & 0.51 & 0.52 & 0.54 & 0 & 1 & 0 & 0 & 10 & 0.24 & 41 & 0.50 & \begin{tabular}{|l|}
0.67 \\
\end{tabular} & 0.66 & 0.61 & 67 & 70.58 & 40 & 0.68 & 0.58 & 0.30 & 0.29 & 0.52 & ( \\
\hline 4 & ERA & 0.24 & 0.27 & 0.13 & 0.27 & 0.21 & 0.16 & 0.44 & 0.25 & 0.24 & -0.07 & 0.26 & 0.15 & \begin{tabular}{|l|}
0.46 \\
\end{tabular} & 0.50 & 0.34 & 0.40 & 0.28 & 0.22 & 0.55 & 5 ( 0.49 & 0.27 & 0.07 & 0.36 & 0.38 \\
\hline 5 & MEI & 0.69 & 0.61 & 0.64 & 0.55 & 0.31 & -0.22 & -0.01 & -0.07 & 0.23 & 0.40 & 0.55 & 0.53 & \begin{tabular}{|l|}
0.71 \\
\end{tabular} & 0.68 & 0.65 & 0.63 & $\begin{array}{ll}3 & 0.53\end{array}$ & 0.26 & 0.42 & 0.40 & 0.36 & 0.48 & 0.56 & \\
\hline 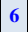 & ER $A$ & 0.51 & 0.52 & 0.53 & 0.45 & 0. & -0.55 & 0.08 & 0.02 & 0.22 & 0.20 & 0.28 & 0.36 & \begin{tabular}{|l|}
0.61 \\
\end{tabular} & 0.65 & 0.60 & 0.65 & 50.44 & 0.42 & 0.62 & 0.50 & 0.45 & 0.24 & 0.36 & \\
\hline 7 & DO & 01 & ( & -0.08 & -0. & -0.09 & 0.07 & 0.34 & 0.29 & 0.13 & -0.02 & 0.12 & 0.04 & \begin{tabular}{|l|}
0.25 \\
\end{tabular} & 0.34 & 0.17 & 0.12 & 0.05 & 0.19 & 0.50 & 0.42 & 0.26 & 0.26 & 0.55 & \\
\hline 8 & & 0. & 0. & o. & o. & & -1 & 0.20 & 0.27 & 0.17 & 0. & -0 . & 0. & 0.20 & 0.30 & 0.28 & 0.35 & 0 & & 0.39 & 0.48 & 0.48 & 0.17 & 17 & \\
\hline 9 & 2 & .26 & -0.17 & -0.16 & -0.04 & -0.20 & -0.54 & -0.10 & -0.4 & -0.42 & -0.38 & -0.55 & -0.09 & -0.03 & 0.07 & -0.11 & -0.03 & $3-0.15$ & -0.26 & 0.00 & 0.01 & -0.09 & -0.16 & 0.11 & \\
\hline 1 & & & 0 & 0 & 0 & 0 & 0 & 6 & 2 & 9 & & & 0.16 & \begin{tabular}{|l|}
0.50 \\
\end{tabular} & 0.47 & & 0 & 6 & & 74 & 0.71 & & & & \\
\hline 2 & 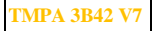 & & ( & 0 & 0 & & & & & & & & 0 & 0 & 55 & & 0 & 30 & & 1 & 37 & 79 & & & \\
\hline 3 & PF & & & 0 & & & & & & & & & & & & & & & & & & & & & \\
\hline 4 & $\mathrm{C}$ & & 0 & 0 & 0 & & & & 0 & 8 & & & 0 & & 4 & & 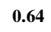 & 9 & & 7 & 44 & 65 & 10 & 3 & 0.31 \\
\hline 5 & $\mathrm{Cl}$ & 0 & 0 & 0 & 0 & & 0 & & 0 & 5 & 0 & 0 & -1 & 29 & 3 & 46 & 55 & 61 & 3 & 86 & 0.83 & 66 & 26 & 7 & 23 \\
\hline 6 & WI & 1 & 0 & 0.07 & 0 & 0 & 0 & 48 & 0 & 48 & 0.03 & 0.24 & -0.07 & .27 & 0.29 & 0.20 & 0.45 & 50.36 & 17 & 0.75 & 50.66 & 0.62 & 0.16 & 0.37 & 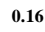 \\
\hline 7 & $\mathrm{CM}$ & 0.16 & 0.14 & 0.14 & 0.29 & -0.02 & 0.10 & 0.20 & 0.19 & 18 & 0.08 & 0.23 & 0.26 & .17 & 0.19 & 0.18 & 0.41 & 10.25 & 0.21 & 0.42 & 0.35 & 0.32 & 0.26 & 0.34 & 0.31 \\
\hline 8 & $\mathrm{CM}$ & .63 & -0.76 & -0.37 & -0.09 & 0.11 & 0.17 & 65 & 0.69 & 0.67 & 0.29 & 0.06 & -0.21 & 0.25 & 0.23 & 0.34 & 0.37 & 7 0.41 & 0.54 & 0.80 & 0.82 & 0.69 & 0.31 & 0.08 & 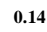 \\
\hline 9 & $\mathrm{CA}$ & 4 & .16 & 0.14 & 0.25 & -0.1 & -0.10 & 0.01 & 0.05 & 0.11 & -0.11 & 0.08 & 0.07 & \begin{tabular}{|l|l} 
\\
\end{tabular} & 0.47 & 0.44 & 0.58 & $\begin{array}{ll}3 & 0.20 \\
\end{array}$ & 0.13 & 0.28 & 0.22 & 0.22 & 0.18 & 0.32 & 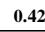 \\
\hline S & & & & & & & & & & & & & & & & & & & & a & & & & & \\
\hline \# & & & & & & & Jun & Jul & & & & & & & & & Apr & y & & ul & I Aug & Sep & ct & ov & \\
\hline 1 & & & & 0 & & & & & & & & & & & 0.80 & & & & & & 1 & & & & \\
\hline 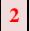 & & & & 69 & 0 & & & & & & & 0.66 & 0 & 86 & 0.77 & 1.04 & 3 & 2 & & 6 & 00 & 96 & 80 & 75 & \\
\hline 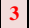 & & & 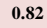 & 34 & & & & & & & & 0 & & .87 & 0.75 & & 0.99 & 0.97 & & 11 & 1.14 & 17 & 6 & & \\
\hline ( & & & & & & & & & & & & & & & & & & & & & 70.66 & 0 & 60 & 4 & \\
\hline 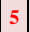 & & 57 & 68 & 0.56 & 76 & & 1 & 88 & 0.94 & 0.86 & 0.59 & 0.63 & 0.43 & \begin{tabular}{|l|}
0.81 \\
\end{tabular} & 0.81 & 0.93 & 0.86 & 60.72 & 91 & 0.81 & 0.79 & 0.78 & 0.72 & 71 & \\
\hline 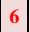 & & & & & & & & & & & & & & & 0.87 & & 0.94 & 40 & & 2 & 0.87 & 1.06 & 87 & 78 & \\
\hline 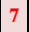 & & & & & & & & & & & & & 0. & \begin{tabular}{|l|}
0.76 \\
\end{tabular} & 0.73 & & 0.82 & 20.70 & & 0.67 & 0.66 & 0.66 & 0.60 & 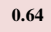 & \\
\hline 8 & & .64 & 0.77 & 0.73 & 0.84 & & & 1.04 & 1.05 & 1.12 & 0.67 & 0.73 & 0.54 & \begin{tabular}{|l|}
0.76 \\
\end{tabular} & 0.73 & 0.77 & 0.82 & 0.70 & 0.78 & 0.67 & 70.66 & 0.66 & 0.60 & 0.64 & 0 \\
\hline 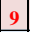 & & & & & & & & & & & & & & \begin{tabular}{|l}
0.76 \\
\end{tabular} & 0.73 & & 0.82 & $2 \quad 0.70$ & & 0.67 & $7 \quad 0.66$ & 0.66 & 60 & 0 & \\
\hline \begin{tabular}{|l|}
1 \\
\end{tabular} & & & & .00 & & & & 42 & & 27 & & & & .74 & 0.80 & & 95 & $5 \quad 1.09$ & & 64 & 56 & 64 & 34 & 77 & \\
\hline 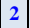 & & & & & & & & & & & & & & 0.65 & 0.75 & & 1.01 & 11 & & 0 & 20.84 & 86 & 0 & 8 & \\
\hline 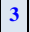 & & & & & & & & & & & & & & \begin{tabular}{|l|}
0.72 \\
\end{tabular} & 0.77 & & 0.80 & 0.74 & & 0.52 & 0.51 & 0.44 & 0.72 & 0.82 & \\
\hline 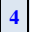 & & 5 & 0.72 & 0.58 & 0.72 & & 1.03 & 1.04 & 1.33 & 0.99 & 0.58 & 0.77 & 0.66 & \begin{tabular}{|l}
0.59 \\
\end{tabular} & 0.55 & 0.61 & 0.69 & 0.69 & 0.68 & 0.67 & 0.56 & 0.78 & 0.70 & 0.70 & 0. \\
\hline 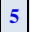 & & & & & & & & & & & & & & & 0.93 & & 1.02 & 20 & & 0.71 & 0.69 & 0 & 30 & 0 & \\
\hline 6 & 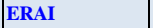 & & 1.23 & 1.17 & 38 & & 2.36 & 1.75 & 1.73 & 38 & 1.22 & 1.31 & & 0.71 & 0.76 & 0.83 & 0.83 & $\begin{array}{ll}3 & 0.68\end{array}$ & & 0.61 & 0.59 & 0.61 & 83 & 0.91 & \\
\hline 7 & & & 1.11 & 0.96 & 1.09 & & 1.00 & 0.77 & 1.06 & 1.09 & 0.95 & 1.02 & 0.83 & \begin{tabular}{|l|}
0.33 \\
\end{tabular} & 0.30 & 0.30 & 0.31 & 10.47 & 0.55 & 0.64 & $4 \quad 0.59$ & 0.56 & 0.30 & 0.40 & 10 \\
\hline 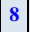 & & & & & & & & 1.06 & & & & & & \begin{tabular}{|l|}
0.88 \\
\end{tabular} & 0.84 & 0.74 & 0.65 & 50.67 & & 0.48 & 0.58 & 0.73 & 0.91 & 0.73 & 0. \\
\hline 9 & $20 C$ & .91 & 0.85 & 0.89 & 1.11 & 1.30 & 1.87 & 1.45 & 1.99 & 1.90 & 1.73 & 2.09 & 1.09 & \begin{tabular}{|l|}
0.28 \\
\end{tabular} & 0.30 & 0.66 & 0.97 & $7 \quad 1.12$ & 0.88 & 0.96 & $\begin{array}{ll}6.98 \\
6\end{array}$ & 1.07 & 0.80 & 0.36 & 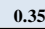 \\
\hline 1 & & & & & & & & & & & & & 0.6 & 0.95 & 1.00 & 1.17 & 1.11 & 10.97 & 1 & 0.90 & 0.90 & 0.96 & 0.97 & 1.21 & \\
\hline 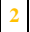 & & & & 0.51 & & & & & & 1.07 & & & 0.3 & \begin{tabular}{|l|}
0.74 \\
\end{tabular} & 0.73 & 0.86 & 0.84 & 40.76 & & 0.77 & 0.76 & 0.73 & 0 & 0.79 & \\
\hline 3 & PER & 0.61 & 0.74 & 0.49 & 0.64 & 0.71 & 1.27 & 1.17 & 1.09 & 1.03 & 0.62 & 0.53 & 0.38 & \begin{tabular}{|l|}
0.43 \\
\end{tabular} & 0.43 & 0.53 & 0.69 & 9.60 & 0.85 & 0.59 & 0.54 & 0.63 & 0.52 & 0.72 & 0. \\
\hline 4 & & & & & & & & & & & & & & \begin{tabular}{|l|}
0.73 \\
\end{tabular} & 0.74 & & 0.87 & $7 \quad 0.88$ & & 0.77 & $\begin{array}{ll}7 & 0.68\end{array}$ & 0.72 & 0.64 & 0.64 & \\
\hline 5 & $\mathrm{CHI}$ & 0.46 & 0.52 & 0.54 & 0.61 & & 0.97 & 0.77 & 0.75 & 0.93 & 0.59 & 0.47 & 0.43 & 0.82 & 0.81 & 0.79 & 1.15 & $\begin{array}{ll}5.17\end{array}$ & 1.09 & 0.86 & 0.88 & 0.94 & 0.86 & 91 & 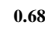 \\
\hline 6 & & & & 0.69 & & & & 0.92 & & 1.07 & & & 0.47 & 0.58 & 0.67 & 0.63 & 0.81 & 10.28 & 0.62 & 0.55 & 50.51 & 0.65 & 0.61 & 0.67 & 0 \\
\hline 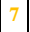 & & & & & & & & & & & & & 1.2 & 0.93 & 0.72 & & 0.69 & 9 0.41 & & 0.54 & 0.55 & 0.58 & 0.55 & 0.62 & 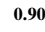 \\
\hline 8 & CMOR & 0.14 & 0.12 & 0.25 & 0.63 & 1.37 & 1.69 & 0.78 & 0.83 & 0.99 & 1.17 & 0.86 & 0.33 & 2.16 & 2.31 & 1.94 & 1.81 & 11.55 & 1.06 & 0.82 & 0.81 & 0.90 & 1.07 & 1.06 & 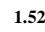 \\
\hline 9 & & 0.39 & 0.50 & 0.44 & 0.42 & 0.53 & 1.56 & 1.53 & 1.36 & 1.30 & 0.52 & 0.42 & 0.37 & 0.53 & 0.58 & 0.66 & 0.77 & $7 \quad 0.41$ & 0.63 & 0.58 & 0.61 & 0.68 & 0.43 & 0.78 & 0.62 \\
\hline
\end{tabular}


Table S-4.2: KGE, correlation, bias and variability ratios for various sub-regions

\begin{tabular}{|c|c|c|c|c|c|c|c|c|c|c|c|c|c|c|c|c|c|c|c|c|c|c|c|c|c|}
\hline \multirow{2}{*}{$\begin{array}{l}\text { S. } \\
\#\end{array}$} & \multirow{2}{*}{$\begin{array}{l}\text { Precipitation } \\
\text { Product }\end{array}$} & \multicolumn{4}{|c|}{ Study Area } & \multicolumn{4}{|c|}{ W-Himalaya } & \multicolumn{4}{|c|}{ Karakoram } & \multicolumn{4}{|c|}{ Kharmong } & \multicolumn{4}{|c|}{ NE-Hindukush } & \multicolumn{4}{|c|}{ SW-Hindukush } \\
\hline & & KGE & $\mathbf{r}$ & $\beta$ & $v$ & GE & $\mathbf{r}$ & $\beta$ & $v$ & KGE & $r$ & $\beta$ & $v$ & KGE & $\mathbf{r}$ & $\beta$ & $v$ & KGE & $\mathbf{r}$ & $\beta$ & $v$ & KGE & $\mathbf{r}$ & $\beta$ & $\gamma$ \\
\hline 1 & GPCC V8 & & & 0.88 & 1.12 & & 0.83 & 0.85 & 1.22 & -0.01 & 0.33 & 0.26 & 0.91 & .71 & 0.72 & 1.05 & 1.00 & 41 & 0.61 & 0.67 & 1.30 & 58 & .76 & 1.24 & \\
\hline 2 & $r$ & or & 1 & 0.88 & & 0 & 0.80 & 0.84 & 1.17 & 81 & 0.32 & 0.26 & 0.90 & 67 & 0.69 & 1.11 & & .41 & 0.60 & 0.66 & 1.28 & 0.58 & 0.76 & 1.23 & \\
\hline 3 & DE & 63 & 67 & 0.90 & & 66 & 0.75 & 0.90 & 2 & 01 & 0. & 6 & & 45 & 0.65 & 1.39 & 0.84 & .30 & 0.58 & 0.58 & 37 & 0.76 & 81 & 1.07 & 0.87 \\
\hline 4 & PH & .62 & .70 & 0.85 & 0.81 & 62 & 0.74 & 0.74 & 0.89 & -0.18 & -0.01 & 0.57 & 0.57 & 0.29 & 0.65 & 1.53 & 0.0 & 0.42 & 0.59 & 0.64 & 1.21 & 0.51 & 0.74 & 1.25 & 0 \\
\hline 5 & PF & .61 & .73 & 0.73 & 0.9 & 59 & 0.78 & 0.66 & 1.04 & -0.05 & 0.25 & 0.38 & 0.58 & 0.47 & 0.60 & 0.94 & 0.0 & 0.40 & 0.59 & 0.63 & 1.24 & 0.57 & 0.75 & 1.20 & 0 \\
\hline 6 & RE & .56 & o & 0.90 & 1.15 & 59 & 0.69 & 0.85 & 1.23 & -0.07 & 0.31 & 0.21 & 0.81 & 0.33 & 0.36 & 1.18 & 0.0 & 0.28 & 0.52 & 0.63 & 1.39 & 0.68 & 0.73 & 1.06 & . \\
\hline 7 & RI & .47 & .61 & 0.76 & 0.74 & 0.43 & 0.64 & 0.56 & 0.93 & 0.05 & 0.33 & 0.37 & 0.77 & 0.09 & 0.28 & 1.20 & 0.4 & 0.52 & 0.62 & 0.74 & 0.85 & 0.43 & 0.78 & 1.38 & \\
\hline 8 & GP & 0.43 & 0.51 & 0.81 & 0 & 0.41 & 0.55 & 0.62 & 0 . & 0.02 & 4 & 0.56 & 0.81 & -0.13 & 0.18 & 1.72 & 8 & 0.46 & 1 & 0.63 & 94 & 0.53 & 65 & 03 & \\
\hline 9 & CPC & 0.41 & 0.65 & 0.54 & 1. & 0.37 & 0.65 & 0.51 & 1.17 & -0.04 & 5 & 0.27 & 0.63 & 0.41 & 0.57 & 0.64 & 0.83 & 0.28 & 0.59 & 0.43 & 1.14 & 0.45 & 0.65 & .90 & \\
\hline 1 & ERA5 & 99 & 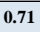 & 19 & 0 & 61 & 0.64 & 4 & 0 & 0 & 7 & 0. & 0 & 0.22 & 5 & 1.63 & 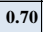 & 0.47 & 3 & 1.18 & 0.85 & 0.33 & 59 & 53 & 0.92 \\
\hline 2 & CFSR & 52 & o. & 1.11 & 0.83 & 5 & 0.49 & 0.81 & 1.10 & 4 & 4 & 1.25 & 0.79 & -0.21 & 0.59 & 2.07 & 0 & 0.50 & 0.51 & 1.01 & 1.10 & 0.51 & 0.58 & 12 & 1.23 \\
\hline 3 & JRA-55 & 0.47 & 0.64 & 1.19 & 0.66 & 0.57 & 0.64 & 0.83 & 0.83 & 0.39 & 0.54 & 1.34 & 0.78 & -0.20 & 0.63 & 2.09 & 0.65 & 0.42 & 0.47 & 1.07 & 0.78 & 0.38 & 0.64 & 1.47 & - \\
\hline 4 & ERA & 0.44 & 0.49 & 0.84 & 0.82 & 0.43 & 0.61 & 0.58 & 1.00 & 0.10 & 0.23 & 0.66 & 0.70 & -0.24 & 0.43 & 2.01 & 0.56 & 0.37 & 0.50 & 0.63 & 0.93 & 0.61 & 0.72 & 0.73 & 1. \\
\hline 5 & MER & 0.42 & 0.53 & 1.33 & 0.94 & 0.47 & 0.48 & 1.07 & 1.05 & 0.56 & 0.59 & 1.15 & 0.92 & -0.02 & 0.53 & 1.88 & 0.82 & 0.38 & 0.43 & 1.25 & 1.00 & 0.34 & 0.47 & 1.39 & 10 \\
\hline 6 & ERAI & 0.38 & 0.59 & 1.40 & 0.77 & 0.61 & 0.67 & 1.02 & 0.79 & 0.24 & 0.32 & 0.87 & 0.69 & -0.20 & 0.65 & 2.10 & 0.65 & 0.17 & 0.42 & 1.59 & 0.92 & -0.05 & 0.54 & 1.94 & 10 \\
\hline 7 & DOE R2 & 0.31 & 0.46 & 1.00 & 0.57 & 0.41 & 0.64 & 0.61 & 0.73 & 0.18 & 0.22 & 0.82 & 0.81 & -0.88 & 0.31 & 2.66 & 0.45 & 0.42 & 0.54 & 0.71 & 0.80 & 0.43 & 0.66 & 1.23 & 0.62 \\
\hline 8 & NCA & 0.26 & 0.40 & 1.33 & 0.70 & 0.40 & 0.47 & 0.95 & 0.71 & 0.04 & 0.20 & 0.49 & 1.14 & -1.17 & 0.31 & 3.01 & 0.58 & 0.27 & 0.31 & 1.18 & 1.17 & -0.11 & 0.44 & 1.93 & 0.77 \\
\hline 9 & $20 \mathrm{Cl}$ & .10 & 0.04 & 1.33 & 1.04 & 0.26 & 0.45 & 0.52 & 1.11 & -0.35 & -0 & 1.69 & 0.61 & -2.91 & -0.06 & 4.76 & 0.76 & 0.23 & 0.32 & 0.65 & 1.09 & 0.51 & 0.56 & 0.86 & 1. \\
\hline 1 & IS & 0.63 & & 8 & & 1 & 0 & 0 & & 3 & 0 & 0.39 & & 0.57 & 0.63 & 0.94 & 0. & 0.57 & 0.58 & 1.06 & 0.94 & 05 & 0.46 & 1.77 & \\
\hline 2 & TMPA: & 0.63 & 0 & 0 . & 0.9 & 3 & 0.78 & 0.71 & & 0.05 & 0.36 & 0.39 & 0.66 & 0.33 & 0.44 & 1.03 & $0.0 \mathrm{~S}$ & 0.39 & 0.56 & 0.61 & 1.15 & 0.69 & 0.77 & 0.92 & \\
\hline 3 & PERS-CDR V1R & 0.56 & 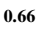 & 78 & 0.82 & 53 & 0.67 & 0.66 & 1.00 & 0.15 & 0.37 & 0.49 & & 0.15 & 0.29 & 1.33 & 0.68 & 0.47 & 0.56 & 0.70 & 0.94 & 0.63 & 0.75 & 1.05 & \\
\hline 4 & CHEI & 0.56 & 0 & .74 & 0.83 & 0.52 & 0.70 & 0.66 & 0.84 & -0.05 & 0.18 & 0.36 & 0.82 & 0.57 & 0.72 & 1.14 & 0.70 & 0.41 & 0.54 & 0.63 & 1.03 & 0.68 & 0.72 & 1.01 & 0.8 \\
\hline 5 & $\mathrm{~V} 2.0$ & 0.51 & $0.0 \%$ & $0.0 \mathrm{~J}$ & 1.00 & 0.42 & 0.71 & 0.53 & 1.19 & 0.06 & 0.33 & 0.35 & & 0.28 & 0.34 & 0.82 & 0.77 & 0.36 & 0.48 & 0.69 & 1.21 & 0.86 & 0.86 & 0.96 & 0.97 \\
\hline 6 & WFDEI & 0. & 0.57 & 0.82 & 0.69 & 0.42 & 0.65 & 0.55 & 0.91 & 0.09 & 0.33 & 0.42 & 0.80 & 0.14 & 0.38 & 1.23 & 0.4 & 0.53 & 0.62 & 0.81 & 0.81 & 0.02 & 0.69 & 1.81 & 0.5 \\
\hline 7 & CMAP & 0.36 & 40 & 1.03 & 0.78 & 0.39 & 0.51 & 0.64 & 0.93 & 0.14 & 0.19 & 1.26 & 0.8 & -0.79 & 0.52 & 2.69 & 0.6 & 0.50 & 0.57 & 0.76 & 0.93 & 0.48 & 0.59 & 1.04 & 0.6 \\
\hline 8 & CMORI & 0.30 & 44 & 0.64 & 1.24 & 0.33 & 0.42 & 0.69 & 1.10 & -0.46 & -0.01 & 0.18 & 1. & -0.35 & -0.24 & 0.51 & 0.7 & -0.14 & 0.04 & 0.70 & 1.53 & 0.25 & 0.28 & 1.03 & 1.23 \\
\hline 9 & CAMSOPI & .25 & 0.29 & 0.78 & 1.07 & 0.30 & 0 & 0.51 & 1.10 & -1 & -0 & 0.62 & 1 & \begin{tabular}{|l|l|} 
& -0.22 \\
\end{tabular} & 0 & 2 & 0.91 & 0.37 & 2 & 0.53 & 32 & 0.40 & 52 & 0.85 & 0.68 \\
\hline
\end{tabular}
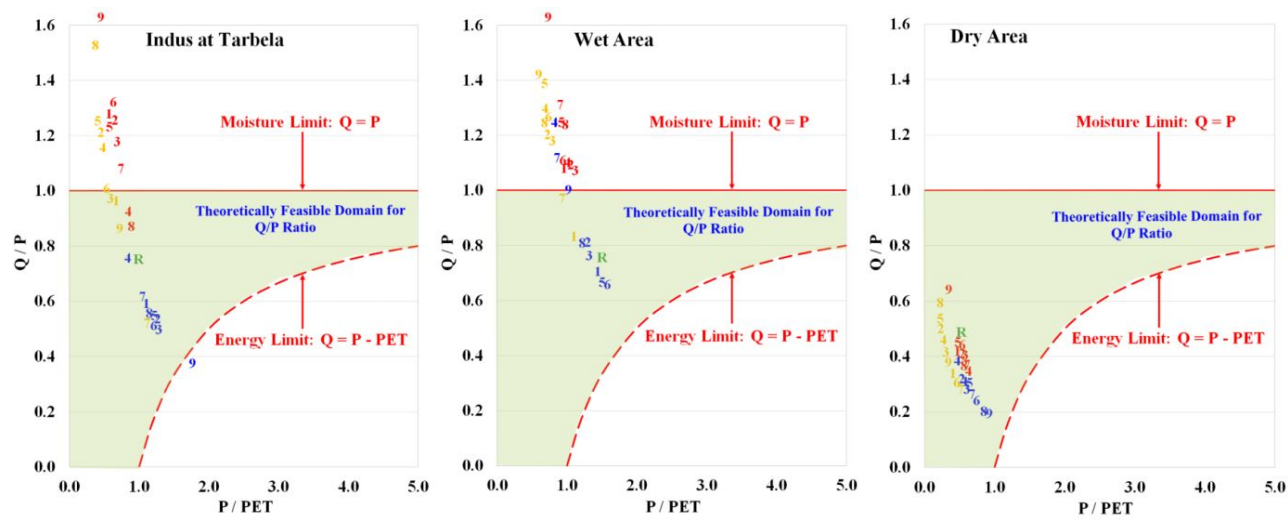

Figure S-4.1: Turc-Budyko representation of gridded precipitation datasets for three regions. Indus at Tarbela includes Khamong, Shigar, Shyok, Hunza, Gilgit \& Astore sub-basins and the catchment area of Indus main upstream of Tarbela dam. Wet area includes W-Himalaya, NE-Hindukush and Karakoram regions, and Dry area consists of SW-Hindukush and Kharmong regions. The colors and numbers in the diagram are same as in Figure 7. 
Table S-4.3: Skill scores and final rankings of gridded precipitation datasets for three regions described in Figure S-1. The colours, numbers and rankings are the same as in Table 4.

\begin{tabular}{|c|c|c|c|c|c|c|}
\hline $\mathbf{R}$ & \multicolumn{2}{|c|}{ Indus at Tarbela } & \multicolumn{2}{|c|}{ Wet Areas } & \multicolumn{2}{|c|}{ Dry Areas } \\
\hline$\#$ & Dataset & SS & Dataset & SS & Dataset & SS \\
\hline 1 & ERA5 & 9.99 & ERA5 & 10.6 & UDEL V5.01 & 7.58 \\
\hline 2 & CFSR & 9.19 & JRA-55 & 9.58 & CHELSA V1.2 & 7.13 \\
\hline 3 & MSWEP V2.2 & 9.08 & CFSR & 8.89 & CHIRPS V2.0 & 6.91 \\
\hline 4 & DOE R2 & 9.00 & MSWEP V2.2 & 8.75 & GPCC V8 & 6.84 \\
\hline 5 & JRA-55 & 9.00 & MERRA-2 & 8.51 & GPCC V7 & 6.79 \\
\hline 6 & CMAP & 8.81 & CMAP & 8.03 & PREC Land & 6.38 \\
\hline 7 & MERRA-2 & 8.44 & ERAI & 7.07 & APH.V1101 & 6.36 \\
\hline 8 & PERS-CDR V1R1 & 8.07 & NCAR R1 & 5.89 & APH.V1801R1 & 6.08 \\
\hline 9 & ERA 20C & 7.97 & & & TMPA 3B42 V7 & 6.00 \\
\hline 10 & APH.V1801R1 & 7.75 & & & CFSR & 5.80 \\
\hline 11 & GPCP V2.3 & 7.49 & & & PERS-CDR V1R1 & 5.71 \\
\hline 12 & NCAR R1 & 6.29 & & & DOE R2 & 5.62 \\
\hline 13 & ERAI & 6.01 & & & ERA $20 \mathrm{C}$ & 5.61 \\
\hline 14 & CAMSOPI & 5.87 & & & CPC Unified & 5.59 \\
\hline 15 & & & & & CRU TS402 & 5.47 \\
\hline 16 & & & & & CMAP & 5.45 \\
\hline 17 & & & & & GPCP V2.3 & 5.35 \\
\hline 18 & & & & & ERA5 & 5.08 \\
\hline 19 & & & & & MERRA-2 & 4.87 \\
\hline 20 & & & & & JRA-55 & 4.80 \\
\hline 21 & & & & & MSWEP V2.2 & 4.71 \\
\hline 22 & & & & & CAMSOPI & 4.49 \\
\hline 23 & & & & & WFDEI-CRU & 4.29 \\
\hline 24 & & & & & CMORPH V1.0 & 4.17 \\
\hline 25 & & & & & NCAR R1 & 3.17 \\
\hline 26 & & & & & ERAI & 3.02 \\
\hline 27 & & & & & 20CR V2C & 2.93 \\
\hline
\end{tabular}




\section{Supplementary Material - Chapter 5}

Table S-5.1: Multiple regressions for maximum and minimum temperatures for the three zones. Tx 1-12 and Tn 1-12 refer to the calendar months for maximum and minimum temperatures respectively. E denotes elevation $(\mathrm{m})$ and $\mathrm{L}$ represents latitude (decimal degrees) of the meteorological stations. $\mathrm{R}^{2}$ is the combined correlation of temperature with $\mathrm{E}$ and $\mathrm{L}$.

\begin{tabular}{|c|c|c|c|}
\hline Regression Equation for $T_{x}$ & $\mathbf{R}^{2}(\%)$ & Regression Equation for $T_{n}$ & $\mathbf{R}^{2}(\%)$ \\
\hline \multicolumn{4}{|c|}{ Northern Zone } \\
\hline $\mathrm{Tx} 1=19.4-0.00763 \mathrm{E}+0.059 \mathrm{~L}$ & 98.9 & $\operatorname{Tn} 1=21.5-0.00632 \mathrm{E}-0.344 \mathrm{~L}$ & 95.2 \\
\hline $\mathrm{T} \times 2=12.7-0.00791 \mathrm{E}+0.325 \mathrm{~L}$ & 98.9 & $\operatorname{Tn} 2=21.1-0.00673 \mathrm{E}-0.241 \mathrm{~L}$ & 96.6 \\
\hline $\mathrm{Tx} 3=6.3-0.00853 \mathrm{E}+0.705 \mathrm{~L}$ & 98.5 & $\operatorname{Tn} 3=14.0-0.00737 \mathrm{E}+0.125 \mathrm{~L}$ & 97.0 \\
\hline $\mathrm{Tx} 4=24.2-0.00867 \mathrm{E}+0.358 \mathrm{~L}$ & 98.7 & $\operatorname{Tn} 4=33.3-0.00719 \mathrm{E}-0.303 \mathrm{~L}$ & 97.5 \\
\hline $\mathrm{Tx} 5=39.7-0.00902 \mathrm{E}+0.103 \mathrm{~L}$ & 98.7 & $\operatorname{Tn} 5=31.8-0.00735 \mathrm{E}-0.125 \mathrm{~L}$ & 97.1 \\
\hline Tx6 $=48.9-0.00914 \mathrm{E}-0.031 \mathrm{~L}$ & 98.7 & $\operatorname{Tn} 6=30.9-0.00730 \mathrm{E}-0.003 \mathrm{~L}$ & 95.4 \\
\hline $\mathrm{Tx} 7=43.6-0.00875 \mathrm{E}+0.152 \mathrm{~L}$ & 98.6 & $\operatorname{Tn} 7=50.4-0.00676 \mathrm{E}-0.519 \mathrm{~L}$ & 94.9 \\
\hline $\mathrm{Tx} 8=42.6-0.00849 \mathrm{E}+0.148 \mathrm{~L}$ & 99.0 & $\operatorname{Tn} 8=53.2-0.00656 \mathrm{E}-0.622 \mathrm{~L}$ & 95.3 \\
\hline $\mathrm{Tx} 9=59.5-0.00835 \mathrm{E}-0.443 \mathrm{~L}$ & 98.3 & $\operatorname{Tn} 9=49.6-0.00636 \mathrm{E}-0.650 \mathrm{~L}$ & 93.9 \\
\hline $\mathrm{Tx} 10=61.0-0.00843 \mathrm{E}-0.635 \mathrm{~L}$ & 98.9 & $\operatorname{Tn} 10=36.6-0.00638 \mathrm{E}-0.439 \mathrm{~L}$ & 94.1 \\
\hline $\mathrm{Tx} 11=49.4-0.00785 \mathrm{E}-0.536 \mathrm{~L}$ & 98.4 & $\mathrm{Tn} 11=31.8-0.00607 \mathrm{E}-0.471 \mathrm{~L}$ & 93.1 \\
\hline $\mathrm{Tx} 12=31.0-0.00757 \mathrm{E}-0.204 \mathrm{~L}$ & 98.5 & $\mathrm{Tn} 12=29.8-0.00605 \mathrm{E}-0.542 \mathrm{~L}$ & 93.5 \\
\hline \multicolumn{4}{|c|}{ Southern Zone } \\
\hline $\mathrm{Tx} 1=33.4-0.00696 \mathrm{E}-0.361 \mathrm{~L}$ & 97.1 & $\operatorname{Tn} 1=27.5-0.00508 \mathrm{E}-0.614 \mathrm{~L}$ & 87.2 \\
\hline $\mathrm{Tx} 2=52.0-0.00700 \mathrm{E}-0.856 \mathrm{~L}$ & 98.5 & $\operatorname{Tn} 2=35.9-0.00549 \mathrm{E}-0.781 \mathrm{~L}$ & 91.9 \\
\hline $\mathrm{Tx} 3=58.3-0.00707 \mathrm{E}-0.887 \mathrm{~L}$ & 97.3 & $\operatorname{Tn} 3=49.6-0.00548 \mathrm{E}-1.05 \mathrm{~L}$ & 93.0 \\
\hline $\mathrm{Tx} 4=65.7-0.00748 \mathrm{E}-0.927 \mathrm{~L}$ & 97.7 & $\operatorname{Tn} 4=60.9-0.00558 \mathrm{E}-1.25 \mathrm{~L}$ & 93.6 \\
\hline $\mathrm{Tx} 5=63.7-0.00796 \mathrm{E}-0.691 \mathrm{~L}$ & 97.9 & $\operatorname{Tn} 5=59.3-0.00591 \mathrm{E}-1.06 \mathrm{~L}$ & 93.8 \\
\hline Tx6 $=24.8-0.00854 \mathrm{E}+0.552 \mathrm{~L}$ & 98.2 & $\operatorname{Tn} 6=56.0-0.00604 \mathrm{E}-0.854 \mathrm{~L}$ & 95.6 \\
\hline $\mathrm{Tx} 7=3.3-0.00739 \mathrm{E}+1.08 \mathrm{~L}$ & 96.4 & $\operatorname{Tn} 7=36.8-0.00583 \mathrm{E}-0.273 \mathrm{~L}$ & 97.4 \\
\hline $\mathrm{Tx} 8=8.7-0.00699 \mathrm{E}+0.869 \mathrm{~L}$ & 96.4 & $\operatorname{Tn} 8=38.3-0.00578 \mathrm{E}-0.333 \mathrm{~L}$ & 98.0 \\
\hline $\mathrm{Tx} 9=17.7-0.00749 \mathrm{E}+0.595 \mathrm{~L}$ & 98.2 & $\operatorname{Tn} 9=60.2-0.00533 \mathrm{E}-1.08 \mathrm{~L}$ & 96.8 \\
\hline $\mathrm{Tx} 10=32.4-0.00778 \mathrm{E}+0.080 \mathrm{~L}$ & 98.6 & $\operatorname{Tn} 10=60.8-0.00513 \mathrm{E}-1.26 \mathrm{~L}$ & 91.8 \\
\hline $\mathrm{Tx} 11=49.7-0.00719 \mathrm{E}-0.606 \mathrm{~L}$ & 97.7 & $\mathrm{Tn} 11=45.1-0.00519 \mathrm{E}-0.941 \mathrm{~L}$ & 89.4 \\
\hline $\mathrm{Tx} 12=44.0-0.00693 \mathrm{E}-0.600 \mathrm{~L}$ & 97.0 & $\mathrm{Tn} 12=24.4-0.00523 \mathrm{E}-0.460 \mathrm{~L}$ & 90.1 \\
\hline \multicolumn{4}{|c|}{ Western Zone } \\
\hline $\mathrm{Tx} 1=17.6-0.00805 \mathrm{E}+0.109 \mathrm{~L}$ & 95.2 & $\operatorname{Tn} 1=-14.2-0.00686 \mathrm{E}+0.652 \mathrm{~L}$ & 94.4 \\
\hline $\mathrm{Tx} 2=24.3-0.00799 \mathrm{E}-0.056 \mathrm{~L}$ & 95.9 & $\operatorname{Tn} 2=-7.5-0.00708 \mathrm{E}+0.511 \mathrm{~L}$ & 96.6 \\
\hline $\mathrm{Tx} 3=7.3-0.00873 \mathrm{E}+0.618 \mathrm{~L}$ & 94.7 & $\operatorname{Tn} 3=6.3-0.00737 \mathrm{E}+0.258 \mathrm{~L}$ & 93.5 \\
\hline $\mathrm{T} \times 4=17.7-0.00904 \mathrm{E}+0.505 \mathrm{~L}$ & 96.3 & $\mathrm{Tn} 4=27.2-0.00700 \mathrm{E}-0.206 \mathrm{~L}$ & 93.7 \\
\hline $\mathrm{Tx} 5=31.1-0.00942 \mathrm{E}+0.309 \mathrm{~L}$ & 94.6 & $\operatorname{Tn} 5=33.6-0.00732 \mathrm{E}-0.247 \mathrm{~L}$ & 91.6 \\
\hline Тх6 $=17.0-0.00922 \mathrm{E}+0.831 \mathrm{~L}$ & 95.0 & $\operatorname{Tn} 6=48.6-0.00722 \mathrm{E}-0.574 \mathrm{~L}$ & 92.3 \\
\hline $\mathrm{Tx} 7=-14.8-0.00850 \mathrm{E}+1.73 \mathrm{~L}$ & 90.8 & $\operatorname{Tn} 7=31.4-0.00703 \mathrm{E}-0.022 \mathrm{~L}$ & 93.6 \\
\hline $\mathrm{Tx} 8=-17.0-0.00813 \mathrm{E}+1.73 \mathrm{~L}$ & 85.0 & $\operatorname{Tn} 8=24.5-0.00680 \mathrm{E}+0.137 \mathrm{~L}$ & 90.2 \\
\hline $\mathrm{Tx} 9=-5.1-0.00895 \mathrm{E}+1.36 \mathrm{~L}$ & 95.1 & $\operatorname{Tn} 9=19.6-0.00667 \mathrm{E}+0.160 \mathrm{~L}$ & 90.5 \\
\hline $\operatorname{Tx} 10=4.1-0.00900 \mathrm{E}+0.967 \mathrm{~L}$ & 96.1 & $\operatorname{Tn} 10=17.0-0.00643 \mathrm{E}+0.055 \mathrm{~L}$ & 90.6 \\
\hline $\mathrm{Tx} 11=20.0-0.00792 \mathrm{E}+0.269 \mathrm{~L}$ & 96.0 & $\operatorname{Tn} 11=5.3-0.00604 \mathrm{E}+0.215 \mathrm{~L}$ & 85.7 \\
\hline $\mathrm{Tx} 12=26.3-0.00779 \mathrm{E}-0.076 \mathrm{~L}$ & 94.7 & $\operatorname{Tn} 12=-16.5-0.00642 \mathrm{E}+0.742 \mathrm{~L}$ & 86.7 \\
\hline
\end{tabular}




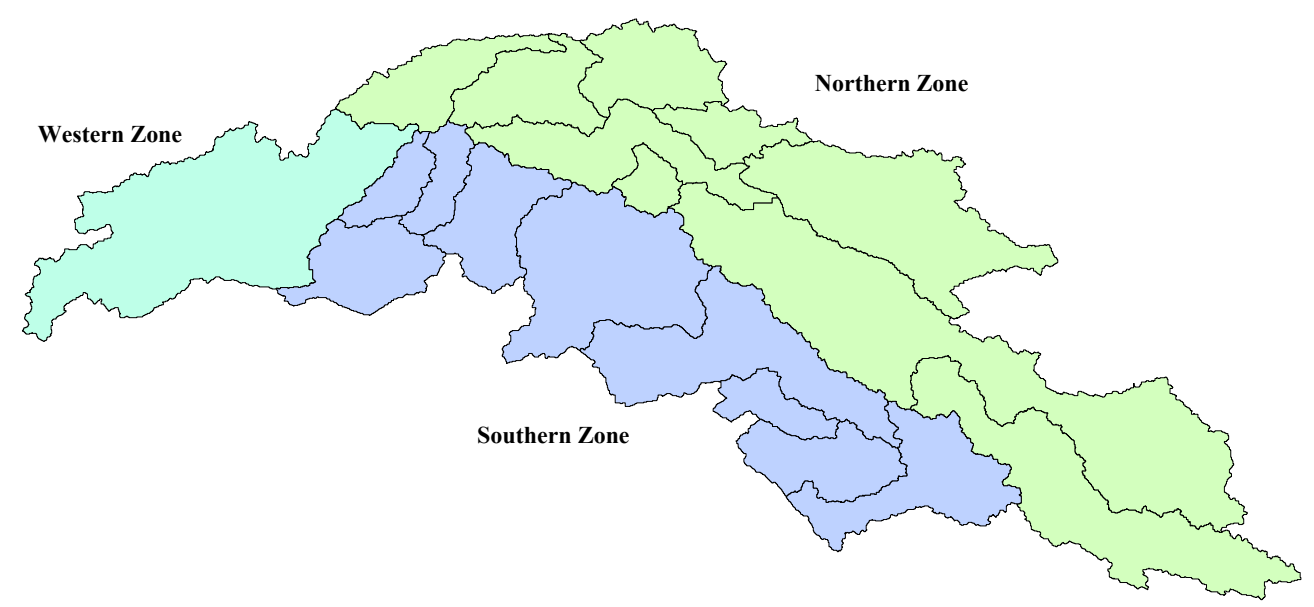

Figure S-5.1: Three zones assumed for calculation of temperature lapse rates 


\section{References}

Aaltonen A, Elomaa E, Tuominen A, Valkovuori P. 1993. Measurement of precipitation, in: Proceedings of the Symposium on Precipitation and Evaporation, edited by: Sevruk B, Lapi M. Slovak Hydrometeorlogical Institute and Swiss Federal Institute of Technology, Bratislava, Slovakia, 42-46.

Ackere SV, Eetvelde GV, Schillebeeckx D, Papa E, Wyngene KV, Vandevelde L. 2015. Wind Resource Mapping Using Landscape Roughness and Spatial Interpolation Methods, Energies 2015, 8, 8682-8703; doi:10.3390/en8088682.

Adam JC, Clark EA, Lettenmaier DP, Wood EF. 2006. Correction of global precipitation products for orographic effects, J. Climate, 19(1), 15-38.

Adam JC, Clark EA, Lettenmaier DP, Wood EF. 2006. Correction of global precipitation products for orographic effects, J. Climate, 19(1), 15-38.

Adam JC, Lettenmaier DP. 2003. Adjustment of global gridded precipitation for systematic bias. J. Geophys. Res.,108, 4257, doi:10.1029/2002JD002499.

ADB. 2010. Technical Assistance Consultant's Report, Part 2 - Main Report, Project Number: 43169 , INDIA: Integrated Water Resources Management Scoping Study for Sutlej River Basin, Himachal Pradesh: Improving Capacity for Climate Change Adaptation.

Adeloye AJ, Remesan R, Soundharajan BS. 2016. Effect of Hedging-Integrated Rule Curves on the Performance of the Pong Reservoir (India) During Scenario-Neutral Climate Change Perturbations, Water Resour Manage (2016) 30:445-470, DOI 10.1007/s11269-015-1171-z.

Adler, R. F., Sapiano, M. R. P., Huffman, G. J., Wang, J.-J., Gu, G., Bolvin, D., Chiu, L., Schneider, U., Becker, A., Nelkin, E., Xie, P., Ferraro, R., and Shin, D.-B. (2018) The Global Precipitation Climatology Project (GPCP) monthly analysis (new version 2.3) and a review of 2017 global precipitation, Atmosphere, 9, 138, https://doi.org/10.3390/atmos9040138.

Adler, R., Sapiano, M., Huffman, G., Bolvin, D., Wang, J., Gu, G., Nelkin, E., Xie, P., Chiu, L., Ferraro, R., et al. (2016) New Global Precipitation Climatology Project monthly analysis product corrects satellite data shifts, GEWEX News, 26, 7-9.

Aghakouchak, A., Mehran, A., Norouzi, H. and Behrangi, A. (2012) Systematic and random error components in satellite precipitation data sets. Geophysical Research Letters, 39(9), 3-6, L09406, doi:10.1029/2012GL051592.

Ahmed, K., Shahid, S., Wang, X., Nawaz, N. and Najeebullah, K. (2019) Evaluation of gridded precipitation datasets over arid regions of Pakistan. Water, 11, 210.

Akhtar M, Ahmad N, Booij MJ. 2008. The impact of climate changes on the water re- sources of Hindukush-Karakorum-Himalaya region under different glacier coverage scenarios. J. Hydrol. 355, 148-163. http://dx.doi.org/10.1016/j.jhydrol.2008.03.015.

Ali, G., Rasul, G., Mahmood, T., Zaman, Q. and Cheema, S.B. (2012) Validation of APHRODITE precipitation data for humid and sub humid regions of Pakistan. Pakistan Journal of Meteorology, 9(17), 57-69.

Ali, J., Syed, K.H., Gabriel, H.F., et al., 2018. Earth Syst. Environ. 2, 437-454. https://doi.org/10.1007/s41748-018-0064-8.

Ali, S., Li, D., Congbin, F., Khan, F., 2015. Twenty first century climatic and hydrological changes over Upper Indus Basin of Himalayan region of Pakistan. Environ. Res. Lett. 10, 014007.

Andermann, C., Bonnet, S. and Gloaguen, R. (2011) Evaluation of precipitation data sets along the Himalayan front, Geochemistry, Geophys. Geosystems, 12(7), 1-16, doi:10.1029/2011GC003513.

Anders, A.M., Roe, G.H., Hallet, B., Montgomery, D.R., Finnegan, N.J. and Putkonen, J. (2006) Spatial patterns of precipitation and topography in the Himalaya. In Willett, S.D., Hovius, N., Brandon, M.T. and Fisher, D. eds., Tectonics, Climate, and Landscape Evolution: Geological Society of America Special Paper 398, p. 39-53, doi: 10.1130/2006.2398(03).

Andreadis K, Storck P, Lettenmaier DP (2009) Modeling snow accumulation and ablation processes in forested environments. Water Resour Res 45:W05429. doi:10.1029/2008WR007042. 
Andréassian, V. and Perrin, C. (2012) On the ambiguous interpretation of the Turc-Budyko nondimensional graph. Water Resour. Res., 48, W10601, doi:10.1029/2012WR012532.

Anjum M.N., Ding, Y., Shangguan, D., Ahmad, I., Ijaz, M.W., Farid, H.U., Yagoub, Y.E., Zaman, M. and Adnan, M. (2018) Performance evaluation of latest integrated multi-satellite retrievals for Global Precipitation Measurement (IMERG) over the northern highlands of Pakistan. Atmospheric Research 205: 134-146. https://doi.org/10.1016/j.atmosres.2018.02.010.

Archer, D. R. and Fowler, H. J. 2004. Spatial and temporal variations in precipitation in the Upper Indus Basin, global teleconnections and hydrological implications, Hydrol. Earth Syst. Sci., 8, 47-61, doi:10.5194/hess-8-47-2004, 2004.

Archer, D. R., 2003: Contrasting hydrological regimes in Indus Basin. Journal of Hydrology, 274: 198-210.

Archer, D. R., 2004: Hydrological implications of spatial and altitudinal variation in temperature in the Upper Indus Basin. Nord. Hydrol, 35, 3. 209-222.

Archer, D. R., Forsythe, N., Fowler, H. J., and Shah, S. M. 2010. Sustainability of water resources management in the Indus Basin under changing climatic and socio-economic conditions, Hydrol. Earth Syst. Sci., 14, 1669-1680, doi:10.5194/hess-14-1669.

Arendt A, Bliss A, Bolch T, Cogley JG, Gardner AS et al. 2015. Randolph Glacier Inventory - A Dataset of Global Glacier Outlines: Version 5.0, GLIMS Technical Report, July 2015.

Arendt, A., Bliss, A., Bolch, T., Cogley, J. G., Gardner, A. S., Hagen, J.-O., Hock, R., Huss, M., Kaser, G., Kienholz, C., Pfeffer, W.T., Moholdt, G., Paul, F., Radić, V., Andreassen, L., Bajracharya, S., Barrand, N.E., Beedle, M., Berthier, E., Bhambri, R., Brown, I., Burgess, E., Burgess, D., Cawkwell, F., Chinn, T., Copland, L., Davies, B., De Angelis, H., Dolgova, E., Earl, L., Filbert, K., Forester, R., Fountain, A.G., Frey, H., Giffen, B., Glasser, N., Guo, W.Q., Gurney, S., Hagg, W., Hall, D., Haritashya, U.K., Hartmann, G., Helm, C., Herreid, S., Howat, I., Kapustin, G., Khromova, T., König, M., Kohler, J., Kriegel, D., Kutuzov, S., Lavrentiev, I., LeBris, R., Liu, S.Y., Lund, J., Manley, W., Marti, R., Mayer, C., Miles, E.S., Li, X., Menounos, B., Mercer, A., Mölg, N., Mool, P., Nosenko, G., Negrete, A., Nuimura, T., Nuth, C., Pettersson, R., Racoviteanu, A., Ranzi, R., Rastner, P., Rau, F., Raup, B., Rich, J., Rott, H., Sakai, A., Schneider, C., Seliverstov, Y., Sharp, M., Sigurðsson, O., Stokes, C., Way, R.G., Wheate, R., Winsvold, S., Wolken, G. and Wyatt, F. (2015) Randolph glacier inventory-a dataset of global glacier outlines: version 5.0 (GLIMS Technical Report).

Arora M, Singh P, Goel NK, Singh RD. 2006. Spatial Distribution and Seasonal Variability of Rainfall in a Mountainous Basin in the Himalayan Region, Water Resources Management 20: 489-508, doi: 10.1007/s11269-006-8773-4

Ashouri, H., Hsu, K.-L., Sorooshian, S., Braithwaite, D.K., Knapp, K.R., Cecil, L.D. and Prat, O.P. (2015) PERSIANN-CDR: Daily precipitation climate data record from multi-satellite observations for hydrological and climate studies. Bulletin of the American Meteorological Society, 96(1), 69-83, doi:10.1175/BAMS-D-13-00068.1.

Barros, A.P., Kim, G., Williams, E., and Nesbitt, S.W., (2004) Probing orographic controls in the Himalayas during the monsoon using satellite imagery: Naturals Hazards and Earth System Science, v. 4, p. 29-51.

Bashir, F, Zeng, X, Gupta, H and Hazenberg, PA (2017) Hydro-meteorological perspective on the Karakoram Anomaly using unique valley-based synoptic weather observations. Geophys. Res. Lett., 44(20), 10,470-10,478 (doi: 10.1002/2017GL075284)

Baudouin, J., Herzog, M., \& A Petrie, C. (2020). Cross-validating precipitation datasets in the Indus River basin. Hydrology and Earth System Sciences, 24 (1), 427-450. https://doi.org/10.5194/hess-24-427-2020.

Beck, H.E., van Dijk, A.I.J.M., Levizzani, V., Schellekens, J., Miralles, D.G., Martens, B. and de Roo, A. (2017) MSWEP: 3-hourly $0.25^{\circ}$ global gridded precipitation (1979-2015) by merging gauge, satellite, and reanalysis data, Hydrol. Earth Syst. Sci., 21, 589-615, https://doi.org/10.5194/hess-21-589-2017. 
Beck, H.E., Wood, E.F., Pan, M., Fisher, C.K., Miralles, D.M., van Dijk, A.I.J.M., McVicar, T.R. and Adler, R.F. 2019. MSWEP V2 global 3-hourly $0.1^{\circ}$ precipitation: methodology and quantitative assessment, B. Am. Meteorol. Soc., in press, https://doi.org/10.1175/BAMS-D-17-0138.1.

Bhutiyani MR. 1999. Mass balance studies on Siachen glacier in the Nubra valley, Karakoram Himalaya, India. J. Glaciol. 45 (149), 112-118.

Biemans H, Hutjes RWA, Kabat P, Strengers BJ, Gerten D, Rost S. 2009. Effects of precipitation uncertainty on discharge calculations for main river basins, J. Hydrometeorology 10, 1011-1025

Biemans, H. et al. Importance of snow and glacier meltwater for agriculture on the Indo-Gangetic Plain. Nat. Sustain. 2, 594-601 (2019).

BIG. 1979. The Batura Glacier in the Karakoram Mountains and its variations, Batura Investigations Group. Sci. Sinica 22, 958-974.

Bindoff NL, Stott PA, AchutaRao KM, Allen MR, Gillett N, Gutzler D, Hansingo K, Hegerl G, Hu Y, Jain S, Mokhov II, Overland J, Perlwitz J, Sebbari R, Zhang X. 2013. Detection and Attribution of Climate Change, Chapter 10 in: Climate Change 2013: The Physical Science Basis. Contribution of Working Group I to the Fifth Assessment Report of the Intergovernmental Panel on Climate Change, edited by: Stocker T. F, Qin D, Plattner G-K, Tignor M, Allen SK, Boschung J, Nauels A, Xia Y, Bex V, Midgley PM. 2013. Cambridge University Press, Cambridge, United Kingdom and New York, NY, USA, 867-952.

BIS. 1992a. IS 5225. 1992. Meteorology - Raingauge, non-recording [PGD 21: Meteorological Instruments], Bureau of Indian Standards, New Delhi.

Bocchiola D, Diolaiuti GA, Soncini A, Mihalcea C, D'Agata C, Mayer C, Lambrecht A, Rosso R, Smiraglia C. 2011. Prediction of future hydrological regimes in poorly gauged high altitude basins: the case study of the upper Indus, Pakistan. Hydrol. Earth Syst. Sci. 15, 2059-2075.

Bocchiola, D. and Diolaiuti, G. 2013. Recent (1980-2009) evidence of climate change in the upper Karakoram, Pakistan, Theor. Appl. Climatol., 113, 611-641.

Boers, N., Bookhagen, B., Marwan, N. and Kurths, J. 2016. Spatiotemporal characteristics and synchronization of extreme rainfall in South America with focus on the Andes mountain range. Clim Dyn 46:601-617.

Bogdanova EG. 1969. A computation method of wind speed averages during rainfall (in Russian), Trans. Voyeykov Main Heophys. Observ., 244, 48-55.

Böhner, J. (2006) General climatic controls and topoclimatic variations in Central and High Asia. Boreas, Vol. 35, 279-295. Oslo. ISSN 0300-9483. DOI10.1080/03009480500456073.

Bokhari SAA, Ahmad B, Ali J, Ahmad S, Mushtaq H, Rasul G (2018) Future climate change projections of the Kabul River basin using a multi-model ensemble of high-resolution statistically downscaled data. Earth Syst Environ. https://doi.org/10.1007/s41748-018-0061-y.

Bolch, T., Kulkarni, A., Kääb, A., Huggel, C., Paul, F., Cogley, J. G.,Frey, H., Kargel, J. S., Fujita, K., Scheel, M., Bajracharya, S. and Stoffel, M. (2012) The state and fate of Himalayan glaciers. Science, 336, 310-314, https://doi.org/10.1126/science.1215828.

Bookhagen B, Burbank DW. 2010. Toward a complete Himalayan hydrological budget: spatiotemporal distribution of snowmelt and rainfall and their impact on river dis- charge. J. Geophys. Res. Earth Surf. 115 (3), 2003-2012.

Bosilovich, M.G., Chen, J., Robertson, F.R. and Adler, R.F. (2008) Evaluation of global precipitation in reanalyses. Journal of Applied Meteorology and Climatology 47: 2279-2299, DOI: 10.1175/2008JAMC1921.1.

Bowling, L. C., J. W.Pomeroy, and D. P.Lettenmaier, 2004: Parameterization of blowing-snow sublimation in a macroscale hydrology model.J. Hydrometeor, 5, 745-762.

Budyko, M.I. (1974) Climate and Life. Orlando, FL: Academic Press, 508 pp.

Businger JA, Yaglom AM. 1971. Introduction to Obukhov's paper 'Turbulence in an atmosphere with a non-uniform temperature'. Bound.-Layer Meteor. 2, pp 3-6.

Businger JA, Yaglom AM. 1971. Introduction to Obukhov's paper 'Turbulence in an atmosphere with a non-uniform temperature'. Bound.-Layer Meteor. 2, pp 3-6. 
Cai, X., Wang, D., Zhu, T., and Ringler, C.: Assessing the regional variability of GCM simulations, Geophys. Res. Lett., 36, L02706, doi:10.1029/2008GL036443, 2009.

Chai, T. and Draxler, R.R. (2014) Root mean square error (RMSE) or mean absolute error (MAE)? arguments against avoiding RMSE in the literature. Geosci. Model Dev., 7, pp. 1247-1250.

Channan, S., K. Collins, and W. R. Emanuel. 2014. Global mosaics of the standard MODIS land cover type data. University of Maryland and the Pacific Northwest National Laboratory, College Park, Maryland, USA. http://www.landcover.org/data/lc/

Chen R, Liu J, Kang E, Yang Y, Han C, Liu Z, Song Y, Qing W, Zhu P. 2015. Precipitation measurement intercomparison in the Qilian Mountains, north-eastern Tibetan Plateau, The Cryosphere, 9, 1995-2008.

Chen, M., P., Xie, P., Janowiak, J.E. and Arkin, P.A. 2002. Global Land Precipitation: A 50-yr Monthly Analysis Based on Gauge Observations, J. of Hydrometeorology, 3, 249-266

Chen, M., Shi, W., Xie, P., Silva, V. B. S., Kousky, V. E., Higgins, R. W., and Janowiak, J. E. 2008. Assessing objective techniques for gauge-based analyses of global daily precipitation, J. Geophys. Res., 113, D04110, https://doi.org/10.1029/2007JD009132.

Chen, R., Liu, J., Kang, E., Yang, Y., Han, C., Liu, Z., Song, Y., Qing, W. and Zhu, P. 2015 Precipitation measurement intercomparison in the Qilian Mountains, north-eastern Tibetan Plateau. The Cryosphere, 9, 1995- 2008.

Ciabatta, L., Massari, C., Brocca, L., Gruber, A., Reimer, C., Hahn, S., Paulik, C., Dorigo, W., Kidd, R. and Wagner, W. (2018) SM2RAIN-CCI: 20A new global long-term rainfall data set derived from ESA CCI soil moisture. Earth System Science Data, 10, 267-280.

Compo, G. P., Whitaker, J. S., Sardeshmukh, P. D., Matsui, N., Allan, R. J., Yin, X., Gleason, B. E., Vose, R. S., Rutledge, G., Bessemoulin, P., et al.: The twentieth century reanalysis project, Quarterly Journal of the Royal Meteorological Society, 137, 1-28, 2011.

Coron, L., Andréassian, V., Perrin, C. and Le Moine, N. (2015) Graphical tools based on TurcBudyko plots to detect changes in catchment behaviour. Hydrol Sci J 60(7-8):1394-1407, doi:10.1080/02626667.2014.964245.

Cruz RV, Harasawa H, Lal M, Wu S, Anokhin Y, Punsalmaa B, Honda Y, Jafari M, Li C and Ninh NH. 2007. Asia. Climate Change 2007: Impacts, Adaptation and Vulnerability, Contribution of Working Group II to the Fourth Assessment Report of the Intergovernmental Panel on Climate Change, M.L. Parry, O.F. Canziani, J.P. Palutikof, P.J. van der Linden and C.E. Hanson, Eds., Cambridge University Press, Cambridge, UK, 469-506.

Cuffey KM, Paterson WSB. 2010. The Physics of Glaciers. Elsevier, Amsterdam, Boston, Heidelberg, London, New York, Oxford, Paris, san Diego, San Francisco, Singapore, Sydney and Tokyo.

CWC NRSC. 2014. Indus Basin, Version 2.0, Central Water Commission and National Remote Sensing Centre, Government of India, Ministry of Water Resources.

D.R. Archer, H.J. Fowler. Spatial and temporal variations in precipitation in the Upper Indus Basin, global teleconnections and hydrological implications. Hydrology and Earth System Sciences Discussions, European Geosciences Union, 2004, 8 (1), pp.47-61. 〈hal-00304788〉

Dahri ZH, Ludwig F, Moors E, Ahmad B, Khan A, Kabat P. 2016. An appraisal of precipitation distribution in the high-altitude catchments of the Indus basin, Science of the Total Environment, 548-549: 289-306.

Dahri, Z.H., Moors, E., Ludwig, F., Ahmad, S., Khan, A., Ali, I. and Kabat, P. 2018. Adjustment of measurement errors to reconcile precipitation distribution in the high-altitude Indus basin. Int $\mathbf{J}$ Climatol. 2018;1-19. https://doi.org/10.1002/joc.5539.

De Scally FA (1994) Relative importance of snow accumulation and monsoon rainfall data for estimating annual runoff, Jhelum basin, Pakistan. Hydrol Sci 39:199-216.

De Souza, K., Kituyi, E., Leone, M., Harvey, B., Murali, K.S. and Ford, J. 2015. Vulnerability to climate change in three hot spots in Africa and Asia: key issues for policy-relevant adaptation and resilience-building research. Reg Environ Change. doi: 10.1007/s10113-015-0755-8. 
Decheng M. 1978. The Map of Snow Mountains in China, K2 (Mount Quogori), Lanzhou, Chinese Academy of Sciences. Lanzhou Institute of Glaciology and Geocryology.

Dee, D. P., Uppala, S., Simmons, A., Berrisford, P., Poli, P., Kobayashi, S., Andrae, U., Balmaseda, M., Balsamo, G., Bauer, d. P., et al. (2011) The ERA-Interim reanalysis: Configuration and performance of the data assimilation system, Quarterly Journal of the royal meteorological society, 137, 553-597.

Diggle PJ, Ribeiro PJ. 2007. Model-based Geostatistics. Springer Series in Statistics.

Ding, Y.H. and Chan, J.C.L. (2005) The East Asian summer monsoon: an overview. Meteorog. Atmos. Phys., 89, 117-142. https://doi.org/10.1007/s00703-005-0125-z.

Dobler, C., S. Hagemann, R. L. Wilby, and J. Stötter (2012), Quantifying different sources of uncertainty in hydrological projections in an alpine watershed, Hydrol. Earth Syst. Sci., 16(11), 4343-4360, doi:10.5194/hess-16-4343-2012.

Eberhardt E, Dickore WB, Miehe G. 2007. Vegetation map of the Batura Valley (North Pakistan), In: Erdkundi 61, 93-112.

Eberhardt E, Dickore WB, Miehe G. 2007. Vegetation map of the Batura Valley (North Pakistan), In: Erdkundi 61, 93-112.

Ebert, E.E., Janowiak, J.E., and Kidd, C. 2007. Comparison of near-real-time precipitation estimates from satellite observations and numerical models. Bulletin of the American Meteorological Society, 88(1), 47-64.

Eckstein, G. 2010. The Greening of Water Law: Managing Freshwater Resources for People and the Environment, UN Environment Programme, Nairobi, Kenya.

Fahrer, C. and Dan Harris, D. 2004. LAMPPOST: A Mnemonic Device for Teaching Climate Variables, J of Geography, 103 (2), http://dx.doi.org.ezproxy.library.wur.nl/ $10.1080 / 00221340408978579$

Farinotti, D.; Immerzeel, W.W.; de Kok, R.J.; Quincey, D.J.; Dehecq, A. Manifestations and mechanisms of the Karakoram glacier Anomaly. Nat. Geosci. 2020, 13, 8-16.

Filippi, L., Palazzi, E., von Hardenberg, J. and Provenzale, A. (2014) Multidecadal variations in the relationship between the NAO and winter precipitation in the Hindu-Kush Karakoram. J Clim. 27(20):7890-7902, 10.1175/JCLI-D-14-00286.1

Fischer G, Nachtergaele F, Prieler S, van Velthuizen HT, Verelst L, Wiberg D. 2008. Global Agroecological Zones Assessment for Agriculture (GAEZ 2008). IIASA, Laxenburg, Austria and FAO, Rome, Italy.

FoDP (Friends of Democratic Pakistan). 2012. A Productive and Water-Secure Pakistan: Infrastructure, Institutions, Strategy. Islamabad, Pakistan: Water Sector Task Force, FoDP. http://metameta.nl/wp-content/uploads/2013/11/FoDP-WSTF-Report-Final-09-29-12.pdf.

Fowler, H.J. and Archer, D.R. (2006) Conflicting Signals of Climatic Change in the Upper Indus Basin. J Clim. 2006; 19: 4276-4293. doi: 10.1175/JCLI3860.1.

Friedl, M. A., Sulla-Menashe, D., Tan, B., Schneider, A., Ramankutty, N., Sibley, A., andHuang, X. 2010. MODIS Collection 5 global land cover: Algorithm refinements and characterization of new datasets. Remote Sensing of Environment, 114, 168-182.

Fujiwara, M., Wright, J.S., Manney, G.L., Gray, L.J., Anstey, J., Birner, T., Davis, S., Gerber, E.P., Harvey, V.L., Hegglin, M.I., Homeyer, C.R., Knox, J.A., Krüger, K., Lambert, A., Long, C.S., Martineau, P., Molod, A., Monge-Sanz, B.M., Santee, M.L., Tegtmeier, S., Chabrillat, S., Tan, D.G.H., Jackson, D.R., Polavarapu, S., Compo, G.P., Dragani, R., Ebisuzaki, W., Harada, Y., Kobayashi, C., McCarty, W., Onogi, K., Pawson, S., Simmons, A., Wargan, K., Whitaker, J.S. and Zou, C.-Z. (2017) Introduction to the SPARC Reanalysis Intercomparison Project (S-RIP) and overview of the reanalysis systems, Atmos. Chem. Phys., 17, 1417-1452, https://doi.org/10.5194/acp-17-1417-2017, 2017.

Funk, C., Peterson, P., Landsfeld, M., Pedreros, D., Verdin, J., Shukla, S., Husak, G., Rowland, J., Harrison, L., Hoell, A., and Michaelsen, J. 2015b. The climate hazards infrared precipitation with 
stations - a new environmental record for monitoring extremes, Scientific Data, 2, 150066, https://doi.org/10.1038/sdata.2015.66.

Funk, C., Verdin, A., Michaelsen, J., Peterson, P., Pedreros, D., Husak, G. 2015a. A global satellite assisted precipitation climatology. Earth Syst. Sci. Data Discuss, 8 (1).

Gampe, D. and Ludwig, R. (2017) Evaluation of gridded precipitation data products for hydrological applications in complex topography. Hydrology, 4, 53, https://doi.org/10.3390/hydrology4040053.

Gao H, Tang Q, Shi X, Zhu C, Bohn TJ, Su F, Sheffield J, Pan M, Lettenmaier DP, Wood EF. 2010. Water budget record from Variable Infiltration Capacity (VIC) model. In Algorithm Theor. Basis Document Terrestrial Water Cycle Data Records.

Gardelle, J., Berthier, E. and Arnaud, Y. (2012) Slight mass gain of Karakoram glaciers in the early twenty-first century. Nat. Geosci., 5, 322-325, doi:10.1038/ngeo1450.

Gardelle, J., Berthier, E., Arnaud, Y. and Kääb A. (2013) Region-wide glacier mass balances over the Pamir-Karakoram-Himalaya during 1999-2011, Cryosph., 7(4), 1263-1286, doi:10.5194/tc-71263-2013.

Gebremichael, M. 2010. Framework for satellite rainfall product evaluation. Geophysical Monograph Series, American Geophysical Union, 191, 265-275, doi:10.1029/2010GM000974.

Gehne, M., Hamill, T.M., Kiladis, G.N. and Trenberth, K.E. 2016. Comparison of global precipitation estimates across a range of temporal and spatial scales. Journal of Climate, 29(21), 7773-7795. https://doi.org/10.1175/JCLI-D-15-0618.1.

Gelaro, R., McCarty, W., Suárez, M. J., Todling, R., Molod, A., Takacs, L., Randles, C. A., Darmenov, A., Bosilovich, M. G., Reichle, R., et al.: The modern-era retrospective analysis for research and applications, version 2 (MERRA-2), Journal of Climate, 30, 5419-5454, 2017.

Ghodichore, N., Vinnarasi, R., Dhanya, C.T. and Roy, S.B. 2018. Reliability of reanalyses products in simulating precipitation and temperature characteristics over India. J Earth Syst Sci 127: 115. https://doi.org/10.1007/s12040-018-1024-2.

Ghulami, M., Babel, M.S. and Shrestha, M.S. 2017. Evaluation of gridded precipitation datasets for the Kabul Basin, Afghanistan. Int. J. Remote Sens. 38 (11) : 3317-3332.

Goodison B E, Louie PYT, Yang D. 1998. WMO solid precipitation measurement intercomparison: Final report. Instruments and Observing Methods Rep. 67, WMO/TD-No. 872, World Meteorological Organization, Geneva, Switzerland.

Gupta, H.V., Kling, H., Yilmaz, K.K. and Martinez-Baquero, G.F. 2009. Decomposition of the mean squared error \& NSE performance criteria: Implications for improving hydrological modelling, J. Hydrol., 377, 80-91, doi:10.1016/j.jhydrol.2009.08.003.

Harding, R.J., Blyth, E.M., Tuinenburg, O.A. and Wiltshire, A. 2013. Land atmosphere feedbacks and their role in the water resources of the Ganges basin. Science of the Total Environment, 468-469, S85-S92. https://doi.org/10.1016/j.scitotenv.2013.03.016.

Harris, I.C. and Jones, P.D. (2019): CRU TS4.02: Climatic Research Unit (CRU) Time-Series (TS) version 4.02 of high-resolution gridded data of month-by-month variation in climate (Jan. 1901Dec. 2017). Centre for Environmental Data Analysis, 01 April 2019, University of East Anglia Climatic Research Unit. doi:10.5285/b2f81914257c4188b181a4d8b0a46bff.

Hasson S.2016. Future water availability from hindukush-karakoram-himalaya upper indus basin under conflicting climate change scenarios. Climate 4(3):40. https://doi.org/10.3390/cli4030040

Hasson S., Saeed F., Böhner J, Schleussner C. 2019. Water availability in Pakistan from HindukushKarakoram-Himalayan watersheds at $1.5{ }^{\circ} \mathrm{C}$ and $2{ }^{\circ} \mathrm{C}$ Paris Agreement targets. Advances in Water Resources 131 (2019) 103365, https://doi.org/10.1016/j.advwatres.2019.06.010.

Hasson, S., Böhner, J., Chishtie, F., 2018. Low Fidelity of present-day climate modelling experiments and future climatic uncertainty over Himalayan watersheds of Indus basin. Clim. Dyn. https://doi.org/10.1007/s00382-018-4160-0. 
Hasson, S., Lucarini, V., and Pascale, S. 2013. Hydrological cycle over South and Southeast Asian river basins as simulated by PCMDI/CMIP3 experiments, Earth Syst. Dynam., 4, 199-217, doi:10.5194/esd-4-199.

Hawkins E, Sutton R. 2009. The potential to narrow uncertainty in regional climate predictions. Bull Am Meteorol Soc 90:1095-1107. doi:10.1175/2009BAMS2607.1

Henn, B., Newman, A.J., Livneh, B., Daly, C. and Lundquist, J.D. 2018. An assessment of differences in gridded precipitation datasets in complex terrain. J. Hydrol., 556, 1205-1219, https://doi.org/10.1016/j.jhydrol.2017.03.008.

Her, Y., Yoo, S.-H., Cho, J., Hwang, S., Jeong, J., \& Seong, C. 2019. Uncertainty in hydrological analysis of climate change: Multi-parameter vs. multi-GCM ensemble predictions. Scientific Reports, 9, 4974. https://doi.org/10.1038/s41598-019-41334-7.

Hersbach, H., de Rosnay, P., Bell, B., Schepers, D., Simmons, A., Soci, C., Abdalla, S., AlonsoBalmaseda, M., Balsamo, G., Bechtold, P., 10 Berrisford, P., Bidlot, J.-R., de Boisséson, E., Bonavita, M., Browne, P., Buizza, R., Dahlgren, P., Dee, D., Dragani, R., Diamantakis, M., Flemming, J., Forbes, R., Geer, A., Haiden, T., Hólm, E., Haimberger, L., Hogan, R., Horányi, A., Janiskova, M., Laloyaux, P., Lopez, P., Munoz-Sabater, J., Peubey, C., Radu, R., Richardson, D., Thépaut, J.-N., Vitart, F., Yang, X., Zsótér, E., and Zuo, H. 2018. Operational global reanalysis: progress, future directions and synergies with NWP, ERA-report, Serie 27, https://doi.org/10.21957/tkic6g3wm, https: //www.ecmwf.int/node/18765.

Hewitt K, Wake C, Young G.J, David C. 1989. Hydrological Investigations at Biafo Glacier, Karakoram Himalaya, Pakistan: An Important Source of Water for the Indus River, Annals of Glaciology 13: 103-08.

Hewitt K. 2005. The Karakoram anomaly? Glacier expansion and the 'elevation effect', Karakoram Himalaya. Mt. Res. Dev. 25, 332-340. http://dx.doi.org/10.1659/02764741(2005)025[0332:TKAGEA]2.0.CO;2.

Hewitt K. 2011. Glacier change, concentration, and elevation effects in the Karakoram Himalaya, Upper Indus Basin. Mt. Res. Dev. 31 (3), 188-200. http://dx.doi.org/10.1659/mrd-journal-d-1100020.1 .

Hewitt, K.: The Karakoram Anomaly? Glacier expansion and the "elevation effect", Karakoram Himalaya, Mt. Res. Dev., 25, 332-340, 2005.

Hodges, K. 2006. Climate and the evolution of mountains. Scientific American, Vol. 295, No. 2, pp. 72-79.

Houghton JT, Jenkins GJ, Ehpraums JJ (eds) 1990. Climate change: the IPCC scientific assessment. Cambridge University Press, Cambridge.

Huffman, G.J., Bolvin, D.T. and Nelkin, E.J. 2018. Integrated Multi-satellitE Retrievals for GPM (IMERG). Technical Documentation, Tech. rep., NASA/GSFC, Greenbelt, MD 20771, USA.

Huffman, G.J., Bolvin, D.T., Nelkin, E.J., Wolff, D.B., Adler, R.F., Gu, G., Hong, Y., Bowman, K.P. and Stocker, E.F. 2007. The TRMM Multisatellite Precipitation Analysis (TMPA): Quasi-global, multiyear, combined-sensor precipitation estimates at fine scales. Journal of Hydrometeorology, $8(1), 38-55$.

Hussain, S., Song, X., Ren, G., Hussain, I., Han, G. and Zaman, M.H. 2017. Evaluation of gridded precipitation data in the Hindu Kush-Karakoram-Himalaya mountainous area. Hydrological Sciences Journal, DOI: 10.1080/02626667.2017.1384548.

Immerzeel WW, Pellicciotti F, Shrestha AB. 2012. Glaciers as a proxy to quantify the spatial distribution of precipitation in the Hunza basin. Mt. Res. Dev. 32 (1), 30-38. http://dx.doi.org/10.1659/MRD-JOURNAL-D-11-00097.1.

Immerzeel WW, Van Beek LP., Bierkens MFP. 2010. Climate change will affect the Asian water towers. Science, 328: 1382 \pm 5. doi: 10.1126/science.1183188 PMID: 20538947

Immerzeel WW, Van Beek LPH, Konz M, Shrestha AB, Bierkens MFP. 2012b. Hydrological response to climate change in a glacierized catchment in the Himalayas, Clim. Change, 110, 721736, doi:10.1007/s10584-011-0143-4, 2011. 
Immerzeel WW, Wanders N, Lutz AF, Shea JM, Bierkens MFP. 2015. Reconciling high altitude precipitation in the upper Indus Basin with glacier mass balances and runoff. Hydrol. Earth Syst. Sci. 19 (4673-4687), 2015. http://dx.doi.org/10.5194/ hessd-12-4755-2015.

Immerzeel, W. W. \& Bierkens, M. F. P. 2012. Asia's water balance. Nature Geosci. 5, 841-842.

Immerzeel, W. W., Droogers, P., De Jong, S. M., and Bierkens, M. F. P., 2009: Large-scale monitoring of snow cover and runoff simulation in Himalayan river basins using remote sensing. Remote Sensing of Environment, 113(1): 40-49

Immerzeel, W. W., Pellicciotti, F. \& Bierkens, M. F. P. 2013. Rising river flows throughout the twenty-first century in two Himalayan glacierized watersheds. Nature Geosci. 6, 1-4.

Immerzeel, W. W., Van Beek, L. P. H. \& Bierkens, M. F. P. 2010. Climate change will affect the Asian water towers. Science 328, 1382-1385.

IPCC, 2007: Climate Change 2007: Impacts, Adaptation and Vulnerability. Contribution of Working Group II to the Fourth Assessment Report of the Intergovernmental Panel on Climate Change, M.L. Parry, O.F. Canziani, J.P. Palutikof, P.J. van der Linden and C.E. Hanson, Eds., Cambridge University Press, Cambridge, UK, 976pp.

IPCC, 2013: Climate Change 2013: The Physical Science Basis. Contribution of Working Group I to the Fifth Assessment Report of the Intergovernmental Panel on Climate Change [Stocker, T.F., D. Qin, G.-K. Plattner, M. Tignor, S.K. Allen, J. Boschung, A. Nauels, Y. Xia, V. Bex and P.M. Midgley (eds.)]. Cambridge University Press, Cambridge, United Kingdom and New York, NY, USA, $1535 \mathrm{pp}$.

IPCC, 2014: Climate Change 2014: Impacts, Adaptation, and Vulnerability. Part A: Global and Sectoral Aspects. Contribution of Working Group II to the Fifth Assessment Report of the Intergovernmental Panel on Climate Change [Field, C.B., V.R. Barros, D.J. Dokken, K.J. Mach, M.D. Mastrandrea, T.E. Bilir, M. Chatterjee, K.L. Ebi, Y.O. Estrada, R.C. Genova, B. Girma, E.S. Kissel, A.N. Levy, S. MacCracken, P.R. Mastrandrea, and L.L. White (eds.)]. Cambridge University Press, Cambridge, United Kingdom and New York, NY, USA, 1132 pp.

Iqbal, M., Dahri, Z., Querner, E., Khan, A. and Hofstra, N. (2018) Impact of climate change on flood frequency and intensity in the Kabul River Basin. Geosciences 8(4), 114; doi:10.3390/geosciences8040114.

Janowiak, J.E. and Xie, P. (1999) CAMS-OPI: A global satellite-rain gauge merged product for realtime precipitation monitoring applications. J. Clim. 12, 3335-3342.

Joseph, R., Smith, T.M., Sapiano, M.R.P. and Ferraro R.R. (2009) A new high-resolution satellitederived precipitation dataset for climate studies. J. Hydrometeorol., 10, 935-952.

Kääb A, Berthier E, Christopher N, Gardelle J, Arnaud Y. 2012. Contrasting patterns of early twentyfirst-century glacier mass change in the Himalayas. Nature 488 (7412), 495-498.

Kabat P, Egerton P, Baddour O, Paterson L, Nullis C, Castonguay S, Walsh M. 2019.United in science: High-level synthesis report of latest climate science information, convened by the science advisory group of the UN climate action summit 2019.

Kalnay, E., M. Kanamitsu, R. Kistler, W. Collins, D. Deaven, L. Gandin, M. Iredell, S. Saha, G. White, J. Woollen, Y. Zhu, A. Leetmaa, R. Reynolds, M. Chelliah, W. Ebisuzaki, W. Higgins, J. Janowiak, K. C. Mo, C. Ropelewski, J. Wang, R. Jenne \& D. Joseph, 1996. The NCEP/NCAR 40-Year Reanalysis Project. Bulletin of the American Meteorological Society 77(3):437-471, doi:10.1175/1520-0477(1996)077<0437:TNYRP>2.0.CO;2.

Kanamitsu, M., Ebisuzaki, W., Woollen, J., Yang, S.-K., Hnilo, J., Fiorino, M., and Potter, G.: Ncepdoe amip-ii reanalysis (r-2), Bulletin of the American Meteorological Society, 83, 1631-1644, 2002.

Karger, D.N., Conrad, O., Böhner, J., Kawohl, T., Kreft, H., Soria-Auza, R.W., Zimmermann, N.E., Linder, H.P. and Kessler, M. (2017) Climatologies at high resolution for the Earth land surface areas. Sci. Data 4:170122 doi: 10.1038/sdata.2017.122.

Kay, A. L., Davies, H.N., Bell, V. A. and Jones, R. G. 2009. Comparison of uncertainty sources for climate change impacts: Flood frequency in England, Clim. Change, 92, 41-63. 
Keith M. 2006. Variation in Temperature With Altitude and Latitude, Journal of Geography Volume 105 (3), http://dx.doi.org/10.1080/00221340608978675

Keli W, Jia S, Guodong C. Hao J. 2011. Effect of Altitude and Latitude on Surface Air Temperature across the Qinghai-Tibet Plateau, J. Mt. Sci. 8: 808-816, DOI: 10.1007/s11629-011-1090-2.

Khan A, Naz BS, Bowling LC. 2015. Separating snow, clean and debris covered ice in the Upper Indus Basin, Hindukush-Karakoram-Himalayas, using Landsat images between 1998 and 2002, Journal of Hydrology 521 (2015) 46-64.

Khan A.J., Koch, M. and Chinchillaet K.M. 2018. Evaluation of Gridded Multi-Satellite Precipitation Estimation (TRMM-3B42-V7) Performance in the Upper Indus Basin (UIB), Climate 2018, 6, 76.DOI: $10.3390 / \mathrm{cli} 6030076$

Khan, A.A., Pant, N.C., Goswami, A., Lal, R. and Joshi, R. 2015. Critical evaluation and assessment of annual precipitation in the Indus, the Ganges and the Brahmaputra Basins, North India. In: Joshi R, Kumar K, Palni LMS (eds) Dynamics of Climate Change and Water resources of Northwestern Himalaya, Springer, Switzerland, pp 67-84.

Khattak, M. S., Babel, M. S., \& Sharif, M. (2011). Hydro-meteorological trends in the Upper Indus River basin in Pakistan. Climate Research, 46, 103-119. doi:10.3354/cr00957.

Kick W. 1980. Material for a glacier inventory of the Indus drainage basin - the Nanga Parbat massif, World Glacier Inventory. (Proceedings of the Riederalp Workshop, September 1978: IAHS-AISH Publ. no. 126, p. 1980.

Kidd, C., Dawkins, E. and Huffman, G. (2013) Comparison of precipitation derived from the ECMWF operational forecast model and satellite precipitation datasets. J. Hydrometeorol., 14, 1463-1482, doi:10.1175/JHM-D-12-0182.1.

Kling, H., Fuchs, M. and Paulin, M. (2012) Runoff conditions in the upper Danube basin under an ensemble of climate change scenarios. J. Hydrol., 424-425, 264-277, doi:10.1016/j.jhydrol.2012.01.001.

Knutti, R., and J. Sedláček (2012), Robustness and uncertainties in the new CMIP5 climate model projections, Nature Climate Change, doi:10.1038/NCLIMATE1716.

Kobayashi, S., Ota, Y., Harada, Y., Ebita, A., Moriya, M., Onoda, H., Onogi, K., Kamahori, H., Kobayashi, C., Endo, H., Miyaoka, K., and Takahashi, K. 2015. The JRA-55 reanalysis: General specifications and basic characteristics, J. Meteorol. Soc. Jpn. Ser. I, 93, 5-48, https://doi.org/10.2151/jmsj.2015-001.

Kochendorfer J, Rasmussen R, Wolff M, Baker B, Hall ME, Landolt S, Jachcik A, Isaksen K, Brækkan R, Leeper R. 2017a. The Quantification and Correction of Wind-Induced Precipitation Measurement Errors, Hydrol. Earth Syst. Sci. Discuss., doi:10.5194/hess-2016-415.

Kochendorfer, J., Nitu, R., Wolff, M., Mekis, E., Rasmussen, R., Baker, B., Earle, M. E., Reverdin, A., Wong, K., Smith, C. D., Yang, D., Roulet, Y.-A., Buisan, S., Laine, T., Lee, G., Aceituno, J. L. C., Alastrué, J., Isaksen, K., Meyers, T., Brækkan, R., Landolt, S., Jachcik, A., and Poikonen, A. 2017b. Analysis of single-Alter-shielded and unshielded measurements of mixed and solid precipitation from WMO-SPICE, Hydrol. Earth Syst. Sci., 21, 3525-3542, https://doi.org/10.5194/hess-21-3525.

Kochendorfer, J., Nitu, R., Wolff, M., Mekis, E., Rasmussen, R., Baker, B., Earle, M. E., Reverdin, A., Wong, K., Smith, C. D., Yang, D., Roulet, Y.-A., Meyers, T., Buisan, S., Isaksen, K., Brækkan, R., Landolt, S., and Jachcik, A. 2017c. Testing and Development of Transfer Functions for Weighing Precipitation Gauges in WMO-SPICE, Hydrol. Earth Syst. Sci. Discuss., https://doi.org/10.5194/hess-2017-228, in review, 2017c.

Kraaijenbrink PDA, Bierkens MFP, Lutz AF, Immerzeel WW (2017) Impact of a $1.5{ }^{\circ} \mathrm{C}$ global temperature rise on Asia's glaciers. Nature 549:257-260. https://doi.org/10.1038/nature23878

Krishnan, R., Shrestha, A.B., Ren, G., Rajbhandari, R., Saeed, S., Sanjay, J., Syed, M.A., Vellore, R., Xu, Y., You, Q. and Ren, Y. 2019. Unravelling climate change in the Hindu Kush Himalaya: rapid warming in the mountains and increasing extremes. In: Wester P, Mishra A, Mukherji A, Shrestha A (eds) The Hindu Kush Himalaya assessment. Springer, Cham, pp 57-97. 
Legates DR, Willmott CJ. 1990. Mean seasonal and spatial variability in gauge-corrected global precipitation, International Journal of Climatology: 10, 111-127.

Legates DR. 1987. A climatology of global precipitation, Publications in Climatology, 40 (1), 85 pp.

Lenderink, G., van Ulden, A., van den Hurk, B. 2007. A study on combining global and regional climate model results for generating climate scenarios of temperature and precipitation for the Netherlands. Clim Dyn 29, 157-176. https://doi.org/10.1007/s00382-007-0227-z.

Liang, X., Lettenmaier, D. P., Wood, E. F., and Burges, S. J.: 1994, 'A Simple Hydrologically Based Model of Land Surface Water and Energy Fluxes for General Circulation Models', J. Geophys. Res. 99, 14415-14428.

Liang, X., Wood, E. F., and Lettenmaier, D. P.: 1996, 'Surface Soil Moisture Parameterization of the VIC-2L Model: Evaluation and Modifications', Global Plan. Change 13, 195-206.

Lin, Z. and Wu, X. (1990) A preliminary analysis about the tracks of moisture transport on the Qinghai-Xizang Plateau Geogr. Res., 9 (1990), pp. 33-40 1990.

Lohmann, D., Raschke, E., Nijssen, B., and Lettenmaier, D. P.: 1998, 'Regional Scale Hydrology: I. Formulation of the VIC-2L Model Coupled to a Routing Model',Hydrol. Sci. J. 43, 131-141.

Lundquist, J.D., Minder, J.R., Neiman, P.J. and Sukovich, E. (2010) Relationships between barrier jet heights, orographic precipitation gradients and streamflow in the northern Sierra Nevada. J Hydrometeorol 11:1141-1156.

Lutz AF, Immerzeel WW, Gobiet A, Pellicciotti F, Bierkens MFP. 2013. Comparison of climate change signals in CMIP3 and CMIP5 multi-model ensembles and implications for Central Asian glaciers. Hydrol. Earth Syst. Sci. 17(9): 3661-3677, doi:10.5194/hess-17-3661-2013.

Lutz AF, Immerzeel WW, Kraaijenbrink PDA, Shrestha AB (2016a) Climate change impacts on the upper Indus hydrology: sources, shifts and extremes. PLoS One, PLoS One 11:e0165630. https://doi.org/10.1371/journal.pone.0165630

Lutz AF, Immerzeel WW, Shrestha AB, Bierkens MFP. 2014. Consistent increase in high Asia's runoff due to increasing glacier melt and precipitation. Nat. Clim. Chang. http://dx.doi.org/10.1038/nclimate2237 advance online publication.

Lutz AF, ter Maat HW, Biemans H, Shrestha AB, Wester P, Immerzeel WW. 2016b. Selecting representative climate models for climate change impact studies: an advanced envelope-based selection approach. Int. J. Climatol. 36: 3988-4005. DOI: 10.1002/joc.4608

Lutz, A.F., Maat, H.W., Wijngaard, R.R., Biemans, H., Syed, A., Shrestha, A.B., Wester, P. and Immerzeel, W.W. 2019. South Asian river basins in a $1.5{ }^{\circ} \mathrm{C}$ warmer world. Reg Environ Change 19, 833-847. https://doi.org/10.1007/s10113-018-1433-4

Maggioni, V., Sapiano, M.R.P. and Adler, R.F. (2016) Estimating uncertainties in high-resolution satellite precipitation products: systematic or random error? Journal of Hydrometeorology, 17(4), 1119-1129. https://doi.org/10.1175/jhm-d-15-0094.1.

Mao, D. and K.A. Cherkauer, 2009. Impacts of Land-use Change on Hydrologic Responses in the Great Lakes Region. Journal of Hydrology 374:71-82.

Matsuura, K. and Willmott, C. (2018) Terrestrial Precipitation: 1900-2014, Gridded Monthly Time Series (1900-2014), V5.01, University of Delaware. Available online: https://www.esrl.noaa.gov/psd/data/gridded/data.UDel_AirT_Precip.html\#detail, documentation: http://climate.geog.udel.edu/ climate/html_pages/Global2017/README.GlobalTsP2017.html

Mayer C, Lambrecht A, Belò M, Smiraglia C, Diolaiuti G. 2006. Glaciological characteristics of the ablation zone of Baltoro glacier, Karakoram, Pakistan. Ann. Glaciol. 43 (1), 123-131. http://dx.doi.org/10.3189/172756406781812087.

Mayer, C., Lambrecht, A., Oerter, H., Schwikowski, M., Vuillermoz, E., Frank, N. and Diolaiuti, G. 2014. Accumulation studies at a high elevation glacier site in central Karakoram. Advances in Meteorology, 215162. https://doi.org/10.1155/2014/215162.

Mayewski PA, Lyons WB, Ahmad N, Smith G, Pourchet M. 1984. Interpretation of the chemical and physical time series retrieved from Sentik glacier, Ladakh Himalaya, India. J. Glaciol. 30 (104), 66-76. 
Mayewski PA, Lyons WB, Ahmad N. 1983. Chemical Composition of a High Altitude Fresh Snowfall in the Ladakh Himalayas. Earth Science Faculty Scholarship Paper 187. http:// digitalcommons.library.umaine.edu/ers_facpub/187.

Mekonnen GB, Matula S, Dolez F, Fisak J. 2015. Adjustment to rainfall measurement under-catch with a tipping bucket rain gauge using ground-level manual gauges, Meteorol Atmos Phys (2015) 127:241-256, DOI 10.1007/s00703-014-0355-z.

Michelson, D.B. (2004), Systematic correction of precipitation gauge observations using analyzed meteorological variables, Journal of Hydrology 290 (2004) 161-177.

Miehe S, Cramer T, Jacobsen JP, Winiger M. 1996. Humidity conditions in the west- ern Karakoram as indicated by climatic data and corresponding distribution patterns of the montane and alpine vegetation. Erdkundi. 50, pp. 190-204.

Miehe S, Winiger M, Bohner J, Yili Z. 2001. The climate diagram map of high Asia, In: Erdkundi 55, 94-97.

Miehe, S., Cramer, T., Jacobsen, J.-P., Winiger, M., 1996. Humidity conditions in the west- ern Karakoram as indicated by climatic data and corresponding distribution patterns of the montane and alpine vegetation. Erdkundi. 50, pp. 190-204.

Mishra V. 2015. Climatic uncertainty in Himalayan Water Towers, J. Geophys. Res. Atmos., 120, 2689-2705, doi:10.1002/2014JD022650.

Mondal, A., Lakshmi, V. and Hashemi, H. (2018) Intercomparison of trend analysis of multisatellite monthly precipitation products and gauge measurements for river basins of India. J Hydrol 565:779-790.

Mukhopadhyay B, Khan A. 2015. A reevaluation of the snowmelt and glacial melt in river flows within Upper Indus Basin and its significance in a changing climate. J Hydrol. 527: 119-132.

Mukhopadhyay B. 2012. Detection of dual effects of degradation of perennial snow and ice covers on the hydrologic regime of a Himalayan river basin by stream water avail- ability modeling. $\mathrm{J}$. Hydrol. 412-413 (0), 14-33. http://dx.doi.org/10.1016/j.jhydrol.2011.06.005.

Murphy JM, Sexton DMH, Barnett DN, Jones GS, Webb MJ, Collins M, Stainforth DA .2004. Quantification of modelling uncertainties in a large ensemble of climate change simulations. Nature 430:768-772. doi:10.1038/nature02771

NESPAK-AHT-DELTARES. 2015. Improvement of Water Resources Management of Indus Basin to Enhance the Capacity of Indus River System Authority-Vol-1, Final Report.

Nitu R, Wong K. 2010. CIMO Survey on national summaries of methods and instruments for solid precipitation measurement at automatic weather stations, World Meteorological Organization, Instruments and Observing Methods Report No. 102. WMO/TD-No. 1544.

Obukhov AM.(1971. Turbulence in an atmosphere with a non-uniform temperature. Bound.-Layer Meteor. 2, 7-29.

Pal, I., Robertson, A.W., Lall, U. and Cane, M.A. 2014. Modeling winter rainfall in northwest India using a hidden Markov model: understanding occurrence of different states and their dynamical connections. Clim. Dyn., 44 (3), pp. 1003-1015, 10.1007/s00382-014-2178-5.

Palazzi E, von Hardenberg J, Provenzale A. 2013. Precipitation in the Hindu-Kush Karakoram Himalaya: observations and future scenarios. J. Geophys. Res. Atmos. 118 (1), 85-100. http://dx.doi.org/10.1029/2012JD018697.

Palmer TN, Shutts GJ, Hagedorn R, Doblas-Reyes FJ, Jung T, Leutbecher M. 2005. Representing model uncertainty in weather and climate prediction. - Ann. Rev. Earth Planet. Sci. 33, 4.1-4.31.

Pang, H., Hou, S., Kaspari, S. and Mayewski, P. A. 201.4 Influence of regional precipitation patterns on stable isotopes in ice cores from the central Himalayas. The Cryosphere, 8, 289-301, https://doi.org/10.5194/tc-8-289-2014.

Pellicciotti F, Buergi C, Immerzeel WW, Konz M, Shrestha AB. 2012. Challenges and Uncertainties in Hydrological Modeling of Remote Hindu Kush-Karakoram-Himalayan (HKH) Basins: Suggestions for Calibration Strategies. Mountain Research and Development 32(1): 39-50. doi: http://dx.doi.org/10.1659/MRD-JOURNAL-D-11-00092.1 
Peña-Arancibia, J.L., van Dijk, A.I.J.M., Renzullo, L.J. and Mulligan, M. (2013) Evaluation of precipitation estimation accuracy in reanalyses, satellite products, and an ensemble method for regions in Australia and Sout and East Asia. Journal of Hydrometeorology, 14, 1323-1333, https://doi.org/10.1175/JHM-D-12-0132.1.

Pepin, N. et al. Elevation-dependent warming in mountain regions of the world. Nat. Clim. Chang. 5, 424-430 (2015)

Prakash, S., Mitra, A.K., Aghakouchak, A., Liu, Z., Norouzi, H. and Pai, D.S. (2018) A preliminary assessment of GPM-based multi-satellite precipitation estimates over a monsoon dominated region. Journal of Hydrology 556, 865-876, https://doi.org/10.1016/j.jhydrol.2016.01.029.

Prein, A. F., A. Gobiet, and H. Truhetz. 2011. Analysis of uncertainty in large scale climate change projections over Europe, Meteorol. Z., 20(4), 383-395, doi:10.1127/0941-2948/2011/0286.

Prein, A.F. and Gobiet, A. 2017. Impacts of uncertainties in European gridded precipitation observations on regional climate analysis. International Journal of Climatology, 37, 305-327, https://doi.org/10.1002/joc.4706.

Pritchard, H.D. 2019. Asia's shrinking glaciers protect large populations from drought stress. Nature $569,649-654$.

Putkonen, J. 2004. Continuous snow and rain data at 500 to $4400 \mathrm{~m}$ altitude near Annapurna, Nepal, 1999-2001. Arctic, Antarctic, and Alpine Research, 36, 244-248, doi:10.1657/15230430(2004)036[0244:CSARDA]2.0.CO;2.

Qazi NA. 1973. Water Resources Development in Suru Basin (Ladakh). 51. Vacant Press, Srinagar, India, p. 1973.

Ragettli S, Pellicciotti F. 2012. Calibration of a physically based spatially distributed hydrological model in a glacierized basin: On the use of knowledge from glaciometeorological processes to constrain model parameters, Water Resour. Res., 48, 1-20, doi:10.1029/2011WR010559.

Ragettli, S., F. Pellicciotti, R. Bordoy, and W. W. Immerzeel 2013. Sources of uncertainty in modeling the glacio-hydrological response of a Karakoram watershed to climate change, Water Resour. Res., 49, 1-19, doi:10.1002/wrcr.20450.

Räisänen, J. 2001. $\mathrm{CO}_{2}$-induced climate change in CMIP2 experiments: quantification of agreement and role of internal variability. J Clim 14:2088-2104.

Rasmussen R, Baker B, Kochendorfer J, Meyers T, Landolt S, Fischer AP, Black J, Theriault JM, Kucera P, Gochis D, Smith C, Nitu R, Hall M, Ikeda K, Gutmann E. 2012. How well are we measuring snow: the NOAA/FAA/NCAR winter precipita- tion test bed. Bull. Am. Meteorol. Soc. 93, 811-829. http://dx.doi.org/10.1175/ BAMS-D-11-00052.1.

Reggiani, P. and Rientjes, T.H.M. (2015) A reflection on the long-term water balance of the Upper Indus basin. Hydrology Research, 46, 446-462. https://doi.org/10.2166/nh.2014.060.

Ren Z, Li M. 2007. Errors and correction of precipitation measurements in China, Adv. Atmos. Sci., 24, 449-458, doi:10.1007/s00376-007-0449-3.

Ruane, A.C. and Roads, J.O. (2007) 6-hour to 1-year variance of five global precipitation sets. Earth Interactions, 11. [Available online at http://EarthInteractions.org.].

S. Dutt, A.K. Gupta, B. Wünnemann, D. Yan. 2018. A long arid interlude in the Indian summer monsoon during: 4,350 to 3,450 cal. yr BP contemporaneous to displacement of the Indus valley civilization. Quat. Int., 482 (2018), pp. 83-92

Saha, S., Moorthi, S., Pan, H.-L., Wu, X., Wang, J., Nadiga, S., Tripp, P., Kistler, R., Woollen, J., Behringer, D., Liu, H., Stokes, D., Grumbine, R., Gayno, G., Wang, J., Hou, Y.-T., Chuang, H.Y., Juang, H.-M. H., Sela, J., Iredell, M., Treadon, R., Kleist, D., Van Delst, P., Keyser, D., Derber, J., Ek, M., Meng, J., Wei, H., Yang, R., Lord, S., Van Den Dool, H., Kumar, A., Wang, W., Long, C., Chelliah, M., Xue, Y., Huang, B., Schemm, J.-K., Ebisuzaki, W., Lin, R., Xie, P., Chen, M., Zhou, S., Higgins, W., Zou, C.-Z., Liu, Q., Chen, Y., Han, Y., Cucurull, L., Reynolds, R. W., Rutledge, G., and Goldberg, M. (2010) The NCEP climate forecast system reanalysis, B. Am. Meteorol. Soc., 91, 1015-1057. 
Sapiano, M.R P. and Arkin, P.A. (2009) An intercomparison and validation of high-resolution satellite precipitation estimates with 3-hourly gauge data. J. Hydrometeor., 10, 149-166.

Schabenberger O, Gotway CA. 2005. Statistical Methods for Spatial Data Analysis, Chap- man an. CRC Press.

Schaefli B, Hingray B, Niggli M, Musy A. 2005. A conceptual glacio-hydrological model for high mountainous catchments. Hydrol. Earth Syst. Sci., 9(1/2), 95-109. doi: 10.5194/hess-9-95-2005

Schaefli, B. 2005. Quantification of modelling uncertainties in climate change impact studies on water resources: application to a glacier-fed hydropower production system in the Swiss Alps, Ph.D. thesis, École polytechnique fédérale de Lausanne, Lausanne.

Schneider, U., Becker, A., Finger, P., Meyer-Christoffer, A., Ziese, M. and Rudolf, B. 2014. GPCC's new land surface precipitation climatology based on quality-controlled in situ data and its role in quantifying the global water cycle. Theor. Appl. Climatol. 115, 15-40. doi:10.1007/s00704-0130860-x.

Schneider, U., Becker, A., Finger, P., Meyer-Christoffer, A., Ziese, M. 2018. GPCC Full Data Monthly Product Version 2018 at $0.25^{\circ}$ : Monthly Land-Surface Precipitation from Rain-Gauges built on GTS-based and Historical Data. doi: 10.5676/DWD_GPCC/FD_M_V2018_025

Schwab, K., and Samans, R. 2016. "The Future of Jobs Employment, Skills and Workforce Strategy for the Fourth Industrial Revolution," in Global Challenge Insight Report (World Economic Forum). Available at: http://www3.weforum.org/docs/WEF_Future_of_Jobs.pdf

Sevruk B, Hamon WR. 1984. International Comparison of National Precipitation Gauges with a Reference Pit Gauge. Instruments and Observing Methods Rep., vol. 17. World Meteorol. Org., Geneva. 135 pp.

Sevruk B, Klemm S. 1989. Catalogue of Standard Precipitation Gauges. Instruments and observing methods, Rep, vol. 39. World Meteorol. Org.,WMO, Geneva. WMO/TD No. 328, 52 pp.

Sevruk B. 1982. Methods of correction for systematic error in point precipitation measurement for operational use, in Oper. Hydrol. Rep. 21 Publ. 589, 91 pp.1982.

Sevruk, B. and Hamon, W.R. 1984. International comparison of national precipitation gauges with a reference pit gauge, Instruments and observing methods (Report No. 17). Geneva: World Meteorological Organization, $135 \mathrm{pp}$.

Sheikh, M. M., Manzoor, N., Adnan, M., Ashraf, J., and Khan, A. M.: Climate Profile and Past Climate Changes in Pakistan, GCISC-RR-01, Global Change Impact Studies Centre (GCISC), Islamabad, Pakistan, 2009

Shroder JF, Bishop MP, Copland L, Sloan VF. 2000. Debris-covered glaciers and rock glaciers in the Nanga Parbat Himalaya, Pakistan. Geogr. Annal.: Ser. A Phys. Geogr. 82 (1), 17-31. http://dx.doi.org/10.1111/j.0435-3676.2000.00108.x.

Shroder JF, Bishop MP, Copland L, Sloan VF. 2000. Debris-covered glaciers and rock glaciers in the

SIHP, 1997. Snow and Ice Hydrology, Pakistan Phase-II Final Report to CIDA, Report No. 54, IDRC File No. 88-8009-00. International Development Research Centre, Ottawa, Ontario, Canada.

Singh P, Kumar N. 1997. Impact assessment of climate change on the hydrological response of a snow and glacier melt runoff dominated Himalayan river, Journal of Hydrology, 193 (1-4): 316350 .

Singh P, Ramasastri KS, Kumar N. 1995. Topographical influence on precipitation distribution in different ranges of Western Himalaya, Nordic Hydrology, 26: 259-284.

Sohn, S., Tam, C., Karumuri, A. and Ahn, J. (2012) Quantifying the reliability of precipitation datasets for monitoring large-scale East Asian precipitation variations. Int. J. Climatol., 32, 15201526, doi:https://doi.org/10.1002/joc.2380.

Stepek A, Wijnant IL. 2011. Interpolating wind speed normals from the sparse Dutch network to a high resolution grid using local roughness from land use maps, De Bilt, 2011 | Technical report; TR-321, Royal Netherlands Meteorological Institute.

Stickler, A., S. Brönnimann, M. A. Valente, J. Bethke, A. Sterin, S. Jourdain, E. Roucaute, M. V. Vasquez, D. A. Reyes, R. Allan, and D. Dee, (2014) ERA-CLIM: Historical Surface and Upper- 
Air Data for Future Reanalyses. Bull. Amer. Meteor. Soc., 95(9), 1419-1430, DOI: 10.1175/BAMS-D-13-00147.1.

Su, B., J. L. Huang, M. Gemmer, et al., 2016: Statistical downscaling of CMIP5 multi-model ensemble for projected changes of climate in the Indus River Basin. Atmos. Res., 178-179, 138149, doi: 10.1016/j.atmosres.2016.03.023.

Sun, Q., Miao, C., Duan, Q., Ashouri, H., Sorooshian, S. and Hsu, K.-L. (2018) A review of global precipitation data sets: Data sources, estimation, and intercomparisons. Reviews of Geophysics, 56, 79-107. https://doi.org/10.1002/2017RG000574.

SUPARCO and FAO (2010) Pakistan Floods/Rains 2010:Rapid Crop Damage Assessment, Series No. 1, Available at http://www.suparco.gov.pk/downloadables/PAKISTAN_FLOODRAIN.pdf

Syed, F.S., Giorgi, F., Pal, J.S. and King, M.P. (2006) Effect of remote forcings on the winter precipitation of central southwest Asia part 1: observations. Theor. Appl. Climatol., 86 (1-4), pp. 147-160, 10.1007/s00704-0050217-1.

Tahir AA, Chevallier P, Arnaud Y, Ahmad B. 2011. Snow cover dynamics and hydro- logical regime of the Hunza River basin, Karakoram Range, Northern Pakistan. Hydrol. Earth Syst. Sci. 15 (7), 2275-2290.

Tayyen, R.J. \& Gergan, J.T., 2010. Role of glaciers in watershed hydrology: a preliminary study of a "Himalayan catchment'. Cryosphere 4, 115-128.

Tian, Y.D., Peters-Lidard, C.D., Eylander, J.B., Joyce, R.J., Huffman, G.J., Adler, R.F., Hsu, K.-L., Turk, F.J., Garcia, M. and Zeng, J. (2009) Component analysis of errors in satellite-based precipitation estimates. Journal of Geophysical Research-Atmospheres, 114, D24101.

Timmermans, B., Wehner, M., Cooley, D., O'Brien, T. and Krishnan, H. (2019) An evaluation of the consistency of extremes in gridded precipitation data sets. Clim Dyn. https://doi.org/10.1007/s00382-018-4537-0.

Treydte, K.S., Schleser, G.H., Helle, G., Frank, D.C., Winiger, M., Haug, G.H. and Esper, J. (2006) The twentieth century was the wettest period in northern Pakistan over the past millennium. $\begin{array}{lllll}\text { Nature, } & 440 & \text { (7088), } & \text { 1179-1182. }\end{array}$ (http://www.nature.com/nature/journal/v440/n7088/suppinfo/nature04743_S1.html).

Trzaska, S. and Schnarr, E., 2014. A Review of Downscaling Methods for Climate Change Projections, a report prepared by Tetra Tech ARD for the United States Agency for International Development

Tuinenburg, O., Hutjes, R.W.A. and Kabat, P. 2012. The fate of evaporated water from the Ganges basin. Journal of Geophysical Research, 117, 1-17. https://doi.org/10.1029/2011JD016221.

Turc, L. 1954. Le bilan d'eau des sols: relation entre les précipitations, l'évapotranspiration et l'écoulement. Annales agronomiques, Série A, 5, 491-595, 6, 5-131.

Turner AG, Annamalai H (2012) Climate change and the south Asian summer monsoon. Nature Clim Change 2:1-9. doi:10.1038/NCLIMATE1495.

Ullah, W., Wang, G., Ali, G., Hagan D.F.T. Bhatti, A.S. and Lou, D. (2019) Comparing Multiple Precipitation Products against In-Situ Observations over Different Climate Regions of Pakistan. Remote Sens. 2019, 11, 628; doi:10.3390/rs11060628.

Ushio, T., Kubota, T., Shige, S., Okamoto, K., Aonashi, K., Inoue, T., Takahashi, N., Iguchi, T., Kachi, M., Oki, R., Morimoto, T. and Kawasaki, Z. (2009) A Kalman filter approach to the Global Satellite Mapping of Precipitation (GSMaP) from combined passive microwave and infrared radiometric data. Journal of the Meteorological Society of Japan, 87A, 137-151.

Valéry, A., Andréassian, V. and Perrin, C. (2010) Regionalization of precipitation and air temperature over high-altitude catchments - learning from outliers. Hydrol. Sci. J., 55, 928-940, doi:10.1080/02626667.2010.504676.

Vila, D., Ferraro, R. and Semunegus, H. (2010) Improved global rainfall retrieval using the Special Sensor Microwave Imager (SSM/I). J. Appl. Meteor. Climatol., 49, 1032-1043.

Voisin N, Wood A W, Lettenmaier DP. 2008. Evaluation of precipitation products for global hydrological prediction, J. Hydrometeorol.,9, 388-407. 
Wagnon P, Linda A, Arnaud Y, Kumar R, Sharma P, Vincent C, Pottakkal JG, Berthier E, Ramanathan A, Hasnain SI, Chevallier P. 2007. Four years of mass balance on Chhota Shigri Glacier, Himachal Pradesh, India, a new benchmark glacier in the Western Himalaya.” Journal of Glaciology 53 (183): 603-11.

Wake CP. 1989. Glaciochemical investigations as a tool for determining the spatial and seasonal variation of snow accumulation in the Central Karakoram, northern Pakistan. Ann. Glaciol. 13, 279-284.

Wang B, Bao Q, Hoskins B, Wu G, Liu Y. 2008. Tibetan Plateau warming and precipitation changes in East Asia. Geophys Res Lett 35:L14702.

Wang, B. and Lin, H. 2002. Rainy season of the Asian-Pacific summer monsoon. J Climate 15:386396.

WAPDA. 2003. Annual report of river and climatological data of Pakistan - 2001, Vol. II, Surface Water Hydrology Project, Hydrology and Water Management Organization, WAPDA, Lahore, Pakistan. 2003.

WAPDA. 2012. Annual report of river discharge, sediment and water quality data for the year 2010, Vol. 1, SWHP Publication No. 71.

Weedon, G.P., Balsamo, G., Bellouin, N., Gomes, S., Best, M.J. and Vidale, P.-L. (2014) The WFDEI meteorological forcing dataset: WATCH forcing data methodology applied to ERAInterim reanalysis data. Water Resour. Res., 50, 7505-7514, doi:10.1002/2014WR015638.

Wei J, Dirmeyer PA, Wisser D, Bosilovich MG, Mocko DM. 2013. Where Does the Irrigation Water Go? An Estimate of the Contribution of Irrigation to Precipitation Using MERRA, J. Hydrometeorol., 14, 275-289, doi:10.1175/JHM-D-12-079.1.

Wijngaard, R.R.; Biemans, H.; Lutz, A.F. Shrestha, A.B., Wester, P., Immerzeel, W.W. 2018. Climate change vs. socio-economic development: Understanding the future South Asian water gap. Hydrol. Earth Syst. Sci. 22, 6297-6321.

Willmott, C. J., Robeson, S. M., and Matsuura, K. (2017) Climate and other models may be more accurate than reported, Eos, 98, https://doi.org/10.1029/2017EO074939.

Winiger M, Gumpert M, Yamout H. 2005. Karakorum-Hindukush-Western Himalaya: assessing high-altitude water resources. Hydrol. Process. 19 (12), 2329-2338http:// dx.doi.org/10.1002/hyp.5887.

WMO (2010) Fact-Finding and Need-Assessment Mission to Pakistan, Mission Report 4-8 November 2010, https://www.wmo.int/pages/prog/dra/rap/documents/PakistanMissionReport.pdf

WMO, 2014. Guide to Meteorological Instruments and Methods of Observation, World Meteorological Organization, WMO-No.8.

Wolff MA, Isaksen K, Petersen-Øverleir A, Ødemark K Reitan T. 2015. Derivation of a new continuous adjustment function for correcting wind-induced loss of solid precipitation: Results of a Norwegian field study, Hydrology and Earth System Sciences 19 (2): 951-967.

Wu, G. and Zhang, Y. (1998) Tibetan Plateau Forcing and the Timing of the Monsoon Onset over South Asia and the South China Sea. Monthly Weather Review 126, 913-927, doi:10.1175/15200493(1998)126<0913:TPFATT>2.0.CO;2 (1998).

Xie, P. and Arkin, P.A. (1997) Global precipitation: a 17-year monthly analysis based on gauge observations, satellite estimates, and numerical model outputs. Bulletin of the American Meteorological Society 78(11): 2539-2558.

Xie, P., Janowiak, J.E., Arkin, P.A., Adler, R.F., Gruber, A., Ferraro, R., Huffman, G.J. and Curtis, S. 2003. GPCP Pentad precipitation analyses: An experimental dataset based on gauge observations and satellite estimates. J. Clim., 16, 2197-2214, doi:10.1175/2769.1.

Xie, P., Joyce, R., Wu, S., Yoo, S.-H., Yarosh, Y., Sun, F. and Lin, R. 2017. Reprocessed, biascorrected CMORPH global high-resolution precipitation estimates from 1998. Journal of Hydrometeorology, 18, 1617-1641. 
Yang D, Goodison BE, Ishida S. 1998. Adjustment of daily precipitation data at 10 climate stations in Alaska: Application of World Meteorological Organization intercomparison results, Water Resources Research, 34(2):241-256.

Yang D, Shi Y, Kang E, Zhang Y, Yang X. 1991. Results of solid precipitation measurement intercomparison in the Alpine area of Urumqi River basin, Chinese Sci. Bull., 36, 1105-1109.

Yang D. 1988. Research on analysis and correction of systematic errors in precipitation measurement in Urumqi River basin, Tianshan, PhD thesis, Lanzhou Institute of Glaciology and Geocryology, Chinese Academy of Sciences, Lanzhou, China, 169 pp.

Yao, T., Thompson, L., Yang, W., Yu, W., Gao, Y., Guo, X., Yang, X., Duan, K., Zhao, H., Xu, B., $\mathrm{Pu}$, J., Lu, A., Xiang, Y., Kattel, D.B. and Joswiak, D. (2012) Different glacier status with atmospheric circulations in Tibetan Plateau and surroundings. Nat. Clim. Chang., 2(9), 663-667, doi:10.1038/nclimate1580.

Yatagai, A., Kamiguchi, K., Arakawa, O., Hamada, A., Yasutomi, N. and Kitoh, A. 2012. APHRODITE: Constructing a long-term daily gridded precipitation dataset for Asia based on a dense network of rain gauges, Bulletin of the American Meteorological Society 93(9):1401-1415. doi:10.1175/BAMS-D-11-00122.1.

Yatagai, A., N. Yasutomi, N., Maeda, M., Masuda, M., Khadgarai, S. 2018. Impact of nomixed endof-the-day and station value conservation to represent extremes precipitation over East Asia, (manuscript under preparation http://aphrodite.st.hirosakiu.ac.jp/product_readme/V1801R1_readme.pdf)

Ye B, Yang D, Ding Y, Han T, Koike T. 2004. A bias-corrected precipitation climatology for China. Journal of Hydrometeorology 5 (6), 1147-1160, doi:10.1175/JHM-366.1.

Yin, H., Donat, M.G., Alexander, L.V. and Sun, Y. (2014) Multi-dataset comparison of gridded observed temperature and precipitation extremes over China. International Journal of Climatology, 35(10): 2809-2827, doi: 10.1002/joc.4174.

Yin, S., Xie, Y., Liu, B. and Nearing, M.A. (2015) Rainfall erosivity estimation based on rainfall data collected over a range of temporal resolutions. Hydrol. Earth Syst. Sci., 19, 4113-4126, https://doi.org/10.5194/hess-19-4113-2015.

Yu W, Yang Y-C, Savitsky A, Alford D, Brown C, Wescot J, Debowicz D, Robinson S. 2013. The Indus Basin of Pakistan: The Impacts of Climate Risks on Water and Agriculture. The World Bank, Washington, D.C.

Zaidi, T.H. (2014) The 2010 Pakistan Floods: Environmental and Economic Impact, Pakistan Studies Programme, Academy of International Studies, Jamia Millia Islamia, New Delhi.

Zhang Y, Ohata T, Yang D, Davaa G. 2004. Bias correction of daily precipitation measurements for Mongolia, Hydrol. Process., 18, 2991-3005, doi:10.1002/hyp.5745.

Zhang, K., Kimball, J.S., Nemani, R.R., Running, S.W., Hong, Y., Gourley, J.J. and Yu, Z. (2015) Vegetation greening and climate change promote multidecadal rises of global land evapotranspiration. Nat Scientif Rep. 2015;5:15956. doi: 10.1038/srep15956.

Zhu, X., W. Wang, and K. Fraedrich (2013), Future climate in the Tibetan Plateau from a statistical regional climate model, J. Clim., 26(24), 10,125-10,138, doi:10.1175/JCLI-D-13-00187.1.

Zorita, E. \& von Storch, H., 1999. The analog method as a simple statistical downscaling technique: comparison with more complicated methods. Journal of Climate 12(8), 2474-2489. 


\section{Summary}

The high-altitude Indus basin is one of the most complex and underexplored mountain regions in the World. Scarcity and directional biases of the observed precipitation coupled with measurement errors, high orographic influences and effects of multiple weather systems have prevented a comprehensive and reliable assessment of precipitation distribution in this region. Quantitative and spatiotemporal distributions of precipitation estimated by the previous studies and global/regional scale gridded datasets for this region are highly contrasting and extremely uncertain. Consequently, the studies using these estimates often lead to suboptimal and misleading outcomes regarding hydrometeorological assessments.

This PhD research study integrated precipitation observations from multiple sources with the indirect estimates at the accumulation zones of major glaciers, adjusted these observations for measurement errors and derived spatially distributed fields of mean monthly precipitation using the standard and well-recognized techniques. The resultant reference estimates of precipitation distribution are cross-validated by the corresponding observed river inflows, which were adjusted for contribution of net mass balance. The reference climatologies of mean monthly maximum and minimum temperature are derived through elevation and latitude dependent lapse rates at sub-regional scale. Performance of 27 widely used gridded precipitation products is evaluated against the observational based reference dataset at sub-regional scale. The best performing gridded dataset is statistically downscaled and bias-corrected with respect to the reference datasets to develop long-term historical dataset of precipitation and temperature. Similarly, precipitation estimates of 75 CMIP5 GCM outputs for the historical period are evaluated against the bias-corrected historical dataset. Top 24 ranked GCM outputs are further analysed based on the changes in their climatic means between 1971-2000 and 2071-2100. Four corners of warm-dry, warm-wet, cold-dry and cold-wet spectrum are determined from the range of projected changes in mean annual air temperature and annual precipitation using the $10^{\text {th }}$ and $90^{\text {th }}$ percentile values. Precipitation and temperature data of two GCMs representing warm-wet and cold-dry extremes under three RCPs $(2.6,4.5 \& 8.5)$ are statistically downscaled and bias-corrected against the historical datasets. A fully-distributed physically-based energybalance hydrological model is forced with these datasets at daily timestep to model the subbasin scale hydrologic regime for the historical as well as six scenarios of future climates.

The altitudinal analysis of precipitation distribution in the study domain demonstrated strong orographic influence. However, the available observations are insufficient to infer an accurate relationship of precipitation with altitude. Rather nonlinear trends of precipitation increase with altitude are evident. Generally, precipitation tends to decrease with increasing latitude (from south to north), while longitude has seasonal influence, positive in monsoon and negative in winter season. Monthly distribution of precipitation largely indicates a bimodal weather system, reflecting influence of winter westerlies and summer monsoon. In contrast to previous studies, the results of this study reveal substantially higher precipitation in most of the sub-basins indicating two distinct rainfall maxima; $1^{\text {st }}$ in the western Himalaya along southern and lower most slopes of Chenab, Jhelum, Indus main and Swat basins, and $2^{\text {nd }}$ around north-west corner of Shyok basin in the central Karakoram. Adjustments of measurement errors in precipitation observation and net snow accumulations indicated significant improvements in the quantitative and spatio-temporal distribution of precipitation over the unadjusted case, while adjustment of river flows 
revealed only a marginal contribution of net glacier mass balance to river flows. The study recognized that the higher river flows than the corresponding precipitation estimates by the previous studies are mainly due to underestimated precipitation. The study demonstrated that the gridded precipitation products are prone to significant errors and their direct (uncorrected) use in climate change and hydrological studies will imply erroneous inferences. Gauge-based and merged products performed relatively better in dry regions and during monsoon season, while reanalyses products provided better estimates in wet areas, at higher-altitudes and during winter months. Overall, ERA5 precipitation product provided relatively better and equally acceptable estimates for all sub-regions. Precipitation projections by the GCMs are more uncertain and generally fail to efficiently reflect the bimodal weather system prevailing in the study area. Nevertheless, MIROC5 and MPIESM-LR provided better skill scores to reproduce the past climate of the study area.

In the presence of significant interannual variability, the mean annual air temperature has increased by $0.6{ }^{\circ} \mathrm{C}$ during the last 40 years and is projected to increase further by 1.3 $2.6{ }^{\circ} \mathrm{C}$ during mid-century and between $0.8-5.7^{\circ} \mathrm{C}$ by the end-century. Compared to temperature, precipitation remains much more variable and uncertain in both space and time. Mean annual precipitation experienced considerable decline during the famous and prolonged drought of recent time (1999-2003). It tried to recover thereafter but still fell short by $11.9 \%$ of the mean annual precipitation for the $1^{\text {st }}$ half of the baseline period (1981-2020). Compared to the baseline historical period, the interannual variability of the projected precipitation by all GCM runs is even more pronounced throughout the $21^{\text {st }}$ century. Definite and strong increasing trend in mean annual precipitation is only evident for MIROC5_RCP8.5, while MPI-ESM-LR shows declining trends for RCP4.5 \& RCP8.5. The remainder of GCM runs show mixed trends. River flows are largely modulated by timing, intensity, duration, and form of precipitation; snow and glacial ice reserves; and amount of energy available for melting of seasonal and perennial snow and glacial ice. The basin-scale projections of water availability show an overall increase of river inflows but exhibit significant spatiotemporal variability. MIROC5 outputs generally depict positive changes, while MPI-ESM-LR outputs result in negative changes. Late spring and premonsoon (Apr-Jun) river inflows will be significantly increased, while considerable reduction in late summer flows is very likely due to early melting of seasonal snow cover. Similarly, slight increments are also projected for wintertime baseflows due to increasing temperature. Hydrologic extremes of floods and drought are projected to be more intensive and frequent under all scenarios.

Although, there are still significant uncertainties, this $\mathrm{PhD}$ research attempted to minimize the underlying uncertainties and added to the current knowledge and understanding of hydrometeorology of the high-altitude Indus basin. The results will serve as the basis for design and operation of hydropower plants and hydraulic structures and provide guidelines for planning and management of water resources. 


\section{Acknowledgements}

All praise and thanksgiving to ALMIGHTY ALLAH who bestowed me all the capabilities and passion to achieve this milestone.

I am highly indebted to extend my sincerest thanks to the Dutch Ministry of Foreign Affairs for sponsoring my $\mathrm{PhD}$ research through the Netherlands Fellowship Programmes (NFP) under the budget for development cooperation. I am also thankful to my employing organization PARC for allowing me to avail this opportunity. Due acknowledgement is extended to all the data collecting agencies and the persons involved therein, whose data has been used in this $\mathrm{PhD}$ dissertation.

I have tremendous appreciation and gratitude to my Promotors and research advisors Professor Dr. Pavel Kabat, Professor Dr. Fulco Ludwig and Professor Dr. Eddy Moors for accepting me as their PhD student. Dear Professors, I owe everything that I achieved during my $\mathrm{PhD}$ course. I really enjoyed your invaluable guidance, constructive ideas, positive criticism and constant encouragement during the entire course of my $\mathrm{PhD}$ research. Especial thanks are due to Eddy and Fulco who not only provided day-to-day supervision but also facilitated me in all aspects during my stay and journeys to and from Wageningen. As a sandwich PhD student, I have to go back and work from Pakistan. While resuming my work at PARC, I could not work on my $\mathrm{PhD}$ research at the same pace when I was in Wageningen. This resulted in unwanted delay in my $\mathrm{PhD}$ completion. Here, I must appreciate and thank my promotors for their positive role and confidence on me. Although, I have been wondering whether I tested their patience! Working with you have been a memorable time of my life. I am particularly thankful for ignoring my unscheduled meetings, when I knocked your door and asked for quick guidance. I did this to Eddy during early phase of my $\mathrm{PhD}$ when Eddy was in Wageningen and to Fulco during the last stage. Fulco, I learned so many things from you; your deep understanding of climate and hydrological sciences, your dedication and flexible schedule for student supervision, and your frankness and great sense of humour. Eddy, I found in you a learned scientist, a successful project planner and manger, a kind and cooperative supervisor, and above all a great human being. Working with you during planning, design and formulation of HIAWARE project was also a wonderful experience. Pavel, I must thank you for overall direction and providing useful opportunity to participate in IIASA's Young Scientists Summer Program 2016. I feel greatly honoured to be part of that three months learning program, where I accomplished part of my $\mathrm{PhD}$ research. I also wish to thank the Netherlands Organisation for Scientific Research for their sponsorship and the scientific team of IIASA's Water Program, particularly Peter Burik who extended his supervision during this short training.

I would also like to express my deepest gratitude to Mr. Wietse Franssen for his constant guidance and support on R and VIC related matters. Wietse, without your help I would have required 1-2 more years to complete my $\mathrm{PhD}$. Sincere thanks are also extended to the secretaries of the chair group for their guidance on management related matters. 
Especial thanks are due to my friends and colleagues at the Chair Group; Beatriz, Christian, Dan, Debora, Emma, Evans, Geoffrey, Hester, Long, Marline, Michelle, Somayeh, Tanya, Wilma, Zamira. Many of these great people have found opportunities elsewhere. I do remember your nice company and offer my best wishes to you all.

I am highly indebted to appreciate and thank the great company and memorable times spent with my Pakistani friends in Wageningen and elsewhere in the Netherlands. Worth mentioning are Abbas Shah, Bilal Mirza, G Ali, Hamid Shah, Hasnain Shah, Harris, Imran, Imtiaz, Masood, Mazhar, Munawar, Mustafa Shah, Shafqat, Shahid, and Zeeshan. I can't forget those weekend evenings when we gather for cricket followed by dinners containing delicious Pakistani dishes arranged sequentially by one or more from us and playing cards for the whole night. In fact, that was our only means of entertainment and relaxation during these tense days.

Finally, I owe my deepest gratitude to my family, brothers, sisters and other close relatives for their constant support and encouragements. The courageous role of my wife in nurturing and upbringing a couple kids (Zeeshan and Ehsan) in my absence is unforgettable. She has been splendidly brave, always ready for sacrifice and proved to be a devoted life partner. 


\section{Curriculum Vitae}

Zakir Hussain Dahri opened his eyes in June 1971 in a farmer's house in Naushahro Feroze, Pakistan. He grew up in a typical rural society and learned the basic art and science of agricultural farming. This innate incitement motivated him to study agricultural engineering at Sindh Agricultural University Tando Jam. After graduation in January 1996, he started his professional career as a research assistant in the country's premier water resources research organization (PCRWR). Later, he joined Pakistan Agricultural Research Council (PARC) in 2001 as a professional researcher in the field of agricultural water management. During services in PCRWR and PARC, he got his post-graduation in 2002 from University of Engineering and Technology, Lahore in the field of water resources management. His passion for higher studies and urge for modern techniques brought him at the University of Melbourne, Australia to undertake another post-graduation in GIS \& RS during Jul 2006-Jun 2008. He started $\mathrm{PhD}$ research at the Water Systems and Global Change group of Wageningen University, The Netherlands as a sandwich student. He also continued working at PARC and was instrumental in planning, design, formulation and execution of IDRC-DFID funded HI-AWARE project during 2013-2018. Zakir has solid background in hydrology and hydrological modeling, climate-water-society interactions, water policy and governance, improving water productivity under different agro-ecological conditions, remote sensing and geographic information science. 


\section{Peer-Reviewed Publications}

1. Dahri, Z.H., F. Ludwig, E. Moors, S. Ahmad, B. Ahmad, S. Ahmad, M. Riaz and P. Kabat (2020), Hydrological Regime of the High-Altitude Indus Basin under Extreme Climate Scenarios", Paper under review for publication in the Science of Total Environment (STOTEN-D-20-16914).

2. Dahri, Z.H., F. Ludwig, E. Moors, A. Khan, I. Ali, S. Ahmad and P. Kabat (2020), "Spatio-temporal Evaluation of Gridded Precipitation Products for the HighAltitude Indus Basin” Paper accepted for publication in Int J Climatology 2019, Royal Meteorological Society (JOC-19-0591).

3. Ahmad, B., H. D. Pham, M. Ashfaq, J.A. Memon, R. Bano, Z. H. Dahri, R. N. Mustafa, I. A. Baig and M. A. R. Naseer (2020), "Impact of Institutional Features on the Overall Performance Assessment of Participatory Irrigation Management: Farmers' Response from Pakistan” Water 2020, 12, 497; doi:10.3390/w12020497.

4. Dahri, Z.H., E. Moors, F. Ludwig, A. Khan, I. Ali, S. Ahmad and P. Kabat (2018), "Adjustment of Measurement Errors to Reconcile Precipitation Distribution in the High-Altitude Indus Basin" Int J Climatology 2018;1-19, Royal Meteorological Society, https://doi.org/10.1002/joc.5539.

5. Shoaib, M., Shamseldin, A.Y., Khan, S., Sultan, M., Ahmad, F., Dahri, Z.H., and Ali, I. (2018), "Input Selection of Wavelet-Coupled Neural Network Models for Rainfall-Runoff Modelling" Water Resources Management: 2018, pp 1-19. https://doi.org/10.1007/s11269-018-2151-x. An International Journal - Published by SpringerLink for the European Water Resources Association (EWRA).

6. Iqbal, M.S., Z.H. Dahri, Q. Erik, A. Khan and H. Nynke (2018), "Impact of Climate Change on Flood Frequency and Intensity in the Kabul River Basin", Geosciences 2018, 8 (4), 114; https://doi.org/10.3390/geosciences8040114.

7. Dahri, Z.H., F. Ludwig, E. Moors, B. Ahmad, A. Khan and P. Kabat (2016), “An Appraisal of Precipitation Distribution in the High-Altitude Catchments of Indus Basin", Science of the Total Environment, Vol. 548-549, Pages 289-306. https://doi.org/10.1016/j.scitotenv.2016.01.001.

8. Siyal, A.W., M. Yasin, I. Ali, Z.H. Dahri, M. Aslam and M. Khan (2015), "Performance Evaluation of Fixed dome and Floating type Biogas Digesters for Tubewell Operation with Dual Fuel Approach in Pakistan”, International Journal of Innovation and Applied Studies, Vol. 10 No. 4 Mar. 2015, pp. 1225-1232. http://www.ijias.issr-journals.org/abstract.php?article=IJIAS-14-268-03. 


\section{SENSE}

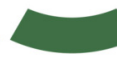

Netherlands Research School for the

Socio-Economic and Natural Sciences of the Environment

\section{I P L O M A}

for specialised PhD training

The Netherlands research school for the Socio-Economic and Natural Sciences of the Environment (SENSE) declares that

\section{Zakir Hussain Dahri}

born on 1 October 1971 in Naushahro Feroze, Pakistan

has successfully fulfilled all requirements of the educational PhD programme of SENSE.

Wageningen, 13 October 2020
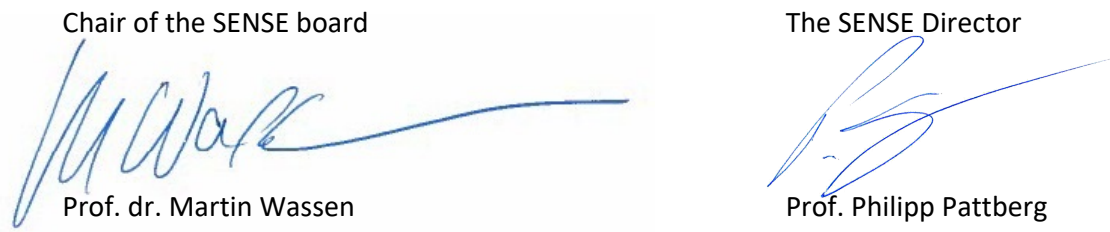

The SENSE Research School has been accredited by the Royal Netherlands Academy of Arts and Sciences (KNAW)

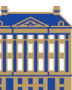

$\begin{array}{llllllllllllllllllllll}K & O & N & I & N & K & L & I & J & K & E & N & E & D & E & R & L & A & N & D & S & E\end{array}$

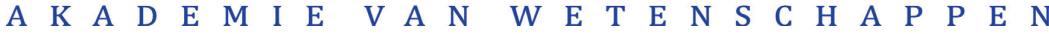




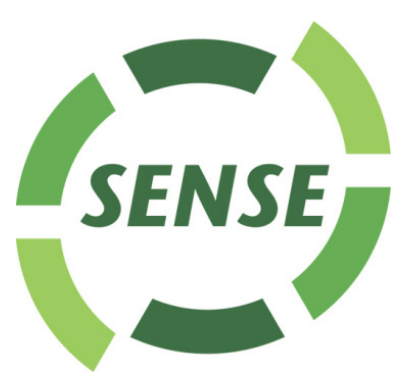

The SENSE Research School declares that Zakir Hussain Dahri has successfully fulfilled all requirements of the educational PhD programme of SENSE with a work load of $42.7 \mathrm{EC}$, including the following activities:

\section{$\underline{\text { SENSE PhD Courses }}$}

- Environmental Research in Context (2012)

- Introduction to R for Statistical Analysis (2012)

- Dealing with Uncertainties in Research for Climate Adaptation (2012)

- Basic Statistics (2014)

- Research in Context Activity: 'Co-organizing the $4{ }^{\text {th }}$ Climate Change Adaptation Policy and Science Conference, Islamabad, Pakistan' (26-27 February 2018)

\section{Other PhD and Advanced MSc Courses}

- Hydrology, Climate Change and Fluvial Systems, Utrecht University (2012)

- Water Governance: Concepts and Practices, Wageningen University (2016)

\section{External Training at a Foreign Research Institute}

o Young Scientists Summer Program (YSSP) 2016, International Institute for Applied System Analysis (2016)

- Water Crisis Management and Conservation, Executive Development Institute (EDI), National School of Public Policy (NSPP), Lahore (2019)

\section{Oral Presentations}

- Time to Adapt-Adaptation Pathways in the Hindu Kush Himalaya, Climate Change Adaptation and Policy Science Conference: Lessons, Achievements and Way Forward, 2 October 2018, Islamabad, Pakistan

- Surface Storage and Groundwater Recharge in Pakistan, National Conference on Water Security: Issues/Way Forward on Aggression and Conservation, 24 October 2018, Islamabad, Pakistan

SENSE coordinator PhD education

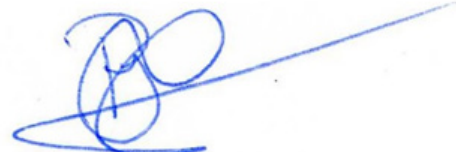

Dr. ir. Peter Vermeulen 
This $\mathrm{PhD}$ research is financially supported primarily by the Dutch Ministry of Foreign Affairs through the Netherlands Fellowship Programme (NFP) under the budget for development cooperation and partially by the Netherlands Organisation for Scientific Research. A part of the research is carried out under the Himalayan Adaptation, Water and Resilience (HI-AWARE) consortium.

The front title cover of this thesis focuses the Indus basin superimposed on google earth image, while the back title cover image is the property of PARC under the HI-AWARE Project.

Cover design: Zakir Hussain Dahri

Printed by: $\quad$ proefschriftmaken (https://www.proefschriftmaken.nl/en/) 
